\title{
Amchitka Island Environmental Anaysis at Idaho National Laboratory
}

G. Elias

W. F. Bauer

J. G. Eisenmenger

C. C. Jensen

B. K. Schuetz

T. C. Sorensen

B. M. White

A. L. Freeman

M. E. Mc Ilwain

June 2005

The INL is a U.S. Department of Energy National Laboratory operated by Battelle Energy Alliance 


\title{
Amchitka Island Environmental Analysis at Idaho National Laboratory
}

\author{
G. Elias, W. F. Bauer, J. G. Eisenmenger, \\ C. C. Jensen, B. K. Schuetz, \\ T. C. Sorensen, B. M. White, A. L. Freeman, \\ M. E. Mc Ilwain
}

June 2005

Idaho National Laboratory

Analytical Chemistry \& Instrumentation Department Idaho Falls, Idaho 83415 


\section{Amchitka Island Environmental Analysis at Idaho National Laboratory}

\section{EXECUTIVE SUMMARY}

The Idaho National Laboratory (INL) provided support to Consortium for Risk Evaluation with Stakeholder Participation (CRESP) in their activities which is supported by the Department of Energy (DOE) to assess the impact of past nuclear testing at Amchitka Island on the ecosystem of the island and surrounding ocean. INL participated in this project in three phases, Phase 1, Phase 2 and Phase 3.

The roles of the INL in phase $1 \mathrm{~A}$ were to provide a method manual which explains the methods and measurement procedures to analyze alpha, beta, and gamma emitting radioisotopes in the environmental samples collected at Amchitka Island, a quality assurance plan that details how samples will be handled, data reporting requirements, calibrations, blank analysis, and setup of a performance demonstration program using blind samples.

In Phase I B, a common set of measurement methods and quality assurance plan were identified. In order to validate the methods identified with the identified sample matrices, INL did some method development work. Under this task, INL purchased samples similar to Amchitka samples from the local stores. These samples included fish (like salmon and halibut), crab, lobsters etc. The INL also purchased some kelp to test the methods. Samples were divided into three categories. (a) Flesh or tissue samples, (b) bone, and (c) kelp. All samples were ground to smaller pieces to make them homogeneous. The analytical scheme for each type of samples is shown in the following flowchart.

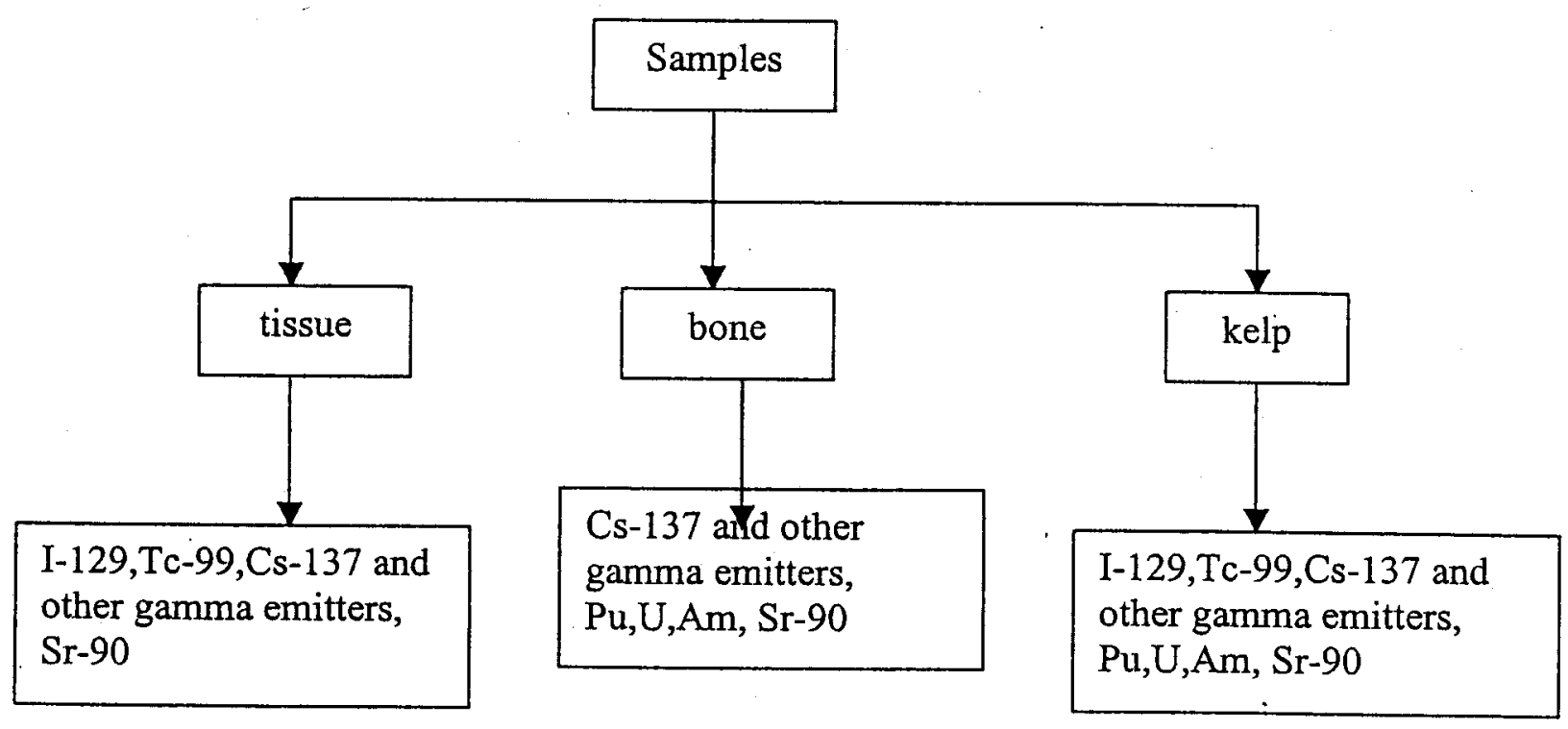


Sample size needed for each analysis to meet the customer detection limit with accuracy and precision in the analysis were tested in Phase 1. A trade off between detection limit, accuracy and precision was needed in terms of availability of the samples from the customer for some analysis. Gamma and ${ }^{129} \mathrm{I}$ systems were calibrated with $100 \mathrm{~g}$ samples of each type with specific geometry. Test results in the method development phase, phase 1, were verified to choose the appropriate amounts of samples for radiochemistry (actinides- $\mathrm{Pu}, \mathrm{U}, \mathrm{Am}$, and ${ }^{90} \mathrm{Sr}$ ) and ${ }^{99} \mathrm{Tc}$. As a result, $100 \mathrm{~g}$ tissue samples for ${ }^{90} \mathrm{Sr}, 15 \mathrm{~g}$ tissue and kelp samples for ${ }^{99} \mathrm{Tc}, 12 \mathrm{~g}$ kelp samples for actinides and ${ }^{90} \mathrm{Sr}$, and $2 \mathrm{~g}$ bone samples for actinides and ${ }^{90} \mathrm{Sr}$ analyses were identified. Gamma spectroscopy was used to measure ${ }^{137} \mathrm{Cs}$, ${ }^{152} \mathrm{Eu}$, and ${ }^{60} \mathrm{Co}$ or any additional isotopes detected. Low Energy Photon Spectrometer (LEPS) was used for $\mathrm{I}^{129}$ analysis. Samples underwent sample pretreatment, digestions and separations for actinides (Plutonium $(\mathrm{Pu})$, Uranium $(\mathrm{U})$, and Americium isotopes), Strontium-90 $\left({ }^{90} \mathrm{Sr}\right)$, and Technetium-99 $\left({ }^{99} \mathrm{Tc}\right)$, and alpha spectrometry, gas flow proportional counter, and Inductively Coupled Plasma Mass Spectrometry (ICP-MS) measured the respective activities of the separated analytes. The Radiological and Environmental Sciences Laboratory (RESL) prepared simulated samples for the project. The developed and tested methods were applied to blind quality control samples from RESL for external quality control or performance audits purposes.

In Phase 2, the screening tests, six batches of samples were shipped from Rutgers University. INL analyzed samples shipped from Rutgers collected for the Amchitka project and QC samples were included in the sample batches. These samples comprised tissues and bones of different species of fish; tissues of other types of marine biota, tissues and bones of a few species of birds, and different species of kelps, analogous to the species identified in the Amchitka Science Plan ${ }^{1}$. INL used the validated methods modified and developed for this project in Phase I B, and analyzed the samples for the requested analyses and reported the results to the customer. INL also provided the completed quality assurance plan', PLN-1719, Quality Assurance Project Plan for the Analysis of Amchitka Island Samples, a supplement to PLN $-153^{3}$, Quality Assurance Project Plan for Analytical Laboratories Department Radioanalytical Activities.

In Phase 3, the customer sent three batches of samples including kelp and bone for actinide analyses using alpha spectrometry.

The details of sample results, $\mathrm{QC}$ results, and other related quality assurance documents are included in appropriate sections. 


\section{CONTENTS}

EXECUTIVE SUMMARY .........................................................................

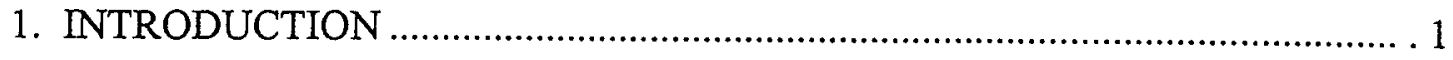

2. EXPERIMENTAL ..............................................................................

3. RESULTS AND DISCUSSION ......................................................... 1

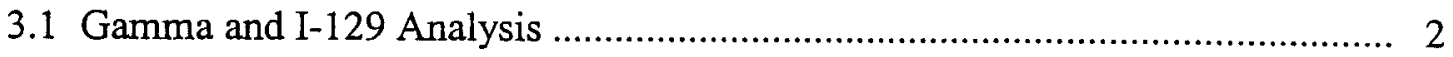

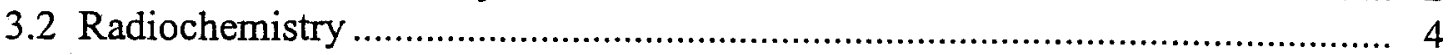

3.3 Technetium-99 (Tc-99) Analysis ........................................................ 5

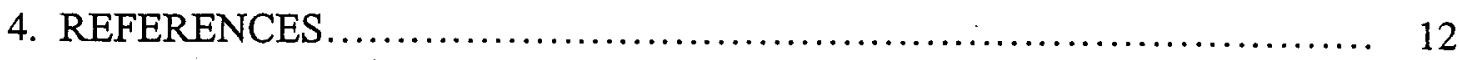

TABLE

Table 3-1. Mean Recovery of Tc-99..................................... 7 


\section{Amchitka Island Environmental Analysis at Idaho National Laboratory}

\section{INTRODUCTION}

The Idaho National Laboratory (INL) operated by Battelle Energy Alliance (BEA) was tasked by Consortium for Risk Evaluation with Stakeholder Participation (CRESP) via CRESP/Department of Energy Idaho Operations Office (DOE-ID) interagency agreements to perform several radioisotope measurements to assess the impact of past nuclear testing at Amchitka Island on the ecosystem of the island and surrounding ocean.

At INL, the Science and Technology organization has the gamma, alpha, and Inductively Coupled Plasma Mass Spectrometry (ICP-MS) capabilities available to measure the activity of these radioisotopes. The target list for $\mathbb{I N L}$ consists of ${ }^{60} \mathrm{Co},{ }^{137} \mathrm{Cs},{ }^{152} \mathrm{Eu}$, or any other gamma emitting nuclides detected with gamma spectroscopy, I-129, Sr-90, Am-241, Pu-238, Pu239/240, U-234, U235, U236 and U238, and Tc-99. Gamma spectroscopy analyzes ${ }^{60} \mathrm{Co},{ }^{137} \mathrm{Cs},{ }^{152} \mathrm{Eu}$, or any other gamma emitting nuclides, low energy photon measurements with LEPS measures I-129, gas flow proportional counter measures the beta activity in Sr-90, alpha spectroscopy is used for counting actinides and ICP-MS is used for measuring Tc-99 activity.

INL provided a method manual ${ }^{4}$, which consists of procedures modified and developed for this project, and a quality assurance plan ${ }^{2}$ to CRESP. The project was done in three phases, Phase 1, Phase 2, and Phase 3. In Phase 1, some new methods were developed, some existing methods were modified, and these methods were evaluated by simulated samples provided by an independent laboratory- Department of Energy Radiological and Environmental Sciences Laboratory (RESL). In Phase 2, and Phase 3, samples from Amchitka were analyzed for target radioisotopes using the different measurement systems following the procedures described in the manual. The data collected for these radioisotopes in different biological matrices (soft tissue, bone, and kelp) from Amchitka and the internal and external' $Q C$ information are documented in this report.

\section{EXPERIMENTAL}

The procedures and documents utilized for the project are in the method manual ${ }^{4}$. For gamma and I-129 measurements, ACLP- $10.10^{5}$, ACLP- $10.31^{6}$, ACLP- $10.41^{7}$, and ACMM $3606^{8}$ were used. In radiochemistry, the analytes in samples were separated by complete ashing and dissolution of the samples using procedures ACMM- $3815^{9}$, and ACMM-3816 ${ }^{10}$. A new procedure, ACMM-3705 ${ }^{11}$, developed for Tc-99, was used for Tc-99 analysis.

\section{RESULTS AND DISCUSSION}

There are three sections in this report. Details of the analyses, data package for the batches, quality assurance/quality control summary (Internal and External QA/QC 
documentation) are found in each section. The data package for the batches is found in Appendix A (Appendix A-1: Gamma and I-129 analysis, Appendix A-2:

Radiochemistry - Alpha and Strontium-90 analysis, and Appendix A-3: Tc-99 Analysis).

The results of the performance Evaluation (PE) standards and the blind QC samples evaluated for this project are in Appendix B. The internal and external quality control (QC) results are also included in this report. Documented in Appendix C (C-1 for gamma, and C-2 for radiochemistry) are the internal QC checks for the detectors used to count these samples. Results show that all relevant parameters were "in control" during the sample counting time frame. Results from the external QC intercomparison program are documented in Appendix D (D-1 for gamma, and D-2 for radiochemistry).

All QA/QC checks were performed in accordance with the QA plan ${ }^{2-3}$ and the RML QA/QC Manual ${ }^{12}$. A copy of these documents is available from the RML Data Analysis Section upon request.

\subsection{Gamma and I-129 Analysis}

Five batches of samples were analyzed for gamma and three batches of samples were analyzed for I-129 from Phase 2. The standard geometry was defined as $125 \mathrm{ml}$ polyethylene bottle. This geometry was used for both gamma and iodine measurement. A mixed National Institute of Standards and Technologies (NIST) traceable gamma ernitting solution was purchased and prepared with homogeneous wet soft tissue, kelp, and bone. Two samples of each material were prepared, one full and one half full in the $125 \mathrm{ml}$ containers of material and frozen. Efficiency curves were generated of each sample type. A similar procedure was followed for iodine except only soft tissue and kelp samples were prepared using a NIST traceable iodine standard. Efficiency curves were prepared for each sample type and geometry.

Performance Evaluation (PE) samples were obtained from RESL, and counted. The results are shown in Appendix B. Some of the activities of the PE samples were well below the minimum detection limit of the detector. Also, the detection of low energy photons of iodine can be interfered with from photons of the same energy from europium and cesium as well as many other isotopes. This effect results in large uncertainties in the quantitative results of some iodine measurements.

\subsubsection{COUNTING AND ANALYSIS INFORMATION}

Included in this report are recount measurements identified by the addition of a "D" to the recounted sample ID. All samples plus the recount measurements were counted in standardized and calibrated geometry for eight hours. The detector counting systems employed were B4 for I-129 measurements and A4, A6 and D4 for regular gamma-ray measurement counting. A4, A6, and D4 are counting systems, which are designed so the detector axis is orthogonal $\left(90^{\circ}\right)$ with respect to the sample axis. Each sample measurement of the aforementioned counting systems were rotated on a turntable in front of the detector during counting duration to help negate the effects of potential inhomogeneity. 
Sample spectra were analyzed by the PCGAP ${ }^{13}$ computer program PCGAP/BATCH_GNUL for Windows using the ENVLIB ${ }^{7}$ decay data library for radionuclide identification with the required radionuclide list of the ENVLIM $^{14}$ library. The ENVLIM ${ }^{14}$ library is a required gamma-ray energy library used to direct the photopeak fitting to the radionuclides of interest. The interference library utilized is ENVINTLIB ${ }^{14}$, which is used to correct for specific photopeak interferences that may occur. A common radionuclide target list from the Plan ${ }^{2}$ for the Analysis of Amchitka Island Samples was utilized. This list contains 3 gamma-emitting radionuclides that are reported on the computer-generated gamma-ray analysis summary. The minimum detectable activity level (MDL) is listed for all results.

Instrumental background spectra were accumulated on the appropriate detector system. A weighted average of four instrumental background spectra for the detector was used in the analysis. Background ID numbers are listed in the sample information section of the Gamma-Ray Analysis Summary.

Data observed in this report are defensible for the samples as analyzed but do not reflect field-sampling uncertainties, representativeness, or survey completeness. Collaboration with RML personnel in data interpretation for end-use is encouraged.

\subsubsection{SUMMARY OF TARGET RADIONUCLIDE RESULTS}

The data report summary in the batches shows gamma-ray measured activities of 3 radionuclides, ${ }^{60} \mathrm{Co},{ }^{137} \mathrm{Cs},{ }^{152} \mathrm{Eu}$, and low energy photon measurements for I-129.

The activities have been decay-corrected to the sample count time and date. The uncertainties shown on the summary pages are for the statistics associated with counting, backgrounds, and photopeak fitting, and estimated uncertainties in the detector efficiency and the sample geometry. Uncertainties are propagated in quadrature and are expressed as one estimated standard deviation.

The computer flags $(+)$ results on the gamma analysis as true positive when the measured activity is greater than two times the measured standard deviation. The plus sign on the outside of the parenthesis around a result indicates the value as true positive where the measured radioactivity is a least two times the measured standard deviation. Either a plus $(+)$ or a minus $(-)$ value within the parenthesis indicates that the net counts in the spectral analysis were either positive or negative with respect to the photopeak fitting or instrument backgrounds. Flagged results determined by the analyst to be true positive and "real" are listed in Analyst's Results of Manmade Gamma-Emitting Radionuclides. The flagged results considered false positive is listed in Analyst's Results of Rejected Gamma-Emitting Radionuclides. Evaluation of these results was done in accordance with the criteria in RML procedure ACLP $10.31^{6}$.

Naturally occurring radionuclides with expected or normal concentrations are usually not reported on the Gamma-Ray Analysis Summary unless the concentrations are abnormally high. 


\subsubsection{ANALYST'S RESULTS OF MANMADE GAMMA-EMUTTING RADIONUCLIDES}

The results that were flagged $(+)$ by the computer as true positive and satisfied the standard selection criteria of the RML or the analyst are reported on Analyst's Results of Manmade Gamma-Emitting Radionuclides summary. These true-positive and "real" results are reported as follows:

(1) Activity (S): The activity with the statistical uncertainty. This uncertainty includes the statistics associated with counting, backgrounds, and photopeak fitting.

(2) Activity (T): The activity with the total uncertainty. This uncertainty includes the statistical uncertainty, plus the estimated uncertainty in the sample geometry and the detector efficiency (Analyst's Results of Manmade GammaEmitting Radionuclides). These uncertainties have been propagated in quadrature and are expressed as one estimated standard deviation.

\subsubsection{ANALYST'S RESULTS OF REJECTED GAMMA-EMITTING RADIONUCLIDES}

The summary of the "Analyst's Results of Rejected Gamma-Emitting Radionuclides," lists the radionuclides that were determined to be false positive. These are the results flagged $(+)$ by the computer as true-positive but did not satisfy standard selection criteria of the RML or the analyst(s). Rejection criteria codes are assigned to each false-positive radionuclide and a key code description is also listed.

\subsubsection{RML GAMMA SPECTROSCOPY RESULTS AND QAQC DATA}

The gamma and I-129 results (data package) are in Appendix A-1. Included also in this report are the internal and external quality control (QC) results. Documented in Appendix C-1 are the internal QC checks for the detectors used to count these samples. Results show that all relevant parameters were "in control" during the sample counting time frame. Results from the external QC intercomparison program are documented in Appendix D-1.

\subsection{Radiochemistry Alpha and Sr-90 analysis}

During this project, there were six batches of samples for radiochemical analysis from Phase 2 and three batches of samples from Phase $3 \mathrm{~A}$. The samples were analyzed for $\mathrm{Sr}-$ 90, Am-241, Pu-238, Pu239/240, U-234, U235, U236 and U238. Complete ashing and dissolution of the samples were achieved and no unusual steps were taken in performing the alpha analyses. The results in this report are the same results that were reported as preliminary results that were sent to CRESP during the period of January 2005 - June 2005. A list of samples with activities $\geq 2$ sigma, with uncertainty @ one sigma, are also included with each batch's results. 
The actinide procedure for small solid sample sizes in ACMM- $3816^{10}$ is used for alpha analysis. ACMM- $3815^{9}$ and ACMM $3816^{10}$ were used for Strontium-90 analysis. For small biological samples, the strontium sulfate precipitation with $10 \% \mathrm{Li}_{2} \mathrm{SO}_{4}$ with $\mathrm{pH}$ adjustment is recommended twice to reduce calcium interference (Step 7.9.7 to 7.9.8). Batch 4 and Batch 5 had $15 \mathrm{~g}$ bone samples. These samples were considered as large samples. The large amount of calcium and other interference like phosphate in bone samples required several clean-up steps to separate strontium. In batch 4, B-L-150-B, B$\mathrm{L}-151-\mathrm{B}$, both $15 \mathrm{~g}$, and B-M-140-B, which was $2.5 \mathrm{~g}$, , produced relatively low yields of strontium because of the complexity of the samples. Batch 2 had some dense soft tissue samples (S-U-76, S-U-77, S-U-90, and S-U-96). These samples also had some interference, causing difficult strontium separation, and some of them had relatively low chemical yield. These soft tissue samples also caused some damage in the platinum dishes, that were used to process these samples. In batch 7, two kelp samples, K-AA210-A, and K-AA-202-A, combusted in the drying process and part of these samples were lost. These samples also produced low actinide yields. The results for the external QC samples or PE samples from RESL from phase 1 and blind QC sample results from phase 2 and phase 3 are included in Appendix B.

Included in this report are results (data package) and the internal and external quality control (QC) results. Appendix A-2 shows the results of each batch. Documented in Appendix C-2 are the internal QC checks for the detectors used to count these samples. Results show that all relevant parameters were "in control" during the sample counting time frame. Results from the external QC intercomparison program are documented in Appendix D-2.

\subsection{Technetium-99 (Tc-99) Analysis}

A method to determine ${ }^{99} \mathrm{Tc}$ at environmental levels in plant tissues, animal tissues and related samples using inductively coupled plasma mass spectrometry (ICPMS) was developed and implemented for this program (ACMM-3705) ${ }^{11}$. The method is based upon several similar methods found in the literature ${ }^{15-19}$. Briefly, samples $(5-25 \mathrm{~g})$ were weighed into preweighed crucibles. The samples were spiked with $100 \mu \mathrm{L}$ of a 200 $\mathrm{ng} / \mathrm{mL} \mathrm{Re}$ solution used as a recovery standard (i.e. yield tracer). One sample in every batch (20 samples) was also spiked with $100 \mu \mathrm{L}$ of a $377 \mathrm{dpm} / \mathrm{mL}$ solution of ${ }^{99} \mathrm{Tc}$ (i.e. $10 \mathrm{ng} / \mathrm{mL})$. All samples were then treated with $10 \mathrm{~mL}$ of ammonia solution $(20 \%)$ in a fume hood to make the samples basic and stabilize the Tc and Re. The samples were then dried for at least $24 \mathrm{hr}$ in an oven or on a hot plate at $<75^{\circ} \mathrm{C}$. The cooled, dried samples were reweighed to determine the dry weight and placed in a furnace at $\sim 75^{\circ} \mathrm{C}$ and the temperature ramped to $550^{\circ} \mathrm{C}$ and held for $3 \mathrm{hrs}$. The ashed samples were removed from the furnace, cooled and reweighed to determine the ash content. The $\mathrm{Tc}$ and Re in the ashed samples was oxidized and "leached" from the ash using $10 \mathrm{~mL}$ of $8 \mathrm{~N}$ $\mathrm{HNO}_{3}$ and $\sim 2 \mathrm{~mL}$ of $\mathrm{H}_{2} \mathrm{O}_{2}$. The "leach" solution containing the ${ }^{99} \mathrm{Tc}$ and Re was decanted from the ash, combined with at least two deionized water rinsates of the ash and diluted to $>200 \mathrm{~mL}$ to dilute the $\mathrm{HNO}_{3}$ in content to $<0.5 \mathrm{~N}$. To eliminate interferences and reconcentrate the analytes of interest, the diluted samples were run through $2 \mathrm{~mL}$ TEVA $^{\circledR}$ cartridges or columns that had been preconditioned with $5 \mathrm{~mL}$ of $8 \mathrm{NHNO}_{3}$ to 
assure that there was no residual analytes of interest on the column and then with $10 \mathrm{~mL}$ of $0.1 \mathrm{~N} \mathrm{HNO}_{3}$ to readjust the column acidity to assure no loss of the analytes of interest. The columns with the trapped analytes were washed with $20 \mathrm{~mL} 1 \mathrm{~N} \mathrm{HNO}_{3}$ to assure removal of interferences ( $\mathrm{Ru}$ in particular) and the analytes subsequently eluted into a clean beaker with $5 \mathrm{~mL}$ of $8 \mathrm{~N} \mathrm{HNO}_{3}$. The volume of the eluted sample was evaproated to near dryness on a hot plate at $<75^{\circ} \mathrm{C}$, spiked with $100 \mu \mathrm{L}$ of a $100 \mathrm{ng} \mathrm{In} / \mathrm{mL}$ solution, and diluted to $10 \mathrm{~mL}$ with $0.1 \mathrm{~N} \mathrm{HNO}_{3}$. Samples were then analyzed with an inductively coupled plasma mass spectrometer (ICPMS) that had been standardized with series of standard solutions containing at most $15 \mathrm{dpm} / \mathrm{mL}^{99} \mathrm{Re}$.

The most probable interferences in the mass spectrometric analysis of ${ }^{99} \mathrm{Tc}$ for are molybdenum hydride $\left({ }^{98} \mathrm{Mo}{ }^{1} \mathrm{H}\right)$ and Ruthenium $(\mathrm{Ru})$ at $\mathrm{m} / \mathrm{z} 99$. The relative abundances for $\mathrm{Ru}$ at $\mathrm{m} / \mathrm{z} 99$ and $\mathrm{m} / \mathrm{z} 101$ are $12.6 \%$ and $17 \%$, respectively. The Ru interference was corrected for by estimating the contribution of Ru at $\mathrm{m} / \mathrm{z} 99$ from the Ru response at $\mathrm{m} / \mathrm{z}$ 101 and subtracting it from the $\mathrm{m} / \mathrm{z} 99$ response.(i.e. Intensity at $\mathrm{m} / \mathrm{z} 99-0.741 *$ Intensity at $\mathrm{m} / \mathrm{z} 101=$ Intensity of ${ }^{99} \mathrm{Tc}$ ). The actual correction factor for Ru was determined from a commercial Ru standard and determined to be 0.725 . The probability of a significant interference by $\mathrm{MoH}$ is not likely the possible possibility of having this interference was determined by monitoring Mo at $\mathrm{m} / \mathrm{z} 95 \mathrm{and} / \mathrm{or} \mathrm{Mo}$ at $\mathrm{m} / \mathrm{z} 98$. No significant Mo was noted.

When natural abundance rhenium was used as the recovery standard, the $\operatorname{Re}$ concentration was simply determined at $\mathrm{m} / \mathrm{z} 185$. When a ${ }^{185} \mathrm{Re}$ enriched standard was used as the recovery standard, natural Re was subtracted by simply using an interelement correction for Re using ${ }^{187} \mathrm{Re}$. The correction factor was determined to be 0.589 from natural abundance Re standards (i.e. Intensity at $\mathrm{m} / \mathrm{z} 185-0.589 *$ Intensity at $\mathrm{m} / \mathrm{z} 187=$ Intensity of $\left.{ }^{185} \mathrm{Re}\right)$. Since tungsten hydrides $\left({ }^{184} \mathrm{~W}^{1} \mathrm{H}\right.$ and $\left.{ }^{186} \mathrm{~W}^{1} \mathrm{H}\right)$ and ${ }^{187} \mathrm{Os}$ may interfere with the Re measurements, $W$ at $\mathrm{m} / \mathrm{z} 182$ was monitored to assess the probability of a significant WH interference and an interelement correction for Os for ${ }^{187} \mathrm{Re}$ was made using the Os response at $\mathrm{m} / \mathrm{z} 189$ (i.e. Intensity at $\mathrm{m} / \mathrm{z} 185-0.589 *$ Intensity at $\mathrm{m} / \mathrm{z} 187=$ Intensity of ${ }^{185} \mathrm{Re}$ ).

Final ${ }^{99} \mathrm{Tc}$ concentrations were determined by subtracting the mean blank value determined from multiple blank analyses during the course of the run for each sample set. The blank corrected concentrations were further corrected for the Re recovery. Errors were propagated for each calculation step. Additional errors were added to propagation of errors to accommodate the variability of the calibration checks during the run and the overall spiked sample recovery for all runs and batches. Detection limits were determined for each sample using the propagated error and a single-sided Student-t value at $p=0.01$ (i.e. is the concentration greater the range of values expected over a concentration of 0 at $p=0.01$ ). The Student-t value was selected at the appropriate degrees of freedom represented by the propagated error. The degrees of freedom for the propagated error was determined using Satterwaite's formula. 


$$
d f=\frac{\left(\sum s_{i}^{2}\right)^{2}}{\sum \frac{s_{i}^{2}}{d f_{i}}}
$$

Table 3-1 represents the mean recovery of $T c$ of all of the spiked samples either submitted for analysis or spiked internal to the laboratory. Low recoveries on the initial performance evaluation samples and the laboratory spikes associated with the analysis of that batch are suspect to have been due to the availability of oxidation during the ashing step. While no quantitative information has been acquired in a controlled fashion, it was noted that allowing some air to enter the furnace always resulted in $10{ }^{99} \mathrm{Tc}$ recoveries while the Re recoveries were either not affected or only slightly diminished. Keeping the furnace closed tightly during the ashing step consistently resulted in higher ${ }^{99} \mathrm{Tc}$ recoveries. While no explanation exists for a $10 \%$ lower $\mathrm{Tc}$ recovery relative to Re in the literature $^{20}$, oxygen availability during ashing could certainly be a contributing factor.

Actual data transmitted to CRESP for the four batches analyzed for ${ }^{99} \mathrm{Tc}$ are given in Appendix A-3.

Table 3-1 Mean Recovery of Tc-99

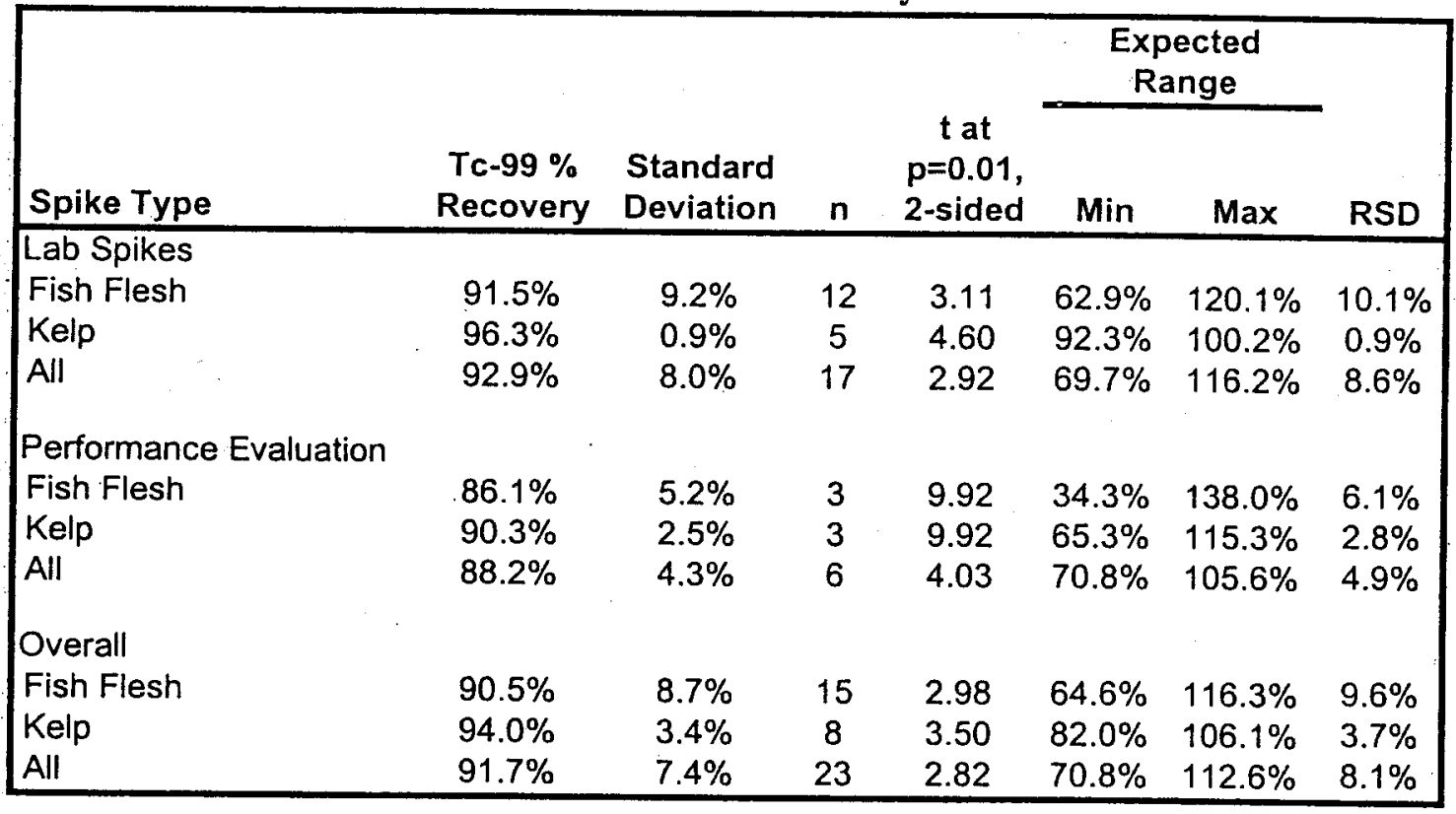

\subsubsection{Case Narrative Batch 1 (soft tissue) - ${ }^{99} \mathrm{Tc}$ Analysis}

- The spreadsheet file with the results from the first batch of Amchitka samples is found in Appendix A-3. Overall all, the analyses appeared to have gone rather smoothly. The following are items are notes and explanations from the analysis. 
- The calibration ${ }^{99} \mathrm{Tc}$ standards were prepared from the $20.2 \mathrm{ng} / \mathrm{mL}$ stock that has been in the laboratory and originated from Isotope Product Laboratories. Three calibration verification solutions were prepared from this standard or from a second standard from Isotope Product Laboratories (Source\# 1052-73). Re in the calibration standards and three verification standards were from commercial stocks (VHG Lot\# PREN32/02 or QCD Analysts lot\# 040875WBS6114Q). Calibration verification standards were run a total of 10 times during the course of the analysis with ${ }^{99} \mathrm{Tc}$ and $R e$ being recovered at $99.4 \pm 2.0 \%$ and $101.9 \pm 0.8 \%$, respectively. Yesterday, we received a ${ }^{99} \mathrm{Tc}$ standard directly from NIST, which we will be using as a calibration verification standard for future analyses.

- One of the calibration verification standards also had $200 \mathrm{pg} \mathrm{Ru} / \mathrm{mL}$ to check for the magnitude of effects on the interelement correction for ${ }^{99} \mathrm{Tc}$. Another simply had $200 \mathrm{pg} \mathrm{Ru} / \mathrm{mL}$. The net effect is that $\mathrm{Ru}$ at $200 \mathrm{pg} / \mathrm{mL}$ could create a negative bias of $\sim-2 \mathrm{pg} / \mathrm{mL}$. No samples had more than $\sim 1.5 \mathrm{pg} \mathrm{Ru} / \mathrm{mL}$ so any residual effects from $\mathrm{Ru}$ are an absolute minimum.

- Five calibration verification blanks were also run during the course of the analysis and the ${ }^{99} \mathrm{Tc}$ was determined to be $0.158 \pm 0.066 \mathrm{pg} / \mathrm{mL}$ in these blanks. This value was subtracted from all sample results. Errors were propagated and the degrees-offreedom of the propagated error determined using Satterwaite's formula.

- Additional errors from the Re correction and the precision of the calibration verification checks were also propagated and the final degrees of freedom determined with Satterwaite's formula. The propagated errors were multiplied by the 1 -sided Student $t$ value at $p=0.01$ to estimate instrument detection limits for each sample.

- Samples of species types "G" and a couple of species type "L" had significant over recoveries of $\mathrm{Re}$ indicating the potential for the natural presence of $\mathrm{Re}$ in the samples. When Re recovery was over $100 \%$ no Re corrections were made. We finally received our ${ }^{185} \operatorname{Re}$ standard and will be doing $\mathrm{Re}$ by isotope dilution in the future to eliminate these types of problems.

- Recoveries for species types "O" and "P" had consistently low Re recoveries indicating a possible matrix effect.

- The laboratory spiked blank recovered only $75.8 \%$. This is slightly out of the range of $80-102 \%$ we have been seeing in the performance evaluation samples and in the laboratory spikes. In short, it appears that the procedure is more variable for flesh than for kelp. When evaluating the actual sample results one must keep in mind that the standard deviations presented only represent the deviations from the instrumental analysis and do not include any sample-to-sample or sample preparation variability. As we progress, an error representing sample-to-sample and sample preparation variability will be included into the propagation of errors. This should have little effect on the samples near and below the detection limit but will cause all samples with significant concentrations of ${ }^{99} \mathrm{Tc}$ to have a total error of $\sim \pm 10 \%$. All positive samples in this batch (i.e. the spike and the blind spike) should be assumed to have a total error of $\sim 10 \%$. It also appears that there may be an average of a 
$\sim 10 \%$ negative bias for ${ }^{99} \mathrm{Tc}$. This is not unexpected based upon the available literature for methods employing $\mathrm{Re}$ as a recovery standard.

\subsubsection{Case Narrative Batch 2 (soft tissue) - ${ }^{99}$ Tc Analysis}

Following is the second set of Tc99 analyses. Notes are:

- We used ${ }^{185}$ Re as the recovery standard. To avoid the lengthy isotope dilution calculation then subtracting the intensity contributions and recalculating the $185_{R e}$ concentration from the corrected intensity, we simply used an interelement correction calculating the ${ }^{185} \mathrm{Re}$ natural intensity from the ${ }^{187} \mathrm{Re}$ intensity. This subtracts all natural Re contributions from the $185_{\mathrm{Re}}$ peak as well as some ${ }^{185_{\mathrm{Re}}}$ in the enriched standards because the Re standard is only $93.6 \%$ enriched, i.e. in effect we are subtracting any enriched Re remaining in the standard from the enrichment process or about an additional $4 \%$ of the ${ }^{185}$ Re peak. It should be noted that in the few analyses we have done where we can measure the actual isotopic ratio on the standard and of a natural Re standard, the enrichment appears to be more like $94.38 \% \pm 0.05 \%$ for the $185 \mathrm{Re}$. In the natural Re standard, the ${ }^{185} \mathrm{Re}$ was measured at $37.2 \% \pm 0.2 \%$ in this run vs the expect $37.4 \%$. Overall, this has no bearing on the analysis other than to demonstrate that the interelement correction is being performed as expected and that determining the natural Re from the ${ }^{187}$ Re residual in the enriched Re standard will be biased somewhat (i.e. 10-20\%). A small error in the isotopic ratio of the enriched standard will have only a small effect on the determination of the natural Re. Indeed, the isotope dilution determination of the natural $R e$ on a standard containing natural and enriched Re was $\sim 95 \%$ while the determination by the standard calibration curve using the $187 \mathrm{Re}$ remaining in the enriched standard was $\sim 114 \%$. We will investigate this further to verify the actual enrichment so that the determination of the natural Re can be optimized. This is probably not that important, however, tissues and species that accumulate Re will probably also accumulate Tc.

- The liquid like samples (jellyfish?) and the bird eggs (mushy samples) had high natural Re concentrations. Having the ${ }^{185}$ Re enriched standard was a definite plus.

- Tc recovery in the laboratory spike was once again lower than expected at $71.4 \%$.

\subsubsection{Case Narrative Batch 3 (kelp and soft tissue) - ${ }^{99}$ Tc Analysis}

Following is the third set of Tc99 analyses. Notes are

- We used ${ }^{185} \operatorname{Re}$ as the recovery standard. To avoid the lengthy isotope dilution calculation then subtracting the intensity contributions and recalculating the $185 \mathrm{Re}$ concentration from the corrected intensity, we simply used an interelement correction calculating the $185 \mathrm{Re}$ natural intensity from the ${ }^{187} \mathrm{Re}$ intensity. This subtracts all natural Re contributions from the $185_{\mathrm{Re}}$ peak as well as some ${ }^{185} \mathrm{Re}$ in the enriched standards because the Re standard is only $93.6 \%$ enriched, i.e. in effect we are 
subtracting any "natural" Re remaining in the standard from the enrichment process or about an additional $4 \%$ of the 185 Re peak. It should be noted that in the few analyses we have done where we can measure the actual isotopic ratio on the standard and of a natural Re standard, the enrichment of the 185 Re standard appears to be more like $94.45 \% \pm 0.06 \%$ this time vs the $94.38 \% \pm 0.05 \%$ we determined in the last set of analyses. Since in the last set of samples we noted that the use of the remaining ${ }^{187} \mathrm{Re}$ in the $185 \mathrm{Re}$ enriched standard to calculate the natural Re content led to an overestimation, this time, we corrected the ${ }^{187} \mathrm{Re}$ and $185 \mathrm{Re}$ concentrations to the enrichment factor we have been measuring (i.e. the ${ }^{185} \operatorname{Re}$ at $94.4 \%$ not $93.6 \%$ as stated by the supplier). This seems to have corrected the problems with using the ${ }^{187} \mathrm{Re}$ for determining natural Re. Once again, this has no bearing on the Tc analysis and only serves to make the natural Re concentrations better. This is still probably not that important, however, tissues and species that accumulate Re will probably also accumulate Tc.

- Indeed, some of the samples in this batch, particularly, the kelp had very significant natural Re concentrations. In some cases, the natural Re was as much as $15 \mathrm{x}$ the

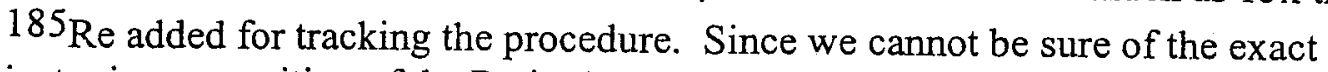
isotopic composition of the Re in these samples, there may be some added error in the Re determinations. In fact, for most of the very high Re containing samples, the spiked ${ }^{185} \mathrm{Re}$ was over recovered slightly and no corrections were made to the Tc concentrations. We don't really have any good data at this time with regards to how the natural Re really affects the analysis due subtraction error or due to variations in the natural Re isotopic ratio. We have not investigated the possibility that geographical variations in the Re isotope ratio exist as they do for other elements. At this time we don't think this is causing a major problem. However, if it can be determined that this is a major problem, we may have to increase the 185 Re spike level to attempt to swamp this effect out, provided there is no effect on column capacity, $\mathrm{Tc}$ recovery, etc.

- Tc recovery in the laboratory spikes for fish flesh and kelp were good at $92 \%$ and $95 \%$, respectively. We are speculating that with more careful study we will find that the low recoveries for Tc can be tied to the quantity of available oxygen during the combustion/charring step.

- Since this batch had both kelp and fish, the overall relative standard deviation of all laboratory spiked samples of fish flesh and kelp has been added to the total uncertainty calculation (8.9).

- Note also that there was one sample with marginally detectable $T c$ at a very low concentration $\left(1.5 \times 10^{-4} \pm 0.3 \times 10^{-4} \mathrm{~Bq} / \mathrm{g}=0.0040 \pm 0.0007 \mathrm{pCi} / \mathrm{g}\right)$. This concentration is well below the required detection limits, only about $2 x$ the estimated $\mathrm{DL}$ and in a sample that, interestingly enough, our lab technician speculates may be the blank control sample. 


\subsubsection{Case Narrative Batch 6 (soft tissue) - ${ }^{99} \mathrm{Tc}$ Analysis}

Following is the fourth set of Tc99 analyses. Notes are

- There were only 8 samples, two of which appear to have been QA samples.

- We used ${ }^{185} \mathrm{Re}$ as the recovery standard. To avoid the lengthy isotope dilution calculation then subtracting the intensity contributions and recalculating the $185_{R e}$ concentration from the corrected intensity, we simply used an interelement correction calculating the ${ }^{185} \operatorname{Re}$ natural intensity from the ${ }^{187} \mathrm{Re}$ intensity. This subtracts all natural $\operatorname{Re}$ contributions from the $185_{\mathrm{Re}}$ peak as well as some $185_{\mathrm{Re}}$ in the enriched standards because the Re standard is only $93.6 \%$ enriched, i.e. in effect we are subtracting any enriched Re remaining in the standard from the enrichment process or about an additional $4 \%$ of the ${ }^{185}$ Re peak. It should be noted that in the few analyses we have done where we can measure the actual isotopic ratio on the standard and of a natural Re standard, the enrichment appears to be more like $94.33 \% \pm 0.02 \%$ this time vs $94.45 \% \pm 0.06 \%$ for set three and $94.38 \% \pm 0.05 \%$ for set two. Since in the sample set two we noted that the use of the remaining ${ }^{187} \mathrm{Re}$ in the $185 \mathrm{Re}$ enriched standard to calculate the natural $\mathrm{Re}$ content led to an overestimation, in sample sets three and four, we corrected the ${ }^{187} \mathrm{Re}$ and $185 \mathrm{Re}$ concentrations to the enrichment factor we have been measuring (i.e. the $185 \operatorname{Re}$ at $94.4 \%$ not $93.6 \%$ ). This seems to have corrected the problems with using the ${ }^{187} \mathrm{Re}$ for determining natural Re. Once again, this has no bearing on the analysis other than to make the natural Re concentrations better. This is still probably not that important, however, tissues and species that accumulate Re will probably also accumulate Tc.

- Natural Re was as expected in the samples.

- Tc recovery in the laboratory spikes for fish flesh $93.0 \%$. Since this batch had only flesh, the overall relative standard deviation of all spiked samples of fish flesh was added to the total uncertainty calculation $(9.6 \%)$. 


\section{REFERENCES}

(1) Amchitka Independent Assessment Science Plan, June 2003.

(2) PLN-1719 "Quality Assurance Project Plan for the Analysis of Amchitka Island Samples", Revision 1, January 2005.

(3) PLN-153 "Quality Assurance Project Plan for Analytical Laboratories Department Radioanalytical Activities", Revision 2, May 2004.

(4) INEEL Method Manual for the Amchitka Environmental Sample Analysis, Revision 1, December 2004.

(5) ACLP-10.10 - TRA Radioanalytical Sample Management.

(6) ACLP-10.31 - Evaluation and Verification of Data for Radionuclide Identification/Selection.

(7) ACLP-10.41 - RML Germanium and Leps Detector Calibration.

(8) ACMM 3606 - Gamma-Ray Analysis of Standardized Samples.

(9) ACMM 3815 - Determination of Selected Actinides and Strontium-90 in Water.

(10) ACMM 3816 - Determination of Selected Actinide Nuclides and Strontium-90 in Filters and Solids.

(11) ACMM-3705-Determination of ${ }^{99 T} \mathrm{c}$ in Biological Samples Using Teva ${ }^{\circledR}$ Resin.

(12) Quality Assurance/Quality Control Program of the Radiation Measurements Laboratory for Gamma Spectroscopy and Direct Gross Alpha/Beta Counting. Reference \# ST-CS-013-89, May 1989.

(13) Radioanalytical Laboratory Group Hardware System and Software Program Application Configuration Management Plan \#PLN-1536 Revision 0, January 2004.

(14) INEEL/EXT-2000-00908 "PCGAP: Users Guide and Algorithm Description", September 2000.

(15) Mas, J. L.; Tagami, K.; Uchida, S. Analytica Chimica Acta 2004, 509, 83-88.

(16) McCartney, M.; Rajendran, K.; Olive, V.; Busby, R. G.; McDonald, P. 
Journal of Analytical Atomic Spectrometry 1999, 14, 1849-1852.

(17) Tagami, K.; Uchida, S. Journal of Radioanalytical and Nuclear Chemistry 2003, 255, 547-551.

(18) Tagami, K.; Uchida, S.; Hamilton, T.; Robison, W. Applied Radiation and Isotopes 2000, 53, 75-79.

(19) Wakoff, B.; Nagy, K. L. Environmental Science \& Technology 2004, 38, 1765-1771.

(20) McCartney, M.; Olive, V.; Scott, E. M. Journal of Radioanalytical and Nuclear Chemistry 1999, 242, 413-418. 


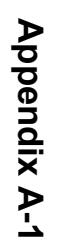


Appendix A-1

Gamma and I-129 Analysis 
BATCH 1

GAMMA AND I-129 

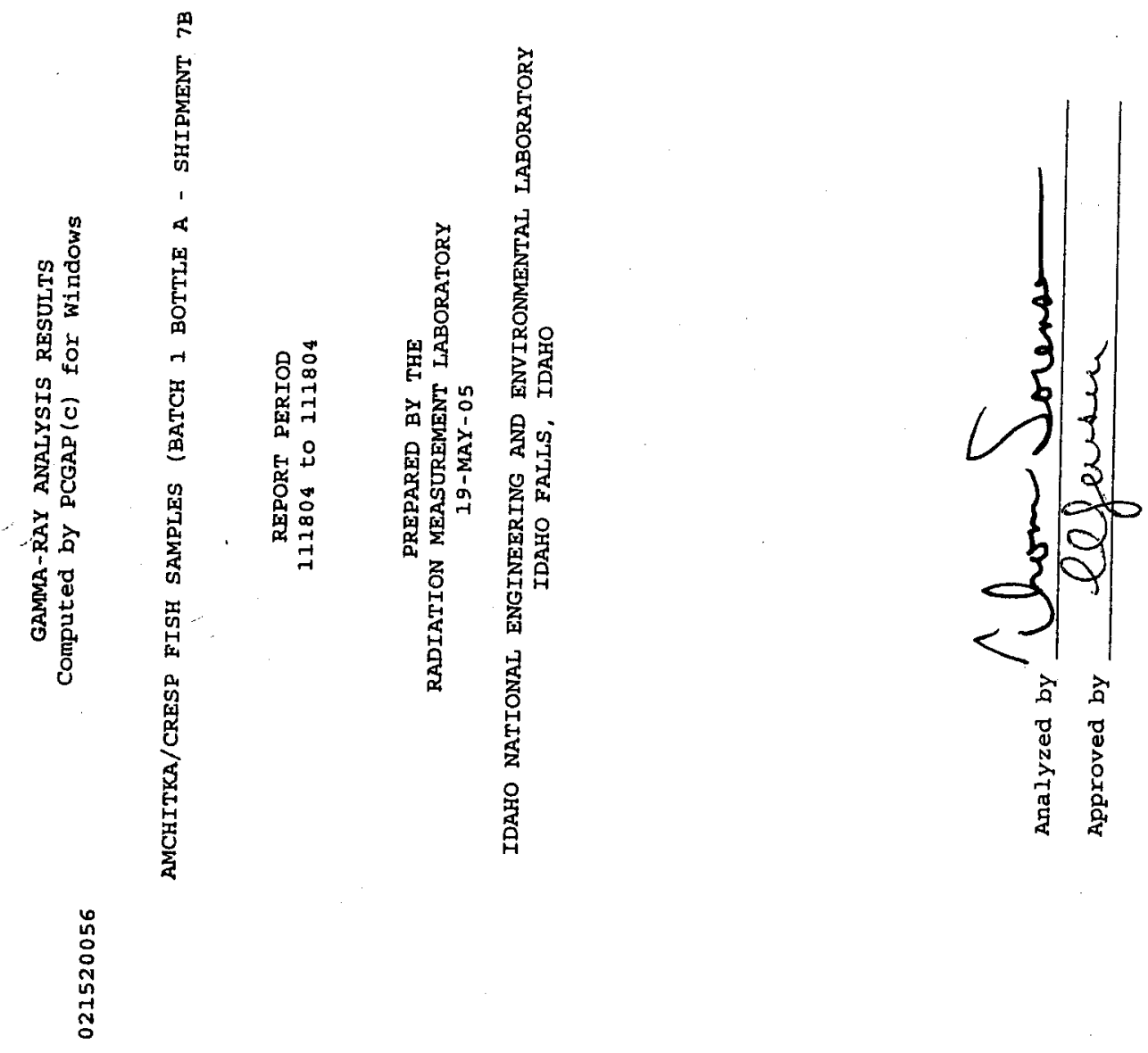


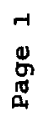

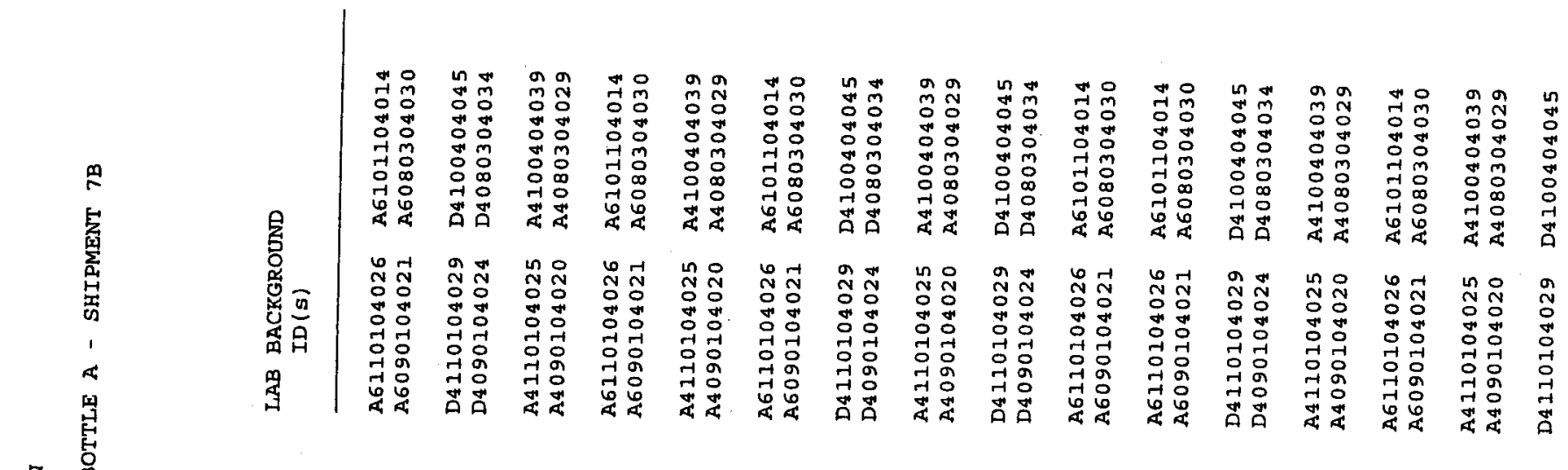

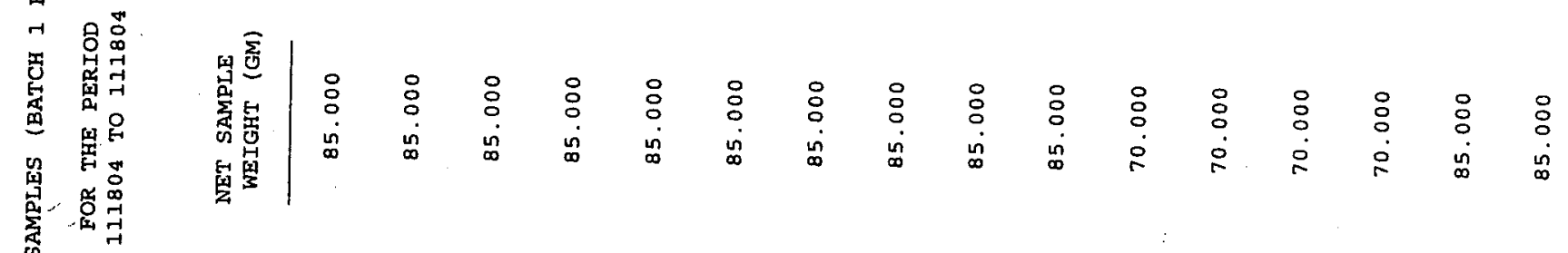

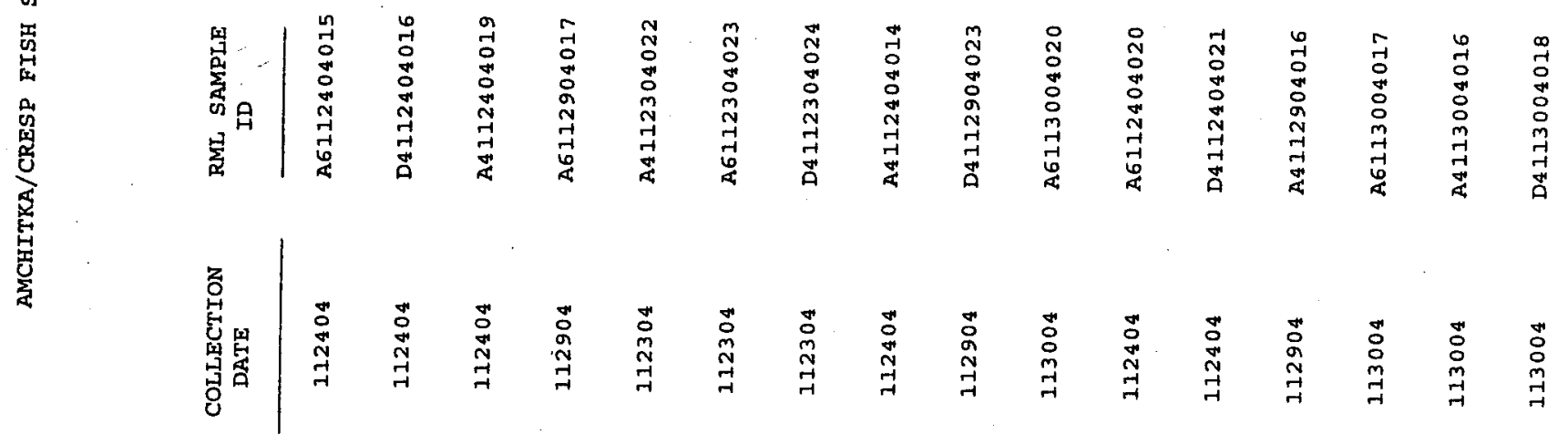

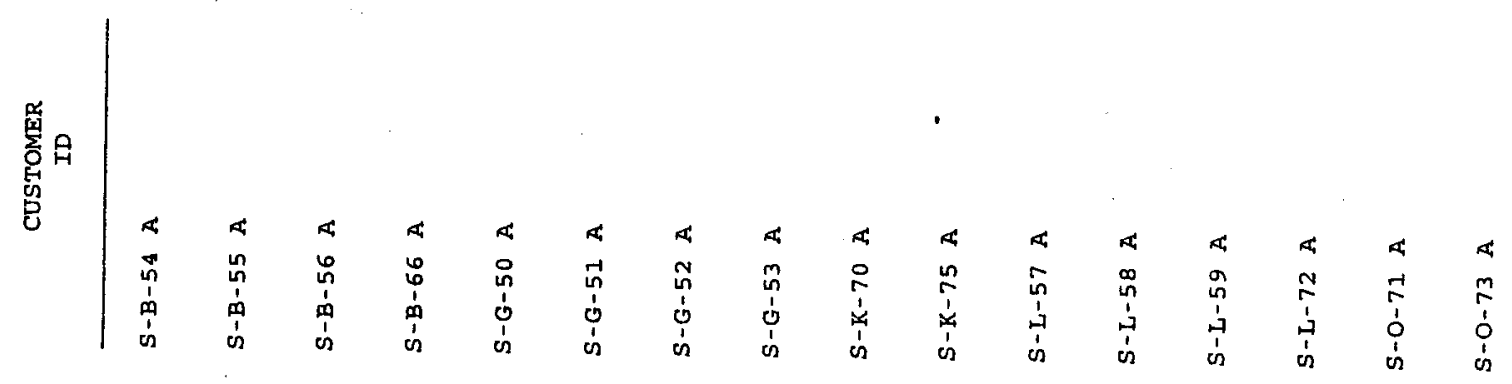




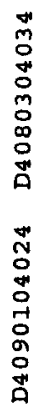




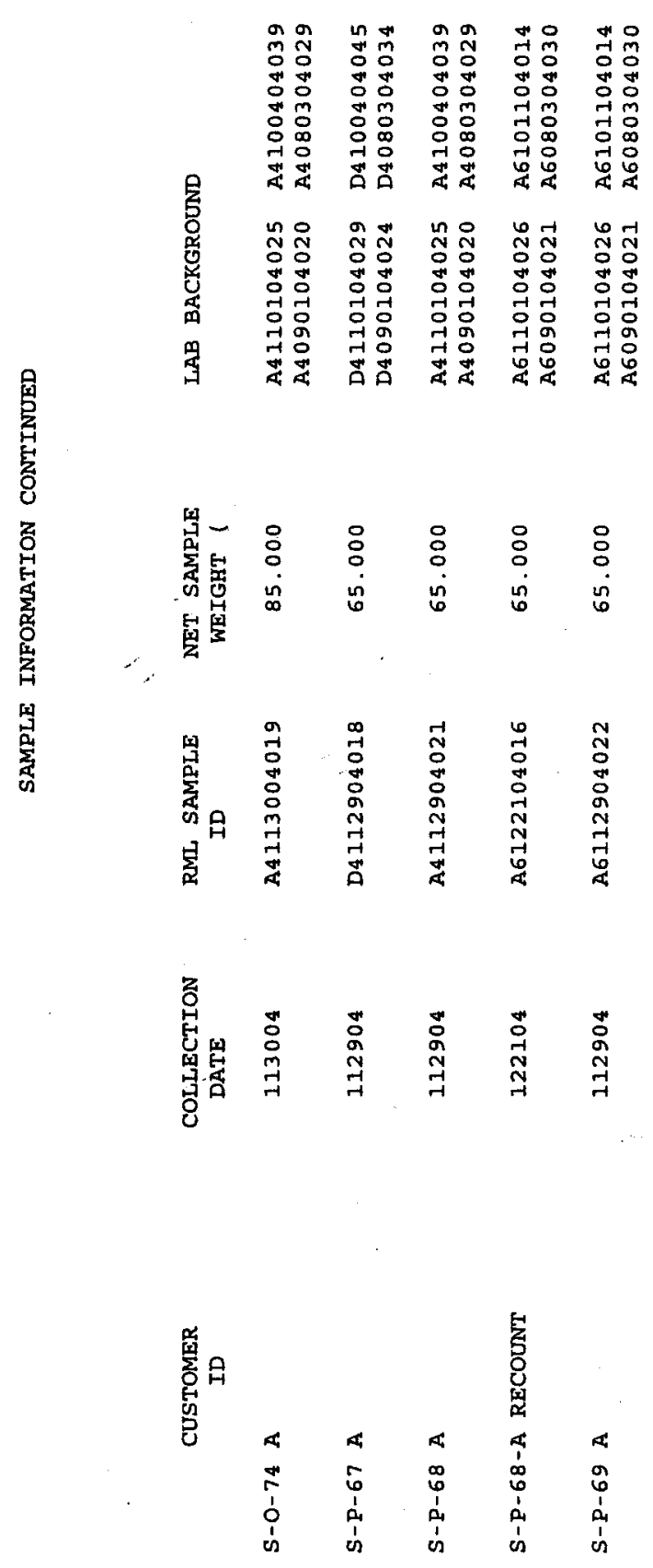



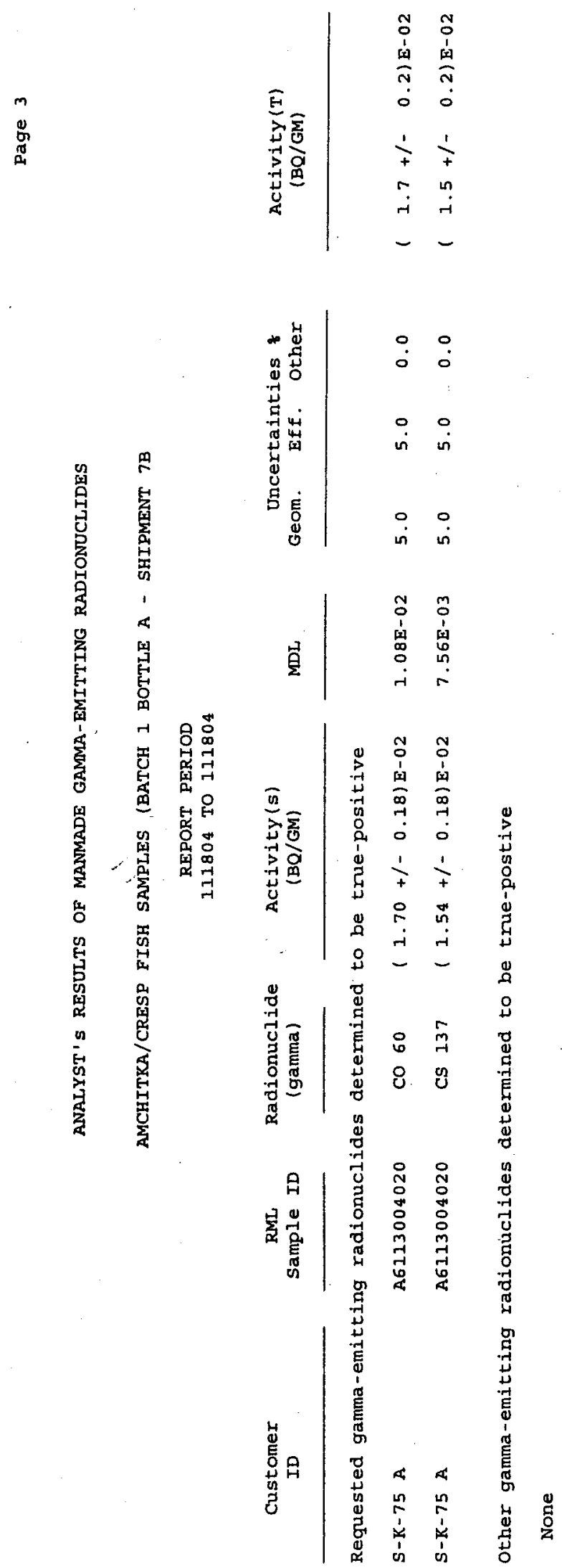


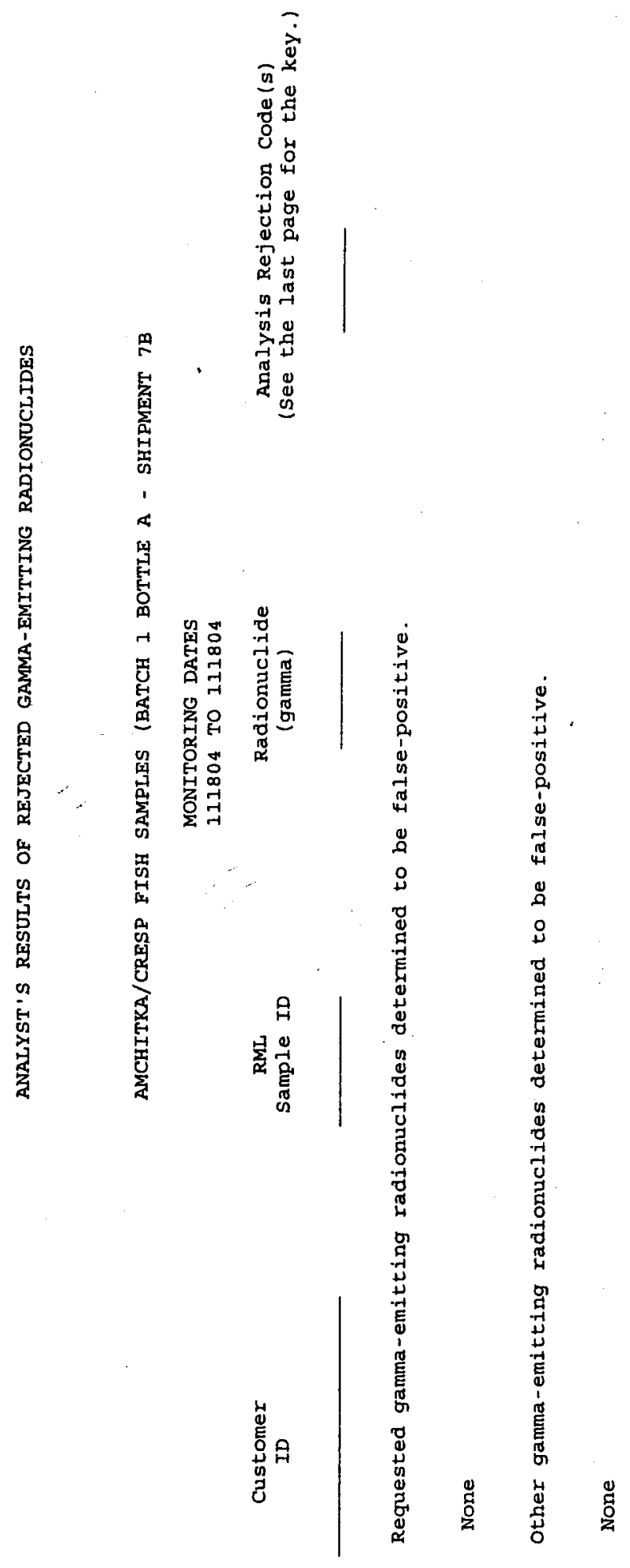


แn
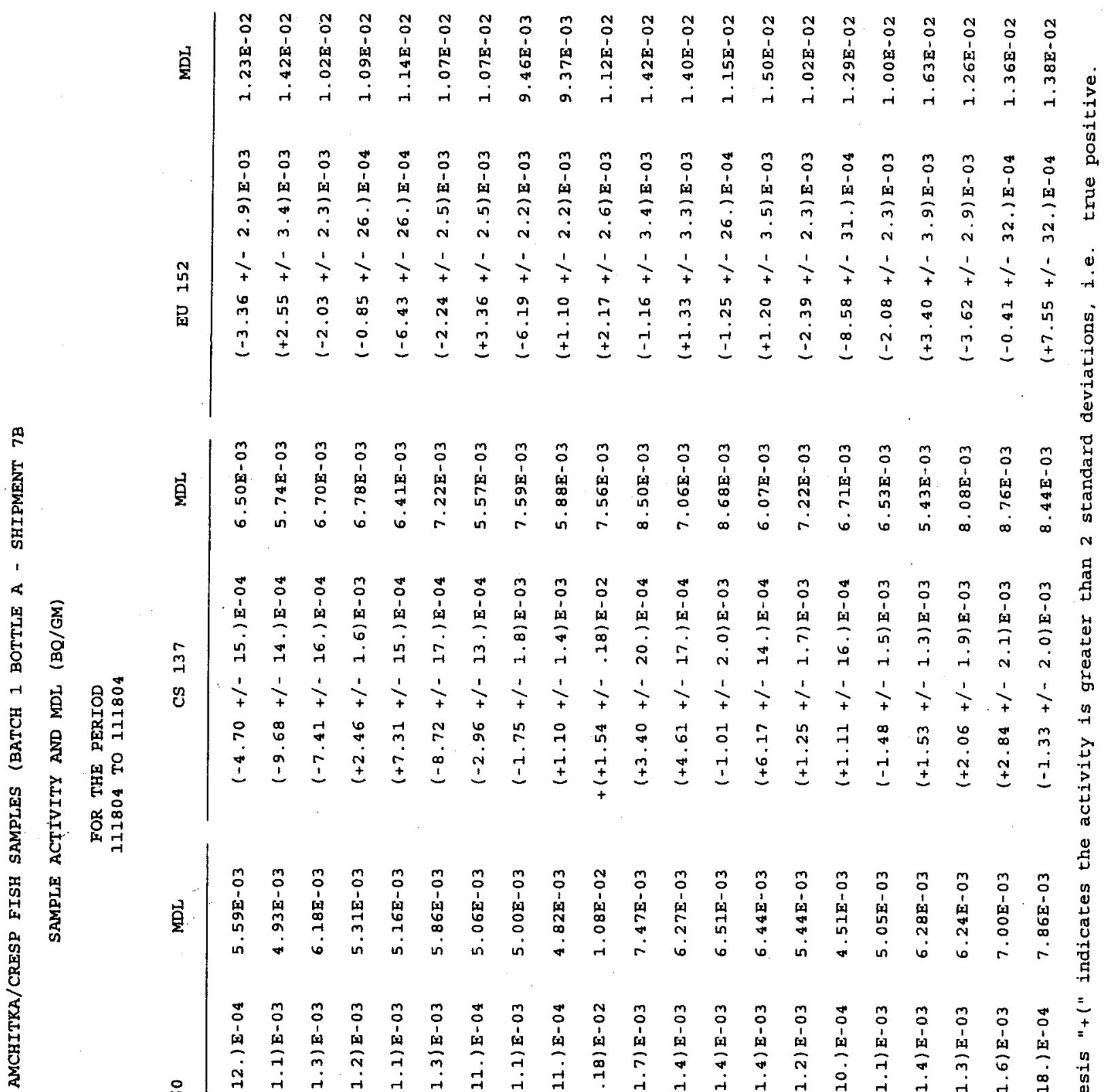

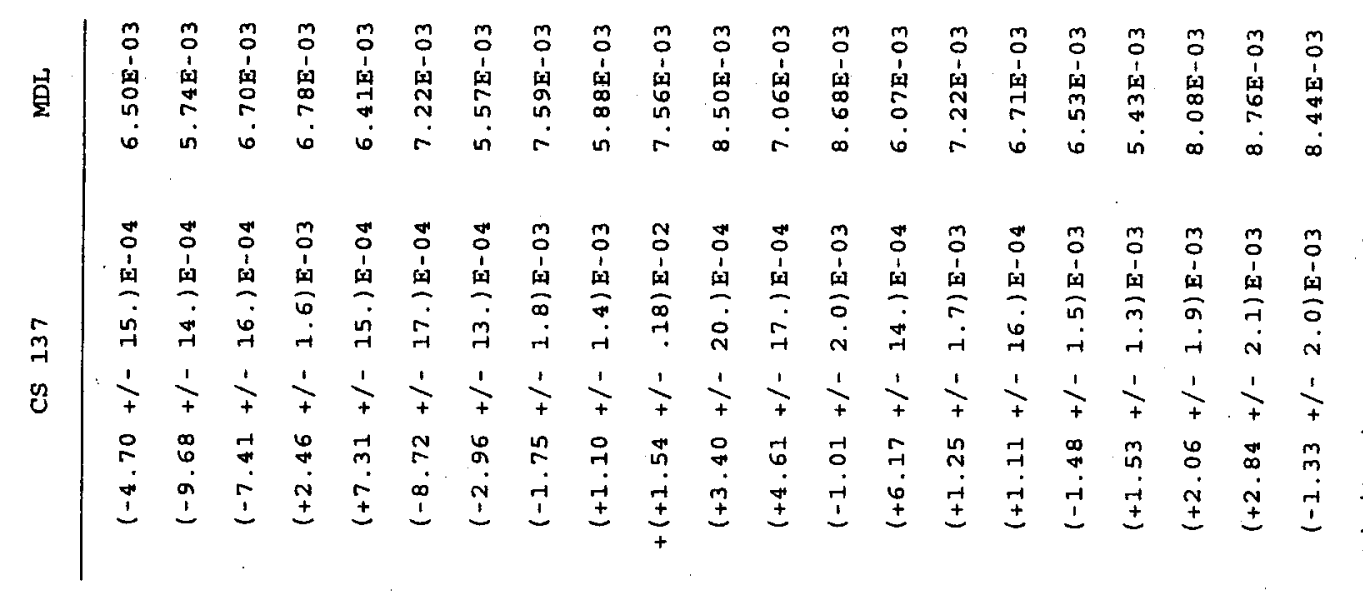

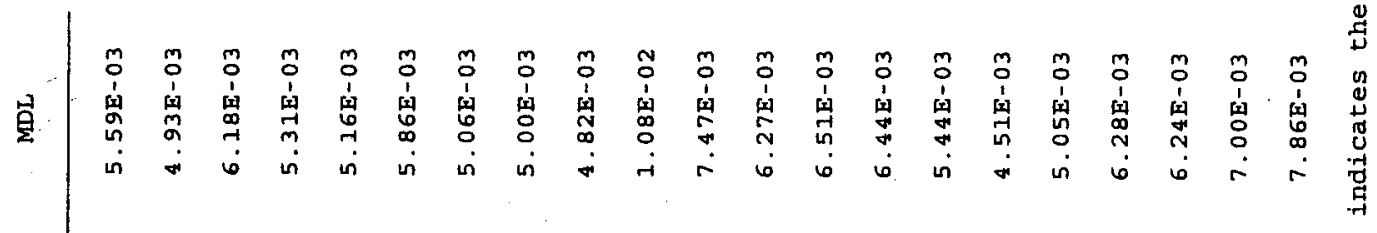

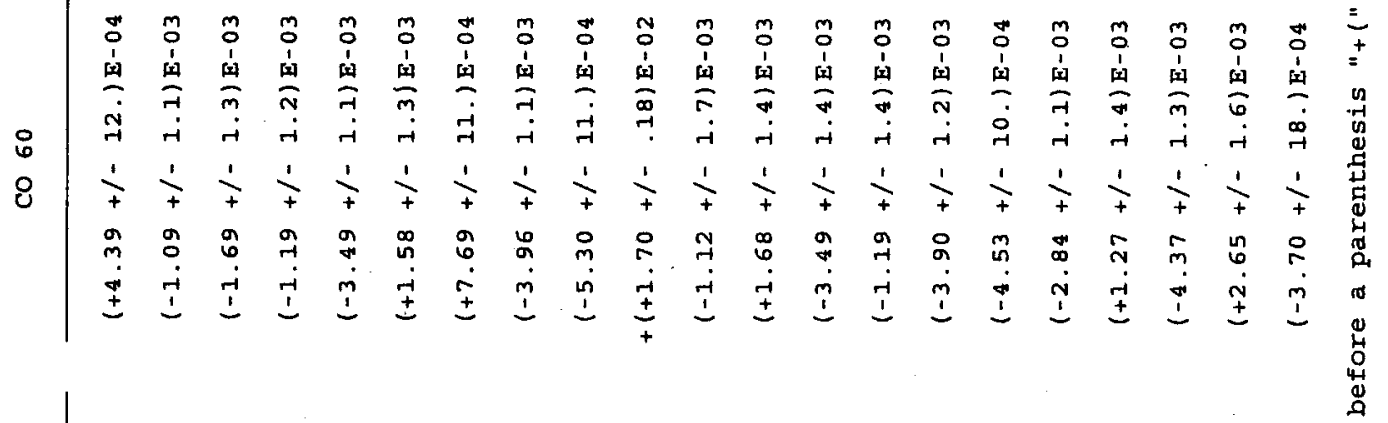

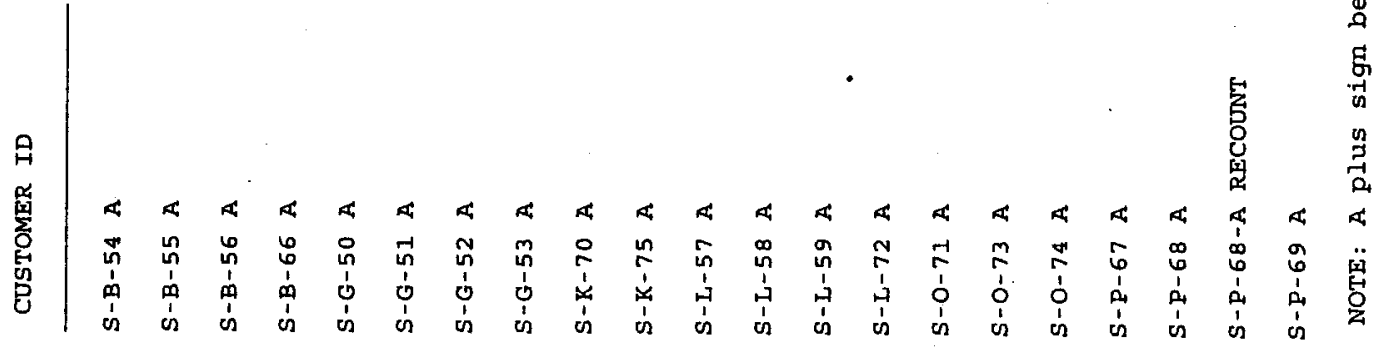


0
0
0
0
0
$n_{1}$

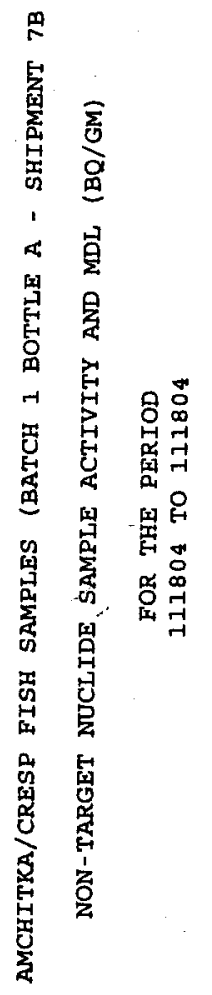



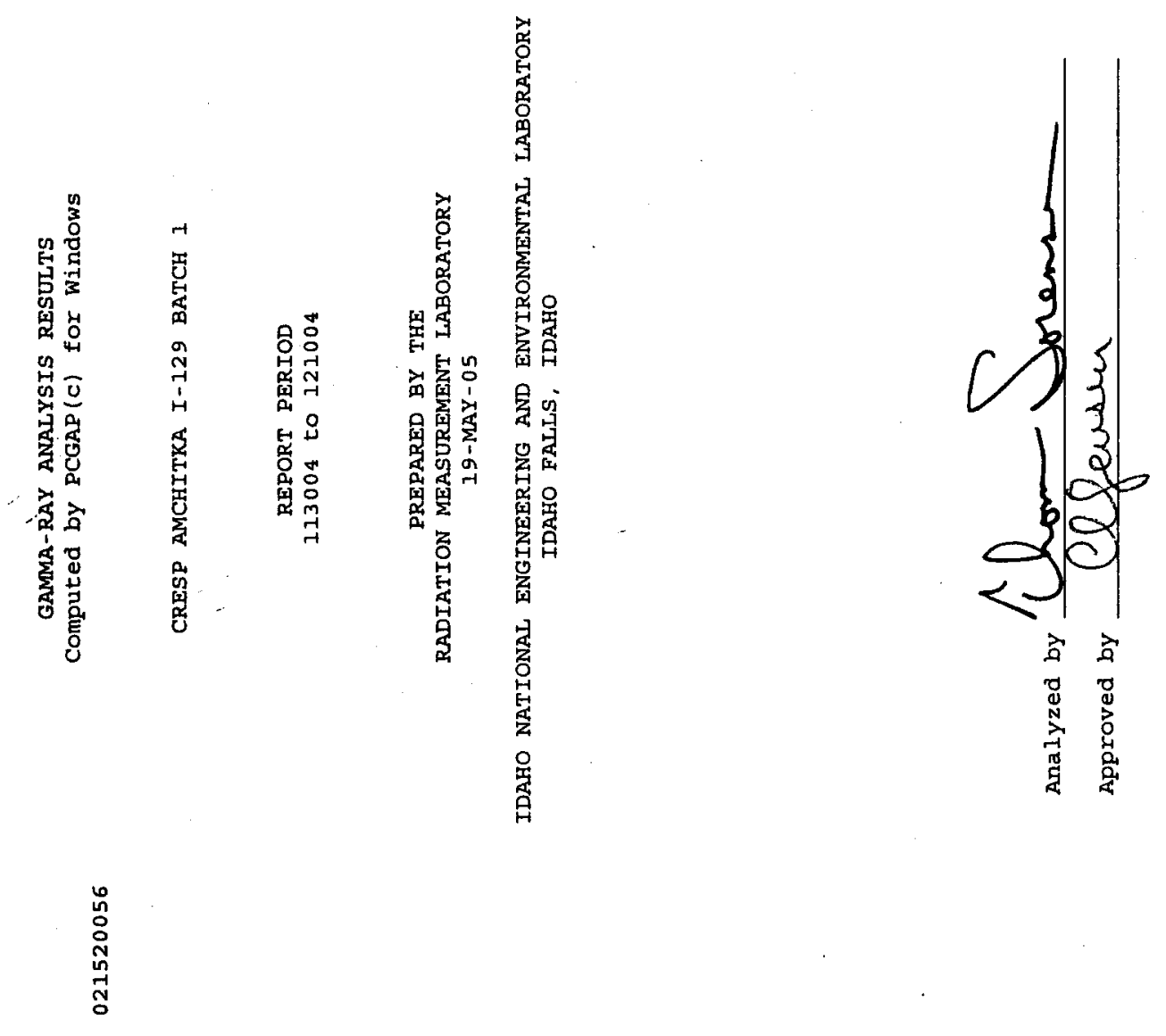


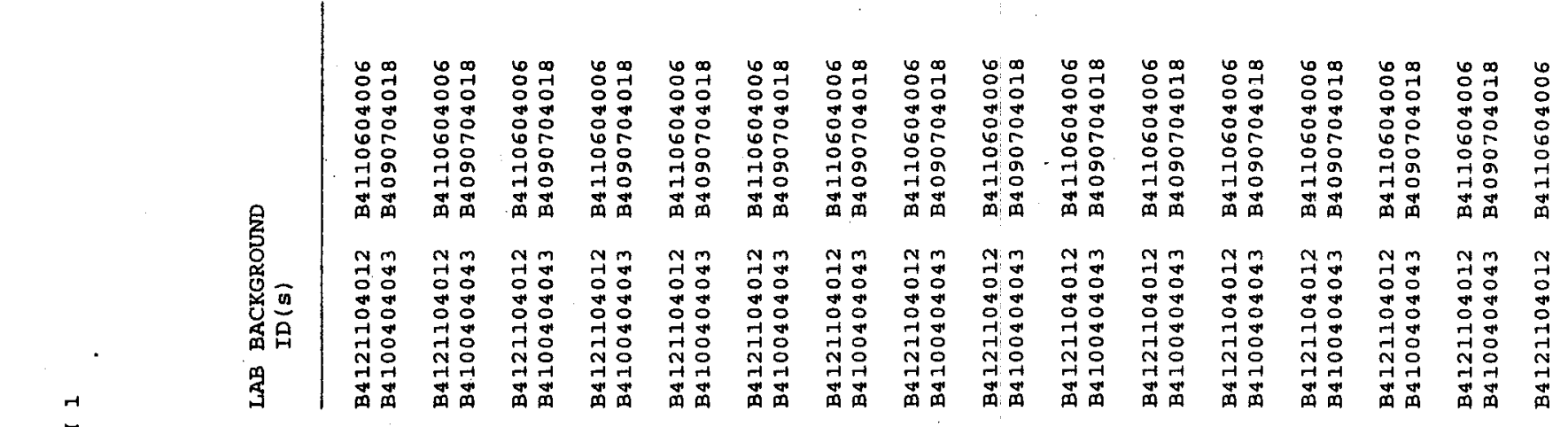

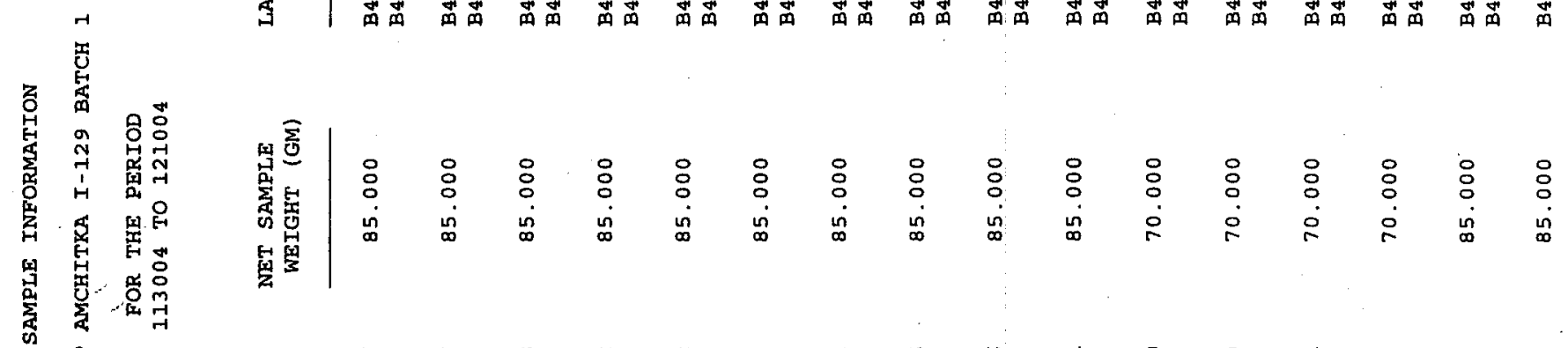

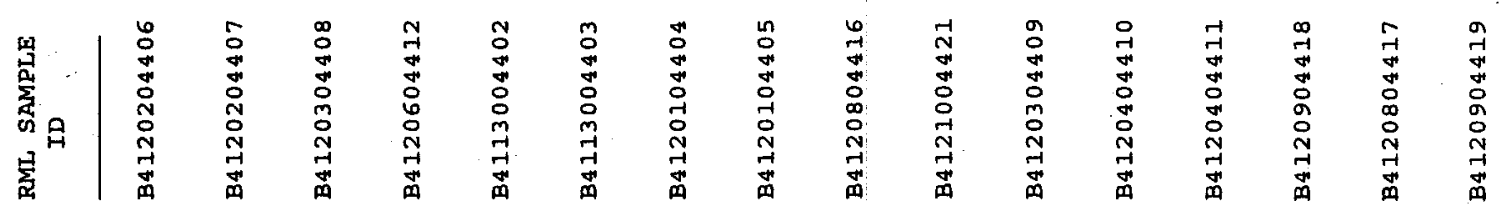

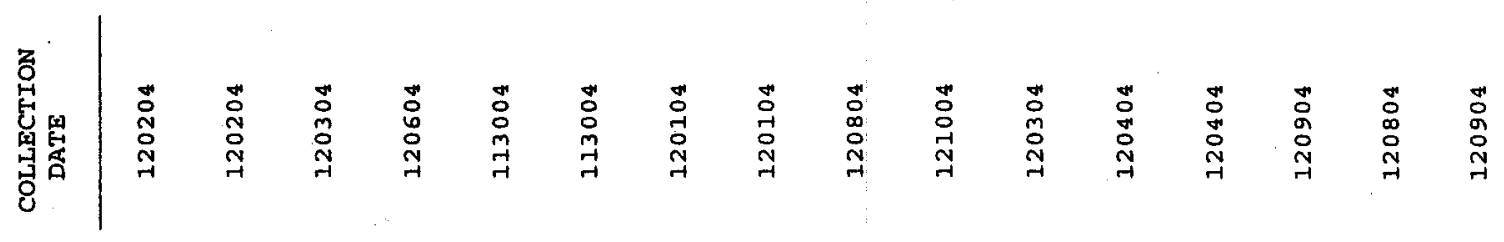

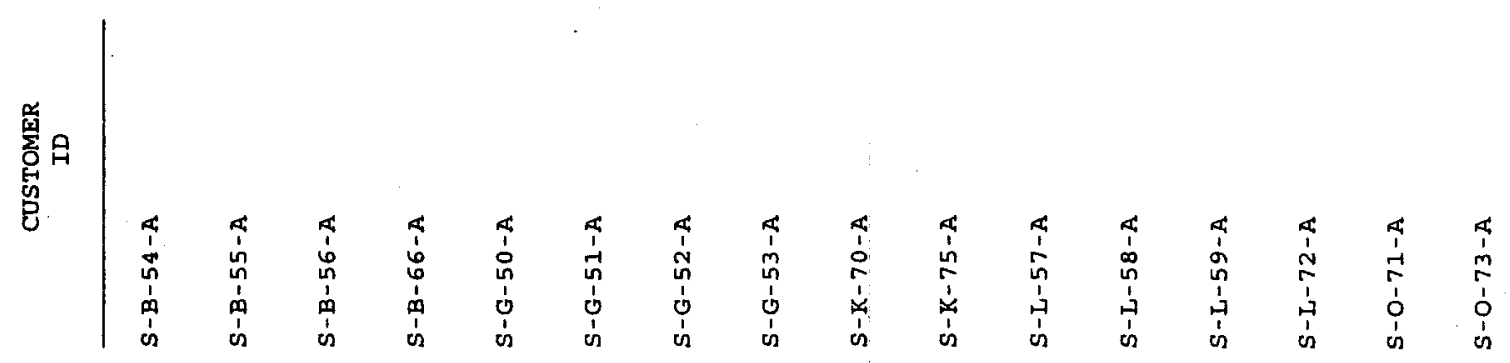


N

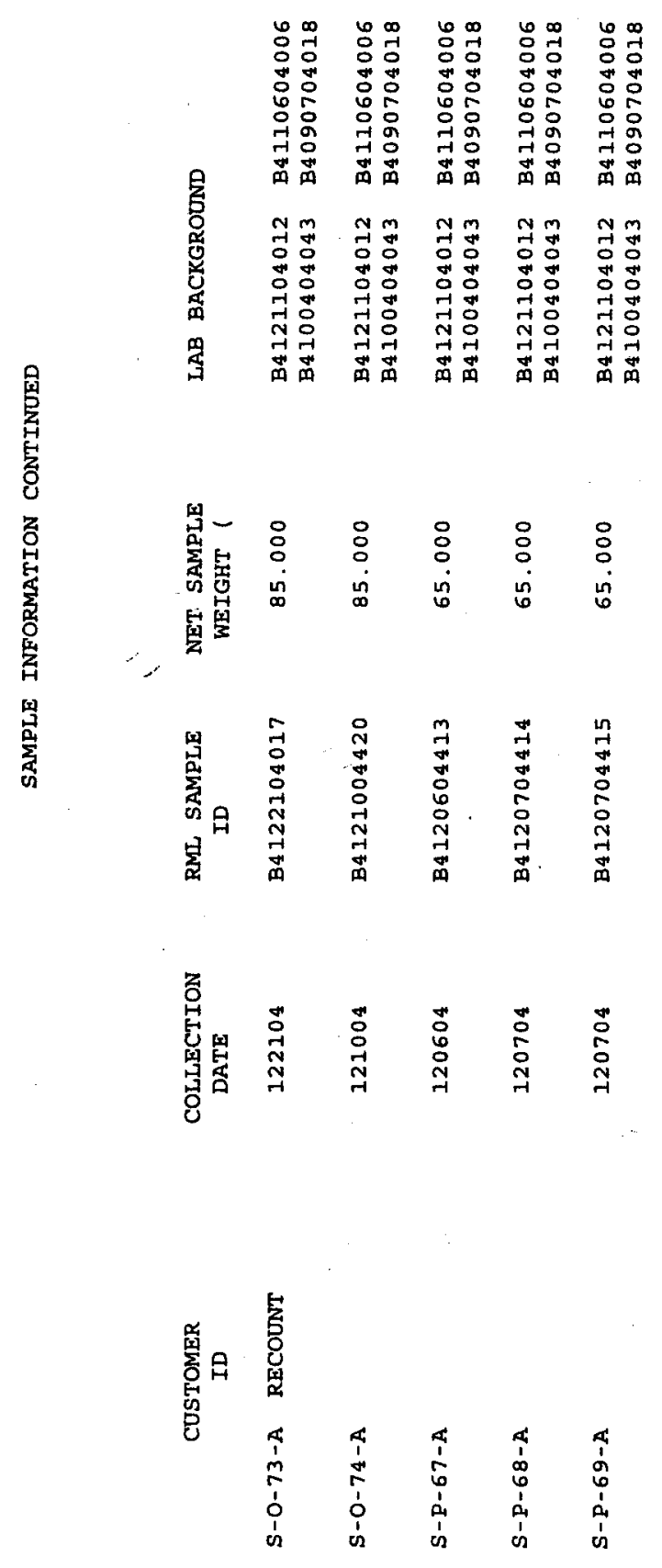




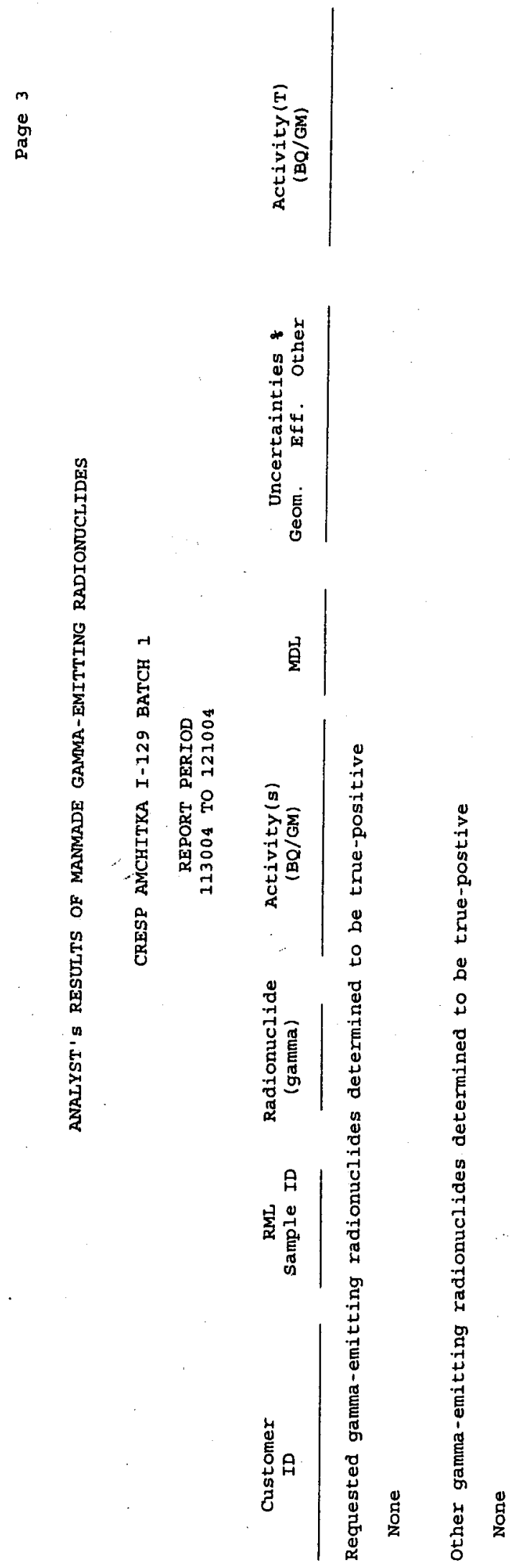


ホ

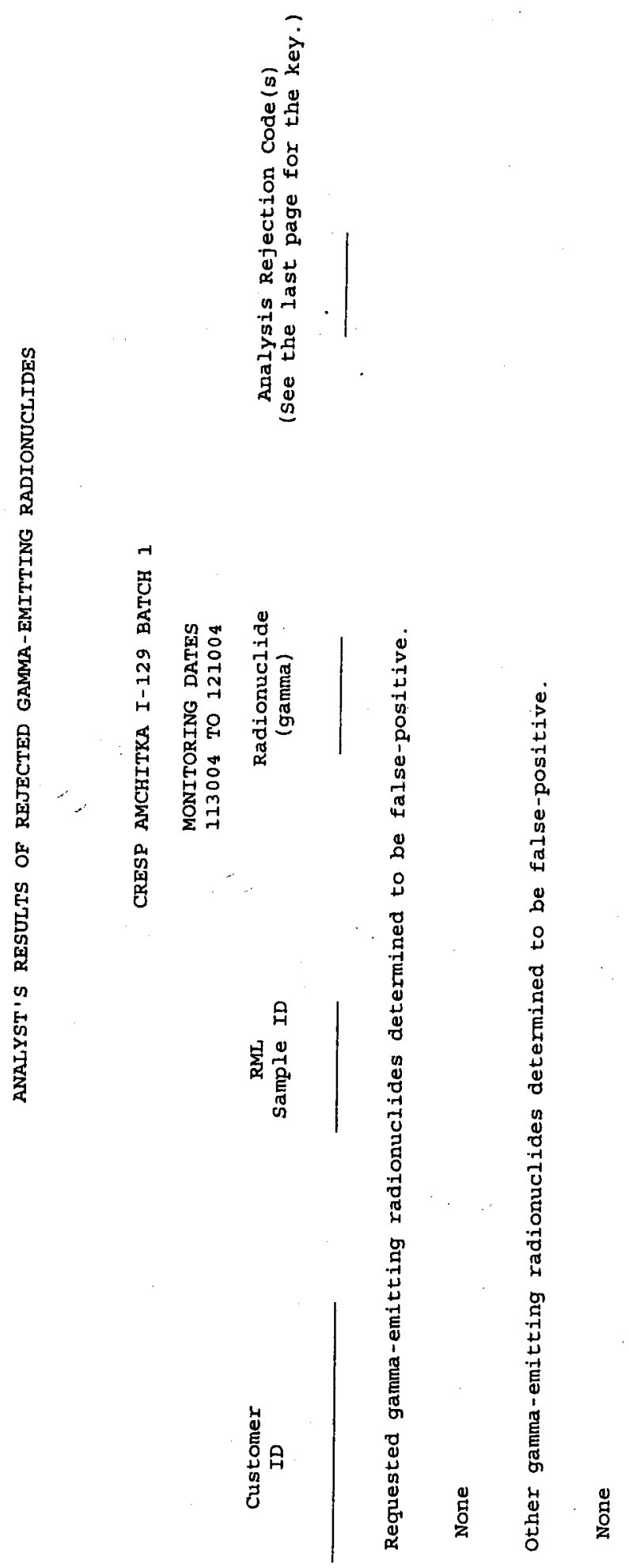


吕
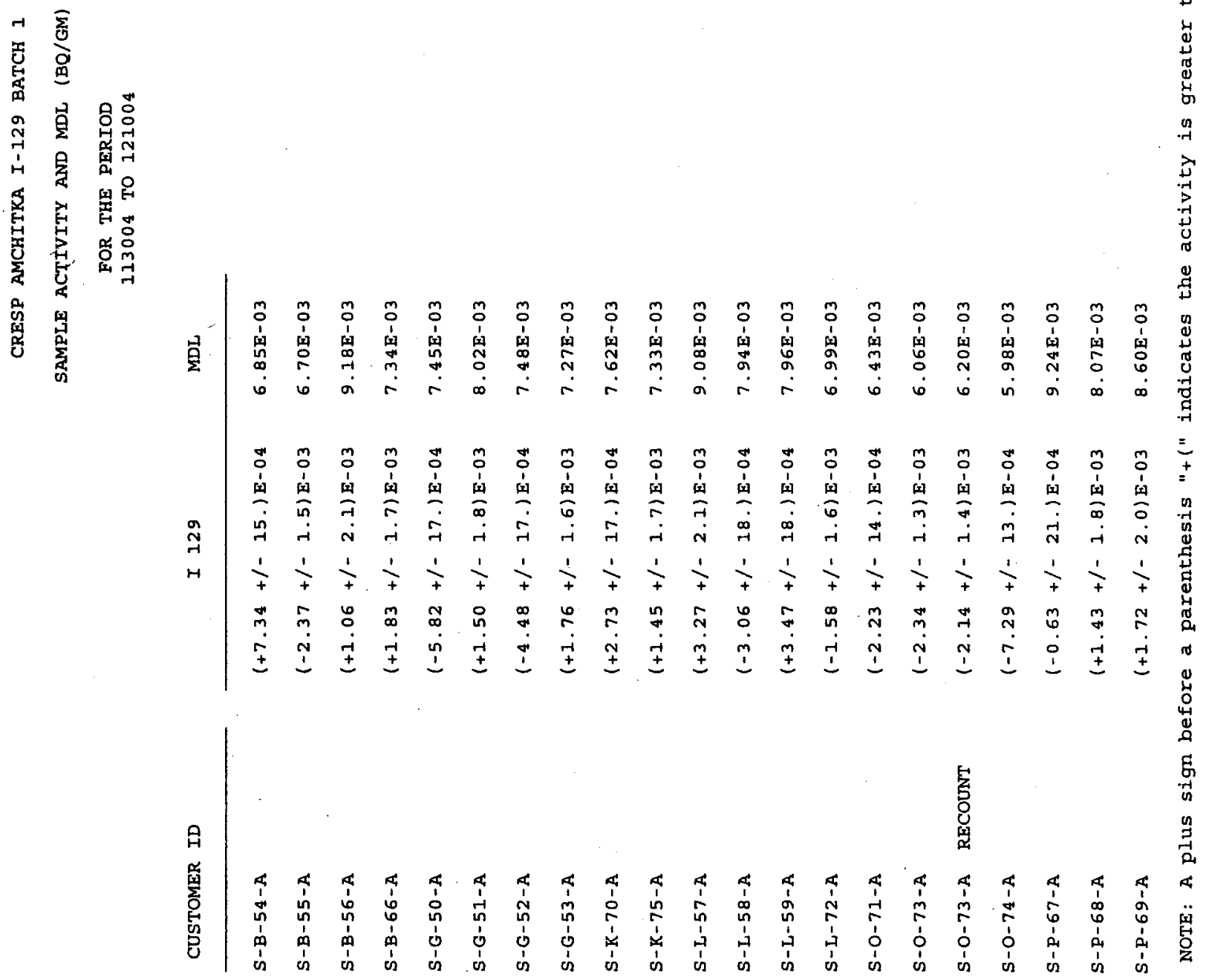
6
0
9
0
0

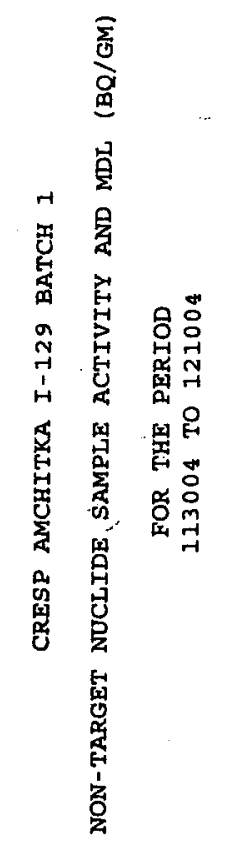


BATCH 2

GAMMA AND I-129 

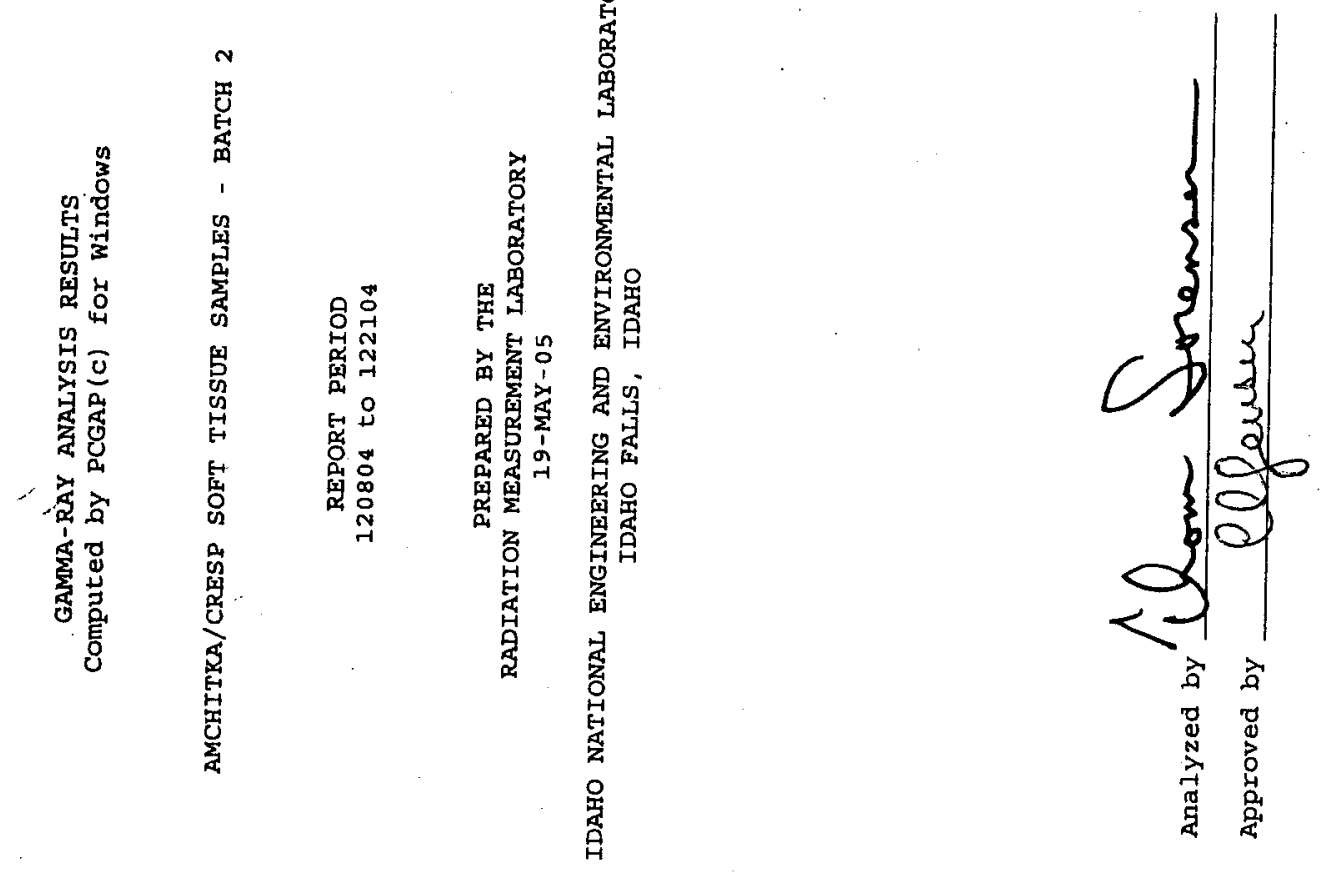

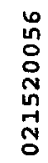




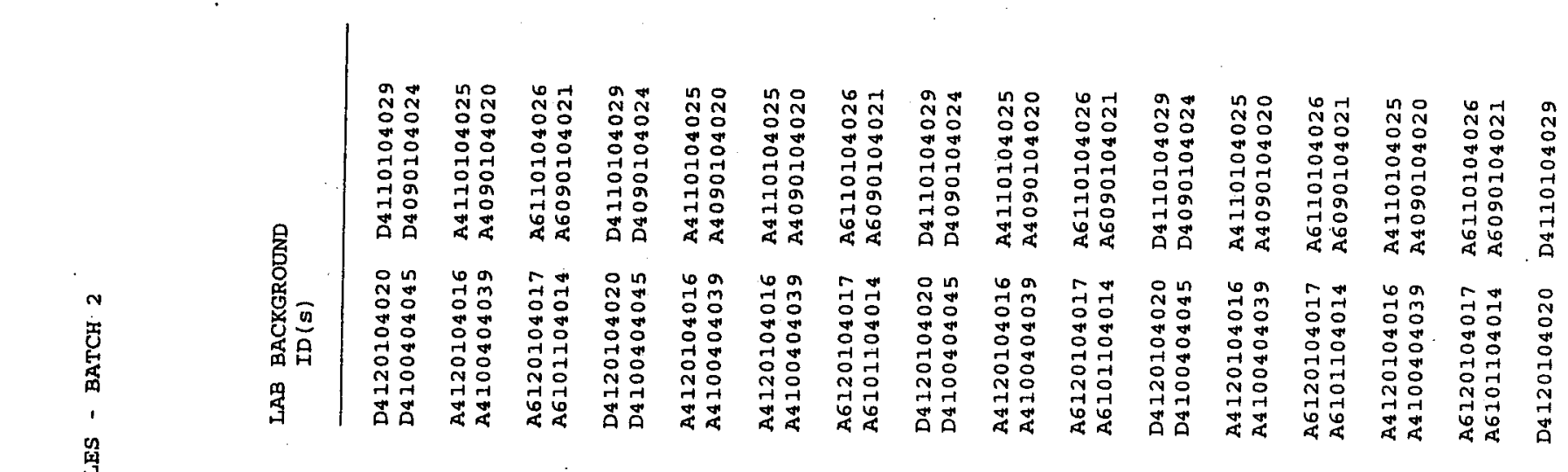

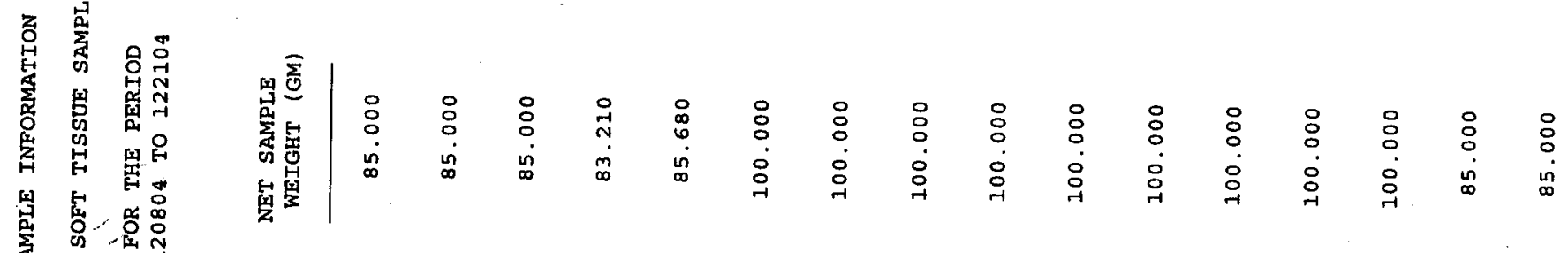
㤅

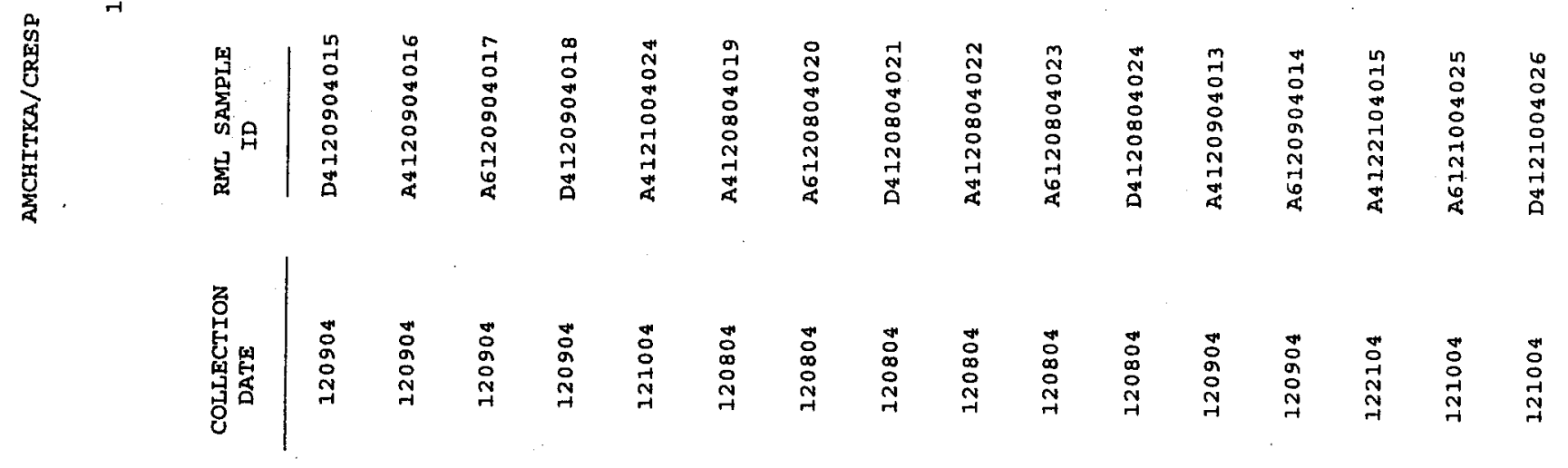

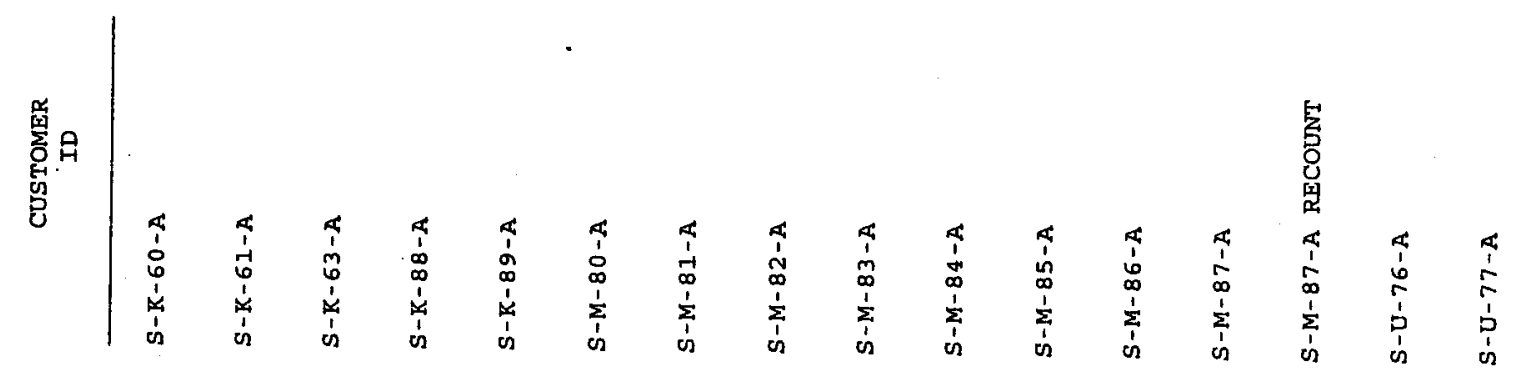




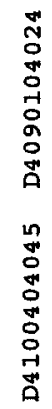




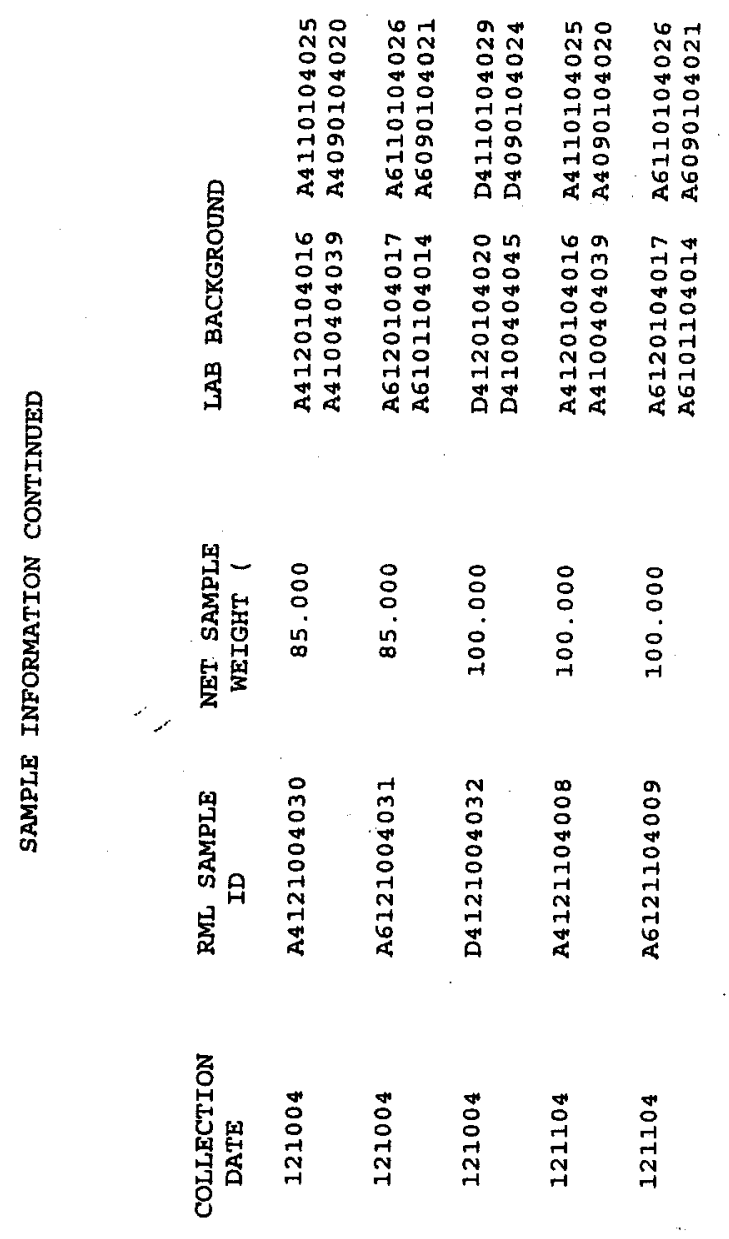

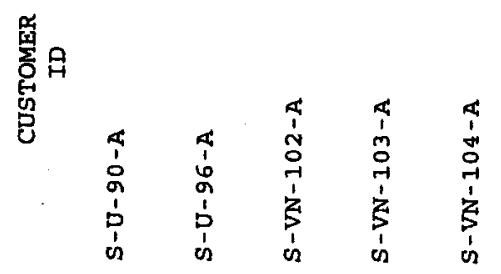



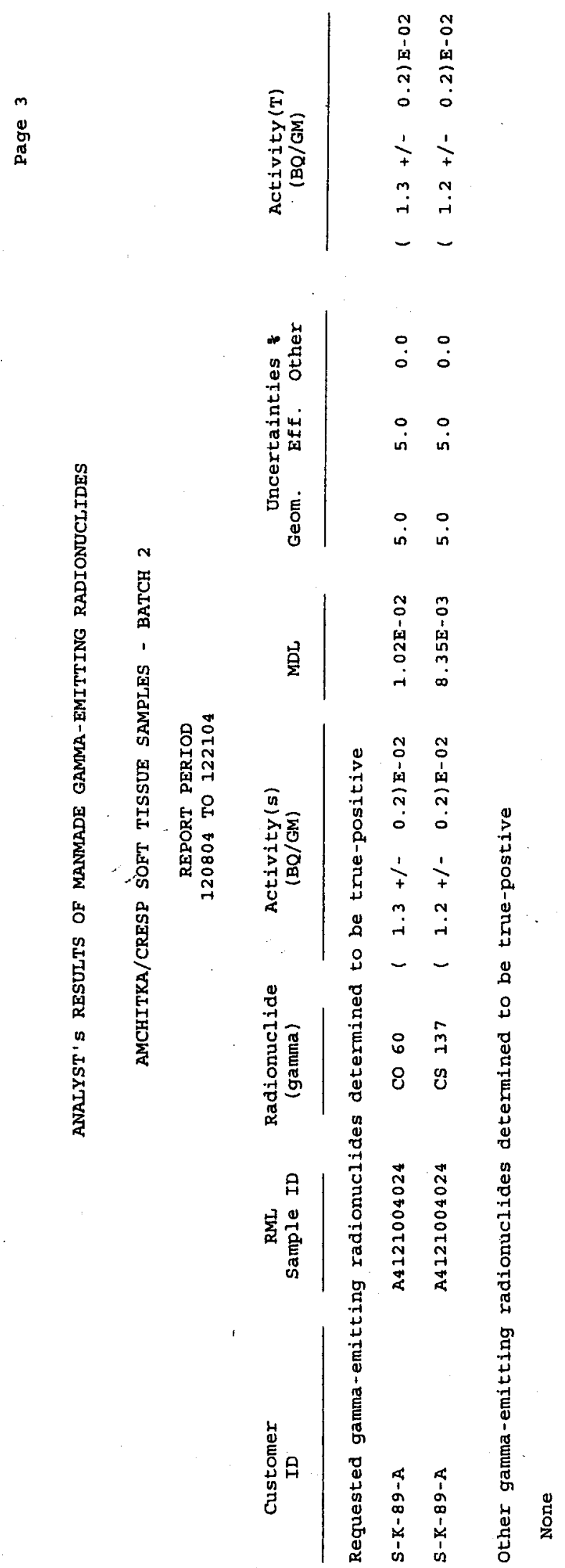


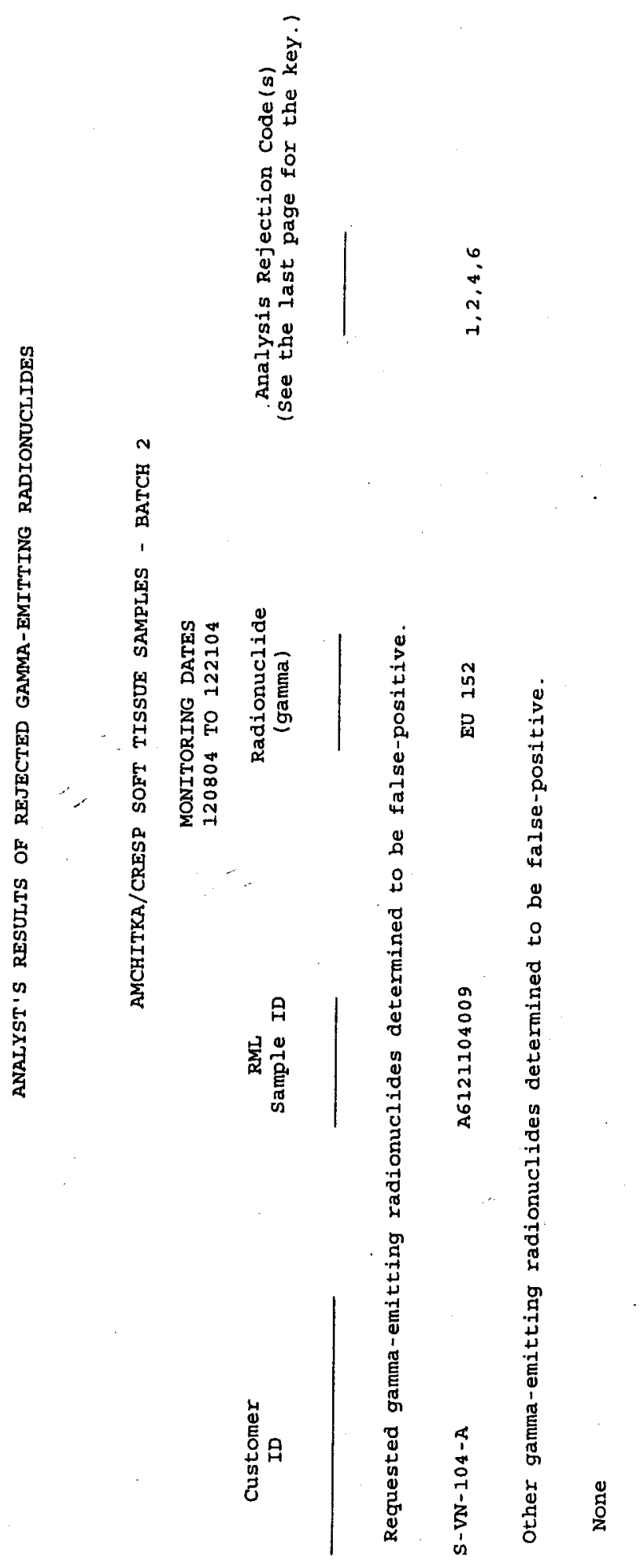




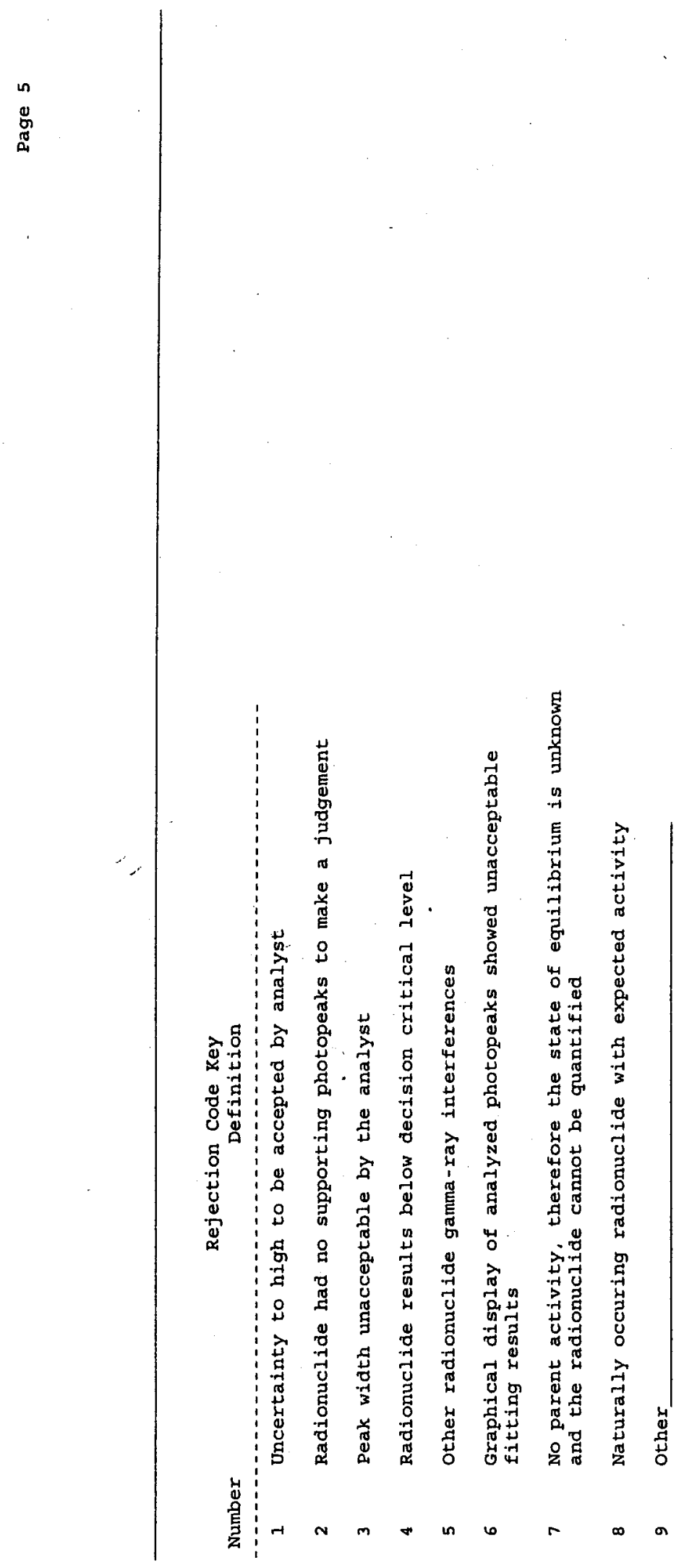




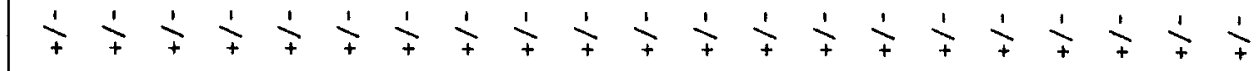

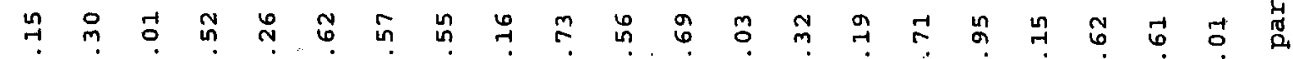

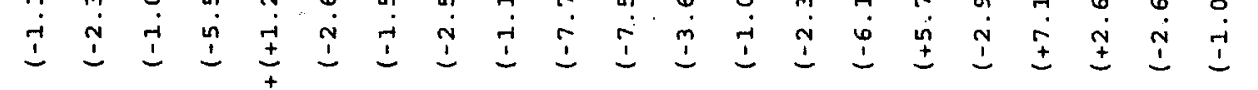

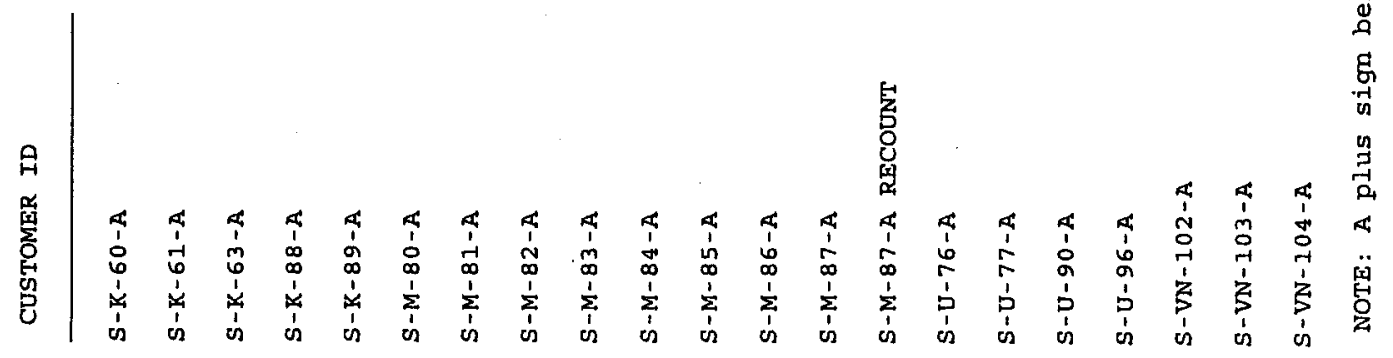


م

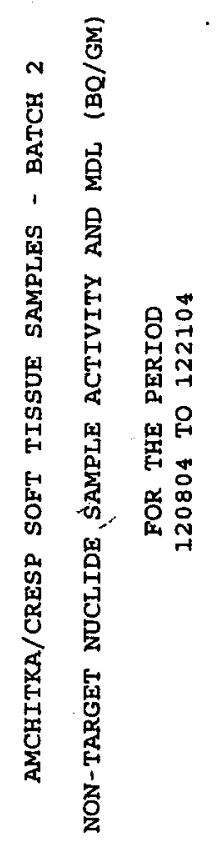



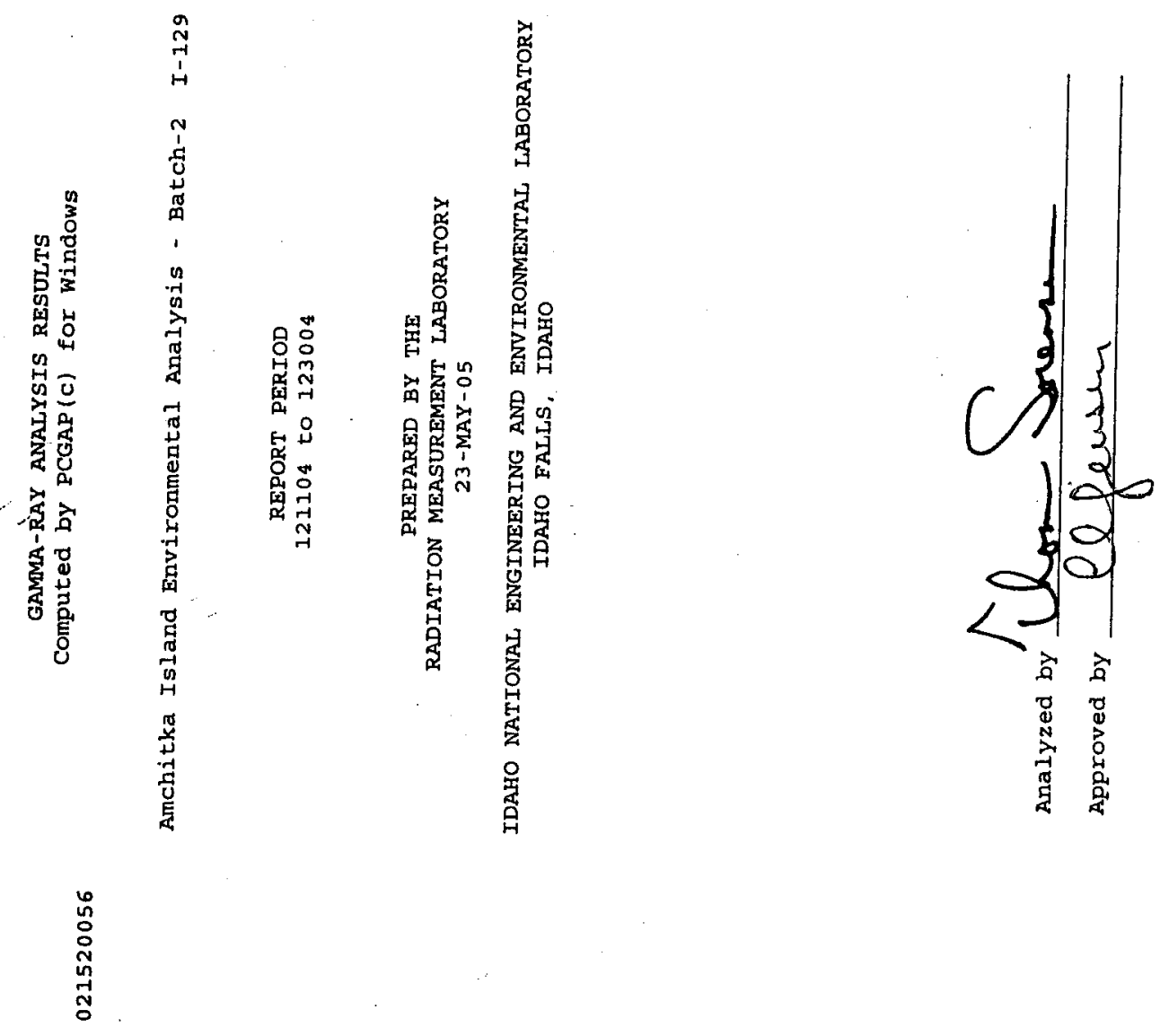


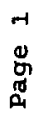

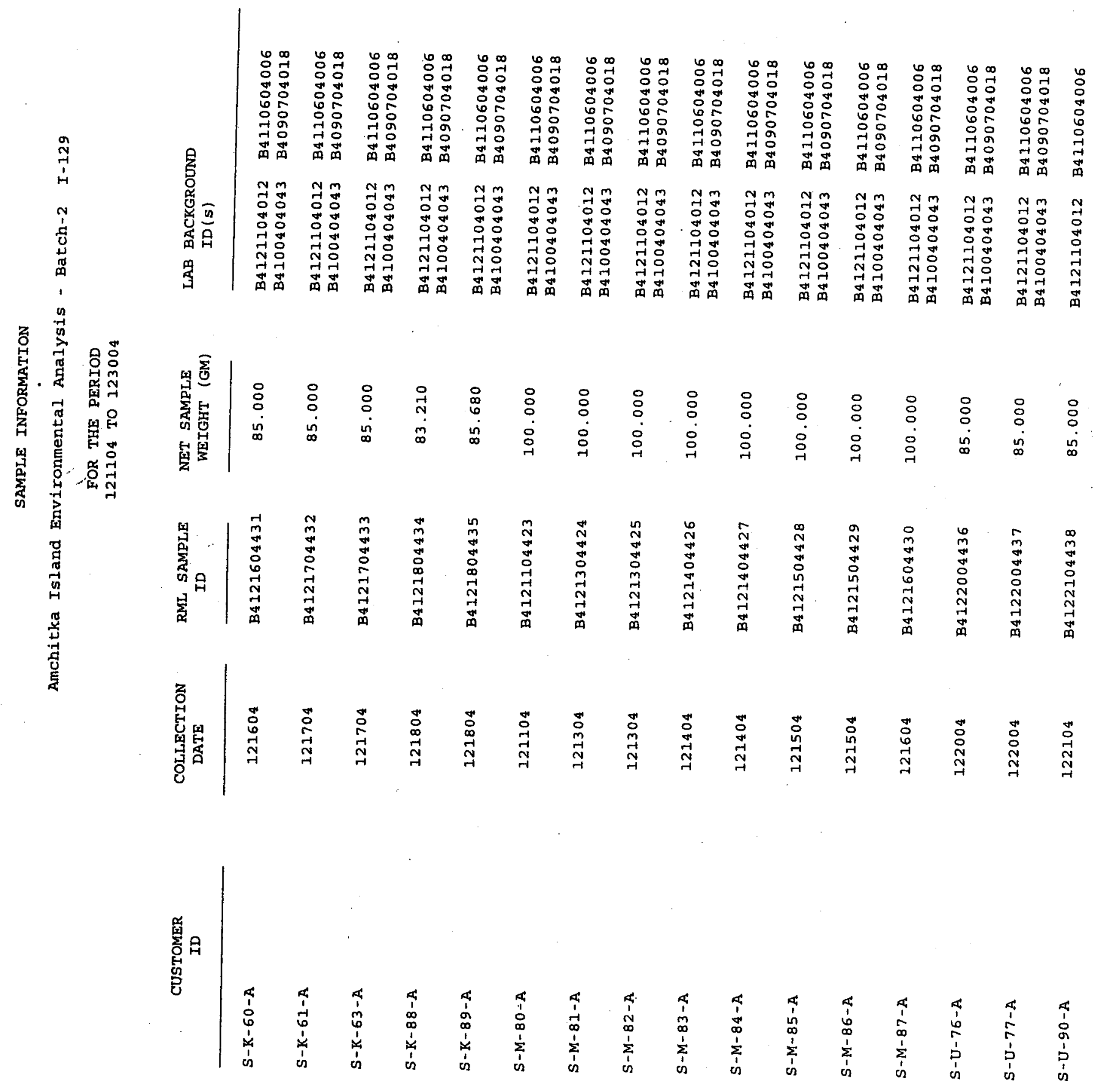




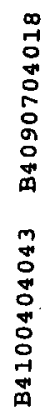




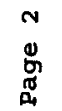

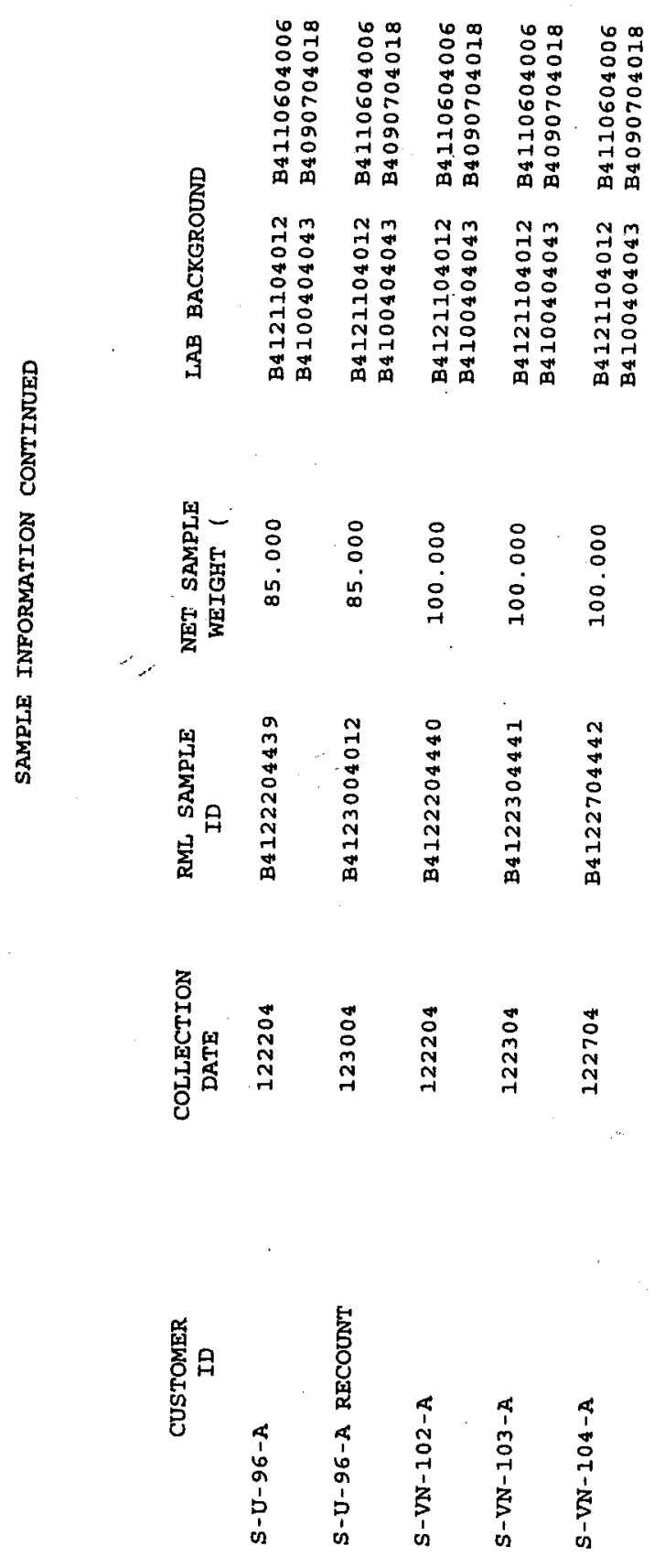




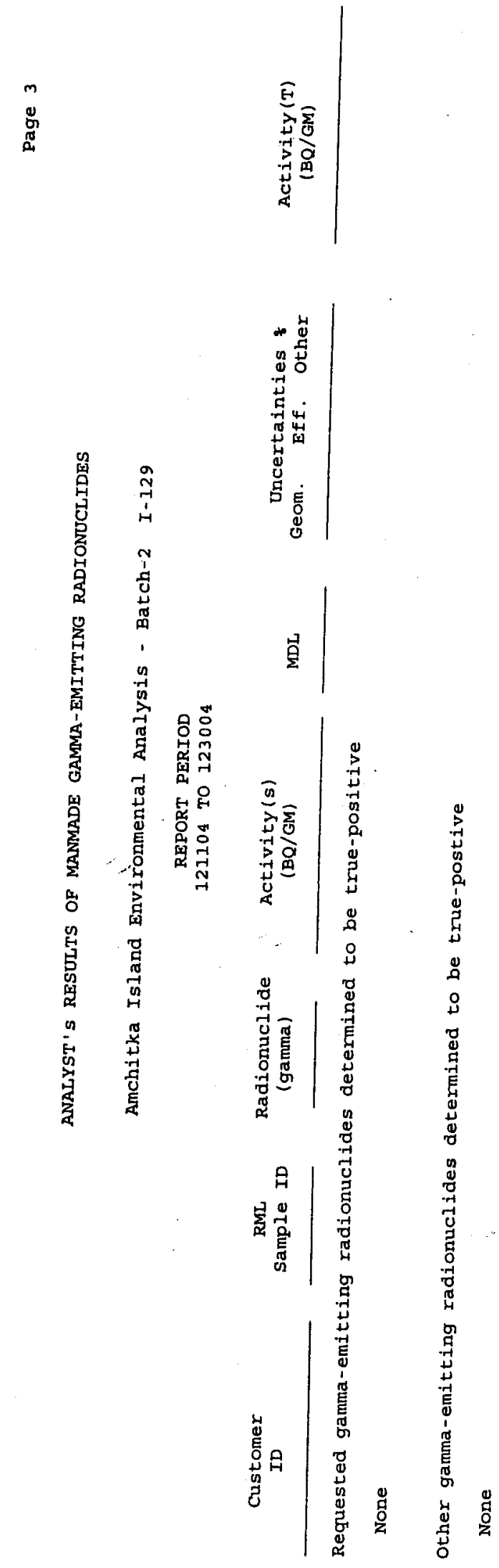


$\stackrel{+}{0}$

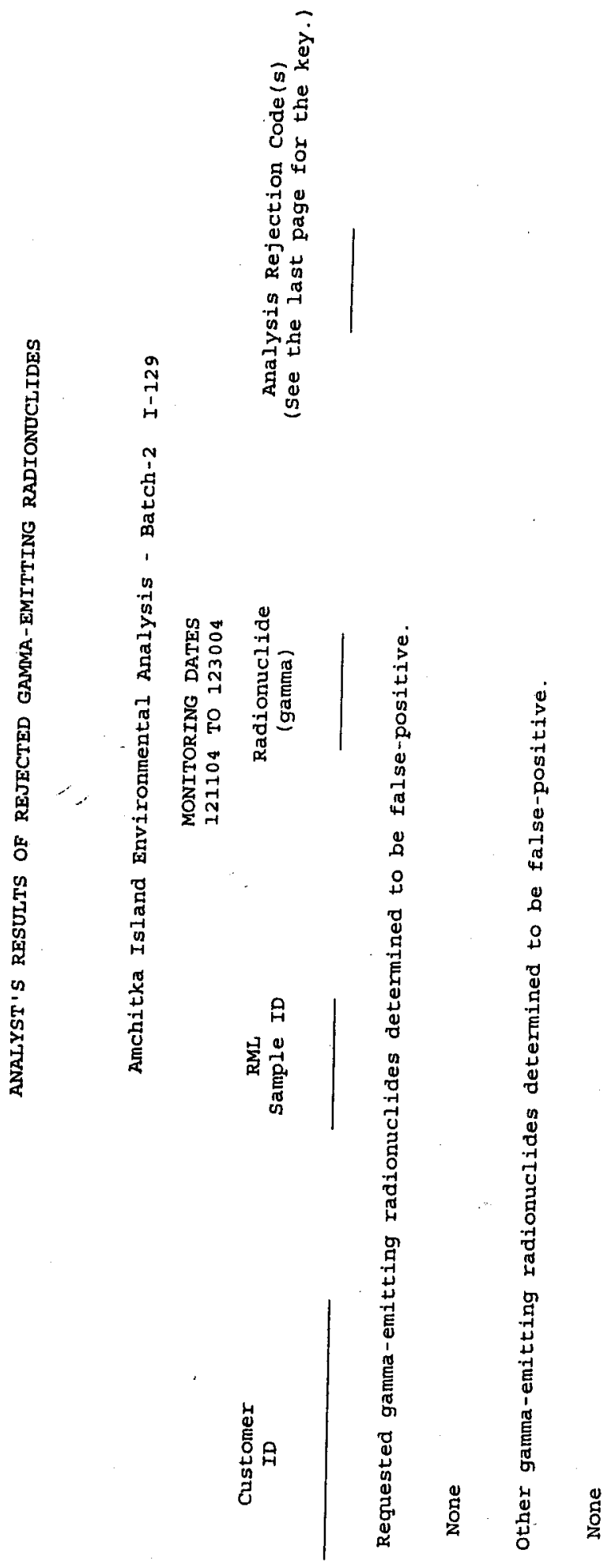




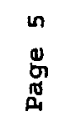

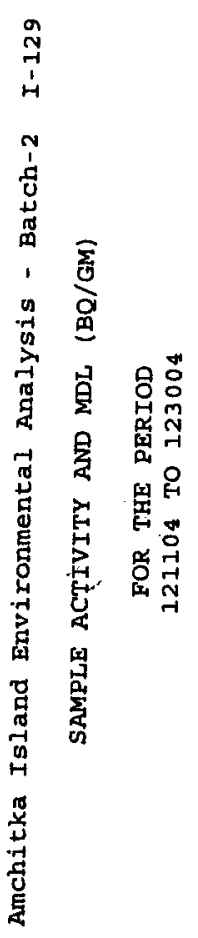

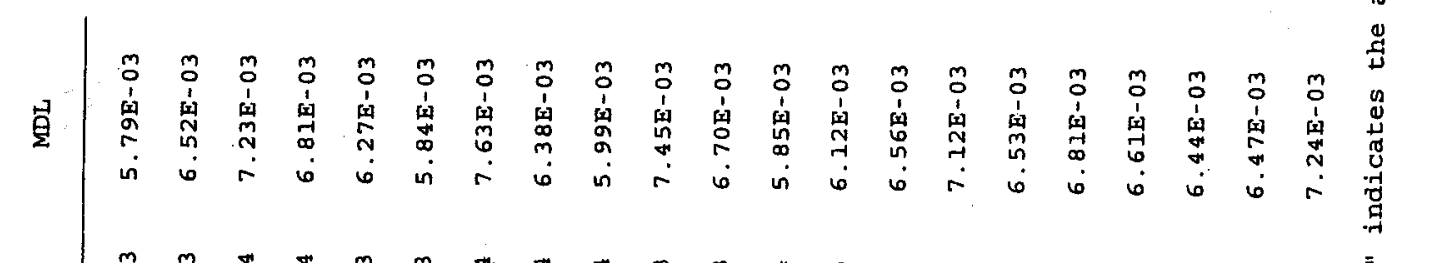

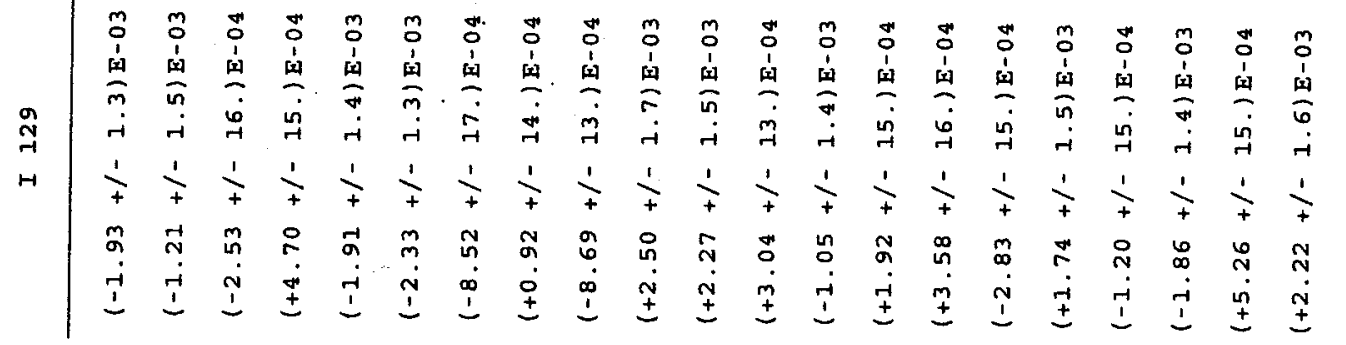

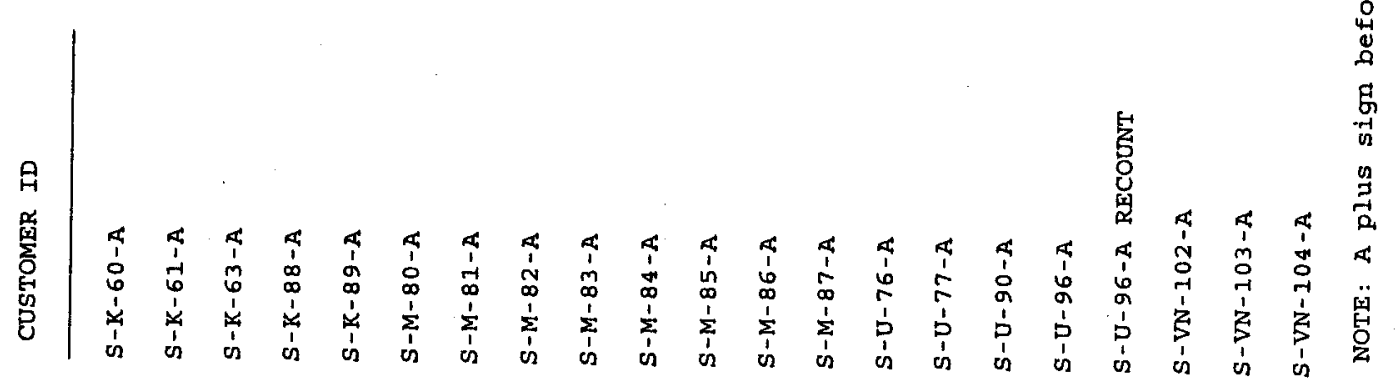


0
0
0
0
0

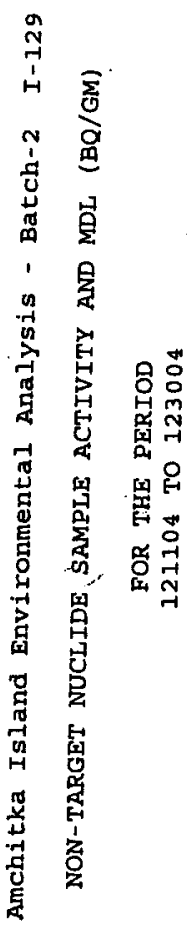

๕ี 
BATCH 3

GAMMA AND I-129 


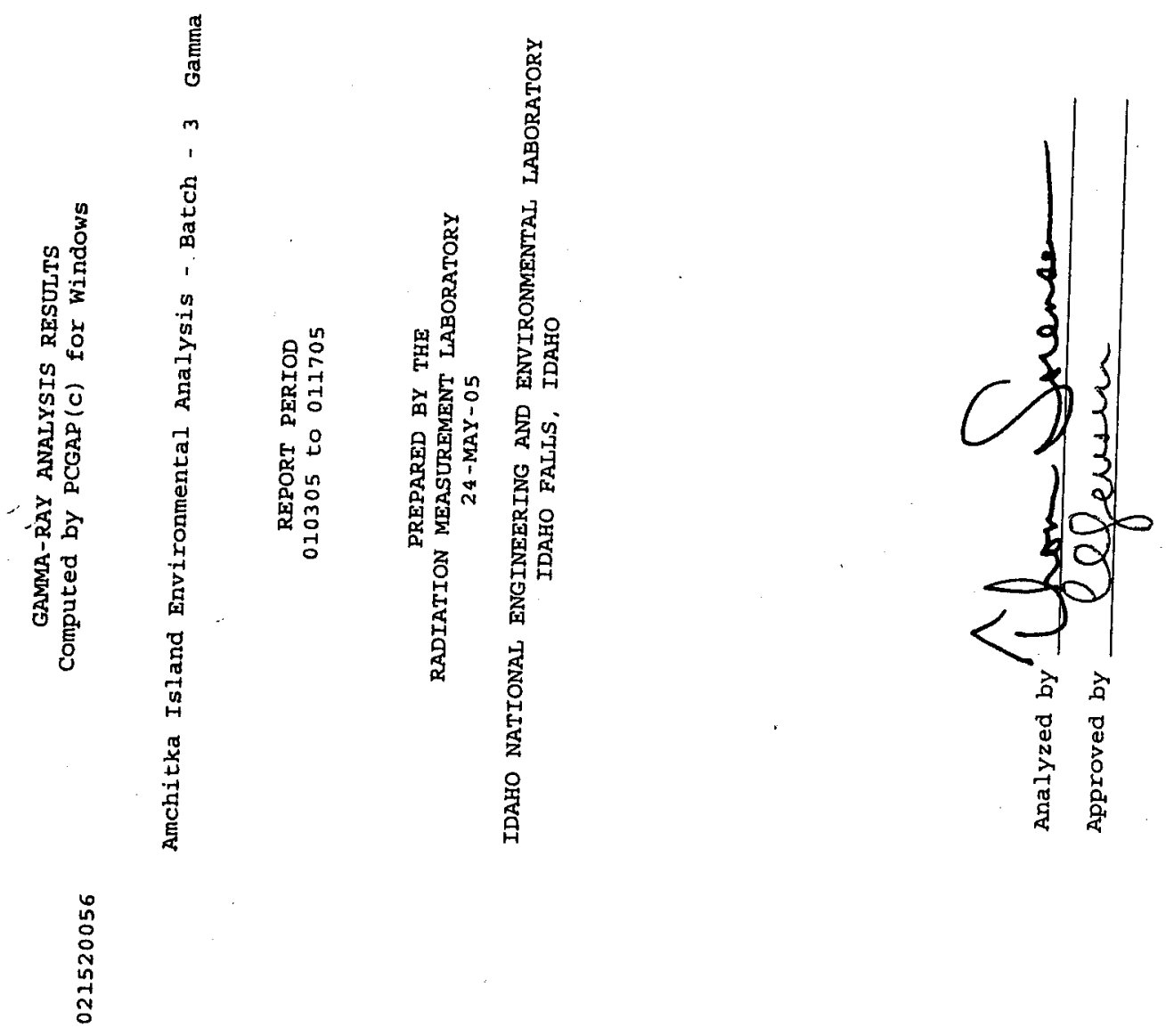


-1
8
8
0
0

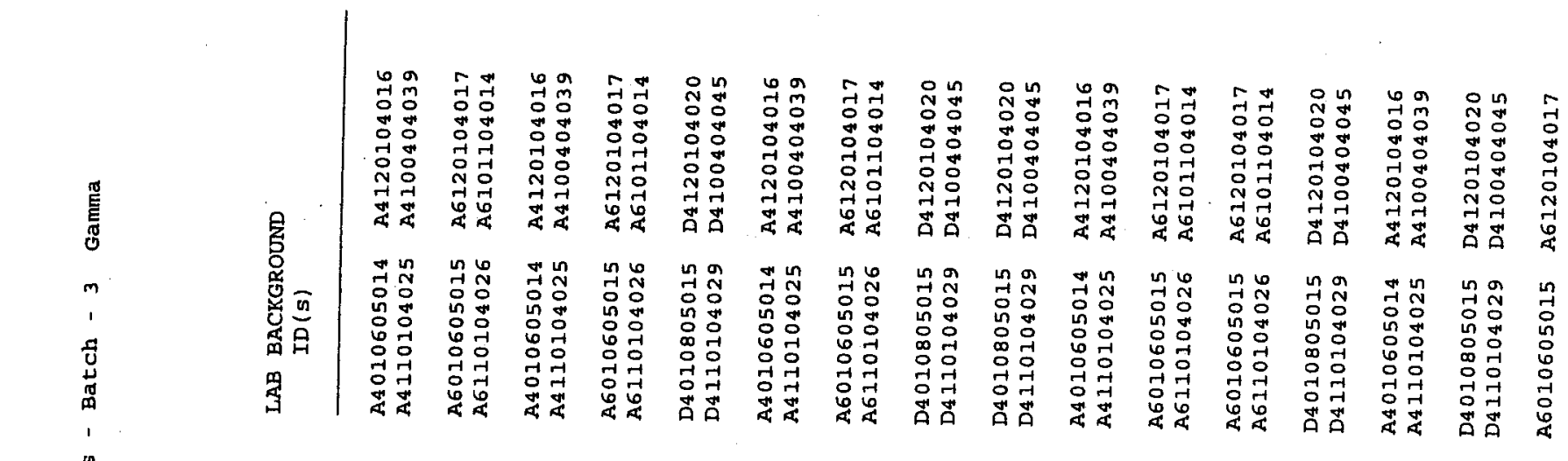

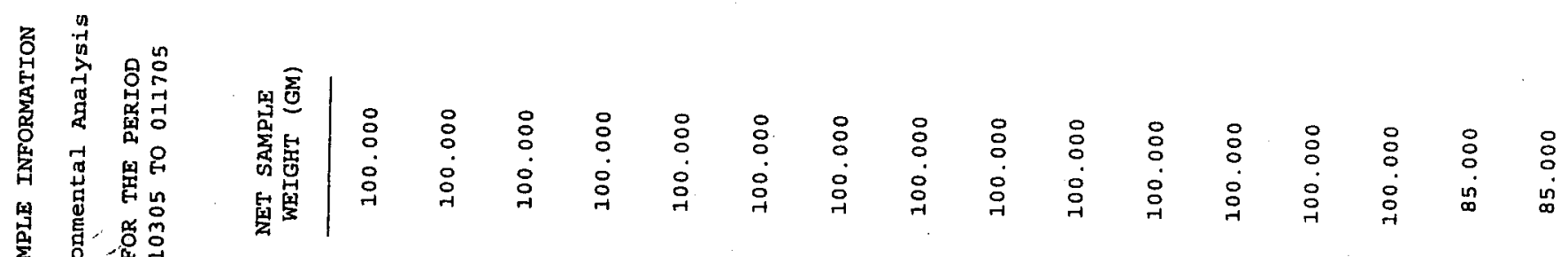

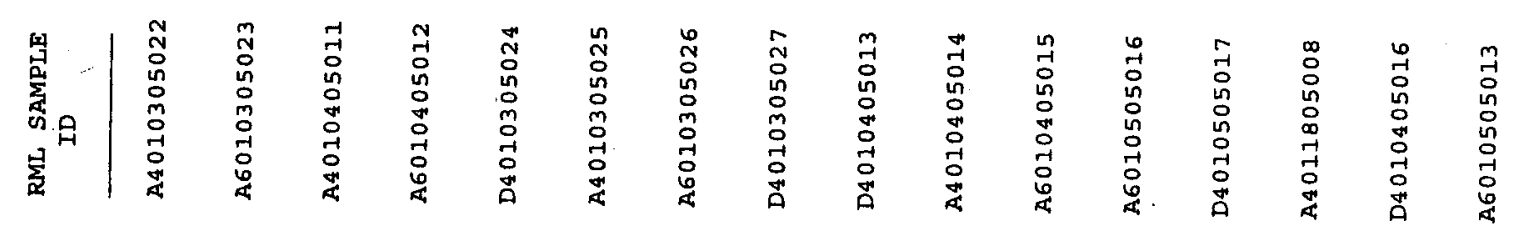

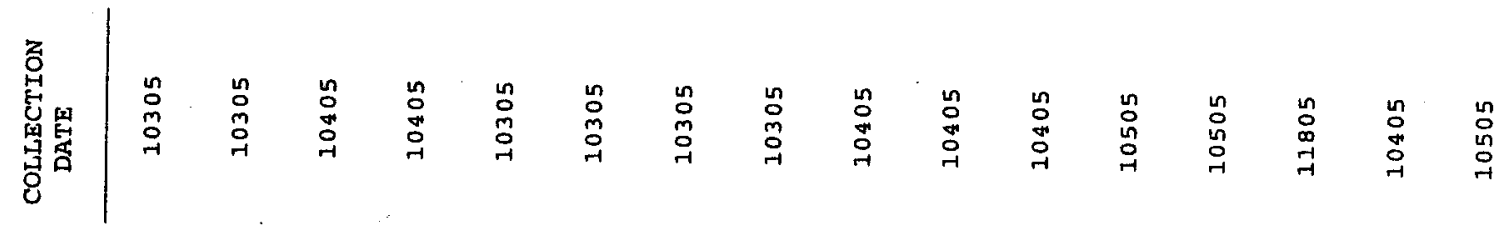

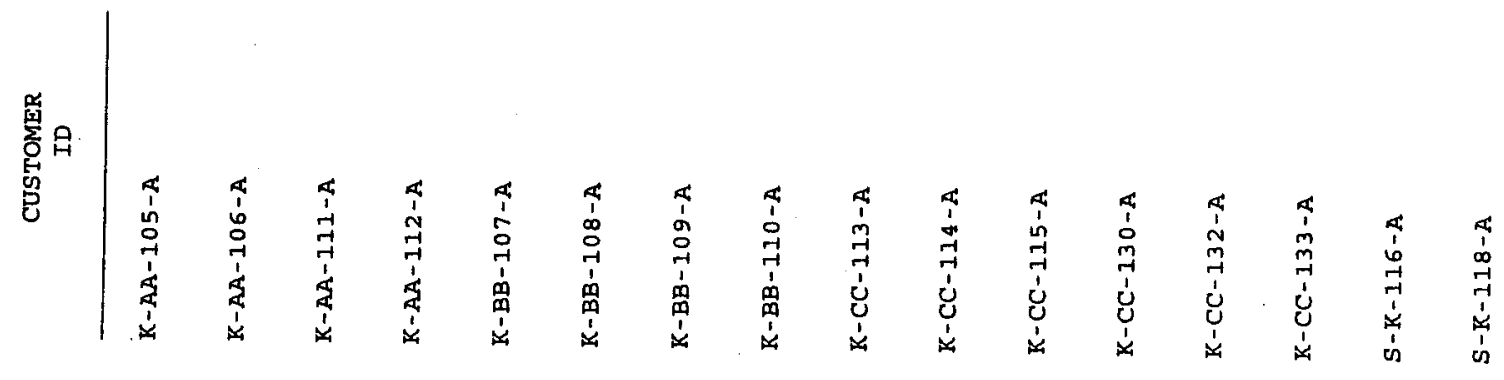




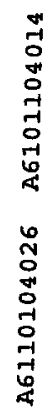


N

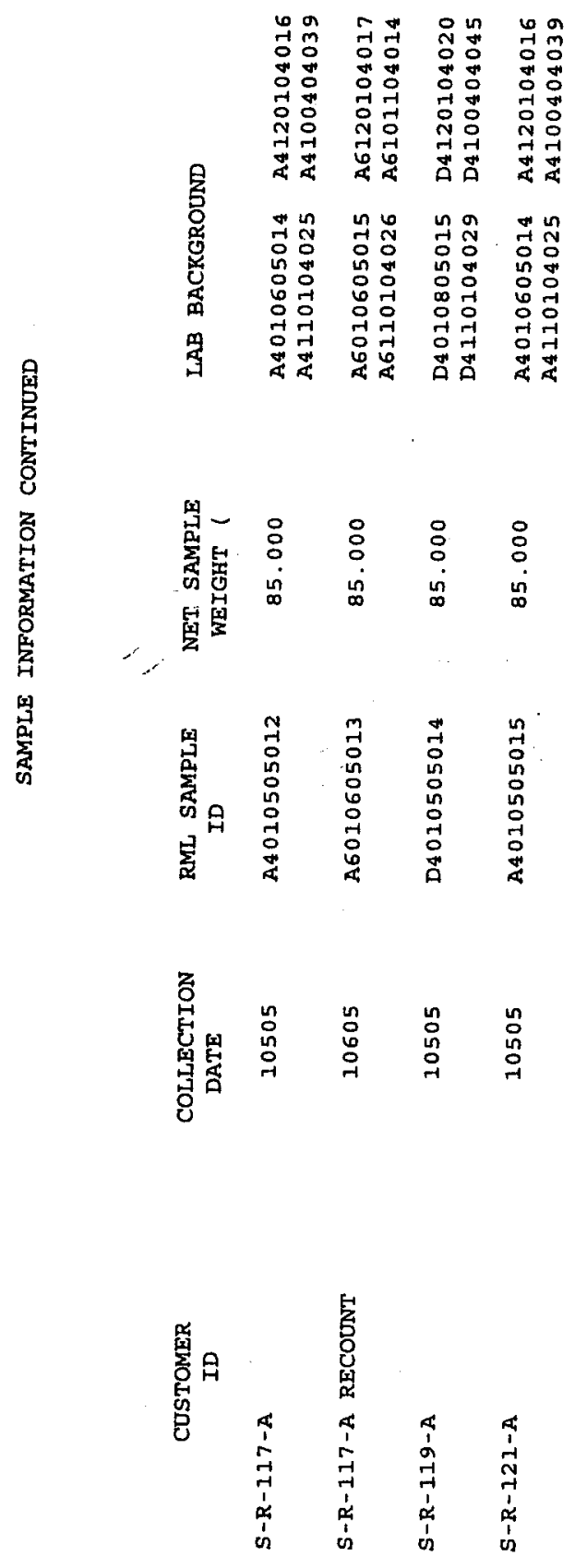



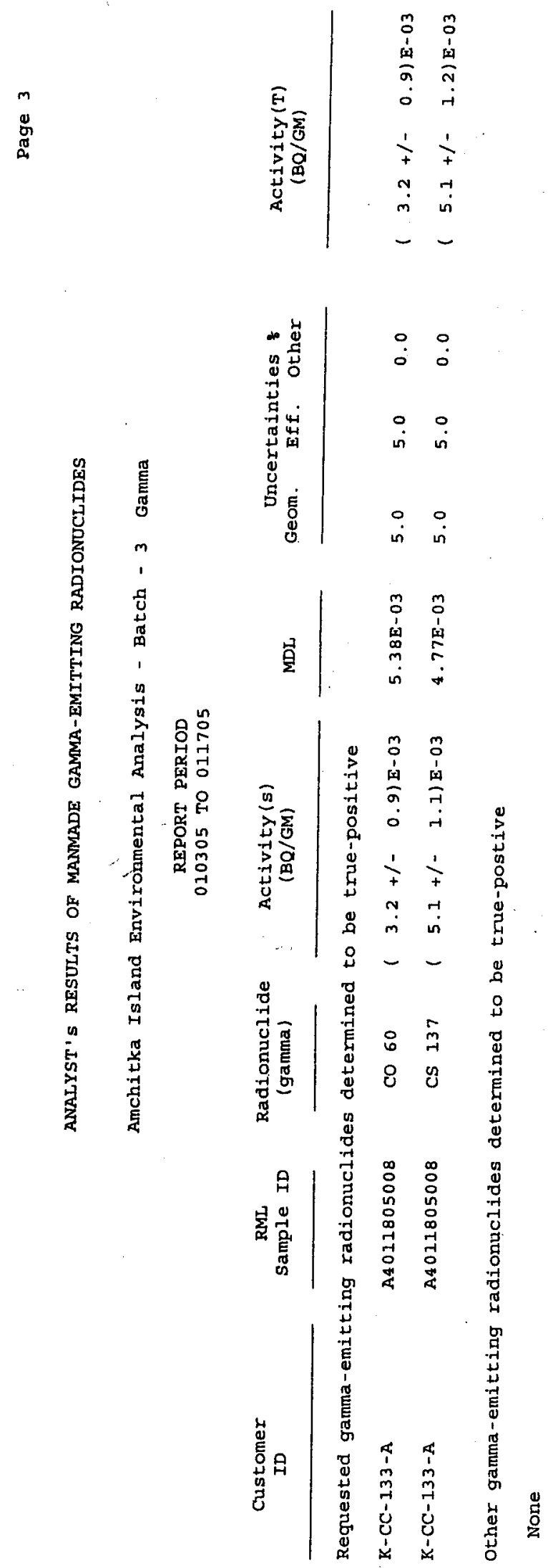
$\frac{1}{2}$

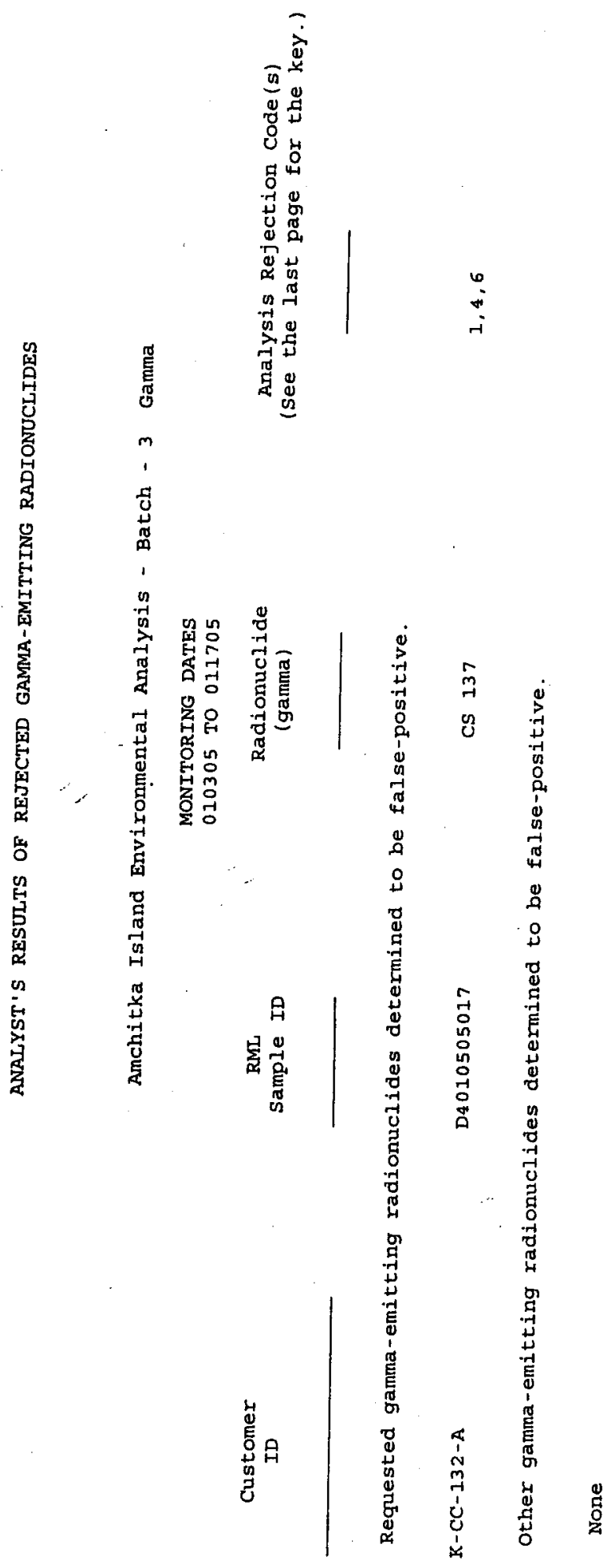




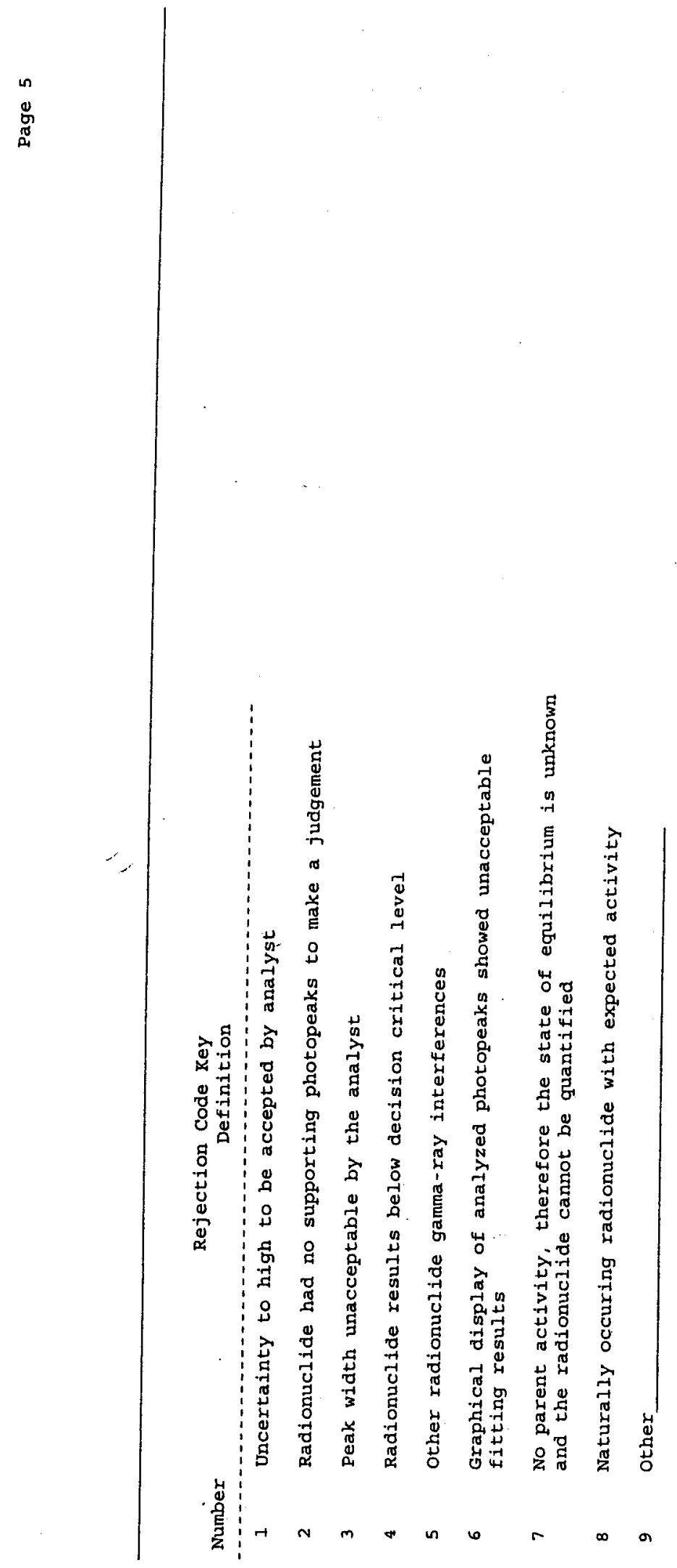


0
0
0
0
0

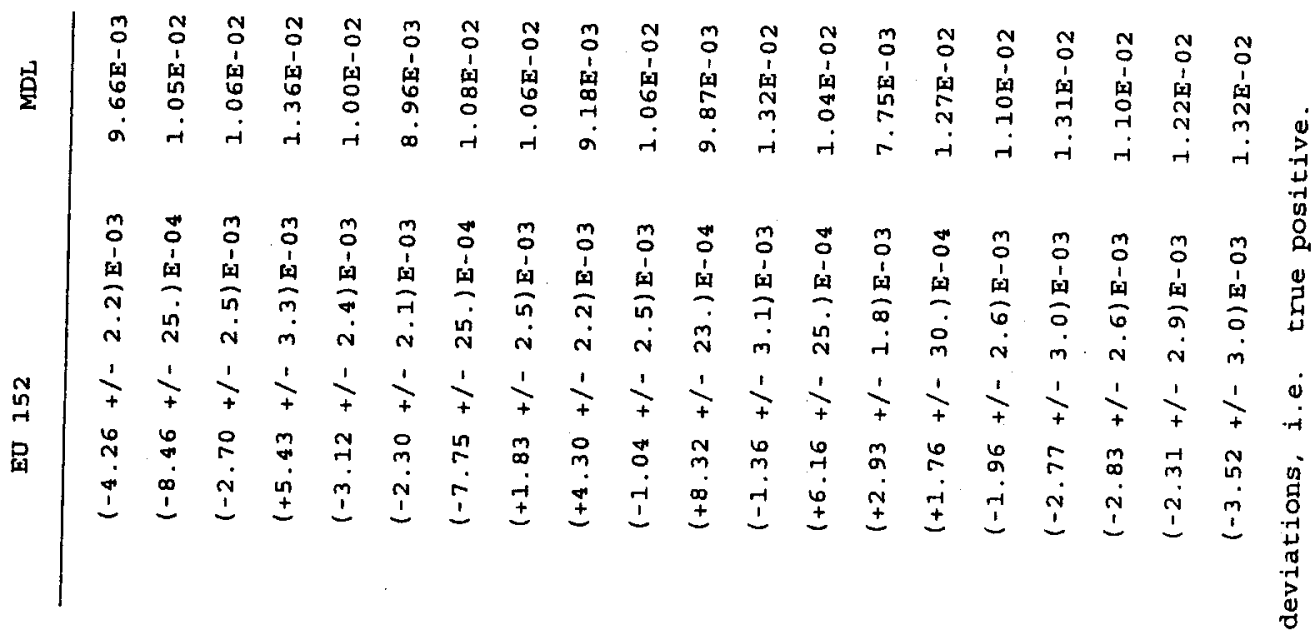

䒿

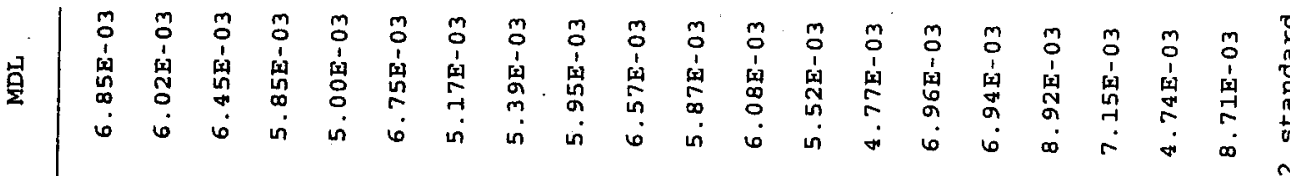

!

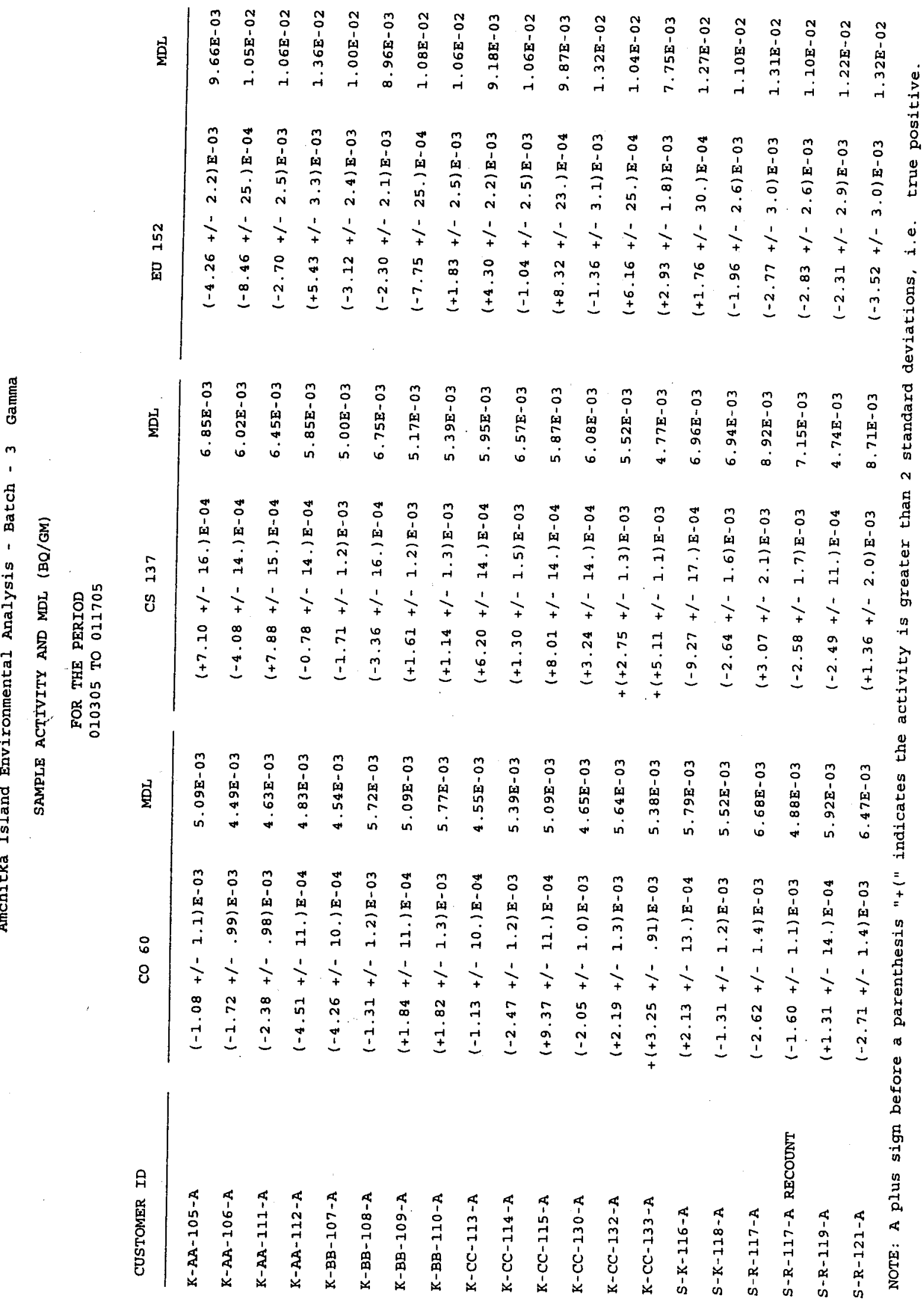


$n$
0
0
0
0

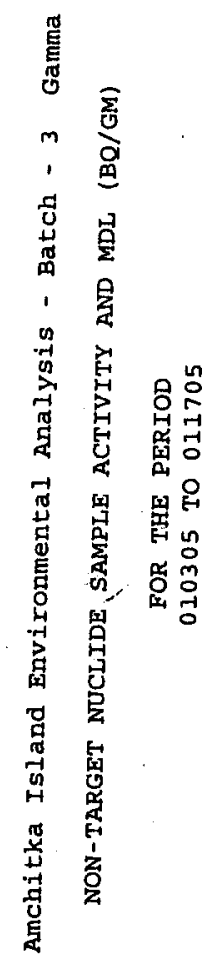

$\stackrel{0}{0}$ 

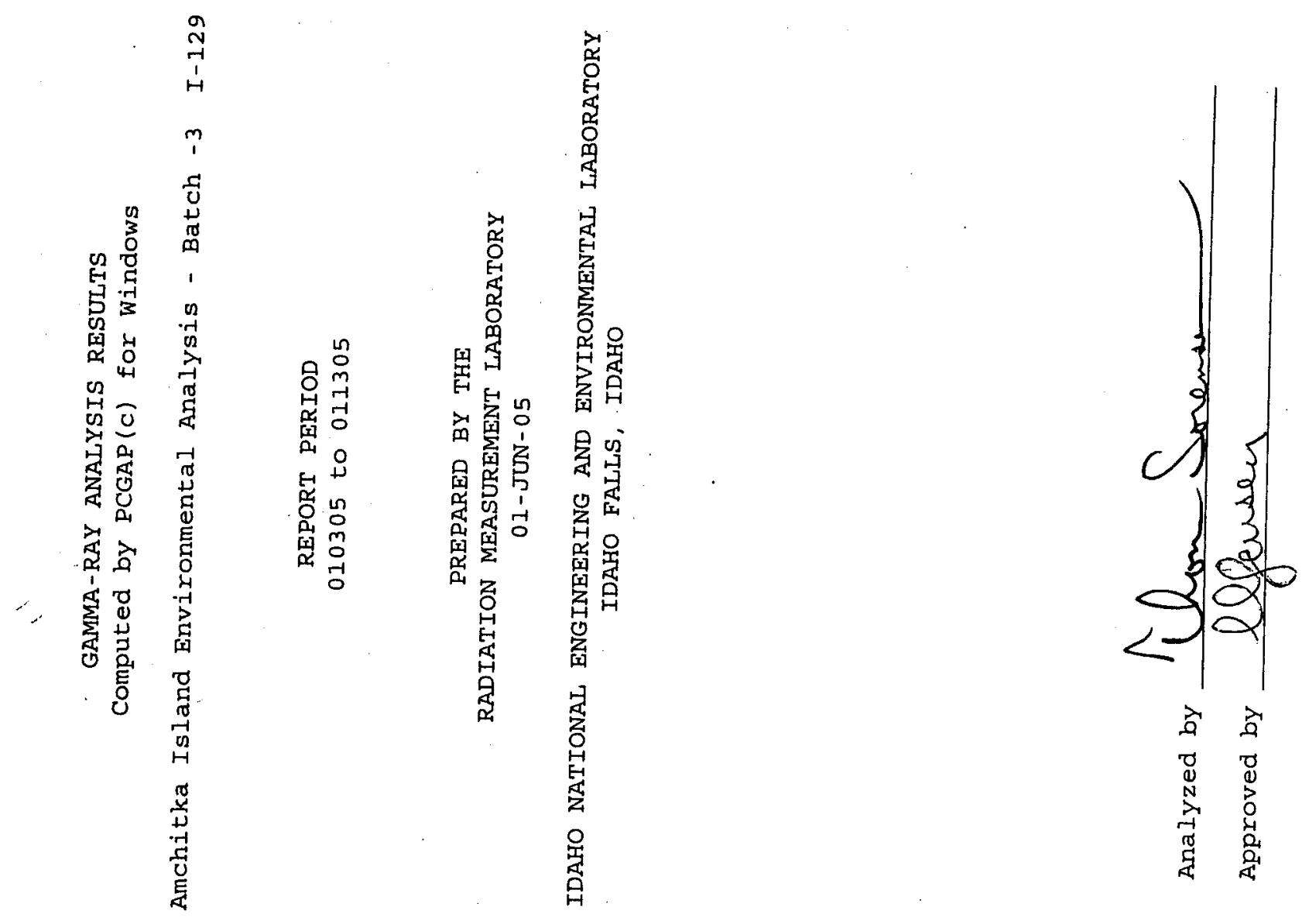

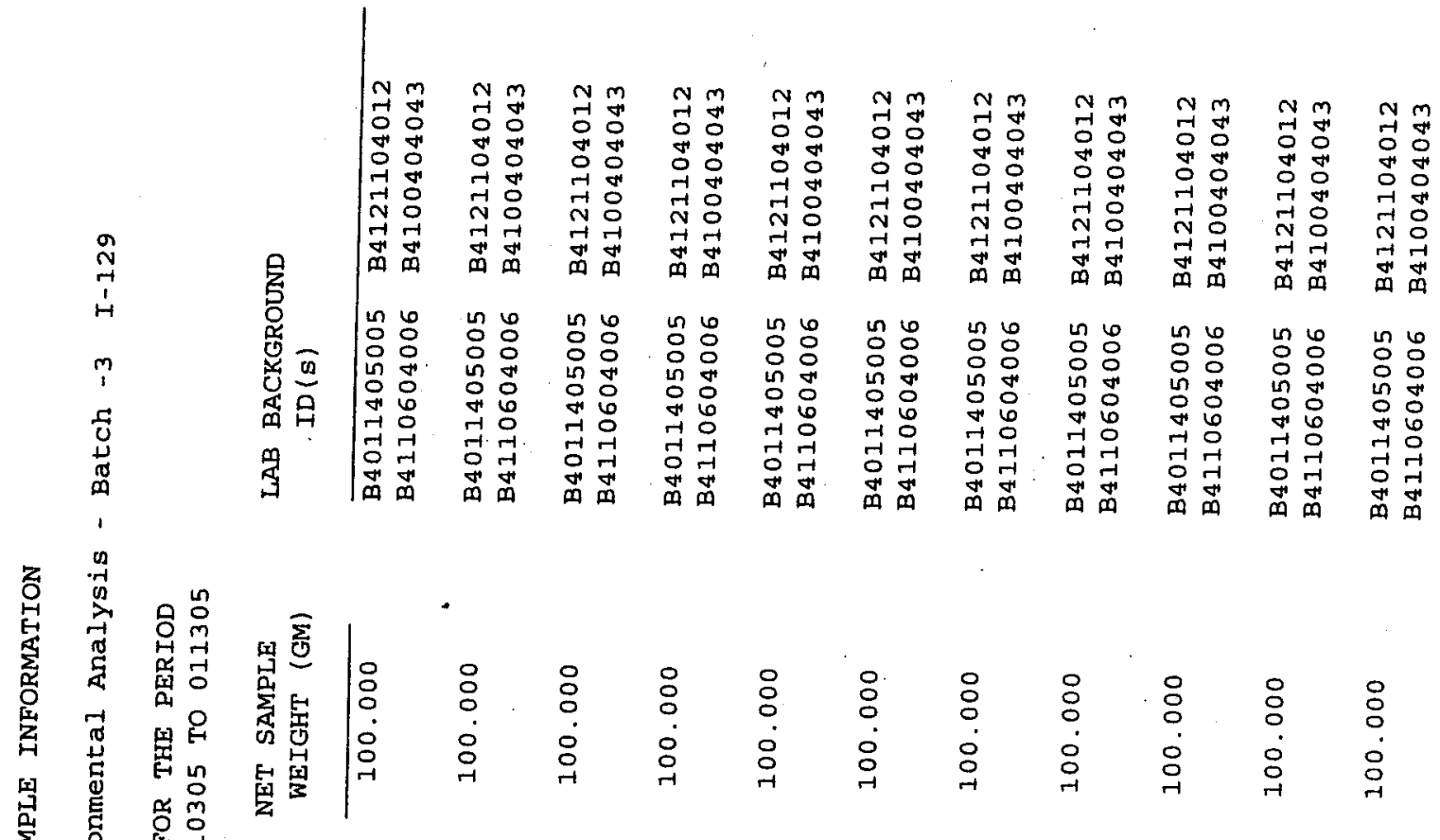
呈年

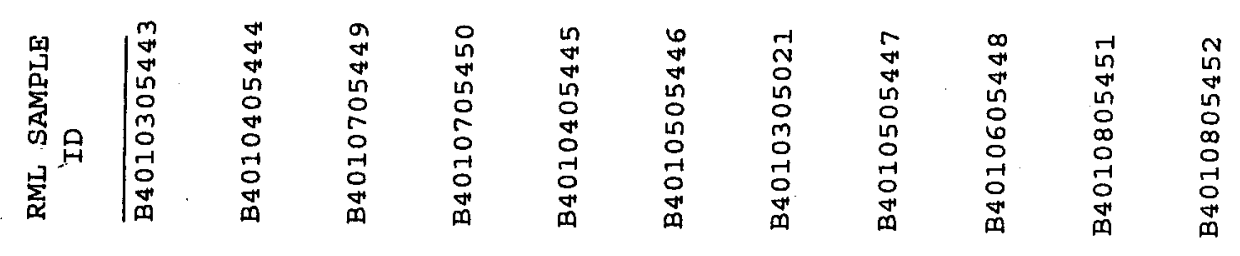

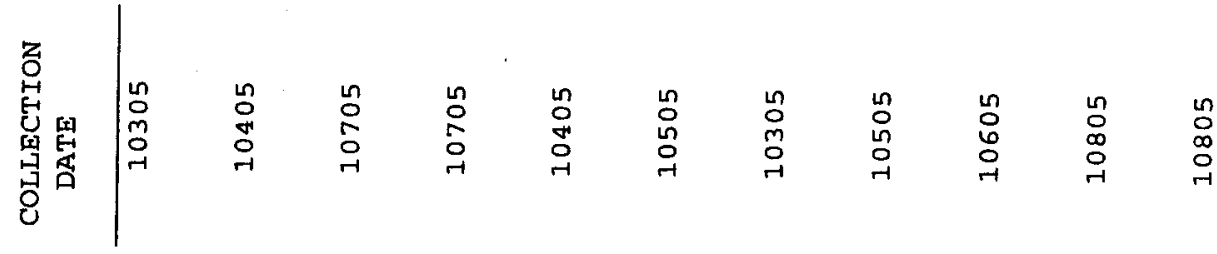

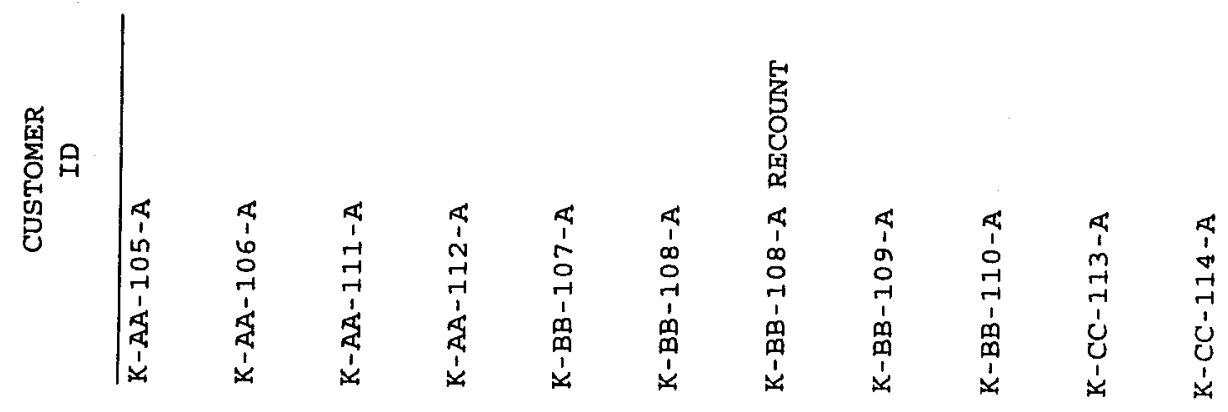




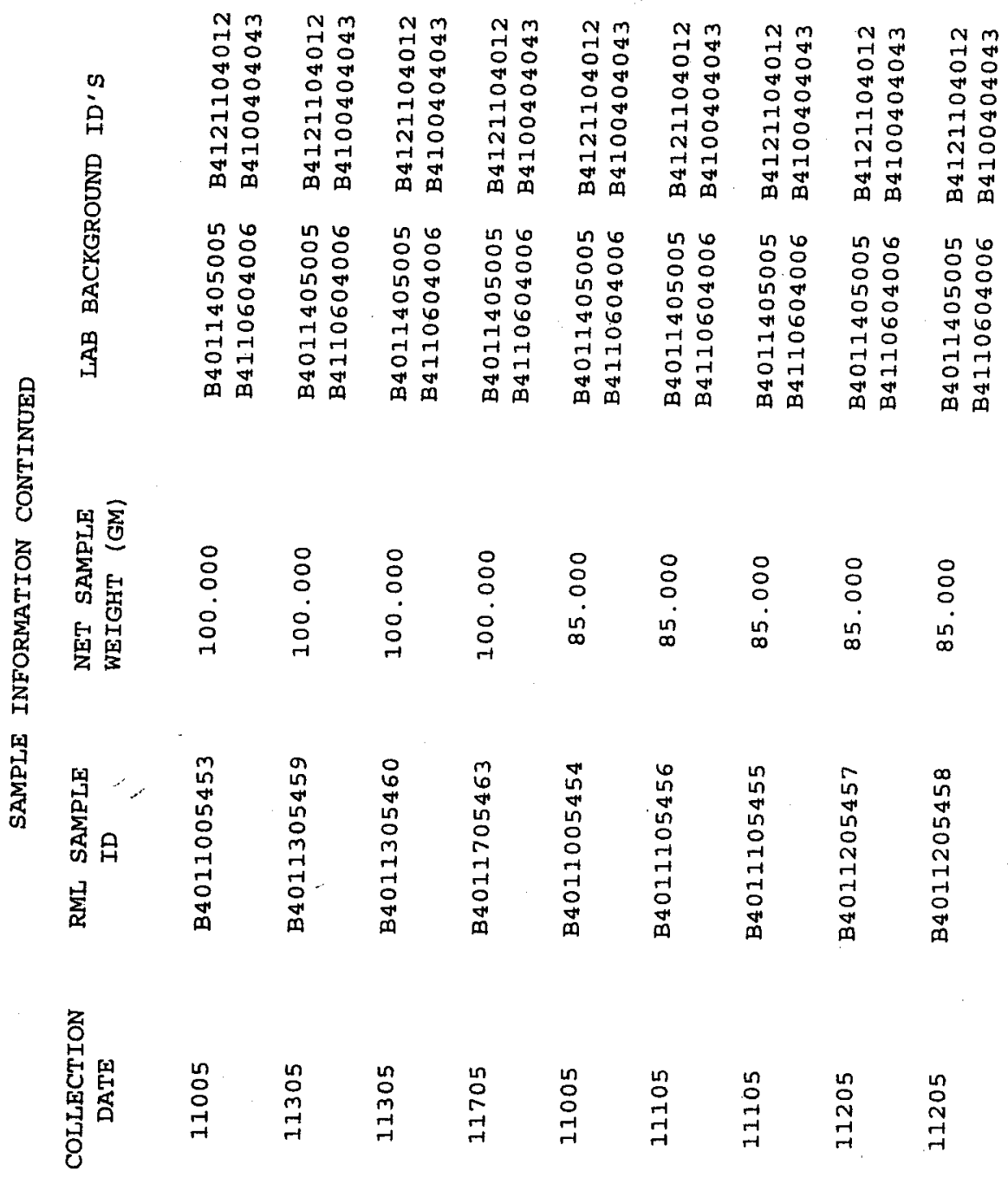

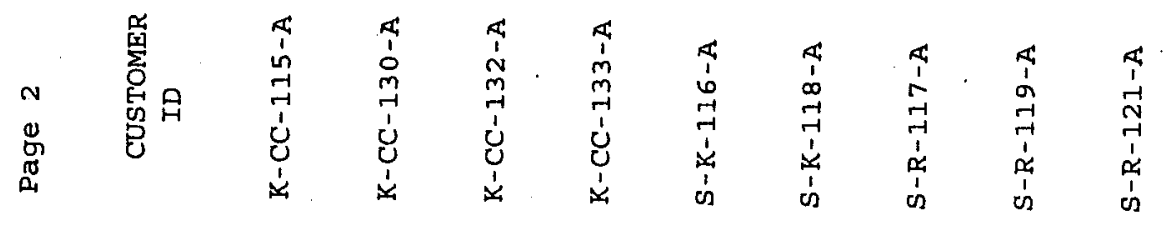




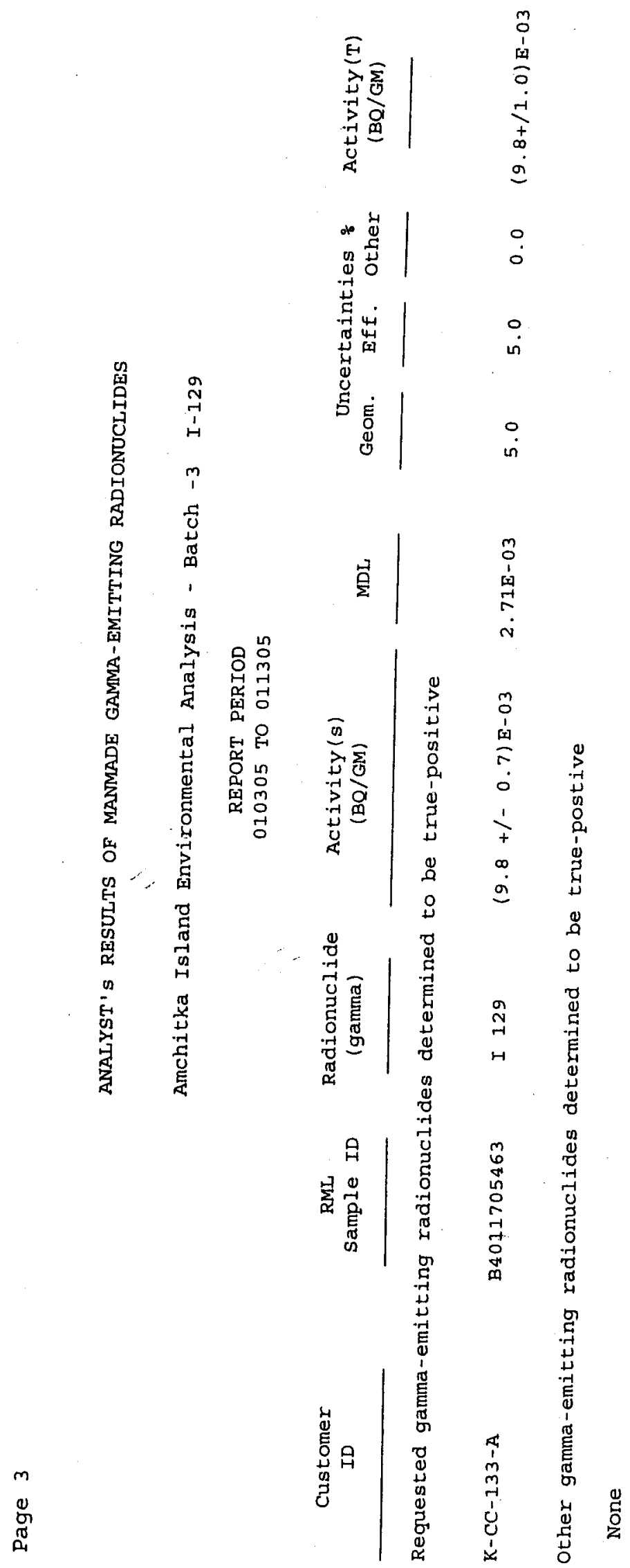




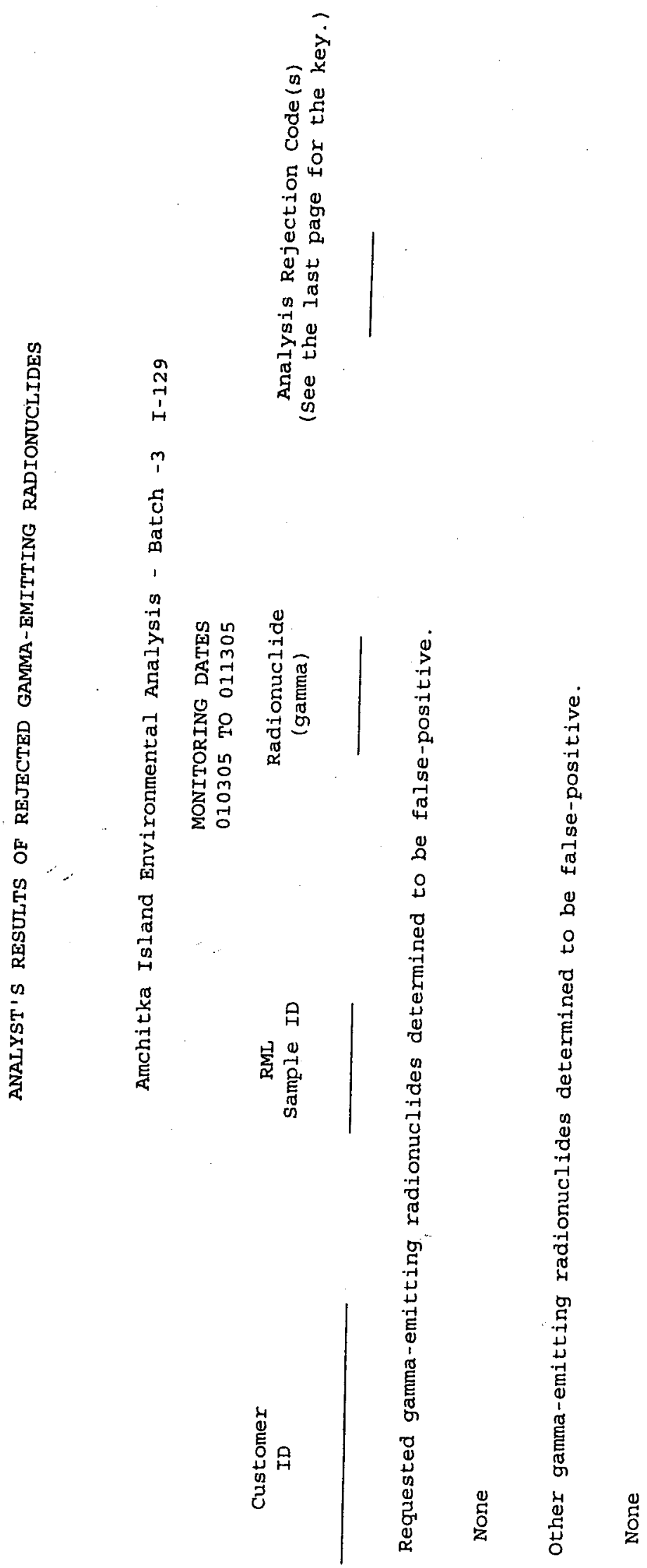


$\omega$
0
$\sigma$
0

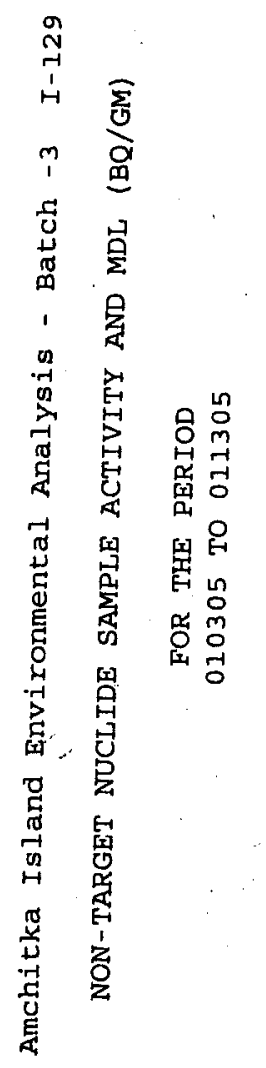

0
0
○ 
BATCH 4

GAMMA 


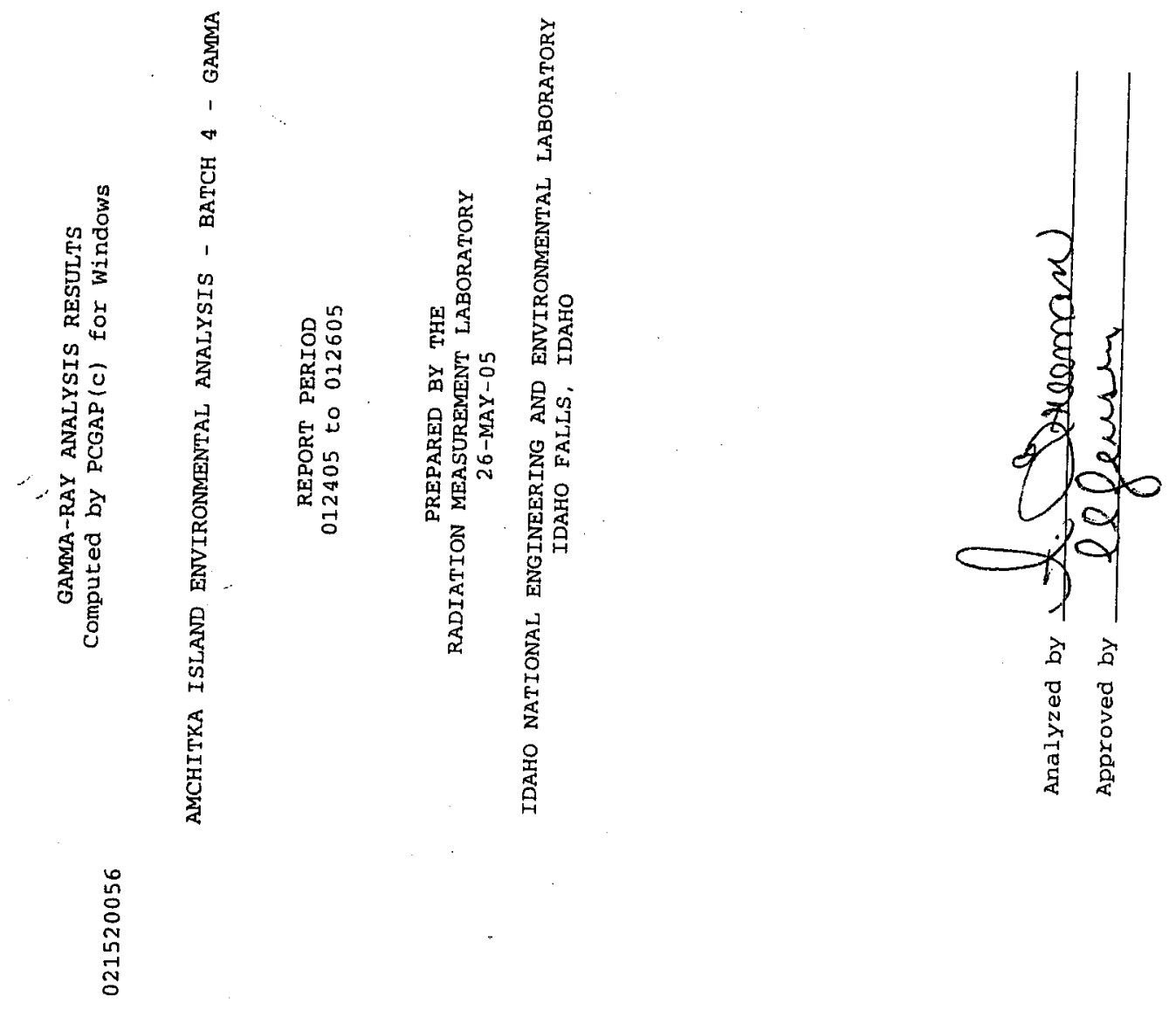


吾

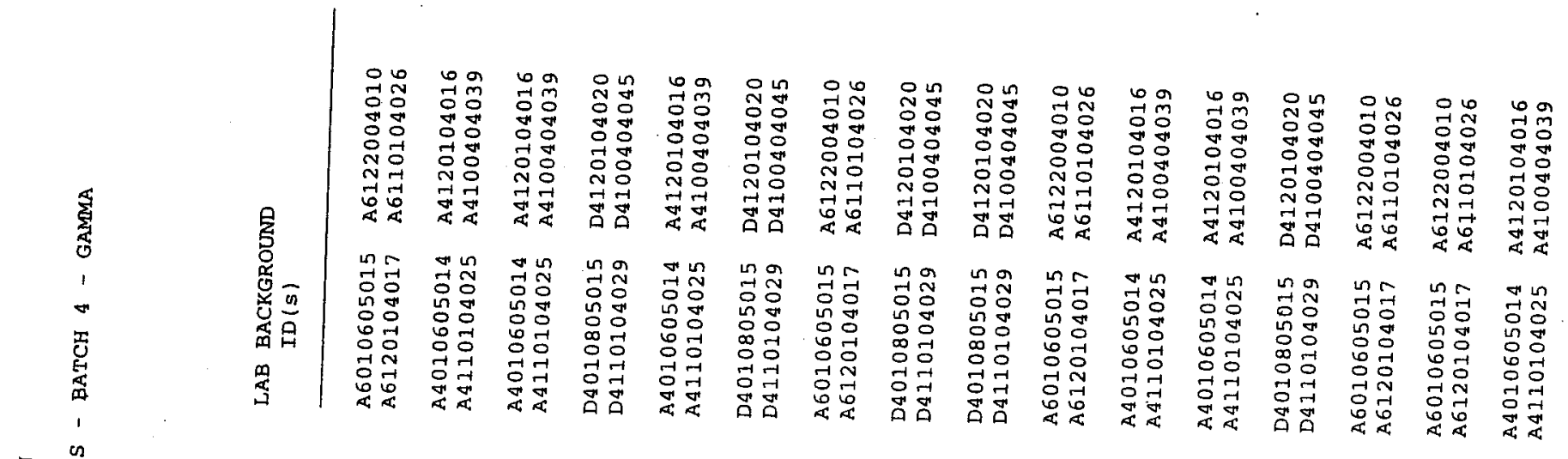

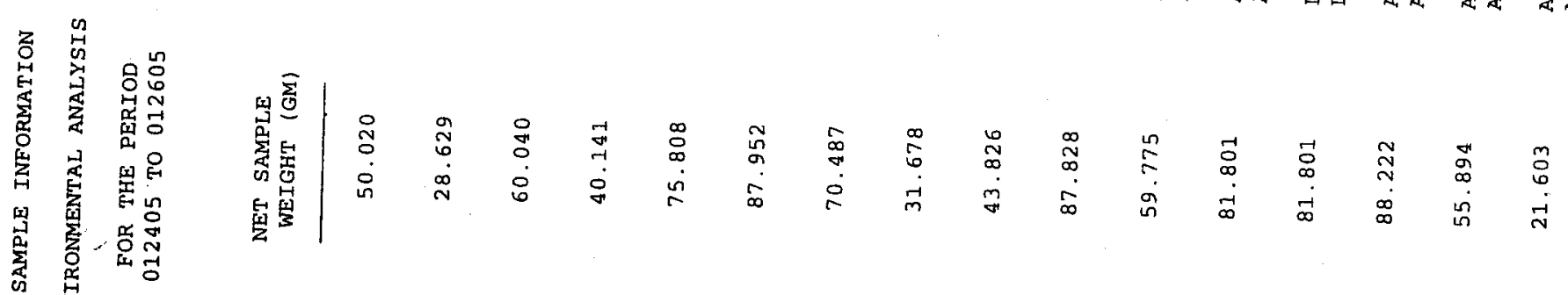

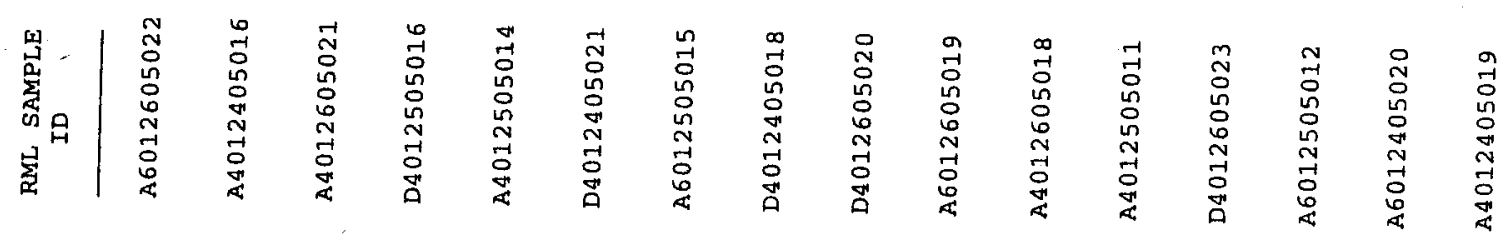

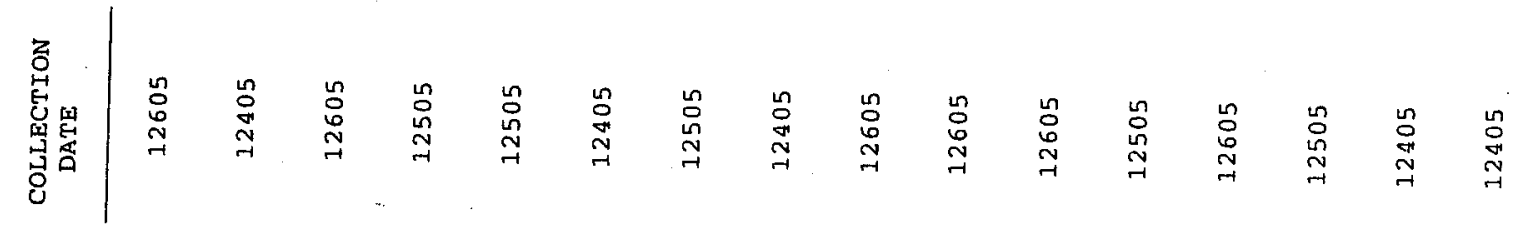

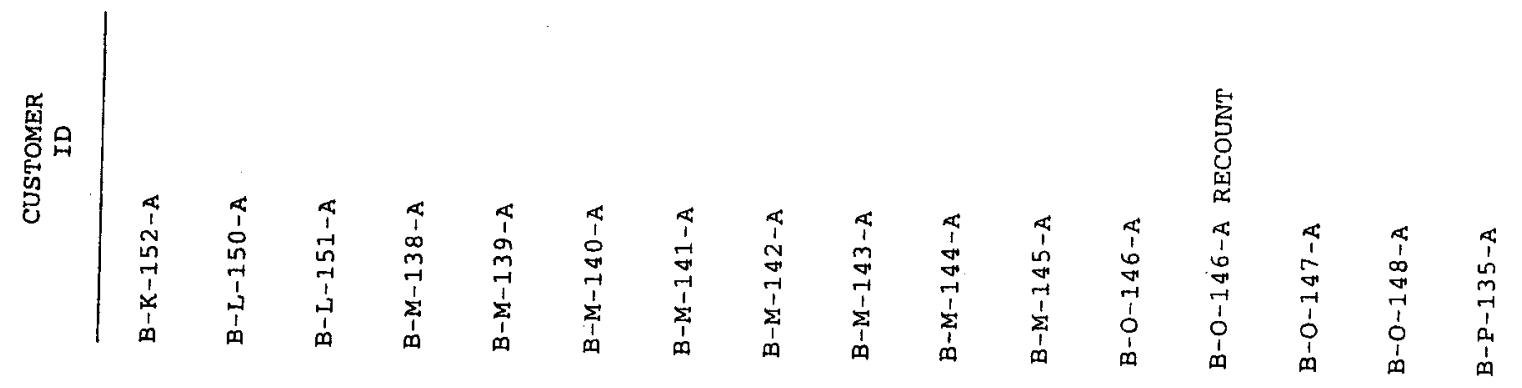


$*$
0
0
0
0
0

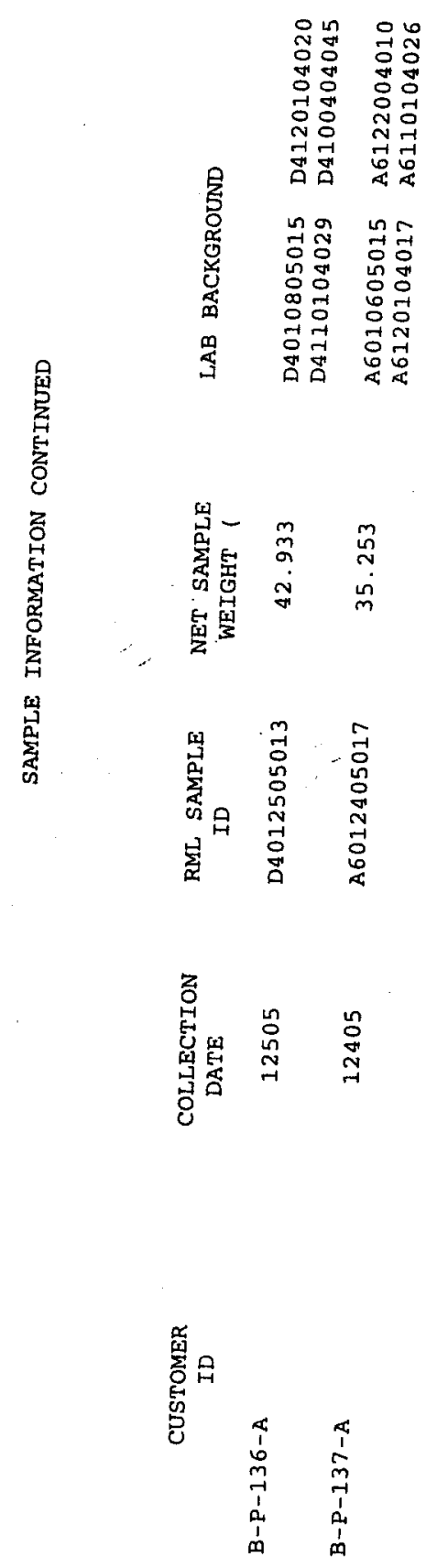




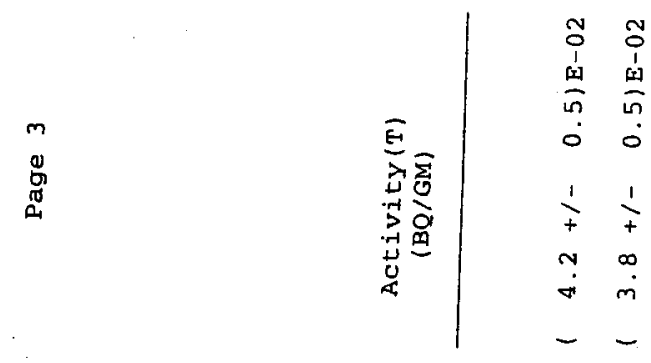

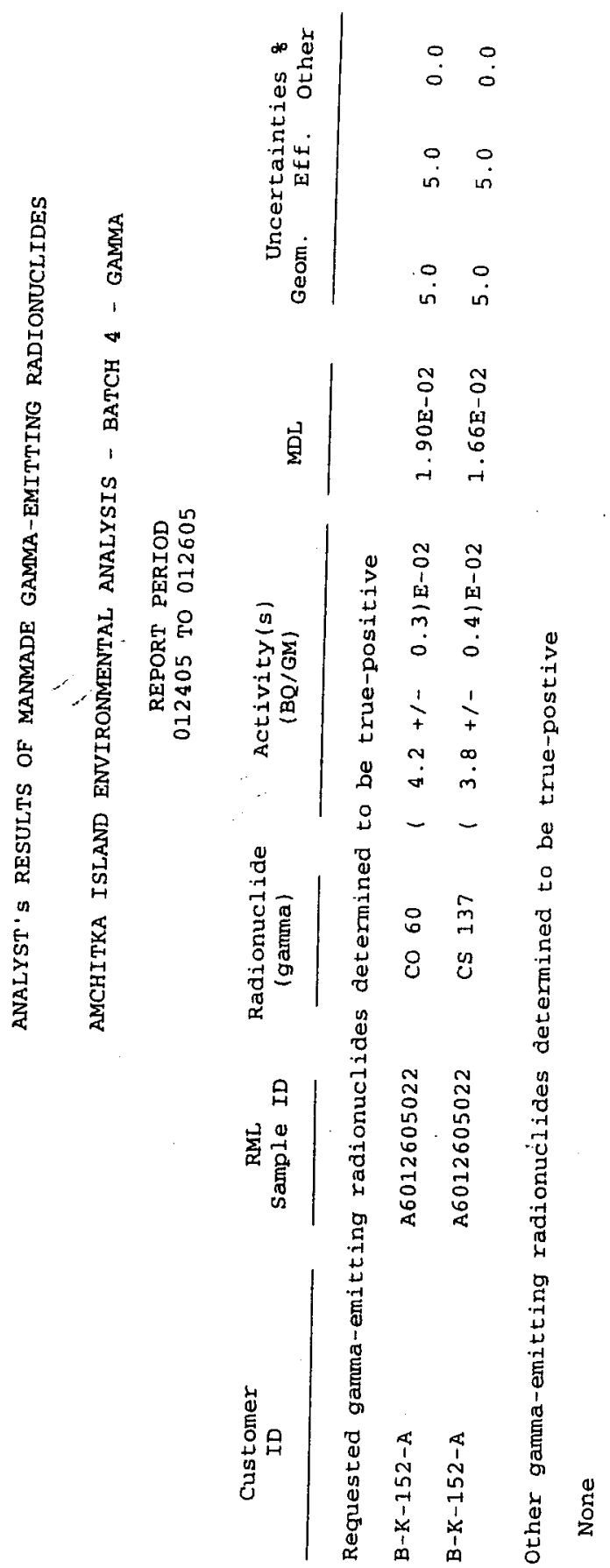


$\pi$
0
0
0
0

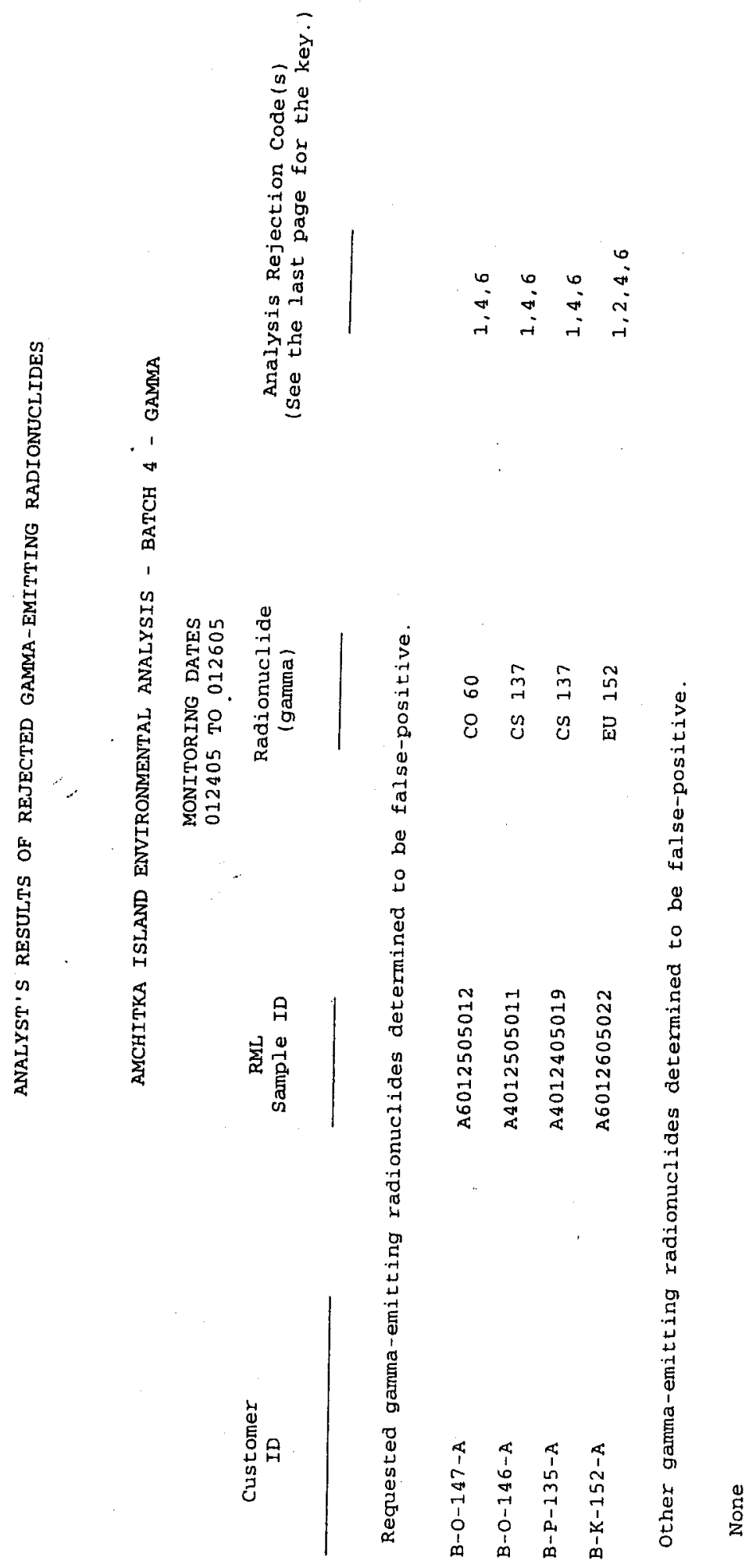




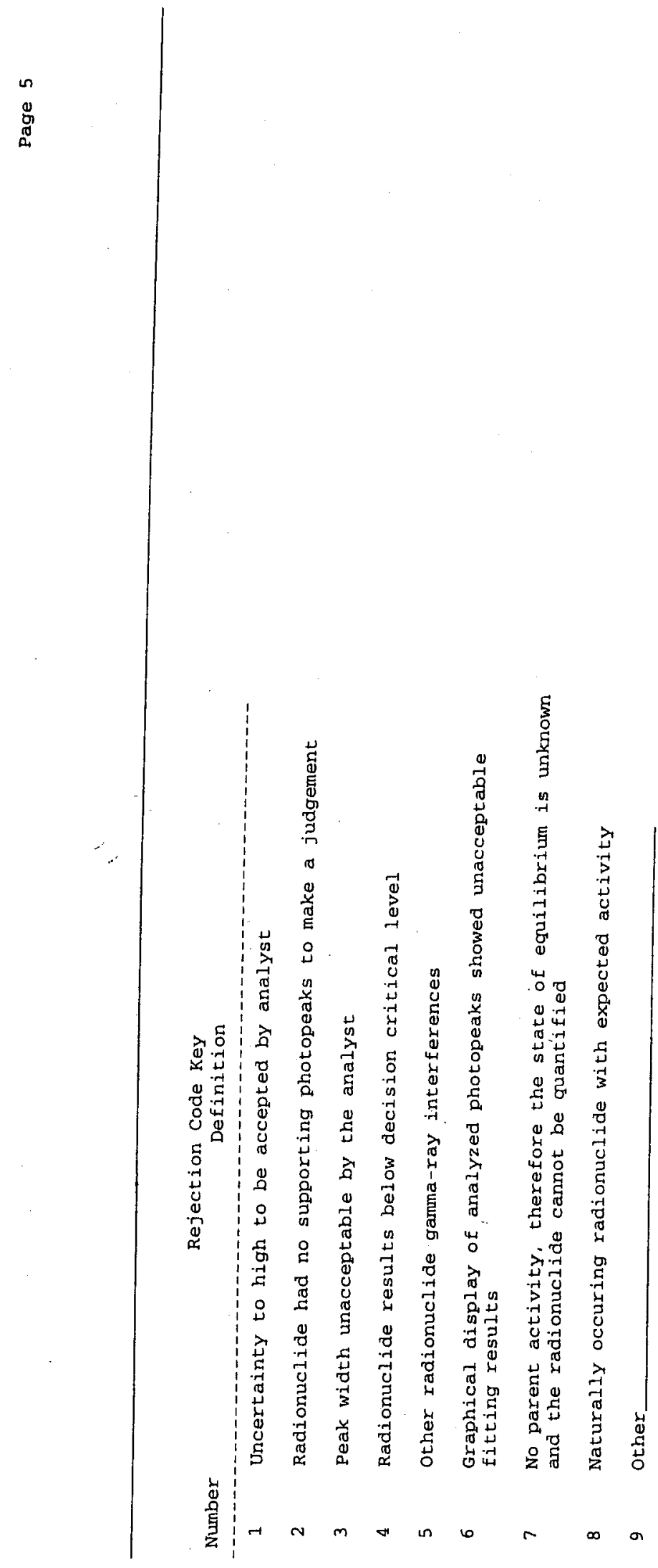


BATCH 5

GAMMA 

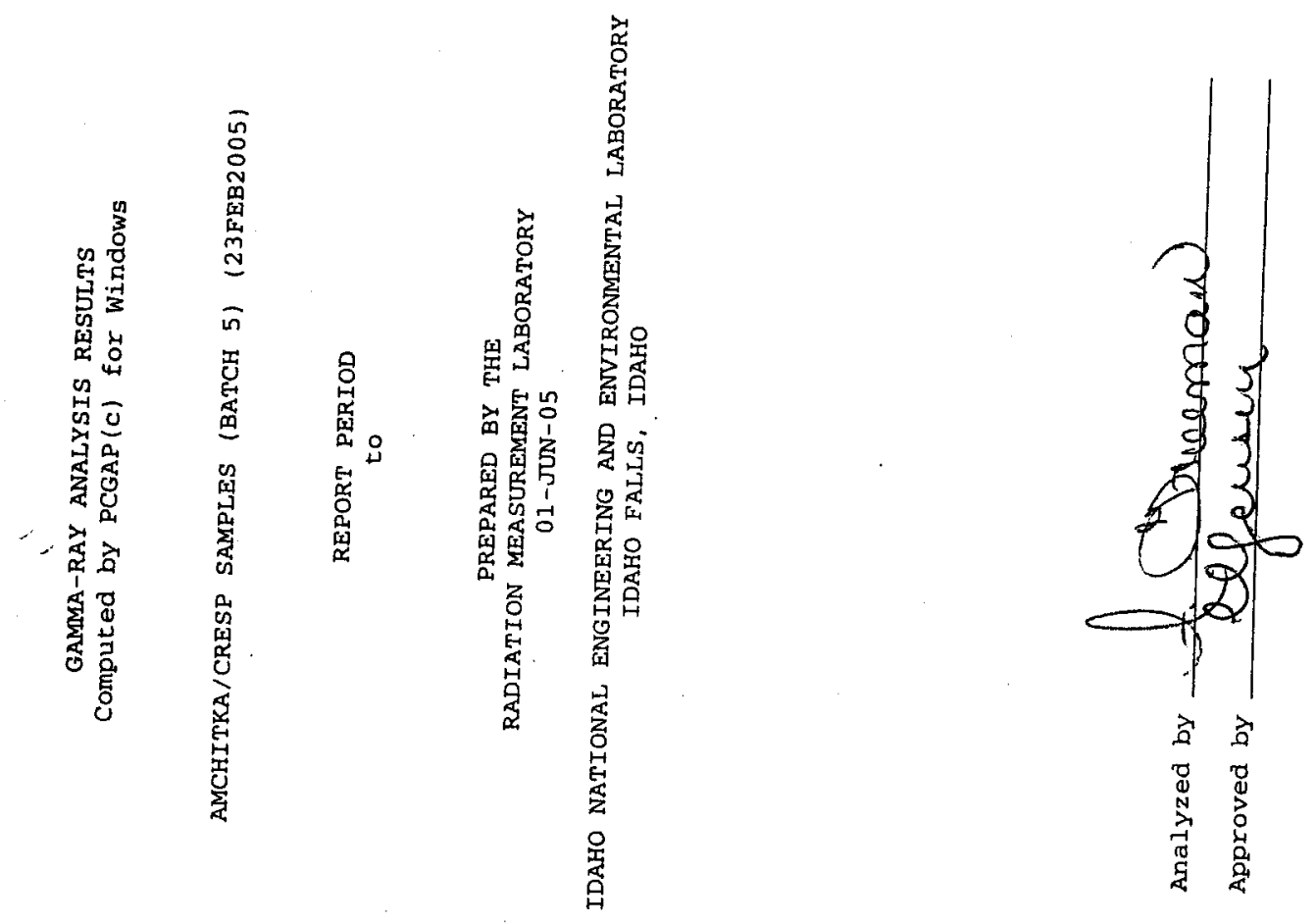

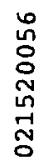




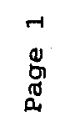

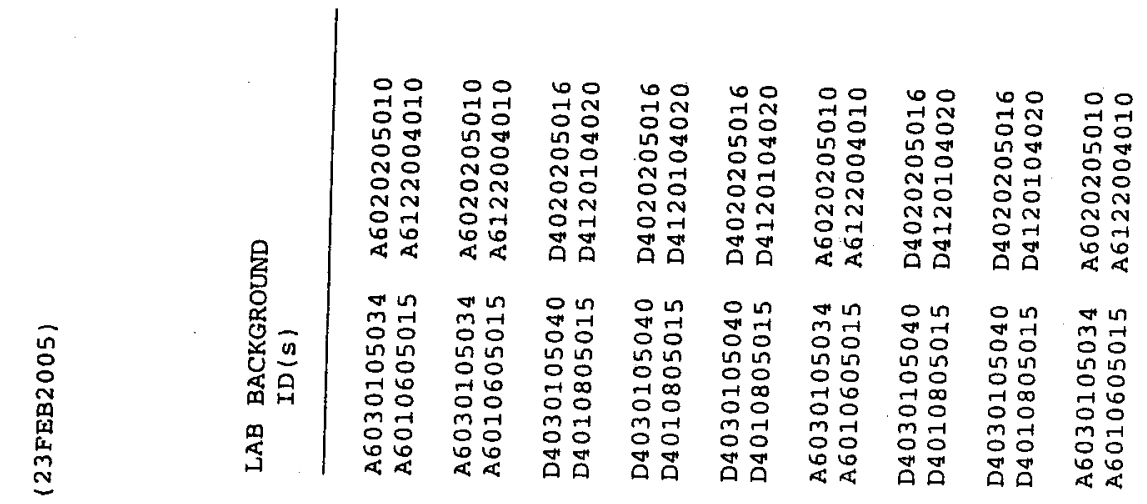

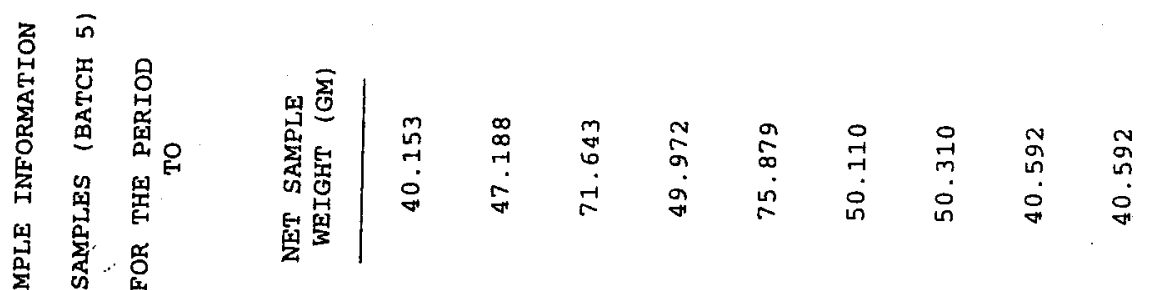

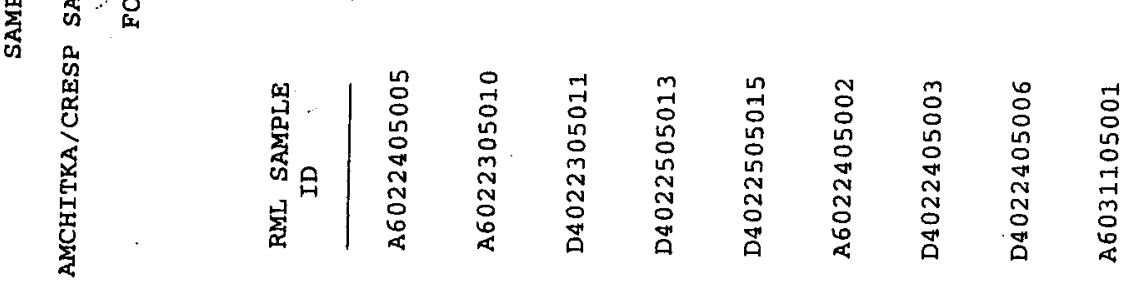

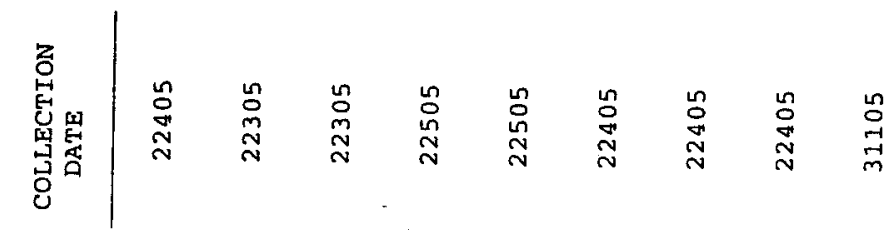

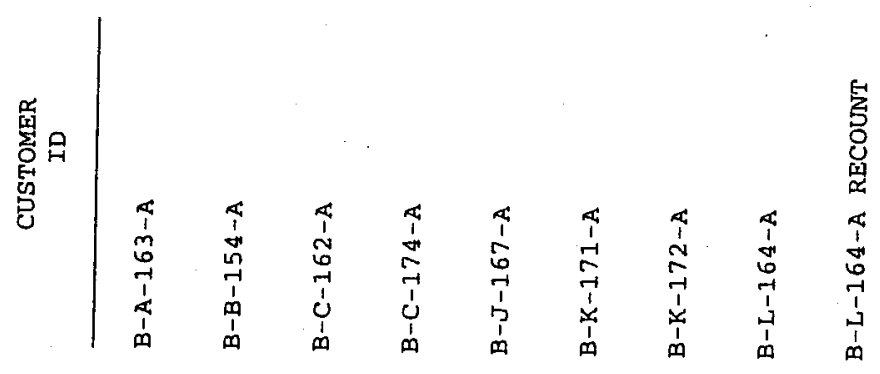




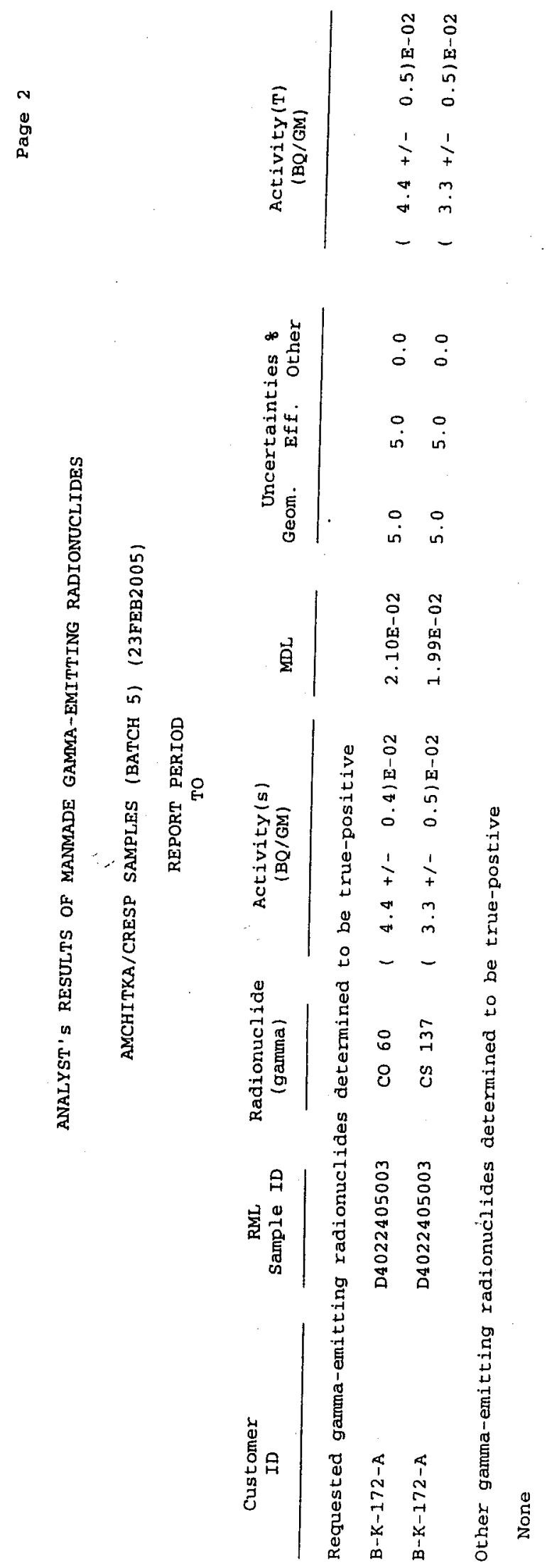


$\frac{1}{2}$

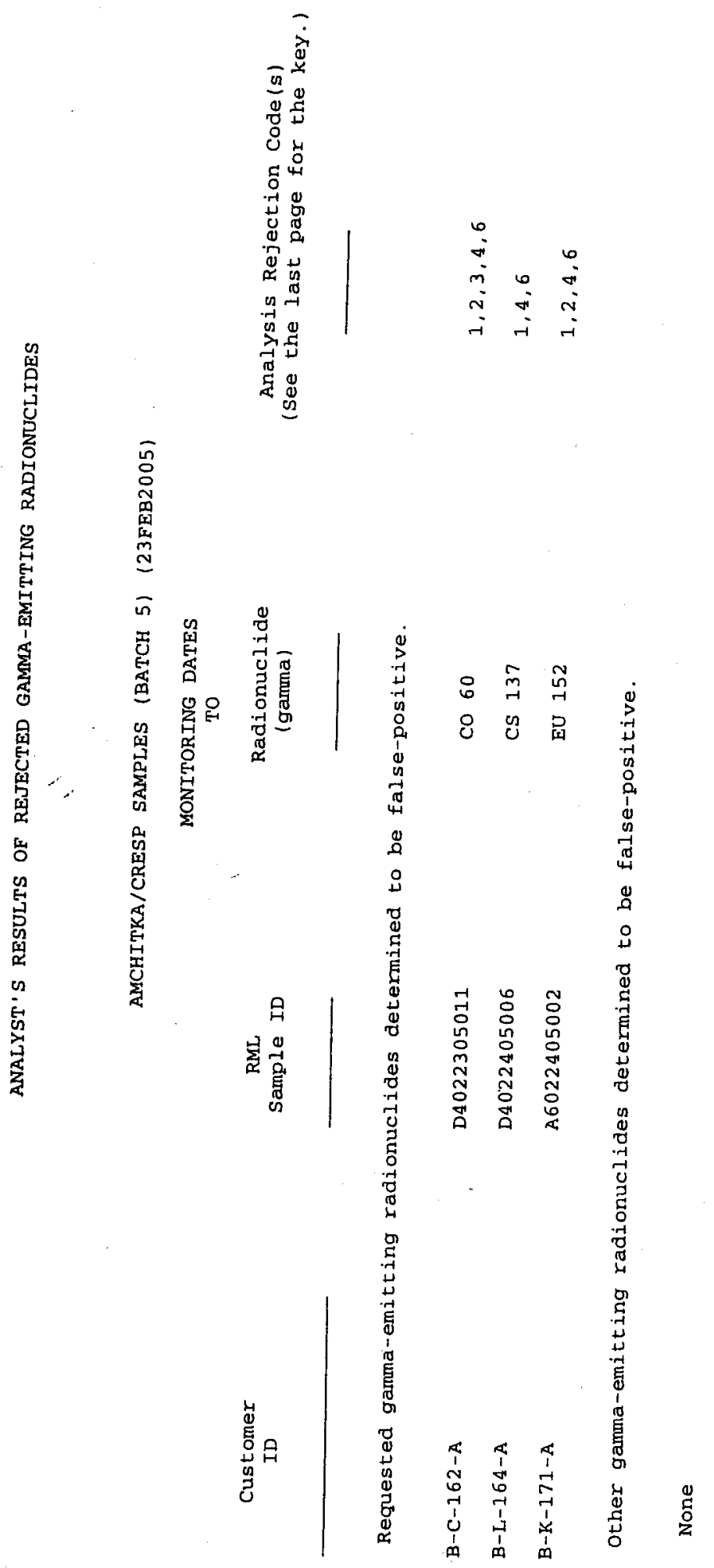




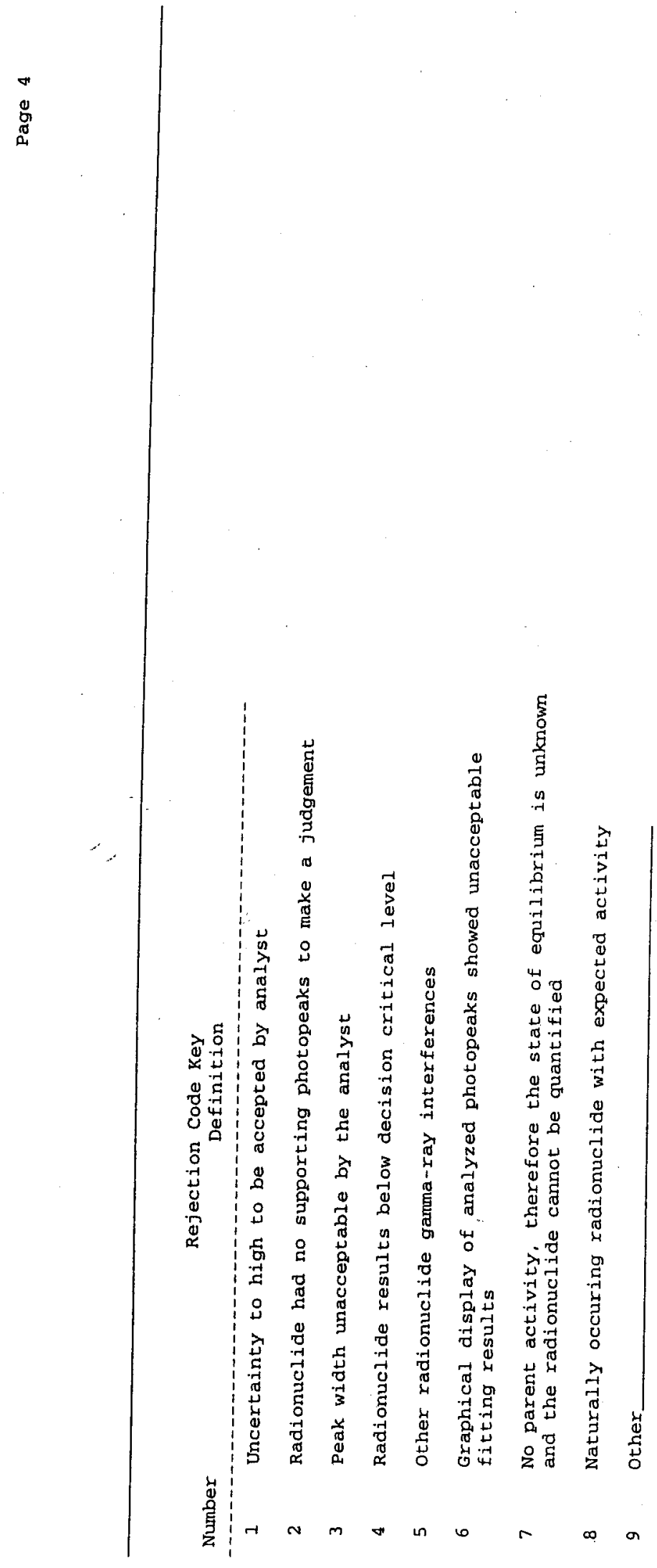




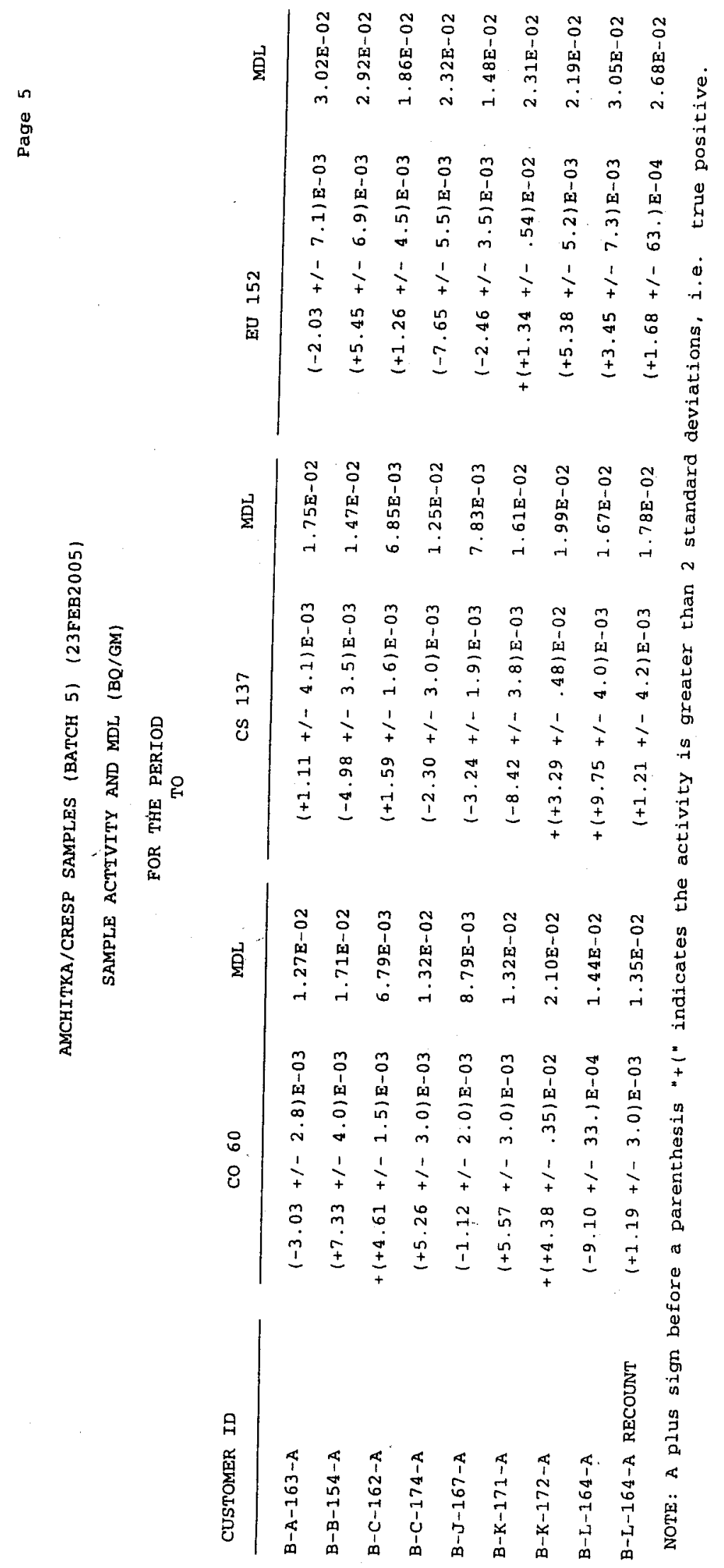


$\vdots$

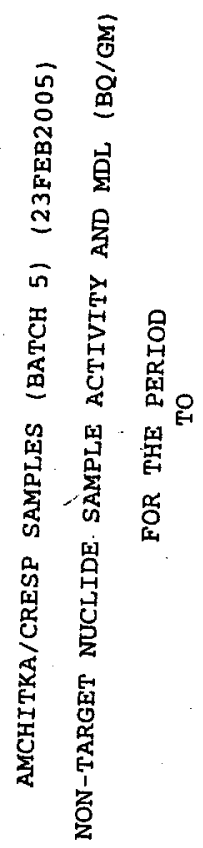

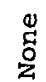


Appendix A-2

Radiochemistry

Alpha and Sr-90 Analysis 
BATCH 1

Sr-90 
Project Title: $\quad$ Sr-90 Analysis for Amchitka Island (Batch 1)

\begin{tabular}{llll} 
Lab Name: & RTC & Case No: & NA \\
Report No.: & AmchitBatchF1 & Method Type: & A/B \\
\cline { 2 - 3 } & Spproved SAP No.: NA & SDG No.: & S-G-50-A \\
\cline { 2 - 4 }
\end{tabular}

\section{SAMPLE NUMBERS}

Customer

Sample ID

S-B-54-A

$S-B-55-A$

S-B-56-A

S-G-50-A

S-G-51-A

$S-G-52-A$

S-G-53-A

S-K-70-A

S-K-75-A

S-L-57-A

$S-L-58-A$

S-L-59-A

S-O-71-A

S.0.73.A

S-0-74-A

S-P-67-A

S-P-68-A

S-P-69-A
Lab Sample

ID

02EQ-05-A

02EQ-06-A

02EQ-07-A

02EQ-01-A

02EQ-02-A

02EQ-03-A

02EQ-04-A

02EQ-15-A

02EQ-20-A

02EQ-08-A

02EQ-09-A

02EQ-10-A

02EQ-16-A

02EQ-18-A

02EQ-19-A

02EQ-12-A

02EQ-13-A

02EQ-14-A

Comments:

Release of the data contained in this data package has been authorized by the laboratory manager or the manager's designee, as verified by the following signature:

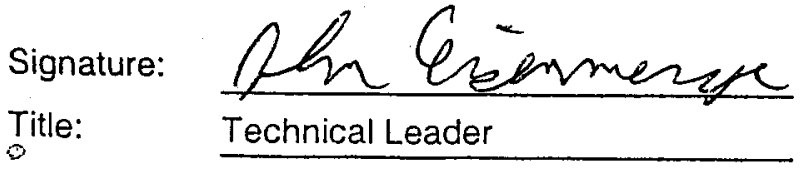

Name: J.G. Eisenmenger

Date: $\quad 05 / 26 / 2005$ 
i

$\checkmark \cong$

बे

a

\begin{tabular}{|c|c|c|c|c|c|c|c|c|c|c|c|c|c|c|c|c|c|c|}
\hline $\begin{array}{l}\text { U } \\
0\end{array}$ & & & & & & & & & & & & & & & & & & \\
\hline 台 & 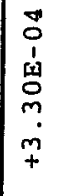 & 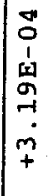 & $\begin{array}{l}0 \\
0 \\
1 \\
1 \\
\omega \\
\sim \\
\sim \\
0 \\
\dot{p} \\
+\end{array}$ & 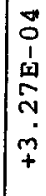 & 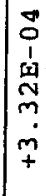 & $\mid \begin{array}{l}\overrightarrow{0} \\
1 \\
0 \\
0 \\
0 \\
0 \\
\dot{p} \\
+\end{array}$ & 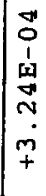 & $\mid \begin{array}{l}0 \\
0 \\
1 \\
\underline{1} \\
2 \\
m \\
m \\
m \\
+\end{array}$ & 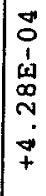 & 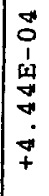 & 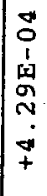 & $\mid \begin{array}{l}0 \\
0 \\
1 \\
0 \\
0 \\
0 \\
0 \\
0 \\
+ \\
+\end{array}$ & 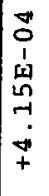 & $\mid \begin{array}{l}0 \\
0 \\
1 \\
1 \\
0 \\
0 \\
0 \\
0 \\
+ \\
+\end{array}$ & 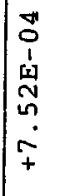 & 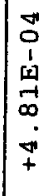 & 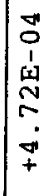 & $\begin{array}{l}0 \\
z\end{array}$ \\
\hline 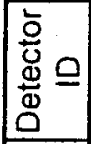 & 盆 & 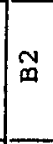 & 向 & $\vec{\alpha}$ & $\tilde{\alpha}$ & 2 & $\$$ & 箇 & $\vec{a}$ & 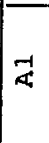 & $\tilde{x}$ & $a$ & $\vec{x}$ & $\approx$ & $\mid \begin{array}{l}\vec{x} \\
\vec{x}\end{array}$ & $\mid \vec{x}$ & $\overrightarrow{10}$ & $m$ \\
\hline 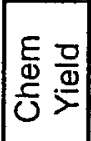 & in & י. & $\begin{array}{l}0 \\
0 \\
0 \\
\infty\end{array}$ & $\begin{array}{c}0 \\
\dot{\alpha} \\
\infty\end{array}$ & $\ddot{\infty}$ & $\begin{array}{l}\tilde{\rho} \\
\dot{\infty} \\
\infty\end{array}$ & $\begin{array}{l}0 \\
0 \\
0 \\
0\end{array}$ & $\begin{array}{l}\sigma \\
\tilde{N} \\
\tilde{\alpha}\end{array}$ & 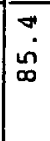 & $\begin{array}{l}\infty \\
0 \\
0 \\
\infty\end{array}$ & $\begin{array}{l}\overrightarrow{1} \\
\infty \\
\infty \\
\infty\end{array}$ & $\begin{array}{l}0 \\
\dot{8} \\
\infty\end{array}$ & $\mid \begin{array}{l}\infty \\
\infty \\
\infty \\
\infty \\
\infty\end{array}$ & $\overrightarrow{5}$ & 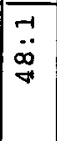 & $\begin{array}{l}n \\
n \\
\infty \\
\infty\end{array}$ & $\begin{array}{l}0 \\
0 \\
0\end{array}$ & $\vec{\sigma}$ \\
\hline$\frac{\pi}{\bar{b}}$ & $\begin{array}{ll}\infty \\
\infty\end{array}$ & 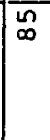 & 夙 & $\infty$ & 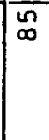 & $\infty$ & $\infty$ & $\begin{array}{l}n \\
\infty \\
\infty\end{array}$ & \begin{tabular}{|l|}
$n$ \\
$\infty$
\end{tabular} & 웅 & I용 & 운 & $\begin{array}{l}\infty \\
\infty\end{array}$ & 原 & $\mid \begin{array}{l}n \\
\infty\end{array}$ & \% & n & 6 \\
\hline 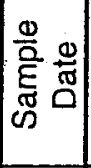 & 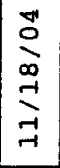 & 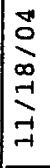 & 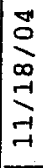 & 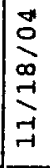 & 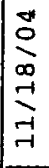 & $\mid \begin{array}{c}\nexists \\
0 \\
\infty \\
0-1 \\
ت \\
-1 \\
ت\end{array}$ & 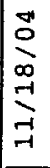 & 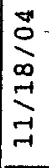 & 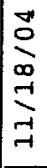 & $\begin{array}{l}\overrightarrow{0} \\
0 \\
\infty \\
\sim-1 \\
\\
-1\end{array}$ & 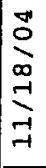 & $\begin{array}{l}0 \\
0 \\
0 \\
0 \\
-1 \\
-1 \\
-1\end{array}$ & $\begin{array}{l}\vec{D} \\
0 \\
\infty \\
-1 \\
-1 \\
-1\end{array}$ & 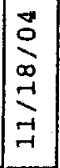 & 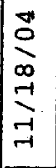 & 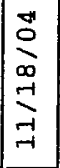 & 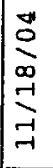 & $\begin{array}{l}\overrightarrow{0} \\
0 \\
0 \\
-1 \\
-1 \\
ન-1\end{array}$ \\
\hline 芫 & $\begin{array}{l}\text { n̊ } \\
\text { o } \\
\text { ò } \\
0 \\
\text { ma } \\
\text { on }\end{array}$ & $\frac{0}{2}$ & $\frac{n}{2}$ & 告 & 告 & 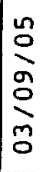 & $\begin{array}{l}n \\
0 \\
o \\
0 \\
0 \\
0 \\
0\end{array}$ & $\begin{array}{l}\text { in } \\
\text { on } \\
\text { o }\end{array}$ & 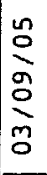 & $\begin{array}{l}n \\
0 \\
0 \\
0 \\
0 \\
0 \\
0\end{array}$ & $\begin{array}{l}\text { no } \\
0 \\
0 \\
0 \\
\text { m. } \\
0\end{array}$ & $\begin{array}{l}\frac{n}{0} \\
0 \\
0 \\
0 \\
\text { ô } \\
0\end{array}$ & $\begin{array}{l}n \\
0 \\
0 \\
0 \\
0 \\
m \\
0\end{array}$ & $\mid \begin{array}{l}n \\
2 \\
o \\
o \\
0 \\
m \\
0\end{array}$ & $\begin{array}{l}n \\
0 \\
0 \\
0 \\
0 \\
0 \\
0\end{array}$ & $\begin{array}{l}n \\
0 \\
0 \\
\rho \\
0 \\
0 \\
0\end{array}$ & 告 & 告 \\
\hline & $\begin{array}{c}0 \\
\dot{0} \\
\end{array}$ & $\theta$ & $\theta$ & on & के & $\begin{array}{l}\text { a } \\
\text { के } \\
\end{array}$ & or & के & o & 家 & के & g & के & \begin{tabular}{|l|}
0 \\
0 \\
\\
\end{tabular} & $\begin{array}{l}0 \\
0 \\
0 \\
9\end{array}$ & $\begin{array}{l}0 \\
0 \\
0 \\
9\end{array}$ & 品 & के \\
\hline 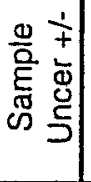 & $\begin{array}{l}0 \\
0 \\
1 \\
1 \\
m \\
0 \\
o \\
o \\
+\end{array}$ & $\mid \begin{array}{c}5 \\
0 \\
0 \\
0 \\
0 \\
0 \\
0\end{array}$ & $\stackrel{\infty}{+}$ & $\begin{array}{l}\text { un } \\
0 \\
1 \\
\text { cus } \\
\text { o }\end{array}$ & $\begin{array}{l}\omega \\
0 \\
\infty \\
\infty \\
\infty \\
\infty \\
+\end{array}$ & $\stackrel{+}{+}$ & $\stackrel{\infty}{+}$ & $\stackrel{9}{+}$ & $\mp$ & $\mp$ & 7 & 7 & 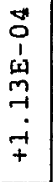 & 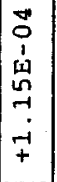 & 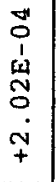 & 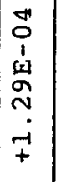 & 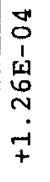 & $\begin{array}{l}\vec{g} \\
1 \\
1 \\
0 \\
0 \\
-1 \\
-1 \\
+ \\
+\end{array}$ \\
\hline$\vec{E}=$ & $\begin{array}{c}0 \\
0 \\
1 \\
1 \\
0 \\
0 \\
n \\
-1 \\
+ \\
\end{array}$ & $\begin{array}{l}5 \\
0 \\
1 \\
0 \\
0 \\
6\end{array}$ & $\begin{array}{l}n \\
0 \\
1 \\
g \\
0\end{array}$ & $\mid \begin{array}{ll}2 \\
0 \\
1\end{array}$ & 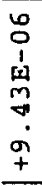 & 号 & ? & $\stackrel{\text { ־ }}{1}$ & 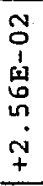 & ? & 16 & N & 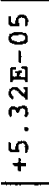 & 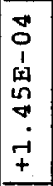 & $\begin{array}{l} \\
0 \\
1 \\
\omega \\
0 \\
0 \\
i \\
i \\
\end{array}$ & \begin{tabular}{|l|}
$n$ \\
0 \\
1 \\
1 \\
1 \\
0 \\
0 \\
0 \\
1 \\
1 \\
\end{tabular} & 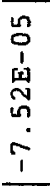 & 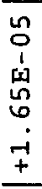 \\
\hline
\end{tabular}

要迢

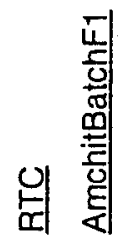

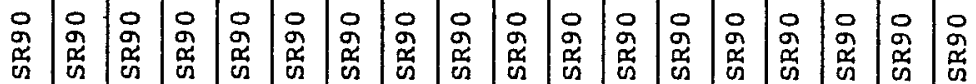

\begin{tabular}{|c|c|c|c|c|c|c|c|c|c|c|c|c|c|c|c|c|c|c|}
\hline & & & & & & & & & & & & C2 & & U & en & & & \\
\hline$\frac{\Phi}{0}$ & 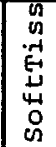 & $\begin{array}{c}0 \\
0 \\
0 \\
-1 \\
4 \\
4 \\
0 \\
0 \\
0\end{array}$ & 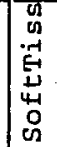 & $\mid \begin{array}{c}n \\
n \\
:-1 \\
-4 \\
4 \\
0 \\
0\end{array}$ & 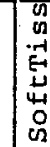 & 蛞 & 要 & 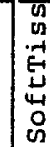 & 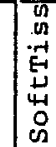 & 吾 & $\begin{array}{l}0 \\
0 \\
0 \\
-1 \\
-1 \\
\end{array}$ & 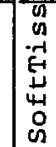 & 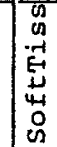 & 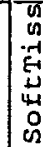 & $\mid \begin{array}{c}n \\
-1 \\
-1 \\
E-1\end{array}$ & & & 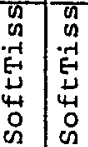 \\
\hline
\end{tabular}

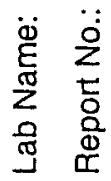

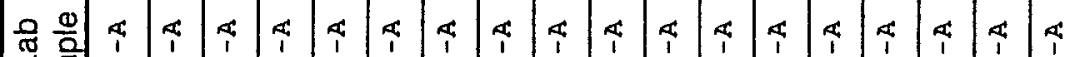
है। c)

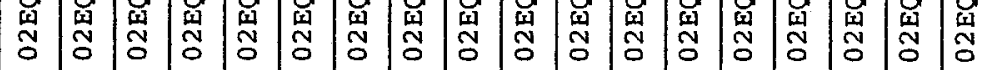




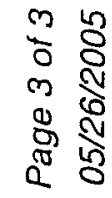
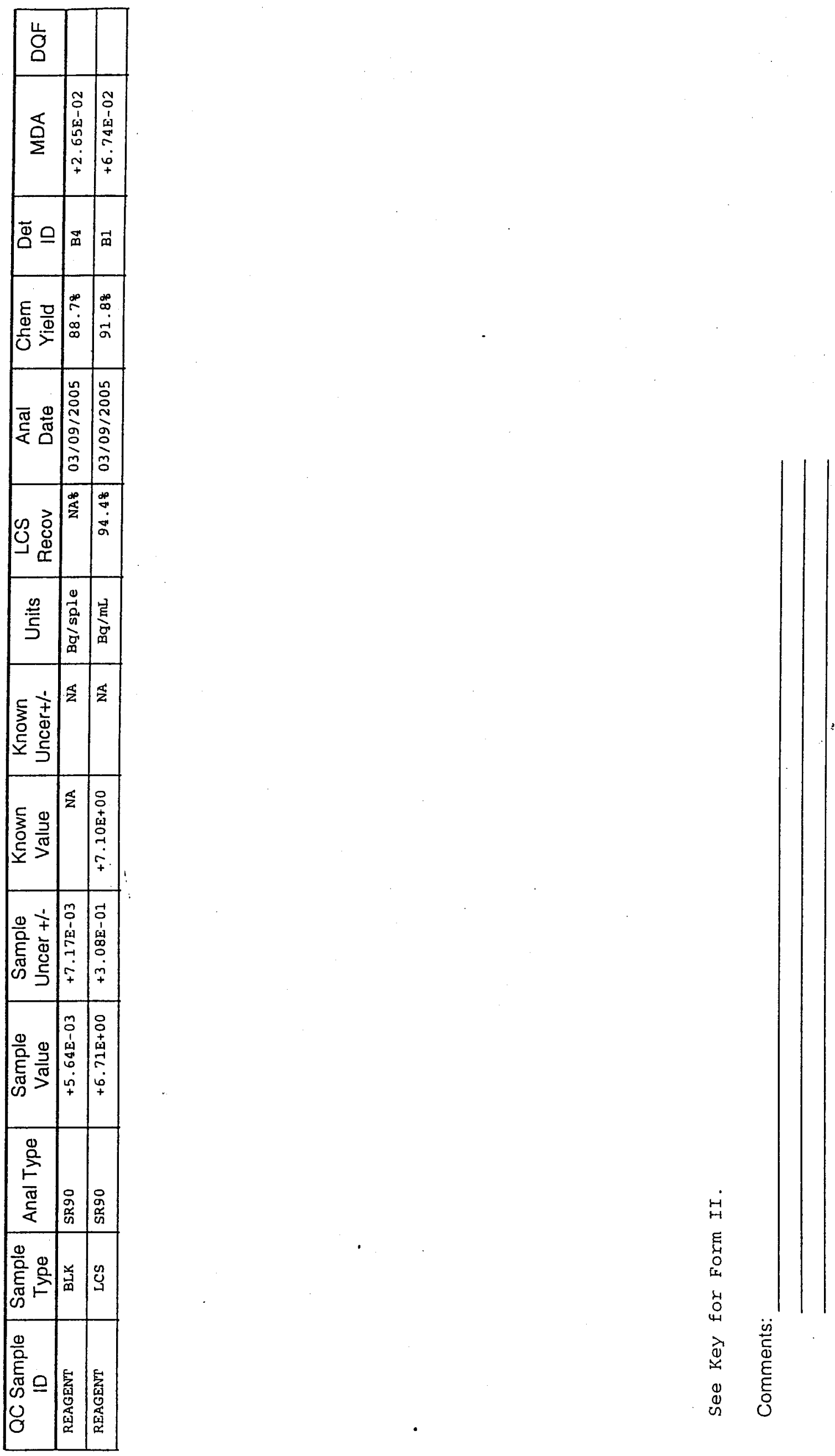


\section{Project: $\quad$ Sr-90 Analysis for Amchitka Island (Batch 1)}

Laboratory: RTC

Report \#: AmchitBatchF1

SDG\#: S-G-50-A

\section{Summary of 2 and 3 sigma activities}

Below are the results for Sr-90 for Batch 1 from the Amchitka Island Project that had a result/uncertainty ratio of 2 or more (uncertainty @ one sigma).

\begin{tabular}{|c|c|c|c|c|c|}
\hline Customer ID & Lab ID & Isotope & $\begin{array}{c}\text { Result } \\
\mathrm{Bq} / \mathrm{g}\end{array}$ & $\begin{array}{c}\text { Uncertainty } \\
\mathrm{Bq} / \mathrm{g}\end{array}$ & $\begin{array}{c}\text { Result } \\
\text { Uncertainty }\end{array}$ \\
\hline S-K-75-A & O2EQ-20-A & SR90 & $2.56 \mathrm{E}-02$ & $1.17 \mathrm{E}-03$ & 21.9 \\
\hline
\end{tabular}

All known sources of uncertainty are included in the uncertainty term. There may be unknown sources of uncertainty that are not accounted for. If the result/uncertainty ratio is more than 3, we have a degree of confidence that the result is positive (i.e. the result is statistically different than zero). A result with the result/uncertainty ratio between 2 and 3 is the first indication that an isotope may be present and further investigation may be warranted.

As with any good science no single data point is used in important decisions (results need to be reproducible). 
BATCH 2

Sr-90 


\section{RADIOANALYTICAL ANALYSES DATA PACKAGE}

Project Title: $\quad$ Sr-90 Analysis for Amchitka Island (Batch 2-1)

Lab Name: $\quad$ RTC

Report No.: $\quad$ AmchitBatchF2-1

Approved SAP No.: NA

Case No: NA

Method Type: $\underline{B}$

SDG No.: $\quad$ S-U-76-C

\section{SAMPLE NUMBERS}

Customer

Sample ID

$\frac{\frac{S-U-76-C}{S-U-77-C}}{\frac{S-U-90-C}{S-U-96-C}}$

Lab Sample

ID

$02 F \times-01-A$

$02 F X-02-A$

$02 F X-03-A$

02FX-04-A

Comments:

Release of the data contained in this data package has been authorized by the laboratory manager or the manager's designee, as verified by the following signature:

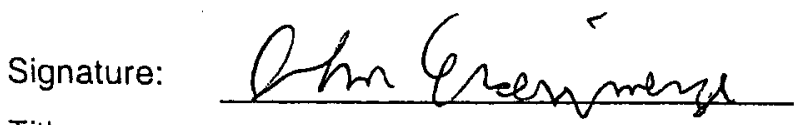

Title:
Name: J.G. Eisenmenger

Date: $\quad 05 / 26 / 2005$ 


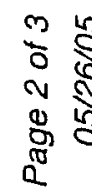
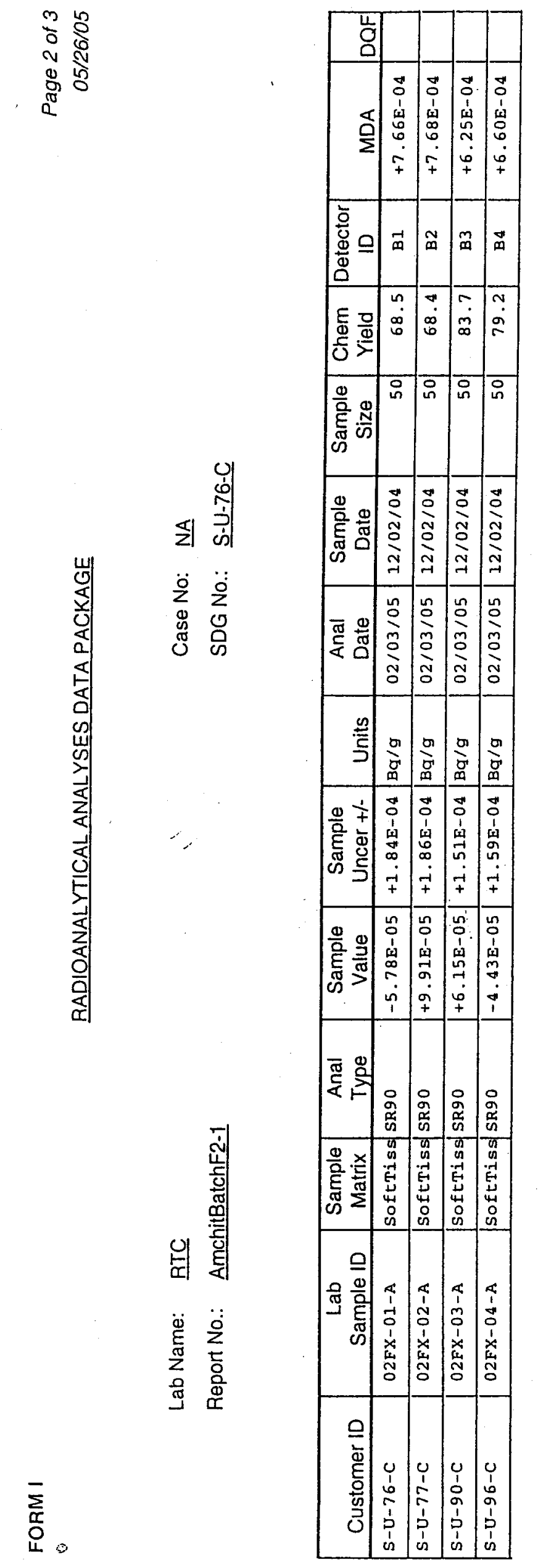

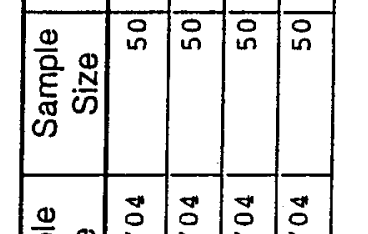

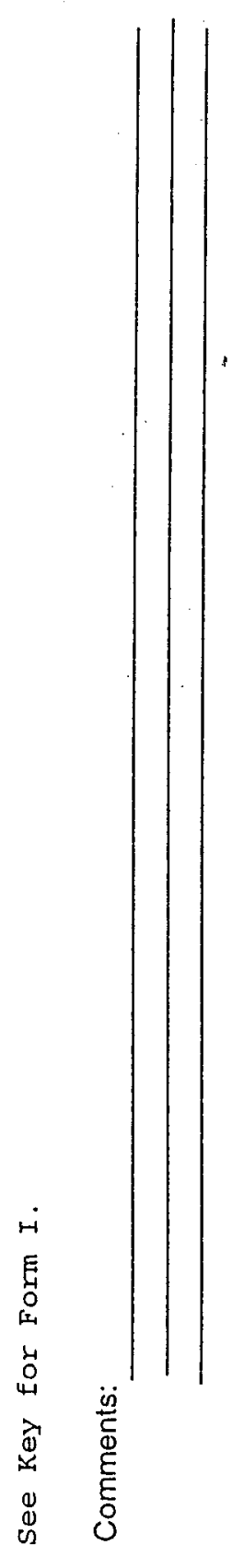


$\begin{array}{ll}3 & 0 \\ 0 & 8 \\ 0 & \mathbb{1} \\ 0 & 0 \\ 0 & \mathbb{1} \\ 0 & 0 \\ 0 & 0\end{array}$

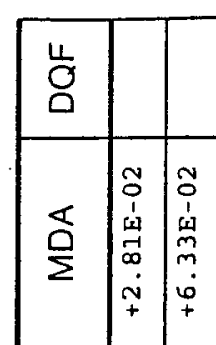

호을

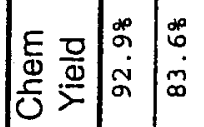

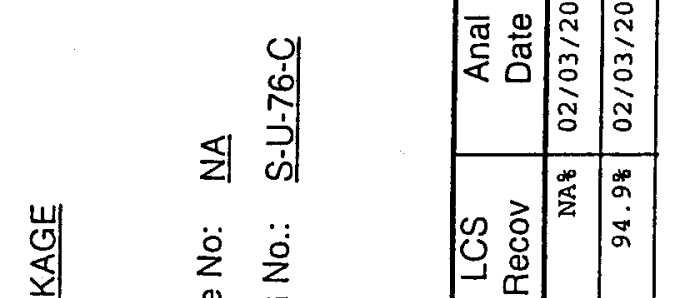

嵌

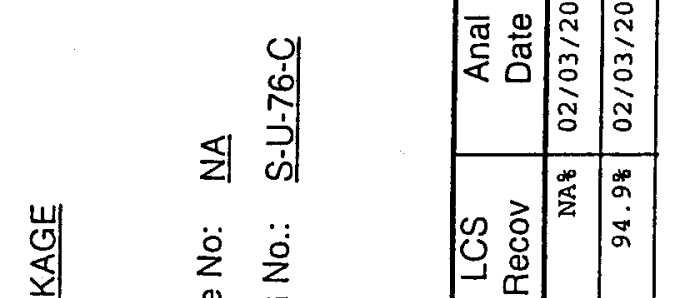

용

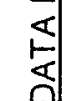

\begin{tabular}{|c|c|c|}
\hline \multicolumn{3}{|c|}{$-\underset{\sim}{\simeq}$} \\
\hline 0 & -1 & \\
\hline- & $\mid \begin{array}{l}\tilde{n} \\
\bar{g}\end{array}$ & 8 \\
\hline
\end{tabular}

崩

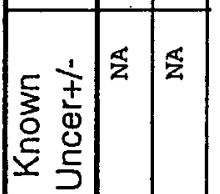

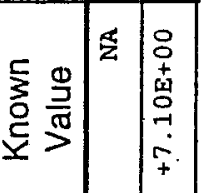

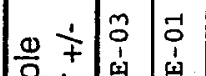

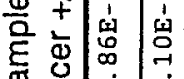

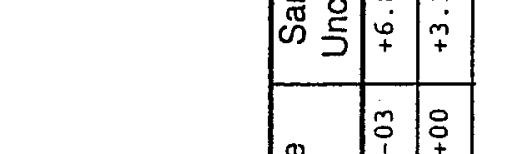

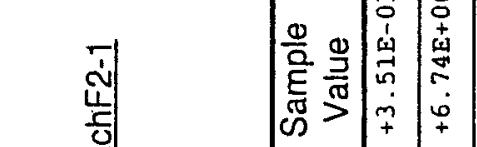

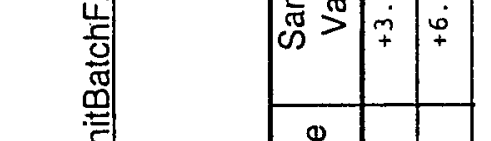

입 홍

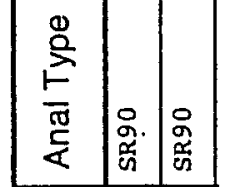

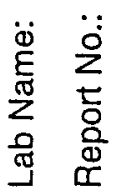

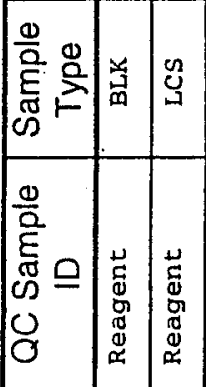

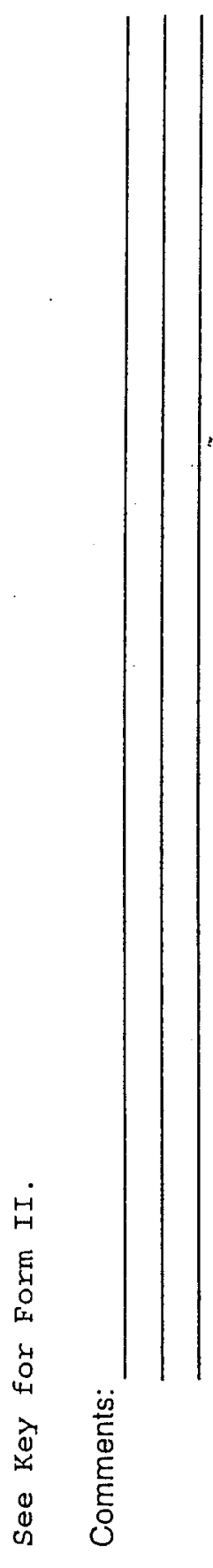


Project: $\quad$ Sr-90 Analysis for Amchitka Island (Batch 2-1)

Laboratory: RTC

Report \#: $\quad$ AmchitBatchF2-1

SDG\#: $\quad$ S-U-76-C

Summary of 2 and 3 sigma activities

There were no results for $\mathrm{Sr}-90$ for Batch 2-1 from the Amchitka Island Project that had a result/uncertainty ratio of 2 or more (uncertainty @ one sigma).

All known sources of uncertainty are included in the uncertainty term. There may be unknown sources of uncertainty that are not accounted for. If the result/uncertainty ratio is more than 3 , we have a degree of confidence that the result is positive (i.e. the result is statistically different than zero). A result with the result/uncertainty ratio between 2 and 3 is the first indication that an isotope may be present and further investigation may be warranted.

As with any good science no single data point is used in important decisions (results need to be reproducible). 


\section{RADIOANALYTICAL ANALYSES DATA PACKAGE}

Project Title: $\quad$ Sr-90 Analysis for Amchitka Island (Batch 2-2)

Lab Name:

RTC

Report No.: $\quad$ AmchitBatchF2-2

Approved SAP No.: NA

Case No: NA

Method Type: AVB

SDG No.: S-K-60-A

\section{SAMPLE NUMBERS}

Customer

Sample ID

S-K-60-A

S-K-61-A

S-K-63-A

S.K-88-A

S-K-89-A

$S \cdot M-80-A$

S-M-81-A

$S-M-82-A$

$S-M-83-A$

$S-M-84-A$

$S-M-85-A$

S-M-86-A

S-M-87-A

S.U-76-A

S-U-77-A

S-U-90-A

S-U-96-A

S.VN-102-A

S-VN-103-A

S-VN-104-A
Lab Sample

ID

02FP-09-A

02FP-10-A

02FP-11-A

02FP-12-A

02FP-13-A

02FP-01-A

02FP-02-A

02FP-03-A

02FP-04-A

02FP-05-A

02FP-06-A

02FP-07-A

02FP-08-A

02FP-14-A

02FP-15-A

02FP-16-A

02FP-17-A

02FP-18-A

02FP-19-A

02FP-20-A

Comments:

Release of the data contained in this data package has been authorized by the laboratory manager or the manager's designee, as verified by the following signature:

Signature:

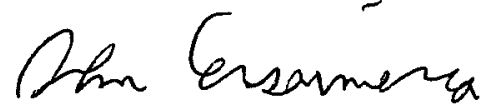

Title:

Technical Leader
Name: J. G. Eisenmenger

Date: $\quad 05 / 26 / 2005$ 
$\begin{array}{ll}n & 0 \\ 0 & 0 \\ 0 & 0\end{array}$

80

a

\begin{tabular}{|c|c|c|c|c|c|c|c|c|c|c|c|c|c|c|c|c|c|c|c|}
\hline $\begin{array}{l}\breve{g} \\
0\end{array}$ & & & & & & & & & & & & & & & & & & & \\
\hline 号 & $\left|\begin{array}{c}7 \\
0 \\
1 \\
\omega \\
0 \\
n \\
n \\
+ \\
+\end{array}\right|$ & 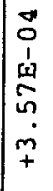 & 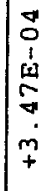 & 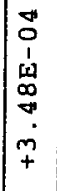 & $\mid \begin{array}{l}0 \\
0 \\
i \\
\dot{\omega} \\
0 \\
\dot{p} \\
+ \\
+\end{array}$ & & 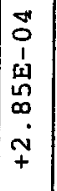 & 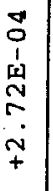 & $\mid \begin{array}{l}\overrightarrow{0} \\
\dot{1} \\
\omega \\
0 \\
0 \\
\dot{p} \\
+\end{array}$ & 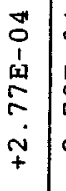 & 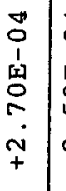 & 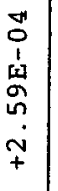 & 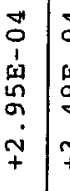 & 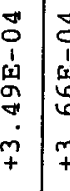 & 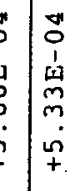 & 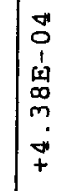 & $\begin{array}{l}07 \\
0 \\
1 \\
0 \\
0 \\
-1 \\
+ \\
+\end{array}$ & 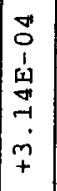 & $\begin{array}{l}\vec{y} \\
0 \\
1 \\
\omega \\
m \\
m \\
m \\
+ \\
+\end{array}$ \\
\hline 蒿 & $\tilde{\alpha}$ & $\frac{m}{\alpha}$ & 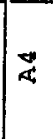 & $\vec{m}$ & $\tilde{m}$ & $\vec{G}$ & $\approx$ & $\vec{x}$ & $\approx$ & $\overrightarrow{\text { |⿰㇒一 }}$ & ल & 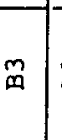 & $\vec{\alpha}$ & 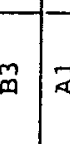 & $\dot{a}$ & q & $\ddot{3}$ & $\vec{m}$ & 跑 \\
\hline 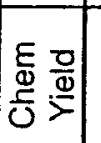 & $\dot{\infty}$ & $\mid \begin{array}{l}m \\
\infty \\
\infty \\
\infty\end{array}$ & $\begin{array}{l}\dot{0} \\
\dot{a} \\
\sigma\end{array}$ & $\begin{array}{l}\bar{a} \\
\dot{a} \\
\infty\end{array}$ & \begin{tabular}{|l|} 
\\
$\vdots$ \\
0 \\
$\infty$
\end{tabular} & \begin{tabular}{l|}
$\vec{a}$ \\
$\dot{a}$
\end{tabular} & \begin{tabular}{|l|}
$n$ \\
$\dot{\infty}$ \\
$\infty$ \\
0
\end{tabular} & $\begin{array}{l}n \\
\vdots \\
0 \\
0\end{array}$ & $\begin{array}{c}+\infty \\
\infty \\
\infty\end{array}$ & $\begin{array}{l}\overrightarrow{1} \\
\infty \\
\infty\end{array}$ & ळ & ִִ & \begin{tabular}{ll|} 
\\
$\dot{\alpha}$ \\
$\dot{\alpha}$
\end{tabular} & \begin{tabular}{c|c}
$\infty$ & $n$ \\
$\infty$ \\
$\infty$ \\
$\infty$ \\
$\tilde{m}$
\end{tabular} & $\dot{0}$ & $\stackrel{\infty}{\stackrel{\infty}{r}}$ & dूं & & \\
\hline 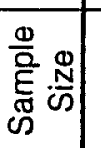 & $\tilde{\infty}_{\mathscr{\infty}}$ & $\begin{array}{l}\mathbb{\Omega} \\
\infty\end{array}$ & $\bar{\infty}$ & \begin{tabular}{|l|}
$\mathbf{\infty}$ \\
$\infty$
\end{tabular} & 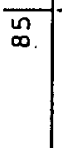 & : & $\begin{array}{l} \\
0 \\
\end{array}$ & $\bar{\Xi}$ & $\bar{O}$ & 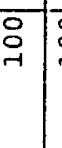 & $\bar{\vdots}$ & 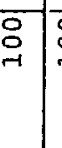 & 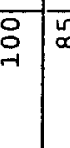 & 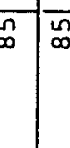 & & $\tilde{\infty}$ & i & \begin{tabular}{l} 
\\
$\vdots$ \\
\hdashline
\end{tabular} & \\
\hline
\end{tabular}

近妾

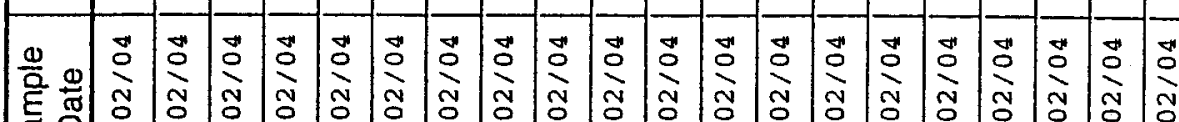

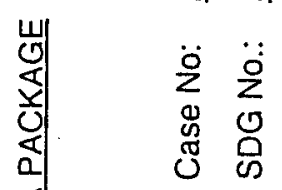
Е

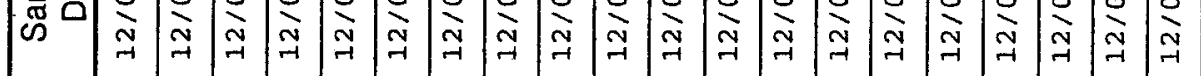

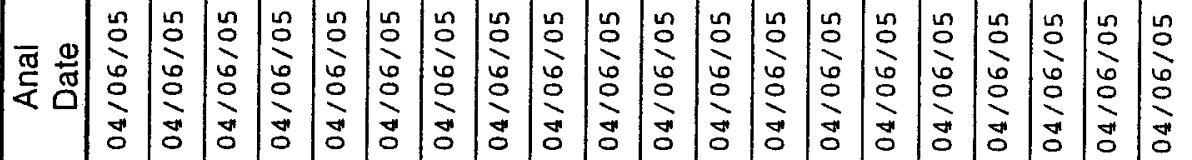

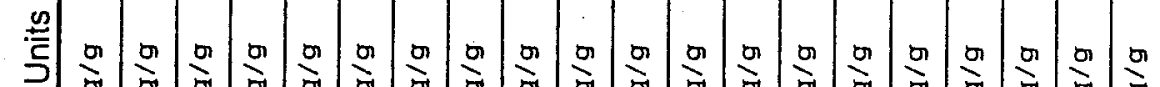

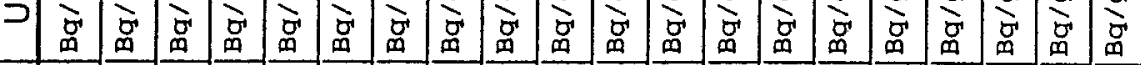

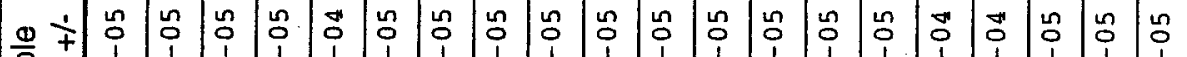

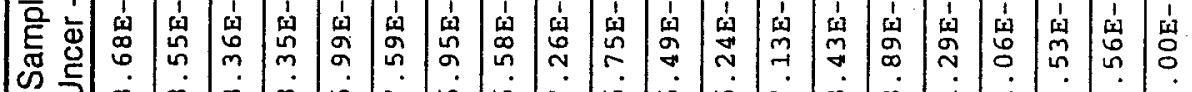

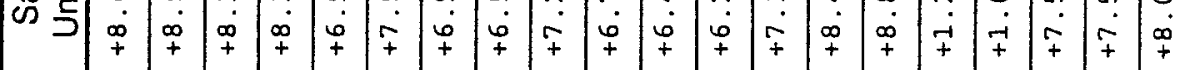

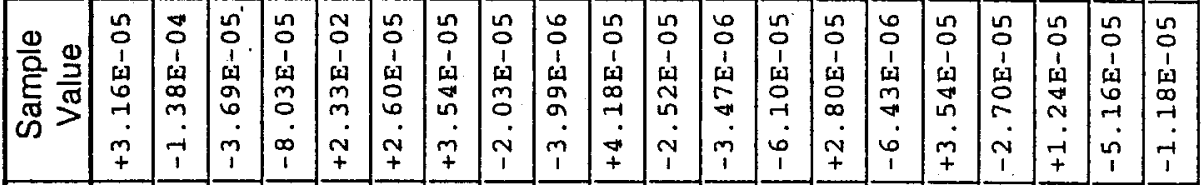

爱学

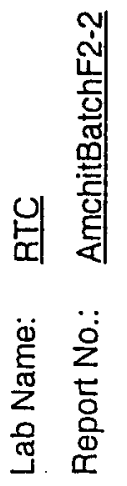

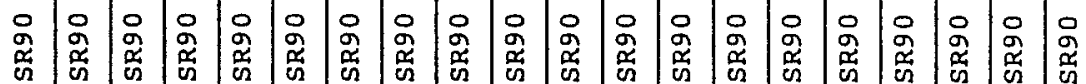

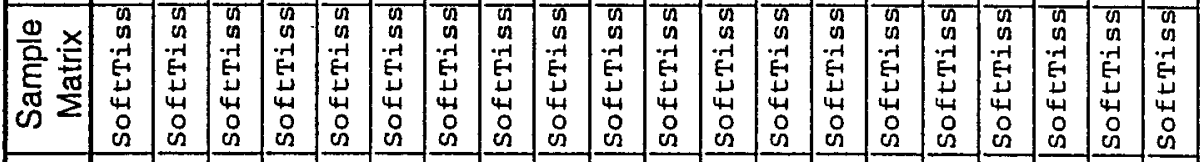

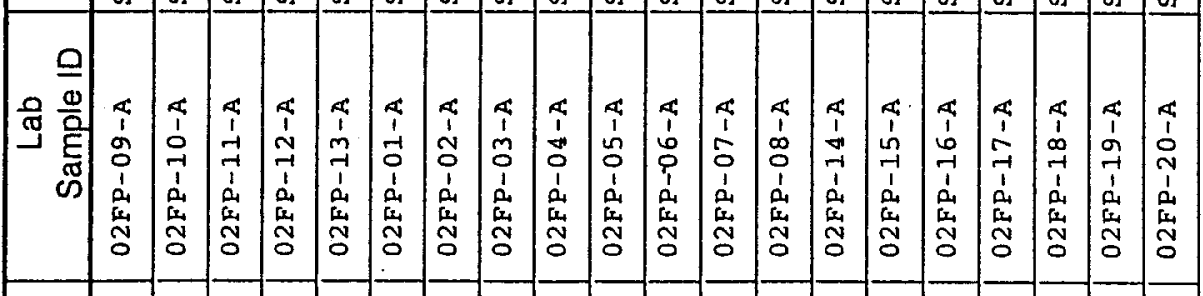




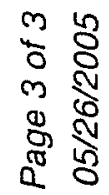
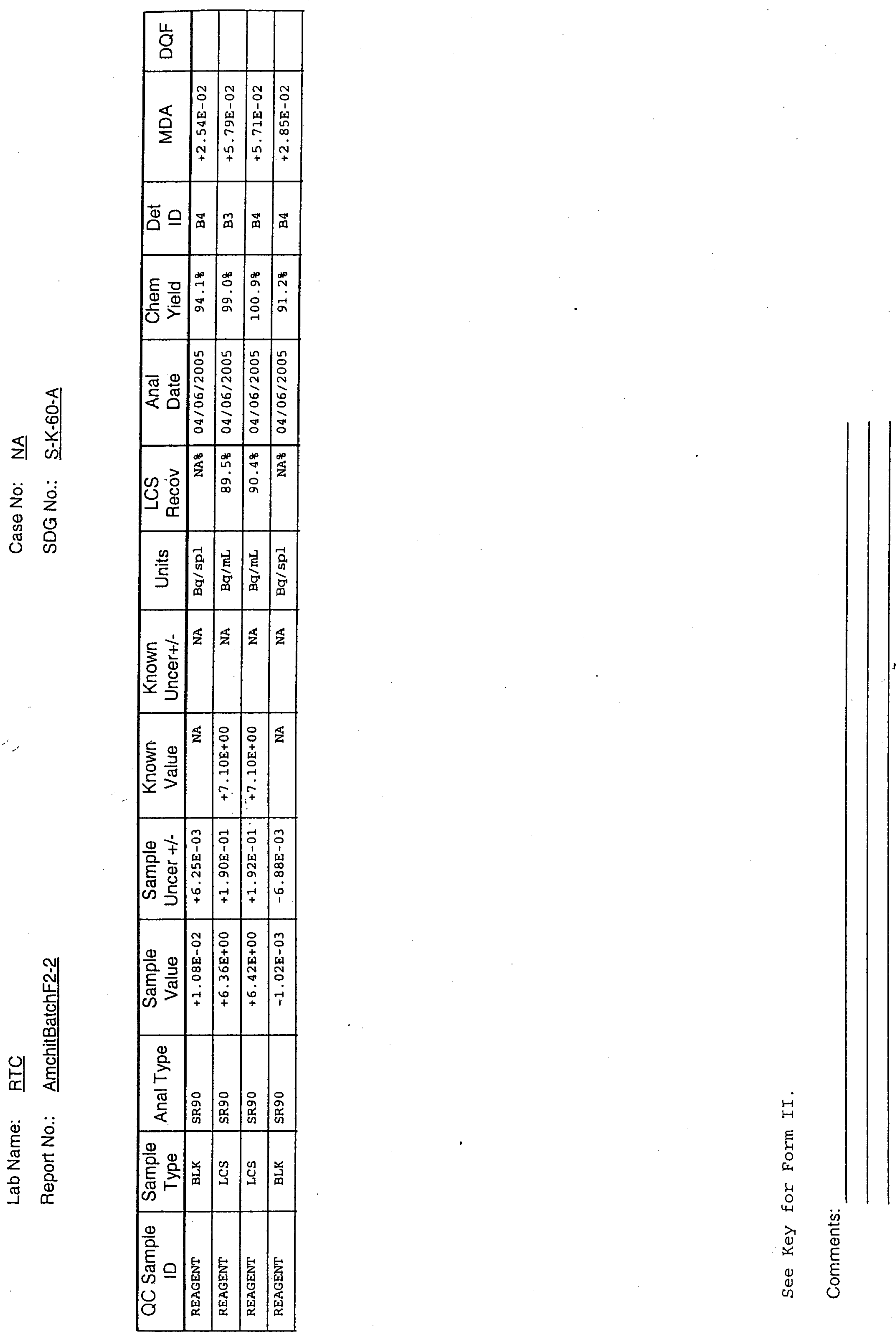
Project: $\quad$ Sr-90 Analysis for Amchitka Island (Batch 2-2)

Laboratory: RTC

Report \#: $\quad$ AmchitBatchF2-2

SDG\#: $\quad$ S-K-60-A

\section{Summary of 2 and 3 sigma activities}

Below are the results for Sr-90 for Batch 2-2 from the Amchitka Island Project that had a result/uncertainty ratio of 2 or more (uncertainty @ one sigma).

\begin{tabular}{|c|c|c|c|c|c|}
\hline Customer ID & Lab ID & Isotope & $\begin{array}{c}\text { Result } \\
\mathrm{Bq} / \mathrm{g}\end{array}$ & $\begin{array}{c}\text { Uncertainty } \\
\mathrm{Bq} / \mathrm{g}\end{array}$ & $\begin{array}{c}\text { Result/ } \\
\text { Uncertainty }\end{array}$ \\
\hline S-K-89-A & O2FP-13-A & SR90 & $2.33 \mathrm{E}-02$ & $6.99 \mathrm{E}-04$ & 33.3 \\
\hline
\end{tabular}

All known sources of uncertainty are included in the uncertainty term. There may be unknown sources of uncertainty that are not accounted for. If the result/uncertainty ratio is more than 3 , we have a degree of confidence that the result is positive (i.e. the result is statistically different than zero). A result with the result/uncertainty ratio between 2 and 3 is the first indication that an isotope may be present and further investigation may be warranted.

As with any good science no single data point is used in important decisions (results need to be reproducible). 
BATCH 3

ALPHA AND Sr-90 


\begin{tabular}{llll} 
Project Title: & Alpha and Sr-90 Analysis for Amchitka Island (Batch 3) & \\
\cline { 2 - 3 } Lab Name: & RTC & Case No: & NA \\
Report No.: & AmchitBatchF3 & Method Type: & A/B \\
Approved SAP No.: NA & SDG No.: & K-AA-105-C
\end{tabular}

\section{SAMPLE NUMBERS}

Customer

Sample ID

K-AA-105-C

K-AA-106-C

K-AA-111-C

K-AA-112-C

$\mathrm{K}-\mathrm{BB}-107-\mathrm{C}$

K-BB-108-C

K-BB-109-C

K-BB-110-C

K-CC-113-C

K-CC-114-C

K-CC-115-C

K-CC-130-C

K-CC-132-C

K-CC-133-C

S-R-117-C

S-R-119-C

S-R-121-C
Lab Sample

ID

02GO-05-A

02G0-06-A

02G0-11-A

02G0-12-A

02G0-07-A

02G0-08-A

02GO-09-A

02G0-10-A

02GO-13-A

02GO-14-A

02G0-01-A

02G0-02-A

02GO-03-A

02G0-04-A

02GO-15-A

02GO-16-A

02GO-17-A

Comments:

Release of the data contained in this data package has been authorized by the laboratory manager or the manager's designee, as verified by the following signature:

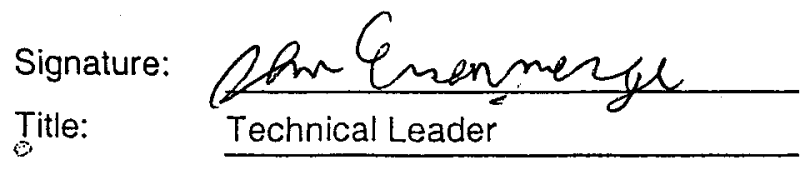

Name: J. G. Eisenmenger

Date: $\quad 05 / 26 / 2005$ 
$\frac{1}{2} \frac{8}{2}$

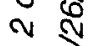

\& 8

a

\begin{tabular}{|c|c|c|c|c|c|c|c|c|c|c|c|c|c|c|c|c|c|c|c|c|c|}
\hline $\begin{array}{l}\square \\
0 \\
\end{array}$ & & & & & & & & & & & & & & & & & & & & & \\
\hline 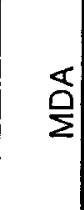 & $\begin{array}{l}m \\
0 \\
1 \\
1 \\
1 \\
2 \\
\infty \\
-1 \\
+\end{array}$ & $\begin{array}{l}n \\
0 \\
1 \\
1 \\
n \\
n \\
2 \\
0 \\
+ \\
+\end{array}$ & $\begin{array}{l}n \\
0 \\
1 \\
1 \\
n \\
n \\
m \\
n \\
+ \\
+\end{array}$ & $\begin{array}{l}n \\
0 \\
0 \\
1 \\
\\
N \\
N \\
\vdots \\
+ \\
+\end{array}$ & $\begin{array}{l}n \\
0 \\
1 \\
1 \\
m \\
m \\
\\
\dot{m} \\
+\end{array}$ & $\begin{array}{l}n \\
0 \\
1 \\
\omega \\
n \\
n \\
n \\
+ \\
+\end{array}$ & $\begin{array}{c}n \\
0 \\
1 \\
1 \\
0 \\
0 \\
0 \\
\sim \\
\sim \\
+\end{array}$ & $\begin{array}{l}n \\
0 \\
1 \\
\omega \\
\omega \\
0 \\
o \\
-1 \\
-1\end{array}$ & $\begin{array}{c}m \\
0 \\
1 \\
\dot{\omega} \\
2 \\
2 \\
\sigma \\
-1 \\
+\end{array}$ & $\begin{array}{l}n \\
0 \\
1 \\
\hat{\omega} \\
m \\
0 \\
0 \\
\dot{0} \\
+\end{array}$ & 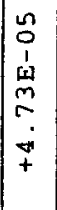 & $\begin{array}{l}n \\
0 \\
1 \\
\omega \\
0 \\
0 \\
0 \\
0 \\
+ \\
+\end{array}$ & $\begin{array}{l}n \\
0 \\
1 \\
\omega \\
\omega \\
a \\
r \\
m \\
+ \\
+\end{array}$ & $\begin{array}{l}n \\
0 \\
1 \\
\omega \\
a \\
6 \\
0 \\
0 \\
+ \\
+\end{array}$ & 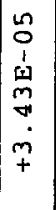 & 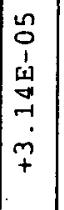 & $\begin{array}{c}m \\
0 \\
1 \\
w \\
q \\
w \\
m \\
\sim \\
+ \\
+\end{array}$ & $\mid$\begin{tabular}{c|}
$n$ \\
0 \\
1 \\
0 \\
0 \\
0 \\
-1 \\
7 \\
+
\end{tabular} & $\mid \begin{array}{c}n \\
0 \\
1 \\
\omega \\
y \\
- \\
m \\
\sim \\
\sim \\
+ \\
\end{array}$ & $\begin{array}{l}n \\
0 \\
1 \\
1 \\
\omega \\
\sigma \\
7 \\
0 \\
+ \\
+\end{array}$ & 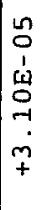 \\
\hline 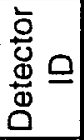 & $\vec{D}$ & $\stackrel{\infty}{0}$ & $\stackrel{\infty}{\circ}$ & ${ }_{0}^{\infty}$ & $\stackrel{m}{\rightarrow}$ & $\vec{m}$ & $\rightarrow$ & $0^{\infty}$ & 管 & ó & $\stackrel{m}{\circ}$ & m & $\vec{\rightarrow}$ & $\vec{I}$ & $\stackrel{-7}{-1}$ & on & $\vec{\alpha}$ & ${ }_{0}^{\circ}$ & 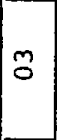 & m & 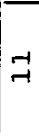 \\
\hline$\frac{5}{0} \frac{0}{0}$ & $\begin{array}{c}+ \\
-1 \\
-1 \\
-1\end{array}$ & $\begin{array}{l}n \\
0 \\
0 \\
\end{array}$ & $\begin{array}{l}n \\
\dot{0} \\
\stackrel{0}{0}\end{array}$ & $\begin{array}{l}n \\
0 \\
0 \\
-1\end{array}$ & . & $\begin{array}{l}\infty \\
1 \\
1 \\
\infty\end{array}$ & $\begin{array}{l}\Upsilon \\
\infty \\
\infty \\
\sigma\end{array}$ & $\begin{array}{l}n \\
0 \\
0 \\
0 \\
\end{array}$ & $\begin{array}{l}\sigma \\
\sigma \\
\sigma\end{array}$ & $\begin{array}{l}0 \\
0 \\
\infty \\
\sigma\end{array}$ & $\mid \begin{array}{l}0 \\
\infty \\
a \\
0\end{array}$ & $\begin{array}{l}0 \\
0 \\
\infty \\
\sigma\end{array}$ & in & $\begin{array}{l}\hat{n} \\
\dot{n} \\
\infty\end{array}$ & $\overrightarrow{\ddot{\sigma}}$ & $\begin{array}{l}0 \\
0 \\
\infty \\
\sigma\end{array}$ & $\begin{array}{l}0 \\
\dot{v} \\
\sigma\end{array}$ & $\begin{array}{l}\infty \\
\dot{\alpha} \\
\dot{\infty}\end{array}$ & \begin{tabular}{|l|} 
\\
$i$ \\
$i$ \\
$\infty$ \\
\end{tabular} & \begin{tabular}{|l|} 
\\
$\vdots$ \\
$i$ \\
$\infty$
\end{tabular} & $\infty$ \\
\hline & $\underset{7}{7}$ & $\theta$ & $\mp$ & $\bar{\sim}$ & $\bar{\sim}$ & $\vec{A}$ & $\approx$ & $\underset{\sim}{\sim}$ & $\vec{T}$ & 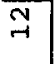 & -1 & $-i$ & 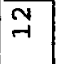 & 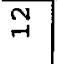 & -1 & $\widetilde{\tau}$ & $\bar{y}$ & $\bar{I}$ & $\overline{\mathcal{F}}$ & $\bar{F}$ & \\
\hline
\end{tabular}

\&

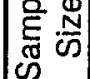

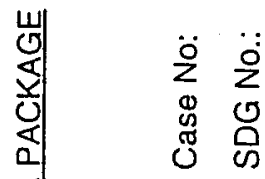

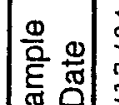

总 F F F

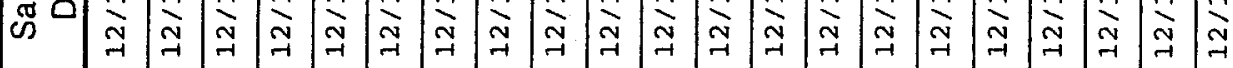

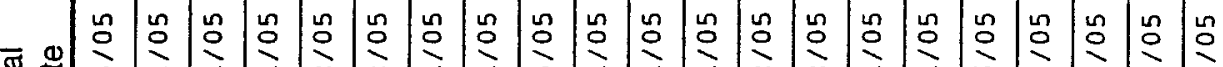

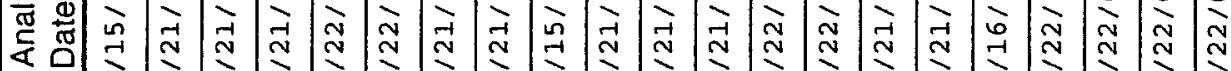

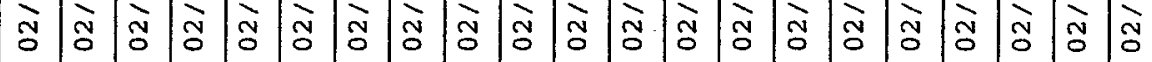

ว.

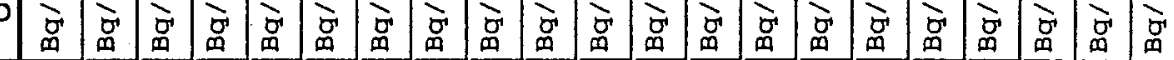

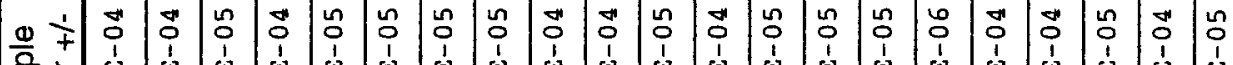

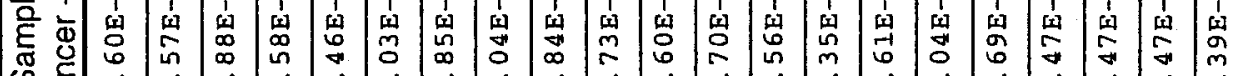

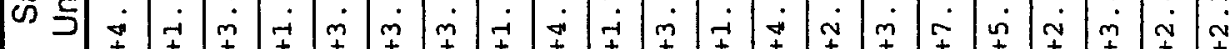

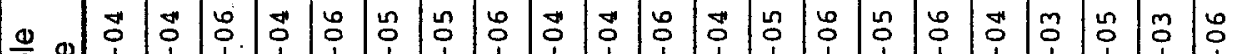

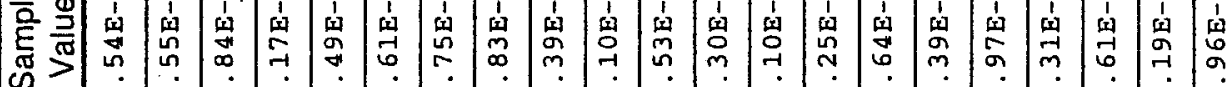

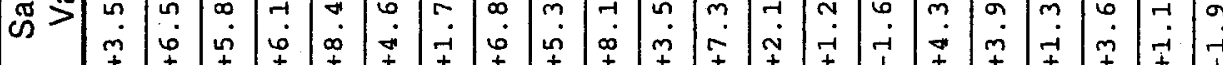

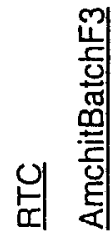

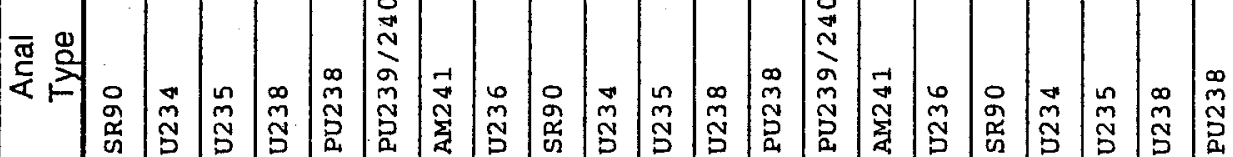
음 즌

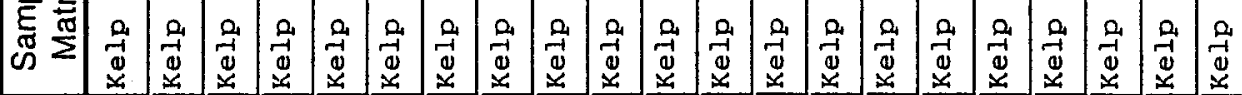

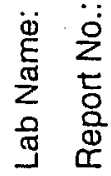

五

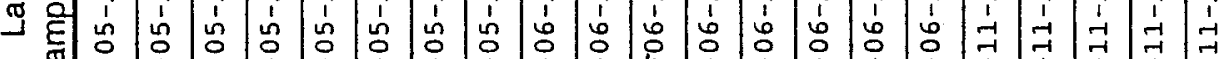
心)

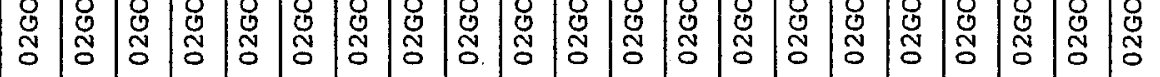

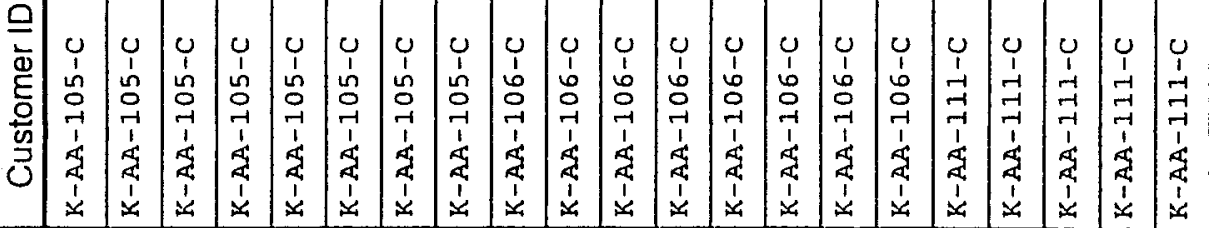




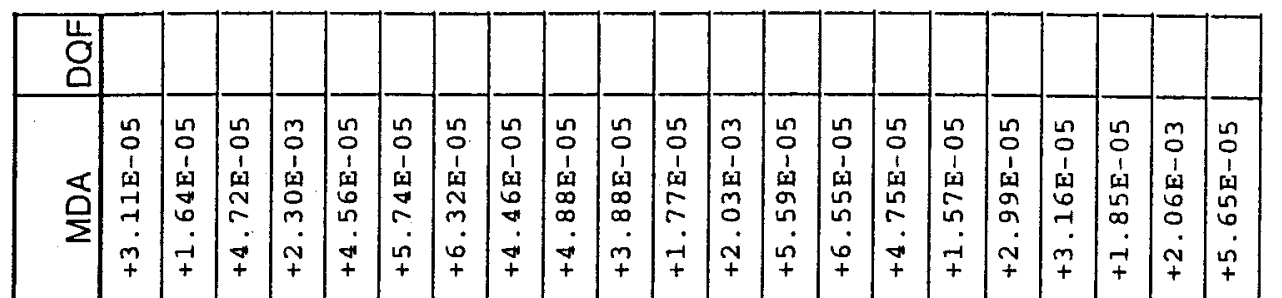

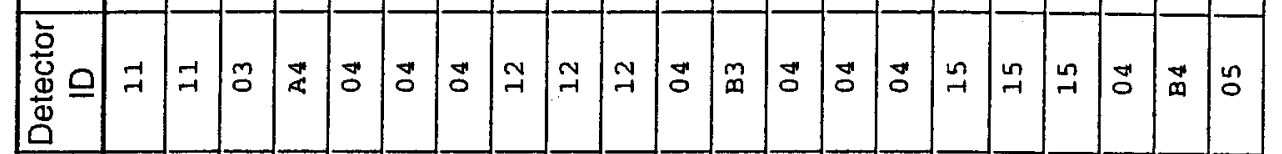

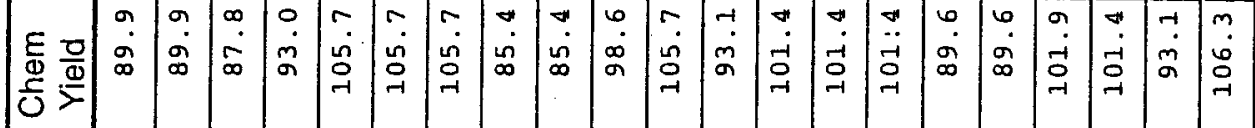

\&1

费 舟 胥

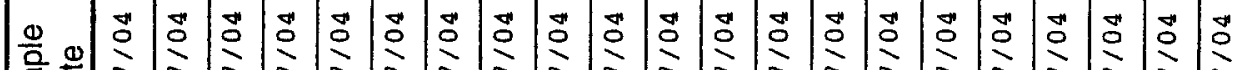

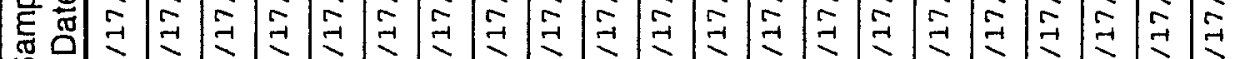

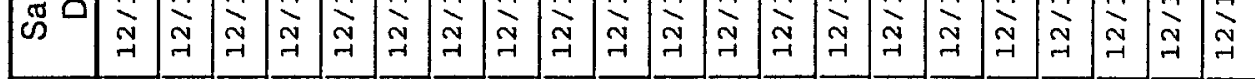

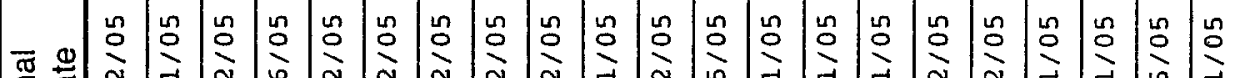

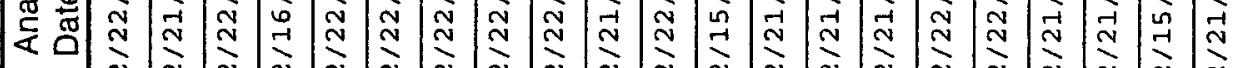
突

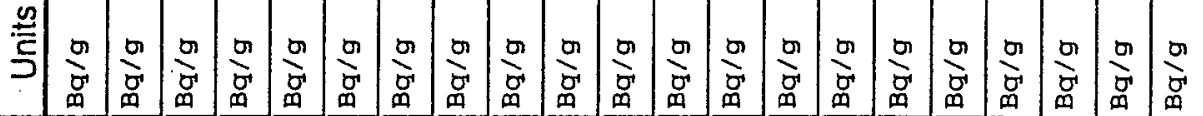

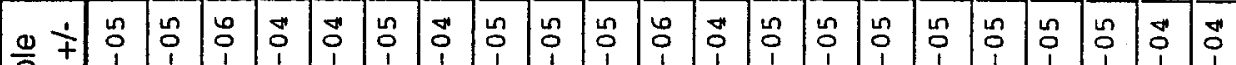

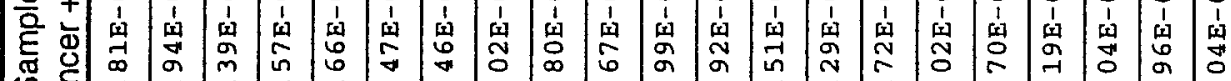

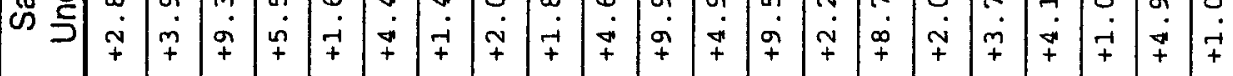

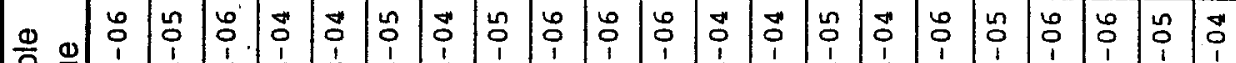

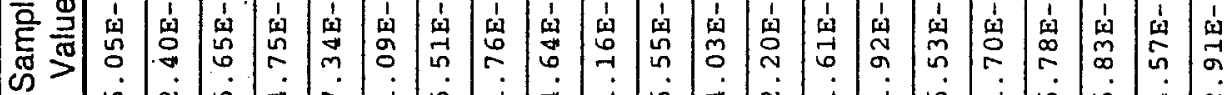

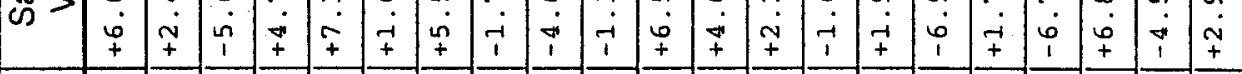

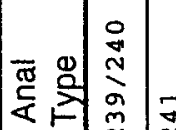

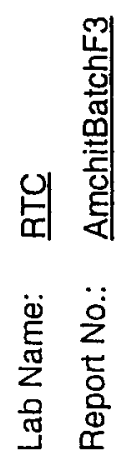

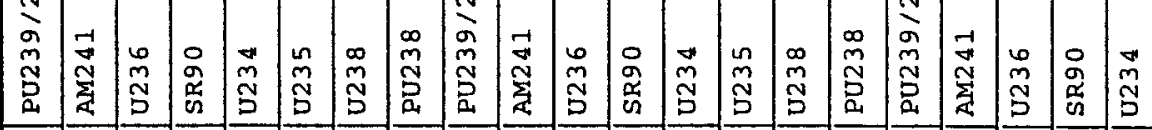
을 즐

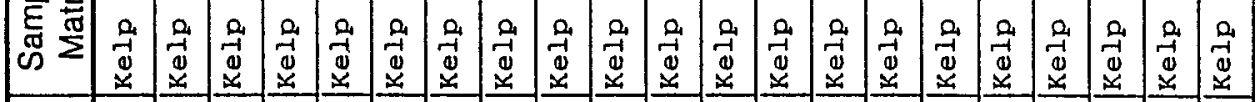
으

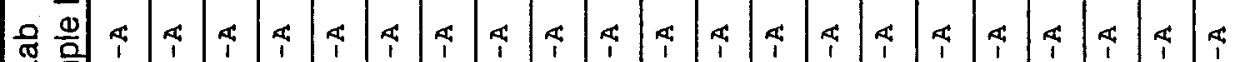
涩烤古

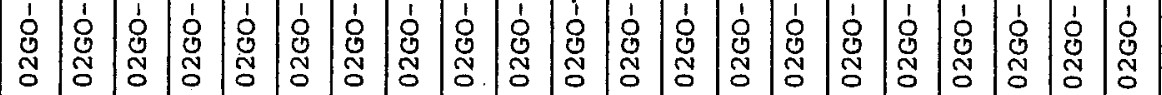




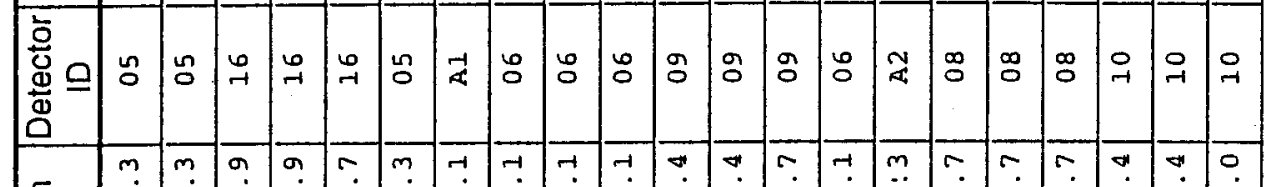

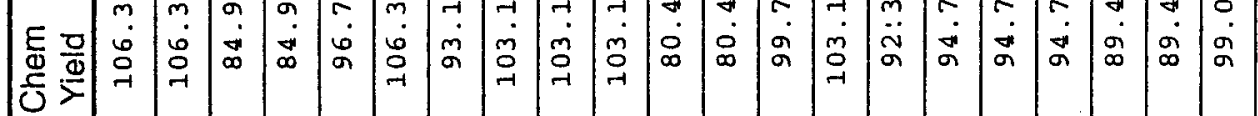
乙)

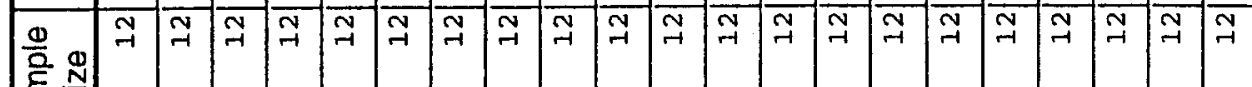

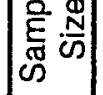

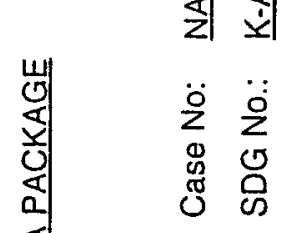

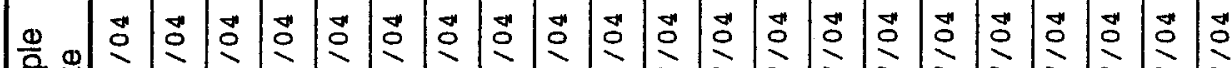

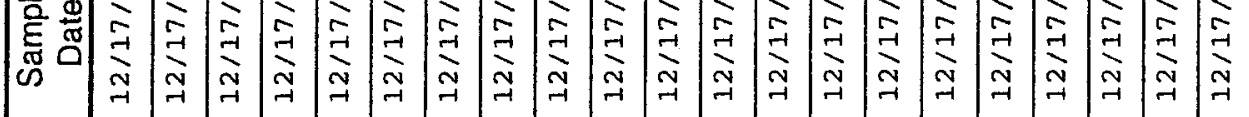

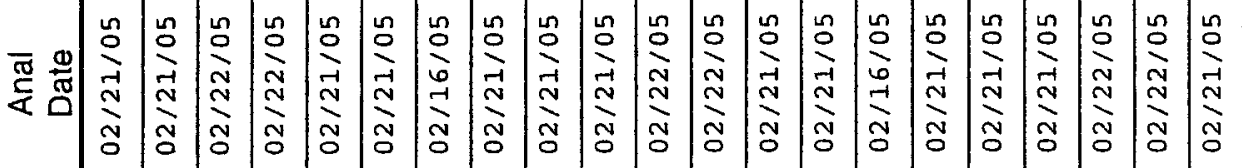

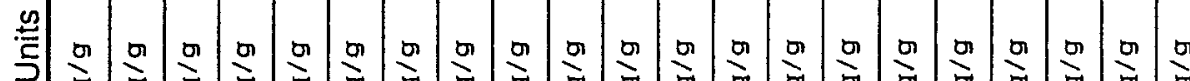

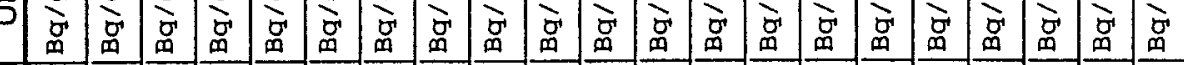

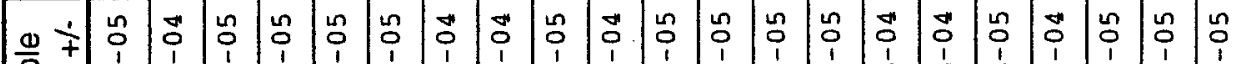

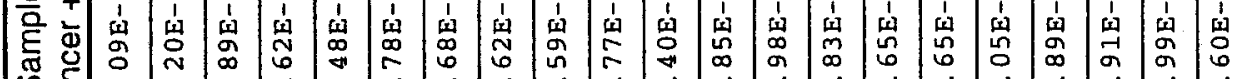

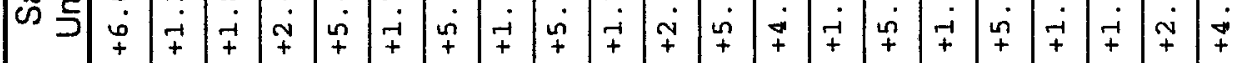

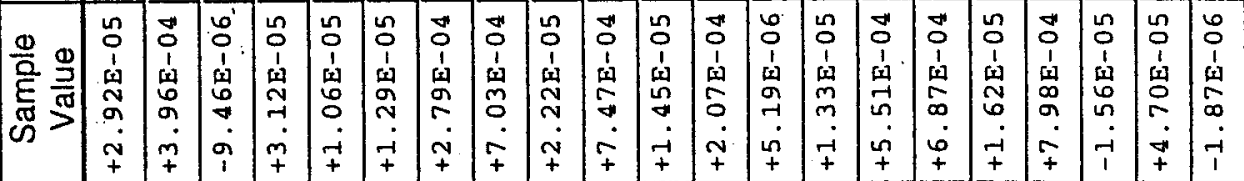

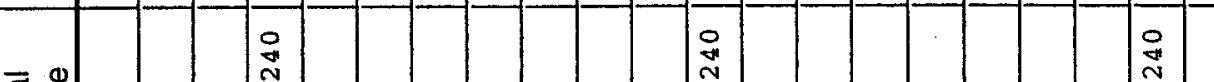

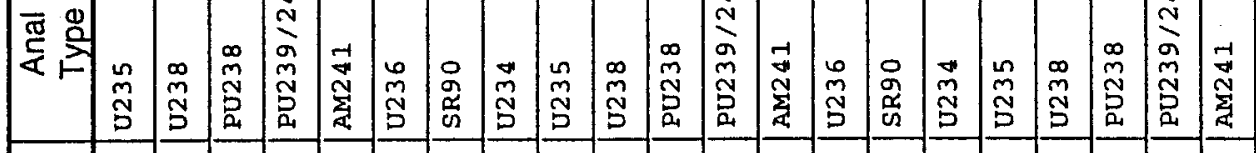
임 음 $\times$

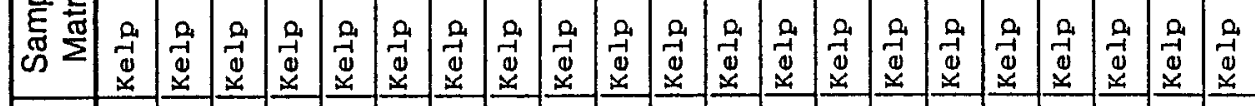

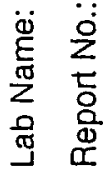

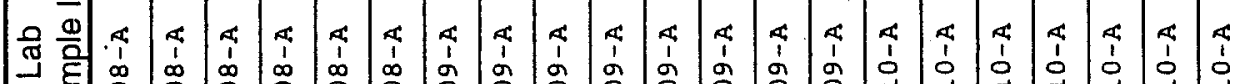
$\exists$ 気

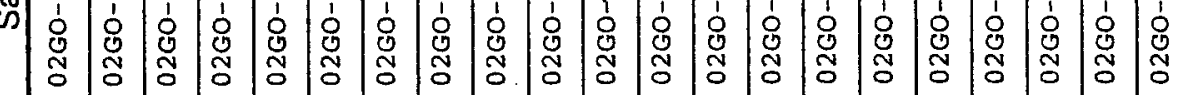




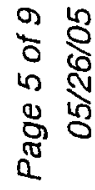

\begin{tabular}{|c|c|c|c|c|c|c|c|c|c|c|c|c|c|c|c|c|c|c|c|c|c|}
\hline 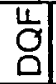 & & & & & & & & & & & & & & & & & & & & & \\
\hline 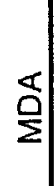 & $\begin{array}{c}n \\
0 \\
1 \\
1 \\
0 \\
0 \\
o \\
\vdots \\
-1 \\
+\end{array}$ & $\mid \begin{array}{c}m \\
0 \\
1 \\
1 \\
\text { dj } \\
\sim \\
\sim \\
\sim \\
+ \\
+\end{array}$ & $\begin{array}{l}n \\
0 \\
1 \\
\omega \\
\alpha \\
\infty \\
0 \\
\dot{m} \\
+\end{array}$ & 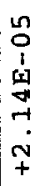 & $\begin{array}{l}n \\
0 \\
0 \\
1 \\
\mu \\
m \\
-1 \\
\dot{0} \\
+ \\
+\end{array}$ & 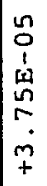 & 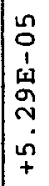 & 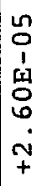 & $\begin{array}{l}n \\
0 \\
1 \\
\omega \\
9 \\
\sim \\
\sim \\
\\
+\end{array}$ & 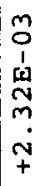 & 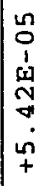 & 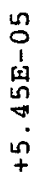 & 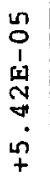 & 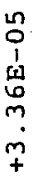 & 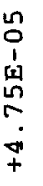 & 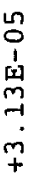 & 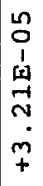 & $\begin{array}{l}m \\
0 \\
1 \\
\hat{\varphi} \\
\sigma \\
\tilde{\sigma} \\
-i \\
+\end{array}$ & $\begin{array}{l}n \\
0 \\
1 \\
1 \\
0 \\
0 \\
0 \\
-0 \\
+ \\
+\end{array}$ & 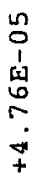 & $\begin{array}{l}n \\
0 \\
1 \\
1 \\
1 \\
0 \\
0 \\
\\
\dot{m} \\
\end{array}$ \\
\hline
\end{tabular}

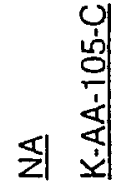

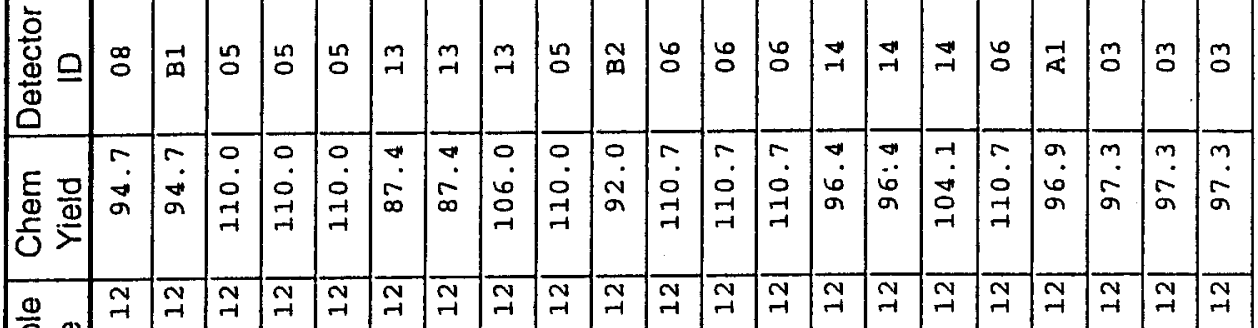
है⿴囗十心 ผ

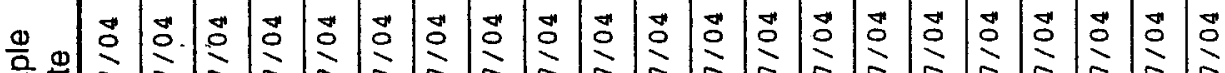

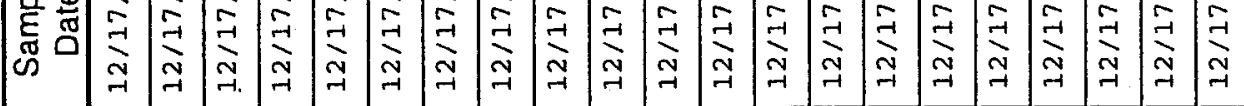

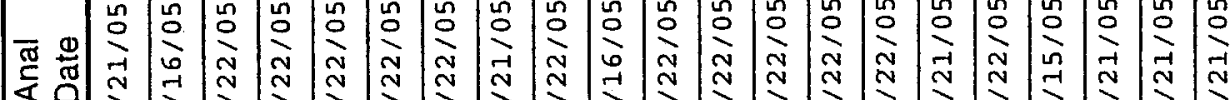

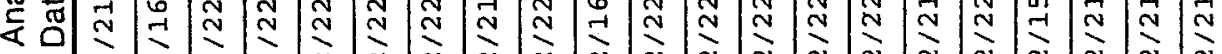

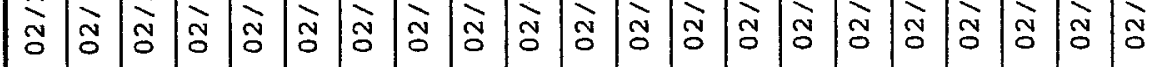

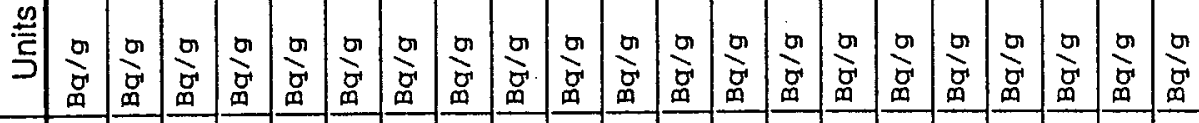

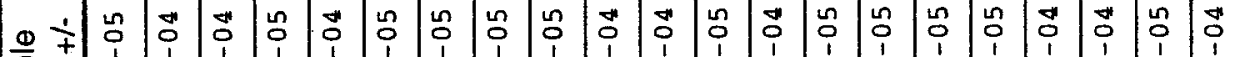

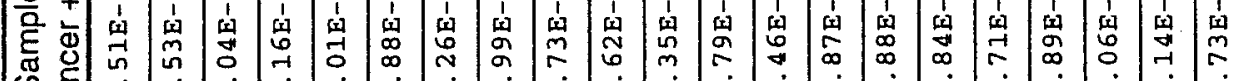
थ

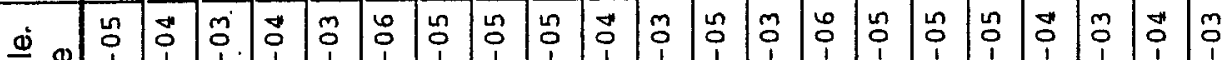

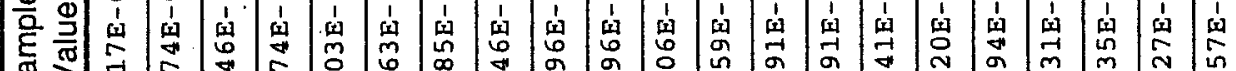
œึ

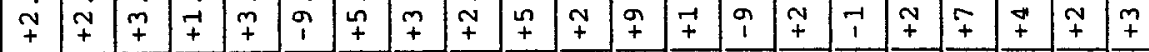

要这

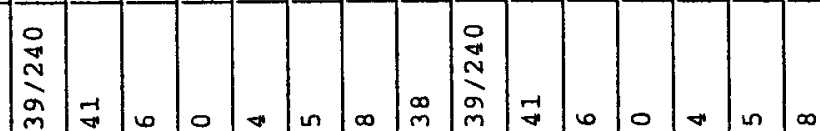

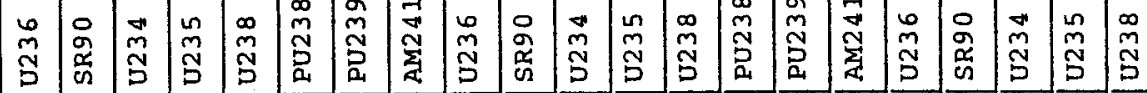

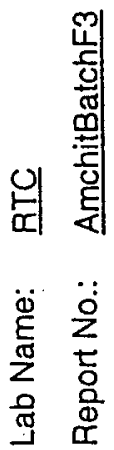
응 즌

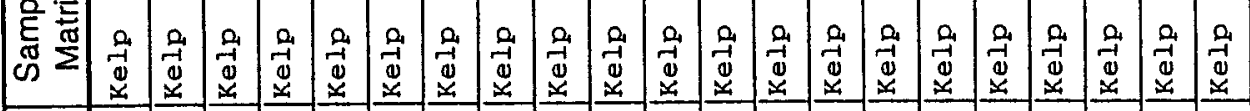
음

品

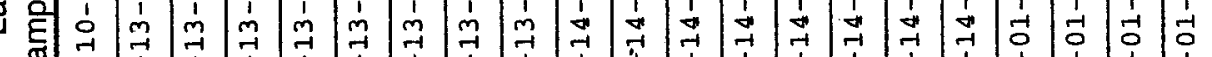
की

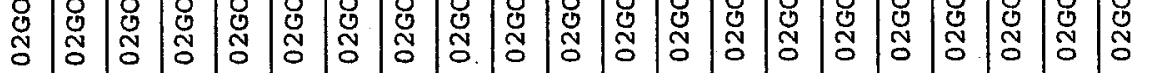

으

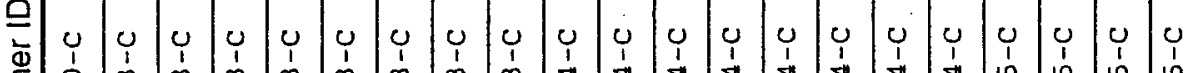

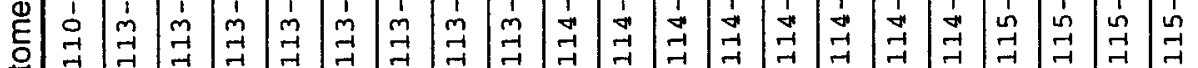


$\frac{1}{9} \stackrel{8}{0}$

เ

a

\begin{tabular}{|c|c|c|c|c|c|c|c|c|c|c|c|c|c|c|c|c|c|c|c|c|c|}
\hline $\begin{array}{l}u \\
0 \\
0\end{array}$ & & & & & & & & & & & & & & & & & & & & & \\
\hline$\stackrel{\mathbb{\alpha}}{\stackrel{0}{2}}$ & $\begin{array}{l}n \\
0 \\
1 \\
1 \\
0 \\
0 \\
0 \\
0 \\
\vdots \\
+\end{array}$ & $\mid \begin{array}{l}n \\
0 \\
1 \\
\omega \\
\hat{p} \\
0 \\
0 \\
m \\
+\end{array}$ & $\mid \begin{array}{l}0 \\
0 \\
1 \\
1 \\
0 \\
0 \\
0 \\
m \\
m \\
0 \\
+\end{array}$ & $\begin{array}{l}u n \\
0 \\
1 \\
1 \\
0 \\
0 \\
0 \\
-1 \\
+\end{array}$ & $\begin{array}{l}m \\
0 \\
1 \\
1 \\
\infty \\
0 \\
\vdots \\
\vdots \\
+ \\
+\end{array}$ & 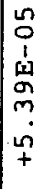 & $\begin{array}{l}n \\
0 \\
0 \\
1 \\
9 \\
9 \\
0 \\
0 \\
0 \\
+\end{array}$ & $\mid \begin{array}{l}n \\
0 \\
1 \\
\vdots \\
0 \\
0 \\
0 \\
0 \\
0 \\
0 \\
+\end{array}$ & $\begin{array}{l}n \\
0 \\
0 \\
1 \\
\omega \\
\\
n \\
n \\
n \\
+\end{array}$ & 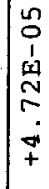 & 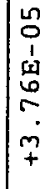 & $\begin{array}{l}n \\
0 \\
1 \\
1 \\
0 \\
0 \\
\vdots \\
\dot{m} \\
+\end{array}$ & $\mid \begin{array}{c}m \\
0 \\
1 \\
1 \\
2 \\
2 \\
\sigma \\
-1 \\
-1 \\
+\end{array}$ & $\mid \begin{array}{c}n \\
0 \\
1 \\
1 \\
\omega \\
0 \\
0 \\
0 \\
n \\
+ \\
+\end{array}$ & $\mid \begin{array}{l}n \\
0 \\
1 \\
1 \\
\sigma \\
\sigma \\
\sigma \\
\sigma \\
+ \\
+\end{array}$ & $\begin{array}{l}0 \\
0 \\
1 \\
1 \\
0 \\
0 \\
\sim \\
n \\
+ \\
+\end{array}$ & \begin{tabular}{|c|}
$n$ \\
0 \\
1 \\
$\omega$ \\
$\sim$ \\
$\sim$ \\
0 \\
+ \\
+
\end{tabular} & 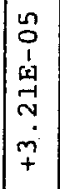 & 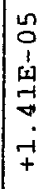 & $\mid \begin{array}{l}n \\
0 \\
1 \\
1 \\
a \\
0 \\
0 \\
-1 \\
+ \\
+\end{array}$ & 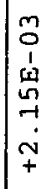 \\
\hline 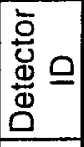 & 18 & g & 18 & ${ }^{m}$ & జ & 8 & $0^{\circ}$ & $\overrightarrow{0}$ & 욱 & 足 & $\stackrel{्}{-1}$ & $0^{*}$ & 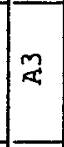 & 年 & | & 足 & $\Rightarrow$ & $\exists$ & $\exists$ & 告 & $\nexists \vec{\alpha}$ \\
\hline 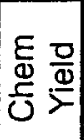 & $\begin{array}{l}0 \\
\dot{0} \\
\infty\end{array}$ & $\begin{array}{l}0 \\
0 \\
0 \\
0\end{array}$ & $\begin{array}{l}0 \\
\sigma \\
\sigma\end{array}$ & $\begin{array}{l}m \\
\text { n. } \\
\text { a }\end{array}$ & $\begin{array}{l}\sim \\
\vdots \\
\sigma\end{array}$ & $\begin{array}{r}n \\
\stackrel{\sim}{0} \\
\stackrel{-}{n}\end{array}$ & 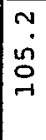 & 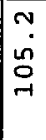 & $\begin{array}{l}n \\
\tilde{N} \\
n\end{array}$ & $\begin{array}{l}\tilde{n} \\
\tilde{\alpha}\end{array}$ & $\begin{array}{l}N \\
a \\
\sigma\end{array}$ & $\begin{array}{l}0 \\
\vdots \\
0 \\
0 \\
-1\end{array}$ & $\begin{array}{l}0 \\
\dot{0} \\
\sigma\end{array}$ & $\begin{array}{l}\infty \\
\alpha \\
\alpha\end{array}$ & $\begin{array}{l}\infty \\
\ddot{\sigma} \\
\sigma\end{array}$ & $\begin{array}{l}\infty \\
\sigma \\
\sigma\end{array}$ & $\begin{array}{l}0 \\
\dot{\alpha} \\
\infty\end{array}$ & $\begin{array}{c}0 \\
\vdots \\
\infty\end{array}$ & $\begin{array}{l}0 \\
\sim \\
0 \\
0 \\
-1\end{array}$ & $\sigma^{\infty}$ & $\begin{array}{l}m \\
\infty \\
\infty\end{array}$ \\
\hline 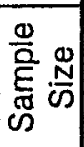 & $\underset{7}{ }$ & $\overline{\mathcal{A}}$ & $\bar{\sim}$ & $\sqrt{\sim}$ & $\underset{\sim}{-}$ & $\bar{T}$ & $\overline{-}$ & $\bar{\gamma}$ & $\overline{\widetilde{N}}$ & $\bar{\sim}$ & $\overline{\mathrm{N}}$ & ㄱ. & $\bar{N}$ & $\bar{\nearrow}$ & $\bar{\Im}$ & $\underset{\sim}{\sim}$ & $\bar{\top}$ & $\bar{\sim}$ & $\bar{\sim}$ & $\bar{\sim}$ & $\bar{I}$ \\
\hline
\end{tabular}

雨

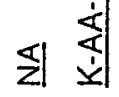

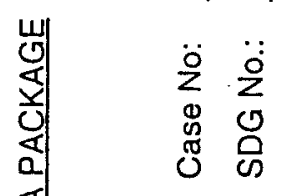

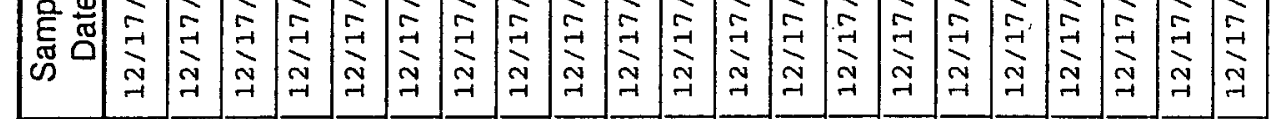

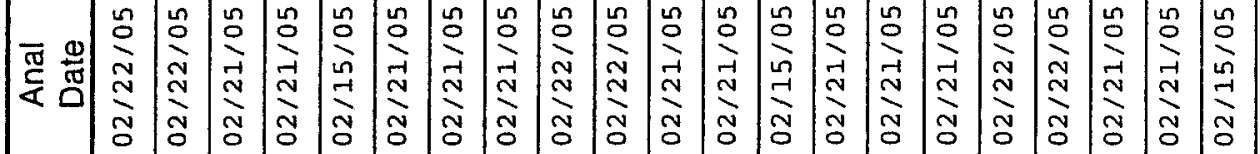
20 a a a a a a a a a a a

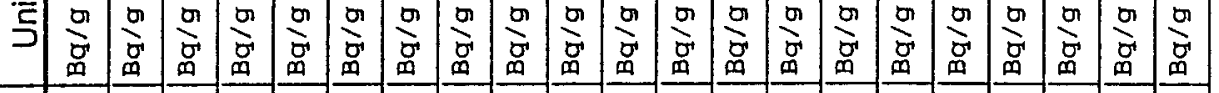

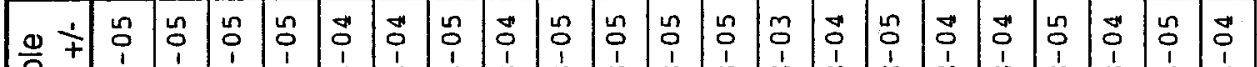

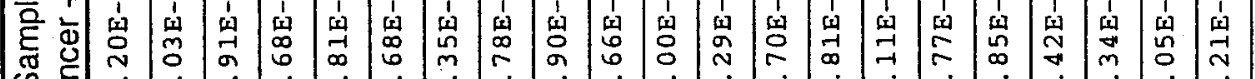

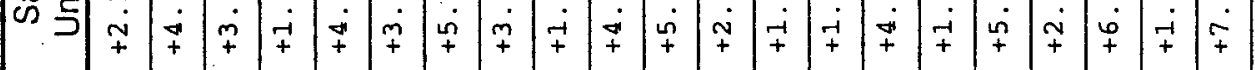

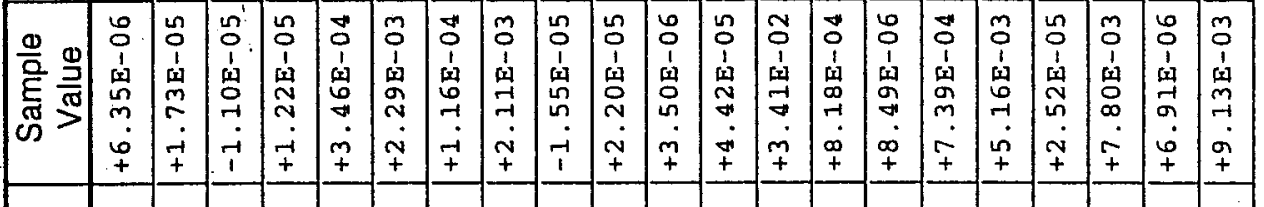

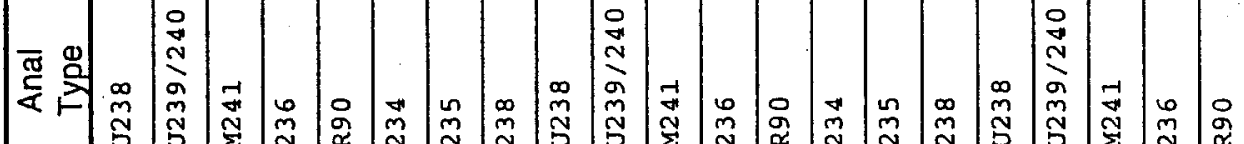

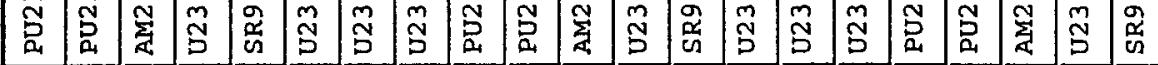

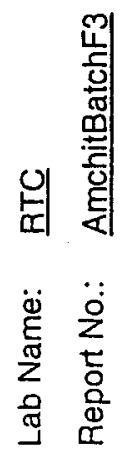

产

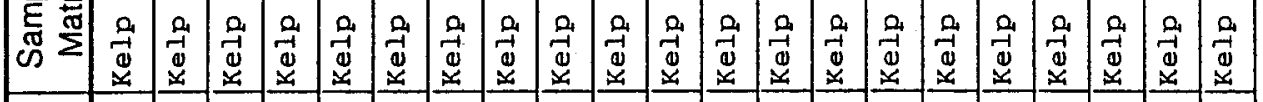
응

兄 00 年

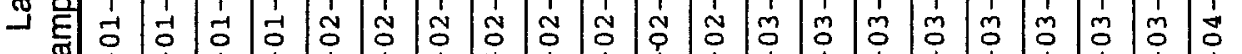

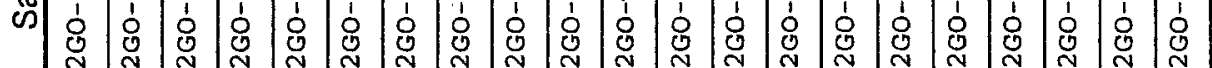

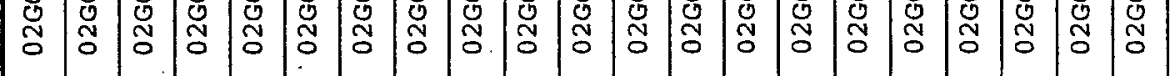

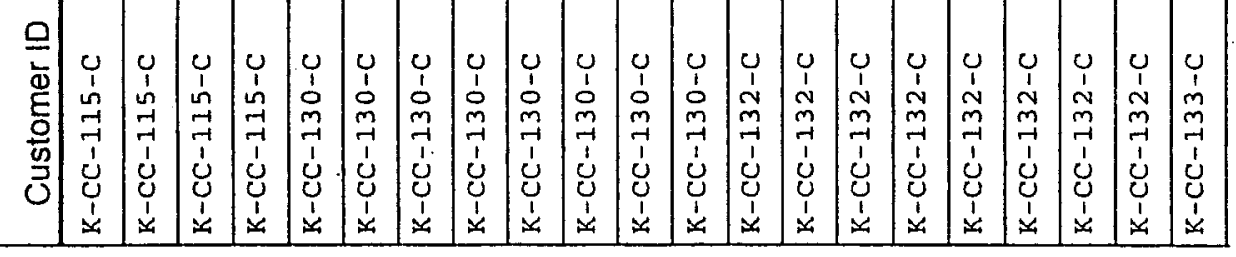


$\begin{array}{lll}0 & 0 \\ 0 & 8 \\ 0 & 0 \\ 1 & 0 \\ 0 & 0 \\ 8 & 0\end{array}$

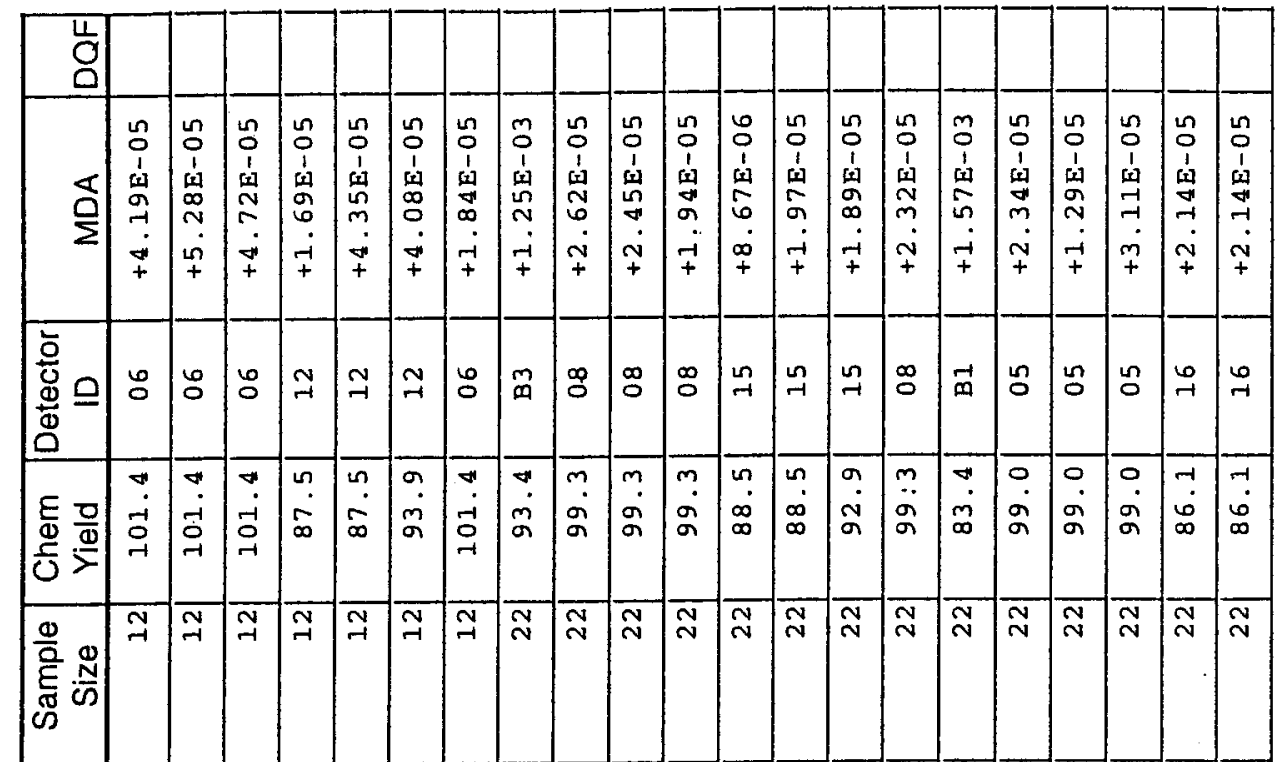

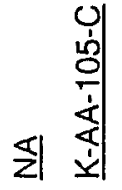

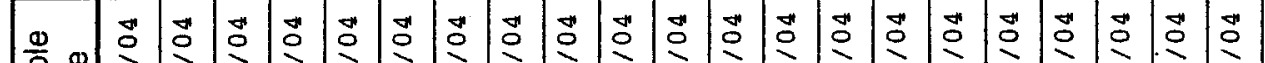
岁|

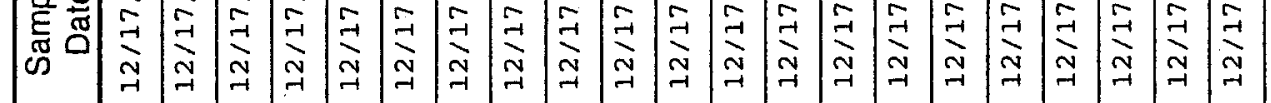
孚

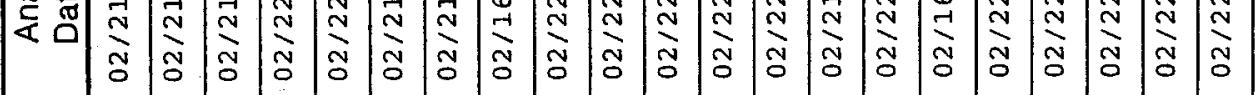

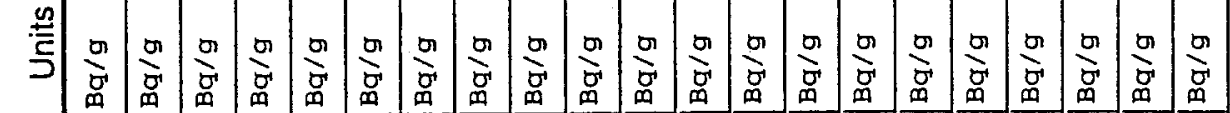

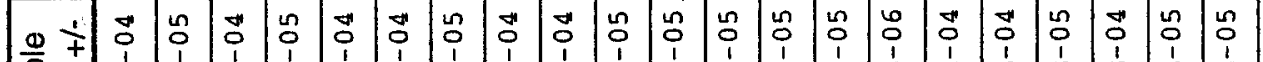

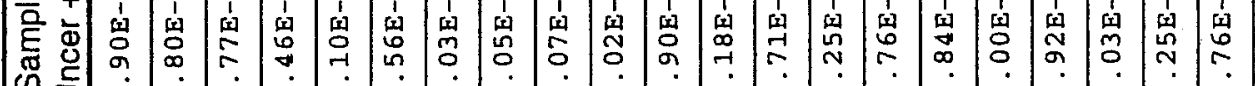

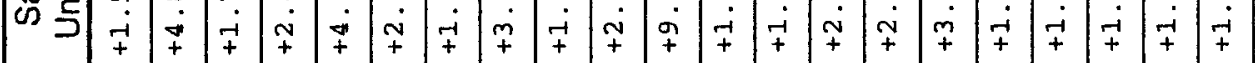
1

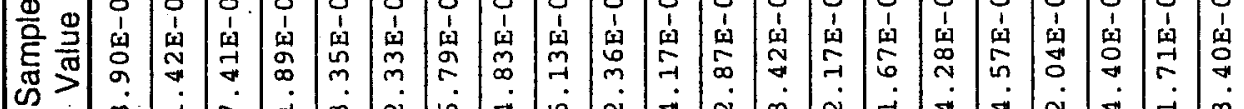

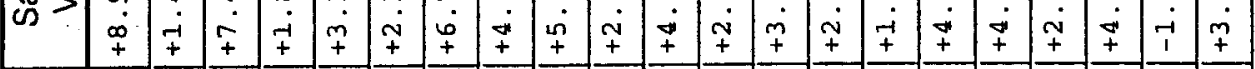

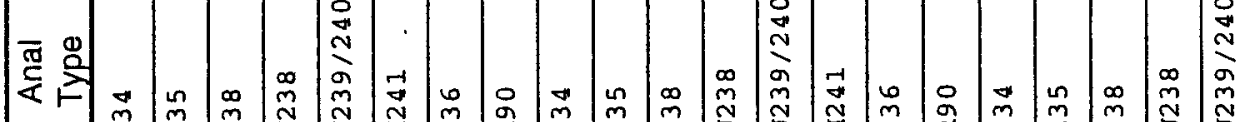
임

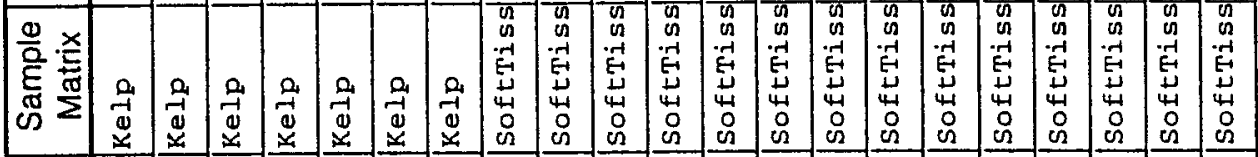

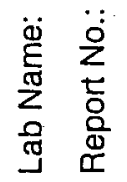

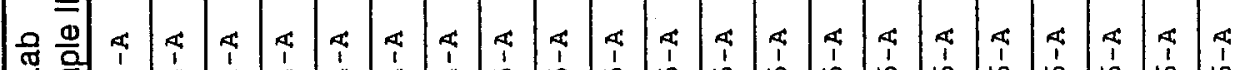

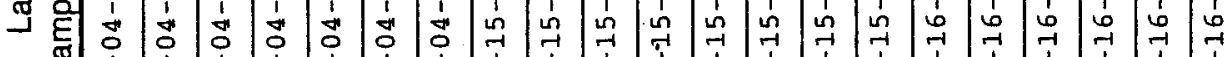
œ

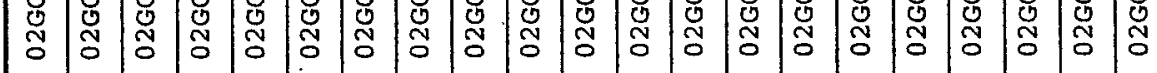


$\begin{array}{ll}9 & 0 \\ 0 & 0 \\ \infty & 5 \\ 0 & 5 \\ 5 & 0 \\ 0 & 0\end{array}$

\begin{tabular}{|c|c|c|c|c|c|c|c|c|c|c|}
\hline $\begin{array}{l} \\
\\
\end{array}$ & & & & & & & & & & \\
\hline $\begin{array}{l}\varsigma \\
\Sigma\end{array}$ & 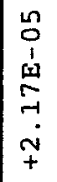 & $\begin{array}{l}n \\
0 \\
1 \\
1 \\
0 \\
0 \\
0 \\
-1 \\
+1\end{array}$ & $\begin{array}{l}m \\
0 \\
1 \\
1 \\
0 \\
0 \\
+4 \\
-1 \\
+\end{array}$ & $\begin{array}{l}n \\
0 \\
1 \\
\omega \\
-1 \\
\sim \\
\dot{n} \\
+\end{array}$ & \begin{tabular}{|c|c}
$n$ \\
0 \\
1 \\
1 \\
2 \\
$\infty$ \\
$\infty$ \\
$\sim$ \\
+ \\
+
\end{tabular} & $\begin{array}{c}n \\
0 \\
1 \\
1 \\
\omega \\
\\
2 \\
m \\
+ \\
+\end{array}$ & $\begin{array}{c}0 \\
0 \\
1 \\
1 \\
1 \\
01 \\
0 \\
0 \\
0 \\
+\end{array}$ & $\begin{array}{l}1 \\
0 \\
1 \\
1 \\
1 \\
0 \\
0 \\
0 \\
+ \\
+\end{array}$ & 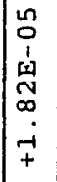 & 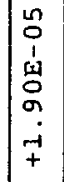 \\
\hline $\begin{array}{l}\overline{\mathrm{g}} \\
\frac{\mathrm{d}}{\mathrm{d}} \\
\mathrm{D}\end{array}$ & $\stackrel{6}{\sim}$ & \& & 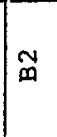 & 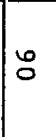 & 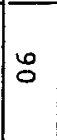 & 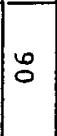 & \% & o & 욱 & $\%$ \\
\hline$\frac{\varepsilon}{\bar{d}} \frac{}{0}$ & $\begin{array}{l}0 \\
0 \\
0\end{array}$ & व. & $\begin{array}{l}\sigma \\
\dot{N} \\
\sigma\end{array}$ & $\begin{array}{c}\sigma \\
- \\
-i \\
-1\end{array}$ & $\begin{array}{c}9 \\
-1 \\
-1 \\
-1\end{array}$ & $\begin{array}{c}0 \\
- \\
- \\
O \\
-1\end{array}$ & $\begin{array}{l}-1 \\
\infty \\
\infty\end{array}$ & $\begin{array}{l}-1 \\
0 \\
\infty \\
\infty\end{array}$ & $\begin{array}{l}0 \\
\sigma \\
\sigma\end{array}$ & $\begin{array}{c}0 \\
- \\
-1 \\
-1 \\
-1\end{array}$ \\
\hline 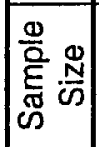 & $\tilde{N}$ & $\bar{N}$ & $\bar{N}$ & $\stackrel{N}{N}$ & $\bar{N}$ & $\bar{N}$ & N & $\bar{N}$ & $\bar{N}$ & $\bar{N}$ \\
\hline 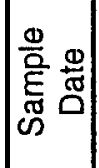 & 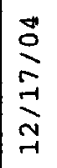 & 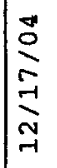 & 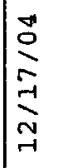 & 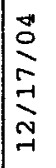 & 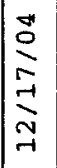 & 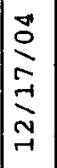 & 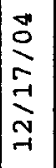 & $\begin{array}{l}\vec{D} \\
\stackrel{2}{a} \\
\vec{f} \\
\stackrel{f}{f}\end{array}$ & 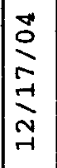 & 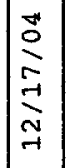 \\
\hline 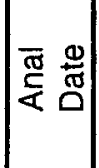 & 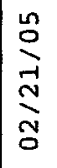 & $\begin{array}{l}n \\
0 \\
N \\
N \\
N \\
0\end{array}$ & \begin{tabular}{l}
$n$ \\
0 \\
0 \\
0 \\
\\
\cline { 1 - 1 } \\
0
\end{tabular} & $\begin{array}{l}n \\
0 \\
N \\
N \\
N \\
0\end{array}$ & $\mid \begin{array}{c}n \\
0 \\
N \\
N \\
N \\
0\end{array}$ & 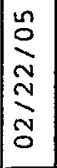 & 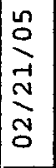 & 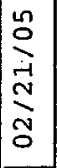 & 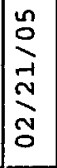 & $\mid \begin{array}{l}n \\
0 \\
\text { N } \\
\text { N } \\
\text { N } \\
\text { o }\end{array}$ \\
\hline$\stackrel{0}{\stackrel{2}{5}}$ & के & 吕 & 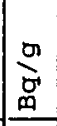 & 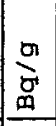 & \begin{tabular}{|l|}
0 \\
0 \\
0 \\
0
\end{tabular} & \begin{tabular}{|c|}
0 \\
0 \\
9
\end{tabular} & \begin{tabular}{|c|c}
0 \\
0 \\
0 \\
0
\end{tabular} & 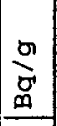 & 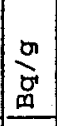 & $\mid \begin{array}{c}0 \\
\dot{9} \\
\end{array}$ \\
\hline 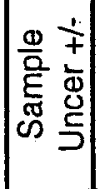 & \begin{tabular}{|l}
$n$ \\
0 \\
1 \\
1 \\
cy \\
0 \\
$m$ \\
$\sim$ \\
$\sim$ \\
+
\end{tabular} & $\mid \begin{array}{l}0 \\
0 \\
1 \\
1 \\
1 \\
\vdots \\
\vdots \\
\vdots \\
+ \\
+\end{array}$ & $\begin{array}{l}0 \\
0 \\
1 \\
1 \\
w \\
0 \\
0 \\
m \\
+ \\
+\end{array}$ & $\begin{array}{l}0 \# \\
0 \\
1 \\
1 \\
0 \\
0 \\
0 \\
-i \\
+ \\
+\end{array}$ & \begin{tabular}{|l|}
$n$ \\
0 \\
1 \\
$\omega$ \\
$\omega$ \\
2 \\
-1 \\
+ \\
+ \\
\end{tabular} & $\begin{array}{c}0 \\
0 \\
1 \\
1 \\
m \\
0 \\
0 \\
-i \\
+ \\
\end{array}$ & 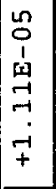 & 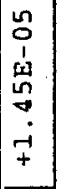 & \begin{tabular}{|c}
$n$ \\
0 \\
1 \\
$\omega$ \\
0 \\
$\sim$ \\
$\sim$ \\
$\sim$ \\
+ \\
+ \\
\end{tabular} & 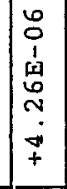 \\
\hline 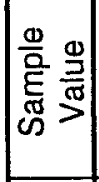 & \begin{tabular}{|l}
$n$ \\
0 \\
1 \\
1 \\
9 \\
0 \\
$\vdots$ \\
$\dot{n}$ \\
+ \\
\end{tabular} & $\begin{array}{l}n \\
0 \\
1 \\
1 \\
0 \\
\vdots \\
-1 \\
-1 \\
+ \\
\end{array}$ & $\begin{array}{l}0 \\
0 \\
1 \\
1 \\
m \\
m \\
m \\
i \\
+ \\
\end{array}$ & $\begin{array}{l}0 \\
0 \\
1 \\
1 \\
0 \\
0 \\
0 \\
0 \\
n \\
+ \\
\end{array}$ & \begin{tabular}{|c|}
0 \\
0 \\
1 \\
0 \\
1 \\
-1 \\
0 \\
0 \\
0 \\
1 \\
\end{tabular} & 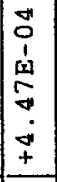 & \begin{tabular}{|c|c}
0 \\
0 \\
1 \\
$\hat{\omega}$ \\
$\infty$ \\
0 \\
$m$ \\
$p$ \\
1 \\
\end{tabular} & 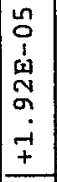 & 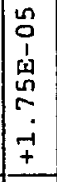 & $\begin{array}{c}0 \\
0 \\
0 \\
1 \\
\dot{\mu} \\
0 \\
0 \\
0 \\
\dot{T} \\
+ \\
+ \\
\end{array}$ \\
\hline$=$ & 离 & $\mid \begin{array}{l}0 \\
\stackrel{N}{S} \\
\text { S. }\end{array}$ & 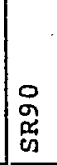 & 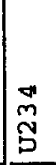 & 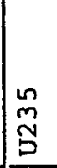 & $\begin{array}{l}\infty \\
\tilde{m} \\
\tilde{S}\end{array}$ & $\mid \begin{array}{c}\infty \\
\stackrel{n}{N} \\
\tilde{D} \\
0\end{array}$ & 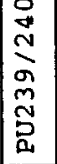 & 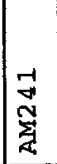 & 兽 \\
\hline 呰 & $\begin{array}{c}0 \\
0 \\
n \\
-1 \\
E \\
4 \\
0 \\
0 \\
0 \\
\end{array}$ & $\begin{array}{l}0 \\
n \\
n \\
-1 \\
E \\
4 \\
0 \\
0 \\
0 \\
0\end{array}$ & $\begin{array}{l}0 \\
0 \\
0 \\
-1 \\
-1 \\
4 \\
0 \\
0 \\
0 \\
0\end{array}$ & $\begin{array}{l}0 \\
0 \\
0 \\
0-1 \\
4 \\
4-1 \\
0 \\
0 \\
0\end{array}$ & $\begin{array}{l}0 \\
0 \\
0 \\
ت-1 \\
4 \\
4 \\
0 \\
0 \\
0\end{array}$ & \begin{tabular}{|l|}
0 \\
0 \\
$0-1$ \\
-1 \\
4 \\
4 \\
0 \\
0 \\
0 \\
\end{tabular} & \begin{tabular}{|l|}
0 \\
0 \\
0 \\
-4 \\
$E$ \\
4 \\
0 \\
0 \\
0 \\
\end{tabular} & \begin{tabular}{|l|} 
\\
0 \\
0 \\
-4 \\
4 \\
4 \\
0 \\
0 \\
0 \\
\end{tabular} & 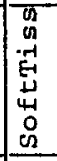 & 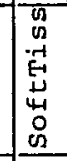 \\
\hline 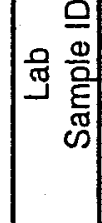 & 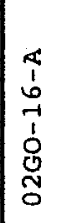 & 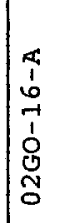 & 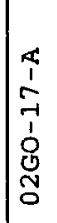 & 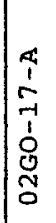 & 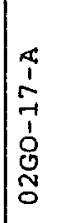 & $\begin{array}{l}\alpha \\
1 \\
1 \\
7 \\
1 \\
0 \\
0 \\
\tilde{N} \\
0\end{array}$ & $\begin{array}{l}r \\
1 \\
1 \\
\\
1 \\
0 \\
0 \\
0 \\
0 \\
0\end{array}$ & $\begin{array}{l}1 \\
1 \\
\vdots \\
7 \\
1 \\
0 \\
0 \\
\tilde{N} \\
0\end{array}$ & 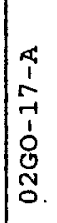 & 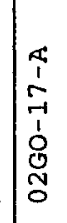 \\
\hline 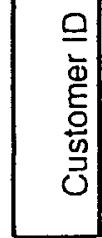 & $\mid \begin{array}{l}0 \\
1 \\
0 \\
-1 \\
-1 \\
1 \\
1 \\
1 \\
1 \\
0\end{array}$ & $\begin{array}{c}0 \\
1 \\
1 \\
a \\
-1 \\
-1 \\
1 \\
\alpha \\
1 \\
0 \\
0\end{array}$ & $\mid \begin{array}{c}0 \\
1 \\
-1 \\
\tilde{n} \\
7 \\
1 \\
x \\
1 \\
1 \\
0\end{array}$ & 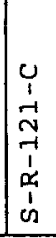 & 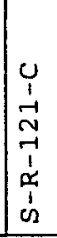 & 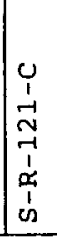 & $\begin{array}{c}0 \\
1 \\
1 \\
-1 \\
-1 \\
i \\
1 \\
0 \\
1 \\
1 \\
02 \\
\end{array}$ & \begin{tabular}{|c|c}
0 \\
1 \\
-1 \\
-1 \\
7 \\
1 \\
0 \\
0 \\
0 \\
0 \\
\end{tabular} & 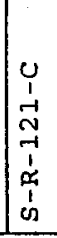 & 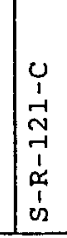 \\
\hline
\end{tabular}

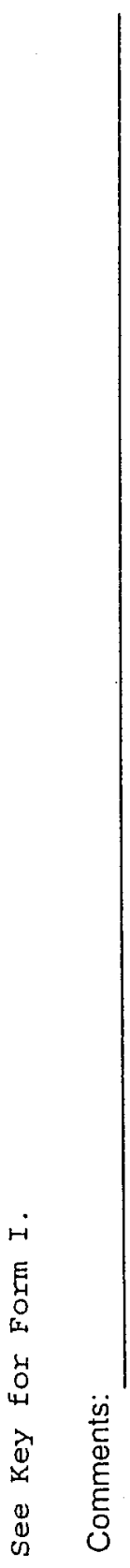


ㅇํㅇ ํํำ

के @े

व

$\sum \mid$

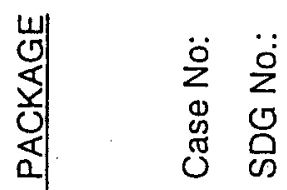

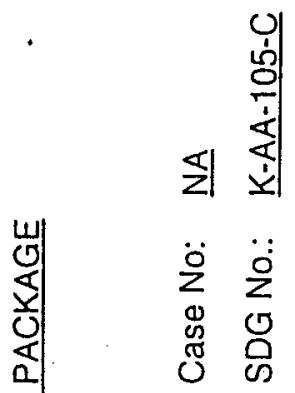

$\frac{1}{8}$

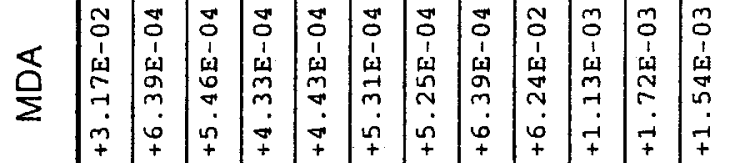
芯

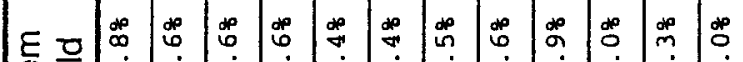

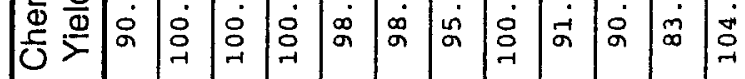

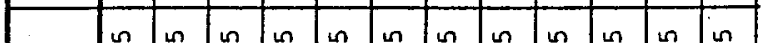

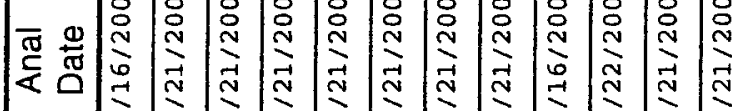
ฟิ

\begin{tabular}{|c|c|c|c|c|c|c|c|c|c|c|c|c|c|}
\hline $\begin{array}{ll}0 \\
\mathcal{~}\end{array}$ & $\underset{\sim}{\alpha}$ & $\sum_{z}^{\infty}$ & $\underset{z}{z}$ & $\mathbb{z}_{z}^{\infty}$ & 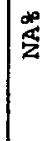 & $\frac{d e}{z}$ & & 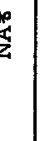 & $\stackrel{+\infty}{\overbrace{}^{\infty}}$ & $\begin{array}{l}\infty \\
\infty \\
\dot{0} \\
\alpha\end{array}$ & $d d^{2}$ & & $\begin{array}{l}\text { dp } \\
\text { m } \\
\text { in } \\
0 \\
r\end{array}$ \\
\hline
\end{tabular}

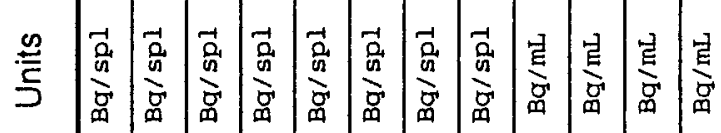
高紊 을 异

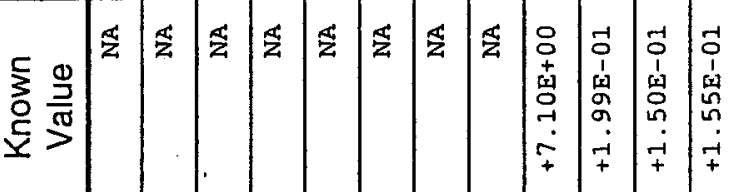
重

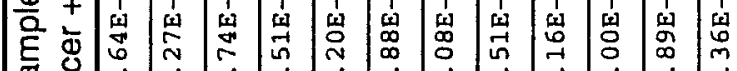

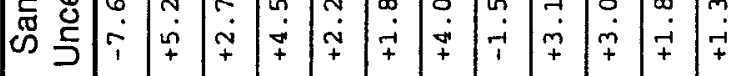

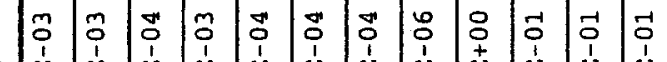

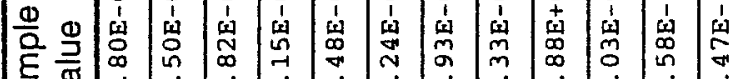

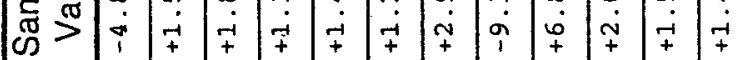

\begin{tabular}{|c|c|c|c|c|c|c|c|c|c|c|c|c|}
\hline$\frac{\stackrel{\Phi}{\frac{0}{2}}}{\frac{\sigma}{\frac{\sigma}{\sigma}}}$ & $\begin{array}{l}0 \\
\sigma \\
\tilde{~} \\
\text { vn }\end{array}$ & $\stackrel{\grave{S}}{\tilde{S}}$ & 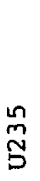 & 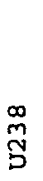 & $\begin{array}{l}\infty \\
\tilde{n} \\
\stackrel{2}{2}\end{array}$ & 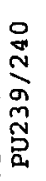 & $\underset{\text { 岕 }}{\stackrel{-1}{*}}$ & $\stackrel{\mathfrak{m}}{\stackrel{S}{S}}$ & $\begin{array}{l}\text { 号 } \\
\text { 药 }\end{array}$ & 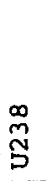 & 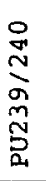 & 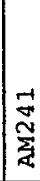 \\
\hline 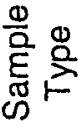 & 苔 & 岁 & 㟔 & 点 & 咍 & 学 & 总 & 岁 & 兄 & 0 & 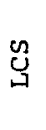 & ภ्త \\
\hline$\underset{\sim}{\tilde{N}} \varrho$ & 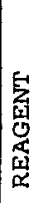 & 蛋 & 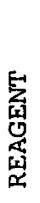 & 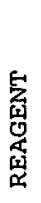 & 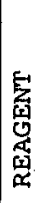 & 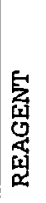 & 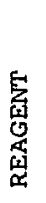 & 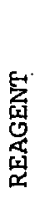 & 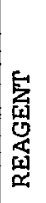 & 息 & 点 & 淠 \\
\hline
\end{tabular}


Project: $\quad$ Alpha and Sr-90 Analysis for Amchitka Island (Batch 3)

Laboratory: RTC

Report \#: $\quad$ AmchitBatchF3

SDG\#: $\quad$ K-AA-105-C

\section{Summary of 2 and 3 sigma activities}

Below are the results for Sr90, U234, U235, U236, U238, Pu238, Pu239/240, and Am241 for Batch 3 from the Amchitka Island Project that had a result/uncertainty ratio of 2 or more (uncertainty@one sigma).

\begin{tabular}{|c|c|c|c|c|c|}
\hline Customer ID & Lab ID & Isotope & $\begin{array}{l}\text { Result } \\
8 q / g\end{array}$ & $\begin{array}{c}\text { Uncertainty } \\
\mathrm{Bq} / \mathrm{g}\end{array}$ & $\begin{array}{c}\text { Result/ } \\
\text { Uncertainty }\end{array}$ \\
\hline K-AA-105-C & 02GO-05-A & U234 & $6.55 \mathrm{E}-04$ & $1.57 E-04$ & 4.2 \\
\hline K-AA-105-C & 02GO-05-A & U238 & $6.17 E-04$ & $1.58 \mathrm{E}-04$ & 3.9 \\
\hline K-AA-106-C & 02G0-06-A & U234 & $8.10 E-04$ & $1.73 E-04$ & 4.7 \\
\hline K-AA-106-C & $02 \mathrm{GO}-06-\mathrm{A}$ & U238 & 7.30E-04 & $1.70 E-04$ & 4.3 \\
\hline K-AA-111-C & $02 \mathrm{GO}-11-\mathrm{A}$ & U234 & $1.31 \mathrm{E}-03$ & $2.47 E-04$ & 5.3 \\
\hline K-AA-111-C & $02 \mathrm{GO}-11-\mathrm{A}$ & U238 & 1.19E-03 & $2.47 \mathrm{E}-04$ & 4.8 \\
\hline K-AA-112-C & $02 \mathrm{GO}-12-\mathrm{A}$ & U234 & $7.34 \mathrm{E}-04$ & 1.66E-04 & 4.4 \\
\hline K-AA-112-C & $02 \mathrm{GO}-12-\mathrm{A}$ & U238 & $5.51 E-04$ & $1.46 \mathrm{E}-04$ & 3.8 \\
\hline K-BB-107-C & 02G0-07-A & U234 & $2.20 \mathrm{E}-04$ & $9.51 E-05$ & 2.3 \\
\hline $\mathrm{K}-\mathrm{BB}-107-\mathrm{C}$ & 02GO-07-A & U238 & $1.92 E-04$ & $8.72 E-05$ & 2.2 \\
\hline K-BB-108-C & 02GO-08-A & U234 & $2.91 \mathrm{E}-04$ & 1.04E-04 & 2.8 \\
\hline K-BB-108-C & 02GO-08-A & U238 & 3.96E-04 & $1.20 \mathrm{E}-04$ & 3.3 \\
\hline K-BB-109-C & $02 \mathrm{GO}-09-\mathrm{A}$ & PU239/240 & 2.07E-04 & $5.85 \mathrm{E}-05$ & 3.5 \\
\hline K-BB-109-C & 02GO-09-A & U234 & $7.03 E-04$ & 1.62E-04 & 4.3 \\
\hline K-BB-109-C & 02GO-09-A & U238. & $7.47 \mathrm{E}-04$ & 1.77E-04 & 4.2 \\
\hline K-BB-110-C & $02 \mathrm{GO}-10-\mathrm{A}$ & U234 & $6.87 \mathrm{E}-04$ & 1.65E-04 & 4.2 \\
\hline K-BB-110-C & $02 G 0-10-A$ & U238 & $7.98 \mathrm{E}-04$ & $1.89 \mathrm{E}-04$ & 4.2 \\
\hline K-CC-113-C & $02 \mathrm{GO}-13-\mathrm{A}$ & U234 & $3.46 \mathrm{E}-03$ & 5.04E-04 & 6.9 \\
\hline $\mathrm{K}-\mathrm{CC}-113-\mathrm{C}$ & $02 \mathrm{GO}-13-\mathrm{A}$ & U235 & $1.74 \mathrm{E}-04$ & $6.16 \mathrm{E}-05$ & 2.8 \\
\hline $\mathrm{K}-\mathrm{CC}-113-\mathrm{C}$ & $02 \mathrm{GO}-13-\mathrm{A}$ & U238 & $3.03 E-03$ & 5.01E-04 & 6.0 \\
\hline K-CC-114-C & $02 \mathrm{GO}-14-\mathrm{A}$ & U234 & $2.06 \mathrm{E}-03$ & 3.35E-04 & 6.1 \\
\hline K-CC-114-C & $02 \mathrm{GO}-14-\mathrm{A}$ & U235 & $9.59 E-05$ & 4.79E-05 & 2.0 \\
\hline $\mathrm{K}-\mathrm{CC}-114-\mathrm{C}$ & 02GO-14-A & U238 & 1.91E-03 & $3.46 \mathrm{E}-04$ & 5.5 \\
\hline
\end{tabular}




\section{Alpha and Sr-90 Analysis for Amchitka Island (Batch 3) Summary of 2 and 3 sigma activities}

Page 2

\begin{tabular}{|c|c|c|c|c|c|}
\hline Customer ID & Lab ID & Isotope & $\begin{array}{l}\text { Result } \\
\mathrm{Bq} / \mathrm{g}\end{array}$ & $\begin{array}{c}\text { Uncertainty } \\
\mathrm{Bq} / \mathrm{g}\end{array}$ & $\begin{array}{c}\text { Result/ } \\
\text { Uncertainty }\end{array}$ \\
\hline $\mathrm{K}-\mathrm{CC}-115-\mathrm{C}$ & $02 \mathrm{GO}-01-\mathrm{A}$ & U234 & $4.35 E-03$ & $6.06 E-04$ & 7.2 \\
\hline $\mathrm{K}-\mathrm{CC}-115-\mathrm{C}$ & $02 \mathrm{GO}-01-\mathrm{A}$ & U235 & $2.27 \mathrm{E}-04$ & $7.14 \mathrm{E}-05$ & 3.2 \\
\hline K-CC-115-C & 02GO-01-A & U238 & $3.57 E-03$ & $5.73 E-04$ & 6.2 \\
\hline $\mathrm{K}-\mathrm{CC}-130-\mathrm{C}$ & 02GO-02-A & U234 & $2.29 \mathrm{E}-03$ & $3.68 \mathrm{E}-04$ & 6.2 \\
\hline K-CC-130-C & $02 \mathrm{GO}-02-\mathrm{A}$ & U235 & $1.16 E-04$ & 5.35E-05 & 2.2 \\
\hline $\mathrm{K}-\mathrm{CC}-130-\mathrm{C}$ & 02GO-02-A & U238 & $2.11 E-03$ & $3.78 E-04$ & 5.6 \\
\hline $\mathrm{K}-\mathrm{CC}-132-\mathrm{C}$ & 02GO-03-A & AM241 & $7.80 \mathrm{E}-03$ & $6.34 \mathrm{E}-04$ & 12.3 \\
\hline K-CC-132-C & 02GO-03-A & PU238 & $5.16 \mathrm{E}-03$ & $5.85 \mathrm{E}-04$ & 8.8 \\
\hline $\mathrm{K}-\mathrm{CC}-132-\mathrm{C}$ & 02GO-03-A & SR90 & $3.41 E-02$ & $1.70 \mathrm{E}-03$ & 20.1 \\
\hline $\mathrm{K}-\mathrm{CC}-132-\mathrm{C}$ & 02G0-03-A & U234 & $8.18 \mathrm{E}-04$ & $1.81 E-04$ & 4.5 \\
\hline K-CC-132-C & 02GO-03-A & U238 & 7.39E-04 & 1.77E-04 & 4.2 \\
\hline K-CC-133-C & $02 \mathrm{GO}-04-\mathrm{A}$ & AM241 & $2.33 \mathrm{E}-03$ & $2.56 \mathrm{E}-04$ & 9.1 \\
\hline K-CC-133-C & 02GO-04-A & PU239/240 & $3.35 \mathrm{E}-03$ & $4.10 \mathrm{E}-04$ & 8.2 \\
\hline K-CC-133-C & 02GO-04-A & SR90 & $9.13 \mathrm{E}-03$ & $7.21 \mathrm{E}-04$ & 12.7 \\
\hline $\mathrm{K}-\mathrm{CC}-133-\mathrm{C}$ & $02 \mathrm{GO}-04-\mathrm{A}$ & U234 & $8.90 \mathrm{E}-04$ & 1.90E-04 & 4.7 \\
\hline K-CC-133-C & $02 \mathrm{GO}-04-\mathrm{A}$ & U238 & 7.41E-04 & 1.77E-04 & 4.2 \\
\hline S-R-117-C & $02 \mathrm{G} 0-15-\mathrm{A}$ & PU239/240 & 3.42E-05 & 1.71E-05 & 2.0 \\
\hline S-R-117-C & 02GO-15-A & U234 & $5.13 E-04$ & $1.07 \mathrm{E}-04$ & 4.8 \\
\hline S-R-117-C & $02 G 0-15-A$ & U238 & 4.17E-04 & $9.90 E-05$ & 4.2 \\
\hline S-R-119-C & $02 G 0-16-A$ & U234 & 4.57E-04 & $1.00 \mathrm{E}-04$ & 4.6 \\
\hline S-R-119-C & 02GO-16-A & U238 & $4.40 E-04$ & $1.03 E-04$ & 4.3 \\
\hline S-R-121-C & $02 \mathrm{GO}-17-\mathrm{A}$ & U234 & $5.05 E-04$ & $1.06 \mathrm{E}-04$ & 4.8 \\
\hline S-R-121-C & $02 \mathrm{GO}-17-\mathrm{A}$ & U238 & $4.47 E-04$ & $1.03 E-04$ & 4.3 \\
\hline
\end{tabular}

All known sources of uncertainty are included in the uncertainty term. There may be unknown sources of uncertainty that are not accounted for. If the result/uncertainty ratio is more than 3 , we have a degree of confidence that the result is positive (i.e. the result is statistically different than zero). A result with the result/uncertainty ratio between 2 and 3 is the first indication that an isotope may be present and further investigation may be warranted.

As with any good science no single data point is used in important decisions (results need to be reproducible). 
BATCH 4

ALPHA AND Sr-90 


\section{RADIOANALYTICAL ANALYSES DATA PACKAGE}

Project Title: $\quad$ Alpha and Sr-90 Analysis for Amchitka Island (Batch 4)

$\begin{array}{llll}\text { Lab Name: } & \text { RTC } & \text { Case No: } & \text { NA } \\ \text { Report No:: } & \text { AmchitBatchF4 } & \text { Method Type: } & \text { A/B } \\ \text { Approved SAP No.: } & \text { NA } & \text { SDG No.: } & \text { B-L-150-B }\end{array}$

\section{SAMPLE NUMBERS}

Customer

Sample ID

B-K-152-B

$B-L-150-B$

$B-M-138-B$

$B-M-139-B$

$B-M-140-B$

$B-M-141-B$

$B-M-142-B$

B-M-143-B

B-M-144-B

B-M-145-B

B-O-146-B

$B-0-147=B$

B-O-148-B

$B-P-135-B$

B-P-136-B

B-P-137-B

\section{Lab Sample}

ID

$021 \mathrm{~K}-17-\mathrm{A}$

02IK-01-A

$021 K-16-A$

$02 \mathrm{~K}-12-\mathrm{A}$

$021 K-10-A$

02IK-06-A

$021 \mathrm{~K}-11-\mathrm{A}$

02IK-03-A

02IK-15-A

02IK-14-A

02IK-13-A

02IK-07-A

$021 K-08-A$

$021 \mathrm{~K}-05-\mathrm{A}$

$021 \mathrm{~K}-04-\mathrm{A}$

02IK-09-A

02IK-02-A

Comments:

Release of the data contained in this data package has been authorized by the laboratory manager or the manager's designee, as verified by the following signature:

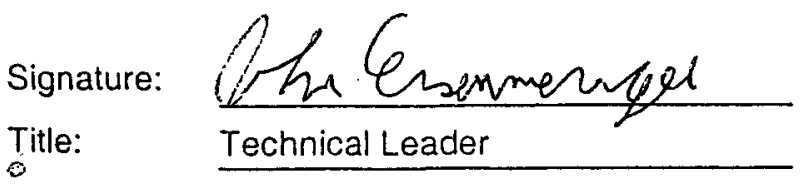




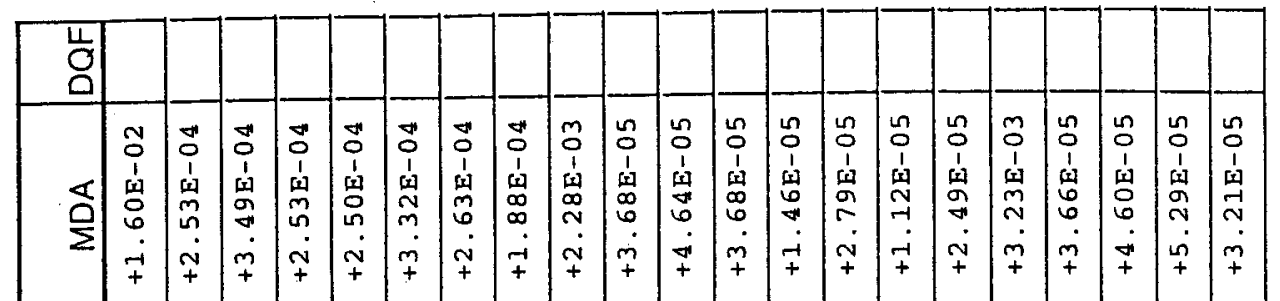

§)

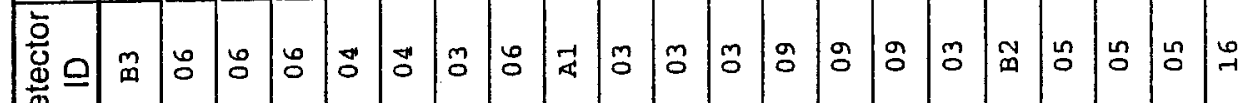
点

过焉

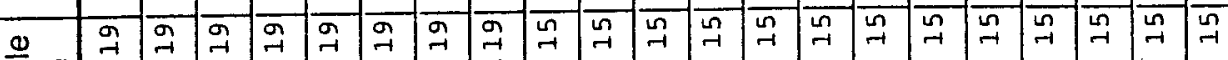

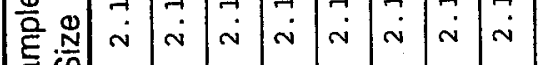
๓

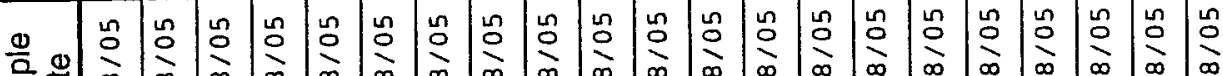

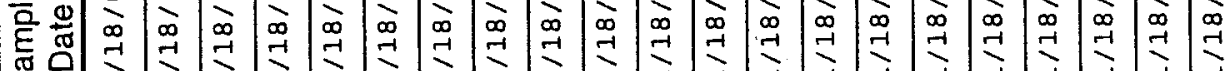
लि

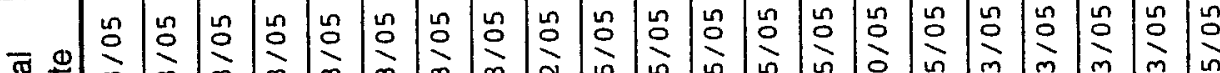

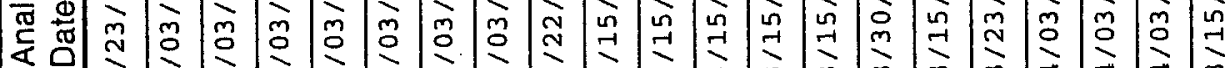
ले

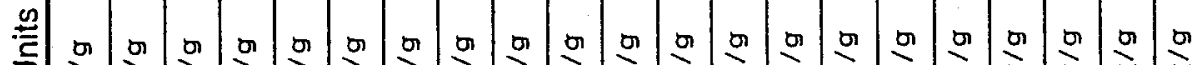

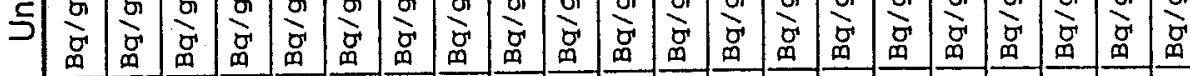

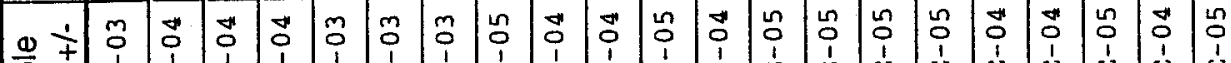

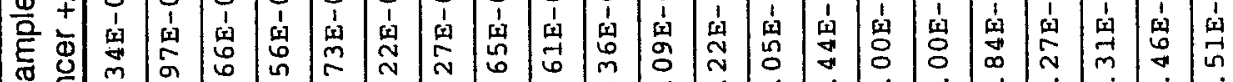

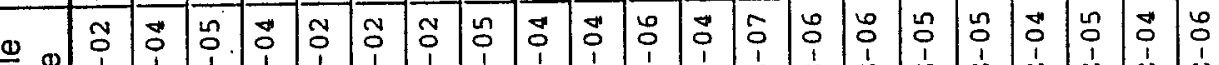

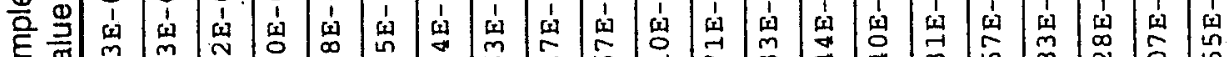
œ

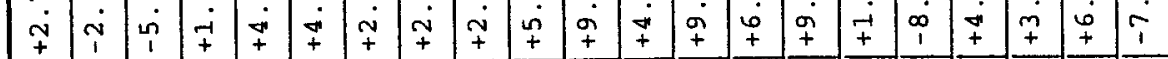
宅 号

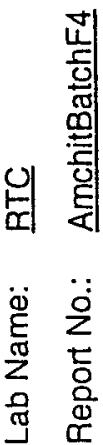

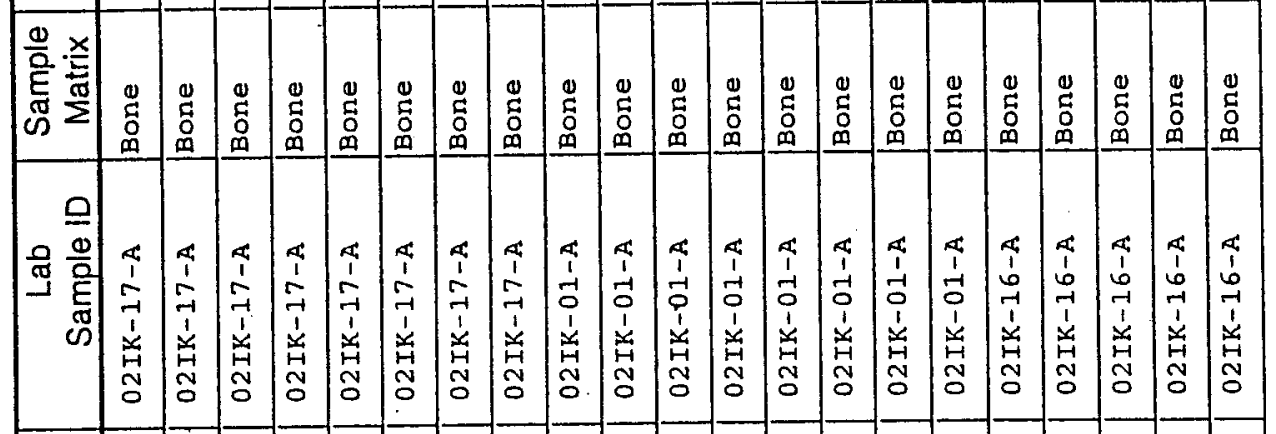

으

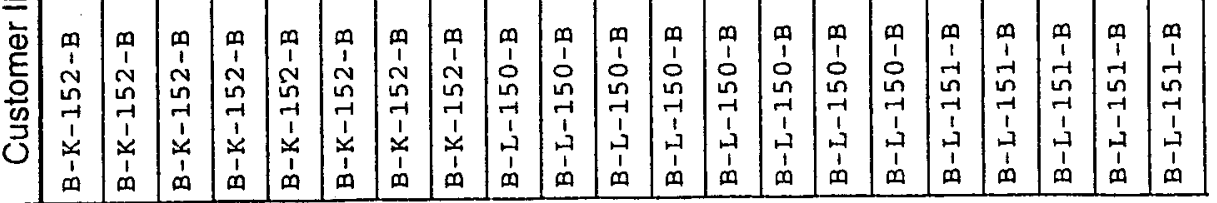

\section{品} D

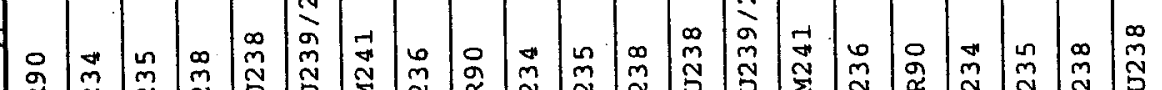

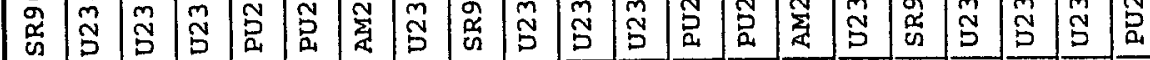


$\frac{0}{2}$

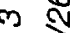

ठै ठे

a

\begin{tabular}{|c|c|c|c|c|c|c|c|c|c|c|c|c|c|c|c|c|c|c|c|c|c|}
\hline \multirow{2}{*}{\multicolumn{22}{|c|}{\begin{tabular}{|l|}
$\mathrm{U}$ \\
\end{tabular}}} \\
\hline & & & & & & & & & & & & & & & & & & & & & \\
\hline 息 & $\begin{array}{l}n \\
0 \\
1 \\
\omega \\
\omega \\
\vdots \\
0 \\
0 \\
+\end{array}$ & $\begin{array}{l}\text { n } \\
0 \\
1 \\
\omega \\
0 \\
0 \\
0 \\
\sim \\
\sim \\
+\end{array}$ & 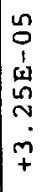 & 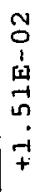 & 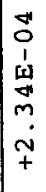 & 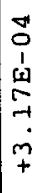 & 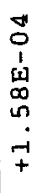 & 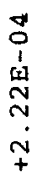 & 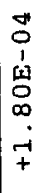 & $\begin{array}{l}\overrightarrow{0} \\
1 \\
1 \\
\hat{1} \\
0 \\
0 \\
\dot{\sim} \\
\sim \\
+\end{array}$ & 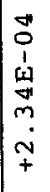 & 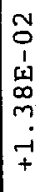 & $\begin{array}{l}\overrightarrow{0} \\
0 \\
1 \\
\hat{\omega} \\
0 \\
0 \\
\dot{p} \\
+\end{array}$ & $\begin{array}{l}0 \\
0 \\
1 \\
0 \\
N \\
N \\
o \\
N \\
+ \\
+\end{array}$ & 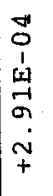 & $\begin{array}{l}n \\
0 \\
1 \\
\omega \\
-1 \\
m \\
0 \\
0 \\
+\end{array}$ & 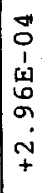 & 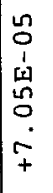 & $\begin{array}{l}\overrightarrow{0} \\
1 \\
\varphi \\
\omega \\
0 \\
0 \\
\sim \\
\sim\end{array}$ & $\begin{array}{l}N \\
0 \\
1 \\
1 \\
m \\
m \\
m \\
-1 \\
+ \\
+\end{array}$ & 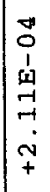 \\
\hline 总 & $\stackrel{0}{-1}$ & $\stackrel{\sim}{\sim}$ & 告 & 同 & 15 & 10 & 18 & $\approx$ & $\underset{\sim}{\stackrel{N}{*}}$ & $\underset{-1}{\beth}$ & 8 & $\stackrel{3}{\alpha}$ & $\stackrel{\infty}{\infty}$ & $\left.\right|_{0} ^{\infty}$ & $\left.\right|_{0} ^{\infty}$ & 只 & Iㄱ & 아 & $\left.\right|_{0} ^{\infty}$ & 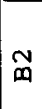 & $\stackrel{m}{o}$ \\
\hline 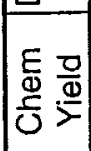 & $\vec{\infty}$ & 虫 & 足. & $\begin{array}{c}N \\
- \\
\infty \\
\infty\end{array}$ & $\begin{array}{l}0 \\
\infty \\
0 \\
-1\end{array}$ & $\begin{array}{l}0 \\
\infty \\
0 \\
- \\
-1\end{array}$ & $\begin{array}{l}0 \\
0 \\
0 \\
0 \\
-1\end{array}$ & 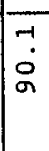 & $\begin{array}{l}-1 \\
0 \\
0\end{array}$ & $\begin{array}{l}\infty \\
\dot{0} \\
0 \\
-1 \\
-1\end{array}$ & $\mid \begin{array}{l}0 \\
0 \\
0 \\
0 \\
-1\end{array}$ & $\begin{array}{l}m \\
\dot{m} \\
\infty\end{array}$ & $\begin{array}{l}0 \\
0 \\
0 \\
0\end{array}$ & $\begin{array}{l}0 \\
0 \\
\infty \\
\sigma\end{array}$ & $\begin{array}{l}0 \\
\ddot{0} \\
0\end{array}$ & $\sum_{\infty}^{\infty}$ & $\dot{m}_{\infty}^{*}$ & $\mid \begin{array}{c}a \\
\vdots \\
0 \\
\sigma\end{array}$ & $\begin{array}{l}0 \\
0 \\
\sigma \\
\sigma\end{array}$ & $\ddot{0}$ & $\begin{array}{l}m \\
m \\
m \\
0 \\
r\end{array}$ \\
\hline 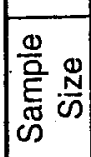 & \begin{tabular}{|l|}
$n$ \\
-1
\end{tabular} & $\stackrel{\sim}{\sim}$ & $\sqrt{\sim}$ & $\vec{\sim}$ & $\tilde{n}$ & $\tilde{\sim}$ & $\begin{array}{l}n \\
\sim \\
\sim\end{array}$ & $\begin{array}{l}n \\
\tilde{n}\end{array}$ & $\tilde{u}$ & $\dot{\tilde{n}}$ & $\tilde{i}$ & $\tilde{\sim}$ & \begin{tabular}{|c|}
$\tilde{n}$ \\
$\tilde{n}$
\end{tabular} & 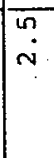 & $\dot{v}$ & $\tilde{v}$ & $\tilde{\tilde{v}}$ & $\tilde{r}$ & in & $\stackrel{n}{n}$ & $\tilde{\sim}$ \\
\hline
\end{tabular}

\&l

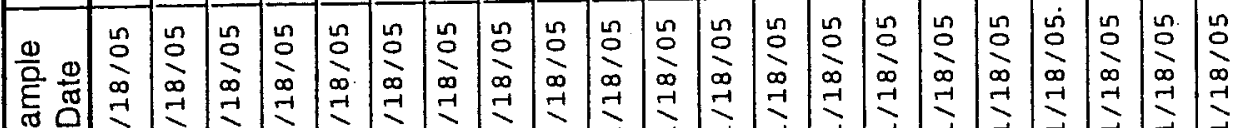

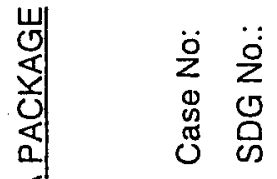

œ

焉弯

a a a a a a a

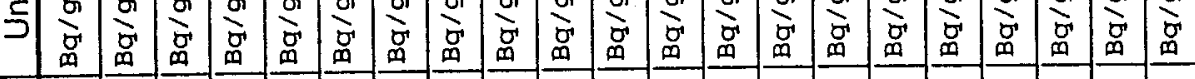

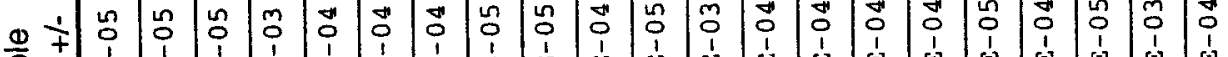

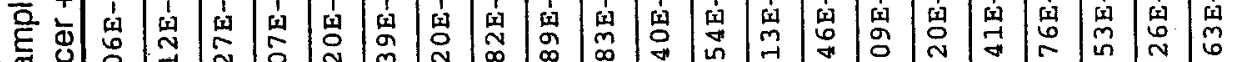

ळ̋

๓

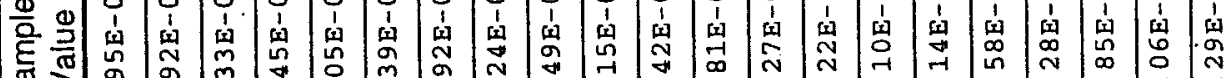

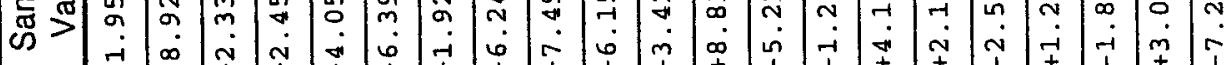

임

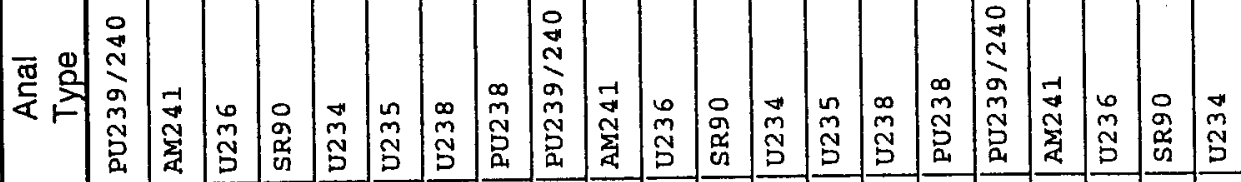

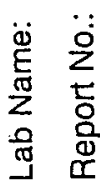

을

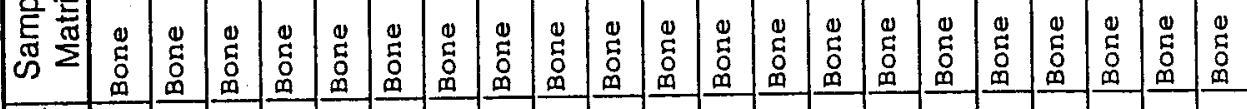

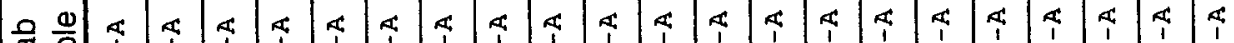

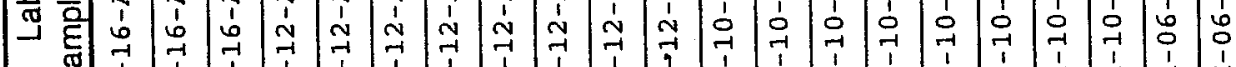

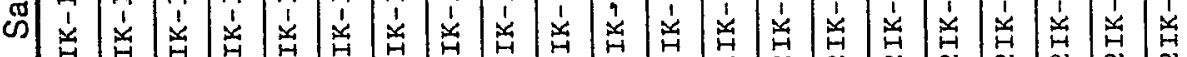
索 


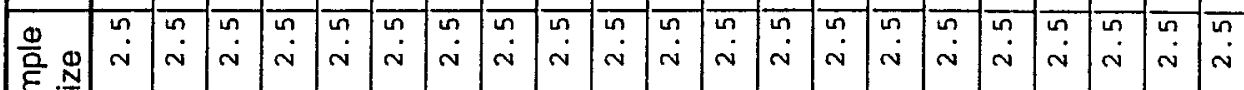
ळ

更

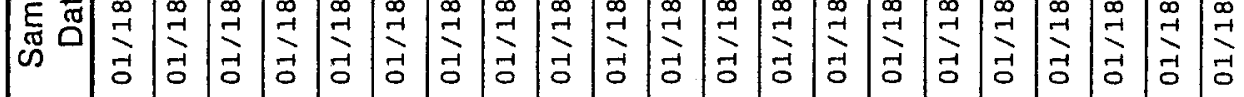

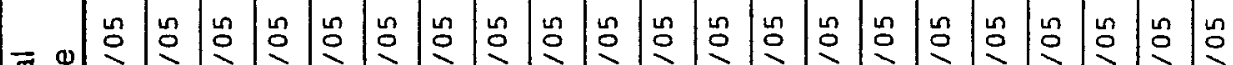
वृ

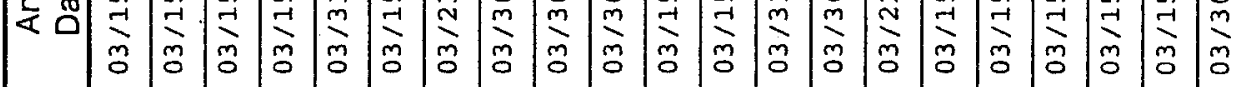
穵

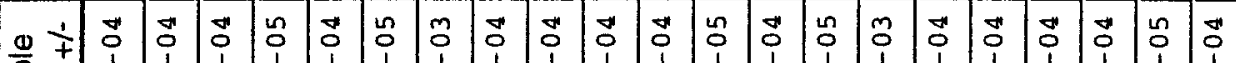

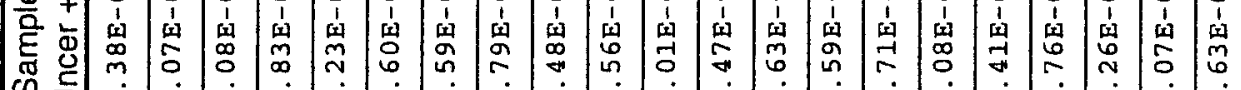

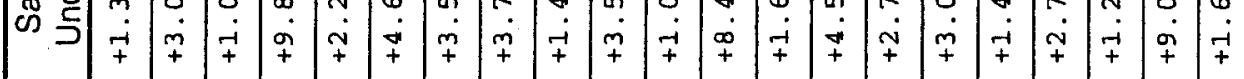

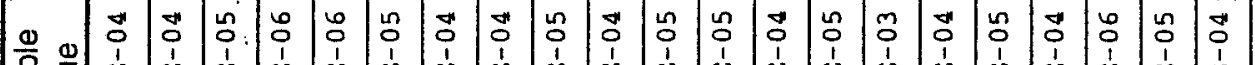

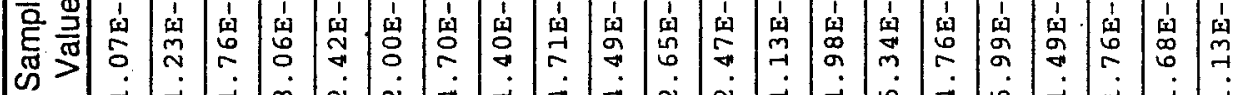

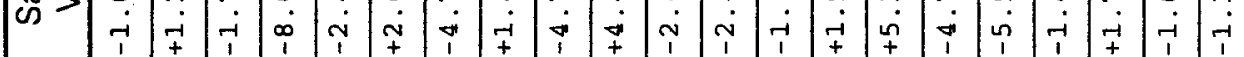

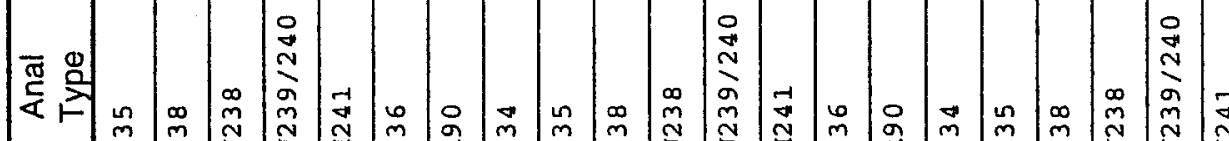

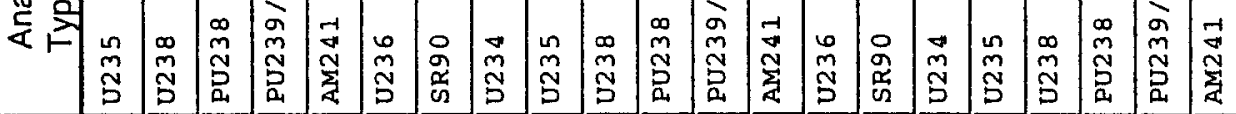

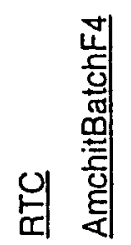
竞:

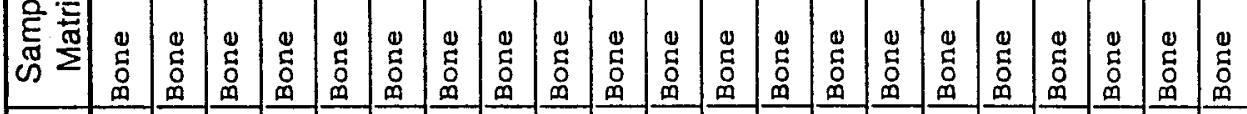
의 윙

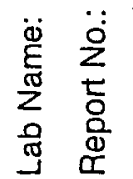

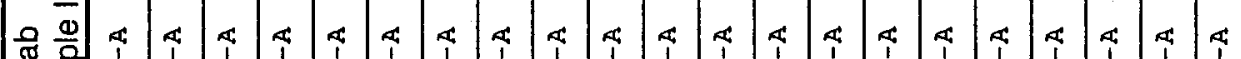

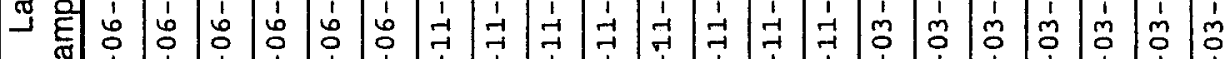

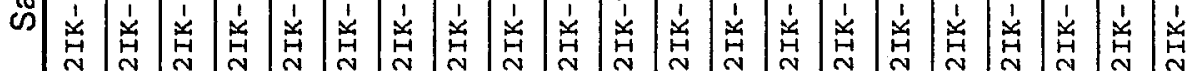
垈 


\begin{tabular}{|c|c|c|c|c|c|c|c|c|c|c|c|c|c|c|c|c|c|c|c|c|c|}
\hline & & & & & & & & & & & & & & & & & & & & & \\
\hline$\stackrel{\Delta}{2}$ & 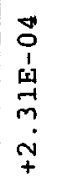 & 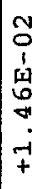 & $\begin{array}{l}\overrightarrow{0} \\
1 \\
1 \\
\dot{c} \\
0 \\
0 \\
\dot{m} \\
+\end{array}$ & $\mid \begin{array}{l}7 \\
0 \\
1 \\
\omega \\
0 \\
0 \\
0 \\
0 \\
+ \\
+\end{array}$ & 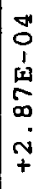 & 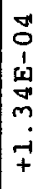 & $\begin{array}{l}\vec{D} \\
1 \\
1 \\
\Phi \\
0 \\
0 \\
-1 \\
+ \\
+\end{array}$ & 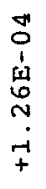 & 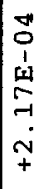 & 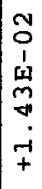 & 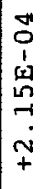 & 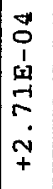 & $\begin{array}{l}\text { d" } \\
0 \\
1 \\
1 \\
0 \\
\sigma \\
o \\
-1 \\
+\end{array}$ & $\begin{array}{l}0 \\
0 \\
1 \\
1 \\
1 \\
0 \\
0 \\
- \\
+ \\
+\end{array}$ & 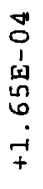 & 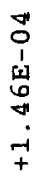 & 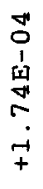 & 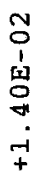 & $\begin{array}{l}\overrightarrow{0} \\
1 \\
1 \\
0 \\
0 \\
0 \\
? \\
-1 \\
+\end{array}$ & 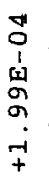 & 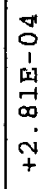 \\
\hline 递 & 㟔 & $\vec{m}$ & $\stackrel{\infty}{\circ}$ & $\stackrel{\infty}{\circ}$ & $0_{0}^{\infty}$ & $\stackrel{n}{7}$ & $\stackrel{n}{\rightarrow}$ & $\stackrel{\sim}{\sim}$ & $\left.\right|_{0} ^{\infty}$ & $\dot{m}$ & $10^{\circ}$ & $\mathscr{0}^{\circ}$ & ${ }^{\circ}$ & $\stackrel{\vec{\sim}}{ }$ & In & $\vec{H}$ & $\stackrel{\circ}{\circ}$ & 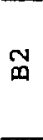 & ฉ̊ & ก & 5 \\
\hline 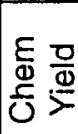 & $\begin{array}{l}\tilde{\sigma} \\
\dot{0} \\
0 \\
-1\end{array}$ & $\dot{m}$ & $\begin{array}{l}\infty \\
\dot{m}\end{array}$ & $\begin{array}{l}\infty \\
\dot{m} \\
\sigma\end{array}$ & $\begin{array}{l}\infty \\
\dot{m} \\
\alpha\end{array}$ & $\begin{array}{l}\dot{\sigma} \\
\dot{n} \\
\sigma\end{array}$ & $\begin{array}{l}\sigma \\
\text { ñ }\end{array}$ & ¿. & $\begin{array}{l}\infty \\
\dot{m} \\
\sigma\end{array}$ & $\ddot{0}$ & $\begin{array}{l}0 \\
\vdots \\
-1\end{array}$ & $\begin{array}{l}0 \\
\dot{7} \\
-1\end{array}$ & $\begin{array}{l}0 \\
\dot{y} \\
=\end{array}$ & ڤ̆ & $\ddot{\sigma}$ & $\begin{array}{l}0 \\
0 \\
0 \\
0 \\
-1\end{array}$ & $\begin{array}{l}0 \\
\dot{1} \\
7\end{array}$ & $\underset{\infty}{\infty}$ & $\begin{array}{l}-1 \\
\infty \\
0 \\
0 \\
-1\end{array}$ & $\begin{array}{l}-1 \\
\infty \\
0 \\
0 \\
-1\end{array}$ & $\begin{array}{l}-1 \\
\infty \\
0 \\
0\end{array}$ \\
\hline 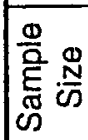 & $\tilde{\sim}$ & $\stackrel{\sim}{\sim}$ & $\stackrel{\sim}{n}$ & $\begin{array}{l}\tilde{N} \\
\tilde{N}\end{array}$ & $\begin{array}{l}\bar{n} \\
\dot{\sim}\end{array}$ & $\stackrel{\tilde{\sim}}{\sim}$ & ?. & 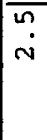 & $\stackrel{\sim}{\sim}$ & $\stackrel{n}{\sim}$ & $\bar{n}$ & $\begin{array}{l}n ? \\
n \\
n\end{array}$ & $\stackrel{\sim}{\sim}$ & $\stackrel{\sim}{\sim}$ & $\dot{\sim}$ & $\stackrel{n}{\sim}$ & $\dot{v}$ & $\dot{m}$ & $\tilde{v}$ & $\stackrel{\tilde{v}}{\tilde{v}}$ & $\stackrel{n}{\sim}$ \\
\hline
\end{tabular}

空

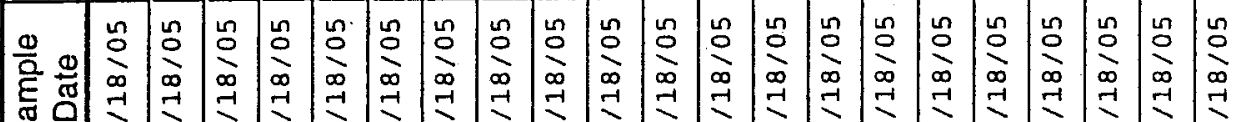

œึ অ

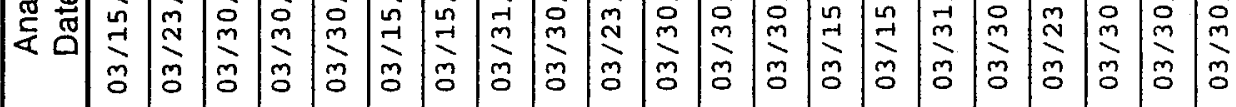

a a a a a a a a a a a

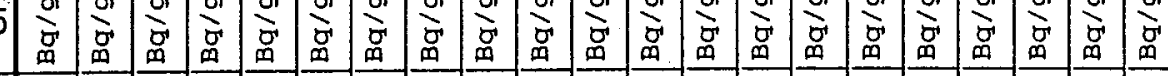

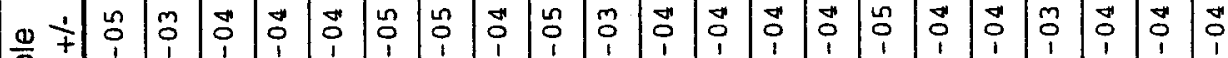
龸

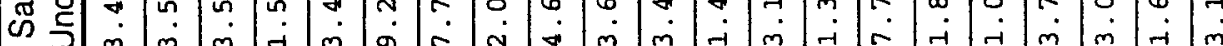

w

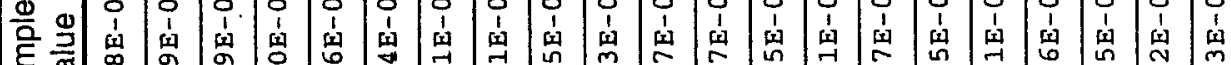
厄ٓ

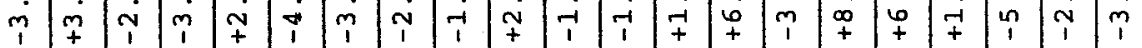

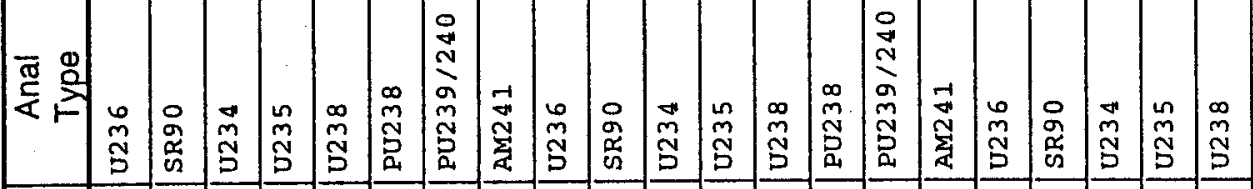

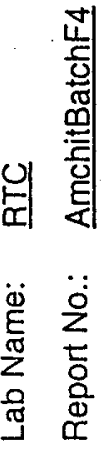

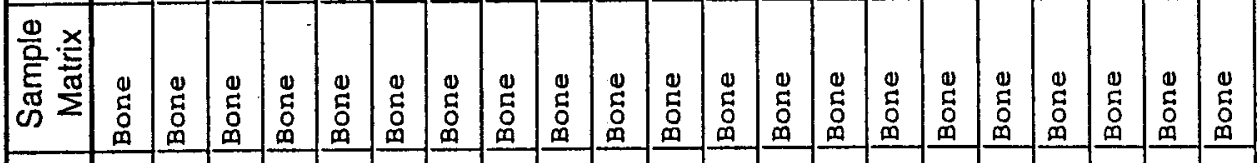
으

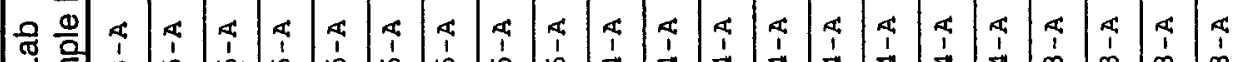

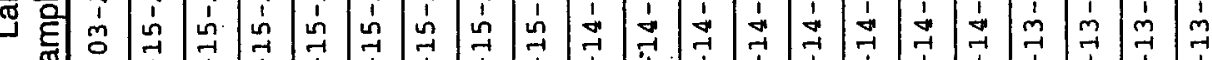
क

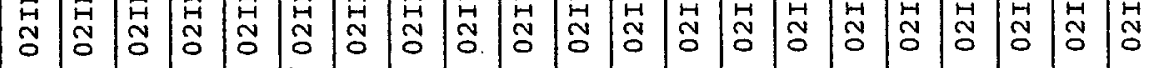

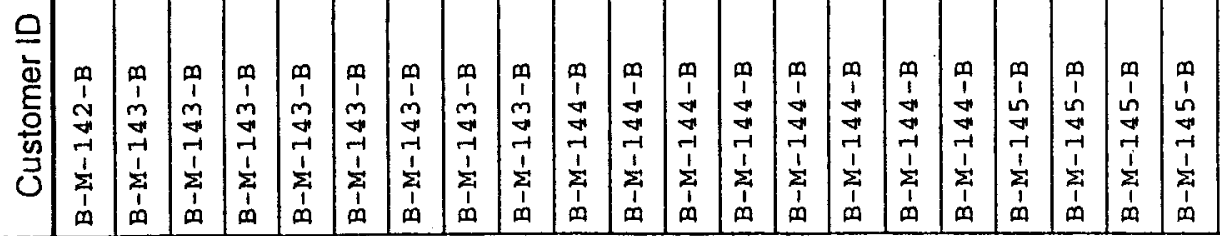


\begin{tabular}{ll}
9 & 2 \\
0 & $\varrho$ \\
0 & \multirow{2}{2}{} \\
0 & 0
\end{tabular}

$\mathbb{R}^{\mathbb{2}}$

\begin{tabular}{|c|c|c|c|c|c|c|c|c|c|c|c|c|c|c|c|c|c|c|c|c|c|}
\hline $\begin{array}{l} \\
\\
\end{array}$ & & & & & & & & & & & & & & & & & & & & & \\
\hline$\stackrel{\nwarrow}{\varrho}$ & 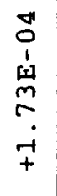 & $\mid \begin{array}{c}\not \\
0 \\
1 \\
1 \\
0 \\
0 \\
r \\
i \\
+ \\
+\end{array}$ & $\begin{array}{l}\not \\
0 \\
1 \\
\omega \\
0 \\
0 \\
\sim \\
-1 \\
+\end{array}$ & 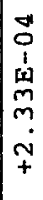 & $\begin{array}{l}\text { N } \\
0 \\
1 \\
\text { wू. } \\
\infty \\
\infty \\
-1 \\
+\end{array}$ & 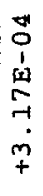 & $\begin{array}{l}8 \\
0 \\
1 \\
\text { w1 } \\
0 \\
0 \\
0 \\
+ \\
+\end{array}$ & 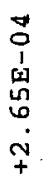 & 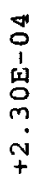 & $\begin{array}{l}\vec{b} \\
0 \\
1 \\
y \\
0 \\
0 \\
\sim \\
\sim \\
+ \\
+\end{array}$ & 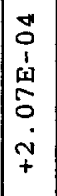 & $\begin{array}{c}\overrightarrow{0} \\
0 \\
1 \\
\omega \\
\vec{a} \\
\vec{\sim} \\
\dot{p} \\
+\end{array}$ & $\begin{array}{l}\tilde{N} \\
0 \\
1 \\
\omega \\
\tilde{n} \\
\sim \\
\sim \\
\sim \\
+ \\
+\end{array}$ & $\begin{array}{l}\vec{c} \\
0 \\
1 \\
\underline{1} \\
5 \\
m \\
m \\
+ \\
+\end{array}$ & 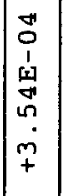 & 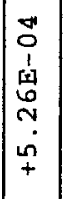 & 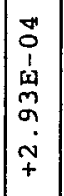 & 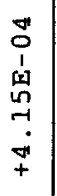 & $\mid \begin{array}{c}\overrightarrow{0} \\
1 \\
1 \\
w \\
\infty \\
0 \\
0 \\
p \\
+ \\
+\end{array}$ & $\begin{array}{l}0 \\
0 \\
1 \\
1 \\
9 \\
2 \\
\vdots \\
\dot{m} \\
+\end{array}$ & 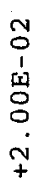 \\
\hline 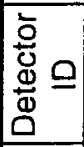 & $\stackrel{m}{-}$ & $\mid \begin{array}{l}m \\
\rightarrow\end{array}$ & $\stackrel{m}{\rightarrow}$ & In & 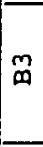 & $\not \nexists$ & $\theta^{*}$ & वే & $\stackrel{n}{\sim}$ & $\stackrel{\sim}{\rightarrow}$ & $\stackrel{\sim}{\neg}$ & I & $\overrightarrow{4}$ & 10 & $\left.\right|^{n}$ & |n & 10 & $\underset{-1}{-1}$ & $\mid \begin{array}{l}6 \\
-1\end{array}$ & 络 & $\vec{m}$ \\
\hline $\begin{array}{l}\frac{c}{0} \\
\frac{0}{0} \\
\frac{0}{0}\end{array}$ & $\begin{array}{l}0 \\
\dot{\theta} \\
0\end{array}$ & के & 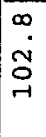 & $\begin{array}{l}-1 \\
0 \\
0 \\
0 \\
-1\end{array}$ & $\begin{array}{l}\infty \\
0 \\
\infty \\
\infty\end{array}$ & $\begin{array}{l}0 \\
0 \\
\vdots \\
\vdots \\
-1\end{array}$ & $\begin{array}{l}0 \\
\vdots \\
\vdots \\
0 \\
-1\end{array}$ & $\begin{array}{l}0 \\
0 \\
\vdots \\
0 \\
0\end{array}$ & $\begin{array}{l}-1 \\
\dot{n} \\
n\end{array}$ & $\begin{array}{l}\vec{r} \\
\dot{m} \\
\sigma\end{array}$ & $\begin{array}{l}\infty \\
\dot{n} \\
0 \\
-1\end{array}$ & $\begin{array}{l}\sigma \\
\tilde{5} \\
5 \\
0 \\
\end{array}$ & $\begin{array}{l}\dot{0} \\
\dot{\tilde{a}} \\
\end{array}$ & $\begin{array}{r}-1 \\
- \\
-1 \\
- \\
-1\end{array}$ & 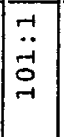 & 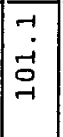 & $\begin{array}{l}0 \\
\tilde{N} \\
\alpha\end{array}$ & $\begin{array}{l}0 \\
\dot{j} \\
\sigma\end{array}$ & $\begin{array}{l} \\
\sigma \\
\sigma\end{array}$ & न- & n. \\
\hline 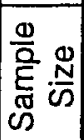 & $\begin{array}{l}\tilde{\sim} \\
\dot{\sim}\end{array}$ & $\begin{array}{l}\text { मे } \\
\dot{\sim}\end{array}$ & $\stackrel{n}{\sim}$ & $\tilde{\sim}$ & $\begin{array}{l}n \\
n \\
r\end{array}$ & $\bar{r}$ & $\tilde{r}$ & ז' & ח- & $\begin{array}{l}n \\
-i\end{array}$ & $\begin{array}{ll}n \\
-i\end{array}$ & \begin{tabular}{|c|}
$n$ \\
$-i$ \\
\end{tabular} & $\begin{array}{l}n \\
-1 \\
-1\end{array}$ & $\begin{array}{l}n \\
-i \\
-i\end{array}$ & \begin{tabular}{|l|}
$\tilde{H}$ \\
$\tilde{r}$
\end{tabular} & \begin{tabular}{|l|}
$\tilde{r}$ \\
$-i$
\end{tabular} & $\begin{array}{ll} \\
-7 \\
-1\end{array}$ & $\tilde{\tau}$ & $\begin{array}{l}n \\
n \\
-1\end{array}$ & $\stackrel{n}{\sim}$ & r \\
\hline
\end{tabular}

导站|

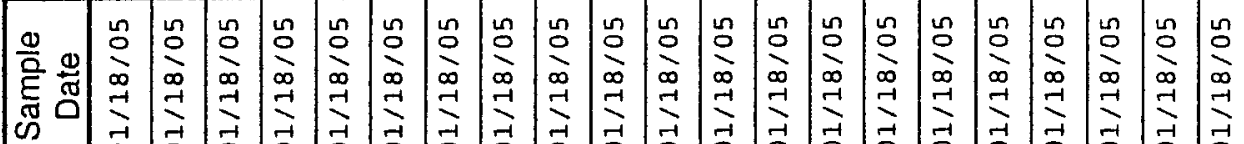
嵌|

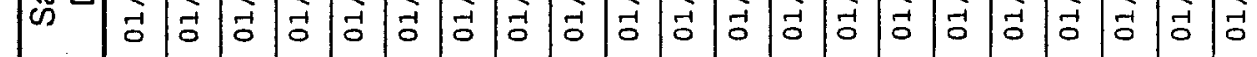

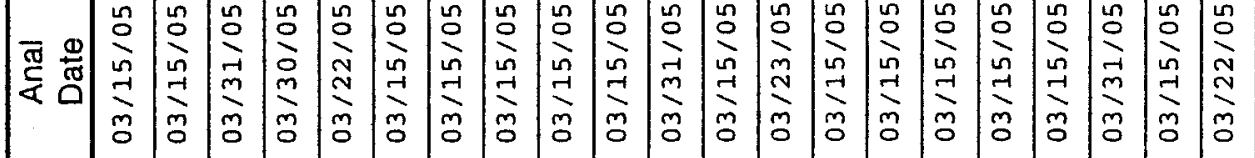
a a a a a a a a a a a

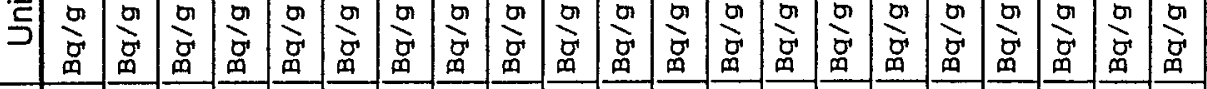

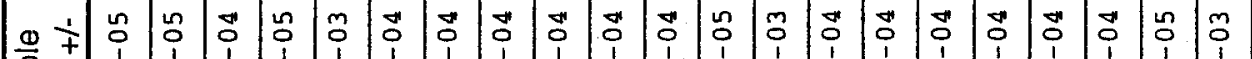

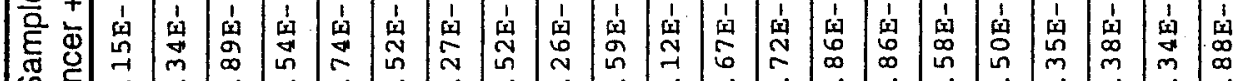
œ̋ ఎ n

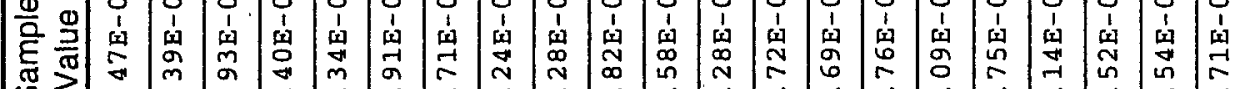
œ

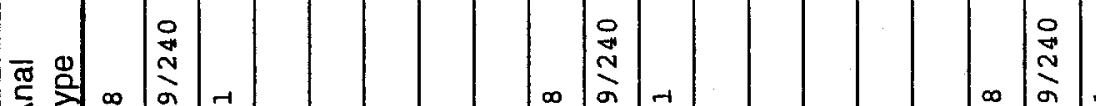

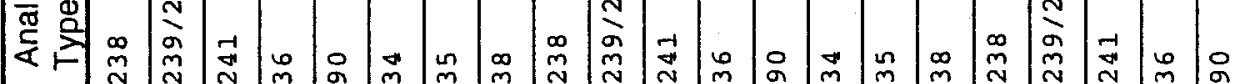

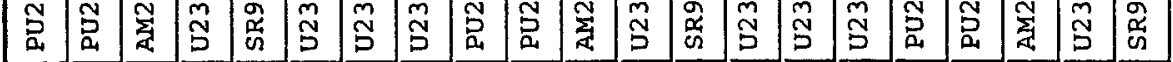

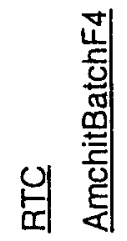
产

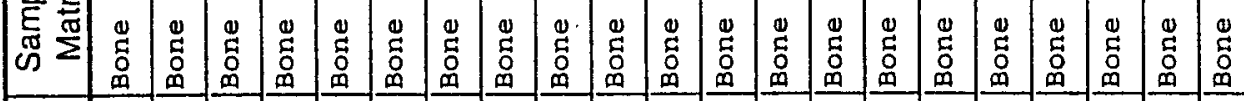

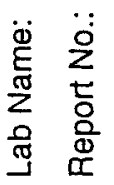

呈前 4 \& 4 a

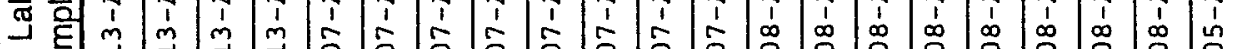

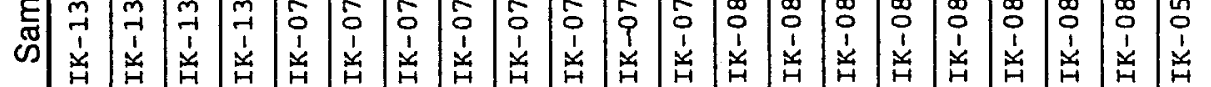
点 
$\frac{1}{0} \frac{1}{8}$

$\wedge \approx$

कृ

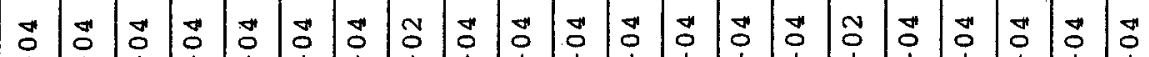

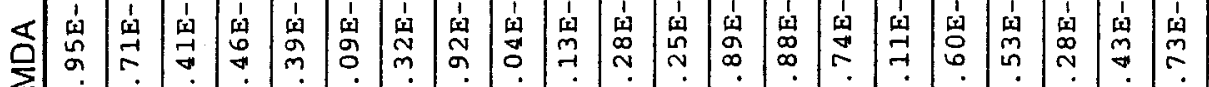

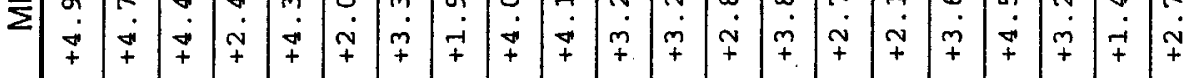

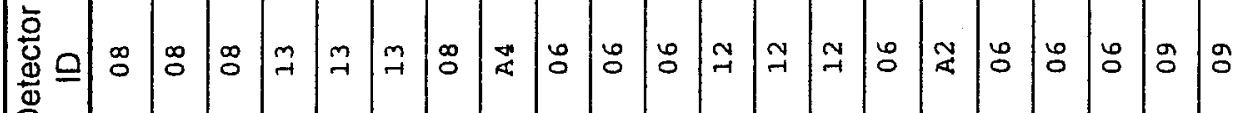
هั

¿ा

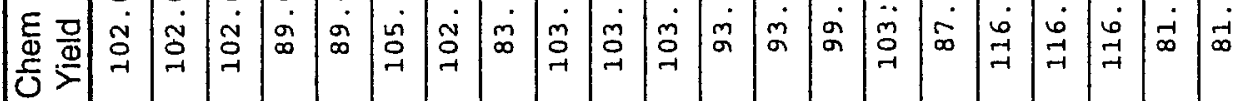

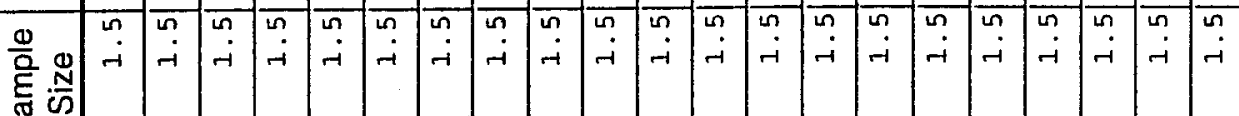
ळं

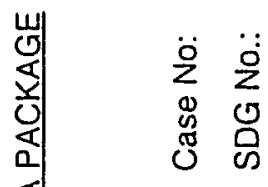

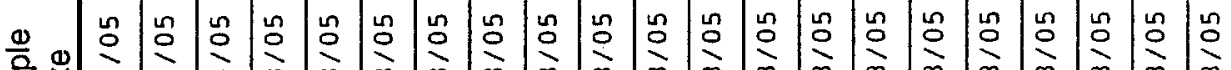
船

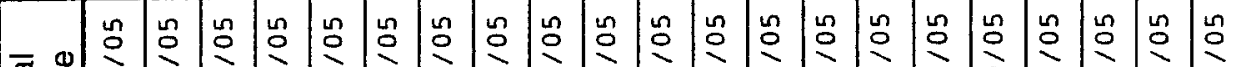

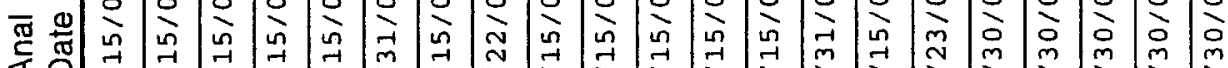
夜 向

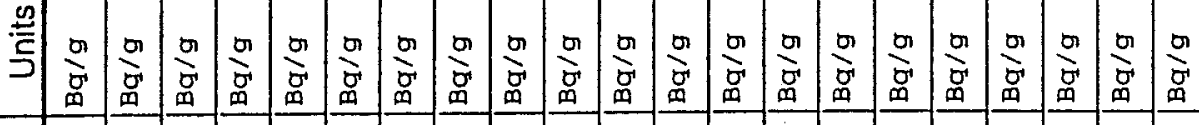

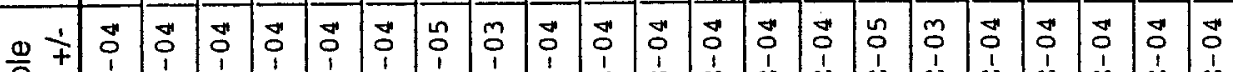
产

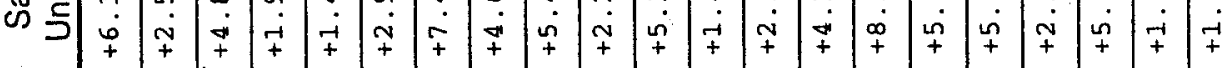
@ n n

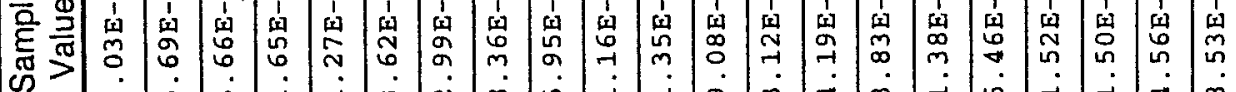

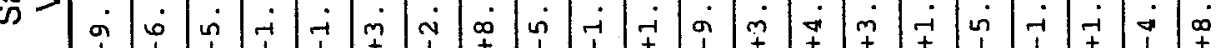

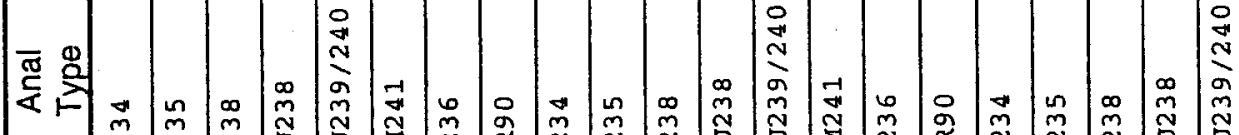

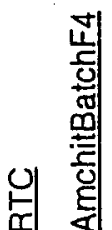

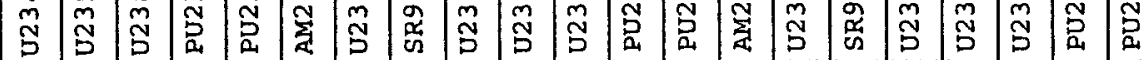
을

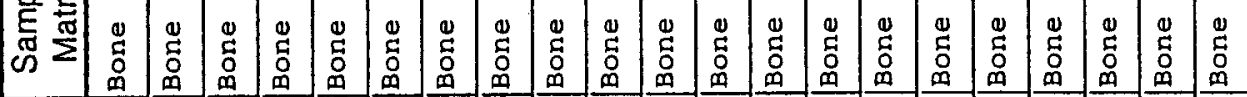

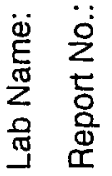

五

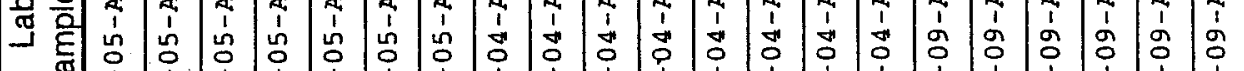

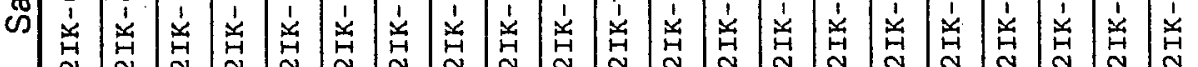
苦

으

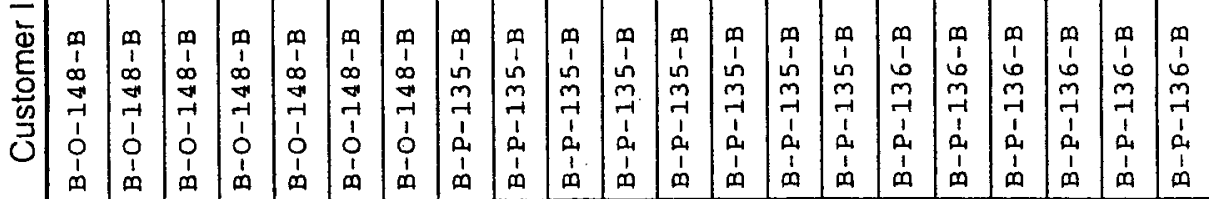




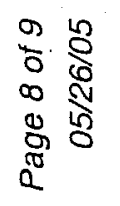

\begin{tabular}{|c|c|c|c|c|c|c|c|c|c|c|}
\hline 岁 & & & & & & & & & & \\
\hline $\begin{array}{l}\text { 号 } \\
\text { 之a }\end{array}$ & $\mid \begin{array}{c}0 \\
0 \\
1 \\
\dot{\theta} \\
0 \\
-1 \\
-i \\
+\end{array}$ & 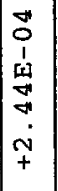 & $\left|\begin{array}{c}\tilde{0} \\
0 \\
1 \\
\hat{\omega} \\
0 \\
0 \\
\tilde{c} \\
+\end{array}\right|$ & 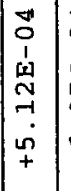 & 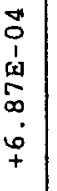 & 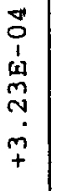 & $\begin{array}{c}0 \\
0 \\
\vdots \\
0 \\
0 \\
0 \\
\dot{0} \\
+ \\
+\end{array}$ & 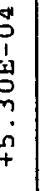 & 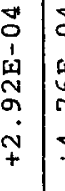 & 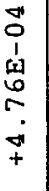 \\
\hline 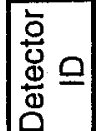 & O & $:$ & $\approx$ & $: 0$ & 8 & 8 & ${ }_{9}^{\circ}:$ & $\stackrel{\circ}{-1}$ & 9 & वै \\
\hline $\mid \begin{array}{l}\frac{5}{9} \\
\frac{\mathrm{d}}{\mathrm{O}}\end{array}$ & \begin{tabular}{|c|}
$m$ \\
$\vec{i}$ \\
$\overrightarrow{0}$ \\
\end{tabular} & \begin{tabular}{|l|} 
\\
0 \\
$\dot{0}$ \\
\\
\end{tabular} & $\begin{array}{l}n \\
\stackrel{n}{n} \\
\therefore\end{array}$ & \begin{tabular}{|c|}
0 \\
$\infty$ \\
$\infty$ \\
$\infty$
\end{tabular} & $\begin{array}{l} \\
6 \\
\infty \\
\infty \\
\infty\end{array}$ & \begin{tabular}{|c|}
0 \\
\\
$\infty$ \\
$\infty$ \\
$\infty$
\end{tabular} & \begin{tabular}{l}
$n$ \\
\hdashline \\
$\vdots$
\end{tabular} & & $\begin{array}{l}n \\
\vdots \\
0\end{array}$ & \begin{tabular}{l|} 
\\
$\dot{0}$ \\
$\infty$ \\
$\infty$
\end{tabular} \\
\hline 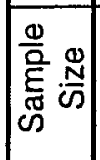 & \begin{tabular}{|l|}
$n$ \\
$\sim$ \\
-
\end{tabular} & $\begin{array}{l}n \\
-i \\
-i\end{array}$ & 品 & $\begin{array}{ll}\pi \\
- \\
-1\end{array}$ & $\begin{array}{l}n \\
i \\
i\end{array}$ & 年 & $\begin{array}{c}n \\
-i \\
-i\end{array}$ & $-i$ & $\begin{array}{r}n-5 \\
-i\end{array}$ & $\stackrel{n}{n}$ \\
\hline 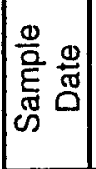 & 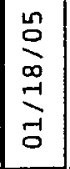 & 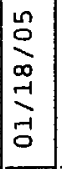 & 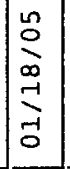 & $\mid \begin{array}{l}n \\
0 \\
0 \\
0 \\
-1 \\
\vdots \\
0 \\
0\end{array}$ & $\begin{array}{l}n \\
0 \\
\infty \\
0-1 \\
-1 \\
0\end{array}$ & $\begin{array}{c}\stackrel{n}{0} \\
\stackrel{0}{0} \\
\stackrel{0}{0} \\
0 \\
0\end{array}$ & $\begin{array}{c}n \\
0 \\
\infty \\
\vdots \\
-1 \\
0\end{array}$ & 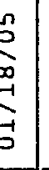 & 号 & 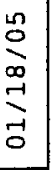 \\
\hline 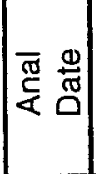 & $\mid \begin{array}{c}\stackrel{n}{0} \\
\stackrel{-}{m} \\
m \\
\tilde{o}\end{array}$ & $\mid \begin{array}{l}n \\
0 \\
0 \\
m \\
m \\
m\end{array}$ & 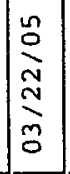 & 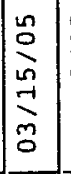 & 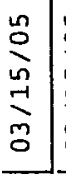 & 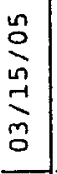 & 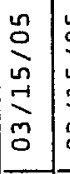 & 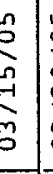 & 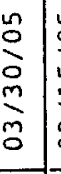 & 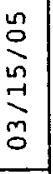 \\
\hline 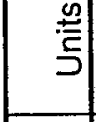 & $\mid \begin{array}{l}0 \\
0 \\
9\end{array}$ & $\mid$\begin{tabular}{r|}
0 \\
$\vdots$ \\
$\vdots$ \\
\end{tabular} & $\mid \begin{array}{l}0 \\
\vdots \\
\vdots \\
\end{array}$ & \begin{tabular}{|c|} 
\\
$\vdots$ \\
0 \\
$m$
\end{tabular} \mid & 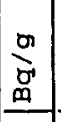 & \begin{tabular}{|l|}
0 \\
$\vdots$ \\
0 \\
\end{tabular} & $\mid \begin{array}{c}0 \\
0 \\
0 \\
9\end{array}$ & $\begin{array}{c}0 \\
0 \\
0 \\
\end{array}$ & 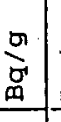 & 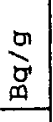 \\
\hline 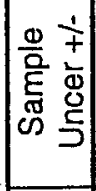 & 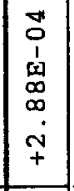 & 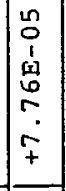 & 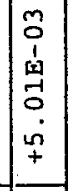 & $\mid \begin{array}{l}\overrightarrow{0} \\
0 \\
1 \\
\mathbf{m} \\
0 \\
0 \\
\dot{\omega} \\
+ \\
\end{array}$ & 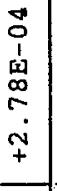 & 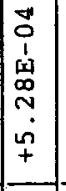 & $\mid \begin{array}{l}0 \\
0 \\
1 \\
0 \\
0 \\
\sigma \\
\dot{+} \\
\dot{+} \\
\end{array}$ & 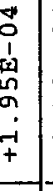 & 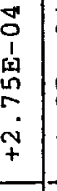 & 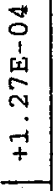 \\
\hline 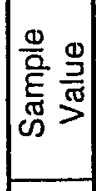 & 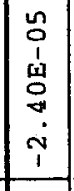 & 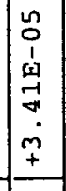 & $\mid \begin{array}{c}0 \\
0 \\
1 \\
\dot{1} \\
0 \\
\dot{m} \\
\dot{1} \\
\end{array}$ & $\mid \begin{array}{l}\overrightarrow{0} \\
1 \\
1 \\
\tilde{\omega} \\
6 \\
0 \\
i \\
i\end{array}$ & 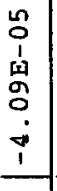 & 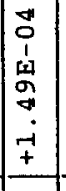 & 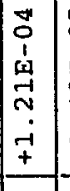 & 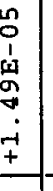 & 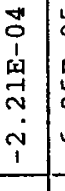 & 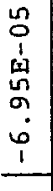 \\
\hline$\overbrace{}^{\bar{\alpha}}$ & 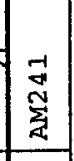 & 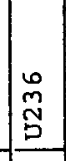 & $\begin{array}{l}0 \\
0 \\
0 \\
0 \\
0 \\
0\end{array}$ & $\mid \begin{array}{l}\vec{j} \\
\tilde{\Xi} \\
\end{array}$ & $\mid \begin{array}{l}n \\
\tilde{m} \\
\underline{\Xi} \\
\end{array}$ & 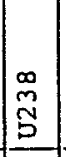 & $\mid$ & 岕 & 节 & 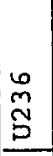 \\
\hline 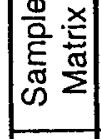 & $\begin{array}{l}0 \\
0 \\
0 \\
\end{array}$ & 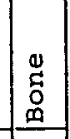 & 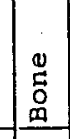 & 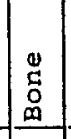 & 竎 & 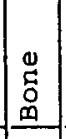 & \begin{tabular}{|l|} 
\\
0 \\
0 \\
0 \\
\end{tabular} & 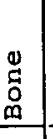 & $\begin{array}{l}0 \\
\vdots \\
0 \\
\\
\end{array}$ & $\begin{array}{l}0 \\
\tilde{0} \\
0 \\
.\end{array}$ \\
\hline 의 & 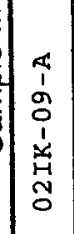 & 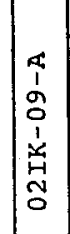 & 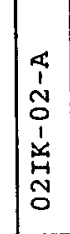 & 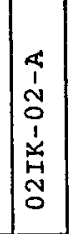 & 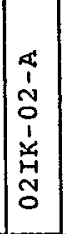 & 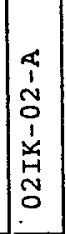 & $\mid$\begin{tabular}{l}
4 \\
1 \\
$\tilde{N}$ \\
0 \\
1 \\
\multirow{y}{H}{} \\
$\tilde{N}$ \\
0
\end{tabular} & 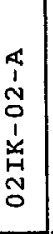 & 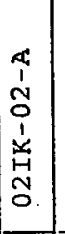 & 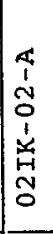 \\
\hline & 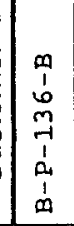 & $\mid \begin{array}{l}0 \\
1 \\
0 \\
0 \\
m \\
1 \\
1 \\
1 \\
1 \\
m\end{array}$ & 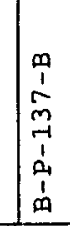 & $\mid \begin{array}{c}0 \\
1 \\
1 \\
m \\
-1 \\
1 \\
0 \\
1 \\
m\end{array}$ & 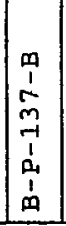 & 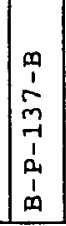 & 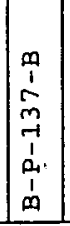 & $\begin{array}{c}m \\
0 \\
1 \\
m \\
m \\
\vdots \\
0 \\
1 \\
m \\
m\end{array}$ & $\mid \begin{array}{c}m \\
1 \\
5 \\
m \\
-1 \\
1 \\
0 \\
1 \\
m \\
\end{array}$ & 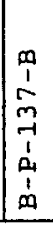 \\
\hline
\end{tabular}




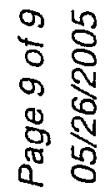

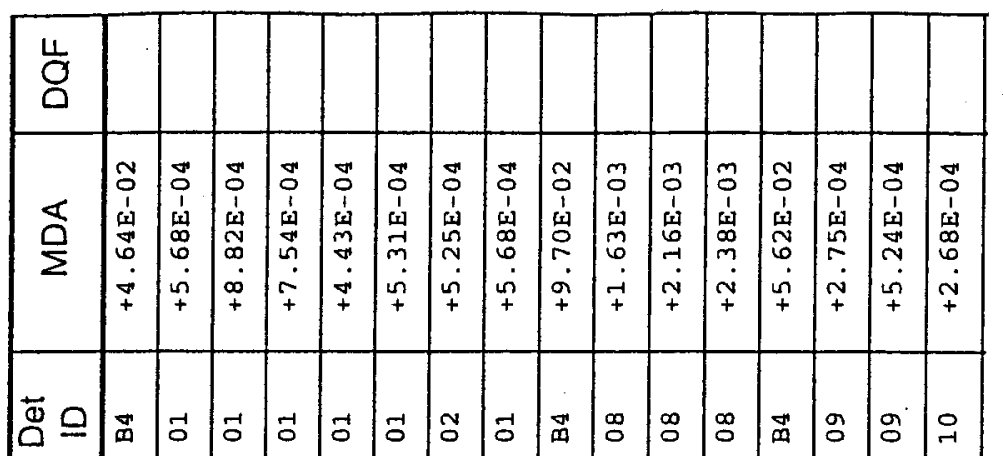

兒

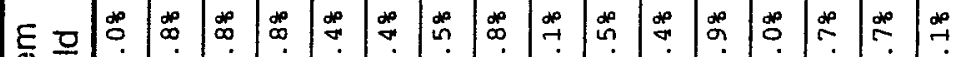

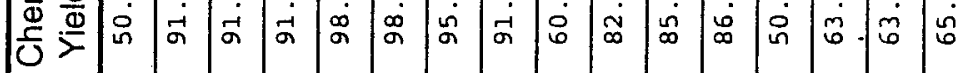

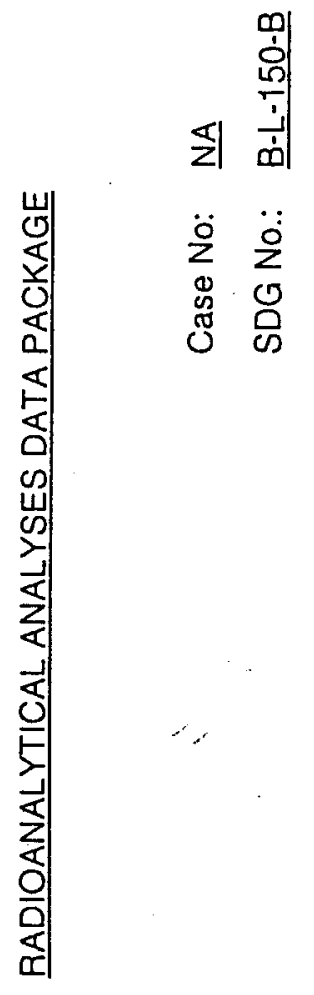

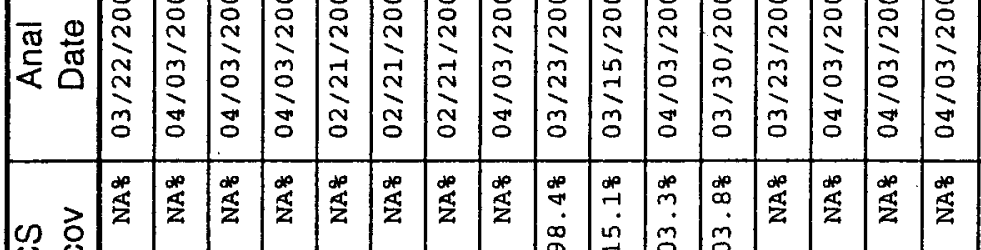
S

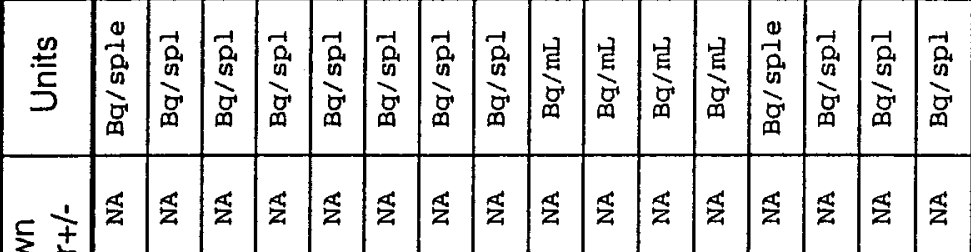

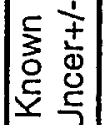

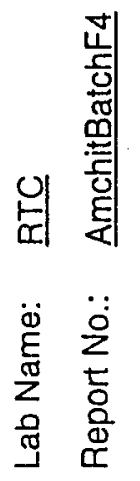

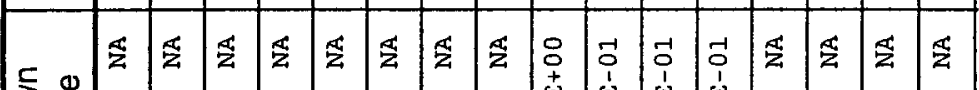

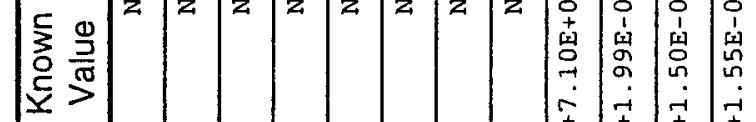

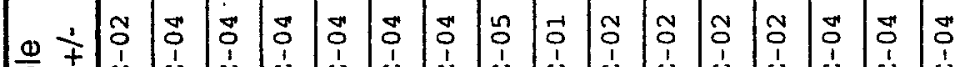

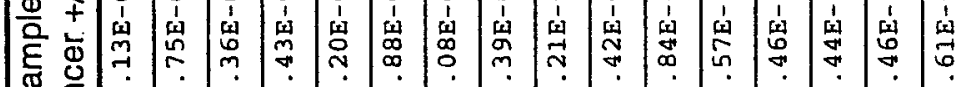
㝴 m

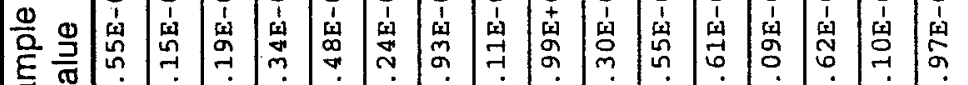
œ̂

\begin{tabular}{|c|c|c|c|c|c|c|c|c|c|c|c|c|c|c|c|c|}
\hline 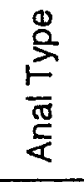 & 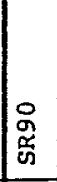 & $\underset{\tilde{S}}{\stackrel{m}{\Xi}}$ & 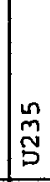 & 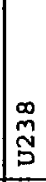 & \begin{tabular}{|l}
$\infty$ \\
$\tilde{N}$ \\
\\
\\
\end{tabular} & 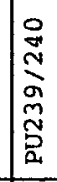 & $\frac{\vec{J}}{\overrightarrow{3}}$ & 怘 & $\begin{array}{l}0 \\
0 \\
0 \\
0 \\
0 \\
0\end{array}$ & $\begin{array}{l}\infty \\
\stackrel{\infty}{S} \\
\end{array}$ & 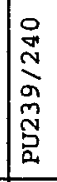 & 䇊 & 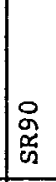 & \begin{tabular}{|l}
$\infty$ \\
$\stackrel{\infty}{N}$ \\
$\stackrel{S}{S}$ \\
\end{tabular} & 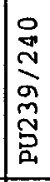 & 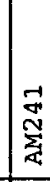 \\
\hline 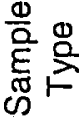 & 首 & 空 & 翻 & 递 & 商 & 㟧 & 总 & 总 & y्u & 号 & 记 & త్ & 剪 & 盖 & 営 & 岁 \\
\hline $\begin{array}{l}\frac{E}{\infty} \\
\text { 感 }\end{array}$ & 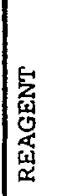 & 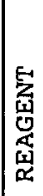 & $\stackrel{E}{z}$ & 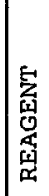 & 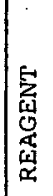 & 谧 & 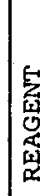 & 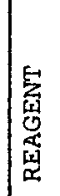 & 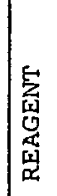 & 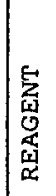 & 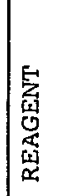 & 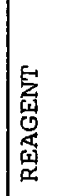 & 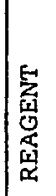 & 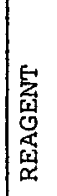 & 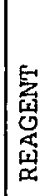 & 兒 \\
\hline
\end{tabular}


Project: $\quad$ Alpha and Sr-90 Analysis for Amchitka Island (Batch 4)

Laboratory: RTC

Report \#: AmchitBatchF4

SDG\#: $\quad$ B-L-150-B

\section{Summary of 2 and 3 sigma activities}

Below are the results for Sr90, U234, U235, U236, U238, Pu238, Pu239/240, and Am241 for Batch 4 from the Amchitka Island Project that had a result/uncertainty ratio of 2 or more (uncertainty @ one sigma).

\begin{tabular}{|c|c|c|c|c|c|}
\hline Customer ID & Lab ID & Isotope & $\begin{array}{c}\text { Result } \\
\mathrm{Bg} / \mathrm{g}\end{array}$ & $\begin{array}{c}\text { Uncertainty } \\
\mathrm{Bq} / \mathrm{g}\end{array}$ & $\begin{array}{c}\text { Result/ } \\
\text { Uncertainty }\end{array}$ \\
\hline$B-K-152-B$ & $021 \mathrm{~K}-17-\mathrm{A}$ & AM241 & 2.44E-02 & 2.27E-03 & 10.7 \\
\hline$B-K-152-B$ & $02 \mathrm{IK}-17-\mathrm{A}$ & PU238 & 4.18E-02 & $4.73 \mathrm{E}-03$ & 8.8 \\
\hline B-K-152-B & $021 \mathrm{~K}-17-\mathrm{A}$ & PU239/240 & 4.65E-02 & $5.22 E-03$ & 8.9 \\
\hline$B-K-152-B$ & $02 \mid K-17-A$ & SR90 & $2.73 \mathrm{E}-02$ & 4.34E-03 & 6.3 \\
\hline$B-L-150-B$ & 02IK-01-A & U234 & $5.67 \mathrm{E}-04$ & 1.36E-04 & 4.2 \\
\hline B-L-150-B & 02IK-01-A & U238 & 4.71E-04 & $1.22 E-04$ & 3.9 \\
\hline B-L-151-B & $021 K-16-A$ & U234 & 4.83E-04 & 1.27E-04 & 3.8 \\
\hline$B-L-151-B$ & $021 \mathrm{~K}-16-\mathrm{A}$ & U238 & $6.07 \mathrm{E}-04$ & $1.46 \mathrm{E}-04$ & 4.2 \\
\hline$B-M-139-B$ & $021 \mathrm{~K}-10-\mathrm{A}$ & SR90 & $8.81 E-03$ & $3.54 \mathrm{E}-03$ & 2.5 \\
\hline$B-M-142-B$ & 02IK-03-A & SR90 & $5.34 \mathrm{E}-03$ & $2.71 E-03$ & 2.0 \\
\hline
\end{tabular}

All known sources of uncertainty are included in the uncertainty term. There may be unknown sources of uncertainty that are not accounted for. If the result/uncertainty ratio is more than 3, we have a degree of confidence that the result is positive (i.e. the result is statistically different than zero). A result with the result/uncertainty ratio between 2 and 3 is the first indication that an isotope may be present and further investigation may be warranted.

As with any good science no single data point is used in important decisions (results need to be reproducible). 
BATCH 5

ALPHA AND Sr-90 


\section{RADIOANALYTICAL ANALYSES DATA PACKAGE}

\begin{tabular}{llll} 
Project Title: & Alpha and Sr-90 Analysis for Amchitka island & (Batch 5) \\
\cline { 2 - 3 } & Rab Name: & Case No: & NA \\
Report No.: & AmchitBatchF5 & Method Type: & A/B \\
Approved SAP No.: NA & SDG No.: & B-A-163-B
\end{tabular}

\section{SAMPLE NUMBERS}

Customer

Sample ID

$B-A-163-B$

$B-B-154-B$

$B-C-162-B$

B-C-174-B

B-J-167-B

$B-K-171-B$

B-K-172-B

B-L-164-B

\section{Lab Sample}

ID

02JJ-03-A

02JJ-01-A

02JJ-02-A

02JJ-08-A

02JJ-05-A

02JJ-06-A

02JJ-07-A

$02 J J-04-A$

Comments:

Release of the data contained in this data package has been authorized by the laboratory manager or the manager's designee, as verified by the following signature:

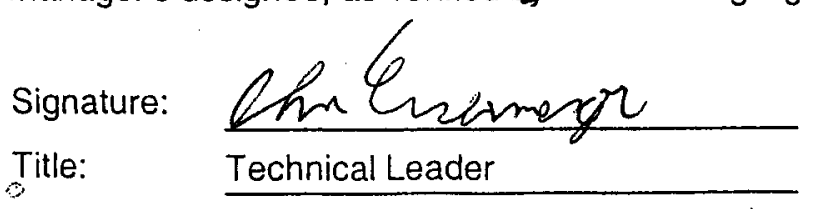

Name: J.G. Eisenmenger

Date: $\quad 05 / 26 / 2005$ 
$\frac{0}{0}$

$\Upsilon$ จ

बु

$a^{\infty}$

\begin{tabular}{|c|c|c|c|c|c|c|c|c|c|c|c|c|c|c|c|c|c|c|c|c|}
\hline $\begin{array}{l} \\
\\
0\end{array}$ & & & & & & & & & & & & & & & & & & & & \\
\hline 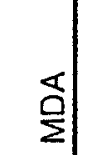 & 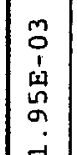 & $\left|\begin{array}{l}n \\
0 \\
1 \\
n \\
n \\
5 \\
0 \\
0\end{array}\right|$ & $\begin{array}{l}n \\
0 \\
1 \\
1 \\
n \\
5 \\
\vdots \\
0\end{array}$ & \begin{tabular}{l}
$n$ \\
0 \\
1 \\
1 \\
$g$ \\
\hdashline \\
$\vdots$
\end{tabular} & 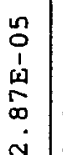 & $\begin{array}{l}n \\
0 \\
1 \\
1 \\
\vdots \\
\vdots \\
\infty \\
\dot{c} \\
\dot{c}\end{array}$ & 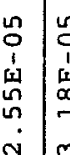 & 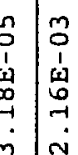 & $\begin{array}{l}n \\
0 \\
1 \\
1 \\
1 \\
\sigma \\
? \\
\vdots \\
0\end{array}$ & \begin{tabular}{|l} 
in \\
0 \\
1 \\
0 \\
0 \\
0
\end{tabular} & $\begin{array}{l}n \\
0 \\
0 \\
1 \\
\dot{a} \\
\sigma \\
\dot{m} \\
m\end{array}$ & $\begin{array}{l}n \\
0 \\
1 \\
\mathfrak{s} \\
0 \\
-1 \\
\dot{m}\end{array}$ & 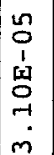 & 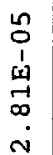 & 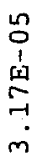 & $\begin{array}{c}0 \\
0 \\
1 \\
\omega \\
m \\
\sigma \\
\sigma \\
-1\end{array}$ & 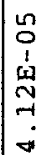 & 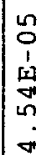 & $\begin{array}{l}n \\
0 \\
1 \\
1 \\
\vdots \\
0 \\
0 \\
m\end{array}$ & \\
\hline
\end{tabular}

产

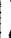

¿

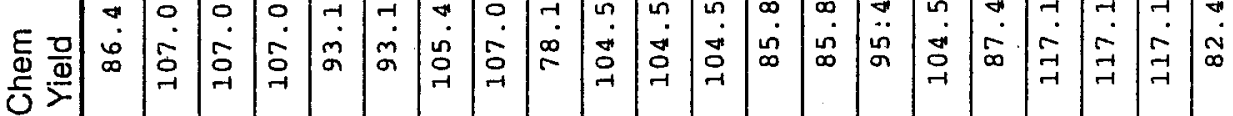

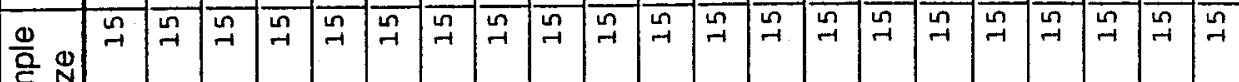

通

密

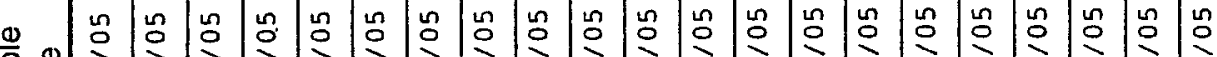

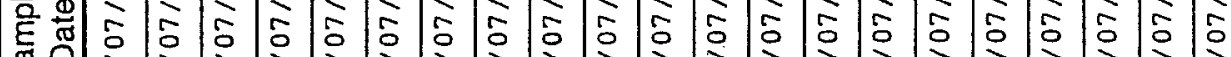

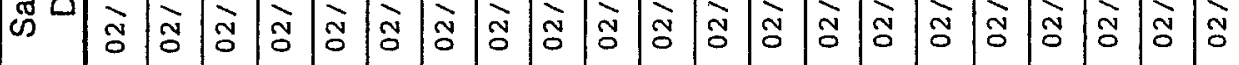

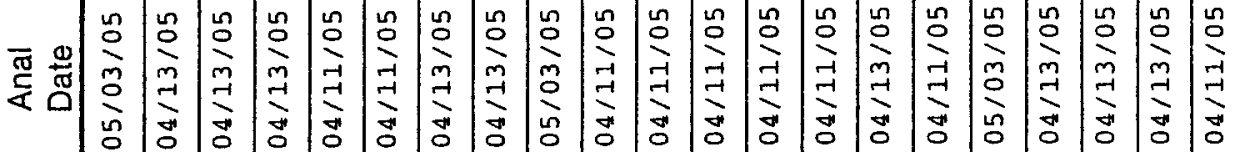

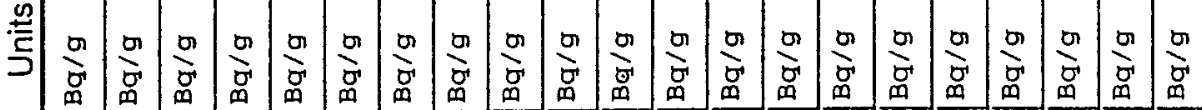

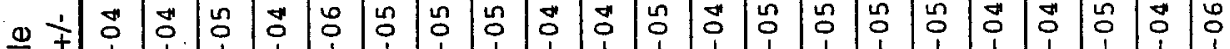

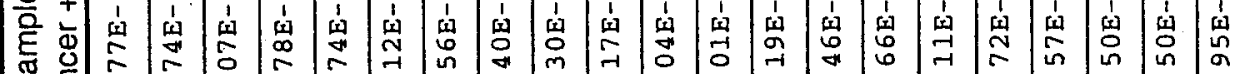

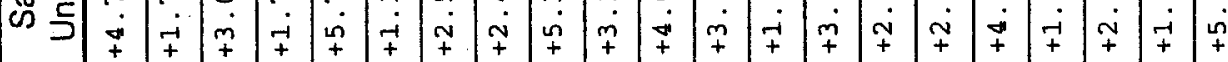

重

E ぶ

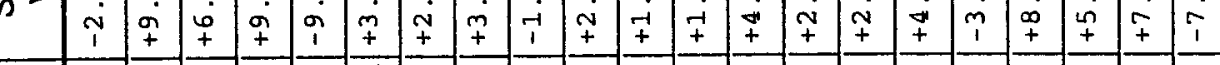

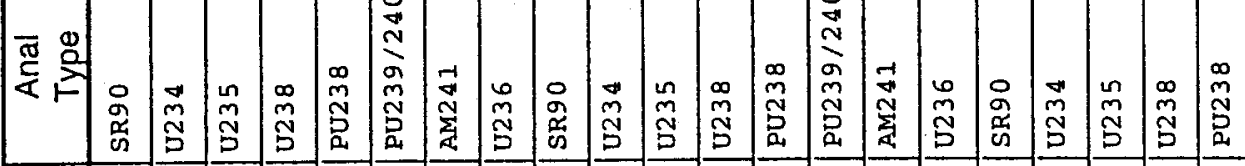

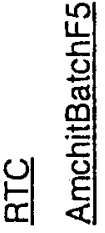

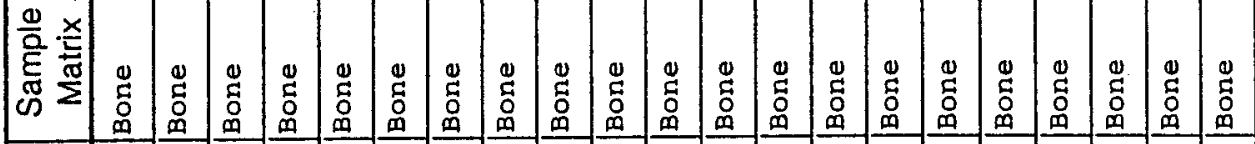

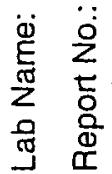

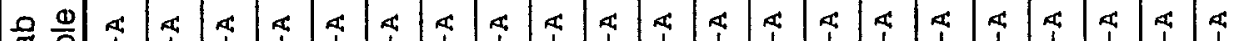

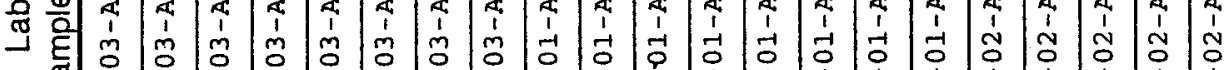

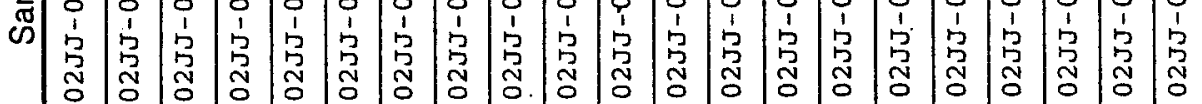

으

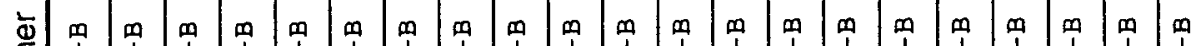

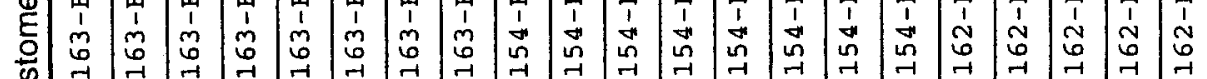

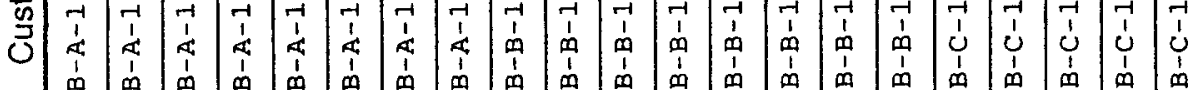


\%

$m$ \&

के ڤొ

Q

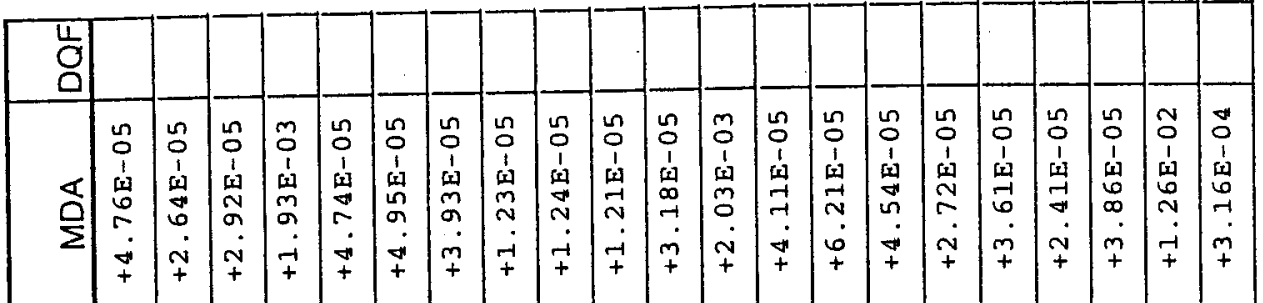

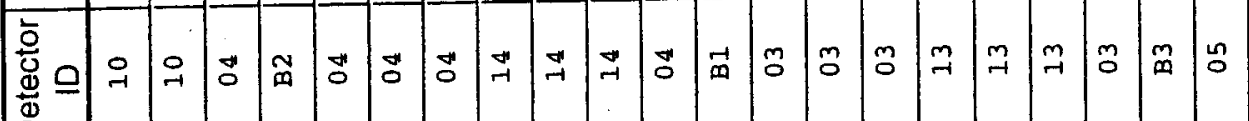

잉

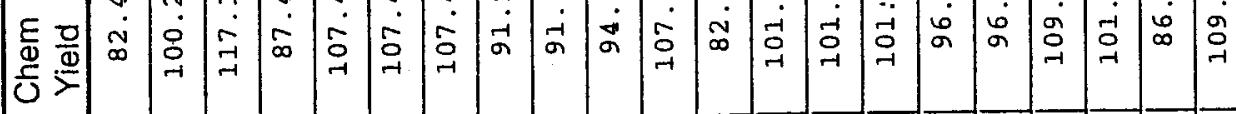

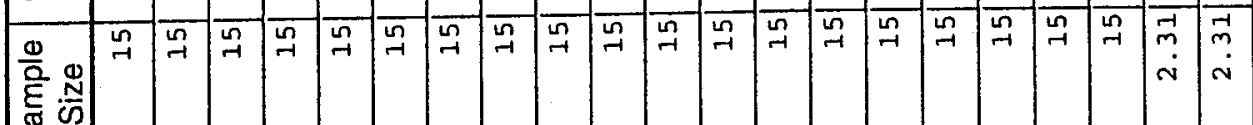

¿

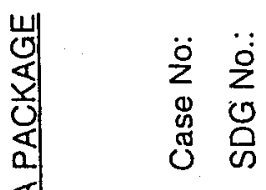

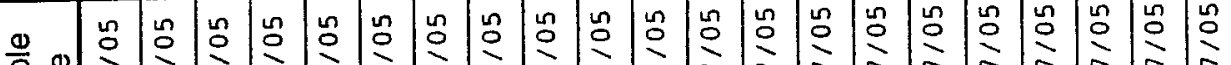

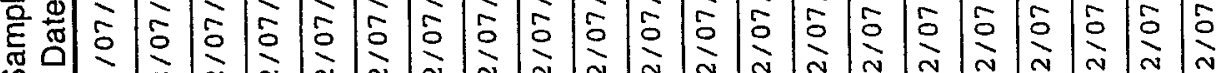
๙্乃

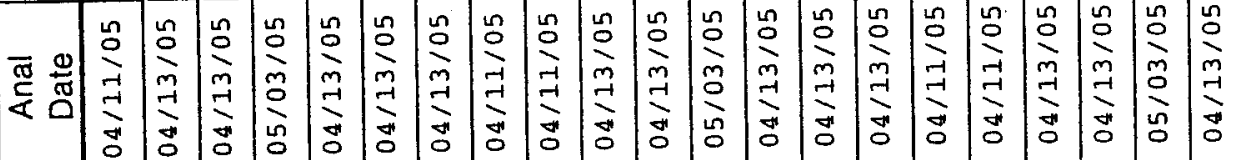

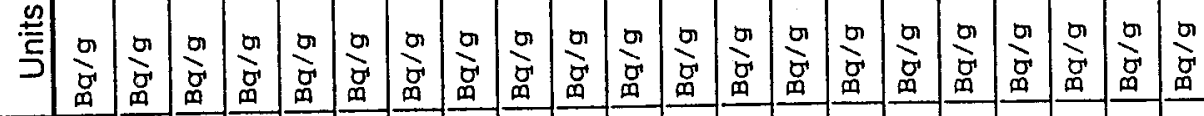

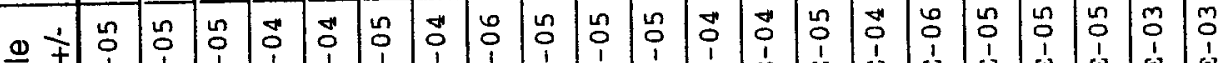

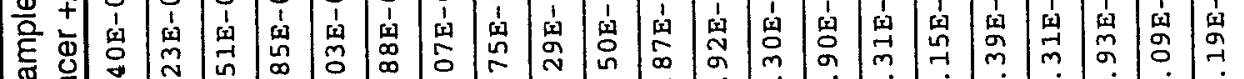

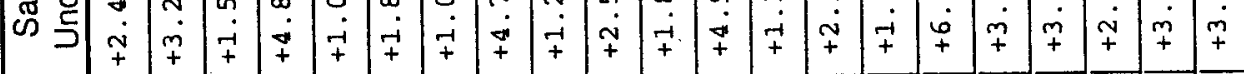

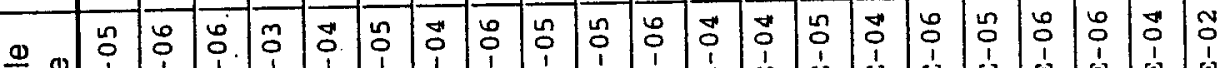

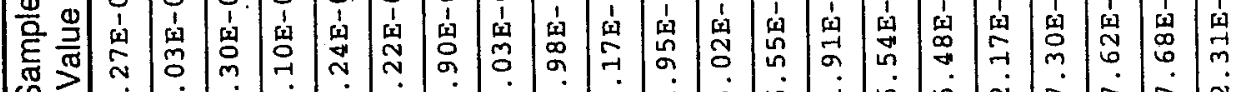

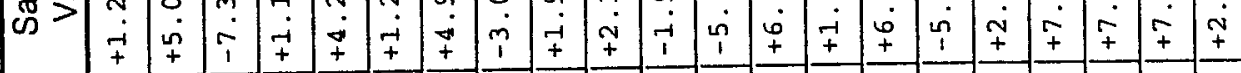

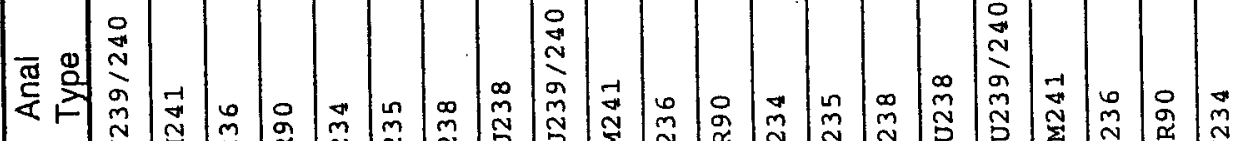

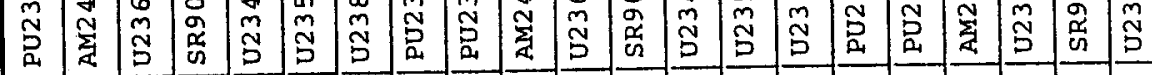

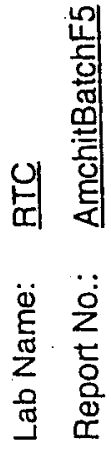

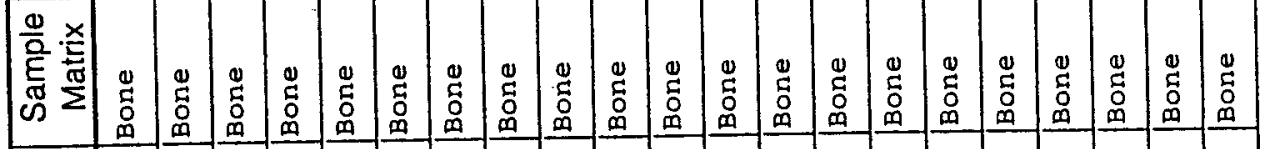
으 员 匹 윌

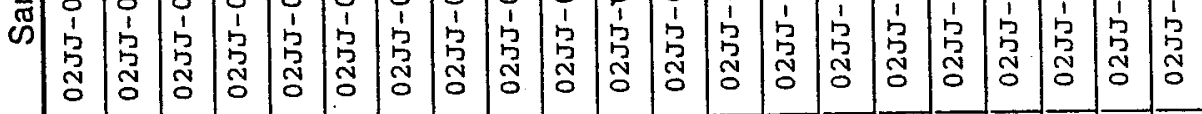

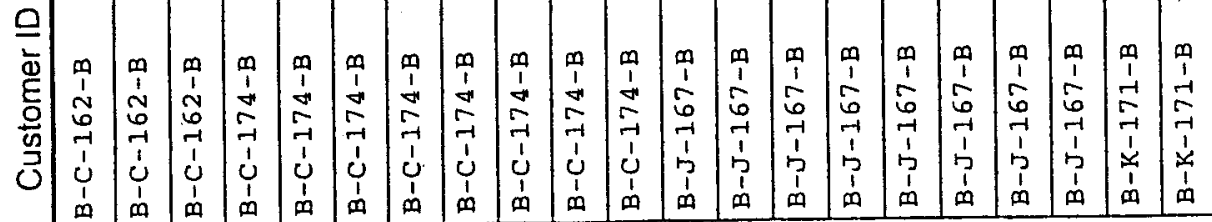


$\begin{array}{lll}0 & 0 \\ 0 & 0 \\ 0 & 0 \\ 0 & 5 \\ 0 & 0 \\ 0 & 0 \\ 0\end{array}$

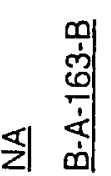

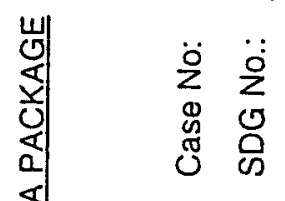

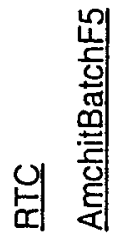

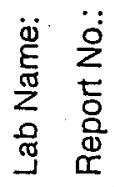

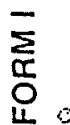

\begin{tabular}{|c|c|c|c|c|c|c|c|c|c|c|c|c|c|c|c|c|c|c|c|c|c|}
\hline $\begin{array}{l}\text { प् } \\
0\end{array}$ & & & & & & & & & & & & & & & & & & & & & \\
\hline 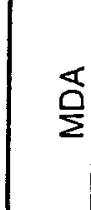 & 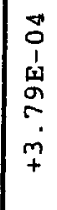 & $\mid \begin{array}{l}\overrightarrow{0} \\
0 \\
1 \\
\hat{1} \\
0 \\
0 \\
0 \\
\sim \\
+ \\
+\end{array}$ & 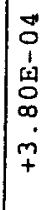 & 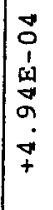 & $\begin{array}{l}\not \\
0 \\
1 \\
1 \\
01 \\
0 \\
0 \\
-1 \\
7 \\
+\end{array}$ & 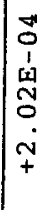 & $\begin{array}{l}2 \\
0 \\
1 \\
1 \\
w \\
w \\
5 \\
-1 \\
+ \\
+\end{array}$ & 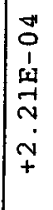 & 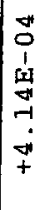 & $\begin{array}{l}\not \\
0 \\
1 \\
1 \\
0 \\
0 \\
\infty \\
\infty \\
-1 \\
+\end{array}$ & $\begin{array}{l}\not \\
0 \\
1 \\
1 \\
1 \\
\infty \\
\infty \\
0 \\
+ \\
+\end{array}$ & \begin{tabular}{l}
8 \\
0 \\
1 \\
1 \\
0 \\
0 \\
\hdashline \\
1 \\
+1 \\
+
\end{tabular} & $\begin{array}{l}n \\
0 \\
1 \\
\omega \\
w \\
m \\
m \\
o \\
+\end{array}$ & 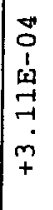 & 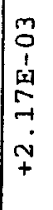 & 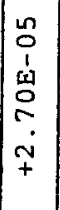 & 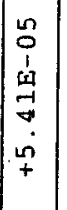 & 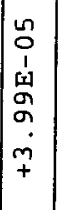 & \begin{tabular}{|c|}
$n$ \\
0 \\
0 \\
$\omega$ \\
$\omega$ \\
$\sim$ \\
$\sim$ \\
$m$ \\
+ \\
\end{tabular} & $\begin{array}{l}n \\
0 \\
1 \\
\omega \\
0 \\
o \\
o \\
\sim \\
+ \\
+\end{array}$ & $\mid \begin{array}{l}n \\
0 \\
1 \\
\dot{w} \\
m \\
0 \\
0 \\
m \\
+\end{array}$ \\
\hline 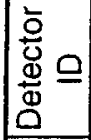 & 号 & ก & $\left.\right|_{0} ^{\infty}$ & $\left.\right|_{0} ^{\infty}$ & $\stackrel{m}{o}$ & I & 7 & $1 \%$ & 10 & 10 & $\infty_{0}^{\infty}$ & $\stackrel{\infty}{\circ}$ & $\ddot{0}$ & $1 \%$ & $\ddot{z}$ & $1 \%$ & 1: & $\%$ & 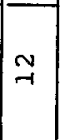 & $\underset{\nearrow}{ }$ & $\underset{\nearrow}{\mp}$ \\
\hline$\frac{\bar{\Phi}}{\mathrm{\Phi}} \frac{\mathrm{o}}{\mathrm{w}}$ & $\begin{array}{l}\tilde{y} \\
\dot{\sigma} \\
0 \\
-1\end{array}$ & 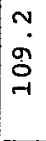 & m. & m. & $\underset{r}{\tilde{m}}$ & 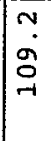 & $\begin{array}{l}a \\
-1 \\
\infty\end{array}$ & ${ }_{0}^{0}$ & $\begin{array}{c}0 \\
0 \\
0 \\
0 \\
-1\end{array}$ & $\begin{array}{l}0 \\
0 \\
0 \\
-1\end{array}$ & 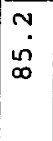 & $\begin{array}{l}N \\
\\
\infty \\
\infty\end{array}$ & 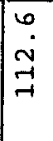 & $\begin{array}{l}0 \\
\dot{0} \\
\dot{0}\end{array}$ & \begin{tabular}{l}
$Z$ \\
$\ddot{0}$ \\
\hdashline
\end{tabular} & 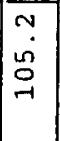 & $\begin{array}{l} \\
\dot{y} \\
\tilde{O} \\
-1\end{array}$ & 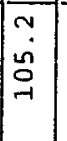 & $\begin{array}{l}m \\
m \\
\sigma\end{array}$ & $\begin{array}{l}m \\
m \\
n \\
0\end{array}$ & $\vec{~} \overrightarrow{.}$ \\
\hline 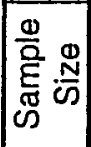 & \begin{tabular}{|l}
$\vec{m}$ \\
$\dot{v}$
\end{tabular} & $\begin{array}{l}\overrightarrow{1} \\
\stackrel{n}{n} \\
\dot{v}\end{array}$ & \begin{tabular}{|l}
$\vec{m}$ \\
$\dot{\sim}$
\end{tabular} & $\begin{array}{l}\vec{r} \\
\tilde{n} \\
\sim\end{array}$ & \begin{tabular}{|l}
$\vec{\mu}$ \\
$\dot{n}$ \\
$\sim$
\end{tabular} & 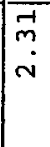 & $\begin{array}{l}0 \\
-1 \\
i\end{array}$ & $\begin{array}{l}0 \\
\stackrel{0}{*} \\
\sim\end{array}$ & $\begin{array}{l}0 \\
\\
\end{array}$ & $\begin{array}{l}0 \\
\stackrel{0}{*} \\
\dot{\sim}\end{array}$ & \begin{tabular}{l}
$\overline{0}$ \\
\hdashline \\
$\sim$ \\
$\sim$
\end{tabular} & $\begin{array}{l}\overline{0} \\
\stackrel{-1}{i} \\
\sim\end{array}$ & $\begin{array}{l} \\
\stackrel{0}{*} \\
\sim\end{array}$ & 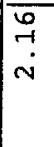 & $\stackrel{\pi}{\sim}$ & $\underset{7}{n-1}$ & ज్ & $\bar{n}$ & $\underset{\sim}{n}$ & $\stackrel{\Omega}{n}$ & $\pi$ \\
\hline
\end{tabular}

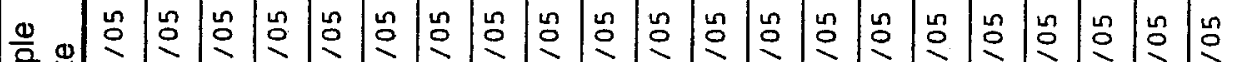

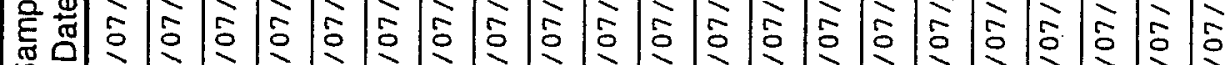

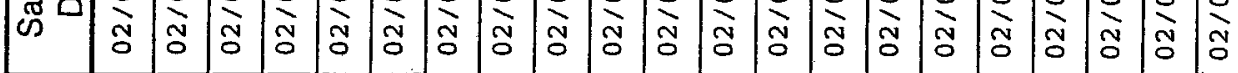
西 包

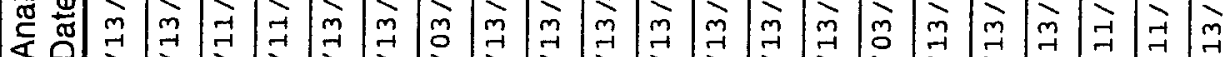
र

¿

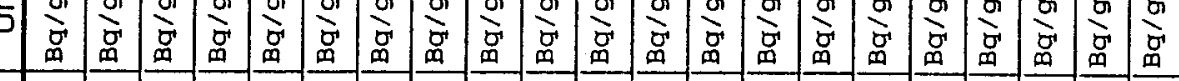

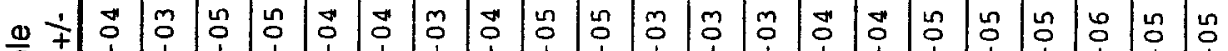

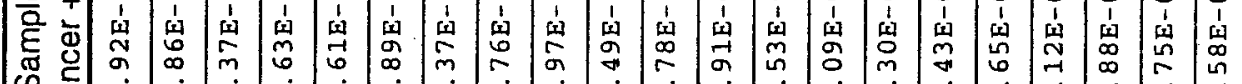

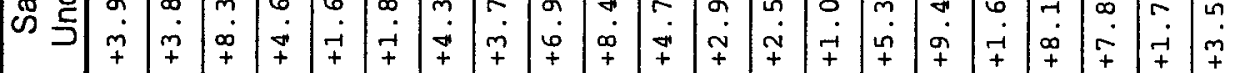
๓

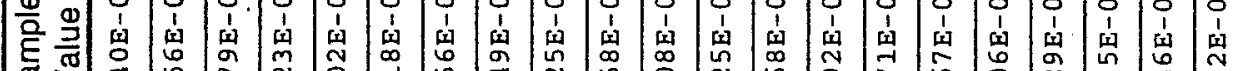

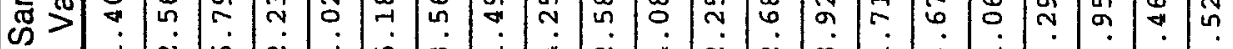
ब

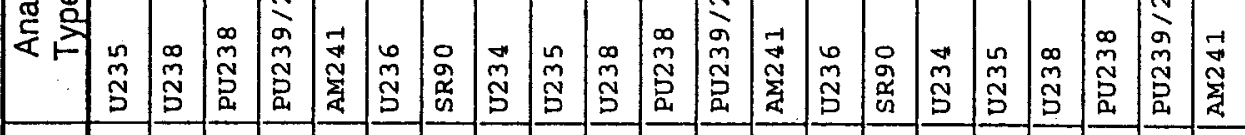

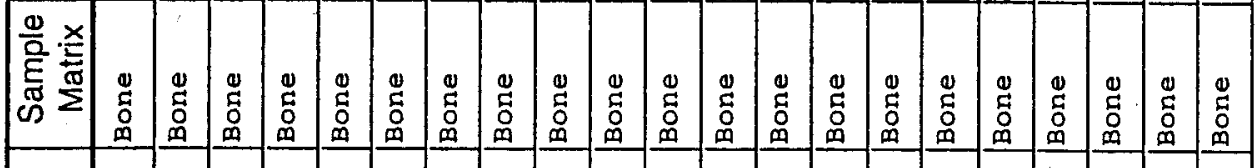

号

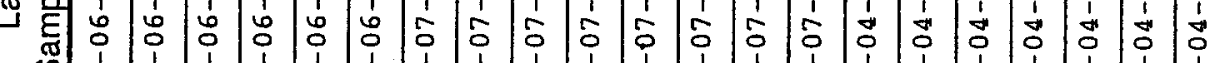

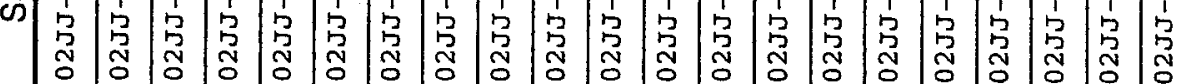


$\begin{array}{ll}0 & 4 \\ 0 & 0 \\ 6 & 0 \\ 0 & 5 \\ 0 & 0 \\ 0 & 0\end{array}$
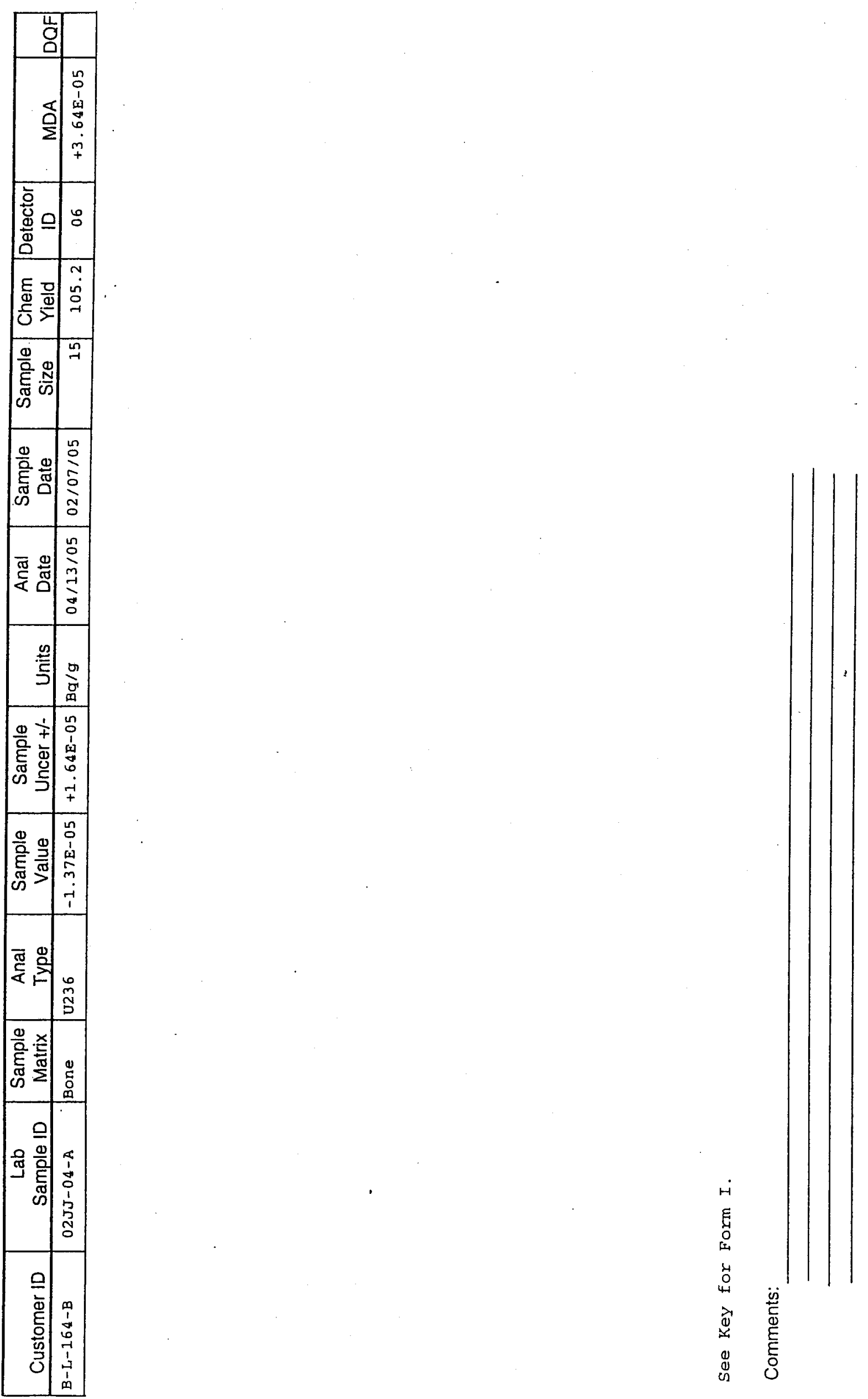


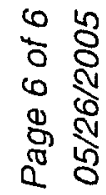

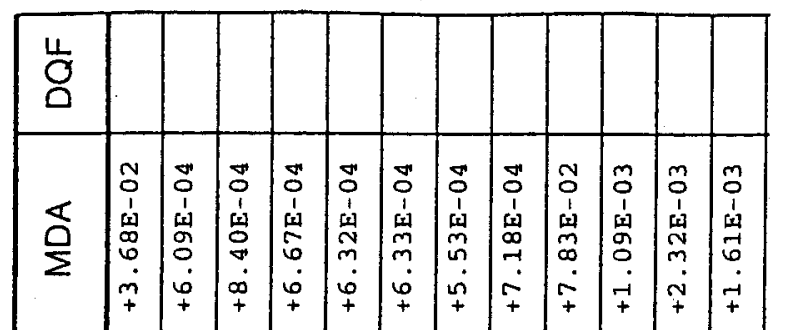

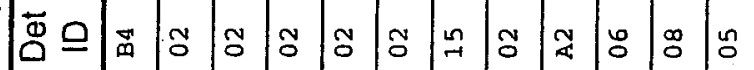

¿l

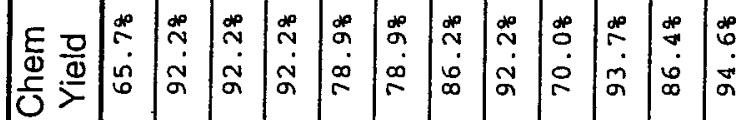

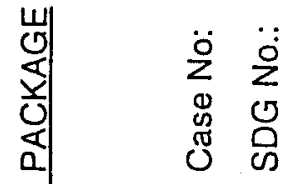

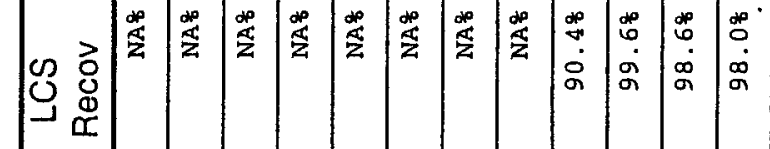

च

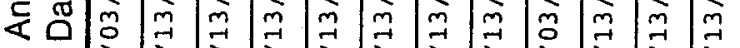

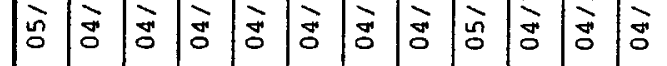

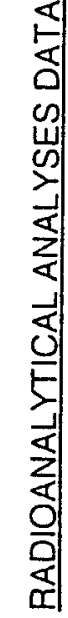

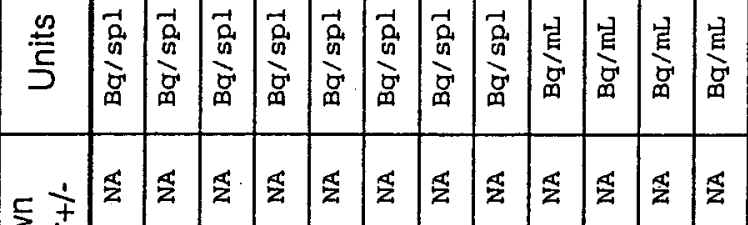

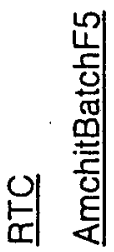

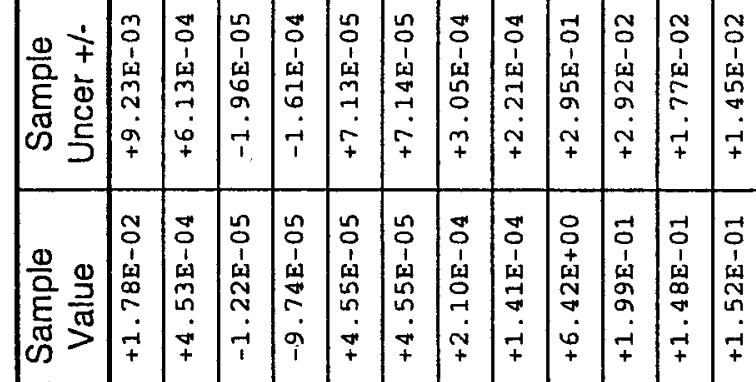

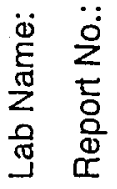

\begin{tabular}{|c|c|c|c|c|c|c|c|c|c|c|c|c|}
\hline 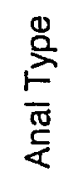 & 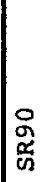 & 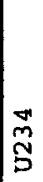 & 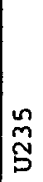 & $\mid$\begin{tabular}{l}
$\infty$ \\
\multirow{S}{S}{}
\end{tabular} & 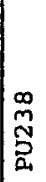 & 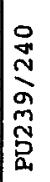 & 雍 & 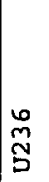 & 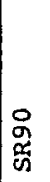 & 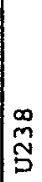 & 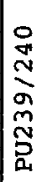 & 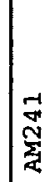 \\
\hline 焉 & $\frac{3}{9}$ & 岁 & 㿞 & 岁 & 㟔 & 总 & 崞 & 㟔 & $\bigcup_{\Xi}^{U}$ & 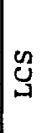 & ఝ્త & U్త \\
\hline 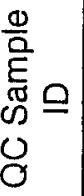 & $\bar{\xi}$ & 舁 & 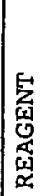 & 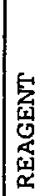 & 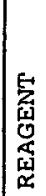 & 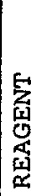 & 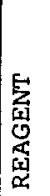 & 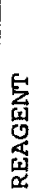 & 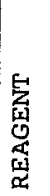 & 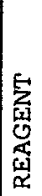 & 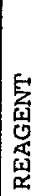 & 点 \\
\hline
\end{tabular}


Project: $\quad$ Alpha and Sr-90 Analysis for Amchitka Island (Batch 5)

Laboratory: RTC

Report \#: AmchitBatchF5

SDG\#: $\quad$ B-A-163-B

Summary of 2 and 3 sigma activities

Below are the results for Sr90, U234, U235, U236, U238, Pu238, Pu239/240, and Am241 for Batch 5 from the Amchitka Island Project that had a result/uncertainty ratio of 2 or more (uncertainty @ one sigma).

\begin{tabular}{|c|c|c|c|c|c|}
\hline Customer ID & Lab ID & Isotope & $\begin{array}{c}\text { Result } \\
\mathrm{Bq} / \mathrm{g}\end{array}$ & $\begin{array}{c}\text { Uncertainty } \\
\mathrm{Bq} / \mathrm{g}\end{array}$ & $\begin{array}{c}\text { Result/ } \\
\text { Uncertainty }\end{array}$ \\
\hline B-A-163-B & 02JJ-03-A & U234 & $9.63 \mathrm{E}-04$ & $1.74 E-04$ & 5.5 \\
\hline$B-A-163-B$ & $02 \mathrm{JJ}-03-\mathrm{A}$ & U235 & $6.53 E-05$ & $3.07 E-05$ & 2.1 \\
\hline B-A-163-B & 02JJJ-03-A & U238 & $9.40 E-04$ & $1.78 E-04$ & 5.3 \\
\hline B-B-154-B & $02 J J-01-A$ & U234 & $2.18 E-03$ & $3.17 E-04$ & 6.9 \\
\hline B-B-154-B & $02 J J-01-A$ & U235 & $1.16 \mathrm{E}-04$ & 4.04E-05 & 2.9 \\
\hline B-B-154-B & 02JJ-01-A & U238 & $1.83 E-03$ & $3.01 \mathrm{E}-04$ & 6.1 \\
\hline B-C-162-B & 02JJ-02-A & U234 & $8.57 E-04$ & $1.57 \mathrm{E}-04$ & 5.5 \\
\hline$B-C-162-B$ & $02 J J-02-A$ & U235 & $5.30 E-05$ & $2.50 \mathrm{E}-05$ & 2.1 \\
\hline B-C-162-B & 02JJ-02-A & U238 & $7.79 E-04$ & $1.50 \mathrm{E}-04$ & 5.2 \\
\hline$B-C-174-B$ & $02 \mathrm{JJ}-08-\mathrm{A}$ & SR90 & $1.10 \mathrm{E}-03$ & $4.85 E-04$ & 2.3 \\
\hline$B-C-174-B$ & $02 \mathrm{JJ}-08-\mathrm{A}$ & U234 & $4.24 \mathrm{E}-04$ & $1.03 E-04$ & 4.1 \\
\hline B-C-174-B & $02 J J-08-A$ & U238 & $4.90 \mathrm{E}-04$ & 1.07E-04 & 4.6 \\
\hline B-J-167-B & $02 \mathrm{JJ}-05-\mathrm{A}$ & U234 & $6.55 E-04$ & 1.30E-04 & 5.0 \\
\hline B-J-167-B & $02 \mathrm{JJ}-05-\mathrm{A}$ & U238 & $6.54 \mathrm{E}-04$ & $1.31 \mathrm{E}-04$ & 5.0 \\
\hline$B-K-171-B$ & $02 \mathrm{JJ}-06-\mathrm{A}$ & U234 & $2.31 E-02$ & $3.19 \mathrm{E}-03$ & 7.2 \\
\hline B-K-171-B & $02 \mathrm{JJ}-06-\mathrm{A}$ & U235 & $1.40 \mathrm{E}-03$ & $3.92 E-04$ & 3.6 \\
\hline $\mathrm{B}-\mathrm{K}-171-\mathrm{B}$ & $02 \mathrm{JJ}-06-\mathrm{A}$ & U238 & $2.56 \mathrm{E}-02$ & 3.86E-03 & 6.6 \\
\hline$B-K-172-B$ & $02 \mathrm{JJ}-07-\mathrm{A}$ & AM241 & $2.68 \mathrm{E}-02$ & $2.53 \mathrm{E}-03$ & 10.6 \\
\hline B-K-172-B & $02 \mathrm{JJ}-07-\mathrm{A}$ & PU238 & $4.08 \mathrm{E}-02$ & $4.78 \mathrm{E}-03$ & 8.5 \\
\hline B-K-172-B & $02 J J-07-A$ & PU239/240 & $2.25 E-02$ & $2.91 E-03$ & 7.7 \\
\hline$B-K-172-B$ & $02 \mathrm{JJ}-07-\mathrm{A}$ & SR90 & $3.56 \mathrm{E}-02$ & $4.37 \mathrm{E}-03$ & 8.1 \\
\hline B-L-164-B & 02JJ-04-A & U234 & 3.67 E-04 & $9.43 \mathrm{E}-05$ & 3.9 \\
\hline$B-L-164-B$ & $02 \mathrm{JJ}-04-\mathrm{A}$ & U238 & $3.29 \mathrm{E}-04$ & $8.12 \mathrm{E}-05$ & 4.1 \\
\hline
\end{tabular}




\section{Alpha and Sr-90 Analysis for Amchitka Island (Batch 5)}

Summary of 2 and 3 sigma activities

Page 2

All known sources of uncertainty are included in the uncertainty term. There may be unknown sources of uncertainty that are not accounted for. If the result/uncertainty ratio is more than 3, we have a degree of confidence that the result is positive (i.e. the result is statistically different than zero). A result with the result/uncertainty ratio between 2 and 3 is the first indication that an isotope may be present and further investigation may be warranted.

As with any good science no single data point is used in important decisions (results need to be reproducible). 
BATCH 6

Sr-90 
COVER PAGE

RADIOANALYTICAL ANALYSES DATA PACKAGE

Project Title: $\quad$ Sr-90 Analysis for Amchitka Island (Batch 6)

Lab Name:

RTC

Case No: NA

Report No::

AmchitBatchF136

Method Type: A/B

Approved SAP No.: NA

SDG No.: $\quad$ S-A-175-A

\section{SAMPLE NUMBERS}

Customer

Sample ID

S-A-175-A

S-A-178-A

S-B-66-A

S-C-170-A

S-C-176-A

S-J-169-A

S-J-177-A

S-K-116-A

S-K-118-A

S-K-180-A

S-K-181-A

S-L-72-A
Lab Sample

ID

02JK-03-A

02JK-06-A

02EQ-11-A

02.JK-02-A

02JK-04-A

02JK-01-A

02JK-05-A

02GM-12-A

02GM-14-A

02JK-07-A

02JK-08-A

02EQ-17-A

Comments:

Release of the data contained in this data package has been authorized by the laboratory manager or the manager's designee, as verified by the following signature:

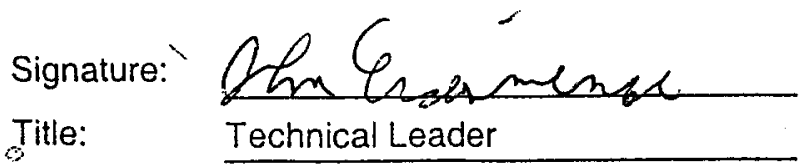

Name: J. G. Eisenmenger

Date: $\quad 05 / 26 / 2005$ 
$\begin{array}{ll}m & h \\ \vdots & 5 \\ 0 & 0 \\ 0 & \mathbb{2} \\ 0 & 8 \\ 0 & 0 \\ 0 & 0\end{array}$

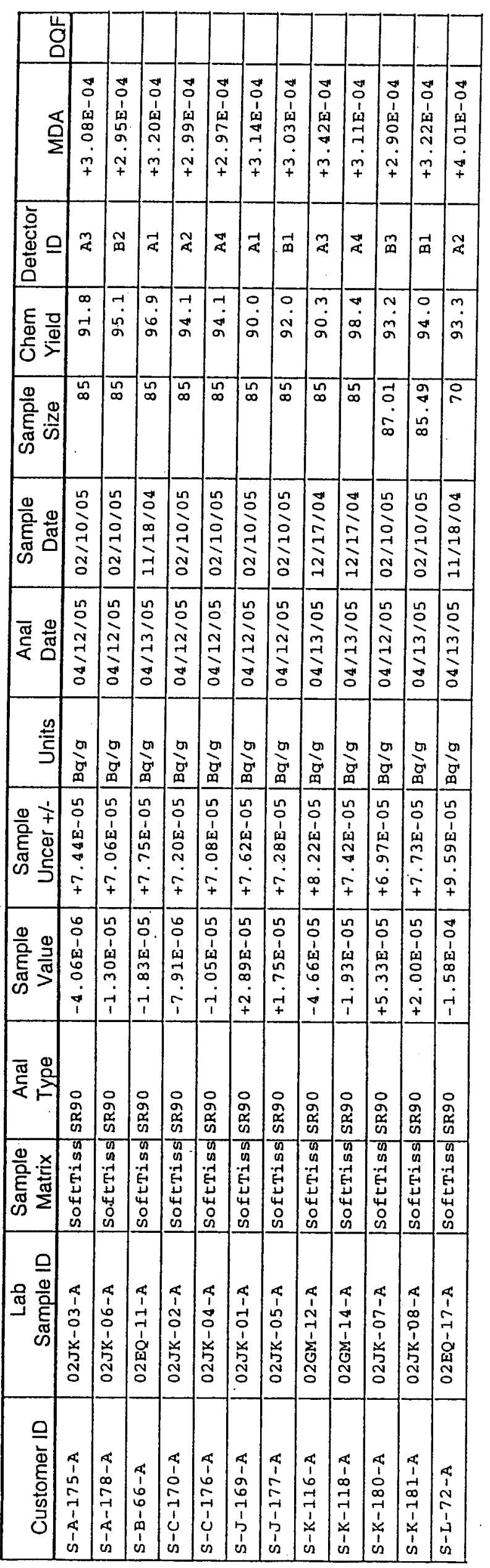




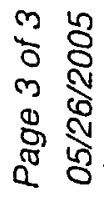

\begin{tabular}{|c|c|c|}
\hline 岁 & & \\
\hline$\stackrel{\nwarrow}{\text { O }}$ & 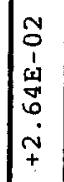 & 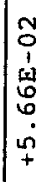 \\
\hline 苂으 & $\ddot{m}$ & 临 \\
\hline 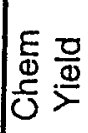 & $\mid \begin{array}{c}\infty \\
m \\
\infty \\
\infty \\
\infty\end{array}$ & $\begin{array}{l}\stackrel{\infty}{*} \\
\stackrel{\sim}{\alpha}\end{array}$ \\
\hline & & \\
\hline
\end{tabular}

岁.

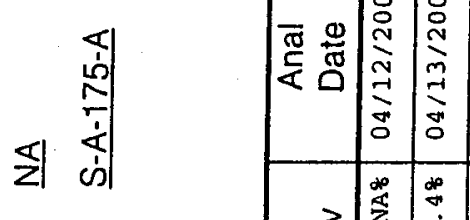

$\stackrel{5}{5}$

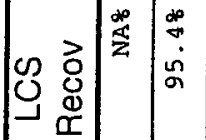

告

点交宗运

文

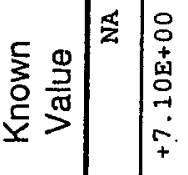

Ð

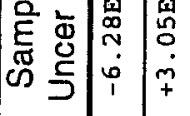

|

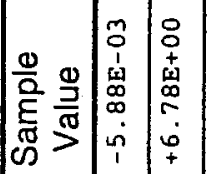

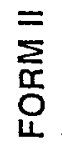

\begin{tabular}{|c|c|c|}
\hline 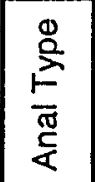 & 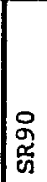 & 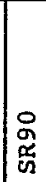 \\
\hline 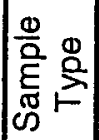 & 总 & $\int_{\square}^{0}$ \\
\hline $\begin{array}{l}\frac{\Phi}{0} \\
\frac{\tilde{\sigma}}{\tilde{E}} \\
\infty \\
\infty \\
0 \\
0\end{array}$ & 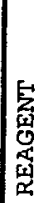 & 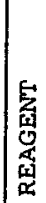 \\
\hline
\end{tabular}

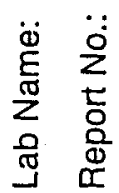


Project: $\quad$ Sr-90 Analysis for Amchitka Island (Batch 6)

Laboratory: RTC

Report \#: AmchitBatchF136

SDG\#: $\quad$ S-A-175-A

Summary of 2 and 3 sigma activities

There were no results for $\mathrm{Sr}-90$ for Batch 6 from the Amchitka Island Project that had a result/uncertainty ratio of 2 or more (uncertainty @ one sigma).

All known sources of uncertainty are included in the uncertainty term. There may be unknown sources of uncertainty that are not accounted for. If the result/uncertainty ratio is more than 3 , we have a degree of confidence that the result is positive (i.e. the result is statistically different than zero). A result with the result/uncertainty ratio between 2 and 3 is the first indication that an isotope may be present and further investigation may be warranted.

As with any good science no single data point is used in important decisions (results need to be reproducible). 
Batch 7

ALPHA 


\begin{tabular}{llll} 
Project Title: & \multicolumn{1}{l}{ Alpha Analysis for Amchitka (Batch 7) } & \\
\cline { 2 - 3 } Lab Name: & RTC & Case No: & NA \\
Report No.: & AmchitBatchF7 & Method Type: & A/B \\
\cline { 2 - 3 } & Spproved SAP No.: NA & SDG No.: & K-AA-198-A
\end{tabular}

\section{SAMPLE NUMBERS}

Customer

Sample ID

\begin{tabular}{|c|}
\hline K-AA-198-A \\
\hline K-AA-200-A \\
\hline K-AA-202-A \\
\hline K-AA-204-A \\
\hline K-AA-207-A \\
\hline K-AA-209-A \\
\hline K-AA-210-A \\
\hline K-AA-211-A \\
\hline
\end{tabular}

K-CC-193-A

K-CC-194-A

K-CC-195-A

K-CC-196-A

K-CC-197-A

K-CC-199-A

$\mathrm{K}-\mathrm{CC}-201-\mathrm{A}$

K-CC-203-A

K-CC-205-A

K-CC-206-A

$\mathrm{K}-\mathrm{CC}-208-\mathrm{A}$

K-CC-212-A
Lab Sample

ID

02MN-06-A

02MN-08-A

$02 M N-10-A$

$02 M N-12-A$

$02 M N-15-A$

$02 M N-17-A$

$02 M N-18-A$

$02 M N-19-A$

$02 M N-01-A$

$02 M N-02-A$

$02 M N-03-A$

02MN-04-A

$02 M N-05-A$

02MN-07-A

02MN-09-A

$02 M N-11-A$

$02 M N-13-A$

$02 M N-14-A$

$02 M N-16-A$

$02 \mathrm{MN}-20-\mathrm{A}$

Comments:

Release of the data contained in this data package has been authorized by the laboratory manager or the manager's designee, as verified by the following signature:

Signature: Phn Cratrmerye

Title: $\quad$ Technical Leader
Name: J.G. Eisenmenger

Date: 05/26/2005 
)

$\sim \triangleq$

के हे

范

\begin{tabular}{|c|c|c|c|c|c|c|c|c|c|c|c|c|c|c|c|c|c|c|c|c|c|}
\hline $\begin{array}{l}\mathrm{U} \\
0 \\
\end{array}$ & & & & & & & & & & & & & & & & & & & & & \\
\hline 卆 & $\begin{array}{l}n \\
0 \\
1 \\
1 \\
0 \\
0 \\
\vdots \\
\dot{n} \\
+\end{array}$ & $\begin{array}{l}n \\
0 \\
0 \\
1 \\
w \\
a \\
0 \\
\dot{m} \\
+\end{array}$ & $\begin{array}{l}n \\
0 \\
1 \\
1 \\
1 \\
2 \\
0 \\
0 \\
0 \\
+ \\
+\end{array}$ & 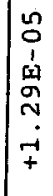 & $\begin{array}{l}n \\
0 \\
0 \\
1 \\
4 \\
-1 \\
9 \\
0 \\
+ \\
+\end{array}$ & $\begin{array}{l}n \\
0 \\
1 \\
1 \\
0 \\
6 \\
6 \\
\dot{y} \\
+\end{array}$ & $\begin{array}{c}n \\
0 \\
1 \\
6 \\
9 \\
\vdots \\
\vdots \\
0 \\
0 \\
+\end{array}$ & 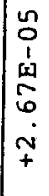 & $\begin{array}{l}n \\
0 \\
1 \\
1 \\
m \\
0 \\
0 \\
0 \\
+ \\
+\end{array}$ & $\begin{array}{l}n \\
0 \\
1 \\
1 \\
0 \\
0 \\
r \\
\dot{m} \\
+\end{array}$ & $\begin{array}{l}n \\
0 \\
1 \\
1 \\
0 \\
5 \\
n \\
0 \\
+ \\
+\end{array}$ & $\begin{array}{l}n \\
0 \\
1 \\
1 \\
0 \\
0 \\
0 \\
\dot{m} \\
+ \\
+\end{array}$ & $\begin{array}{l}n \\
0 \\
1 \\
0 \\
0 \\
0 \\
0 \\
\sim \\
+\end{array}$ & $\begin{array}{l}n \\
0 \\
1 \\
1 \\
0 \\
0 \\
\sim \\
\dot{p} \\
+\end{array}$ & 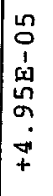 & $\mid \begin{array}{l}n \\
0 \\
1 \\
1 \\
0 \\
\sim 1 \\
\sim \\
0 \\
+ \\
+\end{array}$ & 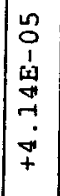 & $\mid \begin{array}{c}n \\
0 \\
1 \\
1 \\
9 \\
w \\
m \\
0 \\
+ \\
+ \\
\end{array}$ & $\begin{array}{l}n \\
0 \\
1 \\
1 \\
1 \\
\infty \\
\infty \\
\vdots \\
+ \\
+\end{array}$ & $\begin{array}{l}n \\
0 \\
1 \\
1 \\
0 \\
0 \\
\vdots \\
+ \\
+\end{array}$ & $\begin{array}{l}n \\
0 \\
1 \\
1 \\
1 \\
1 \\
\alpha \\
0 \\
0 \\
+\end{array}$ \\
\hline 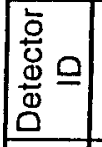 & o & ${ }^{2}$ & o & $\vec{I}$ & 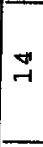 & 우 & on & 농 & \& & $\stackrel{\leftrightarrow}{\circ}$ & $\underset{-1}{\infty}$ & $\underset{-1}{0}$ & 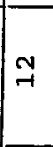 & $1^{n}$ & $\left.\right|^{\infty}$ & ${ }_{a}^{\infty}$ & $\infty_{0}^{\infty}$ & 옥 & 욱 & $\stackrel{\sim}{\sim}$ & $\left.\right|_{0} ^{\infty}$ \\
\hline$\frac{E}{\Phi} \frac{0}{\Phi}$ & $\begin{array}{l}a \\
a \\
a\end{array}$ & के & $\begin{array}{l}\sigma \\
\sigma \\
\sigma\end{array}$ & $\begin{array}{l}9 \\
0 \\
\infty\end{array}$ & $\begin{array}{l}a \\
\vdots \\
\infty\end{array}$ & $\begin{array}{l}0 \\
\alpha \\
\sigma\end{array}$ & $\begin{array}{l}\sigma \\
\sigma \\
\sigma\end{array}$ & $\begin{array}{l}n \\
0 \\
0 \\
0 \\
-1\end{array}$ & $\begin{array}{l}n \\
0 \\
0 \\
0 \\
-1\end{array}$ & $\begin{array}{l}n \\
0 \\
0 \\
0 \\
-1\end{array}$ & $\begin{array}{l}\infty \\
\vdots \\
\vdots \\
\infty\end{array}$ & $\begin{array}{l}\infty \\
\infty \\
\infty \\
\infty\end{array}$ & $\begin{array}{c}0 \\
\dot{y} \\
0 \\
r-1\end{array}$ & $\begin{array}{l}n \\
0 \\
0 \\
0\end{array}$ & \begin{tabular}{|l|} 
\\
0 \\
0
\end{tabular} & $\begin{array}{l}0 \\
0 \\
0 \\
0\end{array}$ & $\begin{array}{l}\overrightarrow{0} \\
\infty \\
0 \\
0\end{array}$ & $\begin{array}{l}0 \\
\dot{0} \\
0\end{array}$ & $\begin{array}{l}0 \\
0 \\
0 \\
0\end{array}$ & $\begin{array}{l}\infty \\
0 \\
0 \\
\end{array}$ & $\begin{array}{l}\overrightarrow{0} \\
\infty \\
0\end{array}$ \\
\hline 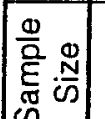 & $\stackrel{\pi}{\pi}$ & $\tilde{r}$ & $\stackrel{\sim}{\sim}$ & חָ & $\stackrel{n}{\pi}$ & जั & $\stackrel{\sim}{\pi}$ & 年 & $\stackrel{\pi}{\pi}$ & $\underset{\sim}{\sim}$ & ${ }_{-1}^{n}$ & $\stackrel{n}{\sim}$ & $\bar{\sim}$ & $\stackrel{\sim}{\sim}$ & In & $\bar{\sim}$ & $\stackrel{\Omega}{\sim}$ & $\stackrel{\Omega}{\rightarrow}$ & $\underset{-1}{n}$ & $\stackrel{\sim}{\sim}$ & $\stackrel{\cong}{\leadsto}$ \\
\hline
\end{tabular}

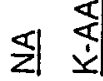

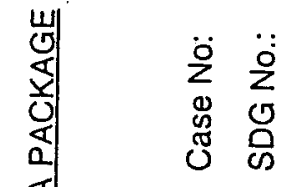

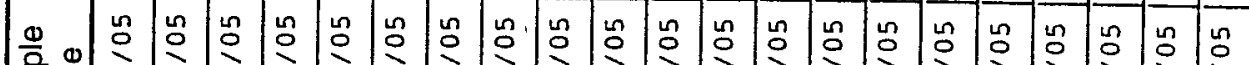
ल

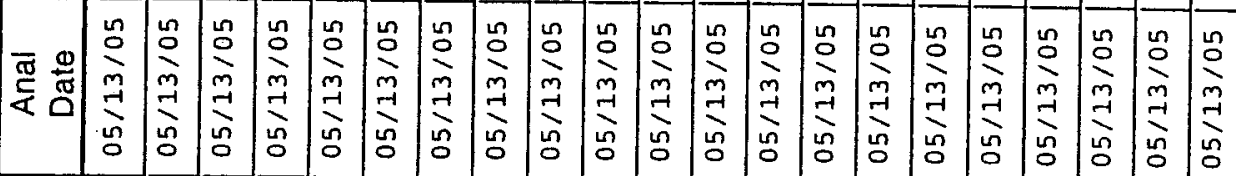

$: \frac{n}{3}$

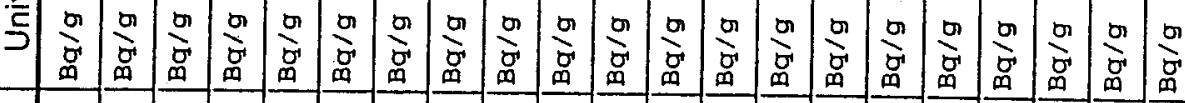

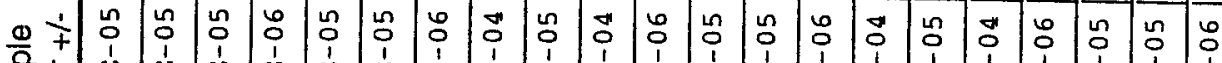

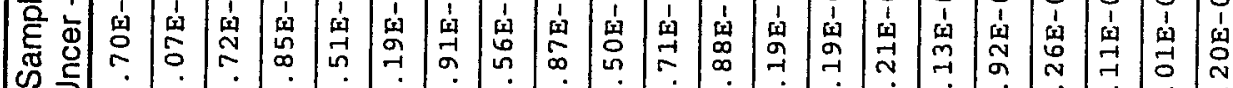

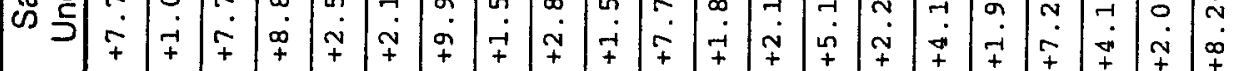

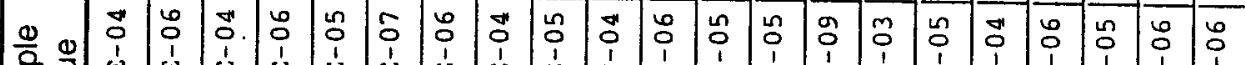

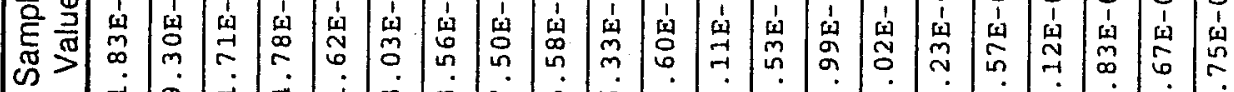

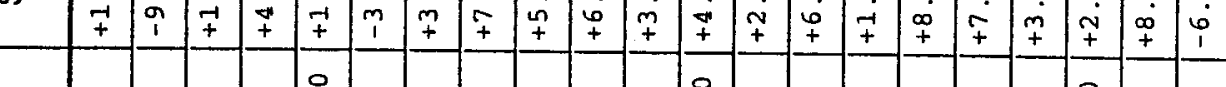

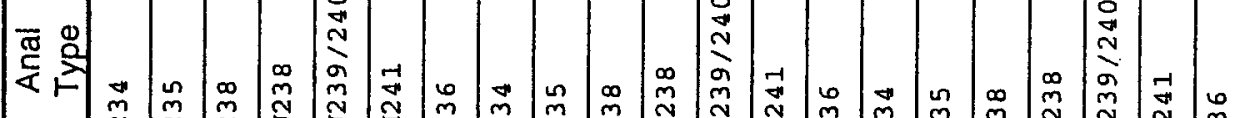

입 홍

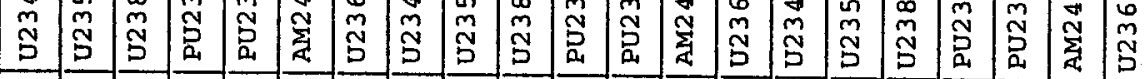

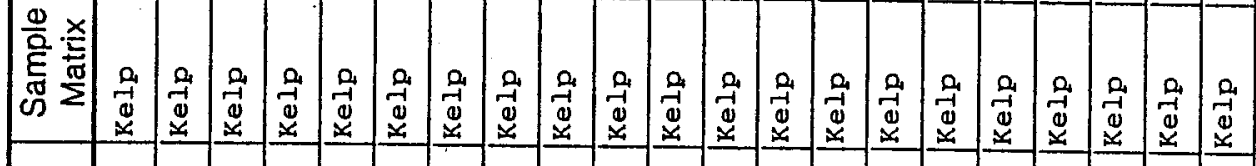
으

而商直

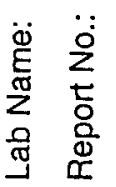

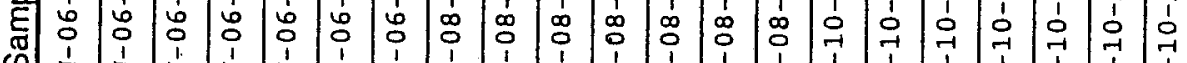

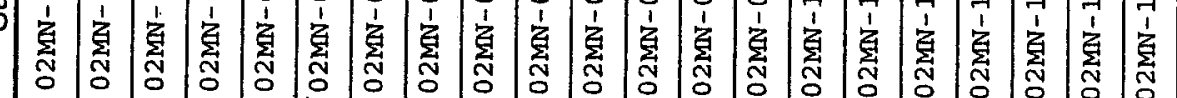

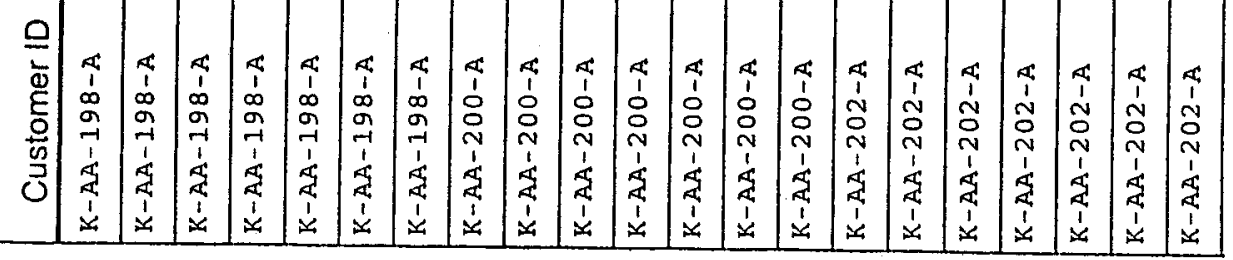




\begin{tabular}{|c|c|c|c|c|c|c|c|c|c|c|c|c|c|c|c|c|c|c|c|c|c|}
\hline $\begin{array}{l}\mathrm{u} \\
\mathrm{O} \\
\mathrm{g}\end{array}$ & & & & & & & & & & & & & & & & & & & & & \\
\hline$\stackrel{\frac{\pi}{2}}{\Sigma}$ & 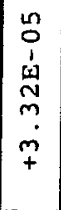 & $\begin{array}{l}n \\
0 \\
1 \\
1 \\
0 \\
\infty \\
-1 \\
\vdots \\
+\end{array}$ & $\begin{array}{l}n \\
0 \\
1 \\
w \\
m \\
n \\
\dot{m} \\
+ \\
+\end{array}$ & 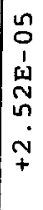 & 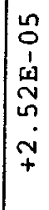 & 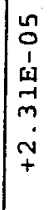 & 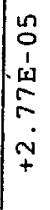 & $\begin{array}{l}n \\
0 \\
0 \\
\omega \\
\omega \\
a \\
-1 \\
+1\end{array}$ & $\begin{array}{l}n \\
0 \\
1 \\
1 \\
\omega \\
\\
\sim \\
\vdots \\
\vdots \\
+\end{array}$ & $\begin{array}{l}n \\
0 \\
1 \\
1 \\
0 \\
\dot{m} \\
m \\
\dot{m} \\
+\end{array}$ & \begin{tabular}{l}
$n$ \\
0 \\
1 \\
1 \\
0 \\
0 \\
\hdashline \\
$\dot{1}$ \\
+ \\
+
\end{tabular} & $\begin{array}{l}n \\
0 \\
1 \\
1 \\
0 \\
0 \\
n \\
\sim \\
+ \\
+\end{array}$ & 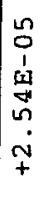 & 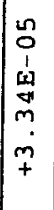 & $\begin{array}{l}n \\
0 \\
1 \\
\vdots \\
0 \\
0 \\
\sim \\
\sim \\
+\end{array}$ & $\begin{array}{l}n \\
0 \\
1 \\
1 \\
0 \\
0 \\
\sim \\
0 \\
+ \\
+\end{array}$ & $\mid \begin{array}{c}n \\
0 \\
1 \\
\omega \\
2 \\
\\
\\
m \\
+ \\
+\end{array}$ & \begin{tabular}{|c|}
$n$ \\
0 \\
1 \\
$\omega$ \\
$n$ \\
$n$ \\
$n$ \\
$\sim$ \\
+ \\
+
\end{tabular} & $\mid \begin{array}{l}n \\
0 \\
1 \\
\text { w } \\
\tilde{y} \\
0 \\
\tilde{p} \\
+\end{array}$ & $\begin{array}{l}n \\
0 \\
1 \\
1 \\
2 \\
\vdots \\
0 \\
\sim \\
+ \\
+\end{array}$ & 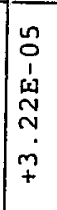 \\
\hline 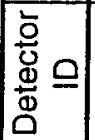 & $\widetilde{O}$ & N & |̃ & $\mathcal{F}$ & 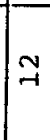 & $\mid-1$ & I & \& & $\ddot{0}$ & \& & $\ln _{\rightarrow \rightarrow}^{n}$ & $\stackrel{\text { n }}{\sim}$ & 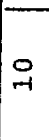 & Ln & ${ }^{m}$ & $\stackrel{m}{o}$ & $\stackrel{m}{o}$ & g & Ig & テ & \% \\
\hline 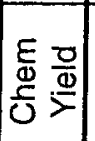 & $\begin{array}{l} \\
\\
0 \\
0 \\
-1\end{array}$ & $\begin{array}{l}+ \\
0 \\
8 \\
0 \\
-1\end{array}$ & 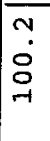 & $\begin{array}{l}\tilde{\alpha} \\
\dot{\infty}\end{array}$ & $\hat{\infty}$ & $\begin{array}{l}0 \\
\infty \\
\infty \\
0\end{array}$ & \begin{tabular}{l}
\multirow{2}{*}{} \\
$\vdots$ \\
$\vdots$ \\
-1
\end{tabular} & ri & 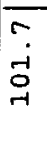 & & $\begin{array}{l}a \\
\infty \\
a\end{array}$ & $\begin{array}{l}\sigma \\
0 \\
\infty \\
\sigma\end{array}$ & $\begin{array}{l}0 \\
\dot{m} \\
\stackrel{0}{0} \\
\stackrel{-1}{2}\end{array}$ & 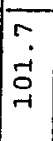 & $\begin{array}{l}9 \\
\ddot{\sim} \\
\tilde{y} \\
-1\end{array}$ & $\begin{array}{c}\tilde{\sigma} \\
\tilde{y} \\
0 \\
-1\end{array}$ & 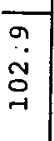 & $\begin{array}{l}a \\
i \\
\infty\end{array}$ & $\begin{array}{l}9 \\
\therefore \\
\infty\end{array}$ & $\begin{array}{l}\tilde{r} \\
\dot{0} \\
0 \\
0\end{array}$ & 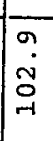 \\
\hline 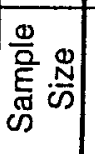 & $\stackrel{n}{7}$ & $\underset{-1}{\mathscr{N}}$ & $\leadsto$ & $\stackrel{\overbrace{}}{-1}$ & $\stackrel{\sim}{\sim}$ & $\stackrel{n}{-1}$ & $\stackrel{n}{r}$ & $\stackrel{n}{\rightarrow}$ & $\stackrel{n}{r}$ & $\stackrel{n}{7}$ & $\stackrel{\sim}{\sigma}$ & $\stackrel{n}{r}$ & $\stackrel{4}{\neg}$ & ${ }_{\tilde{H}}^{n}$ & 4 & $\stackrel{n}{\rightarrow}$ & $\bar{n}$ & $\underset{\sim}{n}$ & $\begin{array}{l}5 \\
\rightarrow\end{array}$ & $\bar{n}$ & $\rightarrow$ \\
\hline
\end{tabular}

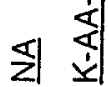

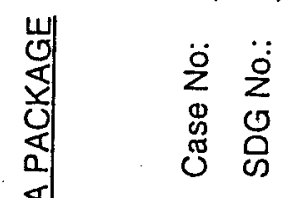

言 先

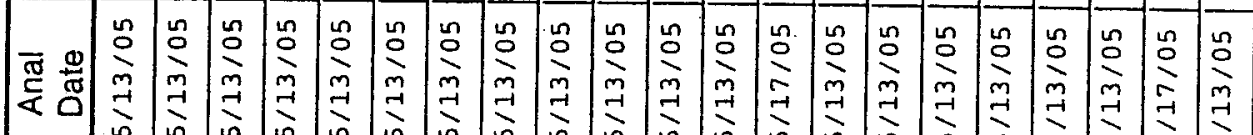

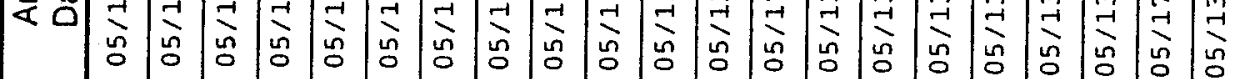

:

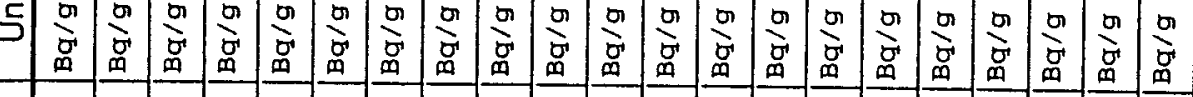
0

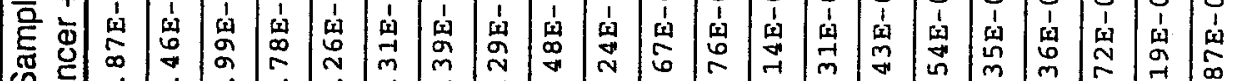
ळ 气

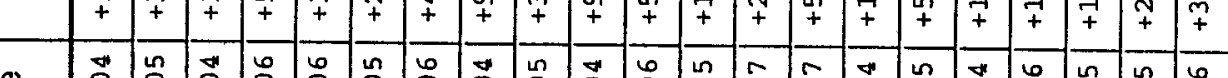

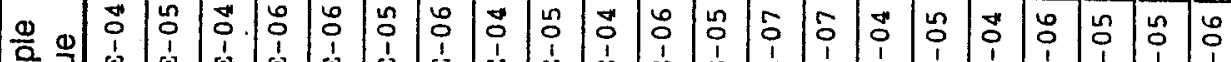

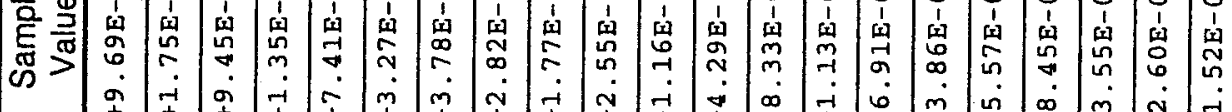

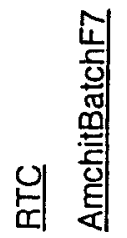

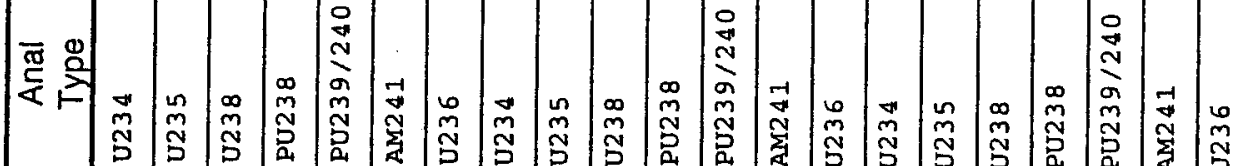

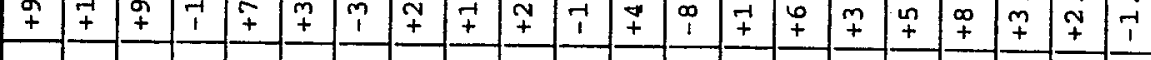
$\stackrel{\Phi}{x}$

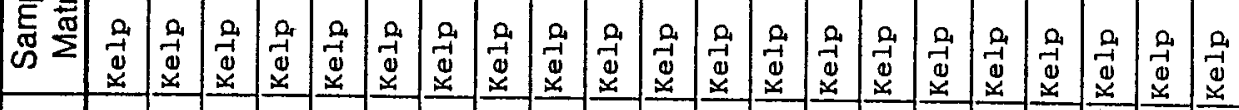

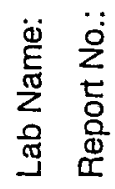

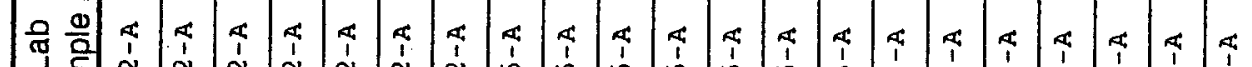

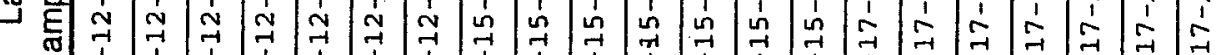

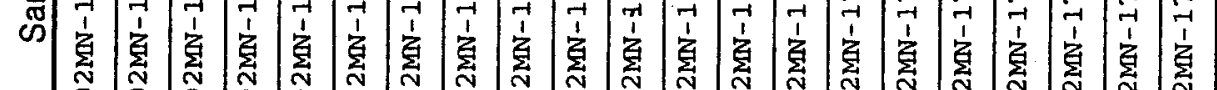

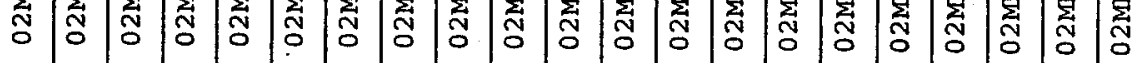




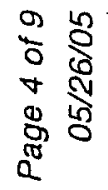

\begin{tabular}{|c|c|c|c|c|c|c|c|c|c|c|c|c|c|c|c|c|c|c|c|c|c|}
\hline $\begin{array}{l} \\
\\
\end{array}$ & & & & & & & & & & & & & & & & & & & & & \\
\hline 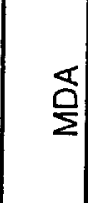 & \begin{tabular}{l}
$\overrightarrow{0}$ \\
1 \\
1 \\
9 \\
0 \\
\multirow{1}{*}{} \\
-1 \\
+
\end{tabular} & 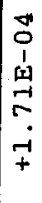 & $\begin{array}{l}\overrightarrow{0} \\
0 \\
1 \\
1 \\
0 \\
\text { N } \\
-1 \\
+\end{array}$ & $\begin{array}{l}\text { In } \\
0 \\
1 \\
1 \\
\text { m } \\
\sim \\
\infty \\
0 \\
\infty \\
+ \\
+\end{array}$ & 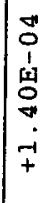 & $\begin{array}{l}n \\
0 \\
0 \\
1 \\
\omega \\
\sim \\
\sim \\
0 \\
\infty \\
+ \\
+\end{array}$ & $\begin{array}{l}0 \\
0 \\
1 \\
1 \\
\vdots \\
0 \\
0 \\
0 \\
-1 \\
+\end{array}$ & $\begin{array}{l}n \\
0 \\
1 \\
1 \\
01 \\
0 \\
\vdots \\
\vdots \\
-i \\
+\end{array}$ & $\begin{array}{l}n \\
0 \\
i \\
i \\
0 \\
0 \\
0 \\
0 \\
\dot{p} \\
+ \\
+\end{array}$ & $\begin{array}{l}n \\
0 \\
1 \\
\mathfrak{w} \\
\tilde{N} \\
m \\
\dot{m} \\
+\end{array}$ & 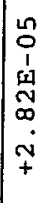 & $\begin{array}{l}n \\
0 \\
1 \\
1 \\
1 \\
0 \\
0 \\
0 \\
m \\
+ \\
+\end{array}$ & $\begin{array}{l}n \\
0 \\
1 \\
1 \\
1 \\
\tilde{a} \\
0 \\
\dot{-} \\
\mp \\
+\end{array}$ & $\begin{array}{c}n \\
0 \\
1 \\
1 \\
w \\
\infty \\
\sim \\
\sim \\
+ \\
+ \\
\end{array}$ & $\begin{array}{l}n \\
0 \\
1 \\
1 \\
\\
0 \\
0 \\
+ \\
+\end{array}$ & $\begin{array}{l}n \\
0 \\
1 \\
1 \\
0 \\
0 \\
\infty \\
n \\
+\end{array}$ & $\begin{array}{l}n \\
0 \\
1 \\
1 \\
0 \\
0 \\
0 \\
0 \\
+ \\
+\end{array}$ & $\begin{array}{l}n \\
0 \\
1 \\
\omega \\
-1 \\
\\
\tilde{y} \\
+ \\
+\end{array}$ & $\begin{array}{l}n \\
0 \\
1 \\
1 \\
0 \\
0 \\
0 \\
0 \\
0 \\
+\end{array}$ & $\begin{array}{l}n \\
0 \\
1 \\
0 \\
0 \\
0 \\
0 \\
\sim \\
+\end{array}$ & 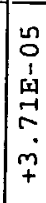 \\
\hline 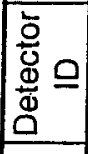 & $\ddot{0}$ & 8 & 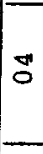 & 19 & ㄱ. & $\rightarrow$ & Dే & 18 & Is & $\approx$ & $\exists$ & $\exists$ & 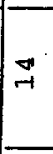 & 里 & ma & ${ }^{m}$ & $\stackrel{m}{\sigma}$ & I & Ig & $\underset{\sim}{m}$ & o \\
\hline & ? & $m$ & m & . & 0 & $\because$ & $\bar{m}$ & $\because$ & $\square$ & $\because$ & $\because$ & 0 & $\because$ & $\because$ & $\because$ & v & $\bar{\Upsilon}$ & ? & ?. & ? & \\
\hline
\end{tabular}

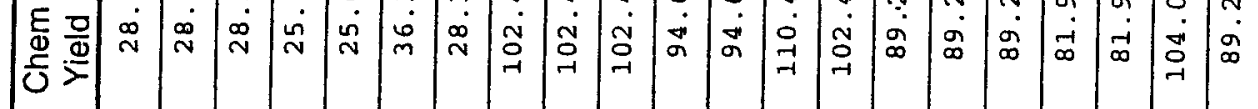

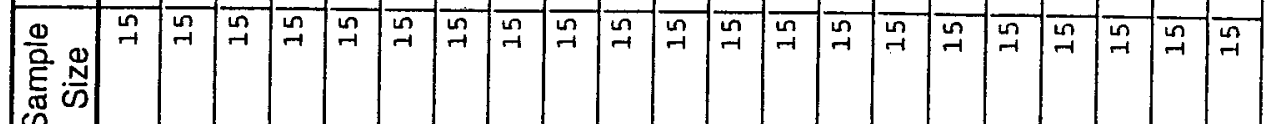

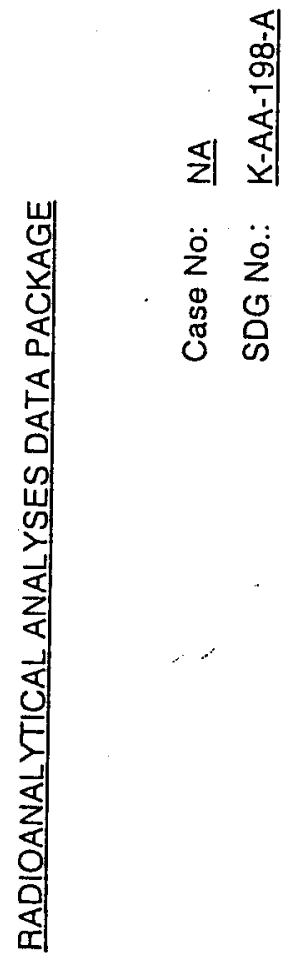

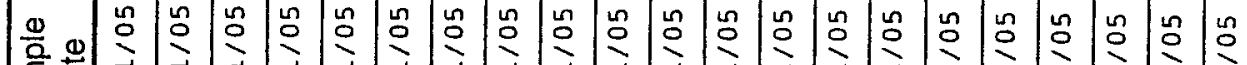

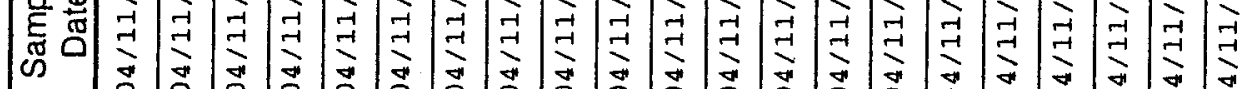

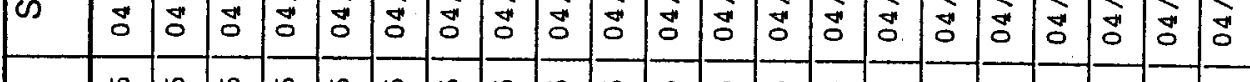

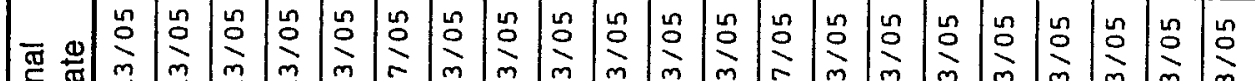

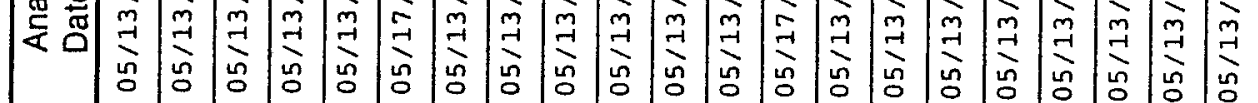

a

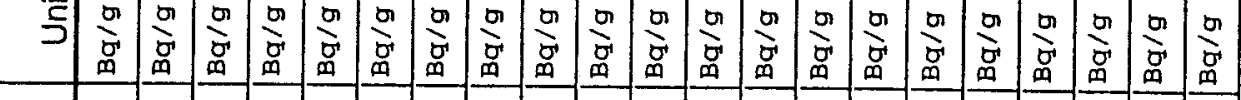

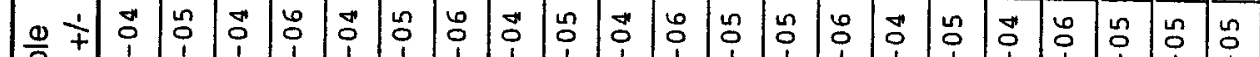

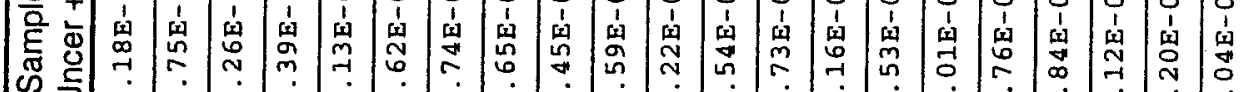

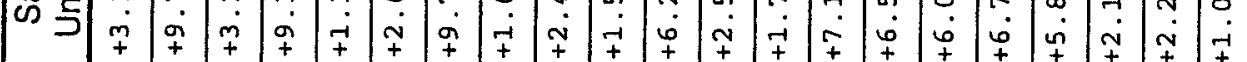

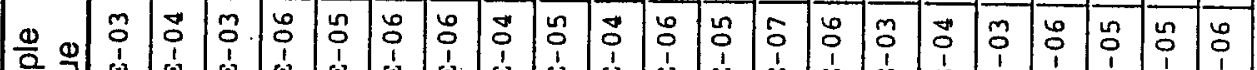

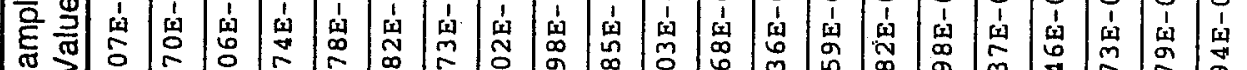

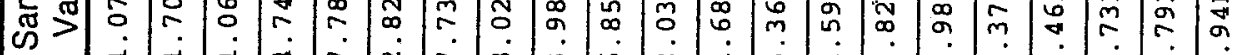

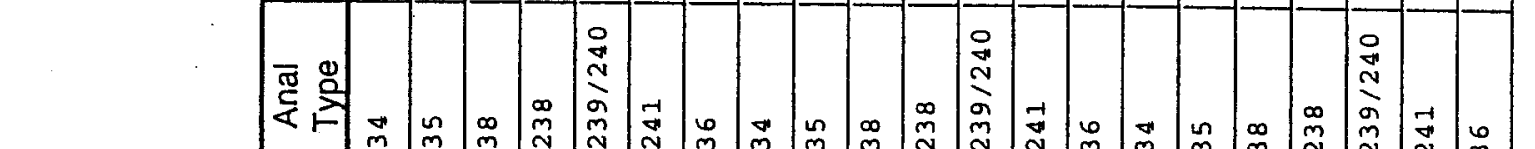

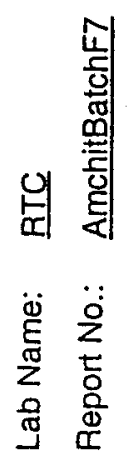

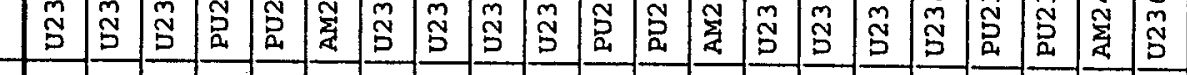
음

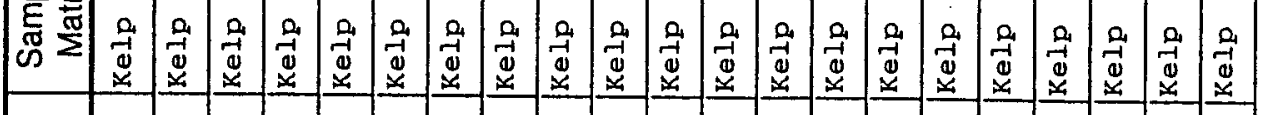

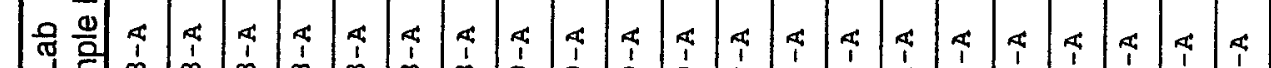

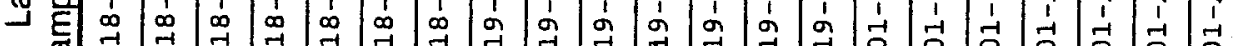

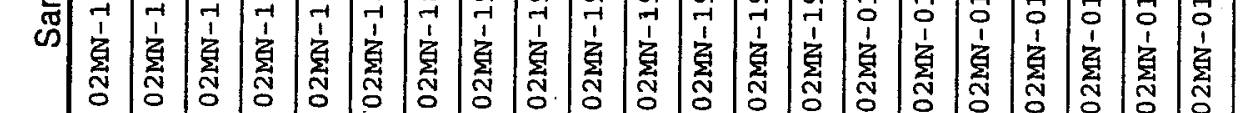


? in $\mathrm{v}$ के है a

学弃

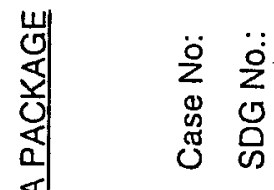
임

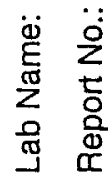

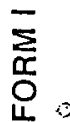

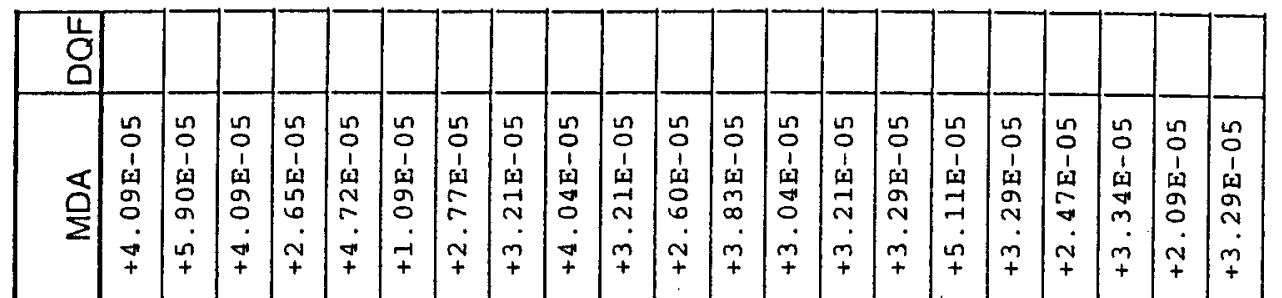

递 口

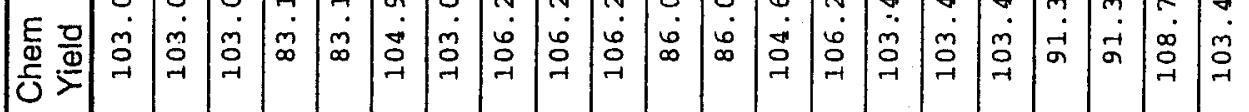
高党 离

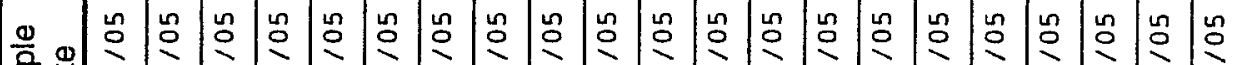
皇 可

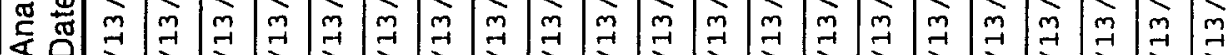

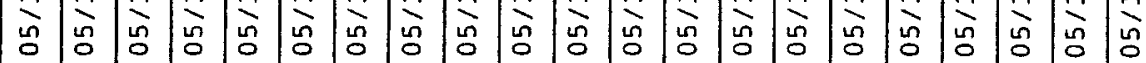

ca

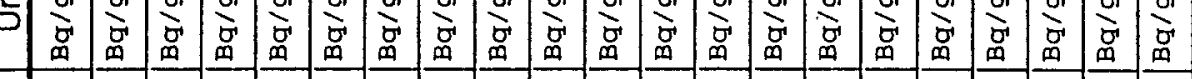

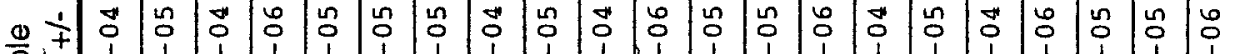
ह气 «

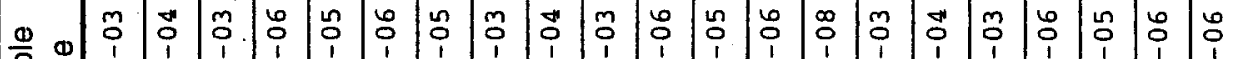

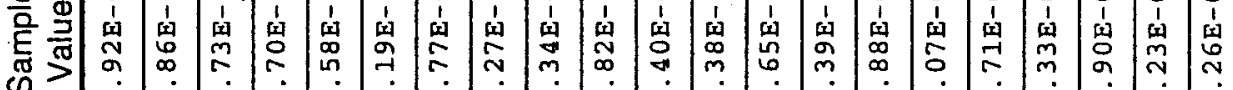

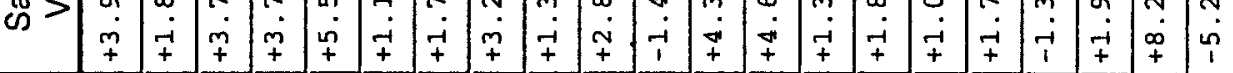

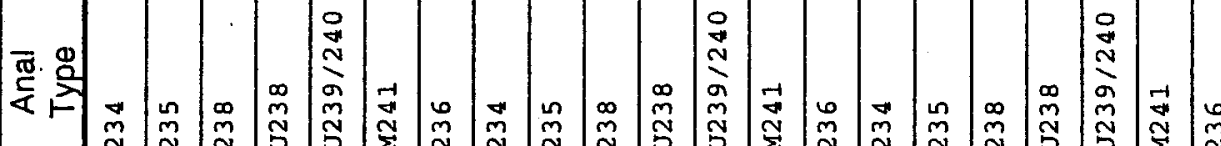

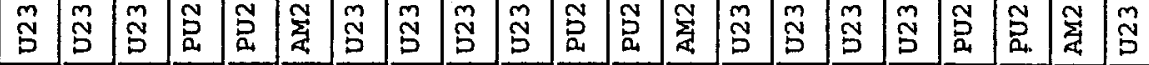
음 $\cdot$ 즌

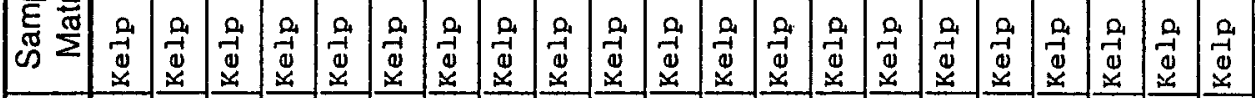
으

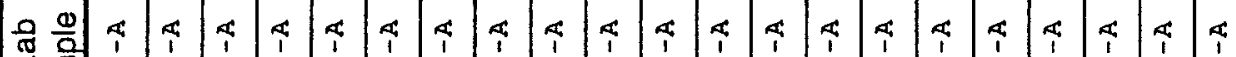

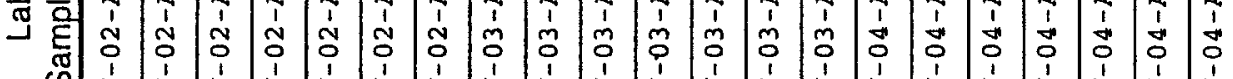
心

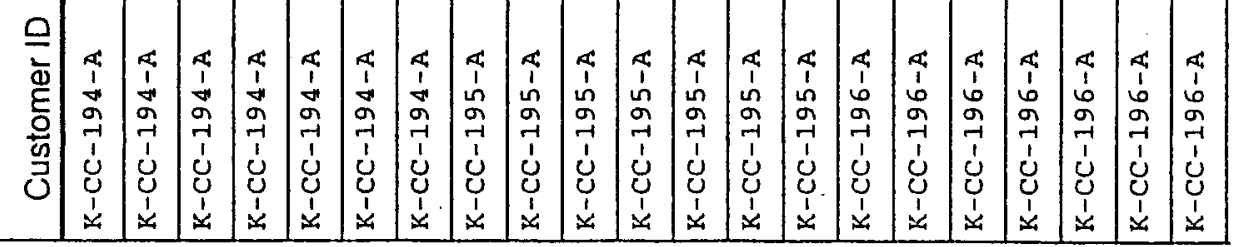

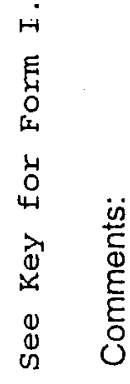




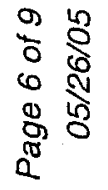

\begin{tabular}{|c|c|c|c|c|c|c|c|c|c|c|c|c|c|c|c|c|c|c|c|c|c|}
\hline $\begin{array}{l}\mathrm{u} \\
\mathrm{O} \\
\mathrm{a}\end{array}$ & & & & & & & & & & & & & & & & & & & & & \\
\hline$\stackrel{\nwarrow}{\mathrm{Q}}$ & \begin{tabular}{l}
$n$ \\
0 \\
1 \\
1 \\
0 \\
0 \\
\multirow{1}{0}{} \\
$\dot{n}$ \\
+
\end{tabular} & $\begin{array}{l}n \\
0 \\
1 \\
1 \\
0 \\
0 \\
o \\
\tilde{m} \\
+ \\
+\end{array}$ & $\begin{array}{l}n \\
0 \\
1 \\
1 \\
4 \\
\\
\sim \\
0 \\
+\end{array}$ & 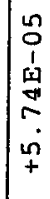 & $\begin{array}{l}n \\
0 \\
1 \\
1 \\
1 \\
\vdots \\
0 \\
0 \\
0 \\
+\end{array}$ & \begin{tabular}{l}
$n$ \\
0 \\
$i$ \\
1 \\
\\
\multirow{2}{*}{} \\
- \\
+ \\
+
\end{tabular} & 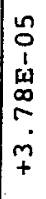 & $\begin{array}{l}\text { in } \\
0 \\
1 \\
0 \\
0 \\
0 \\
\vdots \\
0 \\
+\end{array}$ & $\begin{array}{l}n \\
0 \\
1 \\
0 \\
0 \\
\sigma \\
\sigma \\
0 \\
+1\end{array}$ & $\begin{array}{c}n \\
0 \\
1 \\
1 \\
1 \\
0 \\
\vdots \\
\vdots \\
\vdots \\
+ \\
+\end{array}$ & 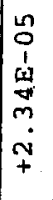 & $\begin{array}{l}n \\
0 \\
1 \\
0 \\
0 \\
0 \\
\stackrel{1}{0} \\
\dot{m} \\
+\end{array}$ & $\begin{array}{l}n \\
0 \\
1 \\
1 \\
4 \\
0 \\
\infty \\
0 \\
\sim \\
+\end{array}$ & $\begin{array}{l}n \\
0 \\
1 \\
1 \\
1 \\
0 \\
o \\
\dot{n} \\
+\end{array}$ & $\begin{array}{l}n \\
0 \\
1 \\
1 \\
w \\
N \\
\sim \\
m \\
+ \\
+\end{array}$ & $\begin{array}{l}n \\
0 \\
1 \\
1 \\
1 \\
0 \\
0 \\
\dot{1} \\
+ \\
+\end{array}$ & $\begin{array}{l}n \\
0 \\
i \\
1 \\
w \\
N \\
N \\
m \\
+ \\
+ \\
\end{array}$ & 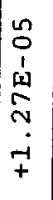 & 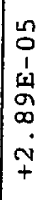 & 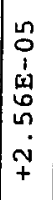 & 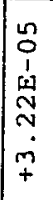 \\
\hline 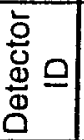 & $\stackrel{\infty}{0}$ & | & $\left.\right|_{0} ^{\infty}$ & $\left.\right|_{0} ^{\infty}$ & $\stackrel{\infty}{\circ}$ & If & $\stackrel{\infty}{\circ}$ & 8 & $\sigma_{0}^{*}$ & 8 & $\stackrel{n}{n}$ & $\stackrel{\sim}{\sim}$ & $\overrightarrow{-1}$ & 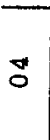 & $\stackrel{\circ}{\circ}$ & \% & : & g & of & $\underset{-7}{m}$ & $1 \%$ \\
\hline
\end{tabular}

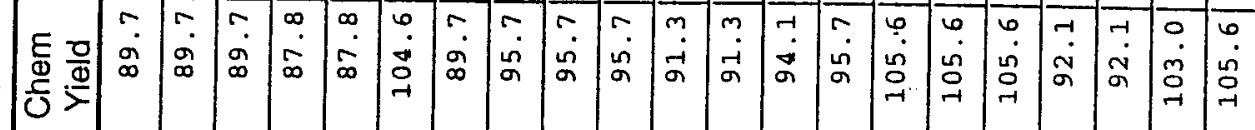

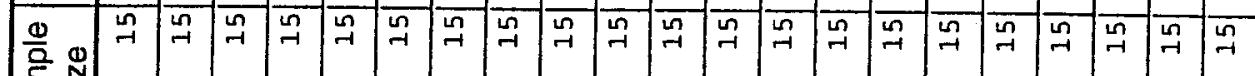

צ|

突

嵌

产恶

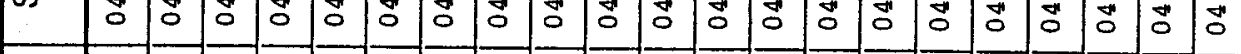

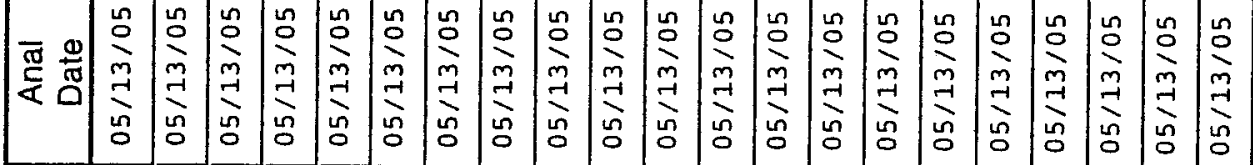

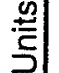

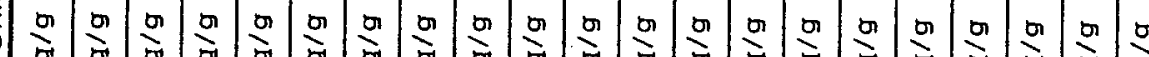
品

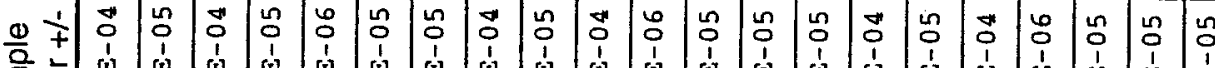

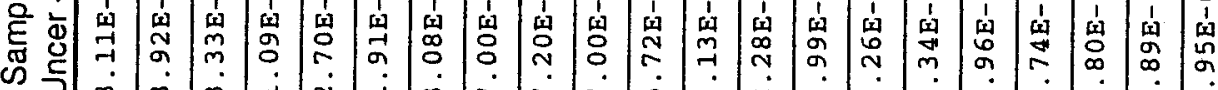

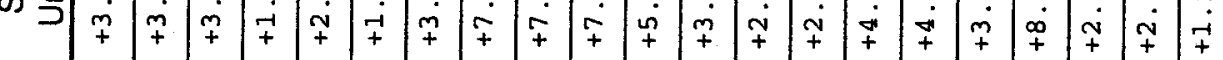

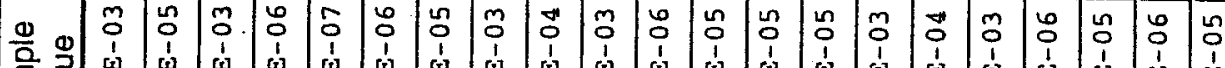

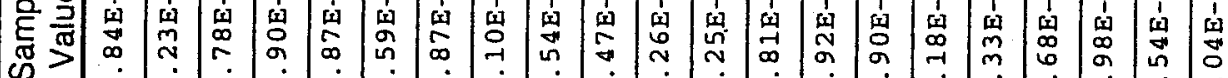

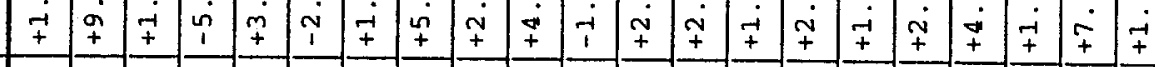

苞罗 n

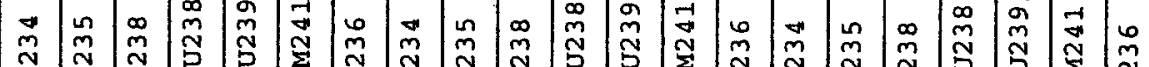

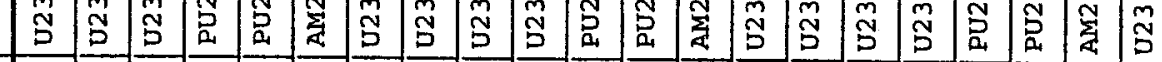

일

응 즌

质 范

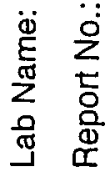

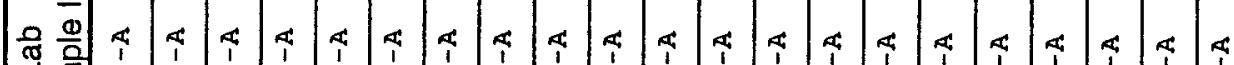

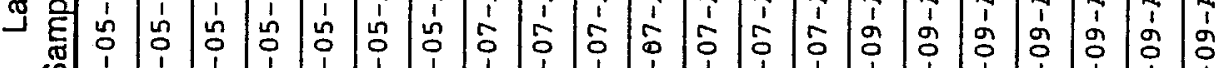

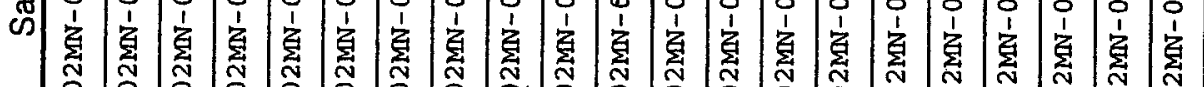
范

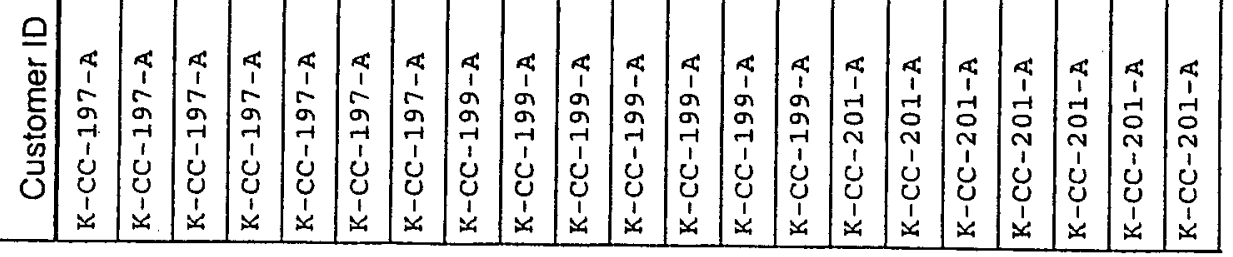




\begin{tabular}{|c|c|c|c|c|c|c|c|c|c|c|c|c|c|c|c|c|c|c|c|c|c|}
\hline $\begin{array}{l} \\
\\
\end{array}$ & & & & & & & & & & & & & & & & & & & & & \\
\hline$\stackrel{\nwarrow}{\stackrel{\Omega}{2}}$ & $\begin{array}{l}n \\
0 \\
1 \\
1 \\
\text { w } \\
m \\
\vdots \\
-1\end{array}$ & $\begin{array}{l}n \\
0 \\
1 \\
w \\
0 \\
0 \\
0 \\
+ \\
+\end{array}$ & 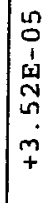 & 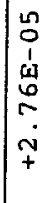 & 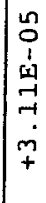 & $\begin{array}{l}n \\
0 \\
1 \\
\omega \\
\vdots \\
\sigma \\
o \\
\sim \\
+\end{array}$ & 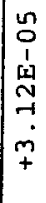 & $\begin{array}{c}n \\
0 \\
i \\
\omega \\
m \\
? \\
m \\
m \\
+\end{array}$ & $\begin{array}{l}n \\
0 \\
1 \\
1 \\
1 \\
0 \\
0 \\
n \\
+ \\
+\end{array}$ & $\begin{array}{l}n \\
0 \\
1 \\
0 \\
-1 \\
6 \\
0 \\
0 \\
+ \\
+\end{array}$ & $\begin{array}{l}n \\
0 \\
1 \\
1 \\
9 \\
\sigma \\
0 \\
0 \\
\dot{m} \\
+\end{array}$ & $\begin{array}{c}n \\
0 \\
1 \\
1 \\
0 \\
0 \\
0 \\
0 \\
+\end{array}$ & $\begin{array}{l}n \\
0 \\
1 \\
1 \\
9 \\
\vdots \\
\vdots \\
\vdots \\
\vdots \\
+\end{array}$ & 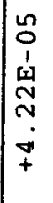 & $\begin{array}{l}n \\
0 \\
1 \\
1 \\
0 \\
0 \\
0 \\
+ \\
+\end{array}$ & $\begin{array}{l}n \\
0 \\
1 \\
1 \\
0 \\
0 \\
m \\
0 \\
0 \\
+\end{array}$ & $\begin{array}{l}n \\
0 \\
1 \\
6 \\
\omega \\
0 \\
0 \\
\vdots \\
+ \\
+\end{array}$ & 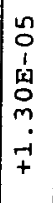 & $\begin{array}{l}n \\
0 \\
1 \\
1 \\
9 \\
m \\
\sigma \\
\dot{m} \\
+ \\
+\end{array}$ & $\begin{array}{l}n \\
0 \\
1 \\
1 \\
9 \\
0 \\
0 \\
\dot{m} \\
+\end{array}$ & $\begin{array}{l}n \\
0 \\
1 \\
1 \\
\text { y } \\
0 \\
0 \\
0 \\
-1 \\
+\end{array}$ \\
\hline 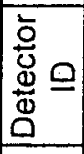 & -1 & 0 & 10 & $\Rightarrow$ & 7 & I & 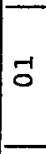 & \% & \% & 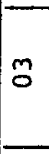 & $\vec{z}$ & $\rightarrow$ & 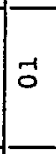 & $\stackrel{m}{0}$ & $\ddot{0}$ & $\overrightarrow{0}$ & $\overrightarrow{0}$ & $\underset{\sim}{-1}$ & 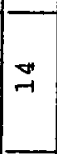 & $\stackrel{m}{o}$ & I \\
\hline 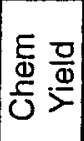 & $\begin{array}{l}m \\
- \\
\exists \\
\exists\end{array}$ & ב. & $\begin{array}{l}m \\
m \\
- \\
-1\end{array}$ & $\left.\right|_{0} ^{\circ}$ & $\left.\right|_{n} ^{\circ}$ & $\begin{array}{l}n \\
\infty \\
0 \\
0 \\
-1\end{array}$ & $\begin{array}{l}m \\
\vec{y} \\
\text { ने }\end{array}$ & m. & $\begin{array}{l}m \\
\dot{m} \\
0 \\
\dot{r}\end{array}$ & $\begin{array}{l}m \\
\dot{m} \\
\dot{0} \\
-1\end{array}$ & $\begin{array}{l}m \\
\dot{m} \\
\sigma\end{array}$ & $\begin{array}{l}m \\
\dot{m} \\
\dot{0}\end{array}$ & $\begin{array}{l}N \\
\vdots \\
0 \\
0\end{array}$ & $\begin{array}{l}3 \\
\dot{0} \\
0 \\
-1\end{array}$ & 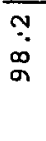 & $\begin{array}{l}n \\
\infty \\
a\end{array}$ & $\vec{n}$ & $\vec{\sigma}$ & $\overrightarrow{5}$ & $\begin{array}{l}\sim \\
\tilde{n} \\
\stackrel{\gamma}{r}\end{array}$ & $\begin{array}{l}\mathfrak{a} \\
\infty \\
\sigma\end{array}$ \\
\hline 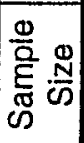 & $\stackrel{\sim}{\sim}$ & $\pi$ & $\tilde{\sim}$ & 品 & $\stackrel{\sim}{\sim}$ & $\tilde{n}_{\rightarrow-1}$ & 尔 & 용 & $\frac{6}{5}$ & $\stackrel{6}{1}$ & $\stackrel{0}{5}$ & $\begin{array}{l}\stackrel{0}{0} \\
\ddot{-}\end{array}$ & $\begin{array}{l}0 \\
\stackrel{0}{-1} \\
-i\end{array}$ & $\begin{array}{l}\infty \\
\sim \\
-1 \\
-1\end{array}$ & $\begin{array}{l}0 \\
\infty \\
\ddot{n} \\
\end{array}$ & $\begin{array}{l}\infty \\
\infty \\
\dot{m} \\
\end{array}$ & $\begin{array}{l}0 \\
\infty \\
\dot{m} \\
m \\
-1\end{array}$ & $\begin{array}{l}0 \\
\infty \\
\dot{m} \\
\dot{r} \\
-1\end{array}$ & $\begin{array}{l}0 \\
\infty \\
\infty \\
m \\
m \\
-1\end{array}$ & & $\begin{array}{l} \\
\infty \\
\dot{n} \\
\dot{\gamma}\end{array}$ \\
\hline
\end{tabular}

긱

\begin{tabular}{|c|c|c|c|c|c|c|c|c|c|c|c|c|c|c|c|c|c|c|c|c|c|}
\hline 荵 & $\frac{n}{0}$ & 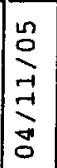 & & $\overrightarrow{0}$ & นn & |n & 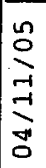 & 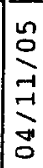 & 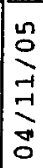 & $\stackrel{\substack{n \\
ٍ}}{ٍ}$ & 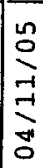 & 㔯 & 告 & 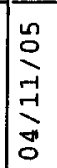 & 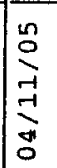 & 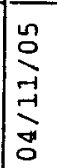 & 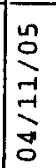 & $\mid \begin{array}{c}n \\
0 \\
0 \\
-1 \\
-1 \\
0 \\
0\end{array}$ & 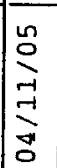 & 告 & 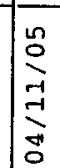 \\
\hline$\pi$ & 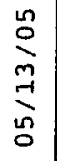 & 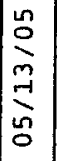 & $\stackrel{0}{9}$ & ${ }_{\text {m }}^{0}$ & ले & ${ }_{\pi}^{m}$ & $m_{-1}$ & $m$ & m & m & $m$ & $\begin{array}{l}n \\
0 \\
m \\
\\
\text { un } \\
0\end{array}$ & 答 & 垈 & 总 & $\stackrel{\substack{0 \\
0}}{\stackrel{m}{9}}$ & 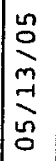 & 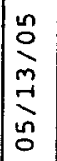 & $\stackrel{\substack{0 \\
0}}{\stackrel{m}{\sigma}}$ & 站 & 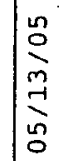 \\
\hline
\end{tabular}

嵌

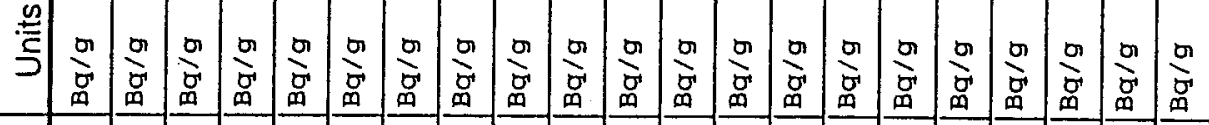

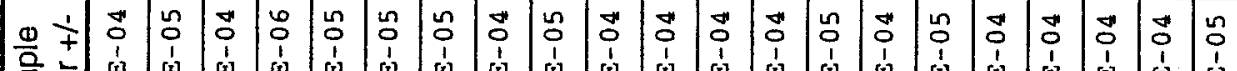

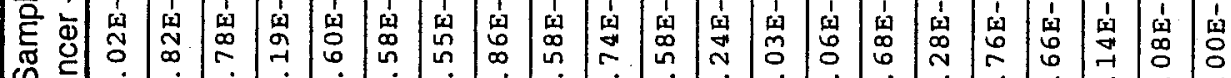

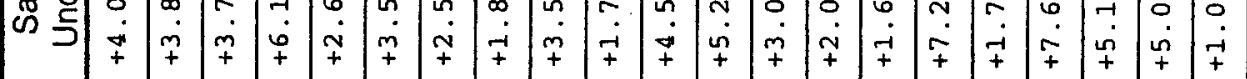

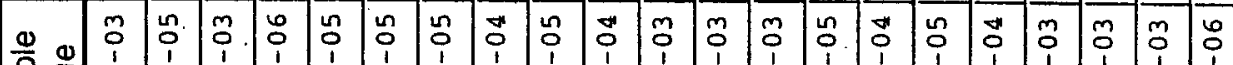

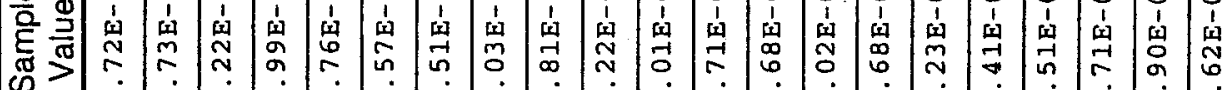

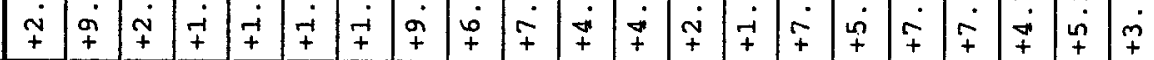

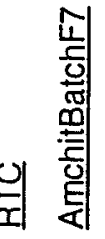

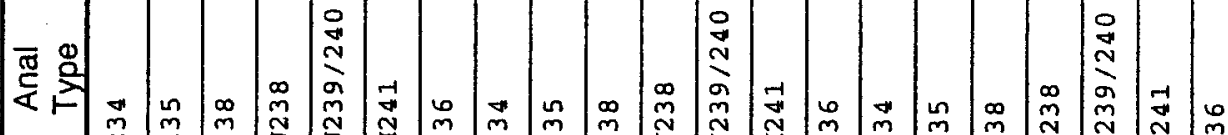

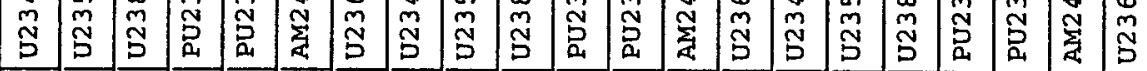

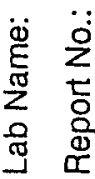

势

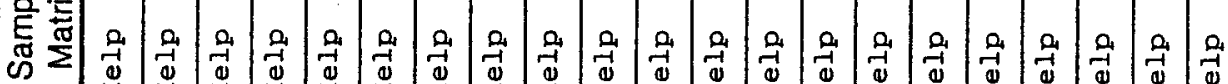

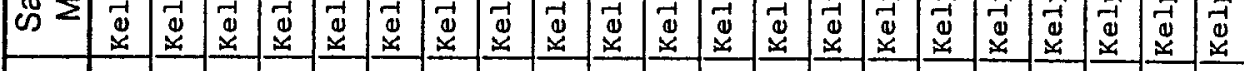
蛋

으

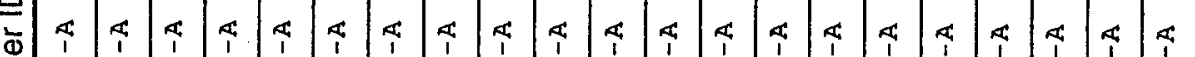

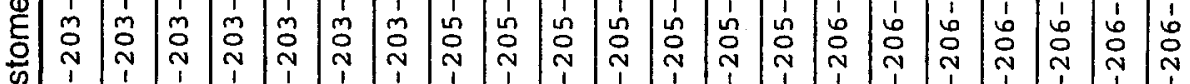

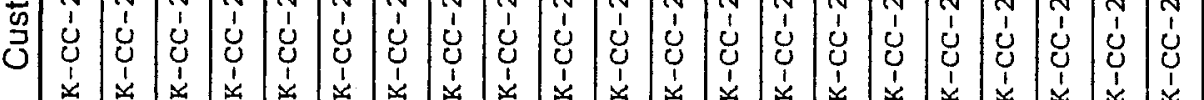




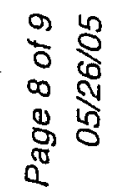

\begin{tabular}{|c|c|c|c|c|c|c|c|c|c|c|c|c|c|c|}
\hline$\frac{1}{0}$ & & & & & & & & & & & & & & \\
\hline 号 & $\mid \begin{array}{l}n \\
0 \\
1 \\
1 \\
0 \\
5 \\
-1 \\
\dot{9} \\
+\end{array}$ & $\mid \begin{array}{l}n \\
0 \\
1 \\
1 \\
01 \\
\vdots \\
7 \\
0 \\
+1 \\
+1\end{array}$ & $\begin{array}{l}n \\
0 \\
1 \\
1 \\
n \\
n \\
? \\
1 \\
m \\
+\end{array}$ & $\begin{array}{l}n \\
0 \\
1 \\
6 \\
n \\
n \\
n \\
4 \\
7 \\
7\end{array}$ & 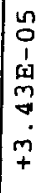 & $\begin{array}{l}n \\
0 \\
1 \\
1 \\
n \\
n \\
-1 \\
\sim \\
+ \\
+\end{array}$ & $\begin{array}{l}n \\
0 \\
0 \\
1 \\
0 \\
n \\
\sim \\
-1 \\
m \\
+\end{array}$ & 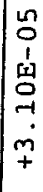 & 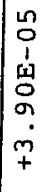 & $\begin{array}{l}n \\
0 \\
1 \\
1 \\
0 \\
-1 \\
\dot{y} \\
+ \\
+\end{array}$ & 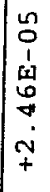 & $\begin{array}{l}n \\
0 \\
1 \\
1 \\
n \\
N \\
m \\
\dot{m} \\
+\end{array}$ & 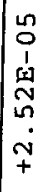 & 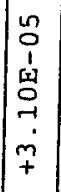 \\
\hline 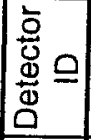 & $\stackrel{\circ}{\circ}$ & $1 \%$ & 10 & 10 & $\stackrel{10}{-1}$ & $F$ & 10 & 10 & 10 & 10 & $\underset{\sim}{\sim}$ & $\approx$ & $\stackrel{n}{n}$ & $1 \%$ \\
\hline $\mid \begin{array}{ll}E & \frac{0}{\Phi} \\
\frac{\Phi}{0} & \frac{0}{\Phi}\end{array}$ & $\begin{array}{l}-1 \\
\infty \\
\infty \\
0 \\
-1\end{array}$ & $\begin{array}{l}-1 \\
\infty \\
0 \\
-1\end{array}$ & . & $\begin{array}{l}0 \\
0 \\
0 \\
0\end{array}$ & $\begin{array}{l}0 \\
0 \\
\infty \\
\infty\end{array}$ & $\begin{array}{l}0 \\
0 \\
0 \\
-1\end{array}$ & $\begin{array}{l}-1 \\
\infty \\
\infty \\
0 \\
-1\end{array}$ & $\begin{array}{l}\infty \\
8 \\
0 \\
-1\end{array}$ & $\begin{array}{l}\infty \\
\infty \\
0 \\
0 \\
-1\end{array}$ & $\begin{array}{l}\infty \\
\infty \\
0 \\
0 \\
-1\end{array}$ & $\begin{array}{r}r \\
-\dot{a}\end{array}$ & ने & $\begin{array}{l}\infty \\
-\dot{0} \\
- \\
-1\end{array}$ & 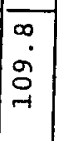 \\
\hline 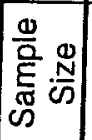 & $\stackrel{-1}{n}$ & In & חָ & $\pi$ & $\bar{\Omega}$ & 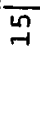 & ฯ & $\stackrel{\sim}{\sim}$ & ก) & $\stackrel{n}{-1}$ & If & $\dddot{\jmath}$ & $\stackrel{\Omega}{\leftrightarrows}$ & $\underset{\sim}{n}$ \\
\hline
\end{tabular}

行题

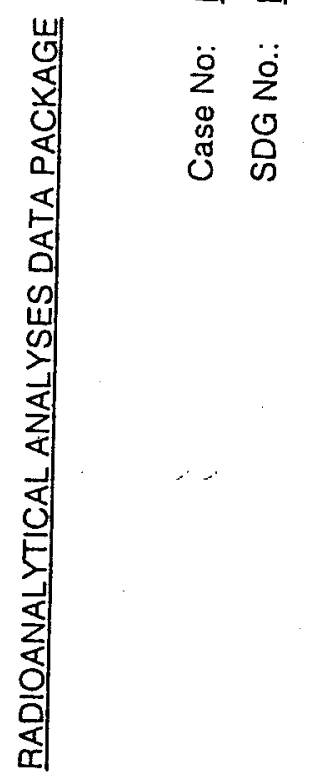

읭

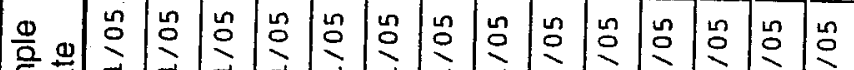

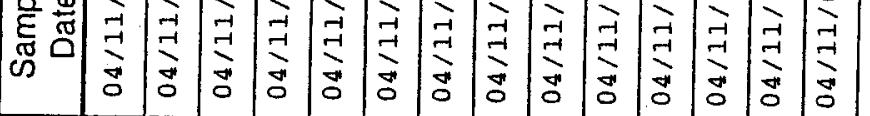

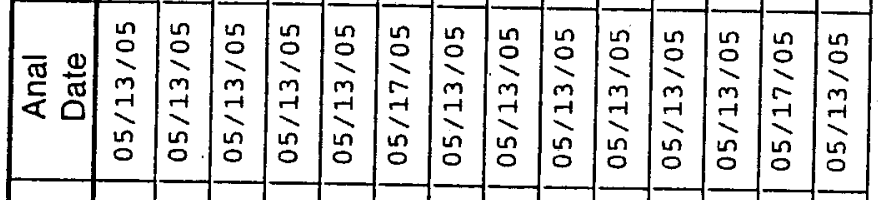

$\stackrel{\text { co }}{\frac{2}{c}}$

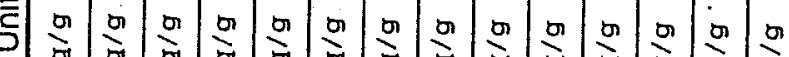

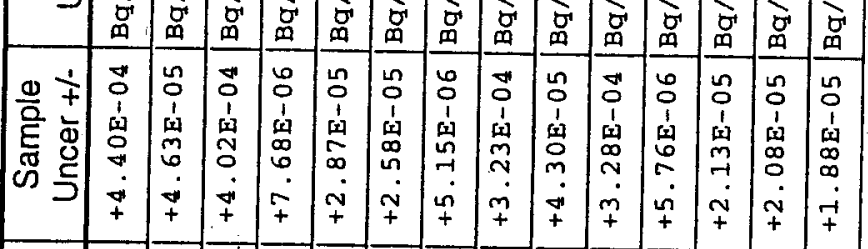

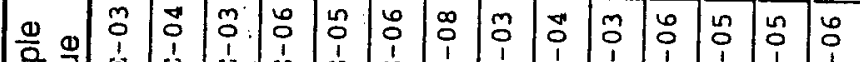

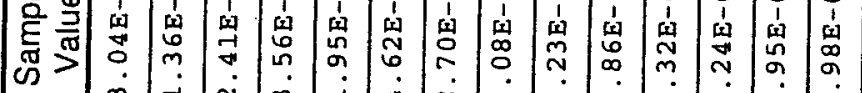

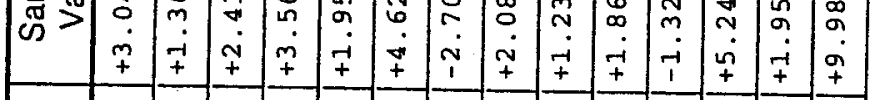

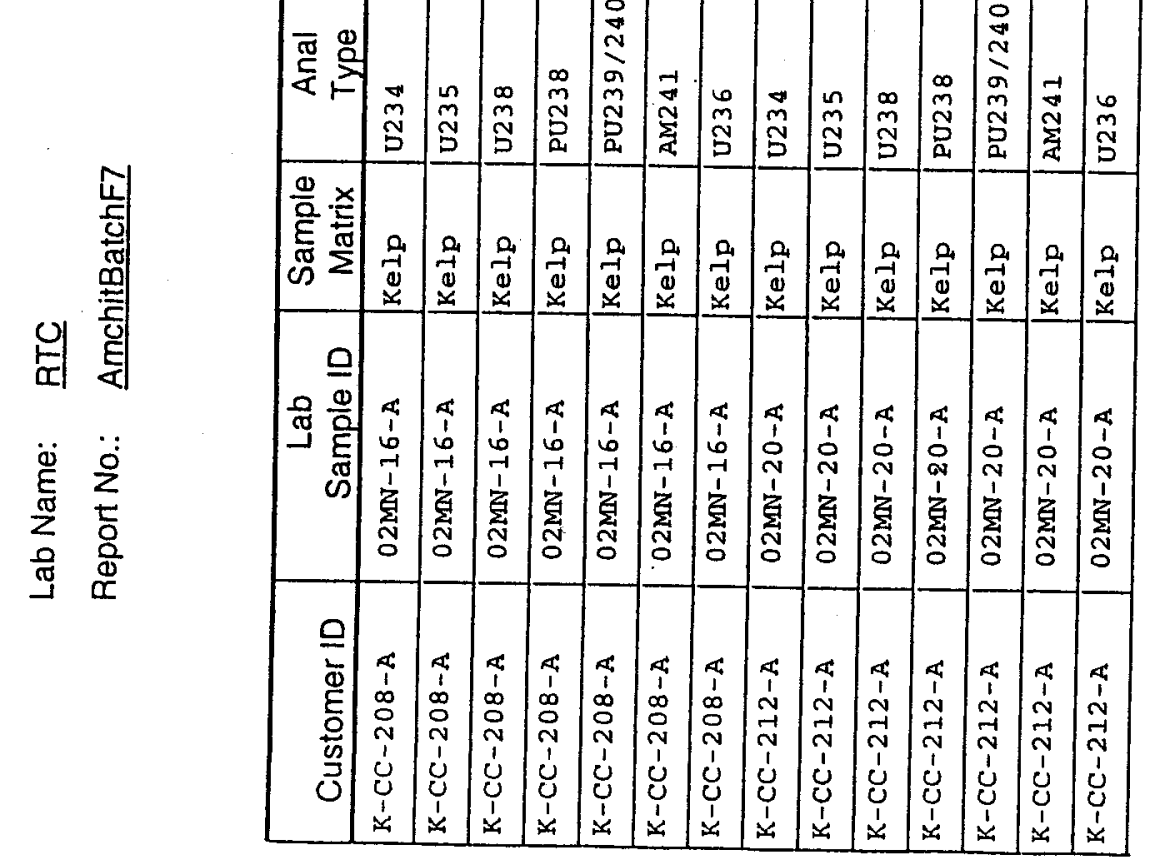




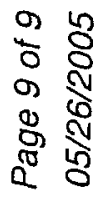

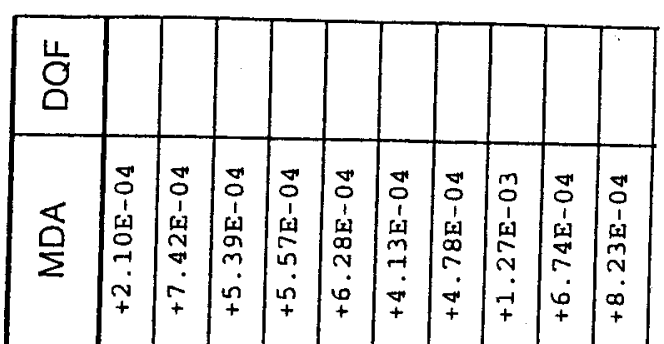

$\square$

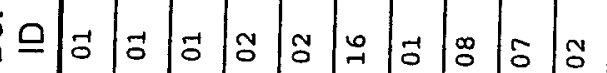

$\varepsilon$ 등

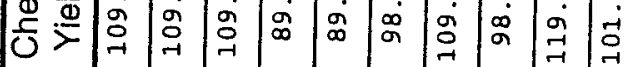

约紊

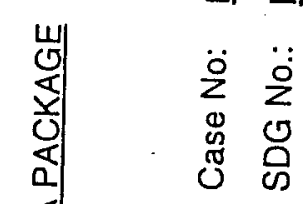

ส

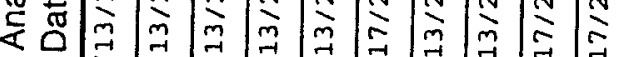

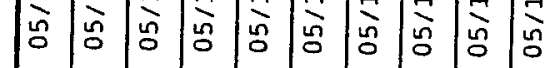

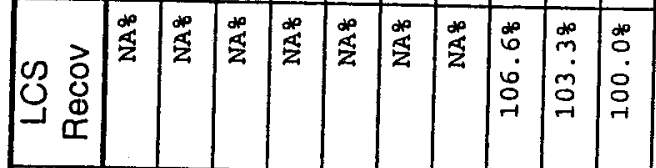

就

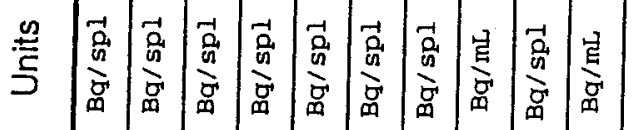

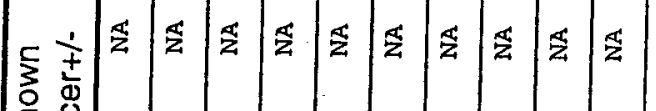

产

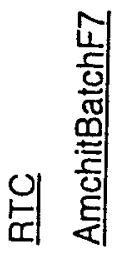

宅 $\frac{0}{5}$

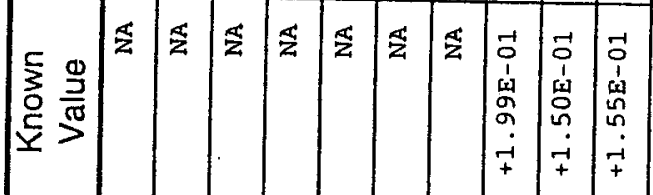

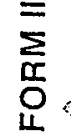

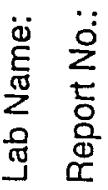

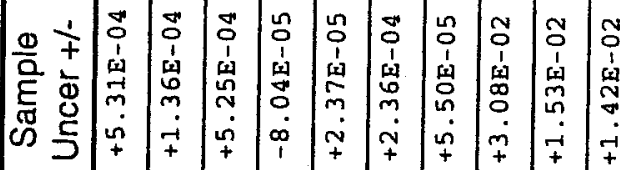

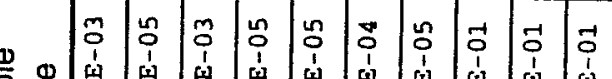

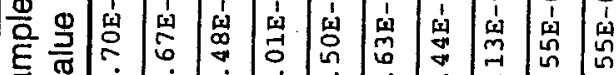

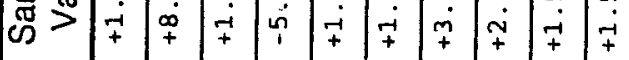

\begin{tabular}{|c|c|c|c|c|c|c|c|c|c|c|}
\hline 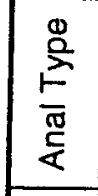 & 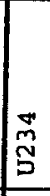 & 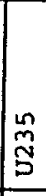 & 离 & 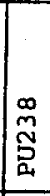 & 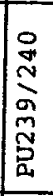 & 趦 & $\begin{array}{l}\stackrel{0}{\sim} \\
\stackrel{S}{S}\end{array}$ & $\stackrel{\infty}{\stackrel{\infty}{S}}$ & 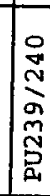 & (\$) \\
\hline$\stackrel{\Phi}{I}$ & 罯 & 盖 & 齐 & 竞 & 亩。 & 总 & 畄 & 包 & $\int_{0}^{\mathscr{y}}$ & 0 \\
\hline $\mid \begin{array}{l}\bar{s} \\
0 \\
0\end{array}$ & 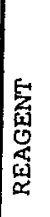 & 焉 & 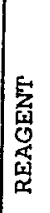 & 点 & $\mid \frac{1}{91}$ & \begin{tabular}{|l}
$\mid$ \\
惫 \\
$\mathbb{4}$
\end{tabular} & 留 & 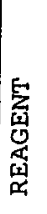 & 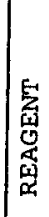 & \\
\hline
\end{tabular}


Project: Alpha Analysis for Amchitka Island (Batch 7)

Laboratory: RTC

Report \#: AmchitBatchF7

SDG\#: $\quad$ K-AA-198-A

\section{Summary of 2 and 3 sigma activities}

Below are the results for U234, U235, U236, U238, Pu238, Pu239/240, and Am241 for Batch 7 from the Amchitka Island Project that had a result/uncertainty ratio of 2 or more (uncertainty@one sigma).

\begin{tabular}{|l|l|l|c|c|c|}
\hline \multicolumn{1}{|c|}{ Customer ID } & \multicolumn{1}{|c|}{ Lab ID } & \multicolumn{1}{c|}{ Isotope } & $\begin{array}{c}\text { Result } \\
\text { Bq/g }\end{array}$ & $\begin{array}{c}\text { Uncertainty } \\
\text { Bq/g }\end{array}$ & $\begin{array}{c}\text { Result/ } \\
\text { Uncertainty }\end{array}$ \\
\hline K-AA-198-A & O2MN-06-A & U234 & $1.83 \mathrm{E}-04$ & $7.70 \mathrm{E}-05$ & 2.4 \\
\hline K-AA-198-A & O2MN-06-A & U238 & $1.71 \mathrm{E}-04$ & $7.72 \mathrm{E}-05$ & 2.2 \\
\hline & & & & & \\
\hline K-AA-200-A & 02MN-08-A & PU239/240 & $4.11 \mathrm{E}-05$ & $1.88 \mathrm{E}-05$ & 2.2 \\
\hline K-AA-200-A & $02 \mathrm{MN}-08-\mathrm{A}$ & $\mathrm{U} 234$ & $7.50 \mathrm{E}-04$ & $1.56 \mathrm{E}-04$ & 4.8 \\
\hline K-AA-200-A & $02 \mathrm{MN}-08-\mathrm{A}$ & $\mathrm{U} 238$ & $6.33 \mathrm{E}-04$ & $1.50 \mathrm{E}-04$ & 4.2 \\
\hline & & & & & \\
\hline K-AA-202-A & $02 \mathrm{MN}-10-\mathrm{A}$ & $\mathrm{U} 234$ & $1.02 \mathrm{E}-03$ & $2.21 \mathrm{E}-04$ & 4.6 \\
\hline K-AA-202-A & $02 \mathrm{MN}-10-\mathrm{A}$ & $\mathrm{U} 235$ & $8.23 \mathrm{E}-05$ & $4.13 \mathrm{E}-05$ & 2.0 \\
\hline K-AA-202-A & $02 \mathrm{MN}-10-\mathrm{A}$ & $\mathrm{U} 238$ & $7.57 \mathrm{E}-04$ & $1.92 \mathrm{E}-04$ & 3.9 \\
\hline & & & & & \\
\hline K-AA-204-A & $02 \mathrm{MN}-12-\mathrm{A}$ & $\mathrm{U} 234$ & $9.69 \mathrm{E}-04$ & $1.87 \mathrm{E}-04$ & 5.2 \\
\hline K-AA-204-A & $02 \mathrm{MN}-12-\mathrm{A}$ & $\mathrm{U} 238$ & $9.45 \mathrm{E}-04$ & $1.99 \mathrm{E}-04$ & 4.7 \\
\hline & & & & & \\
\hline K-AA-207-A & $02 \mathrm{MN}-15-\mathrm{A}$ & $\mathrm{PU} 239 / 240$ & $4.29 \mathrm{E}-05$ & $1.76 \mathrm{E}-05$ & 2.4 \\
\hline K-AA-207-A & $02 \mathrm{MN}-15-\mathrm{A}$ & $\mathrm{U} 234$ & $2.82 \mathrm{E}-04$ & $9.29 \mathrm{E}-05$ & 3.0 \\
\hline K-AA-207-A & $02 \mathrm{MN}-15-\mathrm{A}$ & $\mathrm{U} 238$ & $2.55 \mathrm{E}-04$ & $9.24 \mathrm{E}-05$ & 2.8 \\
\hline & & & & & \\
\hline K-AA-209-A & $02 \mathrm{MN}-17-\mathrm{A}$ & $\mathrm{PU} 239 / 240$ & $3.55 \mathrm{E}-05$ & $1.72 \mathrm{E}-05$ & 2.1 \\
\hline K-AA-209-A & $02 \mathrm{MN}-17-\mathrm{A}$ & $\mathrm{U} 234$ & $6.91 \mathrm{E}-04$ & $1.43 \mathrm{E}-04$ & 4.8 \\
\hline K-AA-209-A & $02 \mathrm{MN}-17-\mathrm{A}$ & $\mathrm{U} 238$ & $5.57 \mathrm{E}-04$ & $1.35 \mathrm{E}-04$ & 4.1 \\
\hline & & & & & \\
\hline K-AA-210-A & $02 \mathrm{MN}-18-\mathrm{A}$ & $\mathrm{U} 234$ & $1.07 \mathrm{E}-03$ & $3.18 \mathrm{E}-04$ & 3.4 \\
\hline K-AA-210-A & $02 \mathrm{MN}-18-\mathrm{A}$ & $\mathrm{U} 238$ & $1.06 \mathrm{E}-03$ & $3.26 \mathrm{E}-04$ & 3.3 \\
\hline & & & & & \\
\hline K-AA-211-A & $02 \mathrm{MN}-19-\mathrm{A}$ & $\mathrm{U} 234$ & $8.02 \mathrm{E}-04$ & $1.65 \mathrm{E}-04$ & 4.9 \\
\hline K-AA-211-A & $02 \mathrm{MN}-19-\mathrm{A}$ & $\mathrm{U} 238$ & $6.85 \mathrm{E}-04$ & $1.59 \mathrm{E}-04$ & 4.3 \\
\hline & & & & & \\
\hline K-CC-193-A & $02 \mathrm{MN}-01-\mathrm{A}$ & $\mathrm{PU} 239 / 240$ & $4.73 \mathrm{E}-05$ & $2.12 \mathrm{E}-05$ & 2.2 \\
\hline K-CC-193-A & $02 \mathrm{MN}-01-\mathrm{A}$ & $\mathrm{U} 234$ & $4.82 \mathrm{E}-03$ & $6.53 \mathrm{E}-04$ & 7.4 \\
\hline K-CC-193-A & $02 \mathrm{MN}-01-\mathrm{A}$ & $\mathrm{U} 235$ & $1.98 \mathrm{E}-04$ & $6.01 \mathrm{E}-05$ & 3.3 \\
\hline K-CC-193-A & $02 \mathrm{MN}-01-\mathrm{A}$ & $\mathrm{U} 238$ & $4.37 \mathrm{E}-03$ & $6.76 \mathrm{E}-04$ & 6.5 \\
\hline
\end{tabular}




\section{Alpha Analysis for Amchitka Island (Batch 7)}

Summary of 2 and 3 sigma activities

page 2

\begin{tabular}{|c|c|c|c|c|c|}
\hline Customer ID & Lab ID & Isotope & $\begin{array}{c}\text { Result } \\
\mathrm{Bq} / \mathrm{g}\end{array}$ & $\begin{array}{c}\text { Uncertainty } \\
\mathrm{Bq} / \mathrm{g}\end{array}$ & $\begin{array}{c}\text { Result/ } \\
\text { Uncertainty }\end{array}$ \\
\hline K-CC-194-A & 02MN-02-A & PU239/240 & $5.58 \mathrm{E}-05$ & $2.44 \mathrm{E}-05$ & 2.3 \\
\hline K-CC-194-A & 02MN-02-A & U234 & $3.92 E-03$ & $5.50 \mathrm{E}-04$ & 7.1 \\
\hline K-CC-194-A & 02MN-02-A & U235 & $1.86 \mathrm{E}-04$ & $5.79 \mathrm{E}-05$ & 3.2 \\
\hline K-CC-194-A & 02MN-02-A & U238 & $3.73 E-03$ & 5.91E-04 & 6.3 \\
\hline K-CC-195-A & $02 \mathrm{MN}-03-\mathrm{A}$ & PU239/240 & $4.38 \mathrm{E}-05$ & $2.04 E-05$ & 2.1 \\
\hline K-CC-195-A & $02 \mathrm{MN}-03-\mathrm{A}$ & U234 & $3.27 E-03$ & 4.69E-04 & 7.0 \\
\hline K-CC-195-A & 02MN-03-A & U235 & $1.34 \mathrm{E}-04$ & $4.58 \mathrm{E}-05$ & 2.9 \\
\hline K-CC-195-A & O2MN-03-A & U238 & $2.82 E-03$ & $4.63 \mathrm{E}-04$ & 6.1 \\
\hline K-CC-196-A & $02 \mathrm{MN}-04-\mathrm{A}$ & U234 & $1.88 \mathrm{E}-03$ & $3.03 E-04$ & 6.2 \\
\hline K-CC-196-A & 02MN-04-A & U235 & 1.07E-04 & 4.16E-05 & 2.6 \\
\hline K-CC-196-A & 02MN-04-A & $\mathrm{U} 238$ & $1.71 \mathrm{E}-03$ & $3.09 \mathrm{E}-04$ & 5.5 \\
\hline K-CC-197-A & $02 \mathrm{MN}-05-\mathrm{A}$ & U234 & $1.84 \mathrm{E}-03$ & $3.11 E-04$ & 5.9 \\
\hline K-CC-197-A & 02MN-05-A & U235 & 9.23E-05 & $3.92 E-05$ & 2.4 \\
\hline K-CC-197-A & $02 \mathrm{MN}-05-\mathrm{A}$ & U238 & $1.78 \mathrm{E}-03$ & 3.33E-04 & 5.3 \\
\hline K-CC-199-A & $02 M N-07-A$ & $\mathrm{U} 234$ & $5.10 \mathrm{E}-03$ & $7.00 \mathrm{E}-04$ & 7.3 \\
\hline K-CC-199-A & \begin{tabular}{|l|}
$02 \mathrm{MN}-07-\mathrm{A}$ \\
\end{tabular} & U235 & $2.54 E-04$ & $7.20 \mathrm{E}-05$ & 3.5 \\
\hline K-CC-199-A & $02 \mathrm{MN}-07-\mathrm{A}$ & $\mathrm{U} 238$ & $4.47 \mathrm{E}-03$ & $7.00 \mathrm{E}-04$ & 6.4 \\
\hline K-CC-201-A & 02MN-09-A & U234 & $2.90 \mathrm{E}-03$ & $4.26 \mathrm{E}-04$ & 6.8 \\
\hline K-CC-201-A & 02MN-09-A & $\mathrm{U} 235$ & $1.18 \mathrm{E}-04$ & 4.34E-05 & 2.7 \\
\hline K-CC-201-A & 02MN-09-A & $\mathrm{U} 238$ & $2.33 E-03$ & $3.96 \mathrm{E}-04$ & 5.9 \\
\hline K-CC-203-A & $02 \mathrm{MN}-11-\mathrm{A}$ & U234 & $2.72 E-03$ & $4.02 E-04$ & 6.8 \\
\hline K-CC-203-A & $02 \mathrm{MN}-11-\mathrm{A}$ & U235 & $9.73 \mathrm{E}-05$ & $3.82 E-05$ & 2.5 \\
\hline K-CC-203-A & $02 \mathrm{MN}-11-\mathrm{A}$ & U238 & $2.22 E-03$ & $3.78 \mathrm{E}-04$ & 5.9 \\
\hline K-CC-205-A & $02 \mathrm{MN}-13-\mathrm{A}$ & AM241 & $2.68 \mathrm{E}-03$ & 3.03E-04 & 8.8 \\
\hline K-CC-205-A & $02 \mathrm{MN}-13-\mathrm{A}$ & \begin{tabular}{|l|} 
PU238 \\
\end{tabular} & 4.01E-03 & $4.58 \mathrm{E}-04$ & 8.8 \\
\hline K-CC-205-A & $02 \mathrm{MN}-13-\mathrm{A}$ & PU239/240 & $4.71 \mathrm{E}-03$ & $5.24 \mathrm{E}-04$ & 9.0 \\
\hline K-CC-205-A & $02 \mathrm{MN}-13-\mathrm{A}$ & U234 & $9.03 E-04$ & 1.86E-04 & 4.9 \\
\hline K-CC-205-A & $02 \mathrm{MN}-13-\mathrm{A}$ & $\mathrm{U} 238$ & $7.22 \mathrm{E}-04$ & $1.74 \mathrm{E}-04$ & 4.1 \\
\hline K-CC-206-A & $02 \mathrm{MN}-14-\mathrm{A}$ & AM241 & $5.90 \mathrm{E}-03$ & $5.08 \mathrm{E}-04$ & 11.6 \\
\hline K-CC-206-A & 02MN-14-A & \begin{tabular}{|l|} 
PU238 \\
\end{tabular} & $7.51 \mathrm{E}-03$ & $7.66 \mathrm{E}-04$ & 9.8 \\
\hline K-CC-206-A & $02 \mathrm{MN}-14-\mathrm{A}$ & PU239/240 & $4.71 \mathrm{E}-03$ & $5.14 \mathrm{E}-04$ & 9.2 \\
\hline K-CC-206-A & $02 \mathrm{MN}-14-\mathrm{A}$ & U234 & $7.68 \mathrm{E}-04$ & $1.68 \mathrm{E}-04$ & 4.6 \\
\hline K-CC-206-A & $02 \mathrm{MN}-14-\mathrm{A}$ & U238 & $7.41 \mathrm{E}-04$ & $1.76 \mathrm{E}-04$ & 4.2 \\
\hline
\end{tabular}




\section{Alpha Analysis for Amchitka Island (Batch 7)}

\section{Summary of 2 and 3 sigma activities}

\section{page 3}

\begin{tabular}{|l|l|l|c|c|c|}
\hline \multicolumn{1}{|c|}{ Customer ID } & \multicolumn{1}{|c|}{ Lab ID } & \multicolumn{1}{|c|}{ Isotope } & $\begin{array}{c}\text { Result } \\
\text { Bq/g }\end{array}$ & $\begin{array}{c}\text { Uncertainty } \\
\text { Bq/g }\end{array}$ & $\begin{array}{c}\text { Result } \\
\text { Uncertainty }\end{array}$ \\
\hline K-CC-208-A & $02 \mathrm{MN}-16-\mathrm{A}$ & $\mathrm{U} 234$ & $3.04 \mathrm{E}-03$ & $4.40 \mathrm{E}-04$ & 6.9 \\
\hline K-CC-208-A & $02 \mathrm{MN}-16-\mathrm{A}$ & $\mathrm{U} 235$ & $1.36 \mathrm{E}-04$ & $4.63 \mathrm{E}-05$ & 2.9 \\
\hline K-CC-208-A & $02 \mathrm{MN}-16-\mathrm{A}$ & $\mathrm{U} 238$ & $2.41 \mathrm{E}-03$ & $4.02 \mathrm{E}-04$ & 6.0 \\
\hline & & & & & \\
\hline K-CC-212-A & $02 \mathrm{MN}-20-\mathrm{A}$ & $\mathrm{PU} 239 / 240$ & $5.24 \mathrm{E}-05$ & $2.13 \mathrm{E}-05$ & 2.5 \\
\hline K-CC-212-A & $02 \mathrm{MN}-20-\mathrm{A}$ & $\mathrm{U} 234$ & $2.08 \mathrm{E}-03$ & $3.23 \mathrm{E}-04$ & 6.4 \\
\hline $\mathrm{K}-\mathrm{CC}-212-\mathrm{A}$ & $02 \mathrm{MN}-20-\mathrm{A}$ & $\mathrm{U} 235$ & $1.23 \mathrm{E}-04$ & $4.30 \mathrm{E}-05$ & 2.9 \\
\hline K-CC-212-A & $02 \mathrm{MN}-20-\mathrm{A}$ & $\mathrm{U} 238$ & $1.86 \mathrm{E}-03$ & $3.28 \mathrm{E}-04$ & 5.7 \\
\hline
\end{tabular}

All known sources of uncertainty are included in the uncertainty term. There may be unknown sources of uncertainty that are not accounted for. If the result/uncertainty ratio is more than 3 , we have a degree of confidence that the result is positive (i.e. the result is statistically different than zero). A result with the result/uncertainty ratio between 2 and 3 is the first indication that an isotope may be present and further investigation may be warranted.

As with any good science no single data point is used in important decisions (results need to be reproducible). 
BATCH 8A

ALPHA 


\section{RADIOANALYTICAL ANALYSES DATA PACKAGE}

Project Title: $\quad$ Alpha Analysis for Amchitka (Batch 8A)

$\begin{array}{llll}\text { Lab Name: } & \text { RTC } & \text { Case No: } & \text { NA } \\ \text { Report No.: } & \text { AmchitBatchF8A } & \begin{array}{l}\text { Method Type: } \\ \text { Ap/B }\end{array} \\ \text { Approved SAP No.: } & \text { NA } & \text { SDG No.: } & \text { B-K-215 }\end{array}$

\section{SAMPLE NUMBERS}

Customer

Sample ID

\begin{tabular}{|c|}
\hline$B-K-215$ \\
\hline B-K-216 \\
\hline B-K-217 \\
\hline B-K-218 \\
\hline B-K-219 \\
\hline$B-K-220$ \\
\hline B-K-221 \\
\hline B-K-222 \\
\hline B-K-223 \\
\hline B-K-225 \\
\hline
\end{tabular}

Lab Sample

ID

02N6-01-A

02N6-02-A

02N6-03-A

02N6-04-A

$02 N 6-05-A$

$02 N 6-06-A$

02N6-07-A

$02 \mathrm{~N} 6-08-\mathrm{A}$

02N6-09-A

02N6-10-A

Comments: All pages Reviewed by: B.K. Jahut 6/17/2005

Release of the data contained in this data package has been authorized by the laboratory manager or the manager's designee, as verified by the following signature:

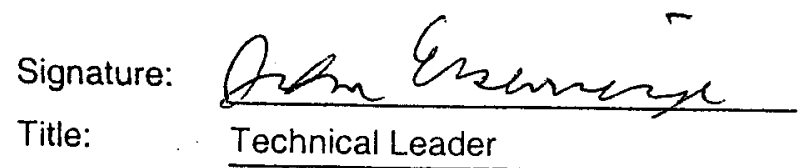

Name: J.G. Eisenmenger

Date: $\quad 06 / 15 / 2005$ 


\begin{tabular}{|c|c|c|c|c|c|c|c|c|c|c|c|c|c|c|c|c|c|c|c|c|c|}
\hline 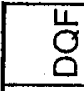 & & & & & & & & & & & & & & & & & & & & & \\
\hline$\frac{\nwarrow}{\mathrm{O}}$ & 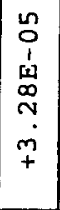 & 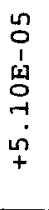 & $\begin{array}{l}n \\
0 \\
1 \\
1 \\
0 \\
0 \\
0 \\
0 \\
+\end{array}$ & 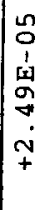 & 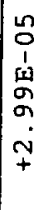 & $\begin{array}{l}n \\
0 \\
1 \\
1 \\
1 \\
0 \\
m \\
m \\
m \\
+\end{array}$ & 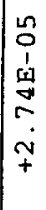 & 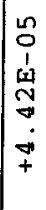 & $\begin{array}{l}n \\
0 \\
1 \\
1 \\
\text { s. } \\
m \\
m \\
m \\
+\end{array}$ & \begin{tabular}{l}
$n$ \\
0 \\
1 \\
1 \\
$w$ \\
\multirow{2}{*}{} \\
$\vdots$ \\
$\vdots$ \\
+ \\
+
\end{tabular} & 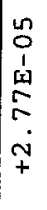 & 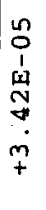 & 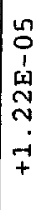 & $\begin{array}{l}n \\
0 \\
1 \\
1 \\
\\
\tilde{0} \\
0 \\
\sim \\
+ \\
+\end{array}$ & $\begin{array}{l}n \\
0 \\
1 \\
1 \\
0 \\
-1 \\
+1 \\
+1\end{array}$ & $\begin{array}{l}\text { n } \\
0 \\
1 \\
\text { cl } \\
0 \\
6 \\
\dot{m} \\
\dot{+} \\
+\end{array}$ & 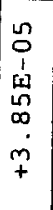 & 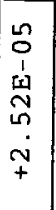 & \begin{tabular}{l}
0 \\
0 \\
0 \\
1 \\
1 \\
\multirow{2}{1}{} \\
0 \\
0 \\
$\dot{p}$ \\
+
\end{tabular} & 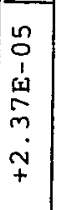 & 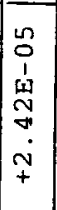 \\
\hline 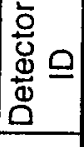 & $\overrightarrow{0}$ & -1 & 0 & 18 & I & 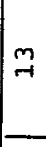 & 0 & O & ON & 次 & iे & i̊ & $\nexists$ & ס̃ & g & $\stackrel{m}{o}$ & $\stackrel{m}{o}$ & $\Rightarrow$ & $\Rightarrow 1$ & $\stackrel{\sim}{\sim}$ & 20 \\
\hline $\begin{array}{l}\frac{E}{\Phi} \\
\frac{\partial}{0} \\
\end{array}$ & 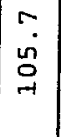 & $\begin{array}{l}r \\
0 \\
0 \\
-1\end{array}$ & 䘾 & $\begin{array}{l}-1 \\
\infty \\
\infty\end{array}$ & a & $\begin{array}{l}-1 \\
0 \\
0 \\
r\end{array}$ & $\begin{array}{r}r \\
\stackrel{2}{0} \\
\stackrel{-}{-1}\end{array}$ & $\begin{array}{l}0 \\
0 \\
0 \\
0 \\
-1\end{array}$ & $\begin{array}{l}0 \\
0 \\
0 \\
0 \\
-1\end{array}$ & $\begin{array}{l}0 \\
0 \\
0 \\
0 \\
-1\end{array}$ & Sa & $\tilde{\sigma}$ & ğ & $\begin{array}{l}0 \\
0 \\
0 \\
0\end{array}$ & $\begin{array}{l}\sim \\
-1 \\
0 \\
-1\end{array}$ & $\begin{array}{c}r \\
-1 \\
0 \\
-1\end{array}$ & $\begin{array}{c}r \\
-1 \\
-1 \\
0 \\
-1\end{array}$ & $\begin{array}{l}0 \\
\infty \\
\infty\end{array}$ & $\begin{array}{l}7 \\
\infty \\
\infty \\
\infty\end{array}$ & $\begin{array}{l}n \\
0 \\
\infty \\
0 \\
-1\end{array}$ & 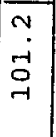 \\
\hline 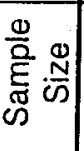 & $\stackrel{n}{n}$ & $\tilde{\sim}$ & 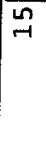 & - & $\tilde{F}$ & ח & 금 & ñ & $\stackrel{n}{\sim}$ & 号 & $\stackrel{n}{\pi}$ & $\tilde{\sigma}$ & $\stackrel{n}{n}$ & ณ & $\sqrt{n}$ & $\bar{n}$ & $\stackrel{\Omega}{n}$ & $\underset{\sim}{n}$ & \begin{tabular}{ll}
\multirow{2}{n}{} \\
$\rightarrow$
\end{tabular} & $\begin{array}{ll}\text { nn } \\
\rightarrow\end{array}$ & ${ }_{n-1}^{n}$ \\
\hline
\end{tabular}

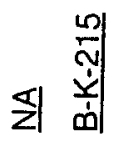

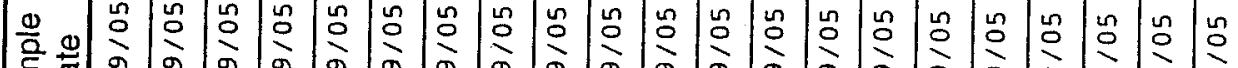
लॉ

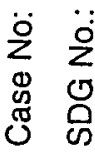

可

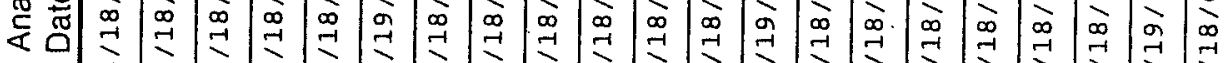

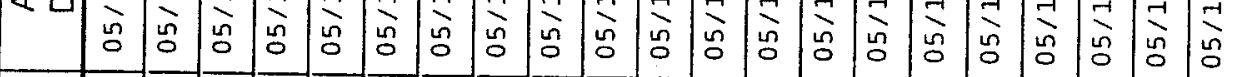

욜

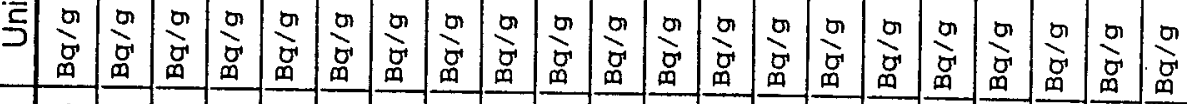

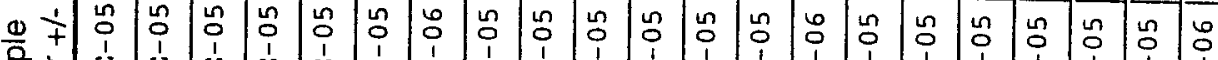

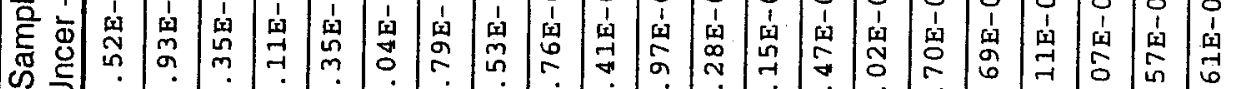

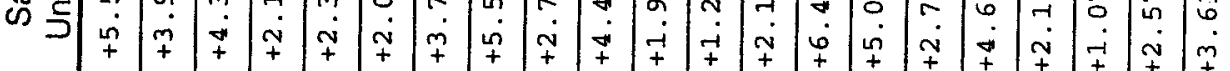

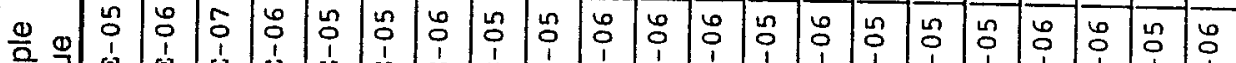

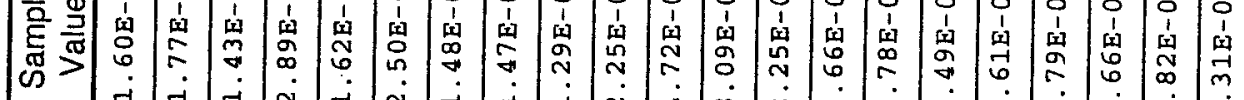

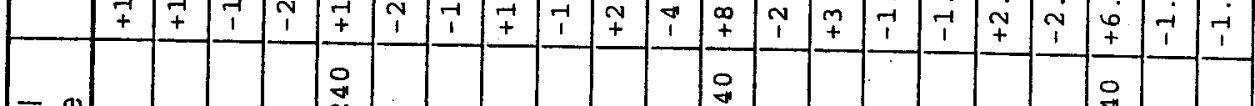

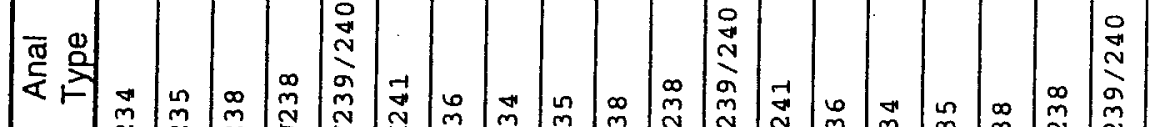

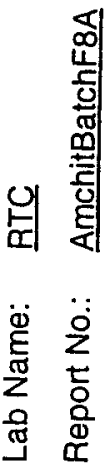

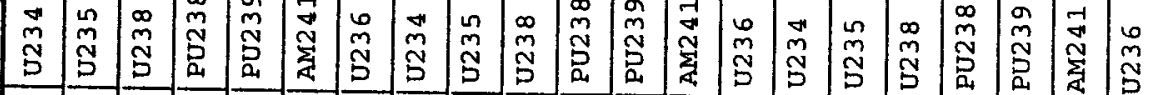
음 $x$

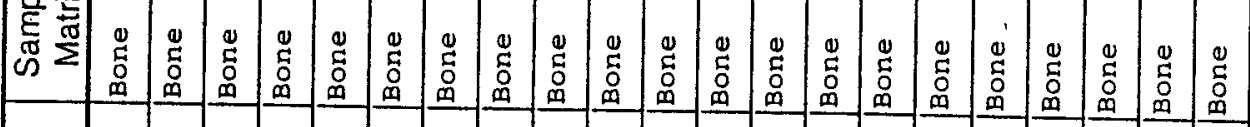
으

西

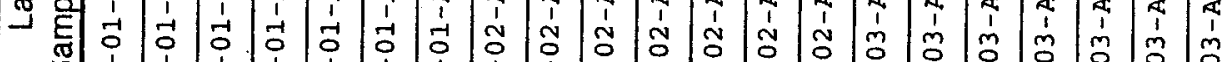
心)

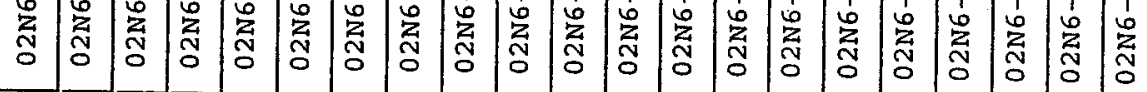

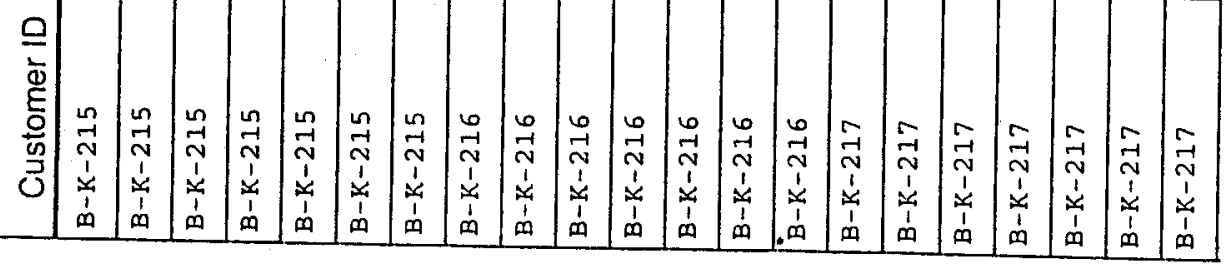




\begin{tabular}{|c|c|c|c|c|c|c|c|c|c|c|c|c|c|c|c|c|c|c|c|c|c|}
\hline $\begin{array}{l} \\
\\
\end{array}$ & & & & & & & & & & & & & & & & & & & & & \\
\hline $\bar{\Sigma}$ & 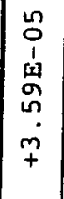 & \begin{tabular}{|l|}
$n$ \\
0 \\
1 \\
1 \\
ch \\
2 \\
$\sigma$ \\
0 \\
+ \\
+
\end{tabular} & $\begin{array}{l}n \\
0 \\
1 \\
1 \\
0 \\
n \\
0 \\
0 \\
+ \\
+\end{array}$ & 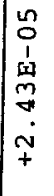 & 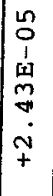 & 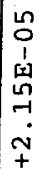 & $\begin{array}{l}n \\
0 \\
1 \\
1 \\
9 \\
7 \\
7 \\
m \\
+\end{array}$ & 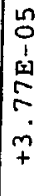 & 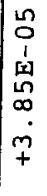 & $\begin{array}{l}n \\
0 \\
1 \\
1 \\
4 \\
n \\
\sim \\
\sim \\
+ \\
+\end{array}$ & $\begin{array}{l}n \\
0 \\
1 \\
1 \\
0 \\
0 \\
0 \\
\dot{m} \\
+\end{array}$ & $\begin{array}{l}n \\
0 \\
1 \\
1 \\
0 \\
0 \\
0 \\
0 \\
+ \\
+\end{array}$ & 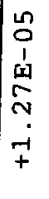 & 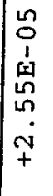 & $\begin{array}{l}n \\
0 \\
1 \\
1 \\
1 \\
0 \\
+1 \\
\dot{m} \\
+\end{array}$ & 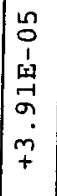 & $\begin{array}{l}u \\
0 \\
1 \\
0 \\
0 \\
2 \\
n \\
\sim \\
+ \\
+\end{array}$ & 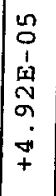 & $\mid \begin{array}{l}n \\
0 \\
1 \\
1 \\
0 \\
0 \\
0 \\
+ \\
+ \\
1\end{array}$ & $\mid \begin{array}{l}n \\
0 \\
1 \\
n \\
0 \\
0 \\
o \\
i \\
+ \\
+\end{array}$ & $\begin{array}{l}n \\
0 \\
1 \\
1 \\
1 \\
0 \\
0 \\
m \\
-i \\
+\end{array}$ \\
\hline 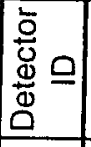 & ठే & 8 & $\overrightarrow{0}$ & $\approx$ & $\exists$ & $L_{-1}^{\circ}$ & 8 & : n & n & \& & $\underset{\gamma}{m}$ & $m$ & ન & $\stackrel{\circ}{\circ}$ & $1 \%$ & 18 & $1 \%$ & $\stackrel{I}{\rightarrow}$ & $\vec{r}$ & $\stackrel{m}{\sim}$ & 10 \\
\hline 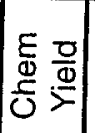 & $\begin{array}{l}-1 \\
\therefore \\
0 \\
-1\end{array}$ & 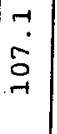 & $\begin{array}{l}-1 \\
0 \\
0 \\
-1 \\
-1\end{array}$ & $\begin{array}{l}\infty \\
\dot{\sim} \\
\sigma\end{array}$ & $\begin{array}{l}\infty \\
\dot{N} \\
\alpha\end{array}$ & $\begin{array}{l}m \\
m \\
n \\
\text { ñ }\end{array}$ & $\begin{array}{l}-1 \\
\vdots \\
0 \\
-1\end{array}$ & $\begin{array}{l}\text { - } \\
-1 \\
-1\end{array}$ & $\begin{array}{l}r \\
- \\
- \\
-\end{array}$ & $\begin{array}{l}\text { T. } \\
- \\
-1 \\
-1\end{array}$ & $\begin{array}{l}0 \\
\dot{0} \\
\infty \\
\infty\end{array}$ & $\begin{array}{l}0 \\
\dot{0} \\
\dot{\infty}\end{array}$ & $\begin{array}{l}\infty \\
\dot{n} \\
\dot{n}\end{array}$ & $\begin{array}{l}\sim \\
-1 \\
- \\
-1\end{array}$ & $\begin{array}{l}n \\
0 \\
0 \\
-1\end{array}$ & $\begin{array}{l}0 \\
0 \\
0 \\
0 \\
r\end{array}$ & $\begin{array}{l}n \\
0 \\
0 \\
0 \\
-1\end{array}$ & $\begin{array}{l}0 \\
0 \\
\square\end{array}$ & $\begin{array}{l}0 \\
\square \\
\end{array}$ & $\begin{array}{l}\vec{H} \\
-1 \\
-1 \\
-1\end{array}$ & $\begin{array}{l}n \\
0 \\
0 \\
-1\end{array}$ \\
\hline 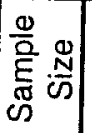 & $\stackrel{\sim}{\sim}$ & $\pi$ & ת & $\stackrel{n}{9}$ & ñ & 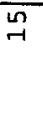 & $\stackrel{n}{\sim}$ & $\stackrel{n}{\sim}$ & 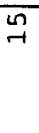 & $\mathbb{n}_{-1}^{n}$ & $\stackrel{n}{n}$ & $\tilde{\sim}$ & 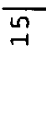 & $\stackrel{\sim}{\rightarrow}$ & $\stackrel{\sim}{\sim}$ & $\stackrel{\sim}{\sim}$ & $\stackrel{n}{\sim}$ & $\underset{r}{n}$ & 告 & $\stackrel{n}{\sim}$ & ? \\
\hline
\end{tabular}

\|

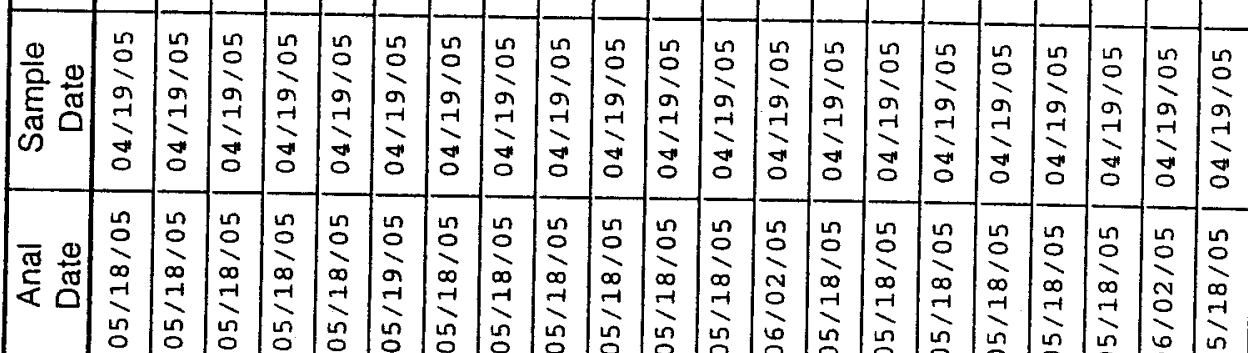

$\begin{array}{ll}\dddot{0} & \ddot{0} \\ 0 & 0 \\ 0 & 0 \\ 0 & 0\end{array}$

$\stackrel{\infty}{=}$

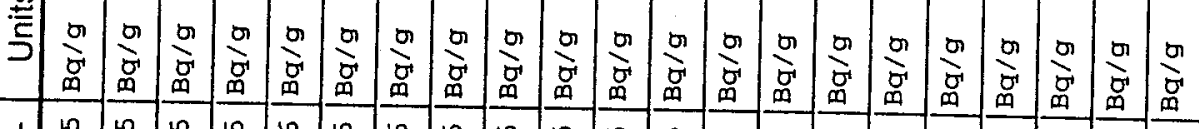

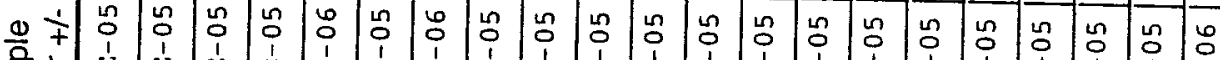

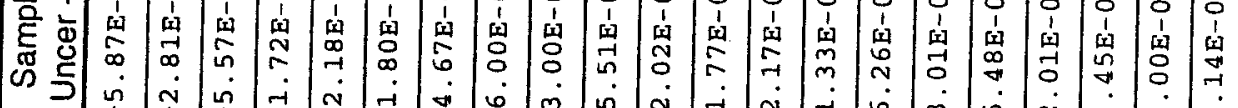

을

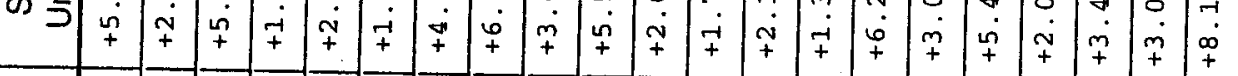

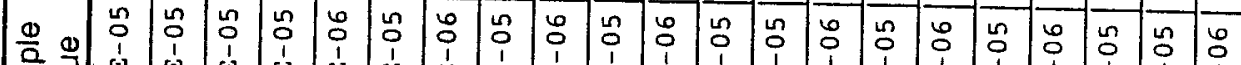

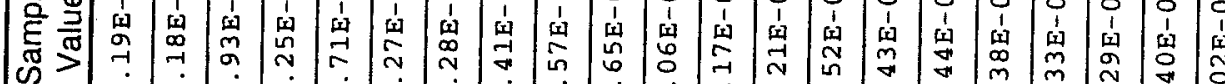

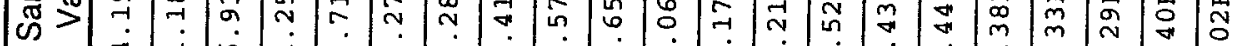

업 훙

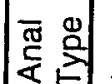

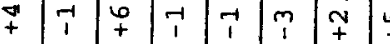

$\infty$
1

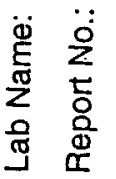

告

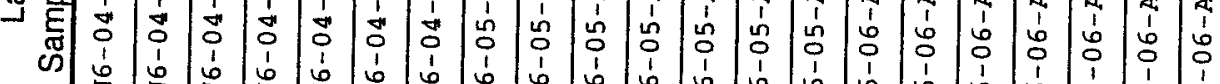

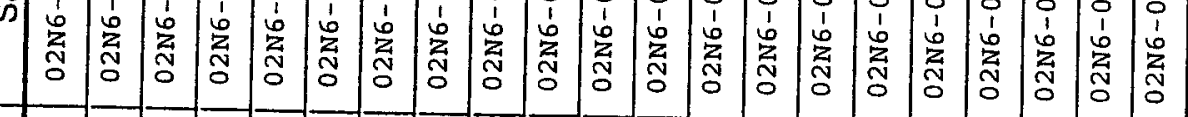
空 음즌 离

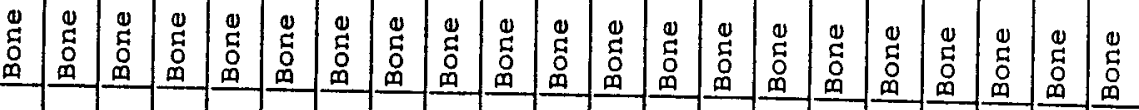




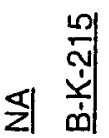

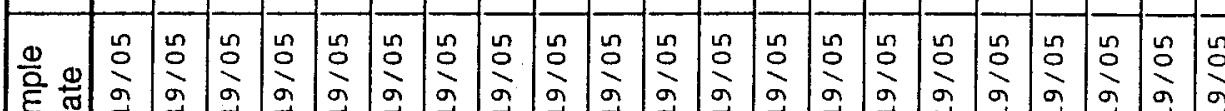

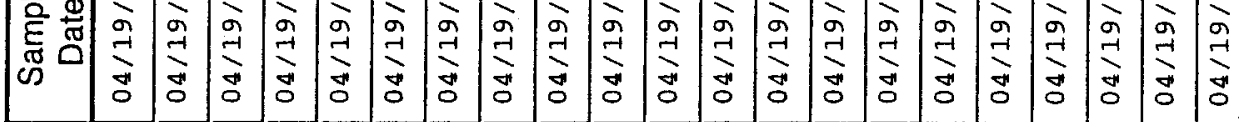

ॠँ

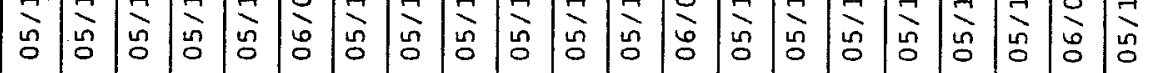

a

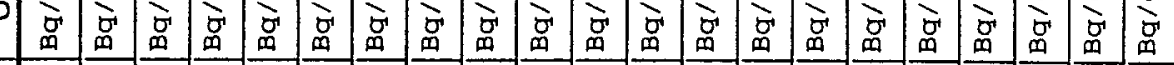

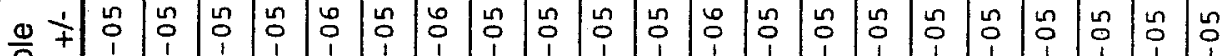
爰

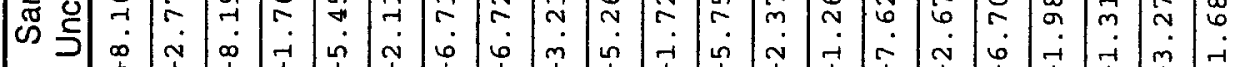
遂

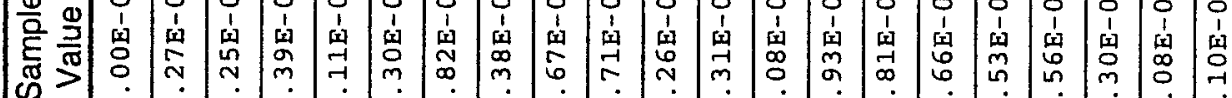
ख ఖ

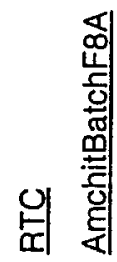

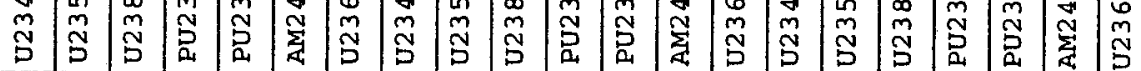

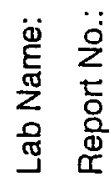

\begin{tabular}{|c|c|c|c|c|c|c|c|c|c|c|c|c|c|c|c|c|c|c|c|c|}
\hline 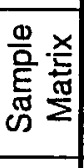 & $\begin{array}{l}0 \\
\\
\end{array}$ & 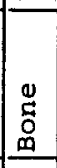 & 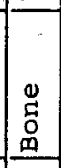 & \begin{tabular}{|l|} 
\\
0 \\
0 \\
0 \\
\end{tabular} & 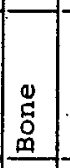 & \begin{tabular}{|l|} 
\\
0 \\
0 \\
\end{tabular} & 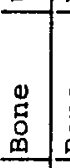 & 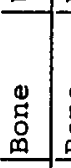 & $\begin{array}{c}0 \\
\stackrel{0}{0} \\
0 \\
\end{array}$ & 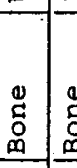 & 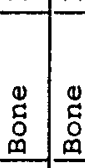 & 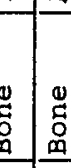 & 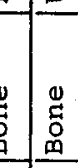 & 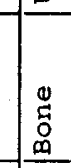 & $\begin{array}{l}0 \\
0 \\
0 \\
0 \\
\end{array}$ & 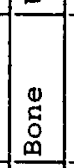 & \begin{tabular}{|l|}
0 \\
0 \\
0 \\
0 \\
\end{tabular} & 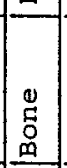 & 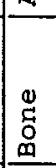 & $\mid \begin{array}{l}\tilde{0} \\
\text { ¿ }\end{array}$ \\
\hline $\begin{array}{l}\frac{0}{\Phi} \\
\frac{0}{0} \\
\text { है⿴囗十 }\end{array}$ & \begin{tabular}{l}
6 \\
1 \\
5 \\
0 \\
1 \\
0 \\
\multirow{2}{7}{} \\
0 \\
0
\end{tabular} & $\mid$\begin{tabular}{l}
4 \\
1 \\
0 \\
0 \\
1 \\
0 \\
\multirow{2}{2}{} \\
0 \\
0
\end{tabular} & 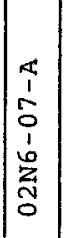 & $\left|\begin{array}{l}1 \\
1 \\
0 \\
0 \\
1 \\
0 \\
z \\
0 \\
0\end{array}\right|$ & $\mid \begin{array}{c}1 \\
1 \\
1 \\
0 \\
1 \\
1 \\
\vdots \\
z \\
0 \\
0\end{array}$ & 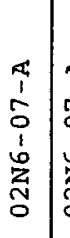 & $\begin{array}{c}1 \\
1 \\
\vdots \\
0 \\
1 \\
0 \\
z \\
0 \\
0\end{array}$ & 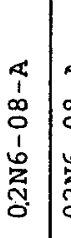 & 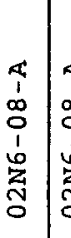 & 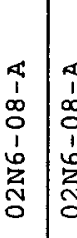 & 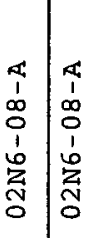 & 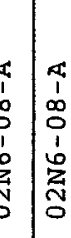 & 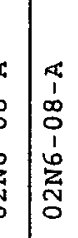 & 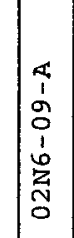 & $\mid$\begin{tabular}{l}
\{ \\
1 \\
$o$ \\
0 \\
1 \\
$\vdots$ \\
\multirow{2}{*}{} \\
0 \\
0
\end{tabular} & 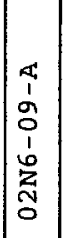 & 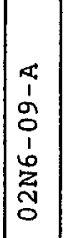 & 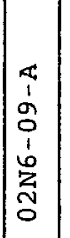 & 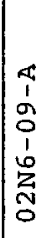 & 4 \\
\hline 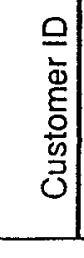 & 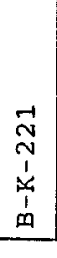 & $\mid \begin{array}{c}n \\
N \\
N \\
1 \\
\vdots \\
1 \\
m \\
\end{array}$ & 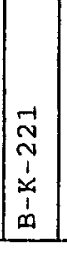 & 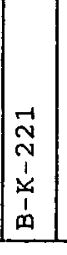 & 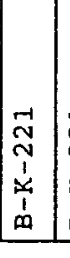 & 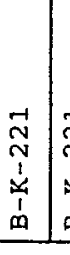 & 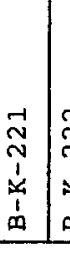 & 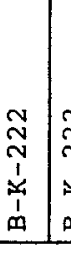 & 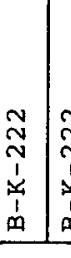 & 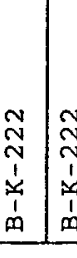 & 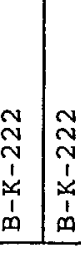 & 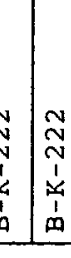 & 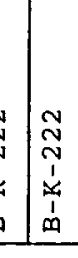 & \begin{tabular}{l}
$m$ \\
\multirow{N}{N}{} \\
$w$ \\
1 \\
$x$ \\
1 \\
$m$
\end{tabular} & $\begin{array}{l}\tilde{n} \\
\tilde{N} \\
1 \\
\underline{u} \\
1 \\
m \\
\end{array}$ & $\left.\mid \begin{array}{c}\tilde{N} \\
\sim \\
\vdots \\
\vdots \\
\vdots \\
m\end{array}\right]$ & 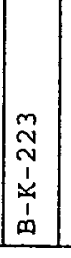 & 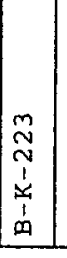 & \begin{tabular}{|c|c}
$\sim$ \\
$\sim$ \\
$N$ \\
1 \\
$w$ \\
1 \\
$\omega$ \\
\end{tabular} & 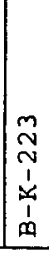 \\
\hline
\end{tabular}




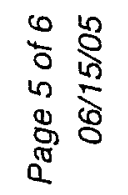

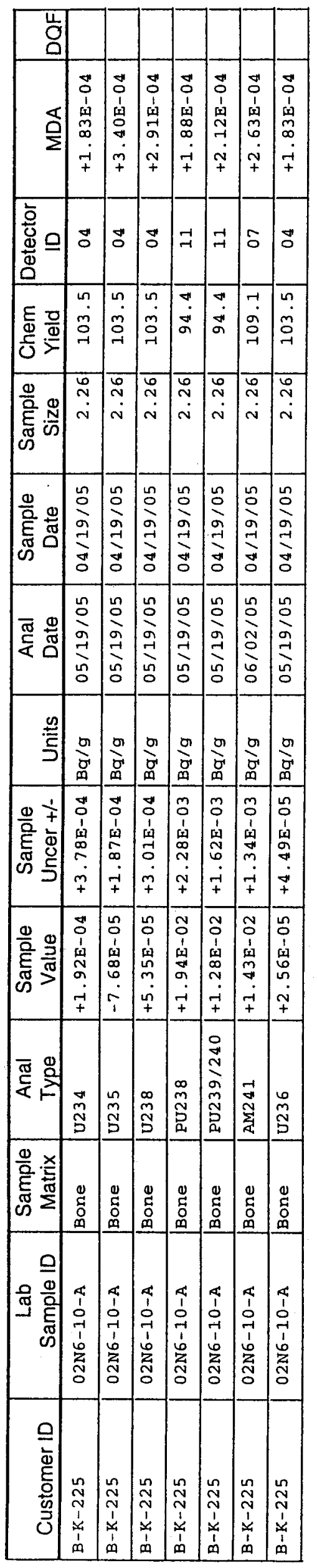

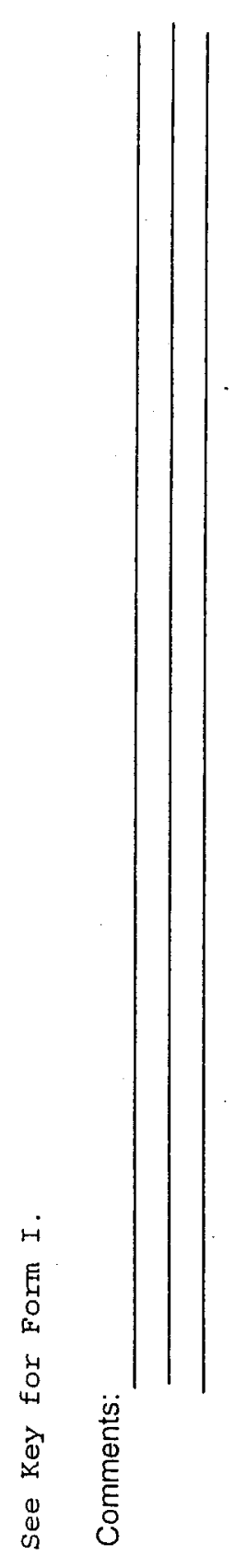


$\begin{array}{ll}0 & 5 \\ 0 & 8 \\ 0 & 8 \\ 0 & 6 \\ 8 & 8 \\ 0 & 8\end{array}$

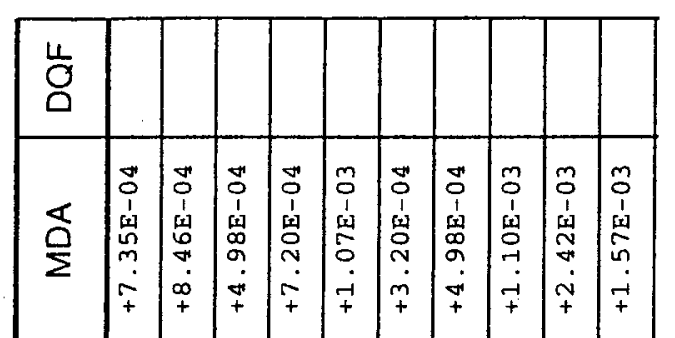

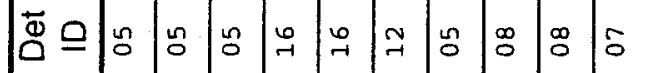

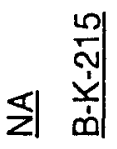

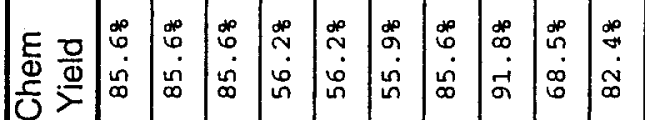

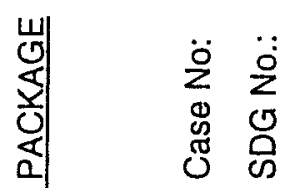

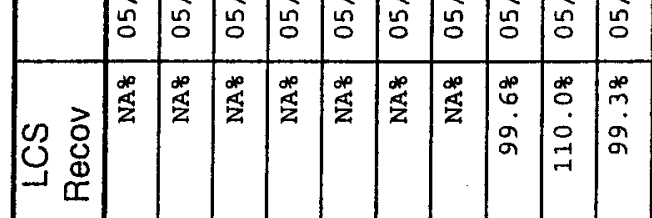

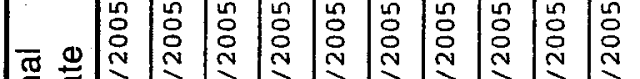

<0|न ने

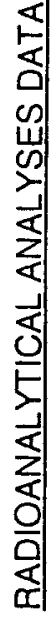

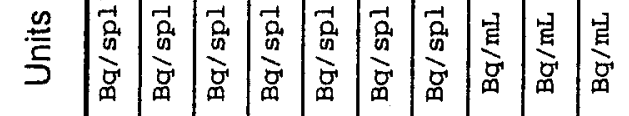

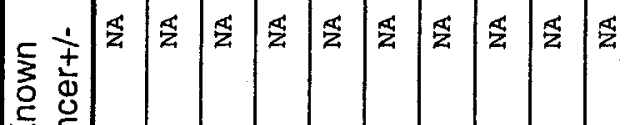

站

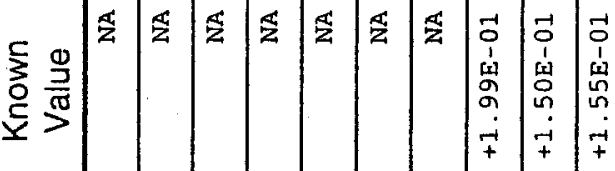

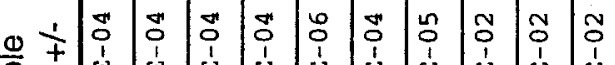

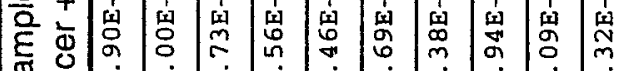

岕

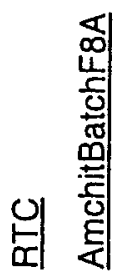

m

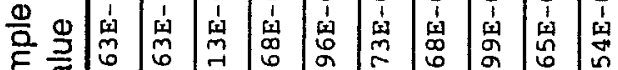

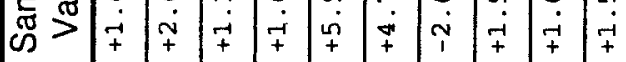

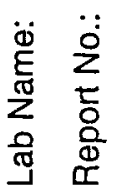

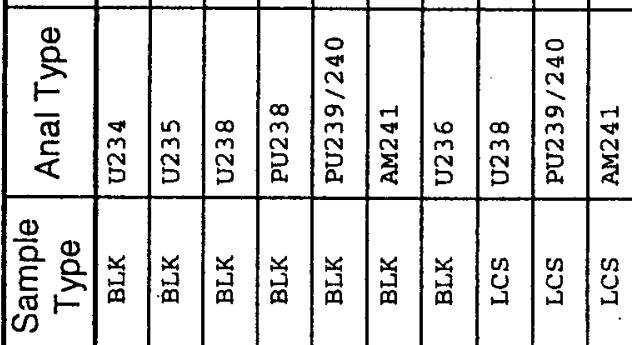

$\sum_{\substack{\text { r } \\ \text { 崩 }}}^{=}$

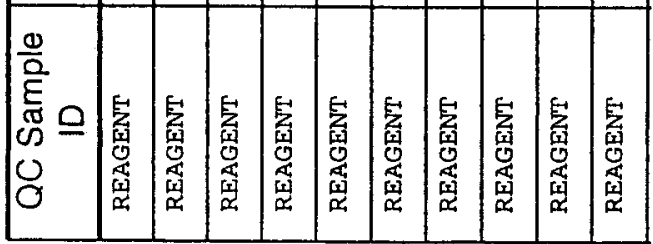


Project: $\quad$ Alpha Analysis for Amchitka Island (Batch 8A)

Laboratory: RTC

Report \#: AmchitBatchF8A

SDG\#: $\quad$ B-K-215

\section{Summary of 2 and 3 sigma activities}

Below are the results for U234, U235, U236, U238, Pu238, Pu239/240, and Am241 for Batch $8 \mathrm{~A}$ from the Amchitka Island Project that had a result/uncertainty ratio of 2 or more (uncertainty @ one sigma).

\begin{tabular}{|l|l|l|c|r|r|}
\hline Customer ID & \multicolumn{1}{|c|}{ Lab ID } & \multicolumn{1}{|c|}{ Isotope } & $\begin{array}{c}\text { Result } \\
\mathrm{Bg} / \mathrm{g}\end{array}$ & $\begin{array}{c}\text { Uncertainty } \\
\mathrm{Bq} / \mathrm{g}\end{array}$ & $\begin{array}{c}\text { Result } \\
\text { Uncertainty }\end{array}$ \\
\hline B-K-221 & 02N6-07-A & U234 & $2.00 \mathrm{E}-04$ & $8.16 \mathrm{E}-05$ & 2.5 \\
\hline B-K-221 & 02N6-07-A & U238 & $2.25 \mathrm{E}-04$ & $8.19 \mathrm{E}-05$ & 2.7 \\
\hline & & & & & 2.4 \\
\hline B-K-223 & 02N6-09-A & U234 & $1.81 \mathrm{E}-04$ & $7.62 \mathrm{E}-05$ & 2.3 \\
\hline B-K-223 & 02N6-09-A & U238 & $1.53 \mathrm{E}-04$ & $6.70 \mathrm{E}-05$ & \\
\hline & & & & & 10.7 \\
\hline B-K-225 & 02N6-10-A & AM241 & $1.43 \mathrm{E}-02$ & $1.34 \mathrm{E}-03$ & 8.5 \\
\hline B-K-225 & O2N6-10-A & PU238 & $1.94 \mathrm{E}-02$ & $2.28 \mathrm{E}-03$ & 7.9 \\
\hline B-K-225 & 02N6-10-A & PU239/240 & $1.28 \mathrm{E}-02$ & $1.62 \mathrm{E}-03$ & \\
\hline
\end{tabular}

All known sources of uncertainty are included in the uncertainty term. There may be unknown sources of uncertainty that are not accounted for. If the result/uncertainty ratio is more than 3, we have a degree of confidence that the result is positive (i.e. the result is statistically different than zero). A result with the result/uncertainty ratio between 2 and 3 is the first indication that an isotope may be present and further investigation may be warranted.

As with any good science no single data point is used in important decisions (results need to be reproducible). 
BATCH 8B

ALPHA 
COVER PAGE

\section{RADIOANALYTICAL ANALYSES DATA PACKAGE}

\begin{tabular}{|c|c|c|c|}
\hline Project Title: & Alpha Analysis for Amchitka (Batch 88) & & \\
\hline Lab Name: & RTC & Case No: & NA \\
\hline Report No.: & AmchitBatchF8B & Method Type: & $\mathrm{A} / \mathrm{B}$ \\
\hline Approved SAP No.: & NA & SDG No:: & $\mathrm{B}-\mathrm{H}-224$ \\
\hline
\end{tabular}

\section{SAMPLE NUMBERS}

Customer

Sample ID

\begin{tabular}{|c|}
\hline B-H-224 \\
\hline B-H-226 \\
\hline $\mathrm{B}-\mathrm{H}-230$ \\
\hline $\mathrm{B}-\mathrm{H}-234$ \\
\hline B-K-227 \\
\hline B-K-228 \\
\hline B-K-229 \\
\hline B-K-231 \\
\hline B-K-232 \\
\hline B-K-233 \\
\hline
\end{tabular}

Lab Sample

ID

02NE-01-A

02NE-02-A

02NE-06-A

02NE-10-A

02NE-03-A

02NE-04-A

02NE-05-A

$02 N E-07-A$

02NE-08-A

02NE-09-A

comments: All Pages Reviewed by! B.K. Johos 6/i7/2005

Release of the data contained in this data package has been authorized by the laboratory manager or the manager's designee, as verified by the following signature:

Signature:

Title:

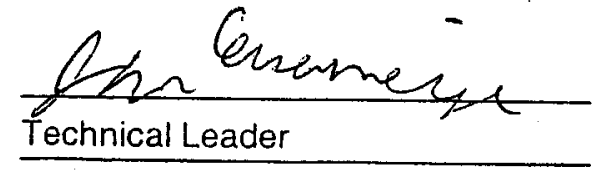

Name: J.G. Eisenmenger

Date: $06 / 15 / 2005$ 
$\begin{array}{lll}0 & 0 \\ 0 & 0 \\ 1 & 5 \\ 0 & 5 \\ 8 & 8 \\ 0 & 0\end{array}$

\begin{tabular}{|c|c|c|c|c|c|c|c|c|c|c|c|c|c|c|c|c|c|c|c|c|c|}
\hline $\begin{array}{l}\frac{1}{9} \\
\end{array}$ & & & & & & & & & & & & & & & & & & & & & \\
\hline 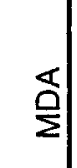 & 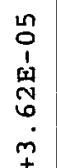 & $\begin{array}{l}n \\
0 \\
1 \\
1 \\
1 \\
\sigma \\
m \\
m \\
m \\
\end{array}$ & 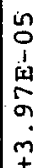 & 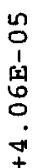 & $\begin{array}{l}n \\
0 \\
1 \\
1 \\
0 \\
0 \\
0 \\
0 \\
+\end{array}$ & $\begin{array}{l}n \\
0 \\
1 \\
1 \\
\infty \\
\infty \\
-1 \\
\\
\text { T. }\end{array}$ & 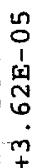 & 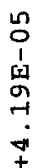 & 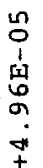 & 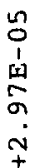 & 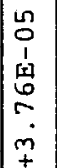 & $\begin{array}{l}n \\
0 \\
1 \\
1 \\
1 \\
0 \\
\infty \\
0 \\
0 \\
+\end{array}$ & $\begin{array}{c}2 \\
0 \\
1 \\
1 \\
9 \\
0 \\
0 \\
-1 \\
+\end{array}$ & $\mid \begin{array}{c}n \\
0 \\
1 \\
1 \\
9 \\
\vec{m} \\
m \\
m \\
+\end{array}$ & $\begin{array}{l}\text { n } \\
1 \\
1 \\
\text { ب1 } \\
6 \\
6 \\
\dot{m} \\
+ \\
+\end{array}$ & $\begin{array}{l}\text { n } \\
\text { 1 } \\
\text { ch } \\
\text { ch } \\
6 \\
\vdots \\
+ \\
+\end{array}$ & $\begin{array}{l}n \\
0 \\
1 \\
1 \\
4 \\
0 \\
\infty \\
0 \\
0 \\
+\end{array}$ & $\begin{array}{l}n \\
0 \\
1 \\
1 \\
1 \\
0 \\
\sim \\
j \\
+ \\
+\end{array}$ & $\mid \begin{array}{c}n \\
0 \\
1 \\
1 \\
2 \\
2 \\
m \\
-1 \\
+\end{array}$ & $\left.\mid \begin{array}{c}n \\
0 \\
1 \\
1 \\
m \\
n \\
\sim \\
\sim \\
+\end{array}\right]$ & $\mid \begin{array}{c}n \\
0 \\
1 \\
1 \\
\omega \\
m \\
\gamma \\
--1 \\
+\end{array}$ \\
\hline
\end{tabular}

旁 $\stackrel{2}{0}$

乙)

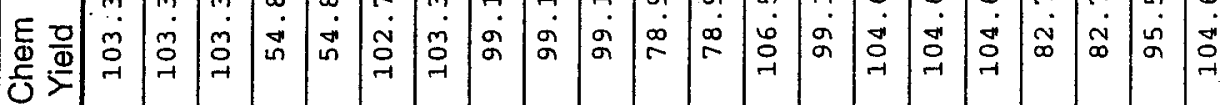

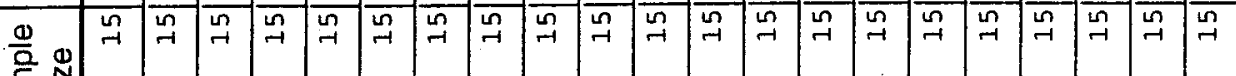
हैं कै

z

总

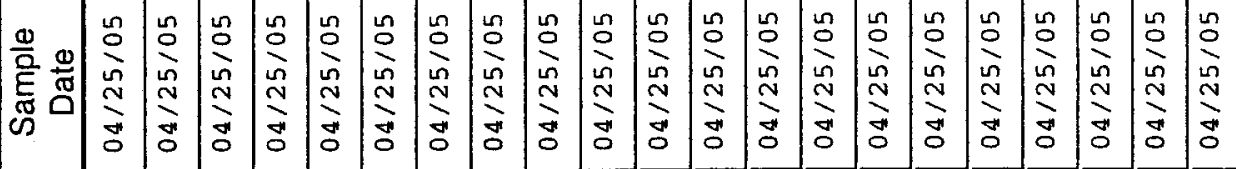

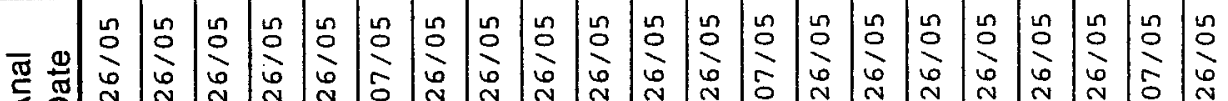

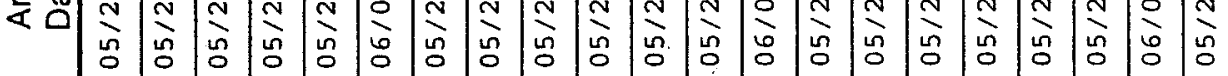

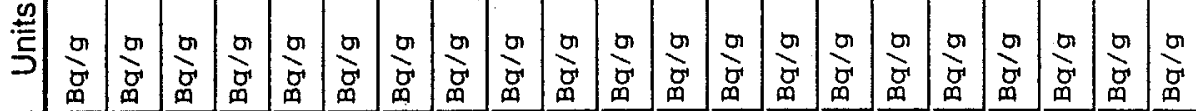

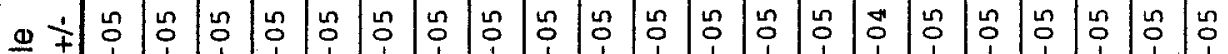

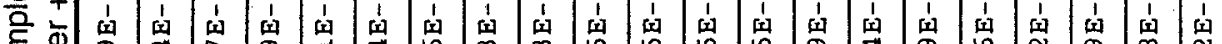

氛

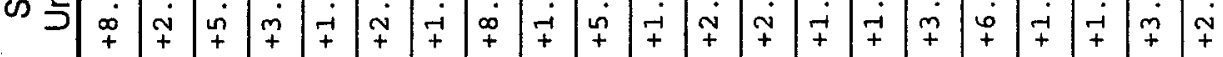

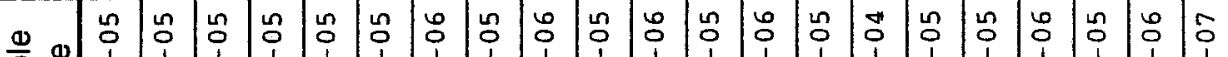

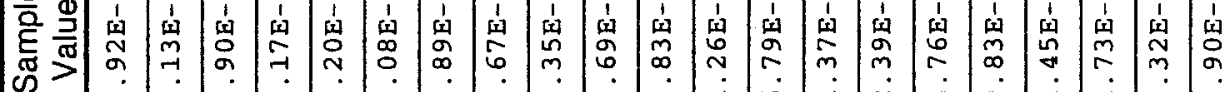

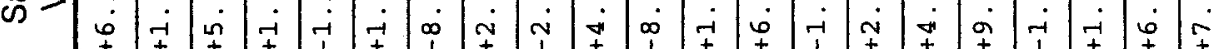

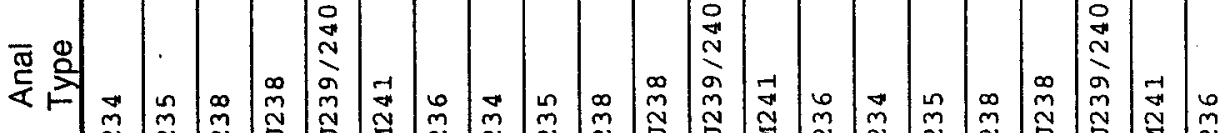

岕

임

음.

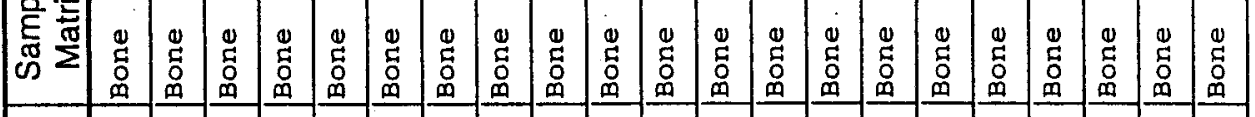

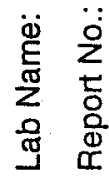

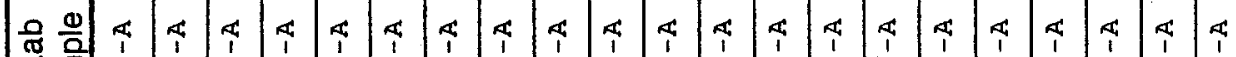
త

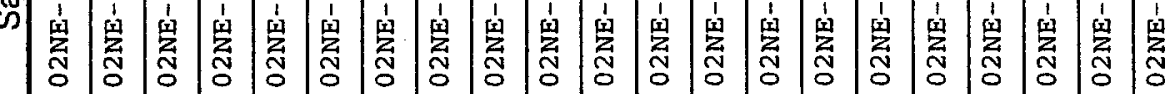




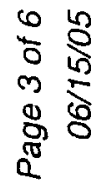

\begin{tabular}{|c|c|c|c|c|c|c|c|c|c|c|c|c|c|c|c|c|c|c|c|c|c|}
\hline $\begin{array}{l} \\
\\
\end{array}$ & & & & & & & & & & & & & & & & & & & & & \\
\hline$\stackrel{\nwarrow}{0}$ & $\begin{array}{l}n \\
0 \\
1 \\
1 \\
6 \\
\vdots \\
\vdots \\
0\end{array}$ & $\mid \begin{array}{l}\text { un } \\
0 \\
1 \\
\text { w } \\
\text { N } \\
0 \\
0 \\
+\end{array}$ & $\begin{array}{l}n \\
0 \\
1 \\
1 \\
0 \\
0 \\
-1 \\
\\
\end{array}$ & $\begin{array}{l}n \\
0 \\
1 \\
1 \\
m \\
2 \\
? \\
0 \\
+\end{array}$ & \begin{tabular}{l}
$n$ \\
0 \\
1 \\
1 \\
$\sigma$ \\
$n$ \\
$n$ \\
\hdashline \\
\end{tabular} & $\begin{array}{l}n \\
0 \\
1 \\
9 \\
m \\
-1 \\
-1 \\
-1\end{array}$ & 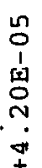 & $\begin{array}{l}n \\
0 \\
1 \\
1 \\
m \\
m \\
m \\
m \\
m\end{array}$ & 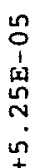 & 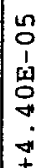 & 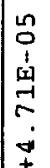 & $\begin{array}{l}n \\
0 \\
0 \\
1 \\
\{ \\
0 \\
m \\
0 \\
0 \\
+\end{array}$ & 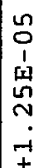 & 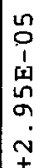 & 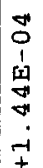 & 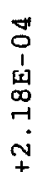 & 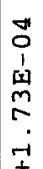 & $\begin{array}{c}n \\
0 \\
1 \\
1 \\
\text { w } \\
0 \\
0 \\
-1 \\
-1\end{array}$ & 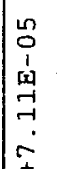 & 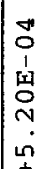 & 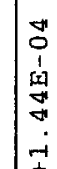 \\
\hline
\end{tabular}
旁

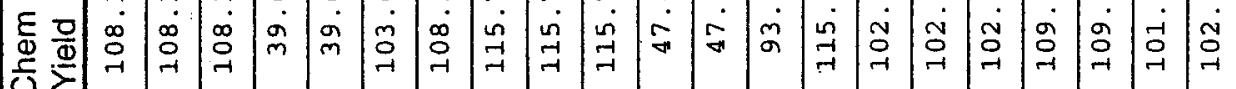
产 政

乏)

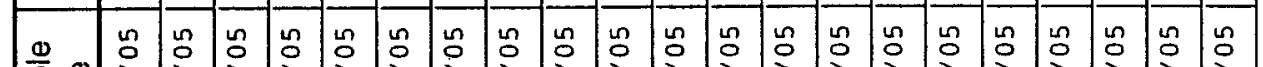

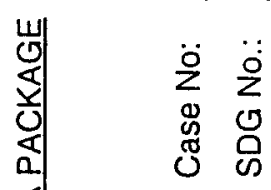

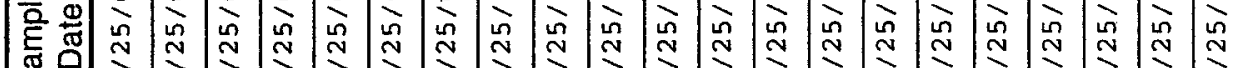
先

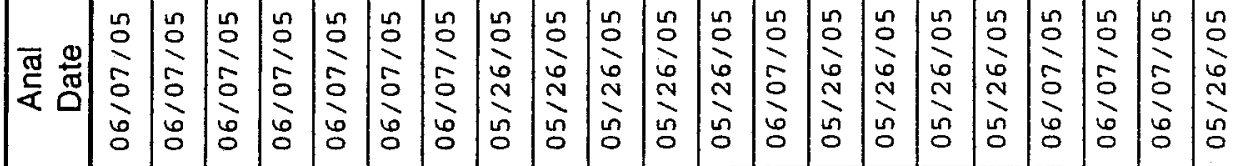

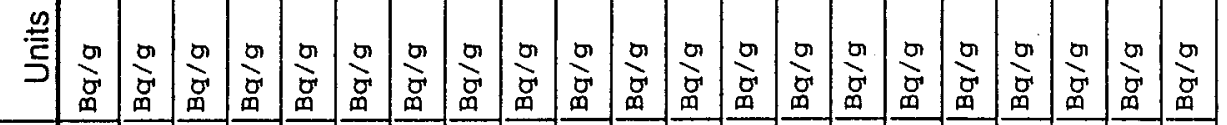

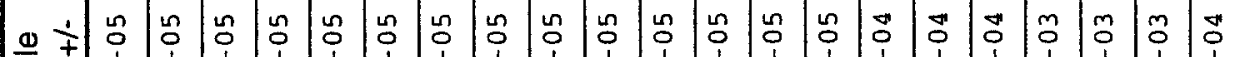

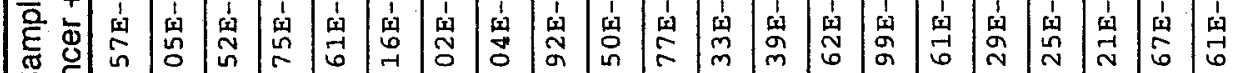

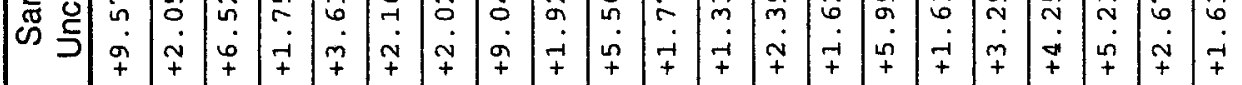
Ð

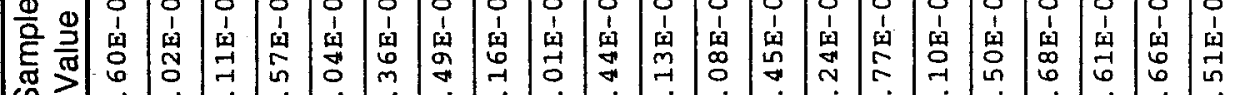

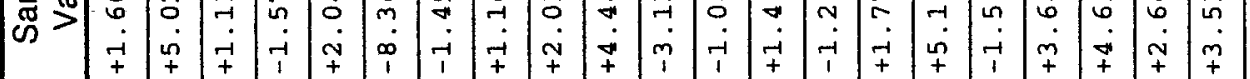

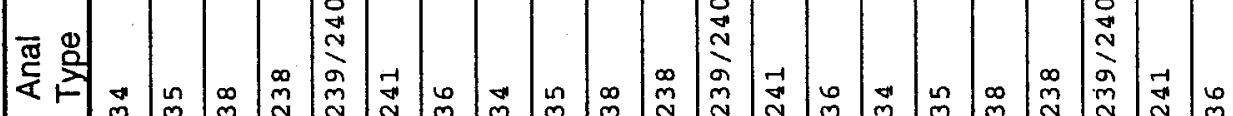

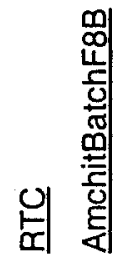

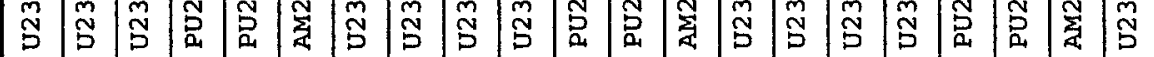

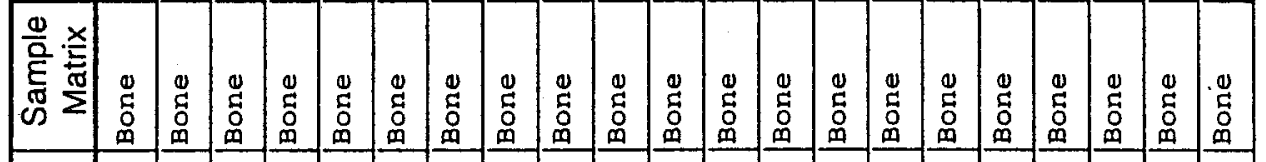

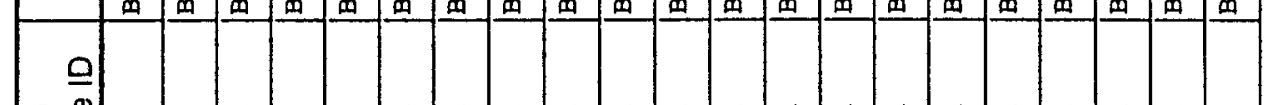

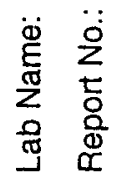
응 응

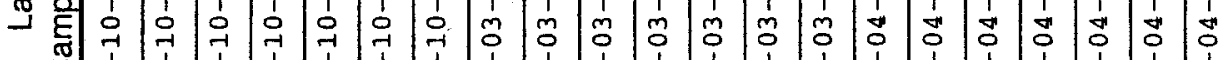

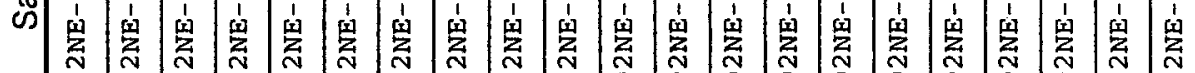

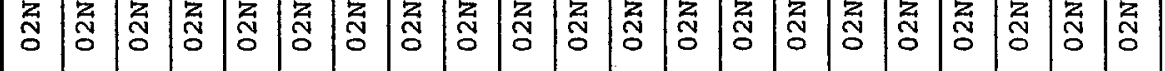

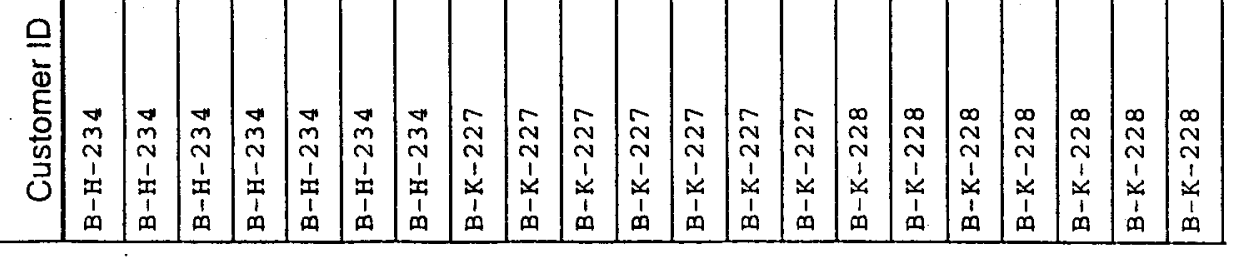




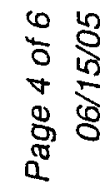

\begin{tabular}{|c|c|c|c|c|c|c|c|c|c|c|c|c|c|c|c|c|c|c|c|c|c|}
\hline $\begin{array}{l} \\
\end{array}$ & & & & & & & & & & & & & & & & & & & & & \\
\hline $\begin{array}{l}\nwarrow \\
\Sigma\end{array}$ & $\begin{array}{l}\stackrel{2}{0} \\
1 \\
1 \\
0 \\
0 \\
0 \\
\stackrel{1}{0} \\
+\end{array}$ & $\mid \begin{array}{l}n \\
0 \\
1 \\
\omega \\
0 \\
0 \\
0 \\
0 \\
+ \\
+\end{array}$ & 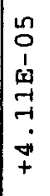 & 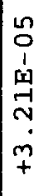 & $\begin{array}{l}n \\
0 \\
1 \\
0 \\
0 \\
0 \\
\infty \\
\dot{p} \\
\stackrel{+}{+}\end{array}$ & 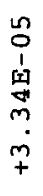 & $\begin{array}{l}n \\
0 \\
1 \\
1 \\
=1 \\
-1 \\
\vdots \\
+ \\
+\end{array}$ & 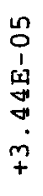 & 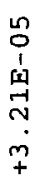 & 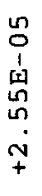 & 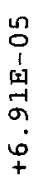 & 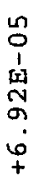 & $\begin{array}{l}n \\
0 \\
1 \\
1 \\
0 \\
-1 \\
-1 \\
-1 \\
+1\end{array}$ & 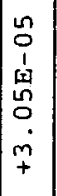 & 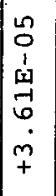 & 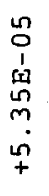 & 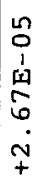 & \begin{tabular}{l}
$n$ \\
0 \\
1 \\
$\mathfrak{w}$ \\
\multirow{1}{1}{} \\
$\stackrel{p}{p}$ \\
+
\end{tabular} & 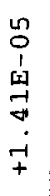 & 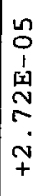 & 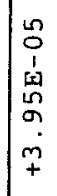 \\
\hline
\end{tabular}

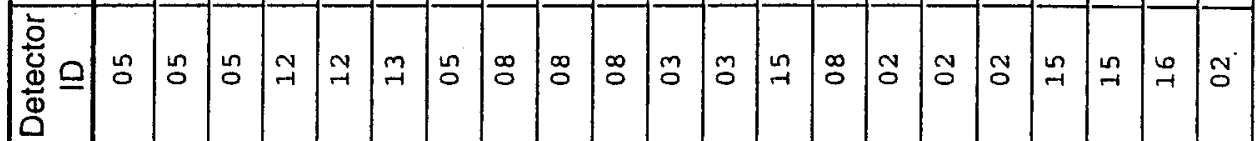

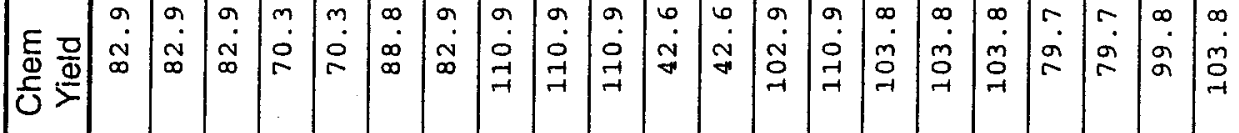

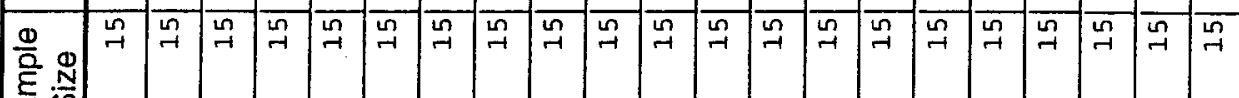
灾

§)

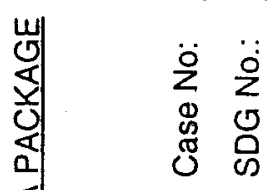

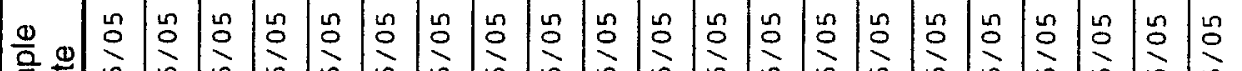

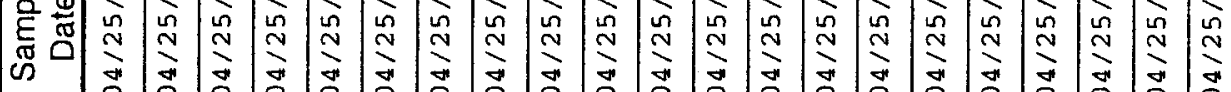

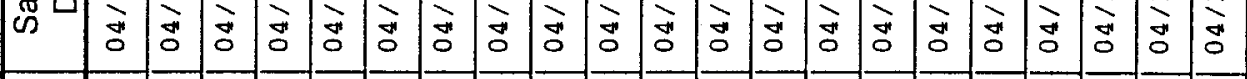

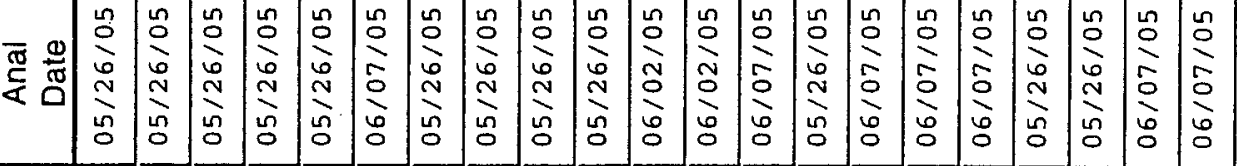

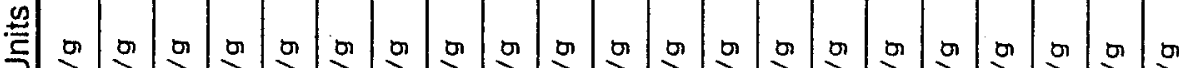

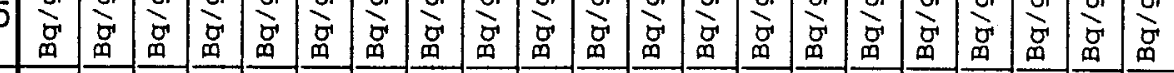

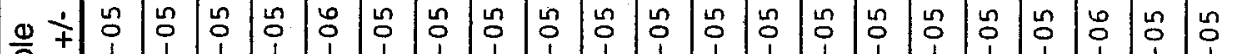
节

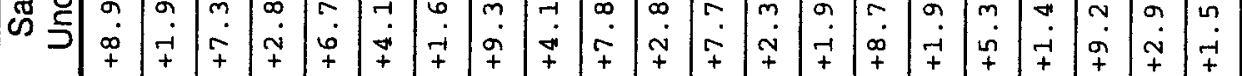
Ф

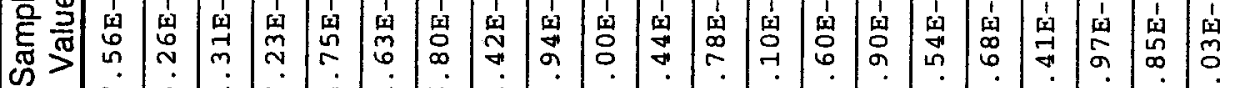

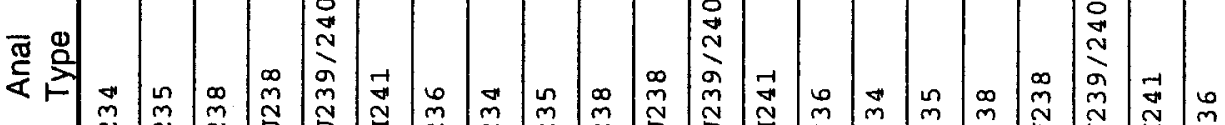
임

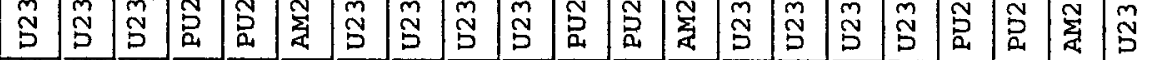
Ф․ㅡ.

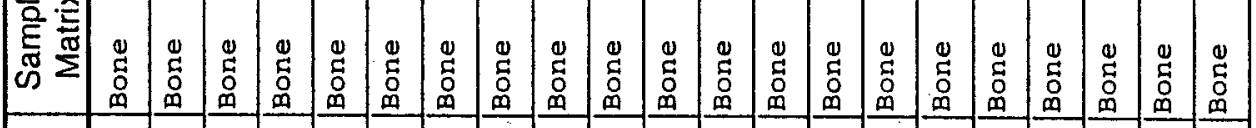
(1) 呈告

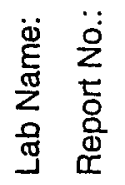

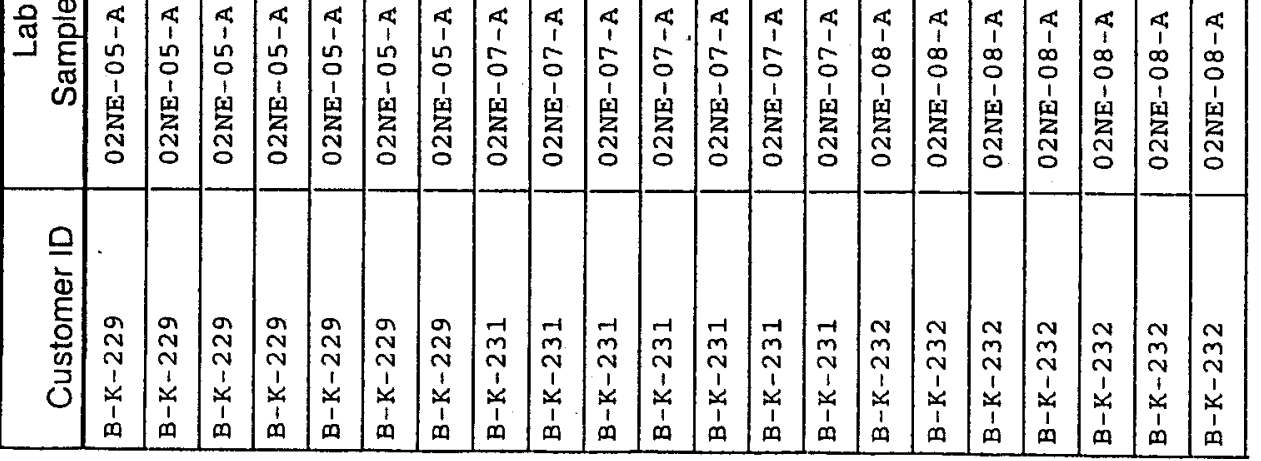




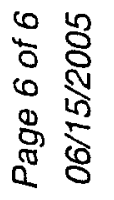
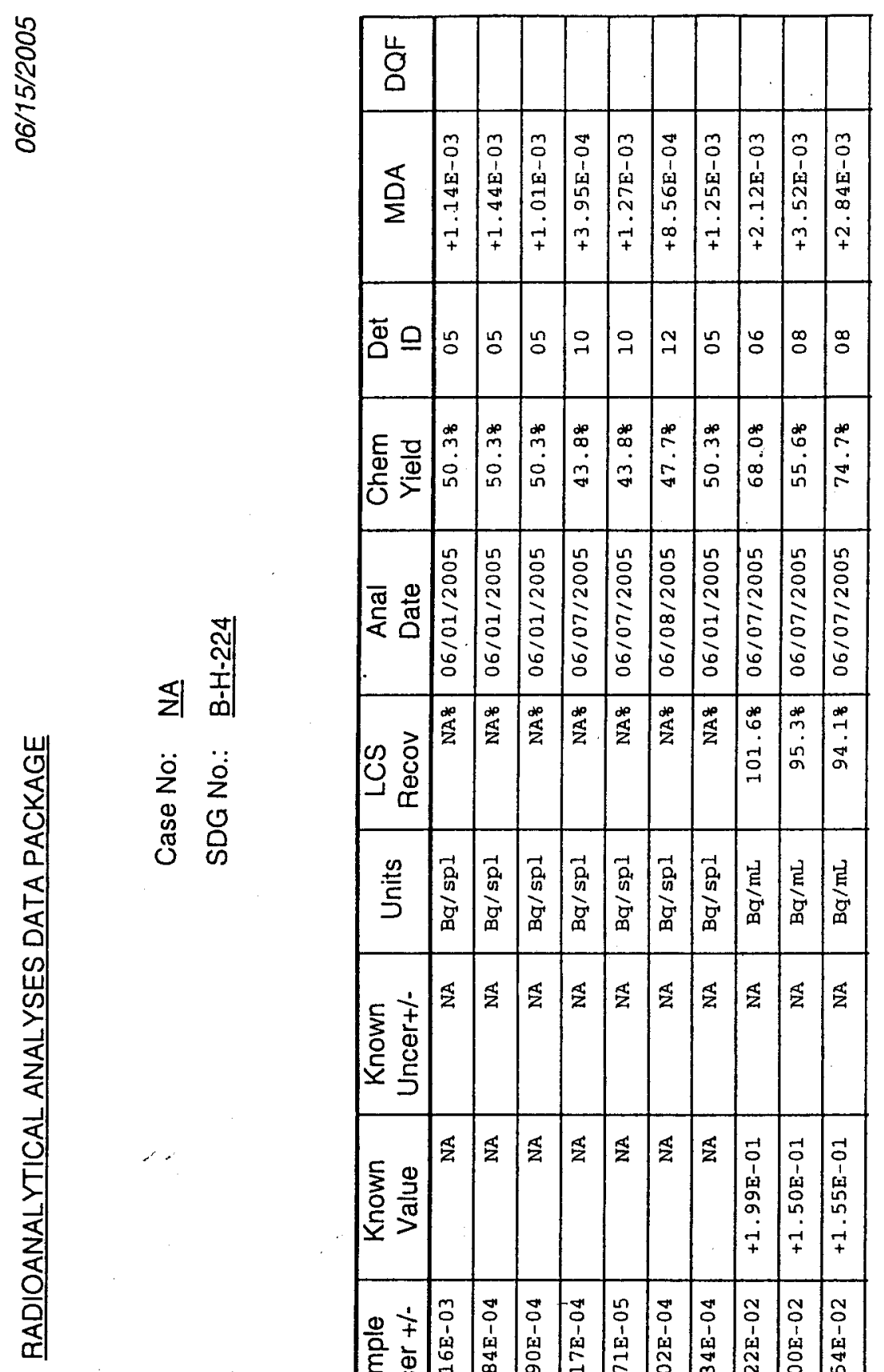

홍으

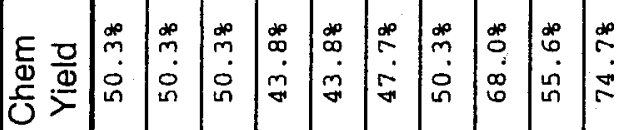

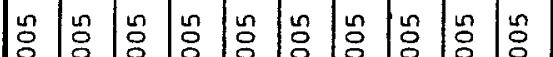

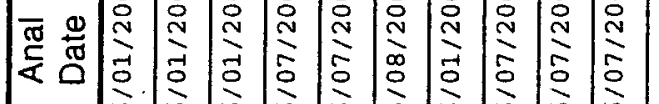

送

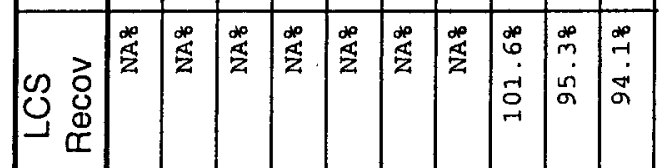

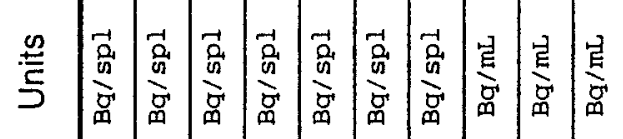

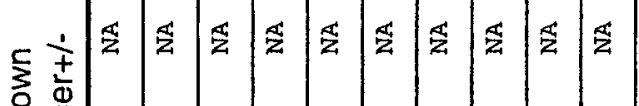

焉

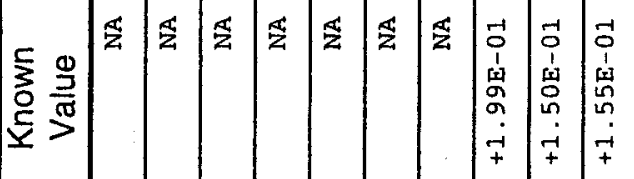

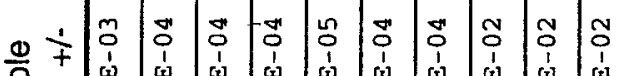

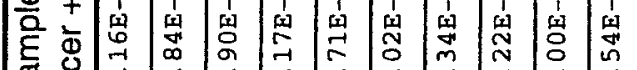

œू

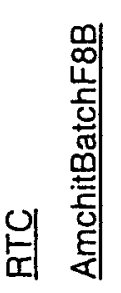

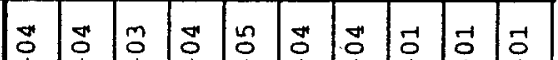

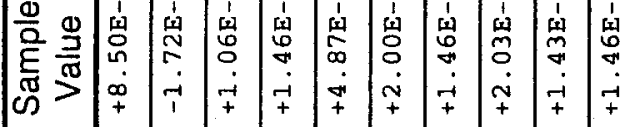

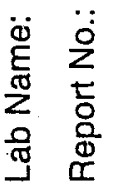

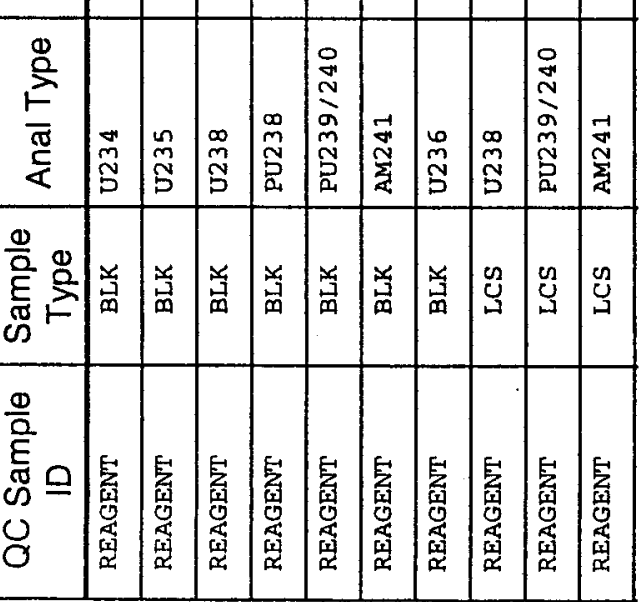


Project: $\quad$ Alpha Analysis for Amchitka Island (Batch 8B)

Laboratory: RTC

Report \#: AmchitBatchF8B

SDG\#: $\quad$ B-K-215

Summary of 2 and 3 sigma activities

Below are the results for U234, U235, U236, U238, Pu238, Pu239/240, and Am241 for Batch 8B from the Amchitka Island Project that had a result/uncertainty ratio of 2 or more (uncertainty @ one sigma).

\begin{tabular}{|c|c|c|c|c|c|}
\hline Customer ID & Lab ID & Isotope & $\begin{array}{c}\text { Result } \\
\mathrm{Bg} / \mathrm{g}\end{array}$ & $\begin{array}{c}\text { Uncertainty } \\
\mathrm{Bg} / \mathrm{g}\end{array}$ & $\begin{array}{c}\text { Result } \\
\text { Uncertainty }\end{array}$ \\
\hline $\mathrm{B}-\mathrm{H}-230$ & 02NE-06-A & U234 & $2.39 \mathrm{E}-04$ & $1.04 \mathrm{E}-04$ & 2.3 \\
\hline B-K-228 & 02NE-04-A & AM241 & 2.66E-02 & 2.67E-03 & 10.0 \\
\hline B-K-228 & O2NE-04-A & PU238 & 3.68E-02 & $4.25 E-03$ & 8.7 \\
\hline B-K-228 & 02NE-04-A & PU239/240 & 4.61E-02 & $5.21 \mathrm{E}-03$ & 8.8 \\
\hline B-K-231 & 02NE-07-A & U238 & 2.00E-04 & $7.86 \mathrm{E}-05$ & 2.5 \\
\hline
\end{tabular}

All known sources of uncertainty are included in the uncertainty term. There may be unknown sources of uncertainty that are not accounted for. If the result/uncertainty ratio is more than 3 , we have a degree of confidence that the result is positive (i.e. the result is - statistically different than zero). A result with the result/uncertainty ratio between 2 and 3 is the first indication that an isotope may be present and further investigation may be warranted.

As with any good science no single data point is used in important decisions (results need to be reproducible). 


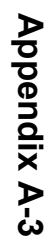


Appendix A-3

Tc-99 Analysis 
BATCH 1

Tc-99 


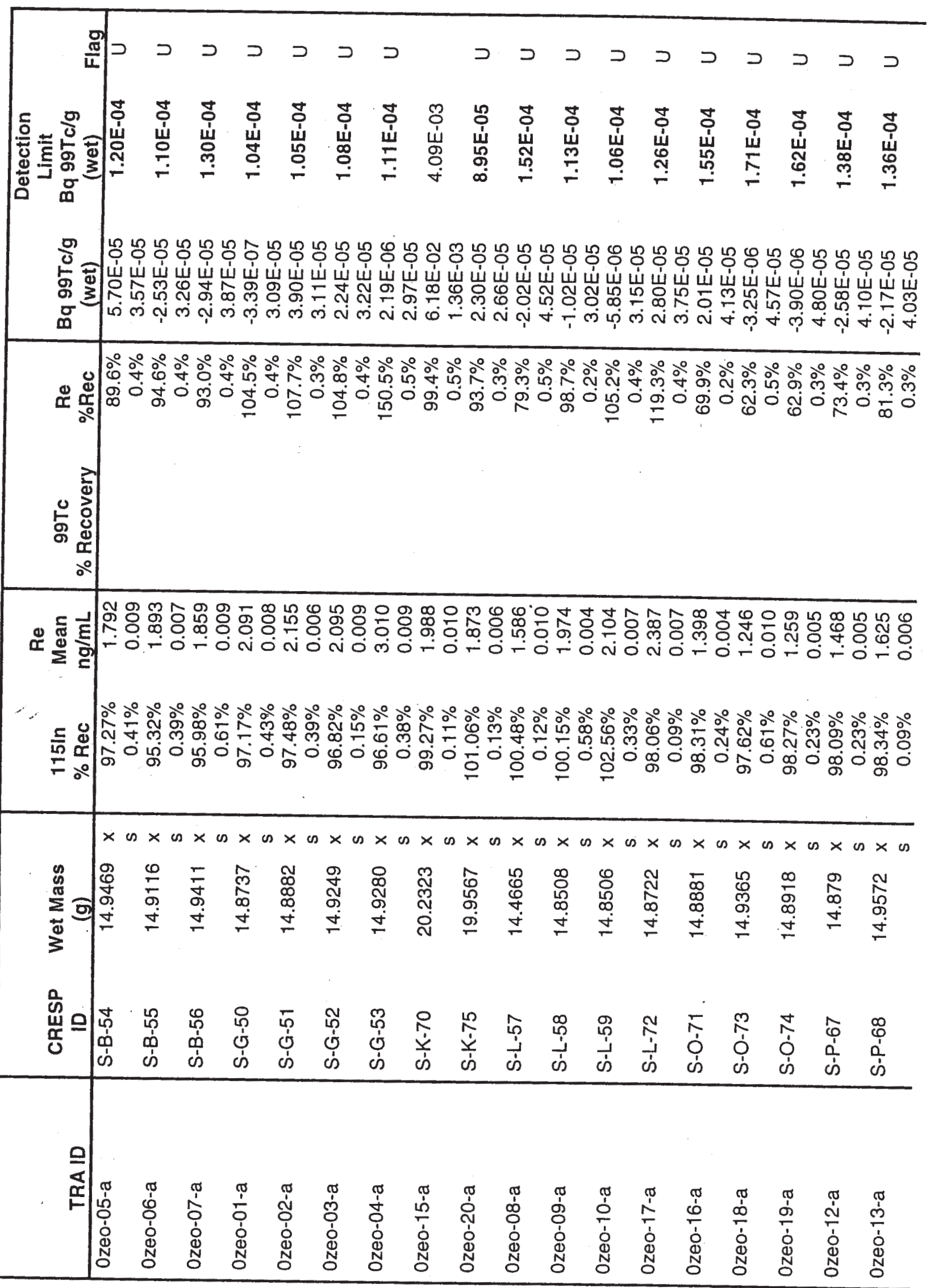




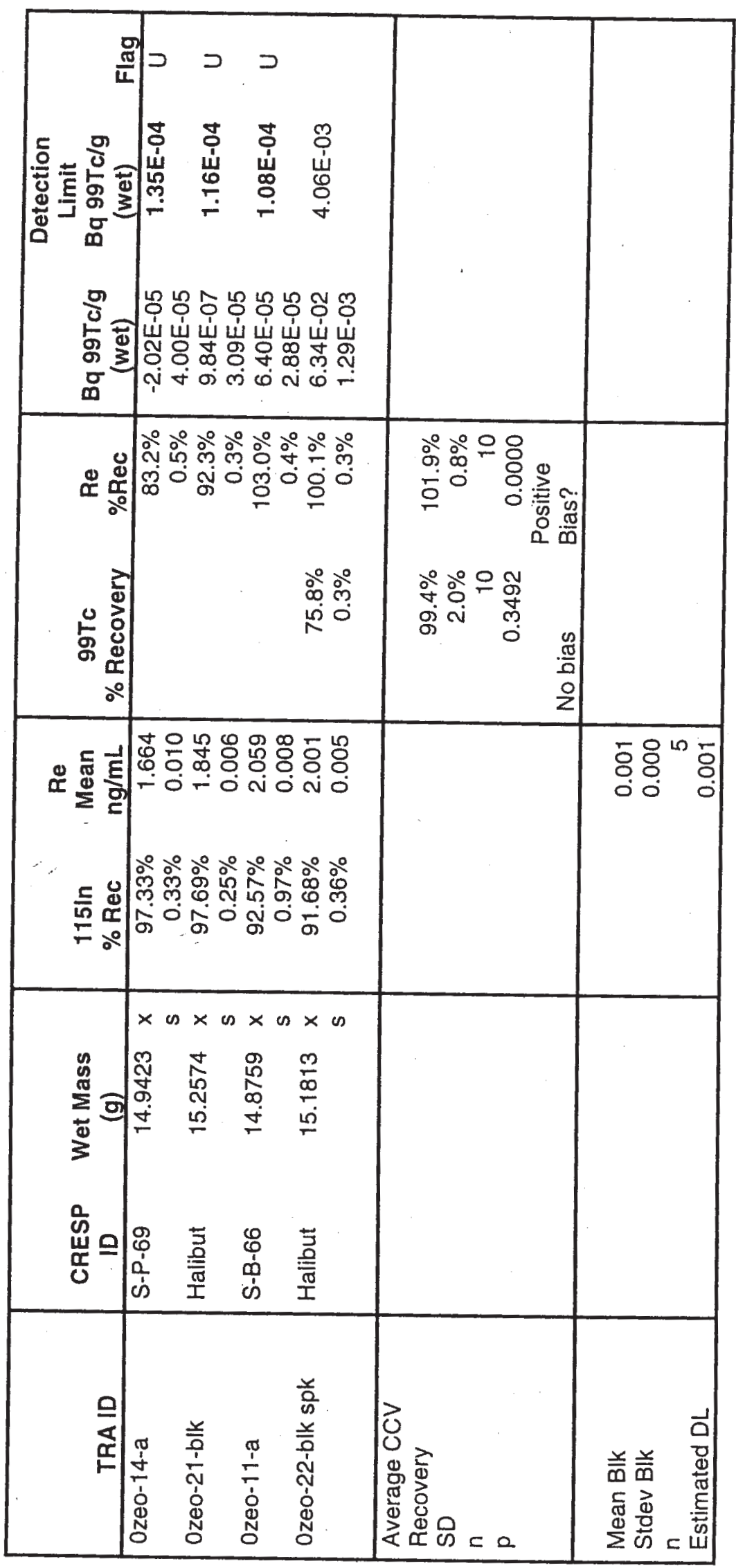




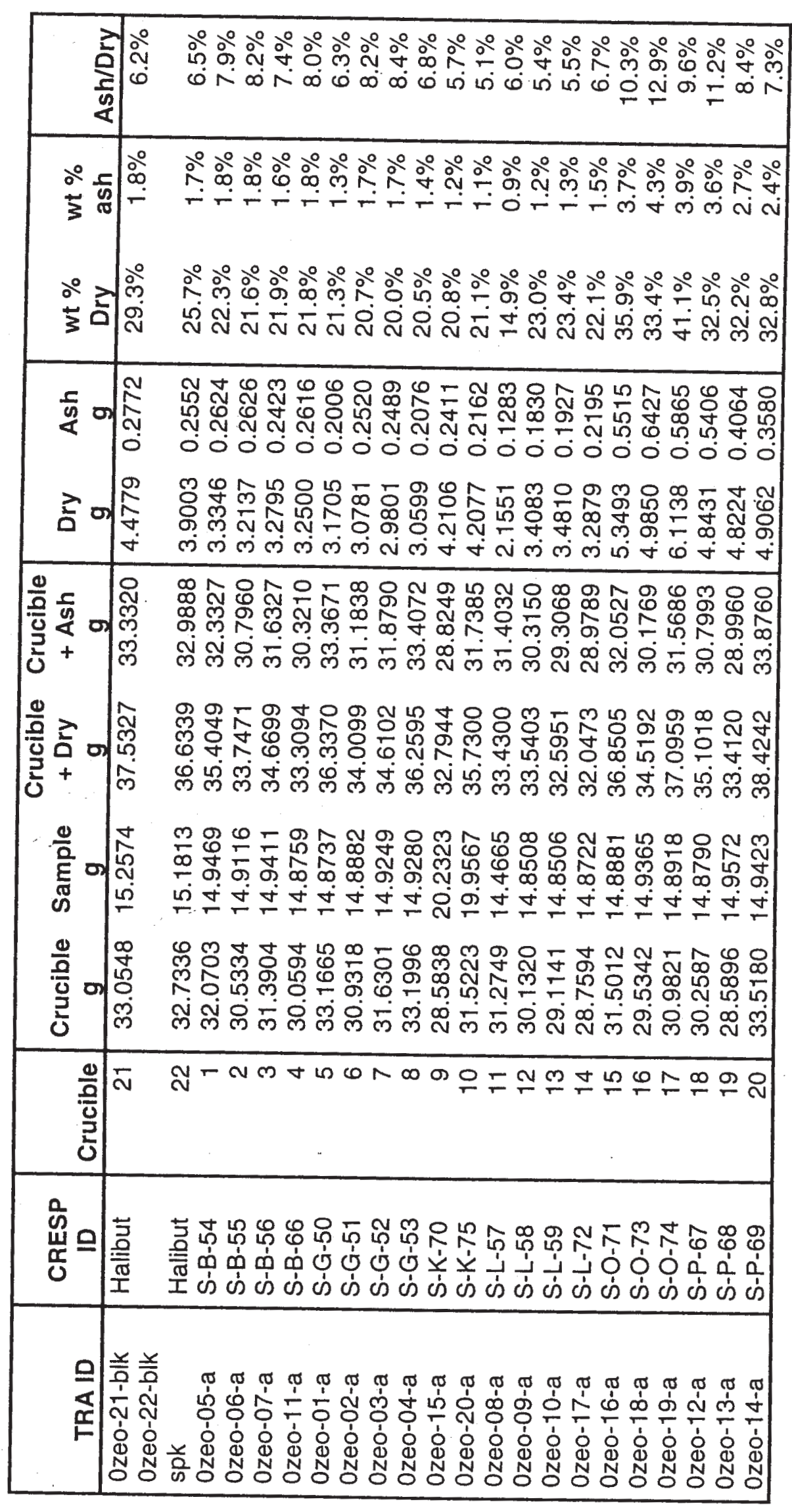




\section{BATCH 2}

Tc-99 


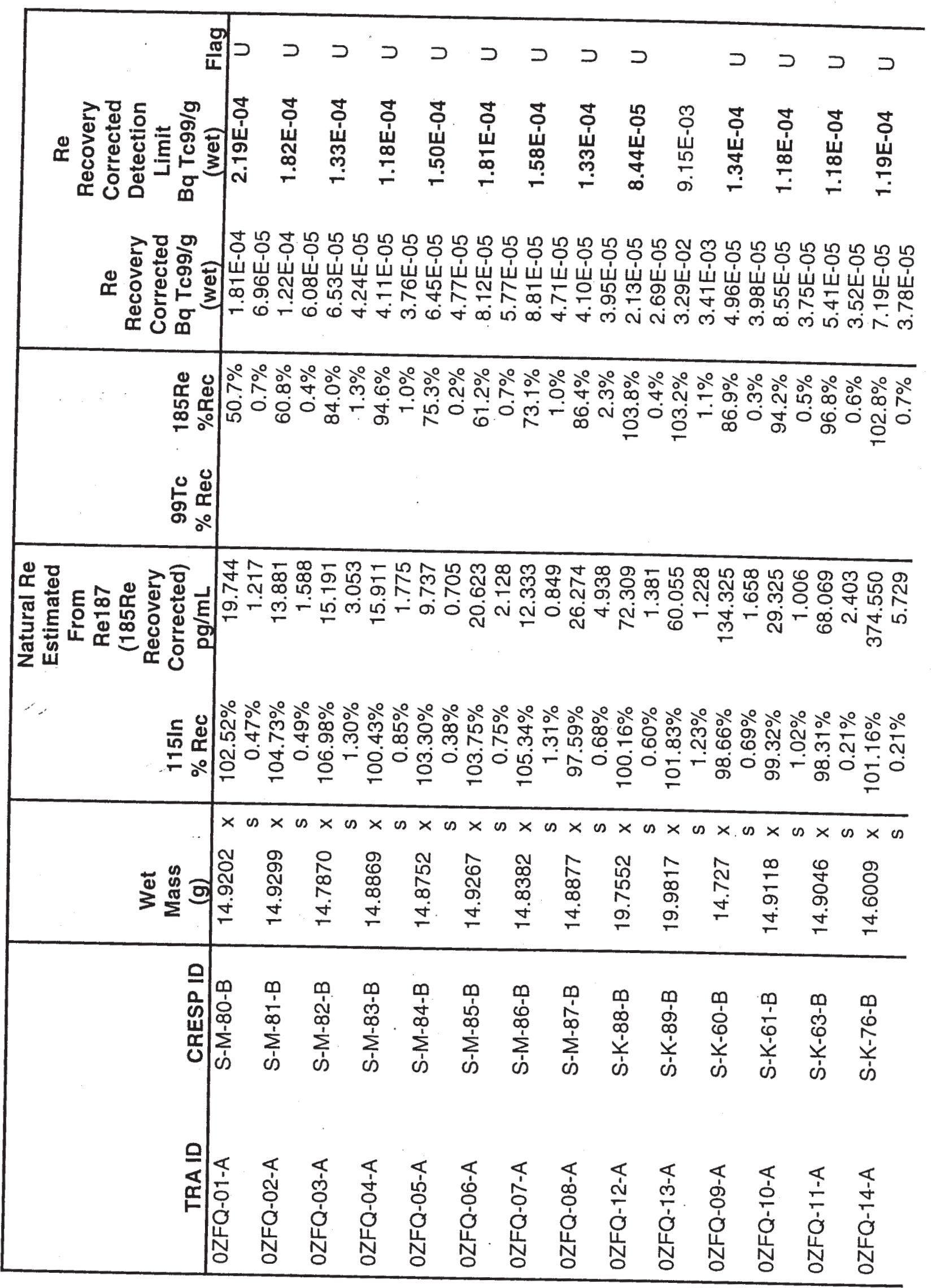




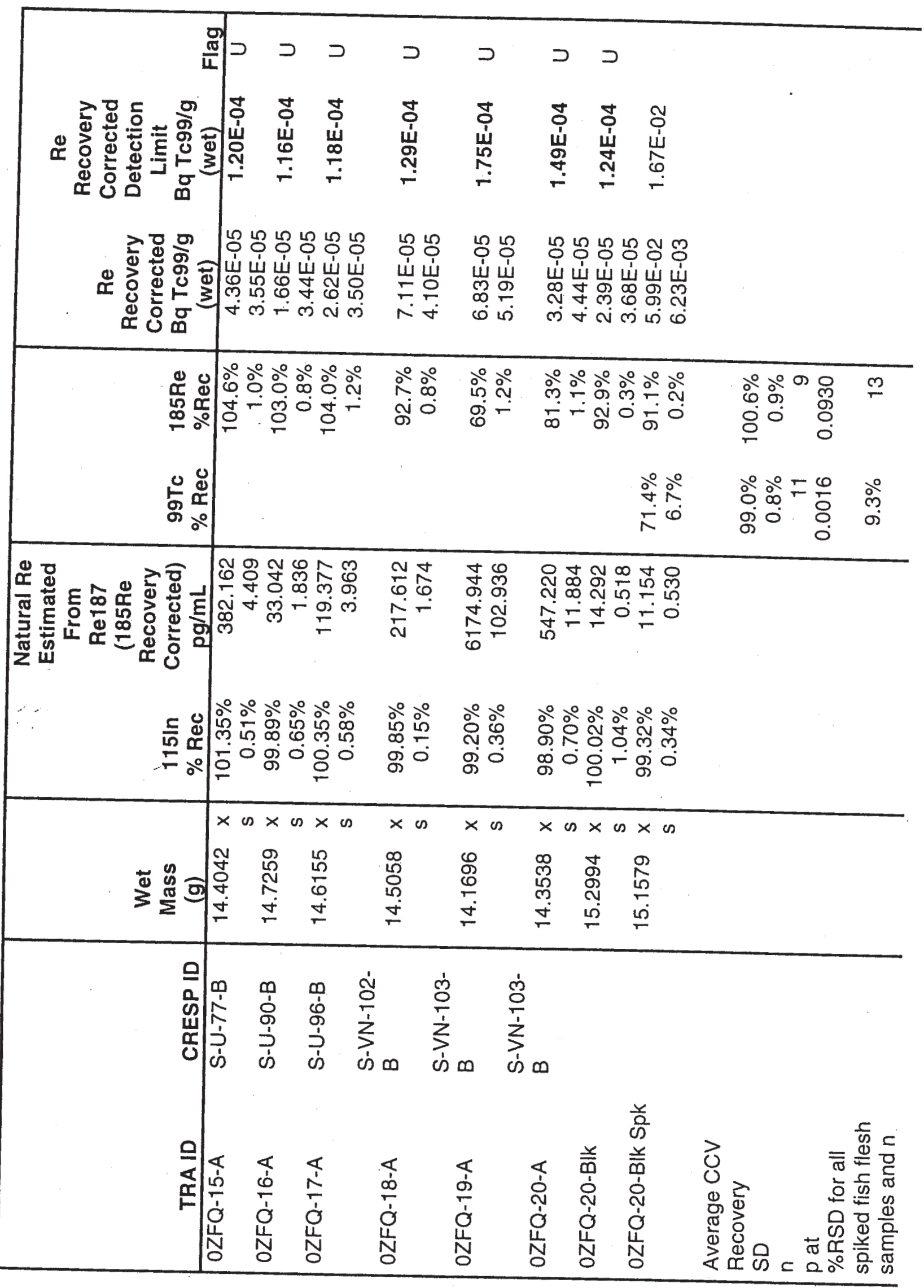




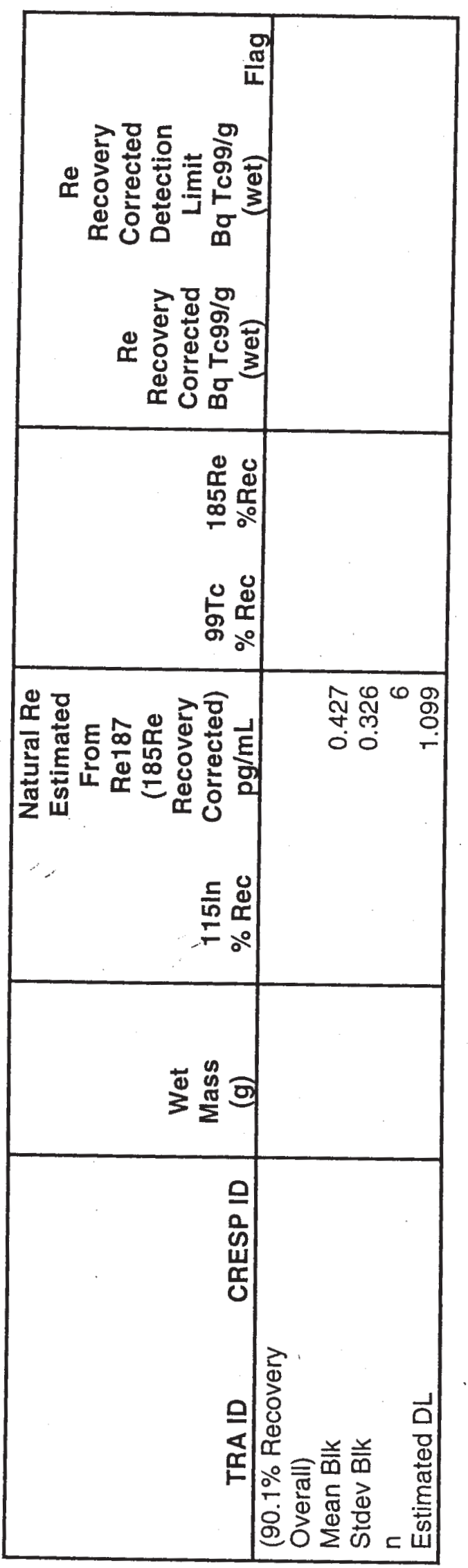




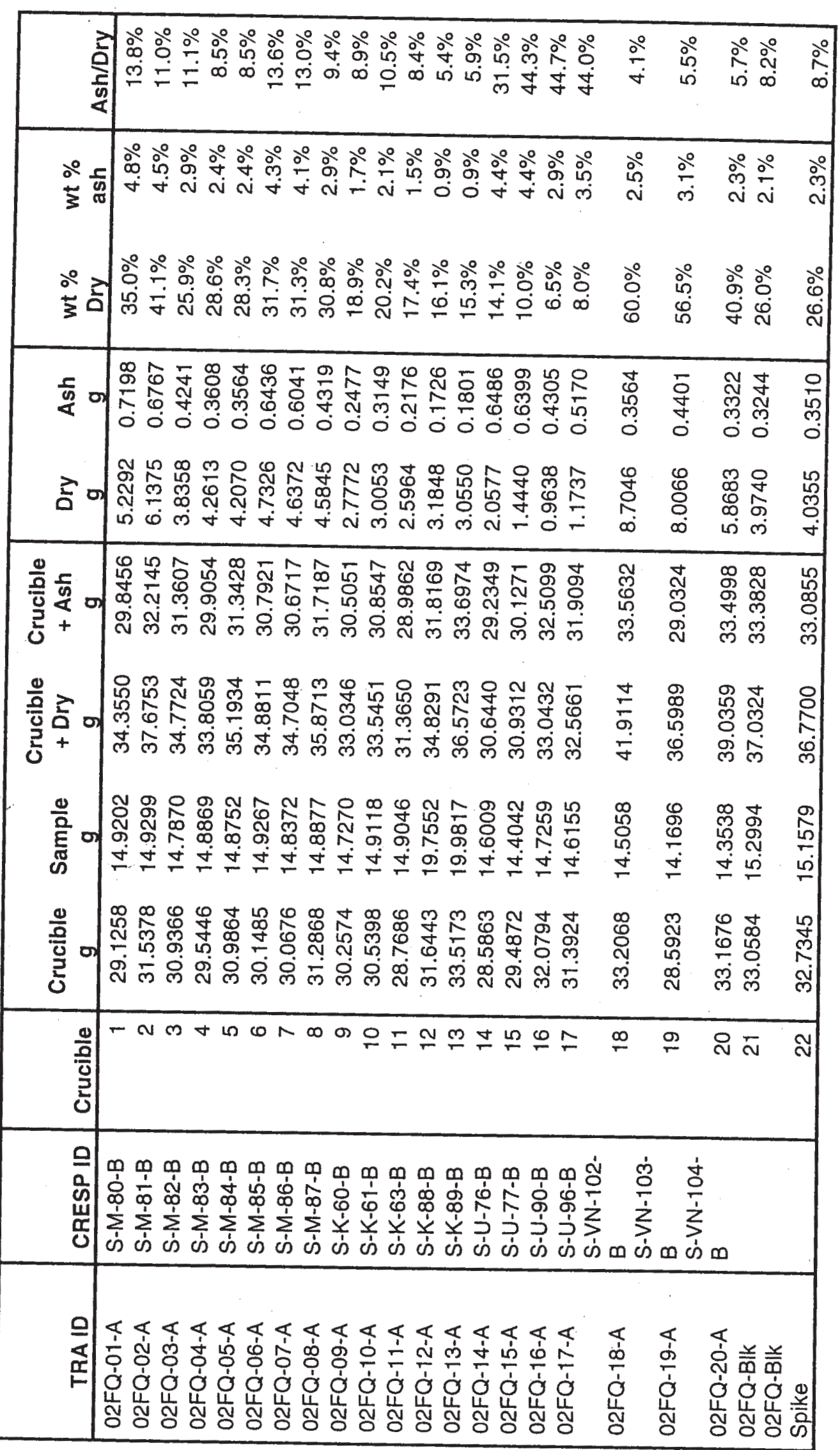


BATCH 3

Tc-99 


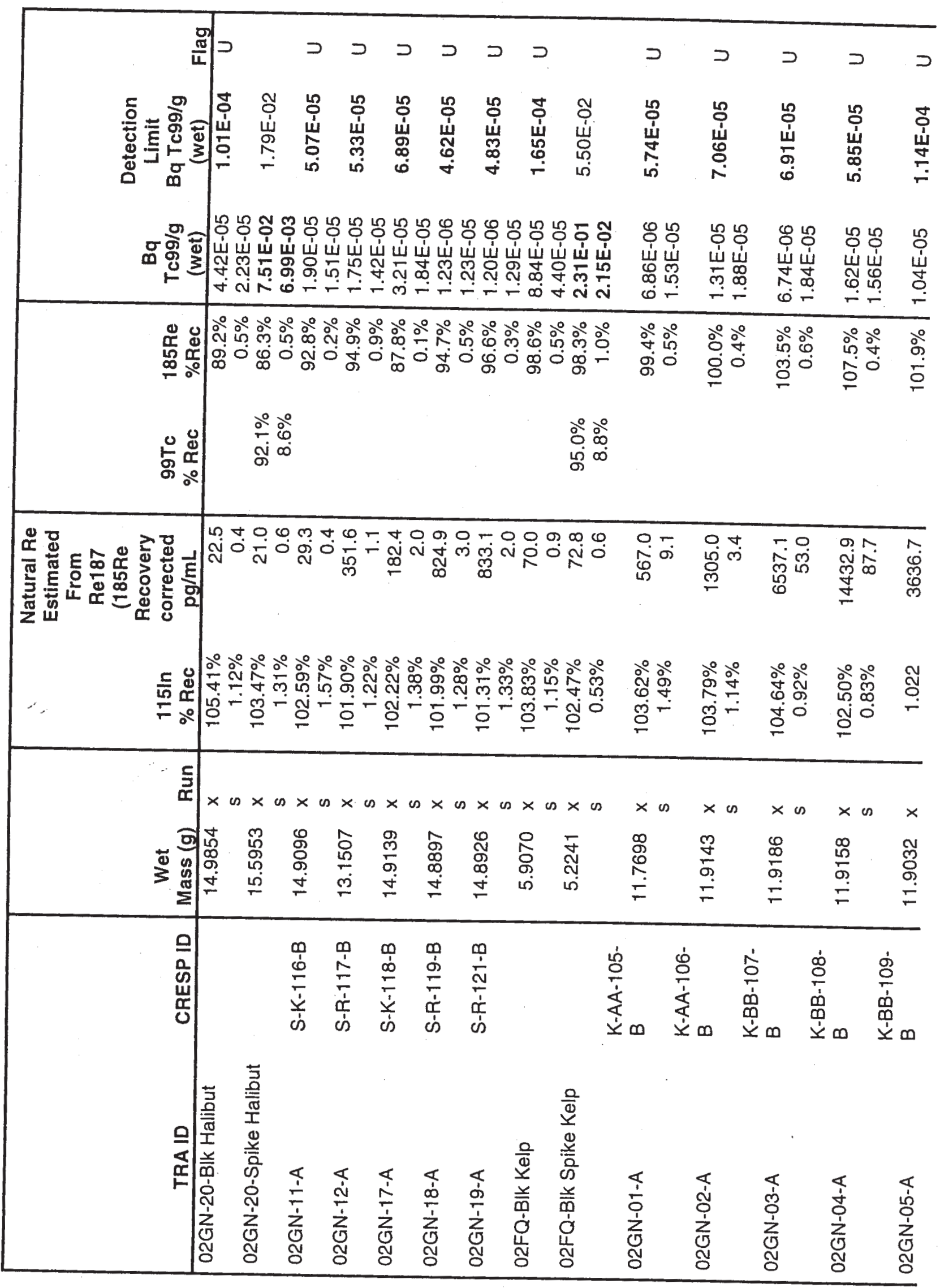




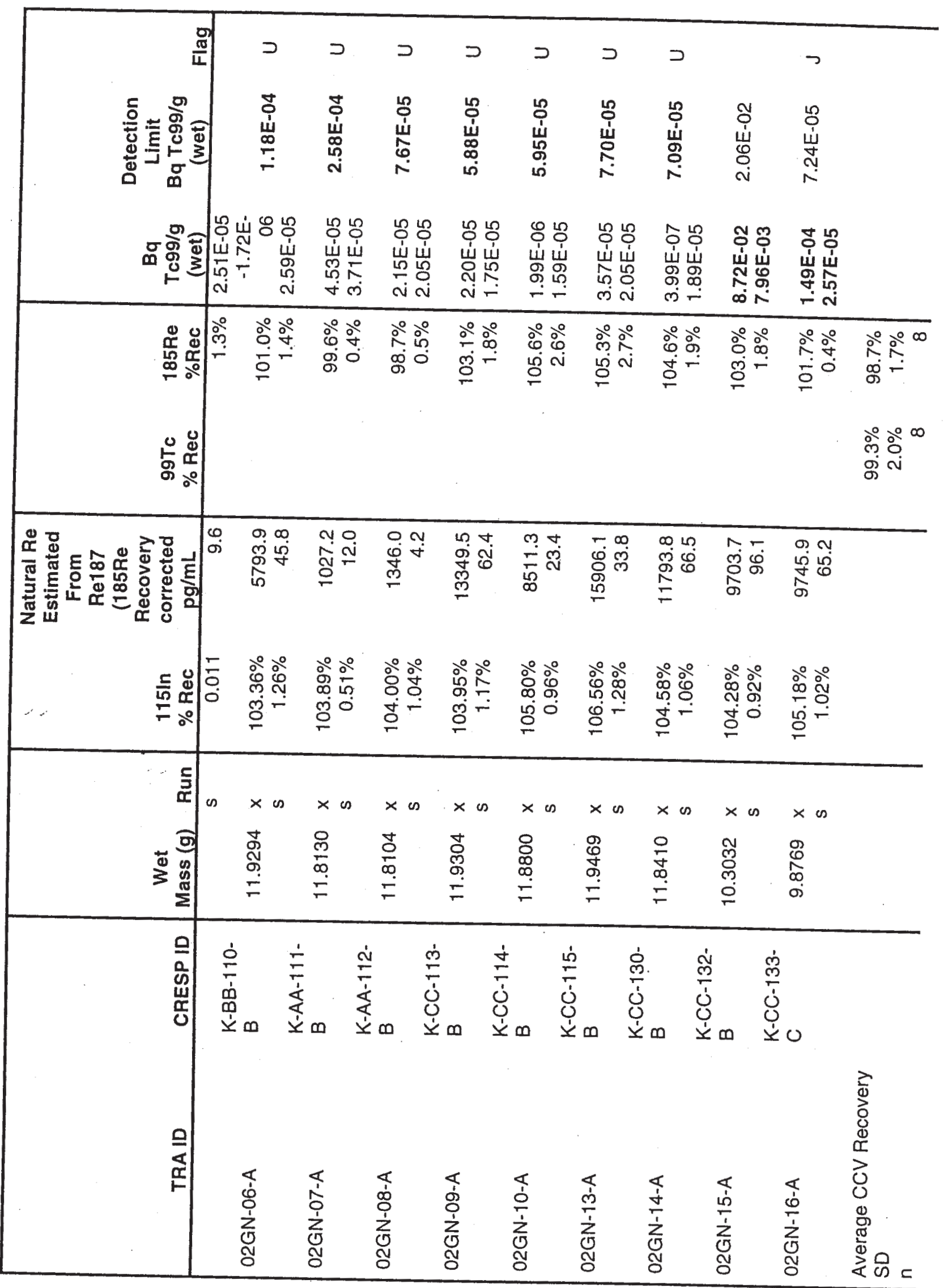




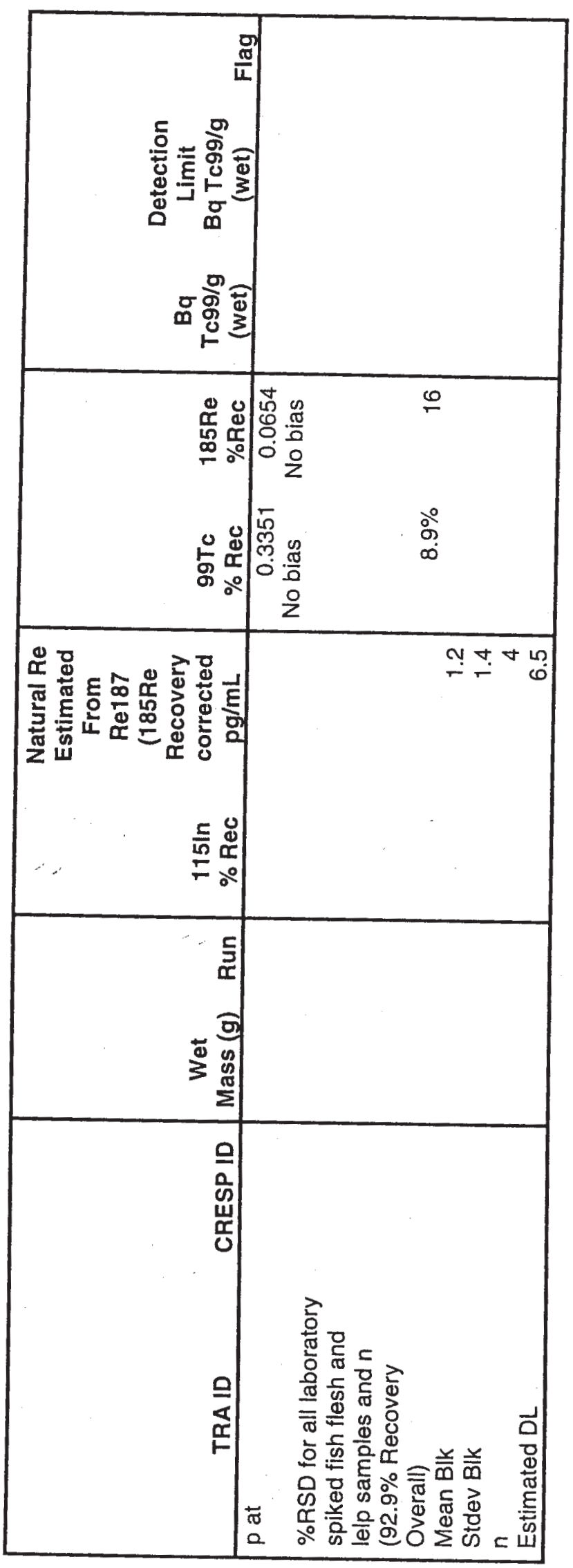




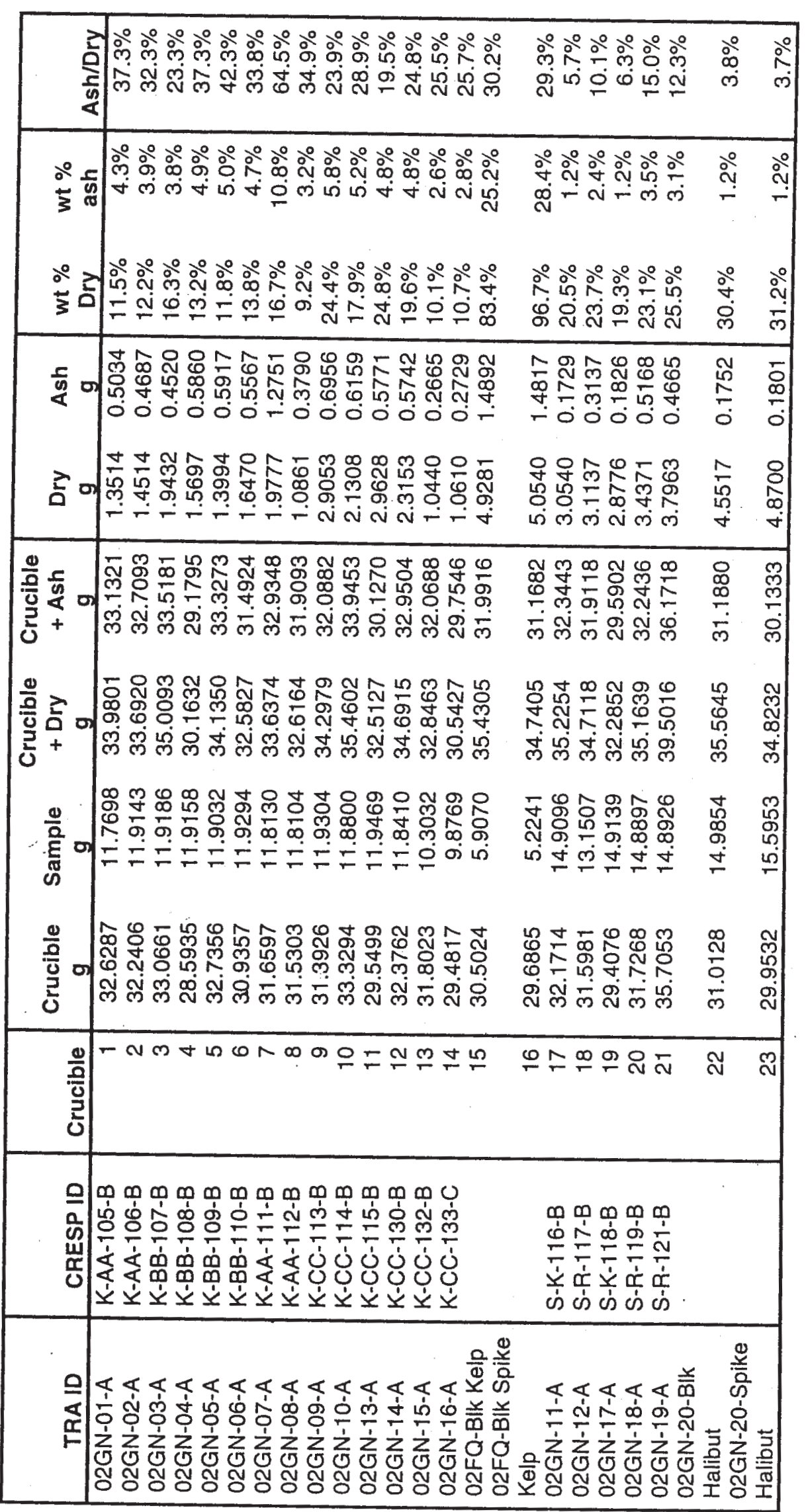


BATCH 6 Tc-99 


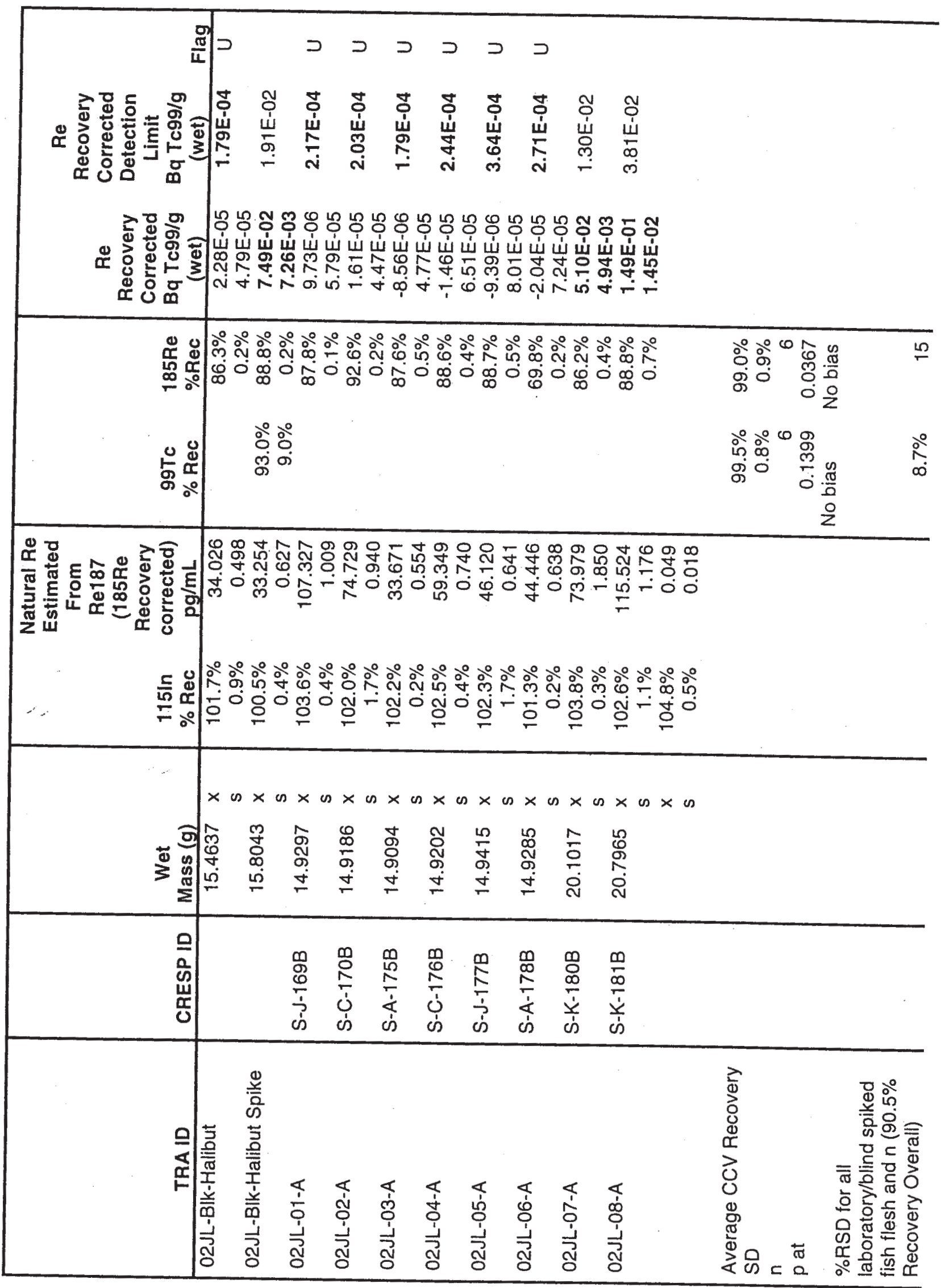




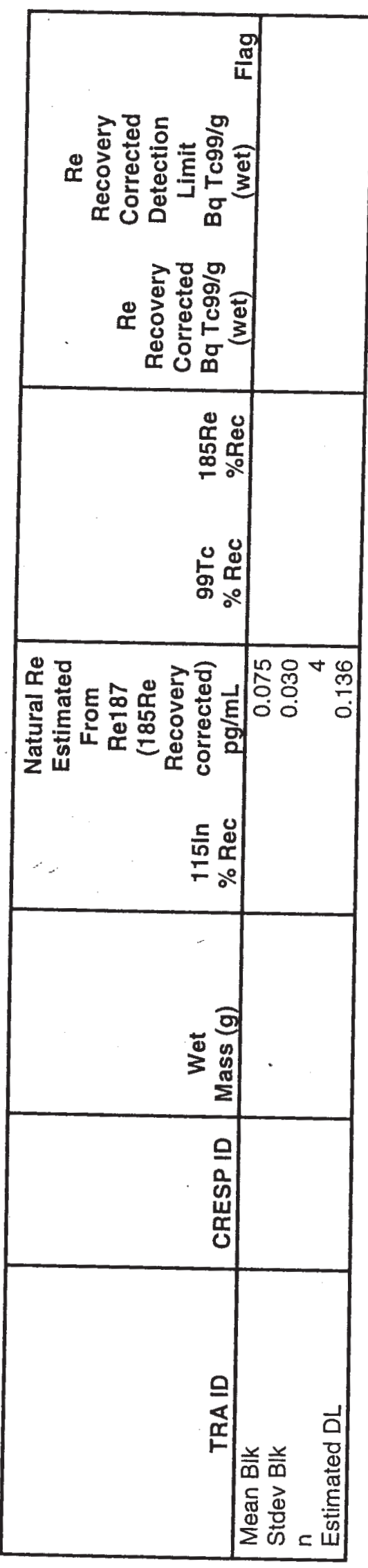




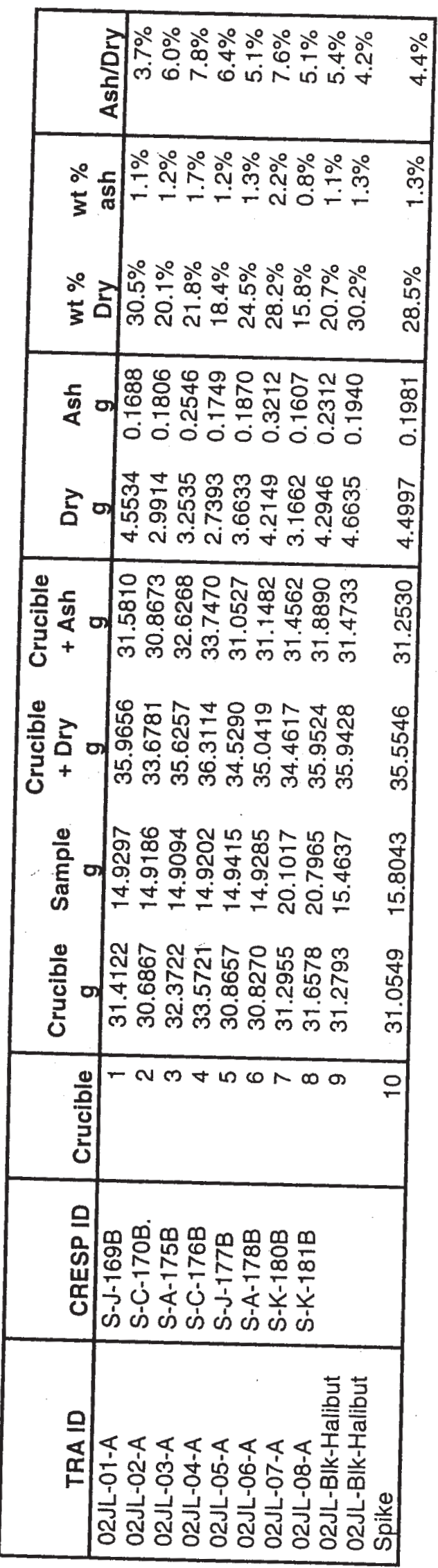


Appendix B

Results of Performance Evaluation Standards And Blind QC Samples 
Results of Performance Evaluation Standards 


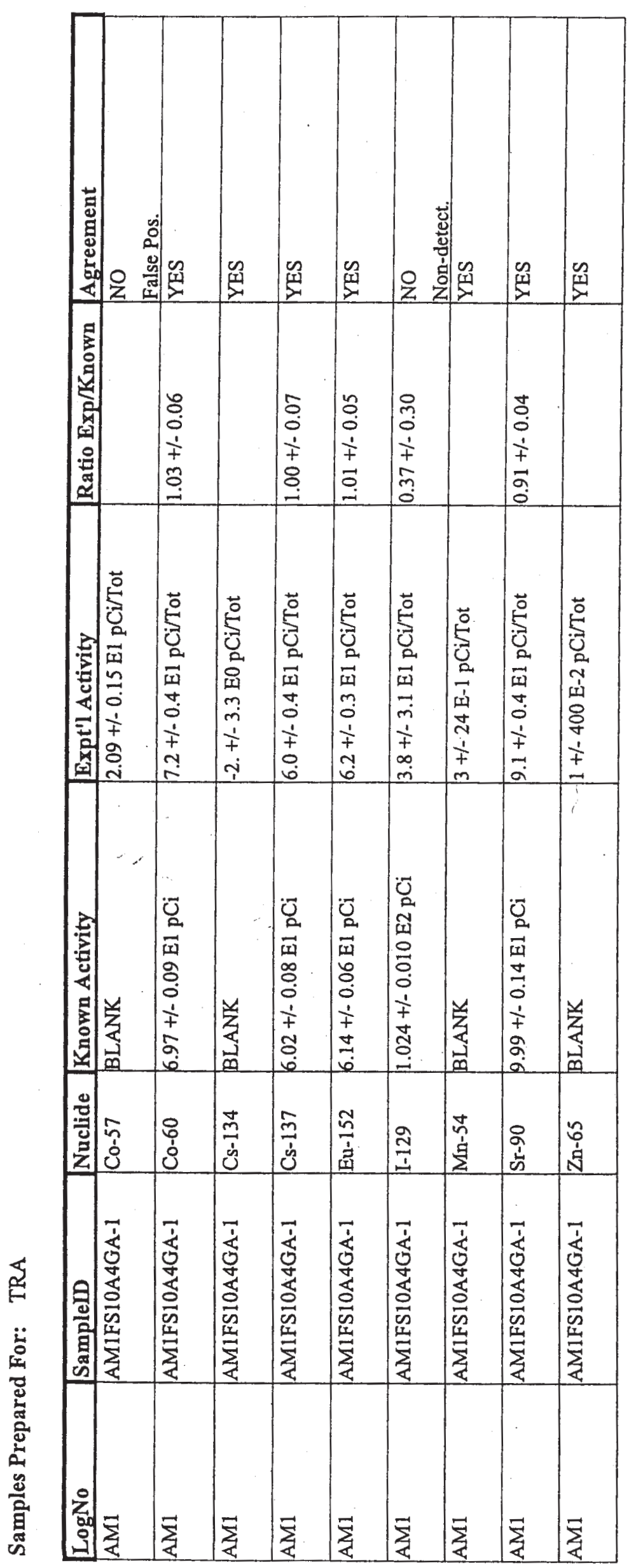

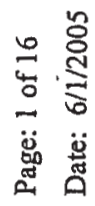

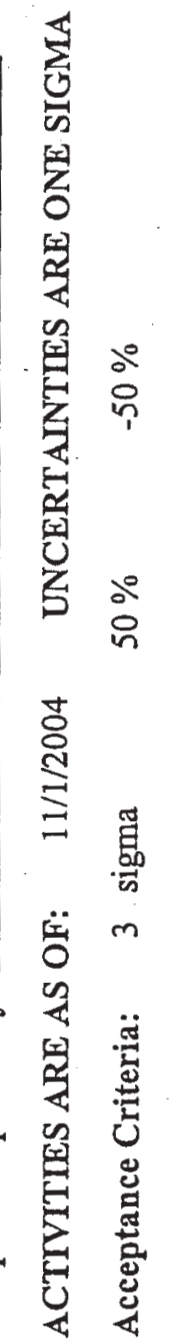




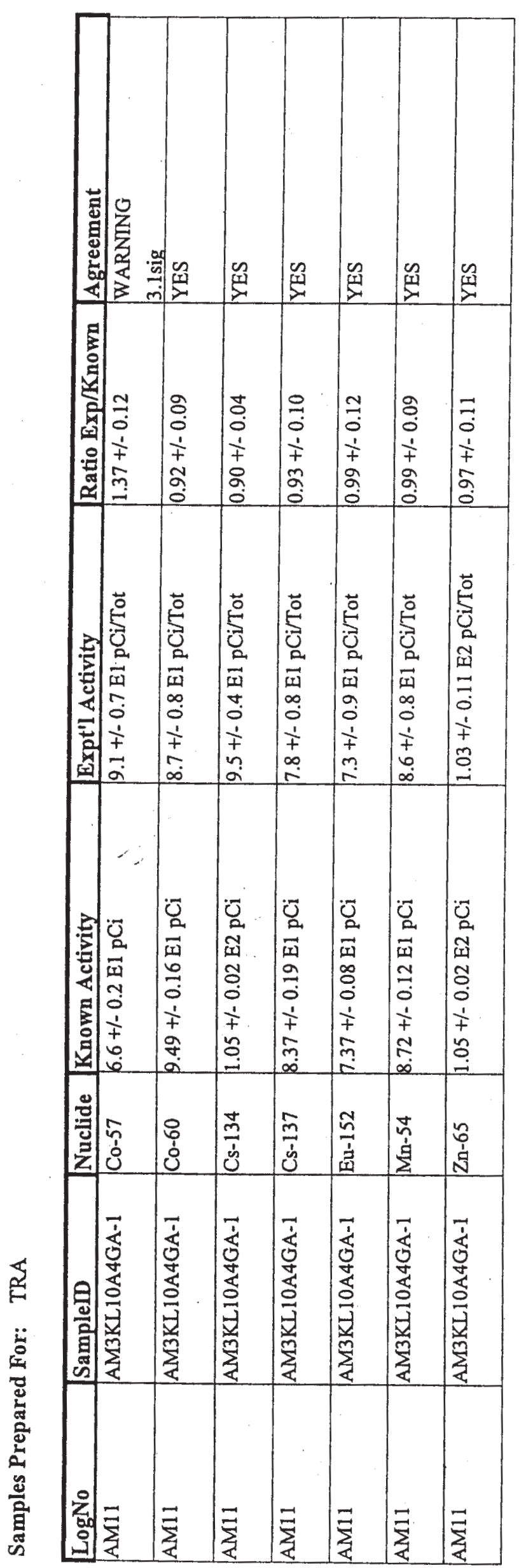

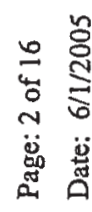

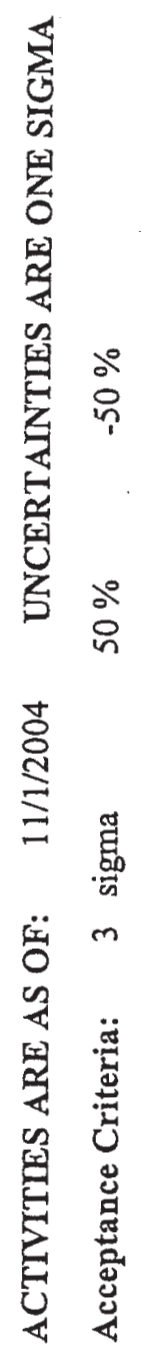




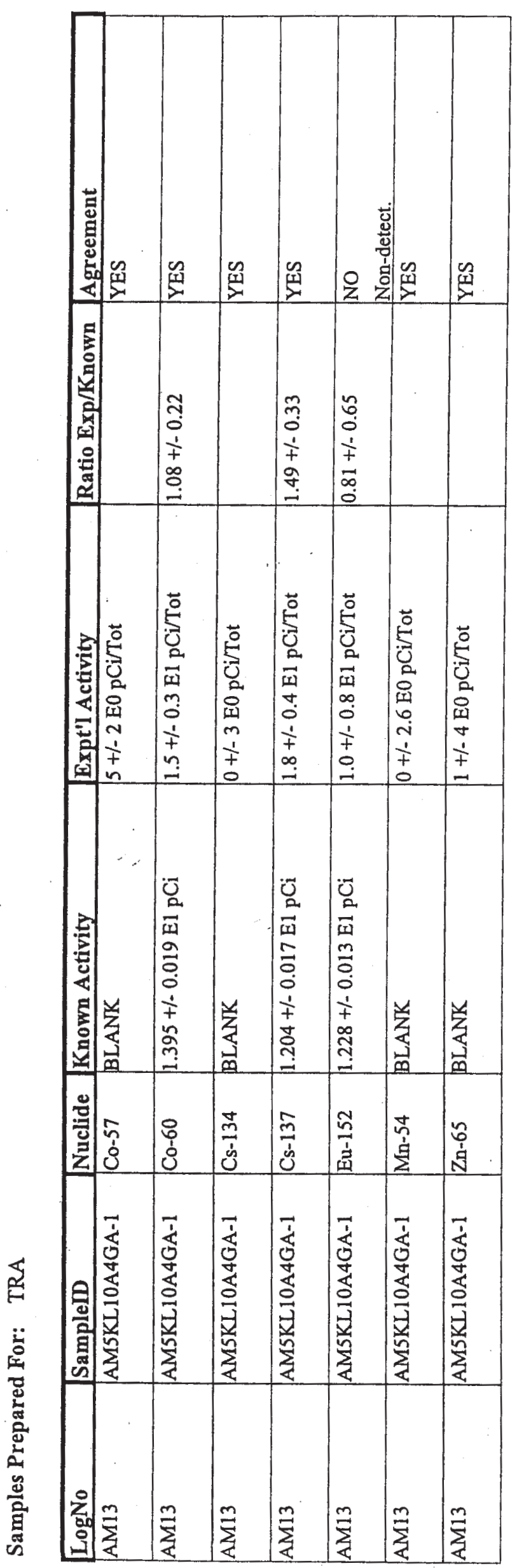

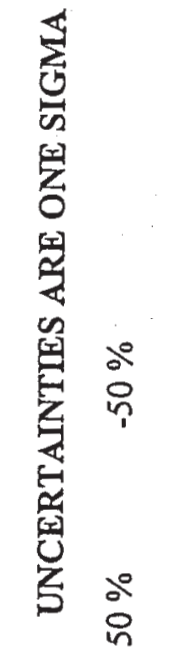

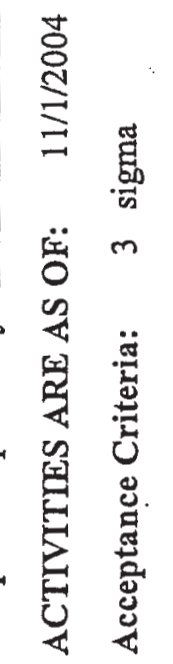



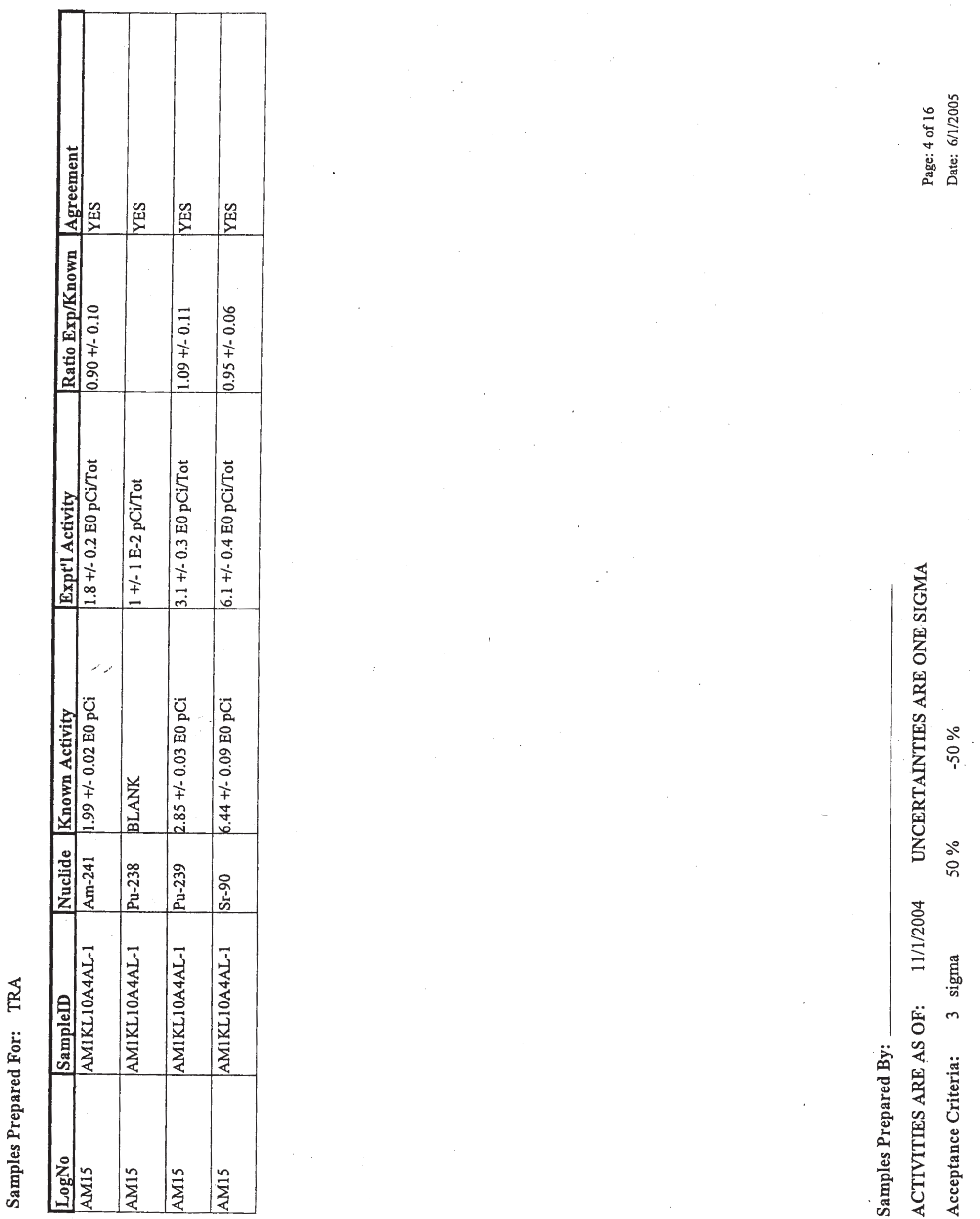

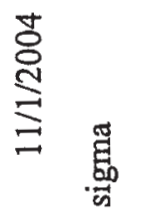

$\ddot{0} m$

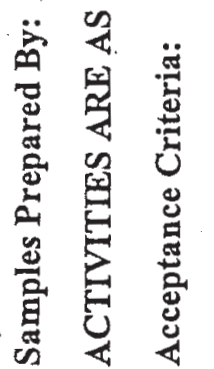



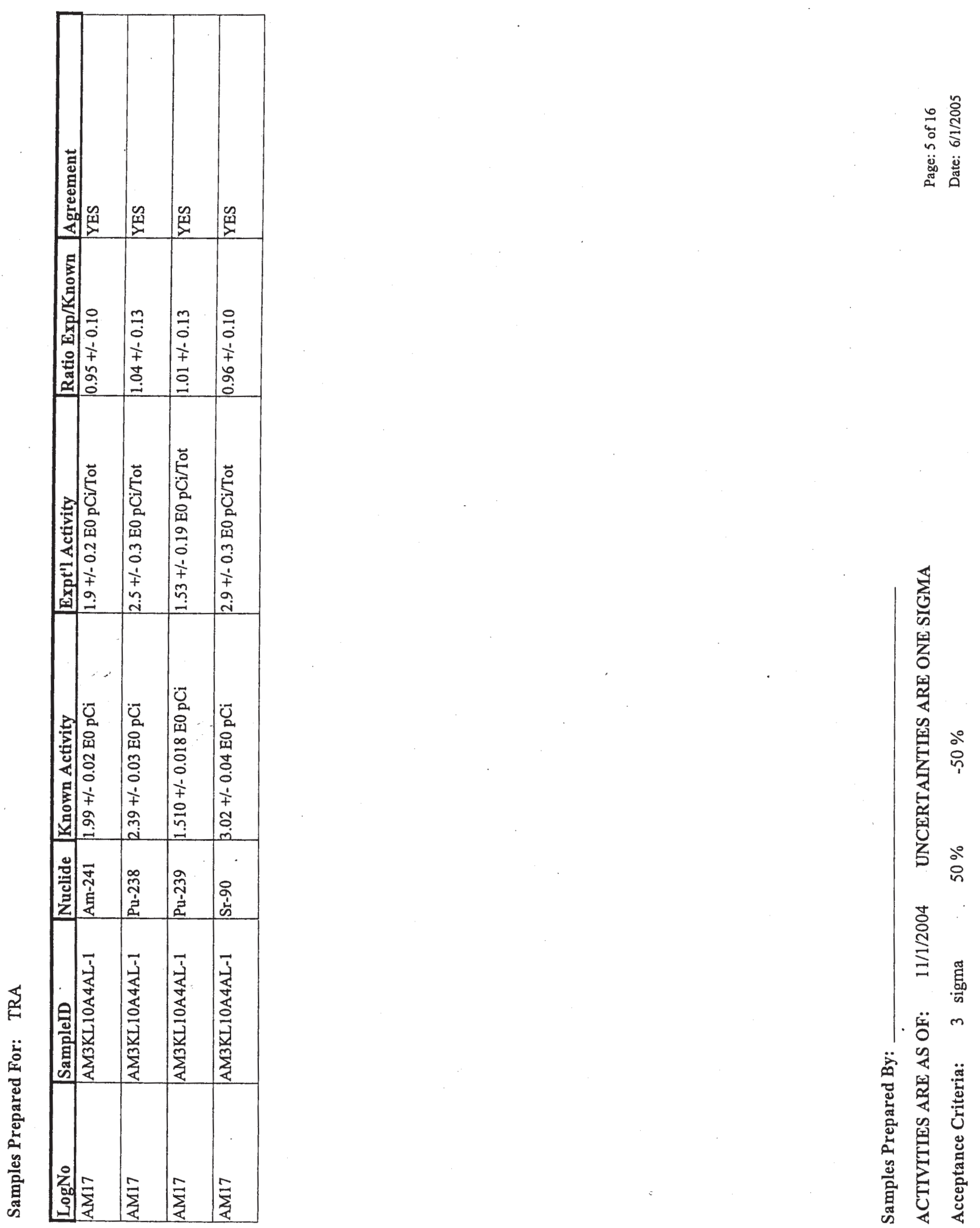

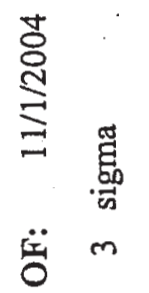

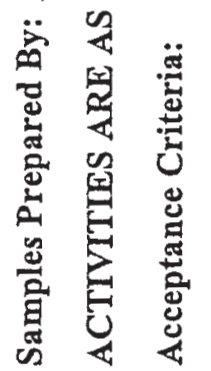



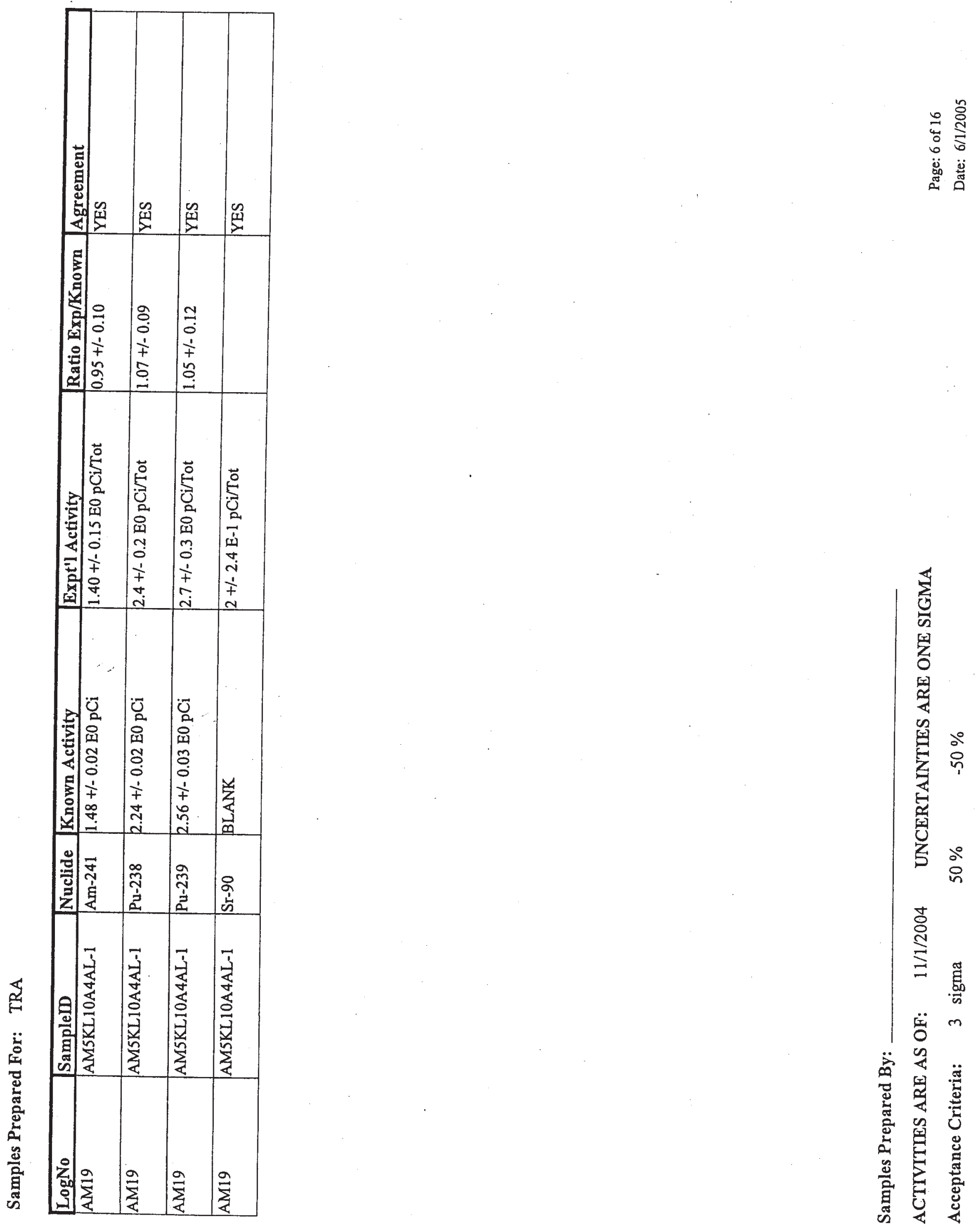

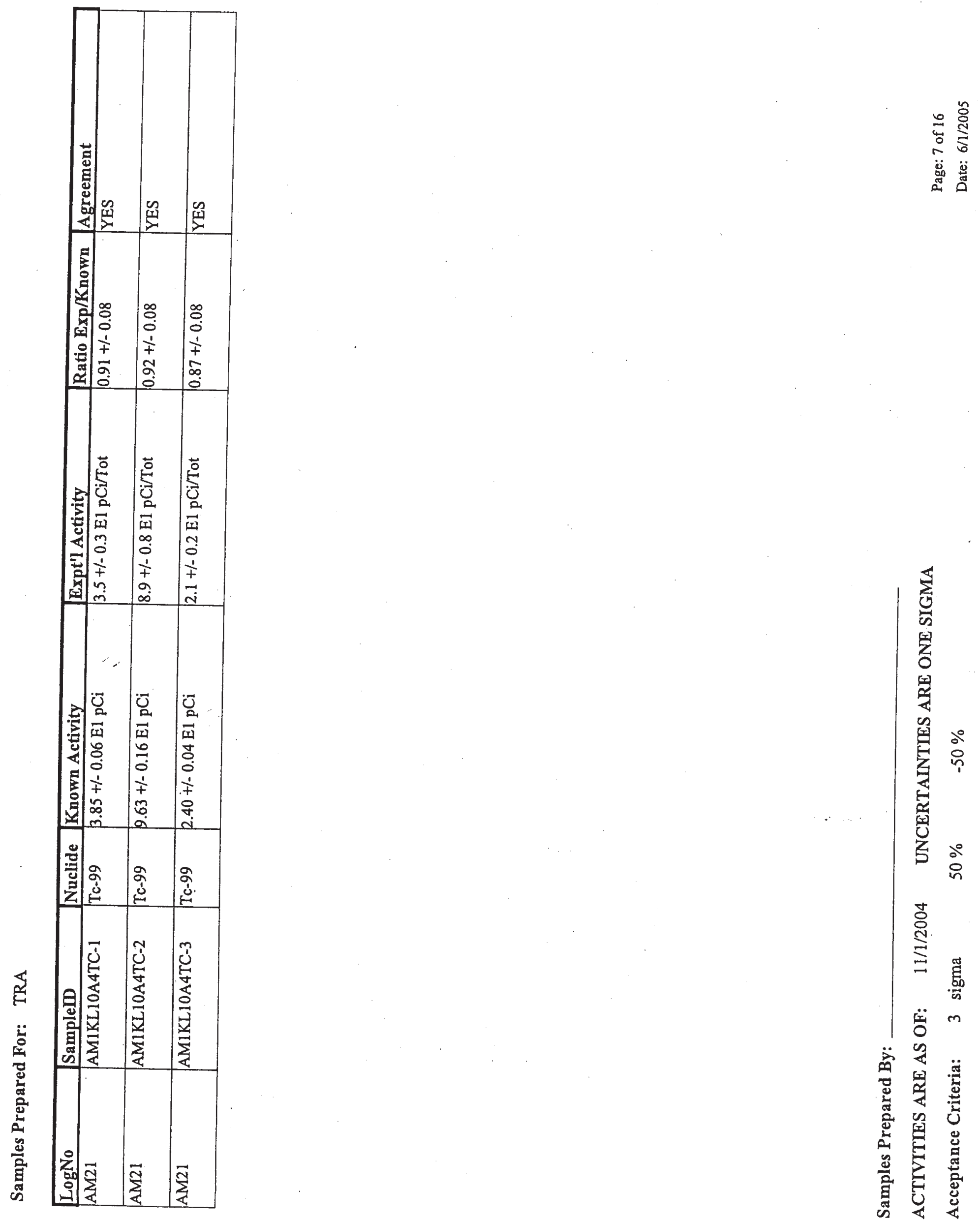

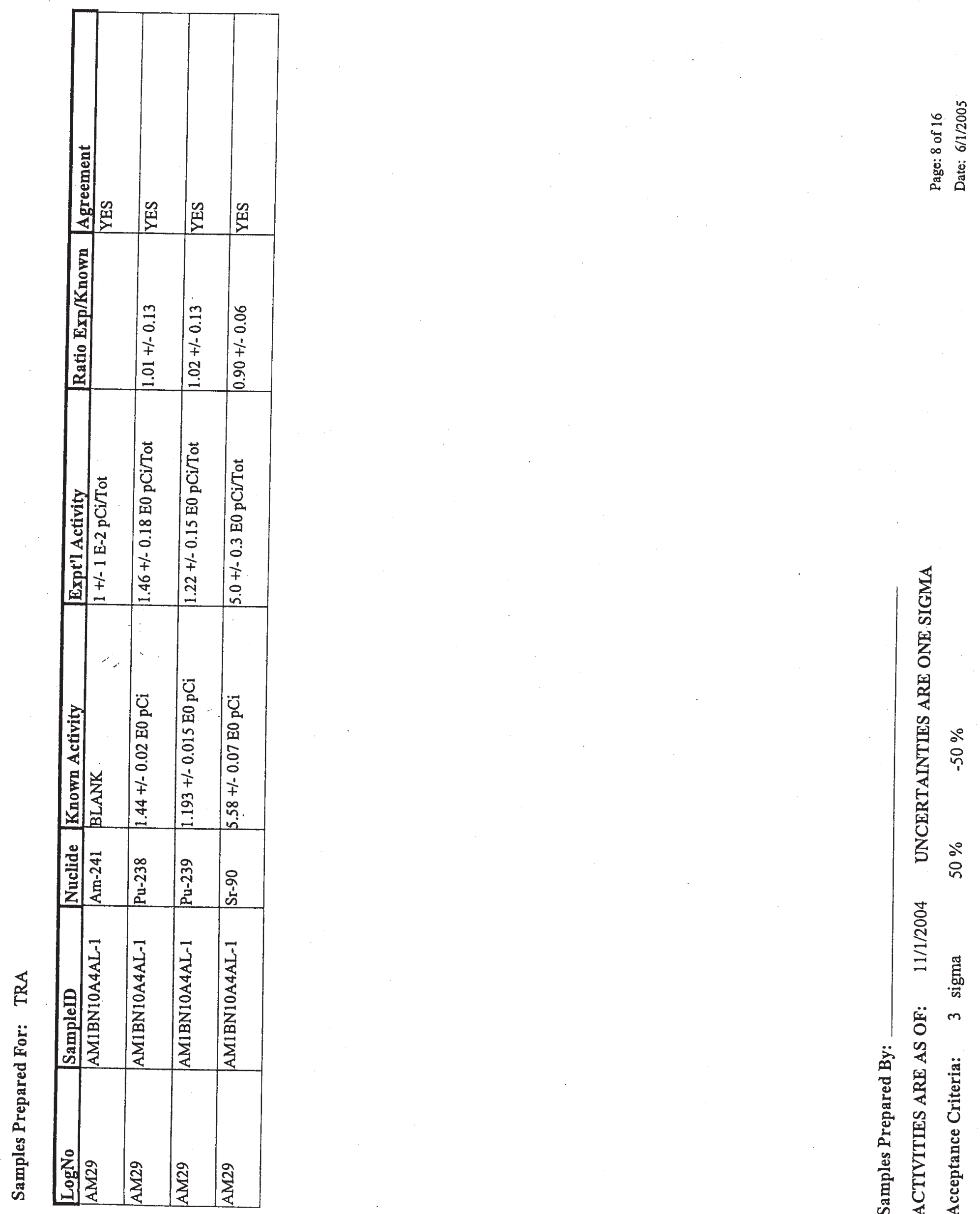

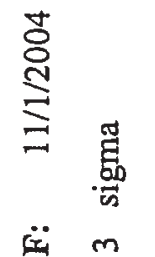

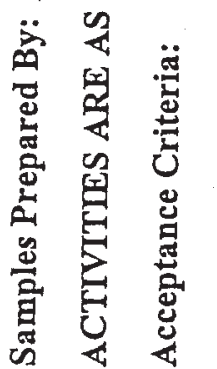




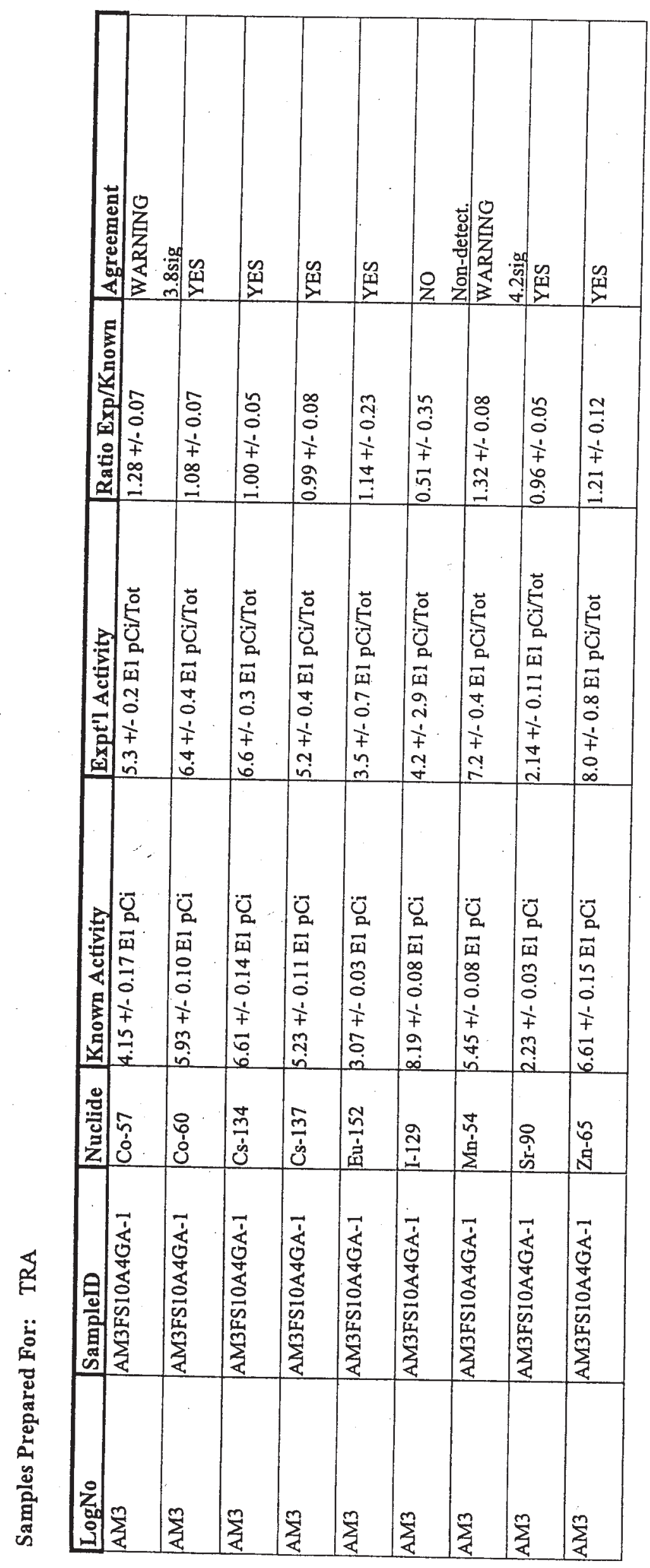



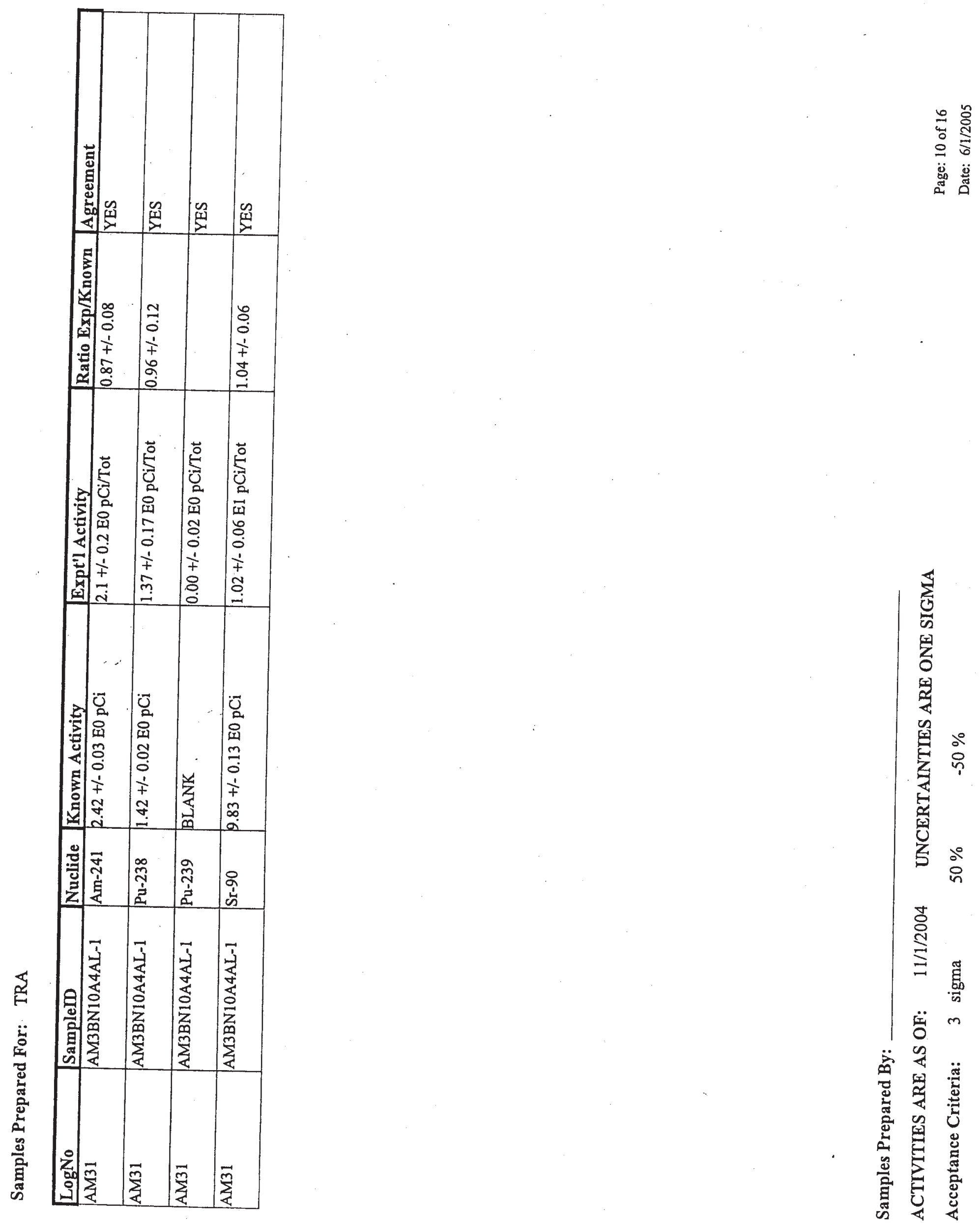

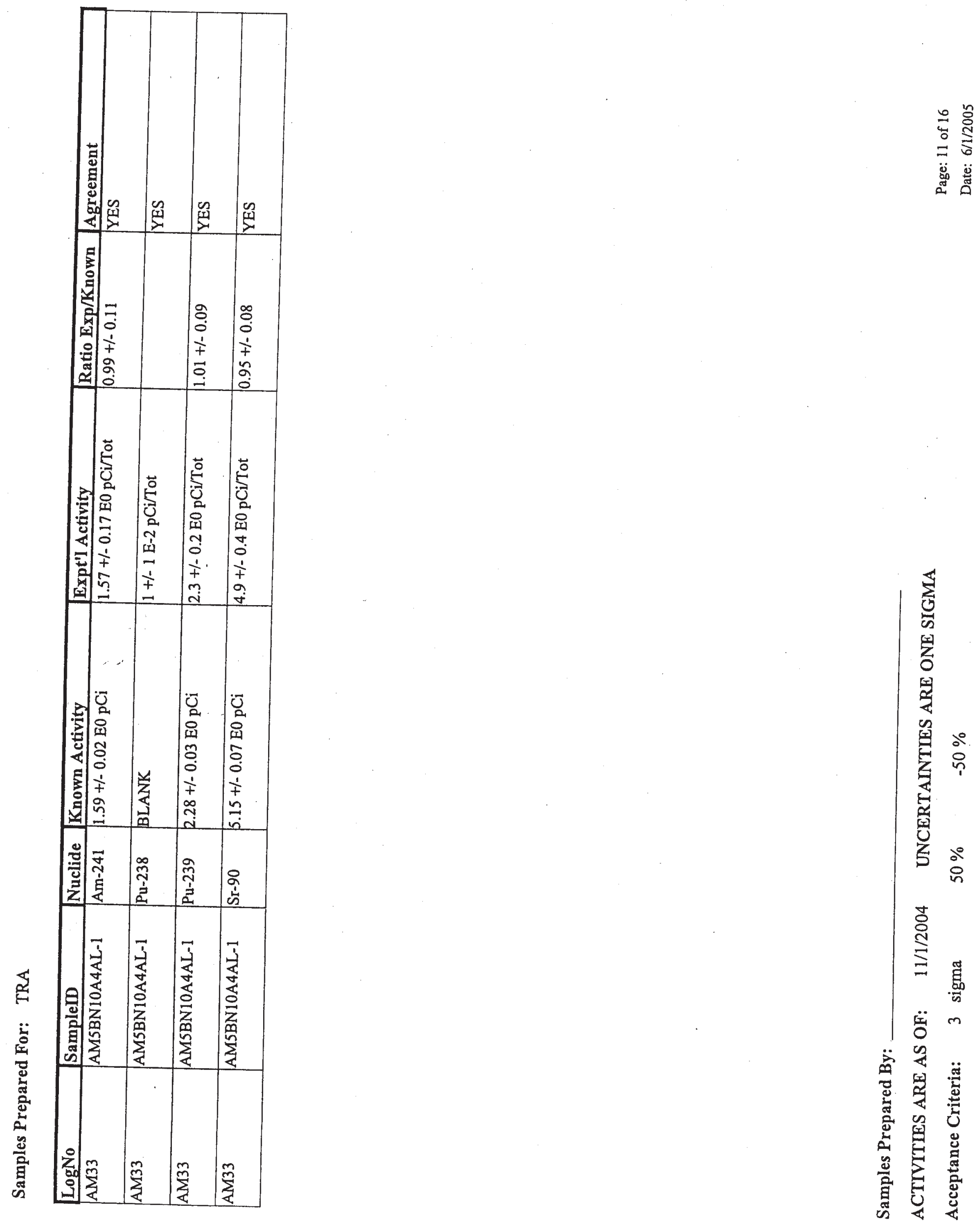


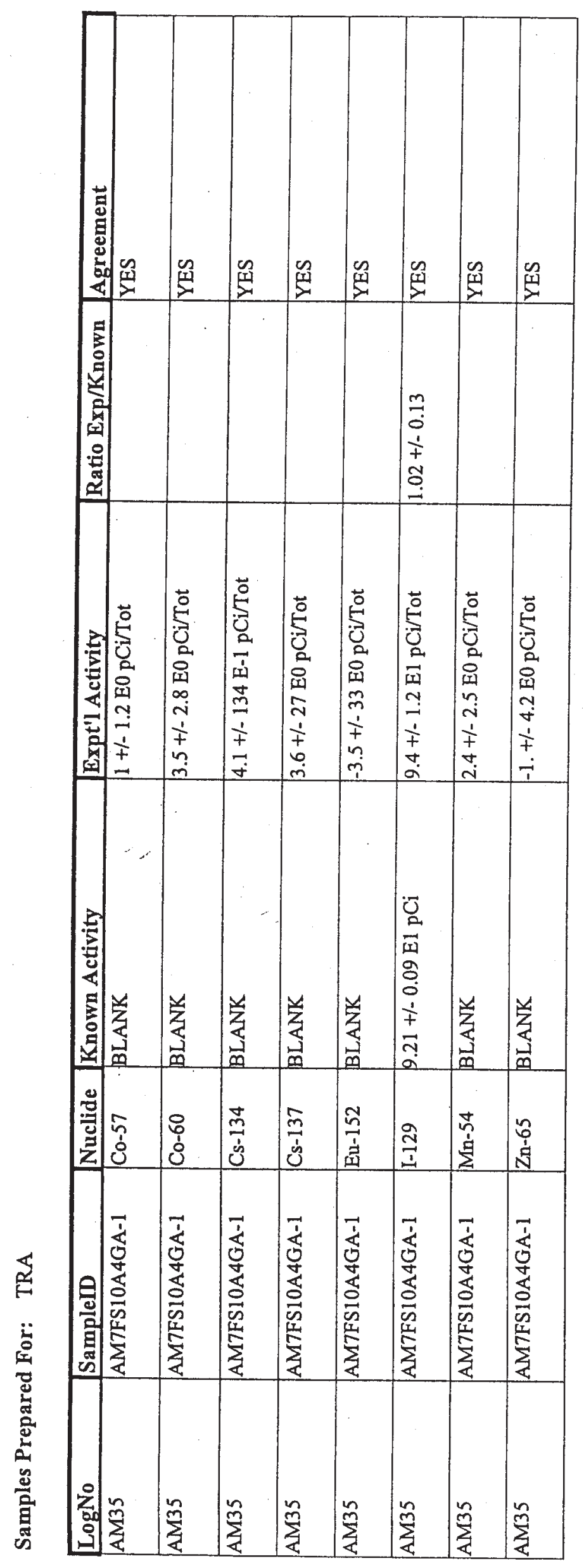

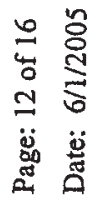

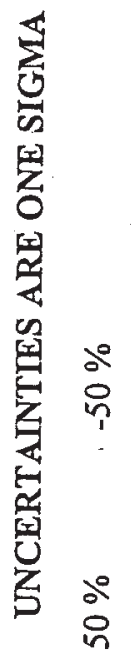

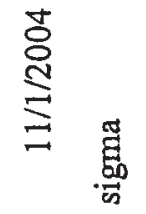

苑 m

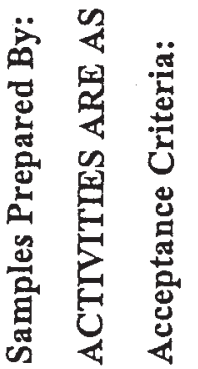



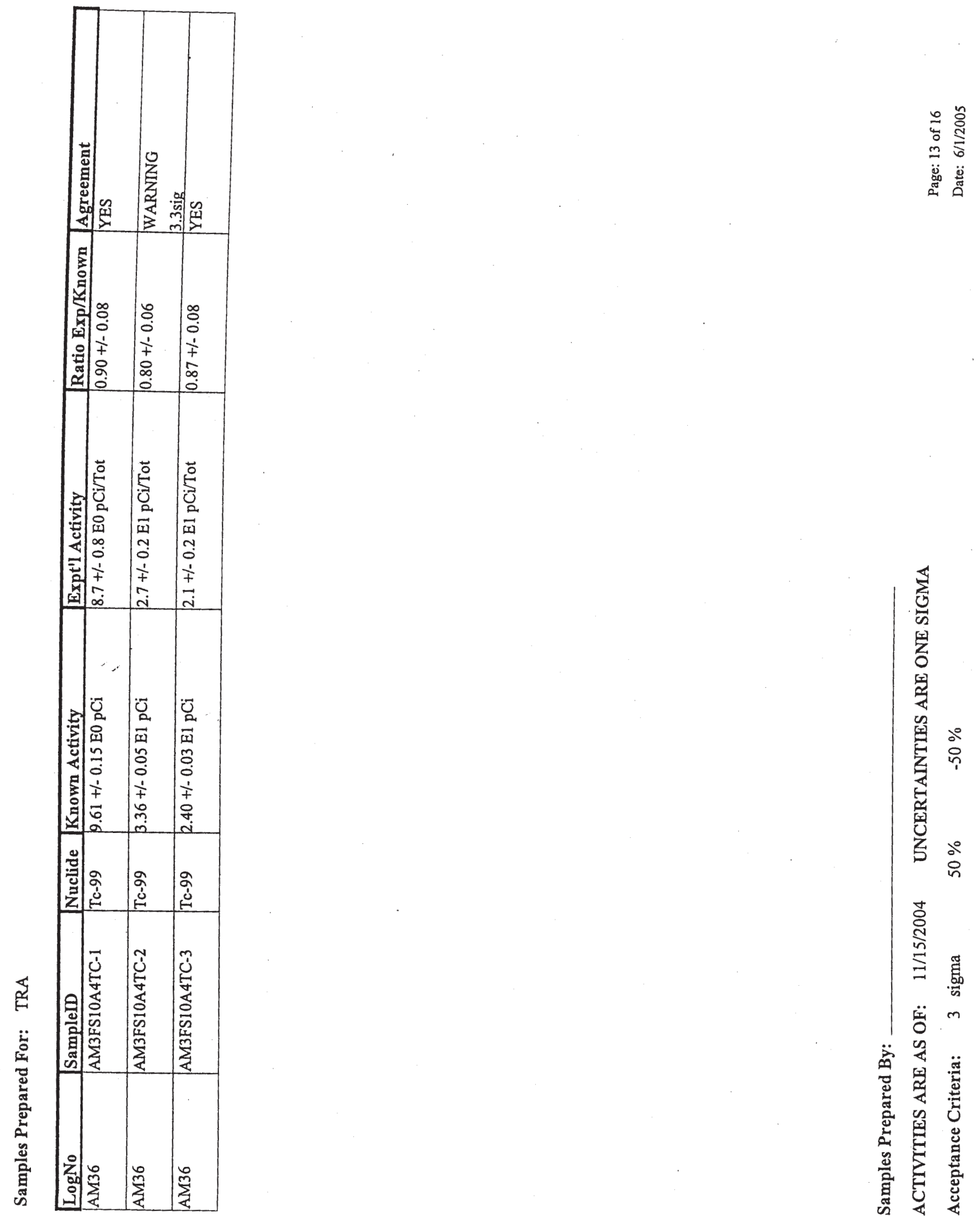


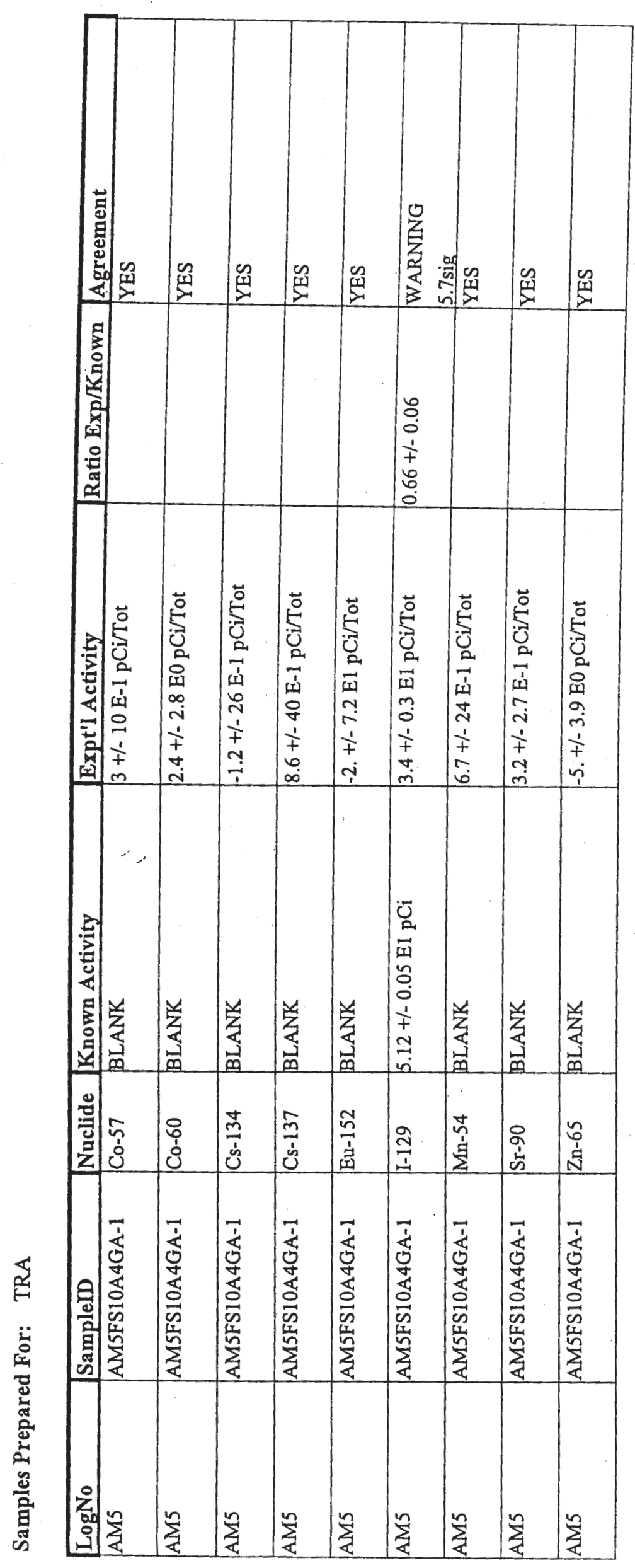



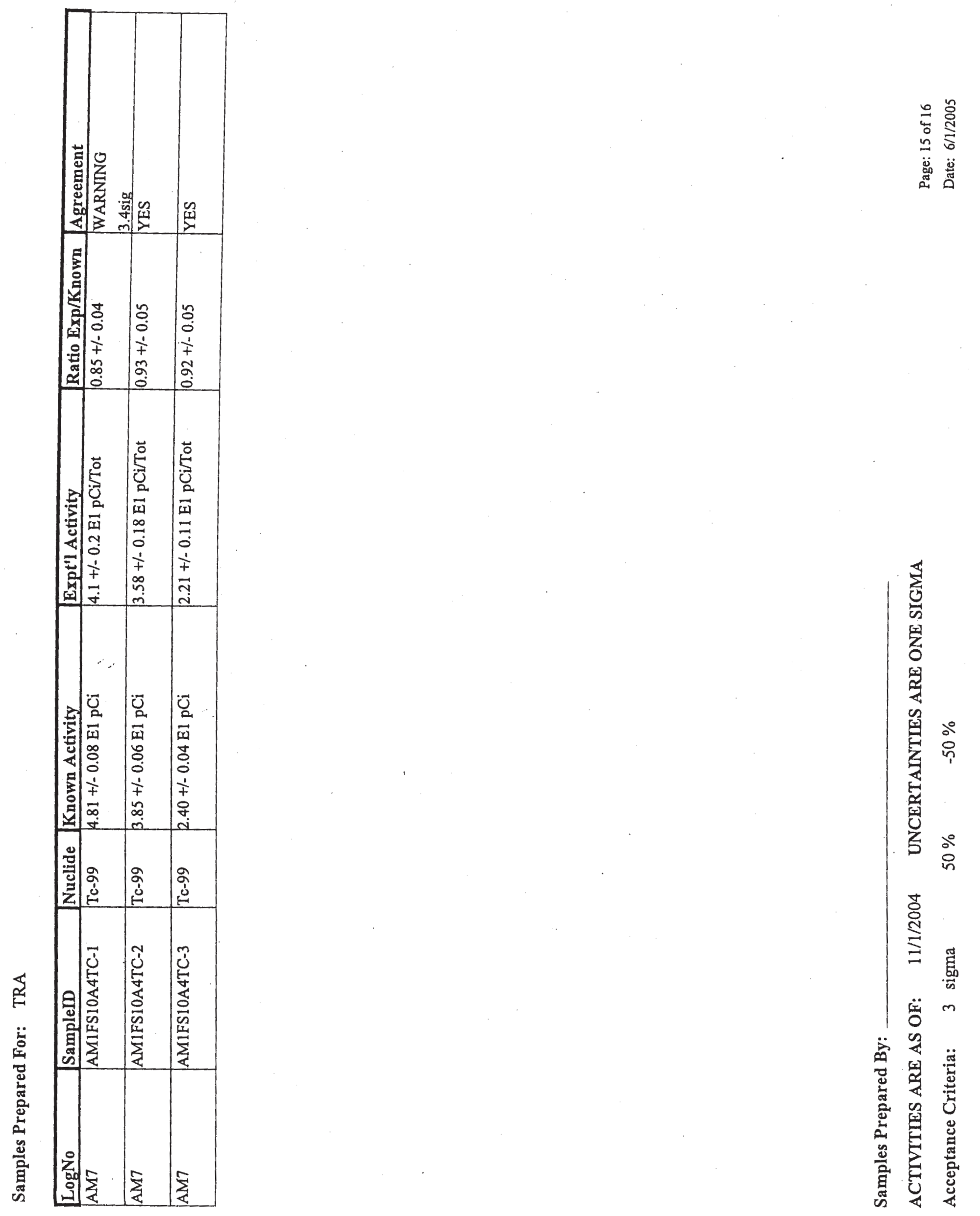

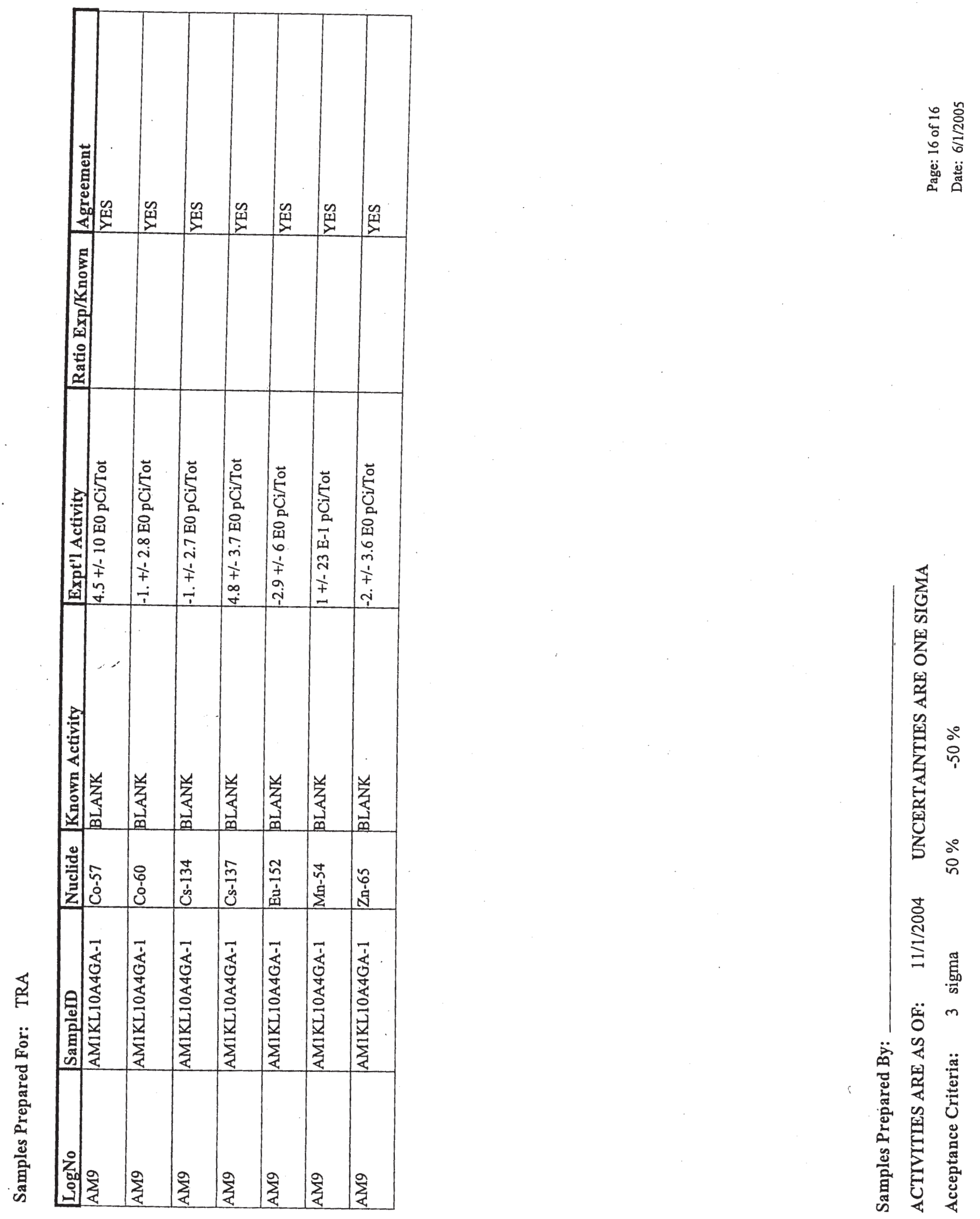


\section{Results of Blind QC Samples}




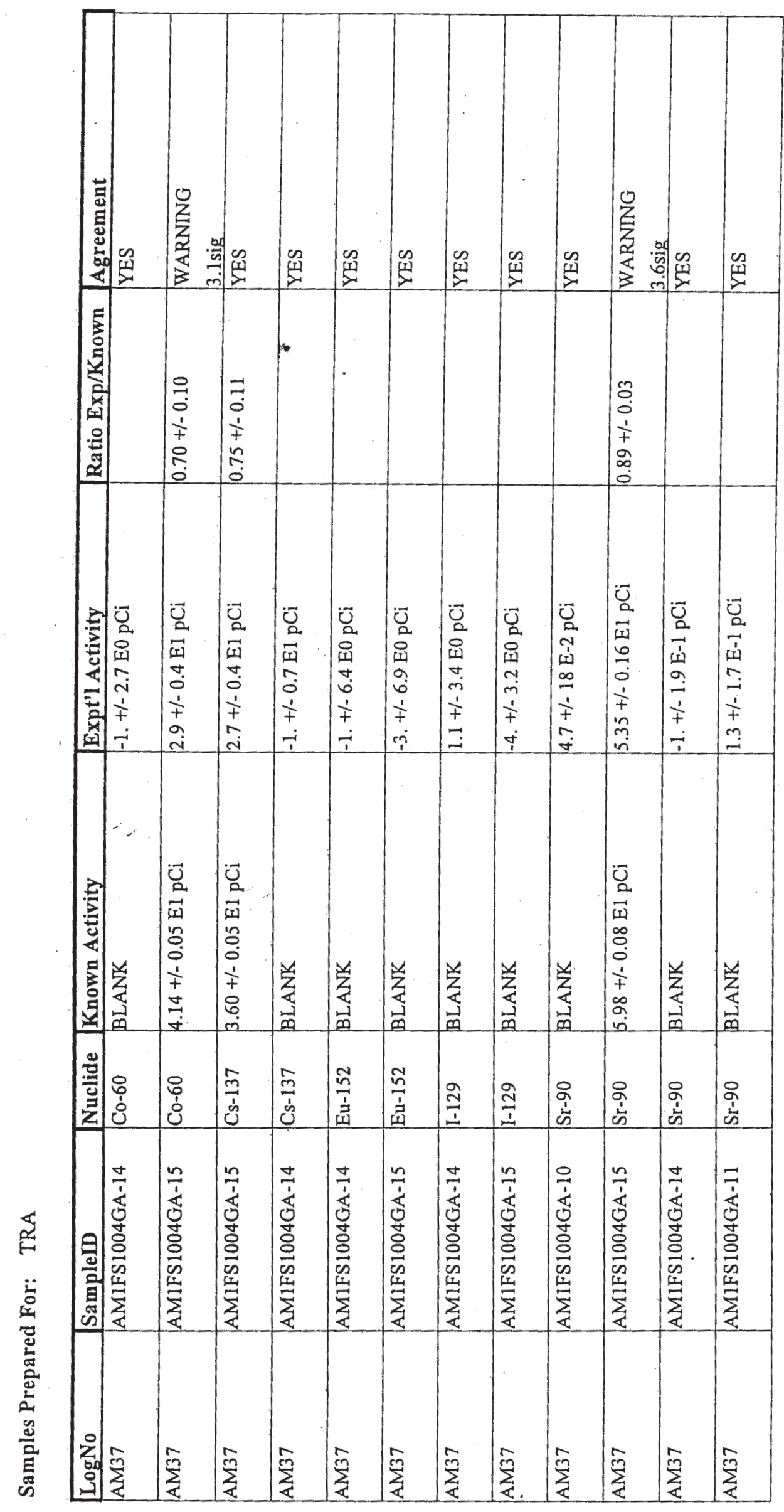

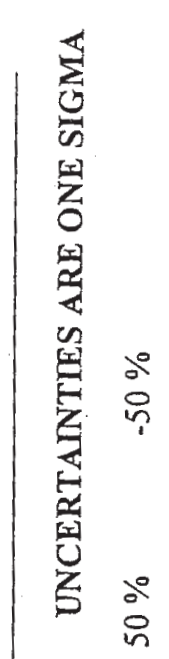

总 范

$\ddot{x}$ m

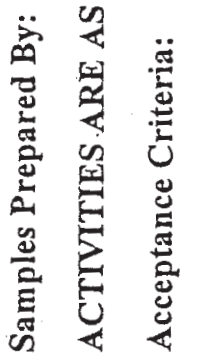



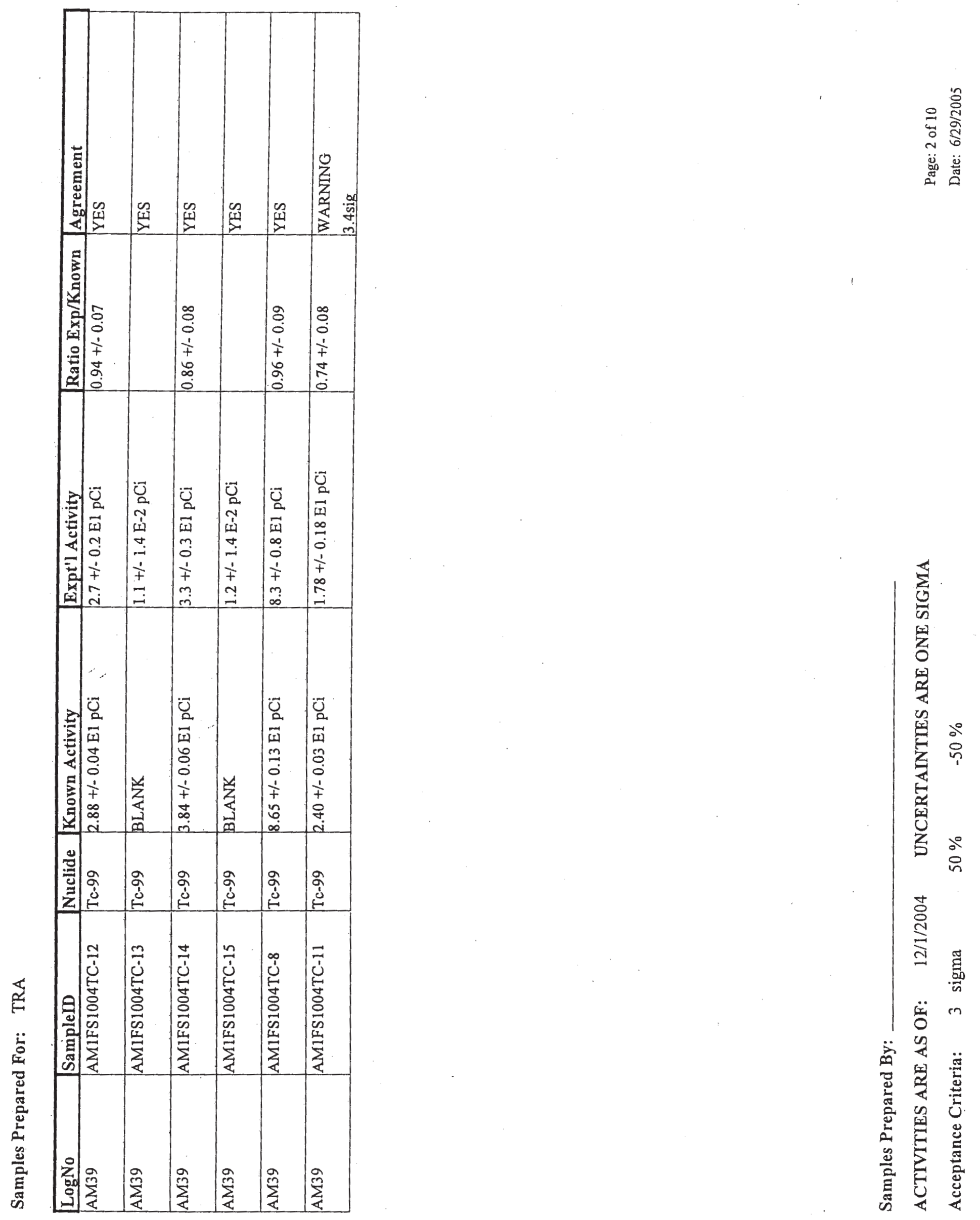

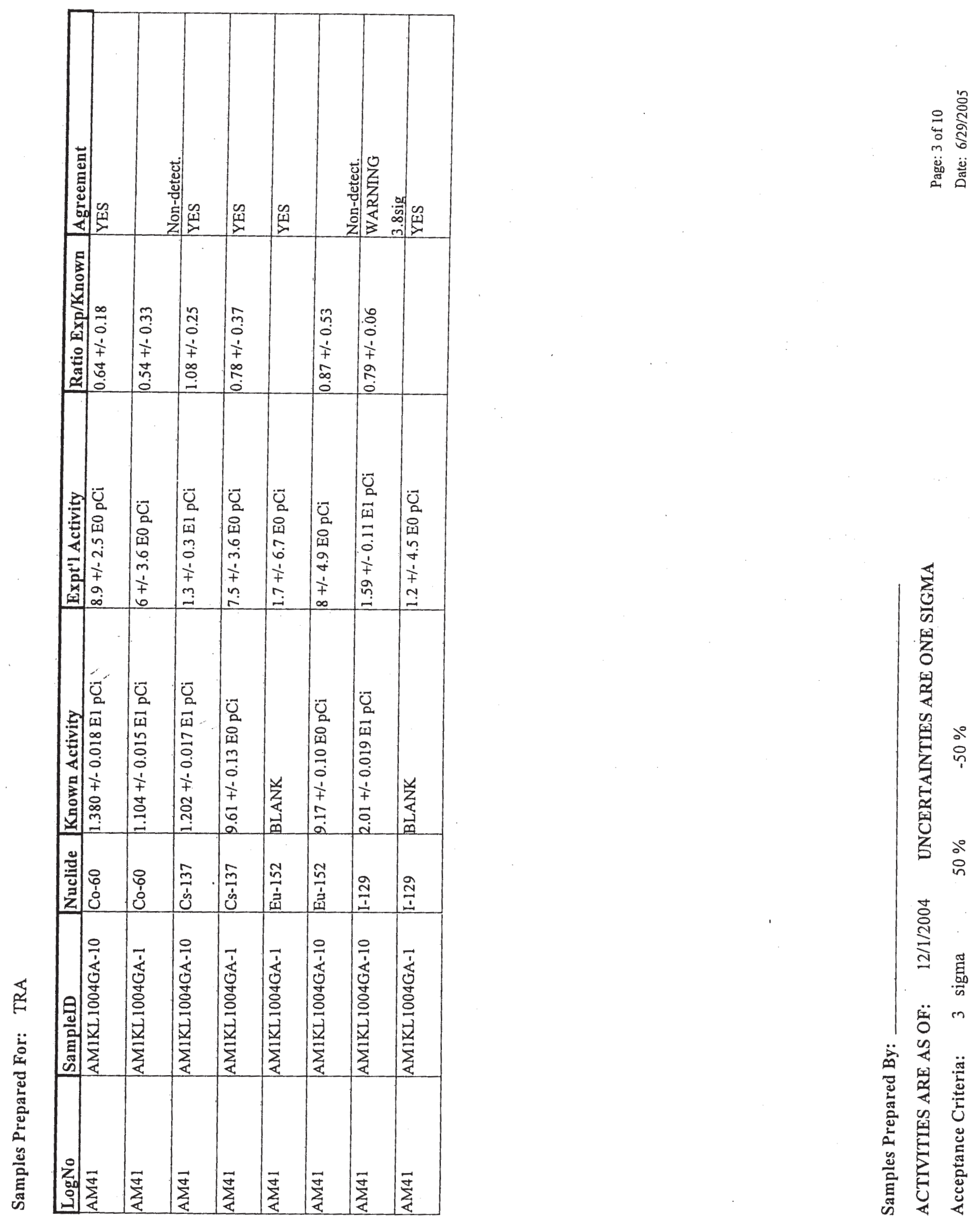


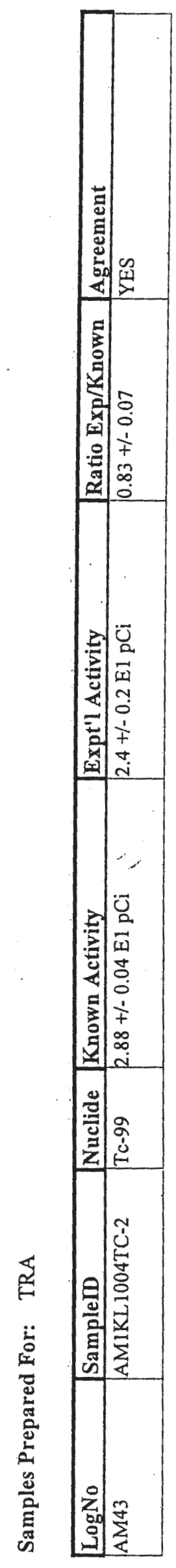

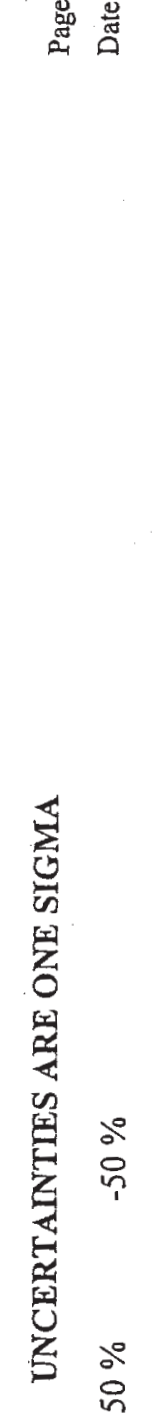

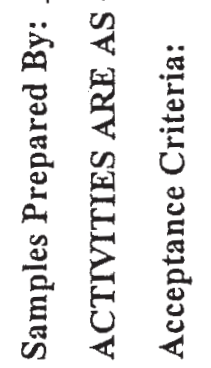




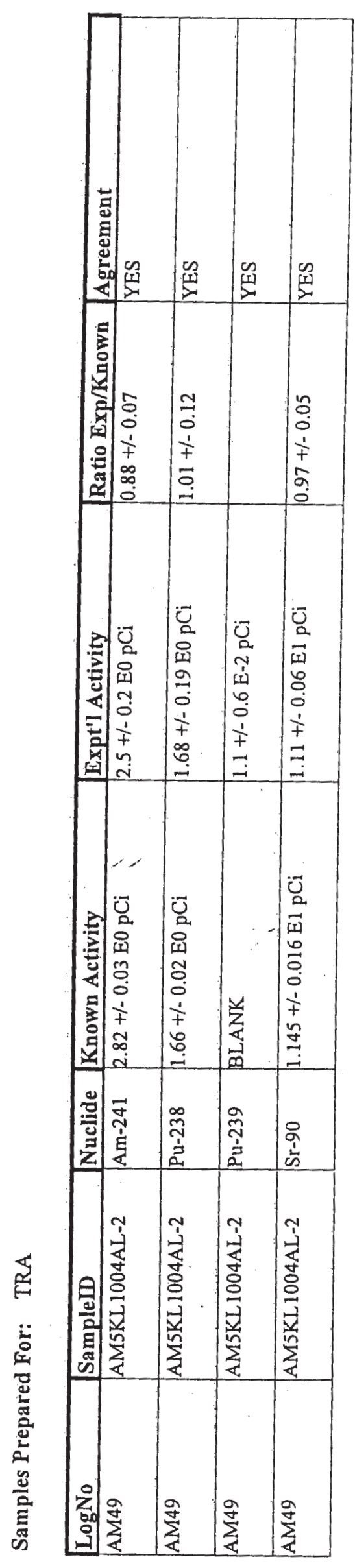

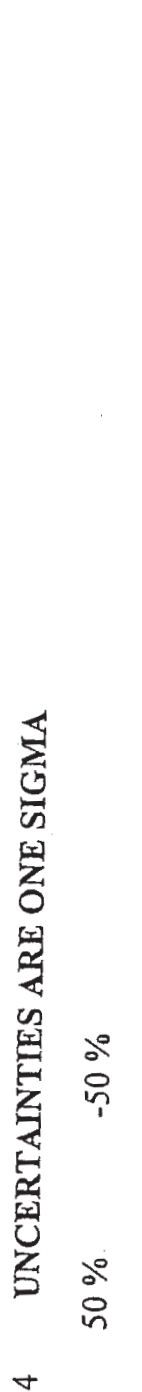

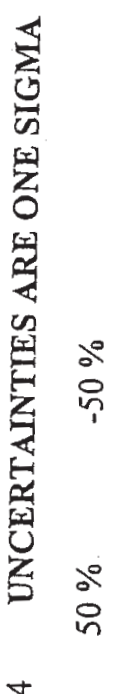



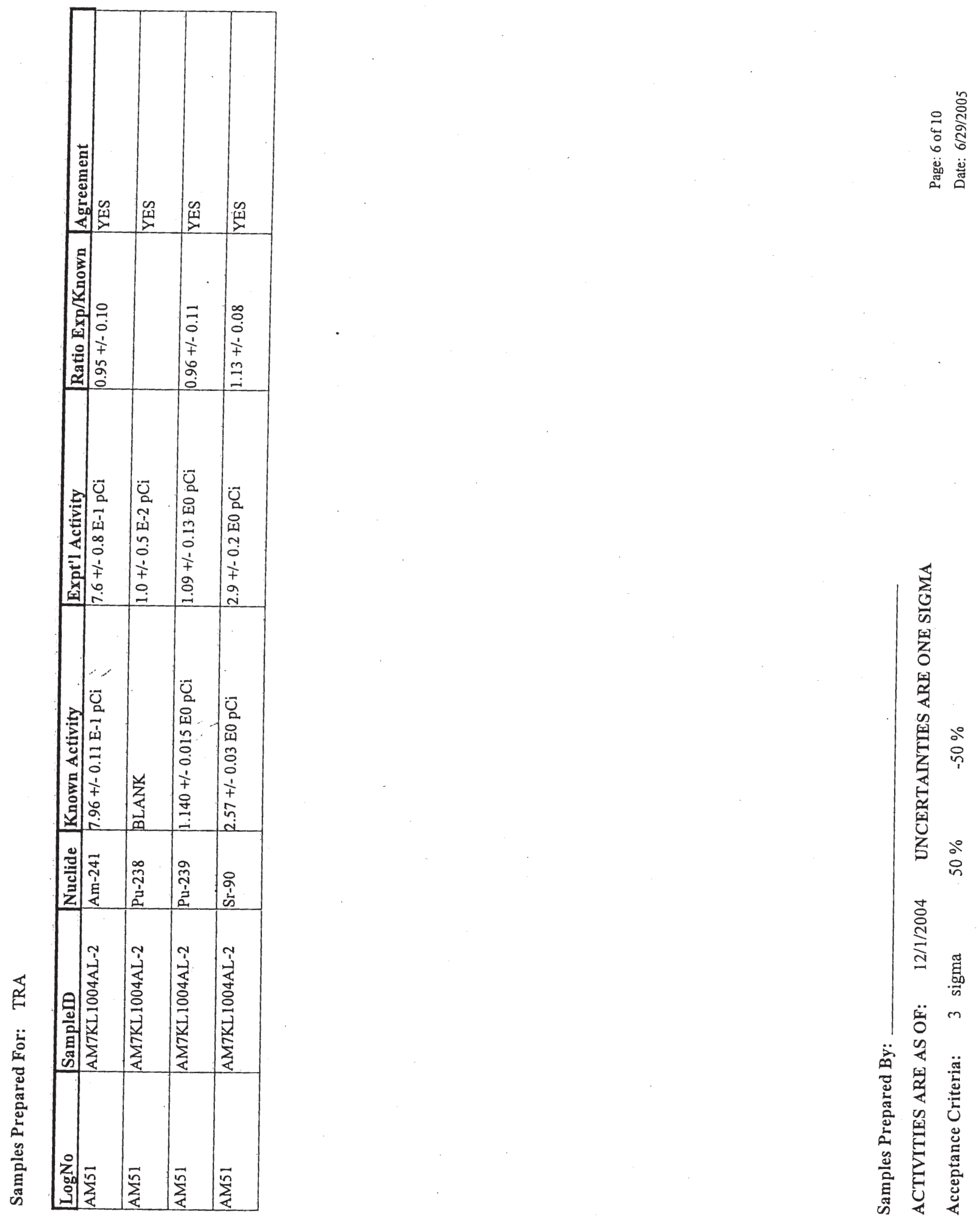

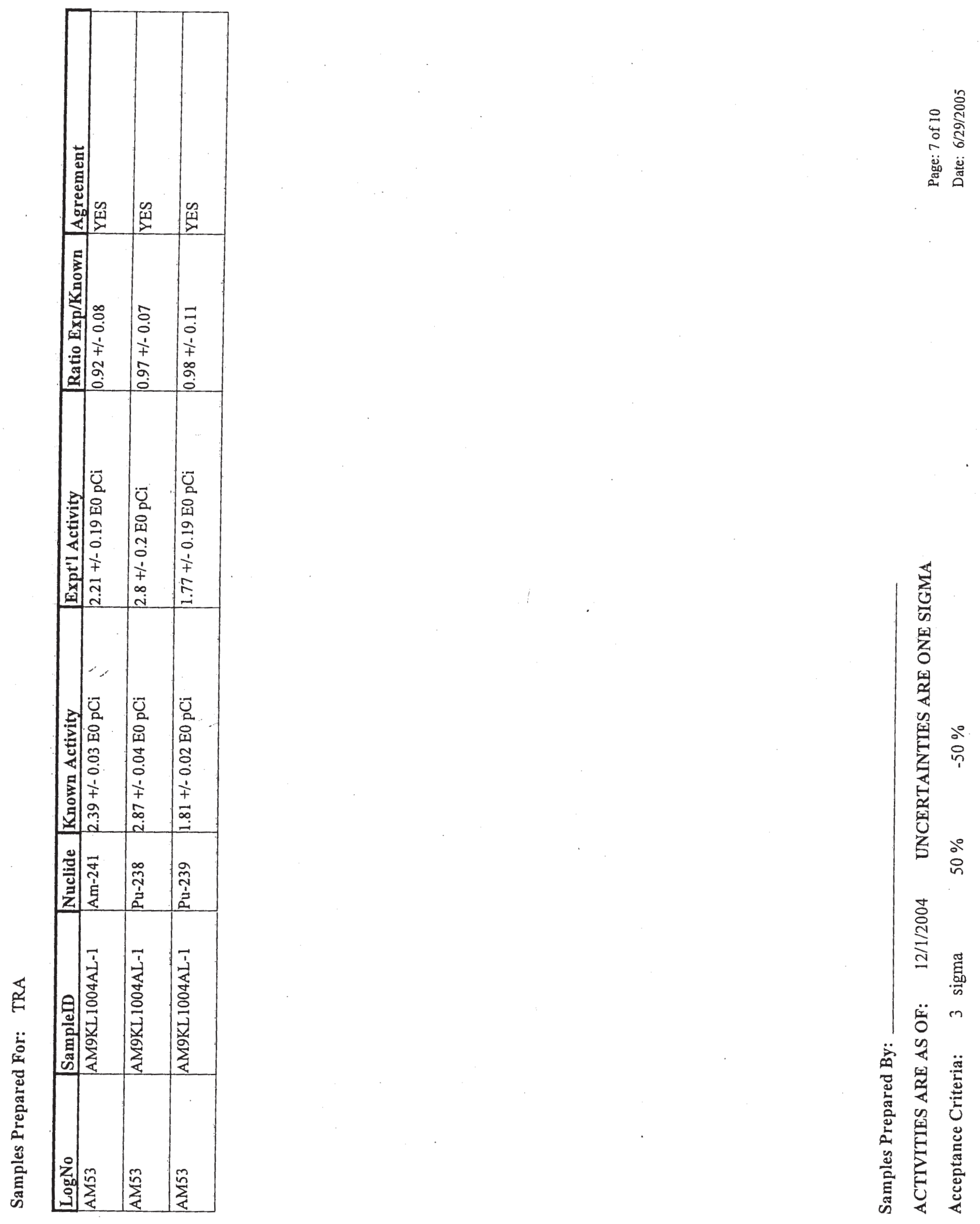

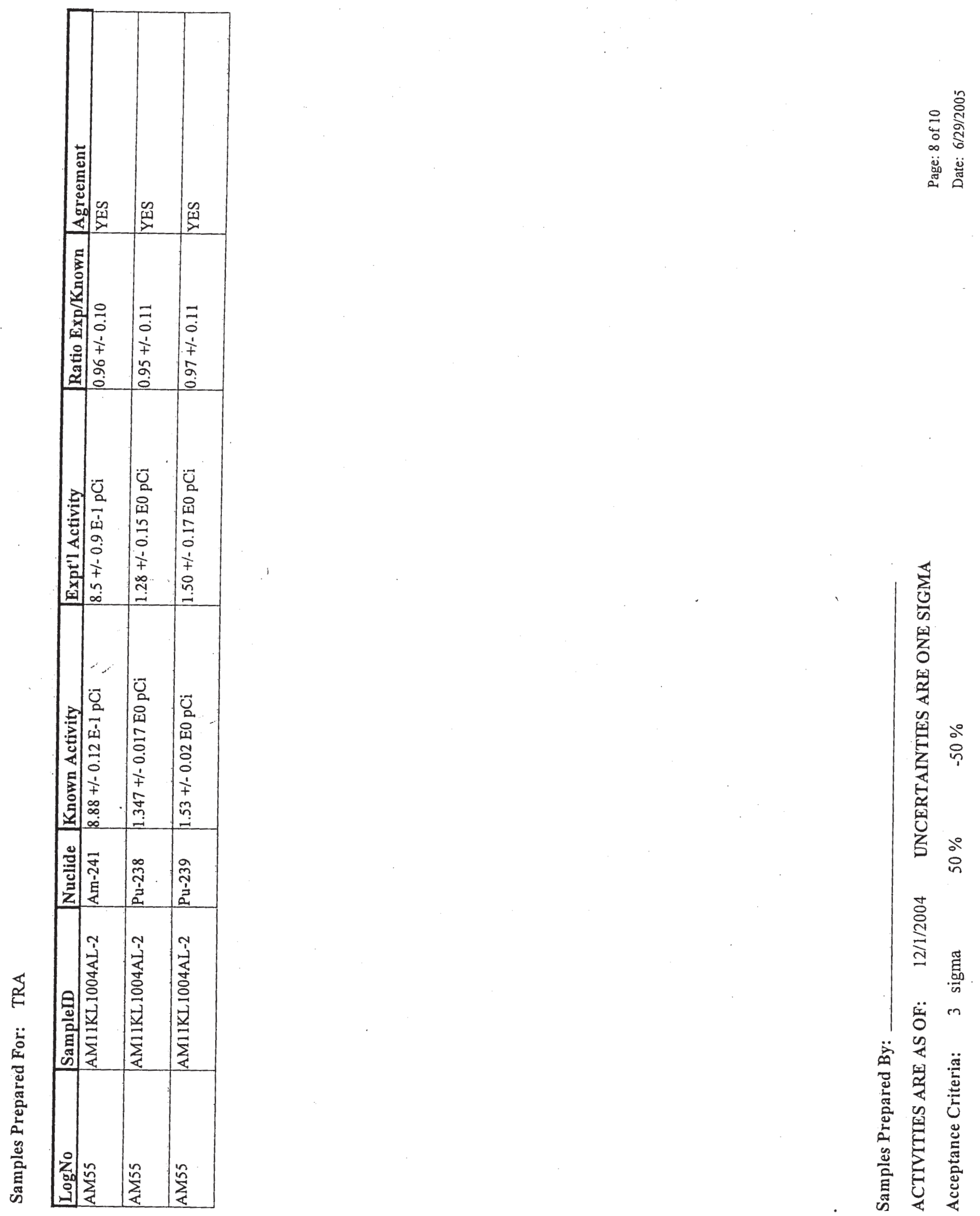

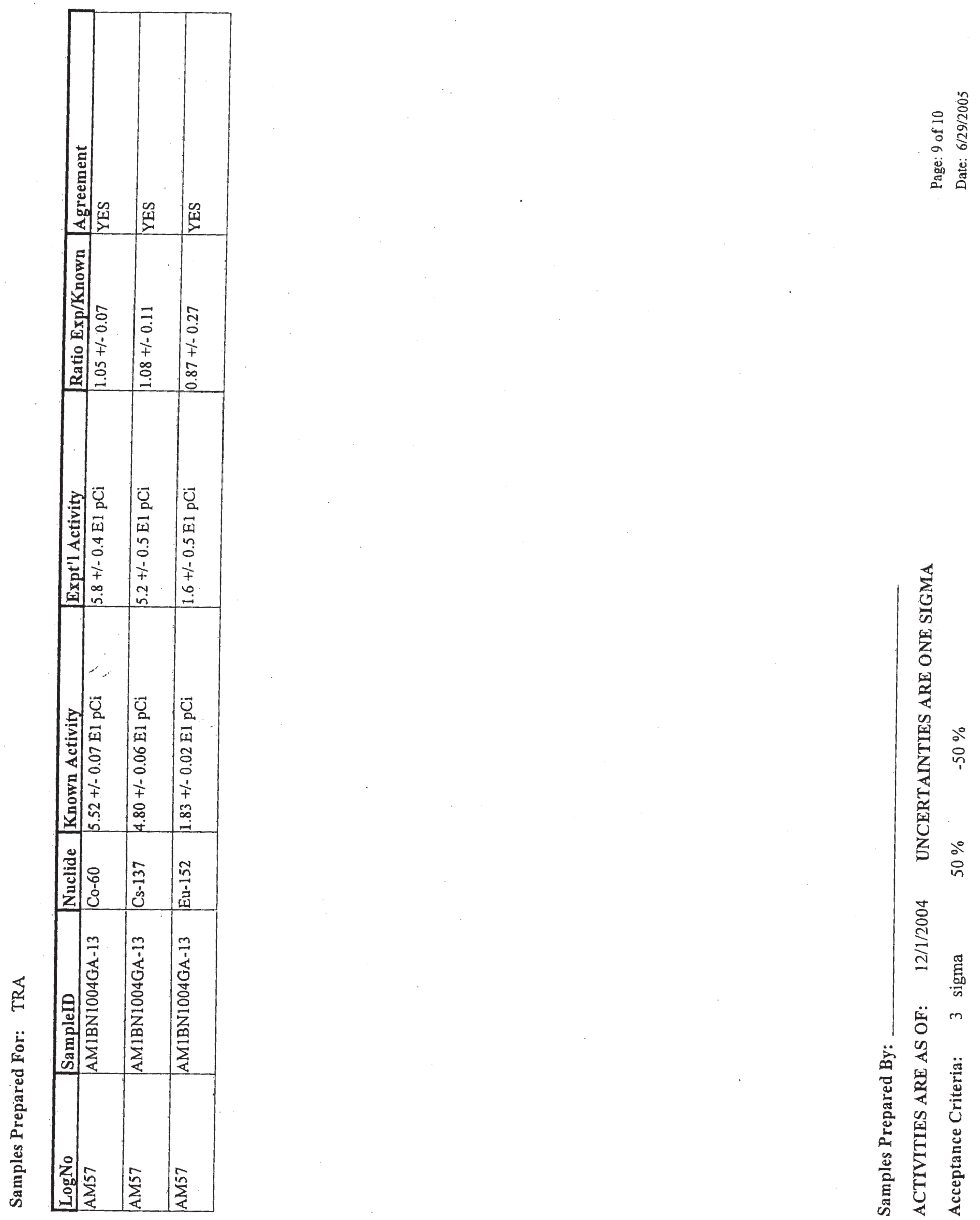


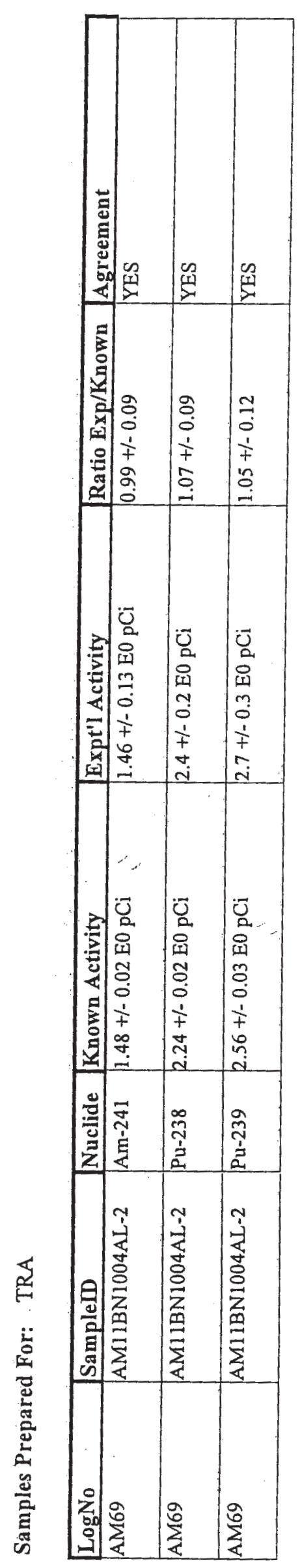

응

融

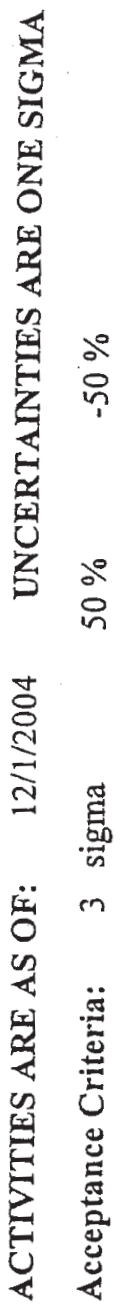


$\frac{1}{0}$
0
0
0
0
$\bar{x}$
$?$
$\stackrel{1}{I}$ 
Appendix C-1

Gamma Spectroscopy Internal QA/QC Program Results 


\section{RADIATION MEASUREMENTS LABORATORY}

\section{Supporting QA/QC Date}

\section{AMCHITKA ISLAND ENVIRONMENTAL ANALYSIS}

For: RML Ge Detector System: A4, Model No. CAN-4

\section{INTERNAL QC CHECKS:}

Gamma-ray energy calibration (Daily or prior to detector use, pulsar equivalents are determined weekly for pulsar based detector systems).

Monthly instrument background check.

Monthly Eu-152 calibration source check.

EXTERNAL QC CHECKS (when applicable):

Mixed Analyte Performance Evaluation Program (MAPEP)

SEE RESULTS FOR RML EXTERNAL QC CHECKS IN APPENDIX D

Analyzed by: Date:

Approved by: Date:

\section{COMMENTS:}

All applicable QA/QC checks demonstrate the RML was "in control" during the time frame the samples were counted and analyzed. 
RADIATION MEASUREMENTS LABORATORY A.4 (CAN-4) COLO AMBIENT BACKGROUND
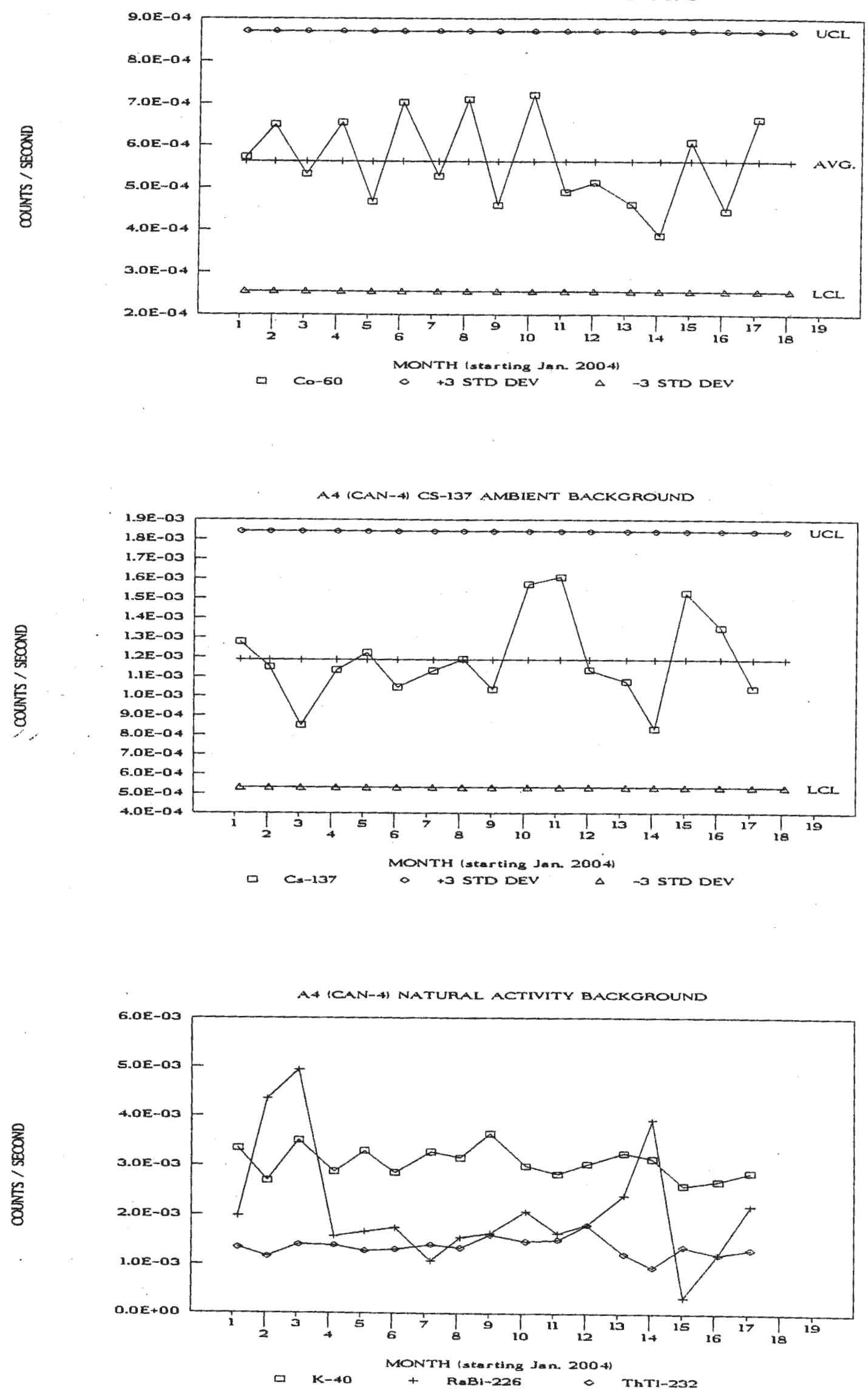


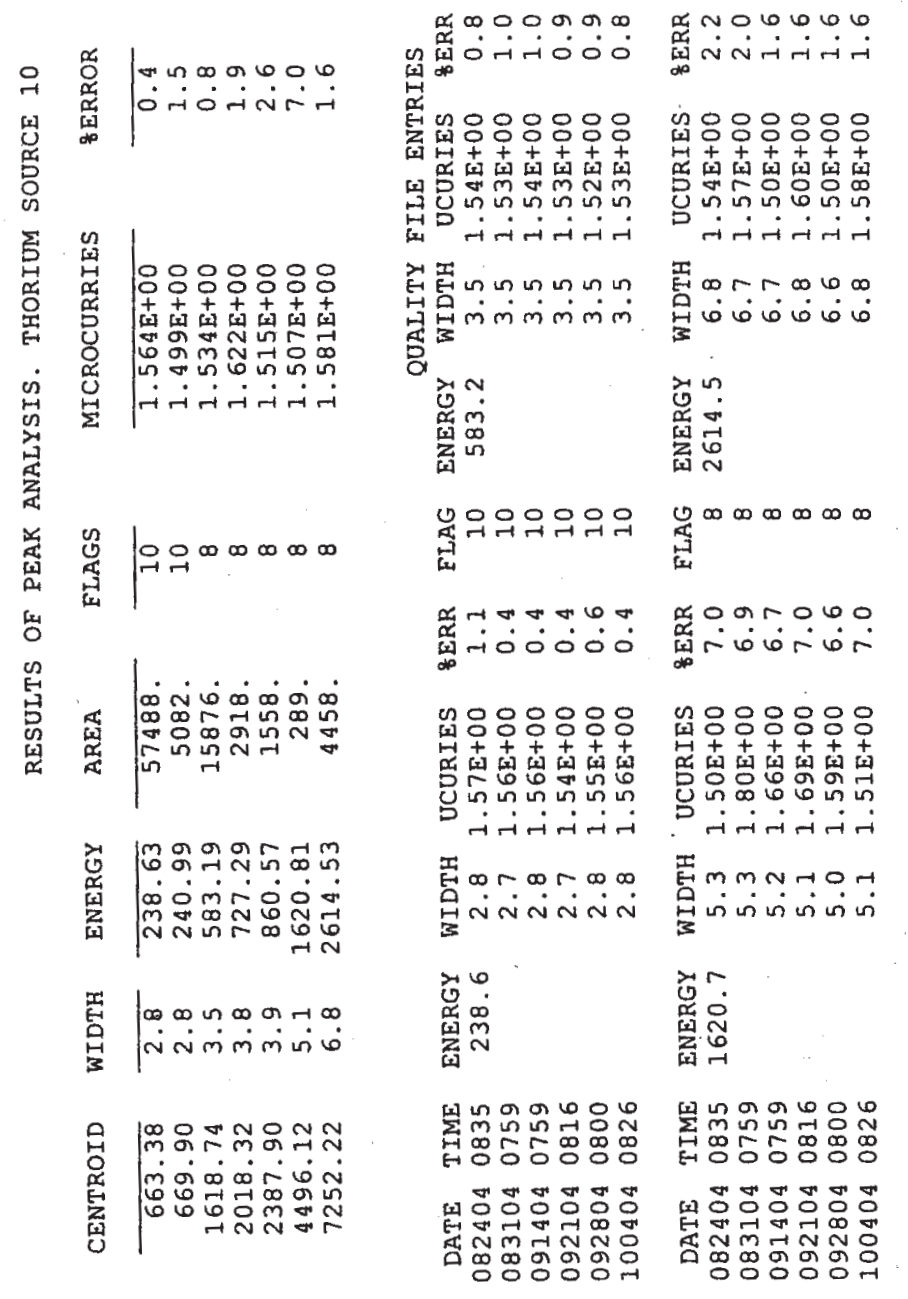



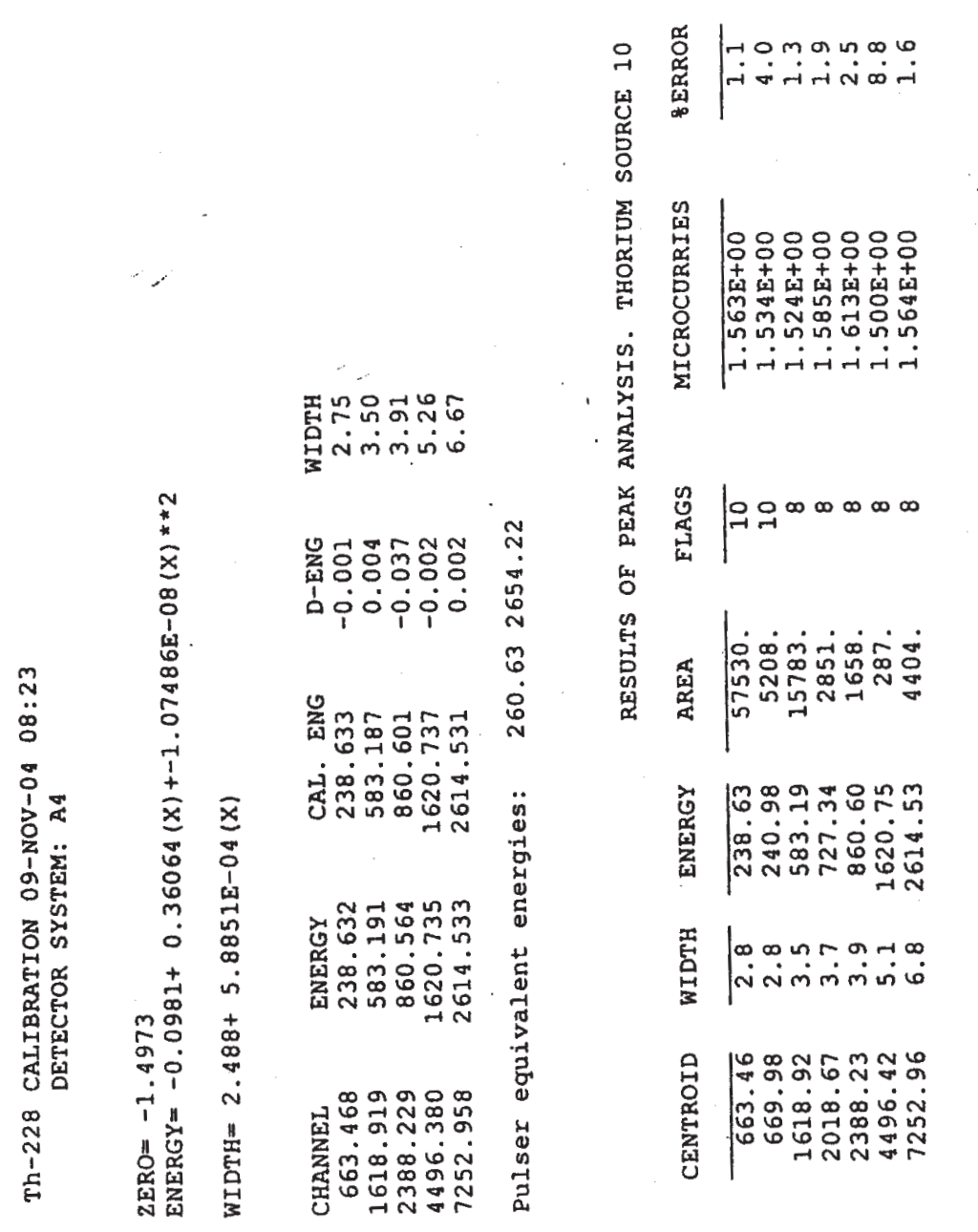

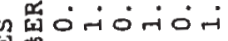
政

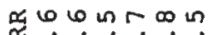

焉

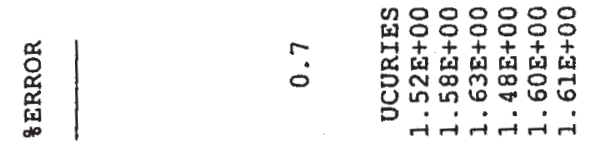

虽

焉00000\%

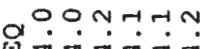

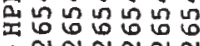

Nin $n$

$0 \infty \infty \infty \infty \infty \infty$

O.

a

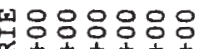

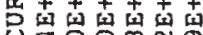

品品品等各

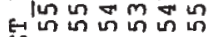

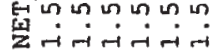

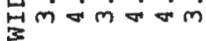

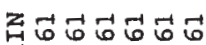

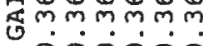

范宫

000000

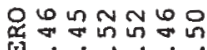

幽 $\infty \infty \infty \infty \infty$

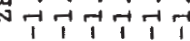

近 $\infty \infty \infty \infty \infty$

出

$\log 2 \pi+10$

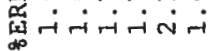

녕응ㅇㅇㅇㅇㅇㅇㅇㅇ

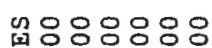

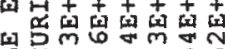

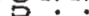

$++t+1$

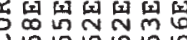

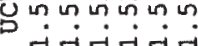

मx

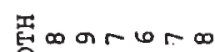

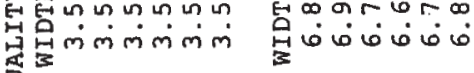

过品

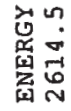

U OOOOOOO $0 \infty \infty \infty \infty \infty \infty$

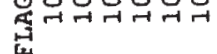

空

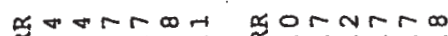

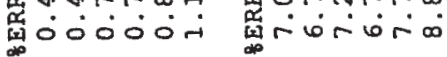

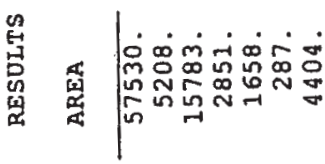

的

兽

\section{in

느응응ㅇㅇ응

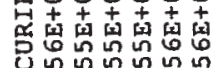
मंन्नंन्तं

동ㅇㅇㅇㅇㅇㅇㅇㅇㅇㅇ

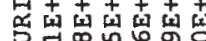

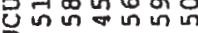

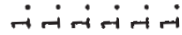

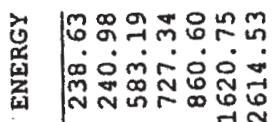

宙 $\infty \infty \infty \infty \infty \infty$.

药 Givinina

ज्ञ

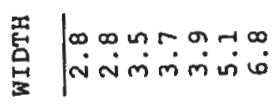

总品

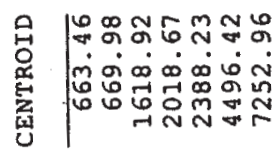

- $\sum_{i=1}$

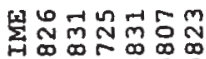

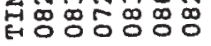

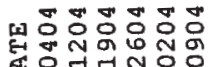

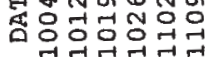

究。

空

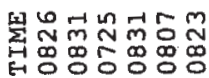

몽ㅁㅇㅇㅇㅇㅇㅇㅇㅇ

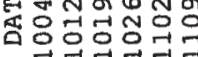



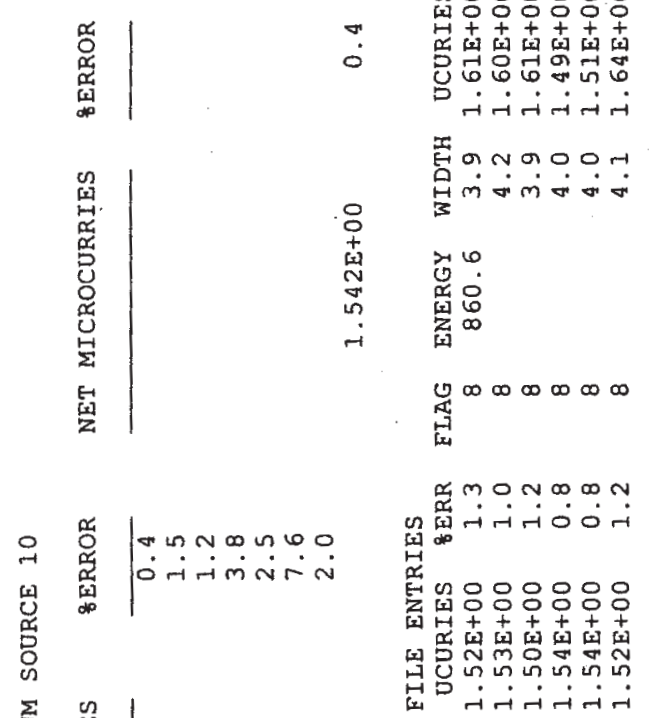

哇

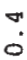

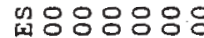

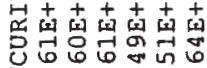
पिंगंबन 每

MONODN

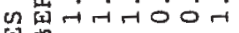

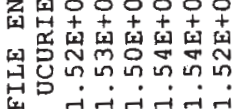

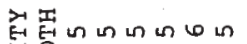

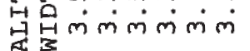
悹

눙

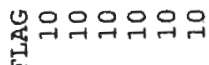

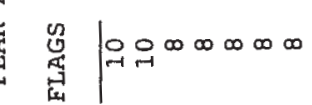
\&ั

留

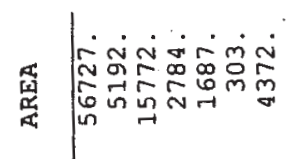

苛 急

焉|

ค แกำำกำำ

مिक

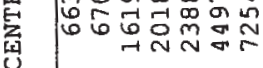

还 $\infty \infty \infty \infty \infty$

แ? 60 .

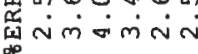

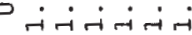

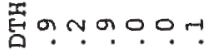

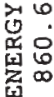

证 $\infty \infty \infty \infty \infty \infty$

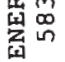

$\alpha$ N

年

N

066660

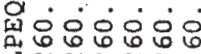

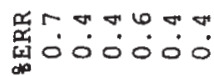

뙹ㅇㅇㅇㅇㅇㅇㅇㅇ

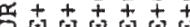

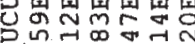

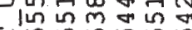

प्añ-

द्युज

बुलmmín

ootoon

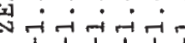

些

$\propto 6 \infty 6 \infty 60$ ज्ञ

또용ㅇㅇㅇㅇㅇ

$+7+千+$

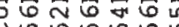

मr-iririr

霓orrar ติ่ง்่อ่

중

淟它

O $\infty 0 \infty \infty \infty \infty$ in

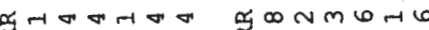

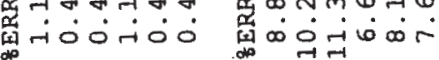

n988880

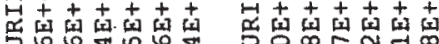

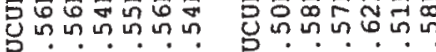
irimiriri Driririri

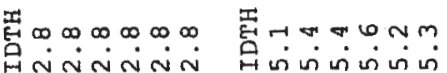

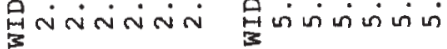

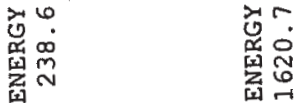

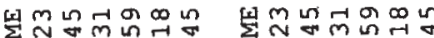

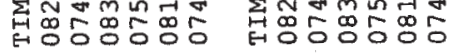

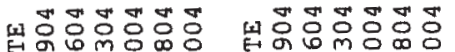

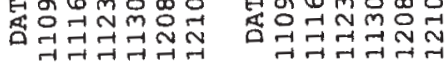




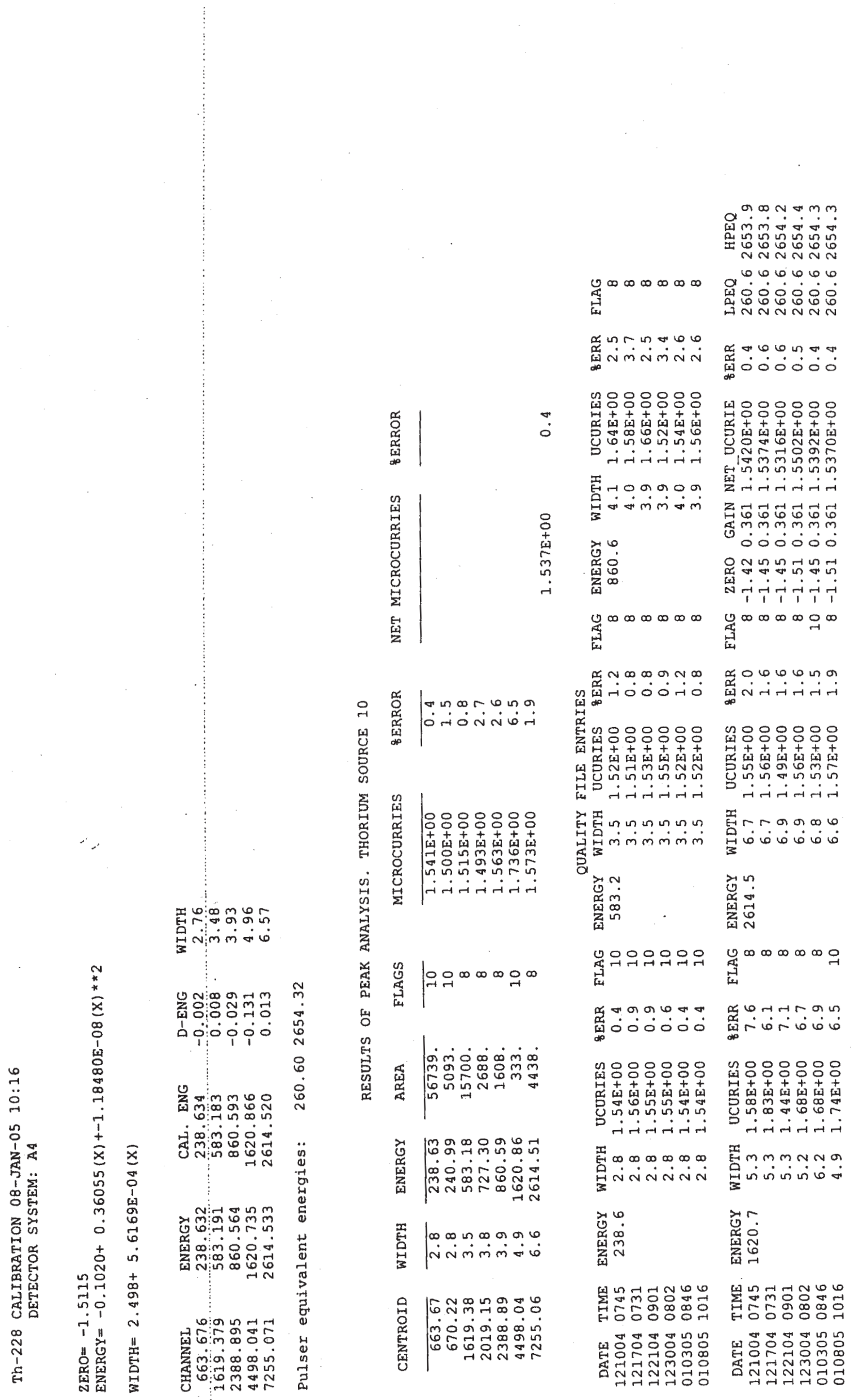




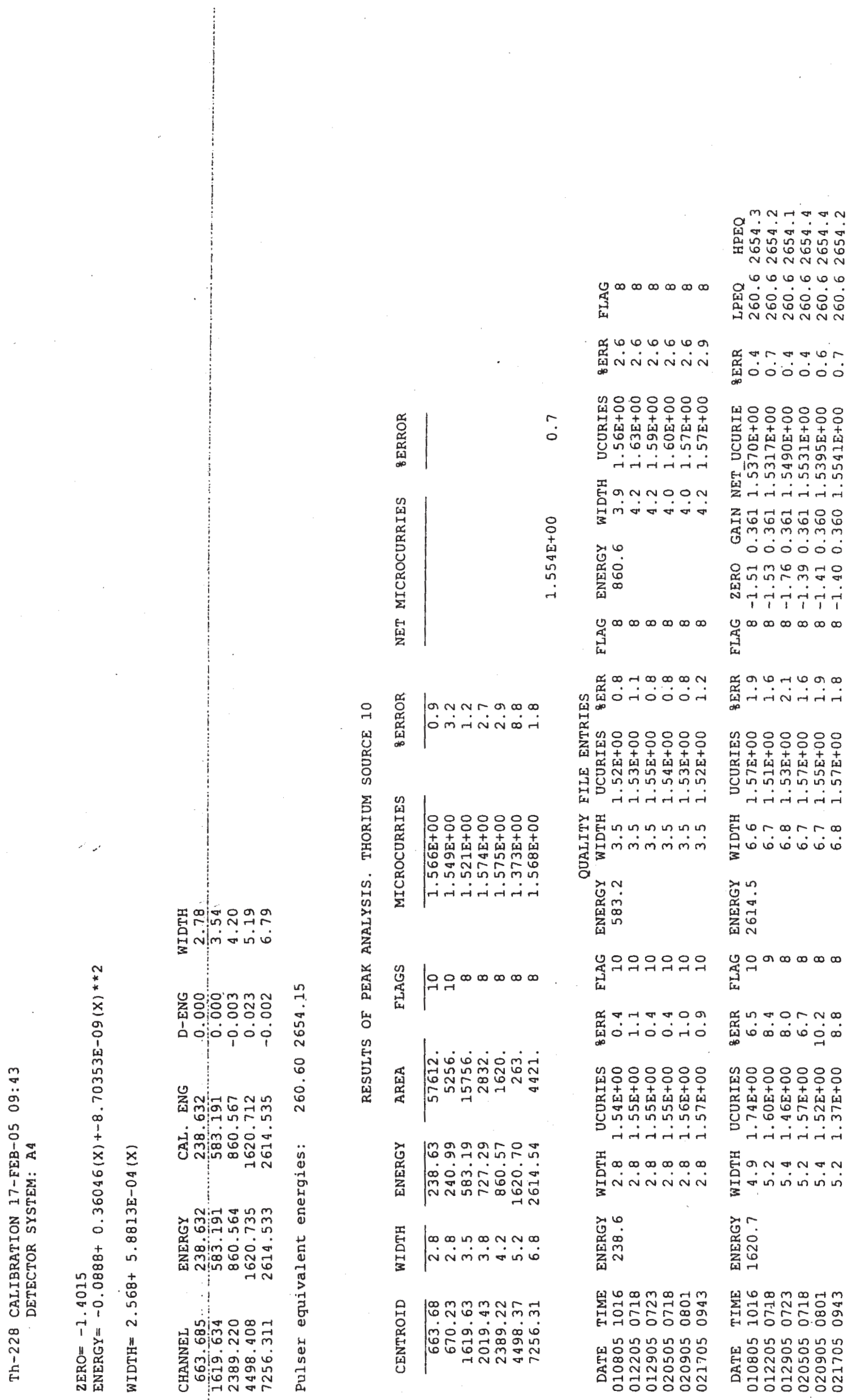




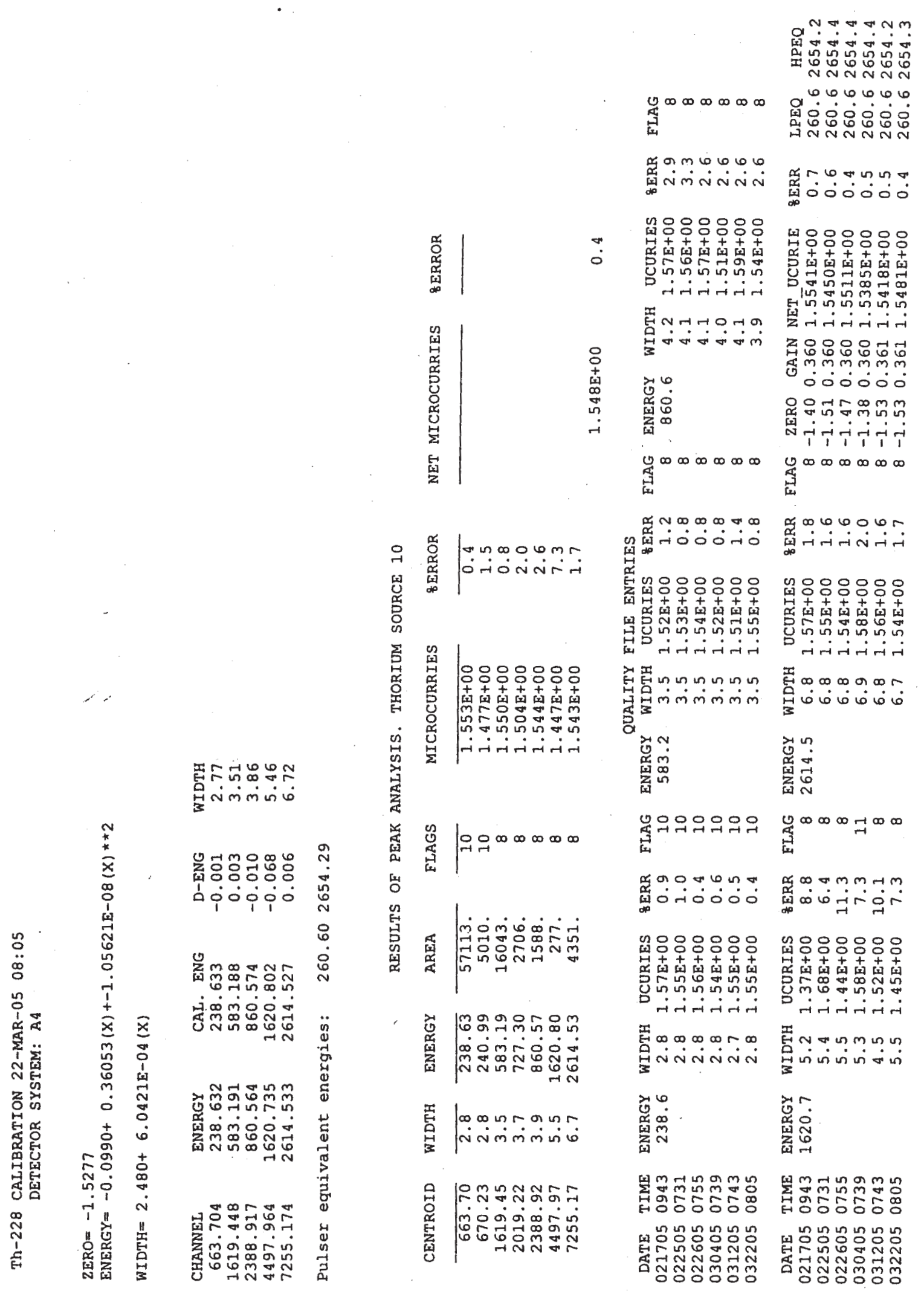



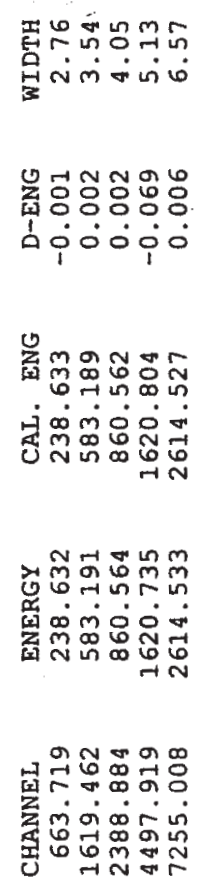

๙ัฒ

(

䓝

\section{$\stackrel{2}{2}$}

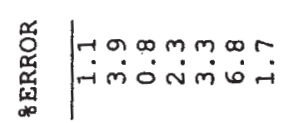

范

虽

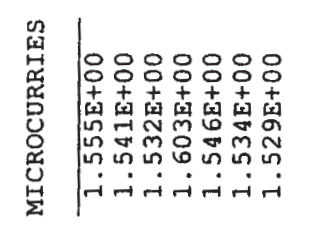

垈

号

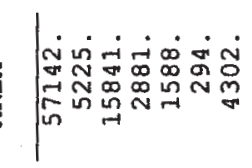

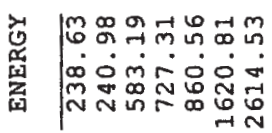

嚊

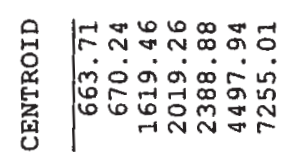

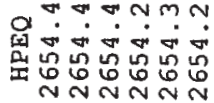

I $\infty 0000006060$

变

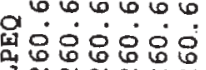

$90606 \mathrm{~m}$

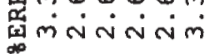

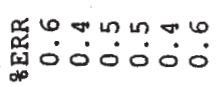

옹용ㅇㅇㅇㅇㅇ

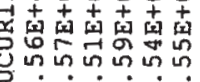

तirinimi

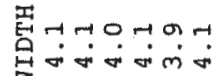

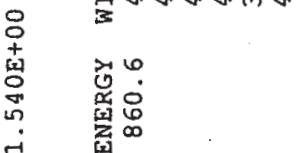

년용ㅇㅇㅇㅇㅇㅇ

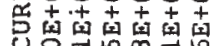

bin

比约约的留

पin

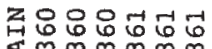

ऊ̛ํำ

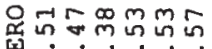

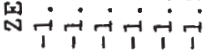

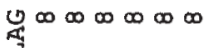

$0 \infty \infty \infty \infty \infty$

t:

$\infty \infty \infty \infty \infty \infty$

㗖

占

뜬응응ㅇㅇㅇ응

细6 $6000 \mathrm{r}$

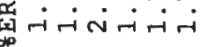

뇡ㅇㅇㅇㅇㅇㅇㅇㅇ응

$4++++$

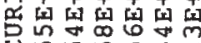

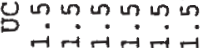

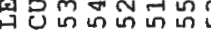

-

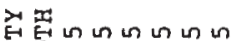

最的的的的

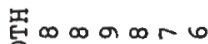
定

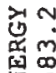

鼠品

落告。

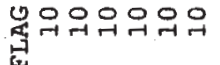

x 000 m n

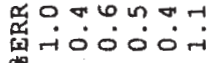

mmmon

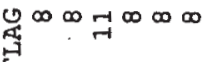

농ㅇㅇㅇㅇㅇㅇㅇㅇㅇㅛ

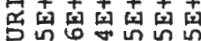

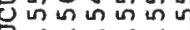
तi

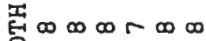

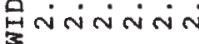

舟

공ㅇㅇㅇㅇㅇㅇ응

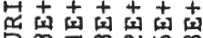

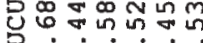

तirimiri

焉? nnum

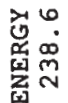

造。

崖

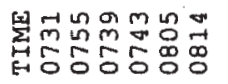

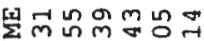

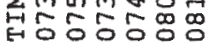

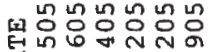

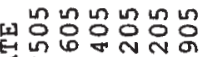

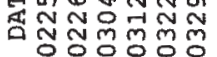




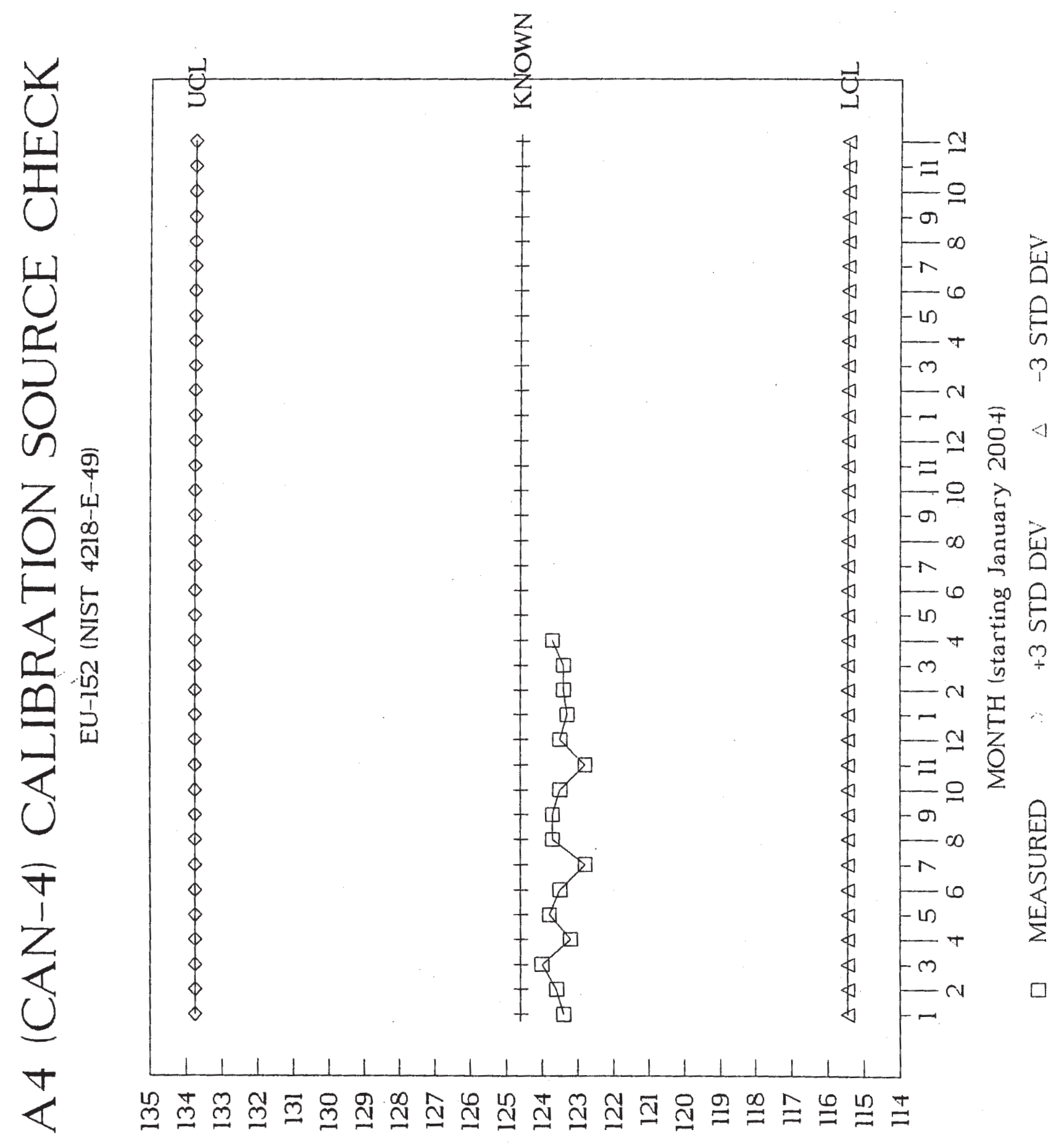

(SPUESnoyL)

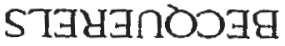




\section{RADIATION MEASUREMENTS LABORATORY}

\section{Supporting QA/QC Date}

\section{AMCHITKA ISLAND ENVIRONMENTAL ANALYSIS}

For: RML Ge Detector System: A6, Model No. PG-13

\section{INTERNAL QC CHECKS:}

Gamma-ray energy calibration (Daily or prior to detector use, pulsar equivalents are determined weekly for pulsar based detector systems).

Monthly instrument background check.

Monthly Eu-152 calibration source check.

EXTERNAL QC CHECKS (when applicable):

Mixed Analyte Performance Evaluation Program (MAPEP)

SEE RESULTS FOR RML EXTERNAL QC CHECKS IN APPENDIX D

Analyzed by: Date:

Approved by: Date:

\section{COMMENTS:}

All applicable QA/QC checks demonstrate the RML was "in control" during the time . frame the samples were counted and analyzed. 
RADIATION MEASUREMENTS LABORATORY A6 (PG-13) CO-60 AMBIENT BACKGROUND
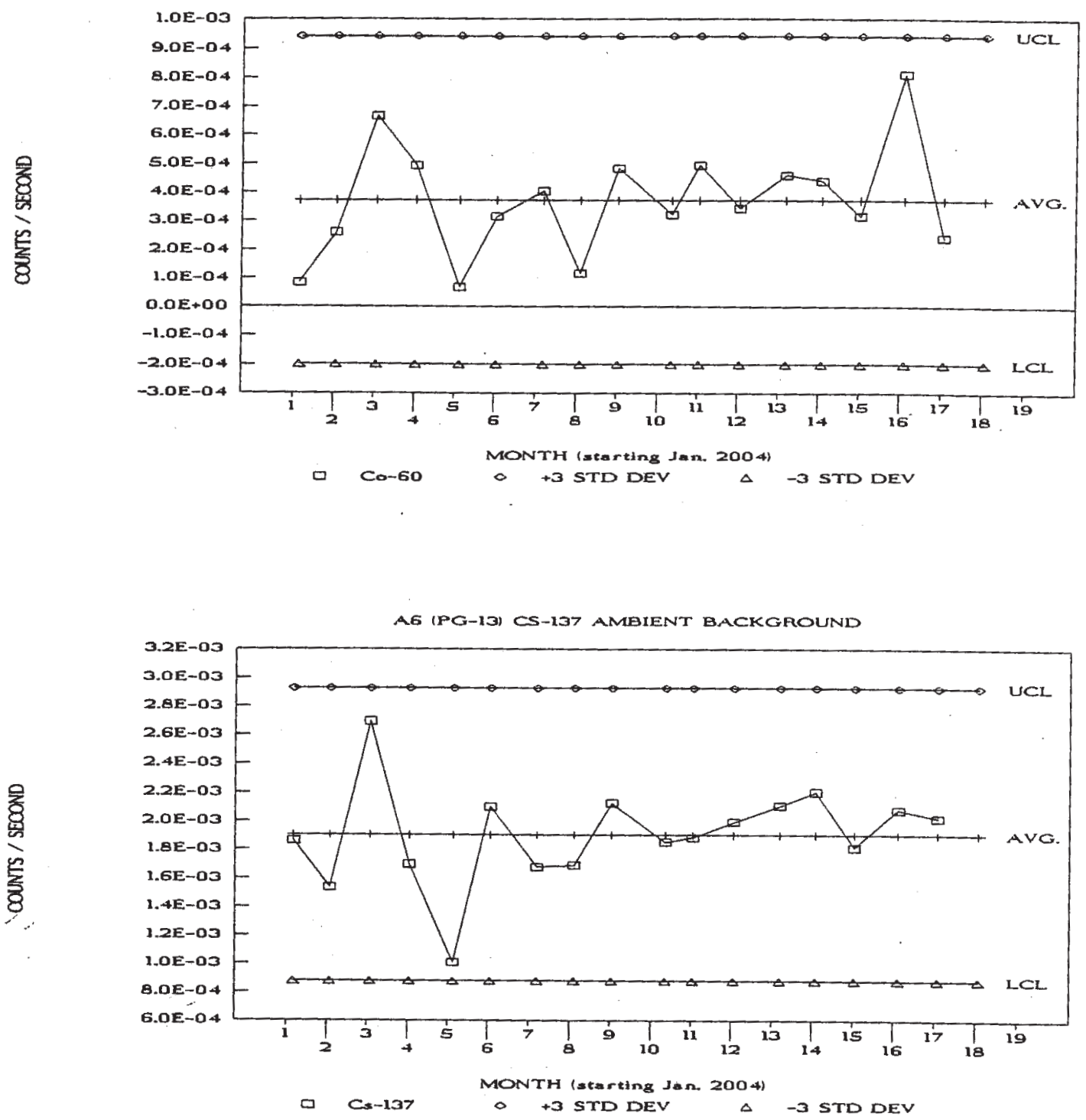

ह

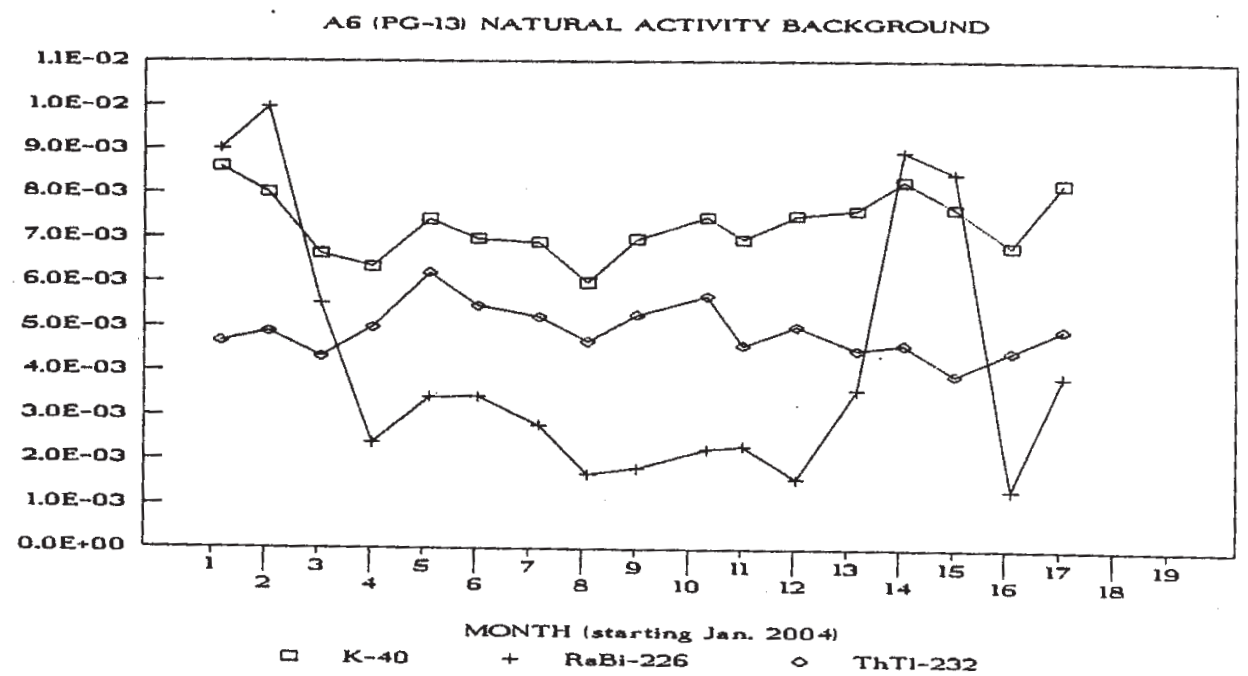



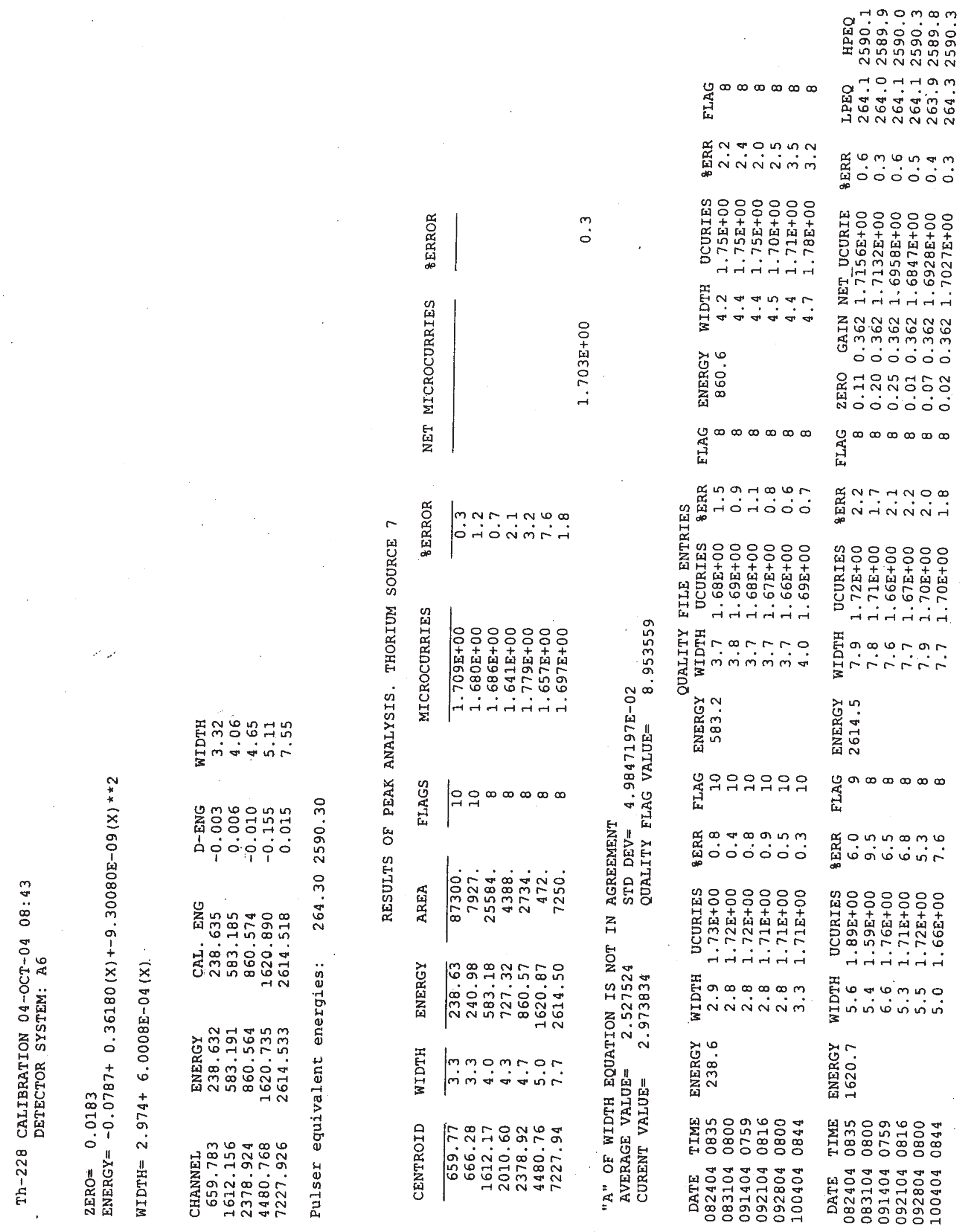

wㅇㅇㅇㅇㅇㅇ

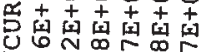

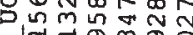

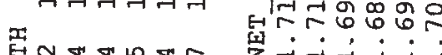

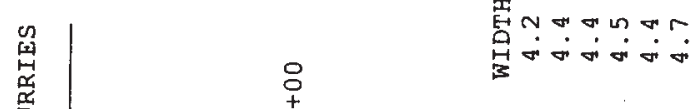

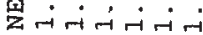

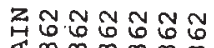

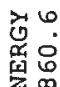

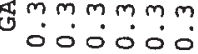

兽

ติ

蒙

通 $\infty \infty \infty \infty \infty$

구규ํำ

N 00000

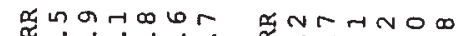

帒 $\infty \infty \infty \infty \infty$

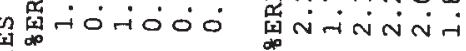
$-1$

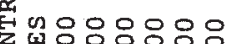

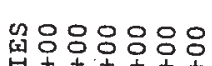

年

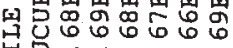

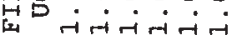

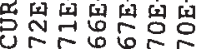

为算roraro

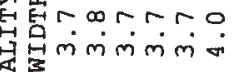

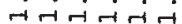
列

겅 N.

密离

Jacorar

tivitita

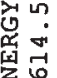

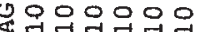
空

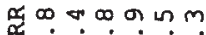

ज्ञ

Q

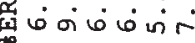

논용ㅇㅇㅇㅛ

গ⿴囗十

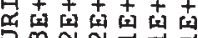

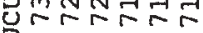
-iनiridi

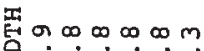

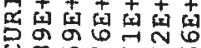

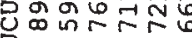

नiनiनiनi

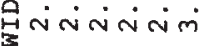

曋oromino

空:

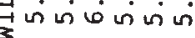

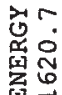

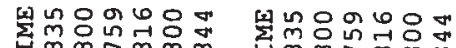

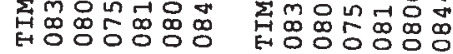

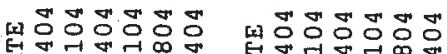

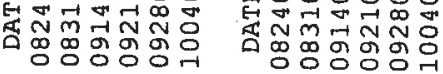



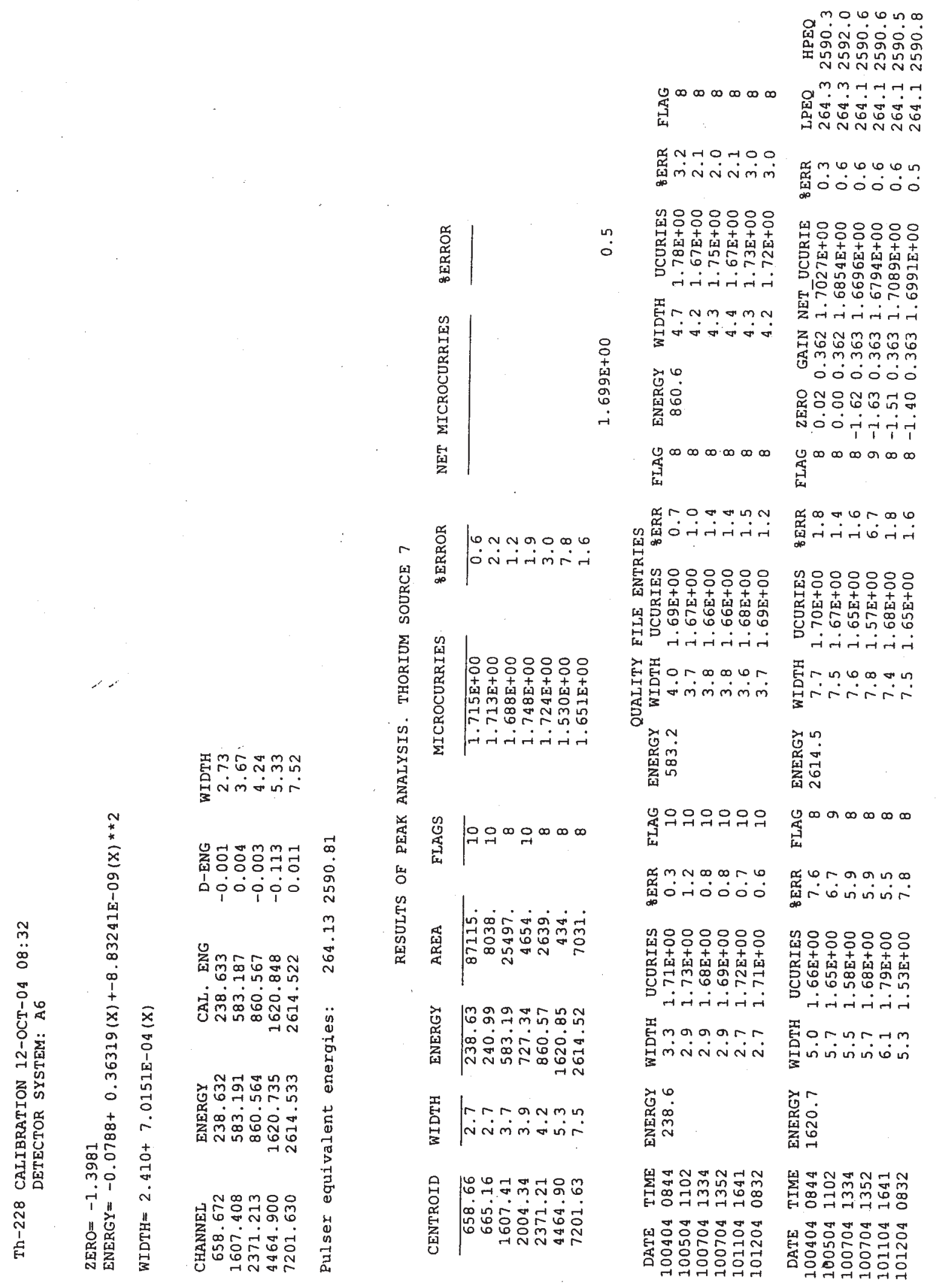

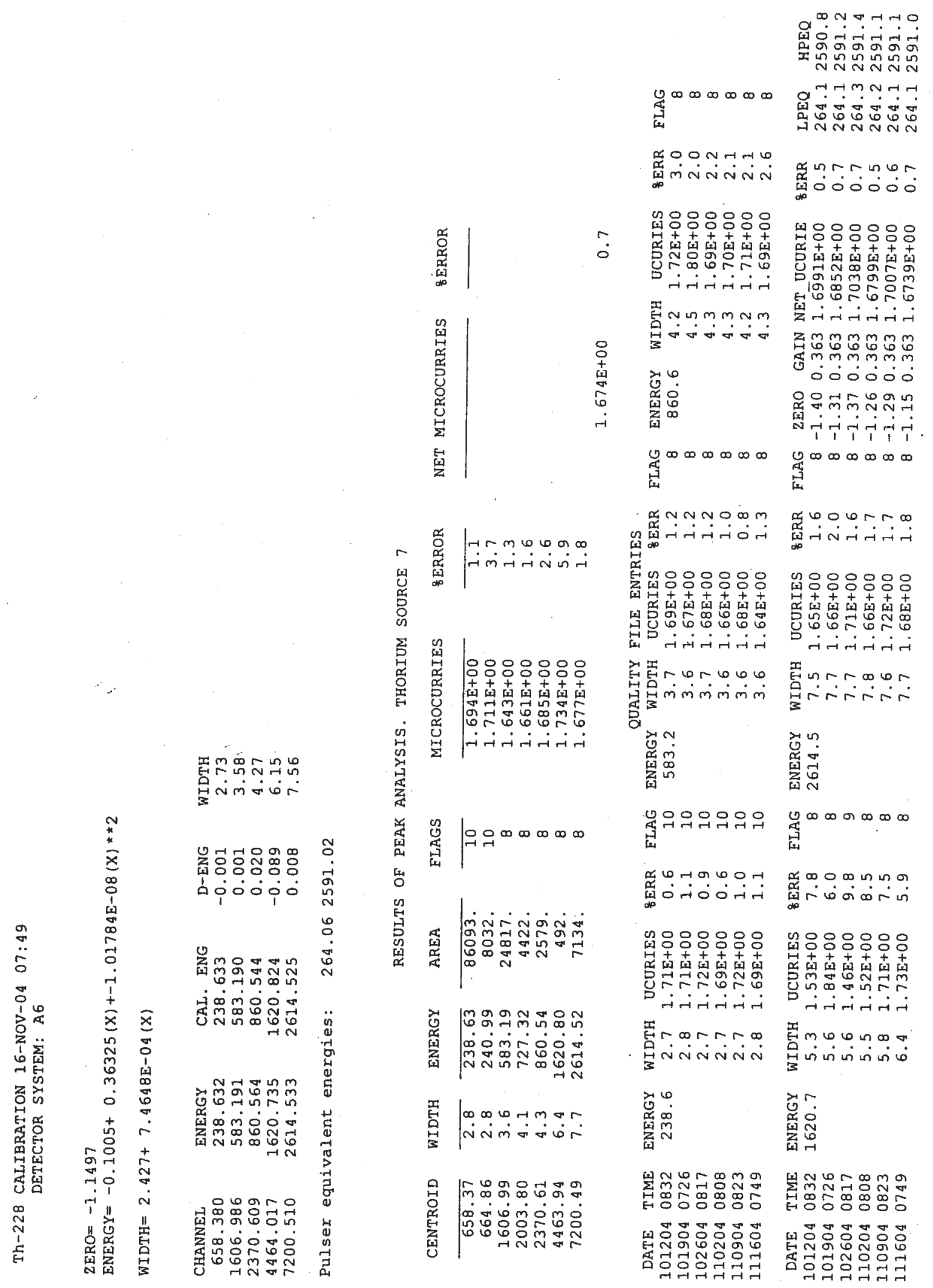

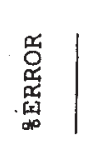

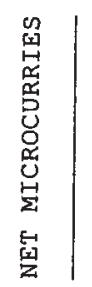

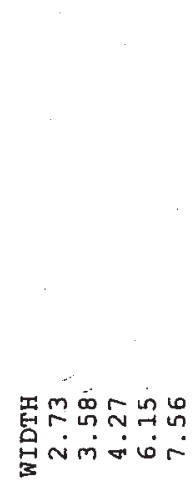

능홍요용

a

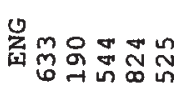

मिंखंड

U

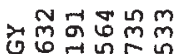

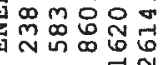

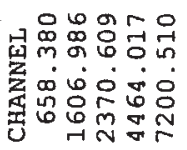

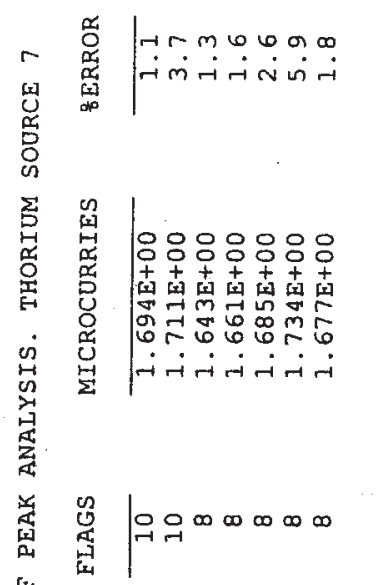

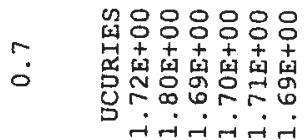

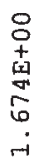

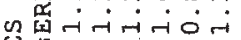

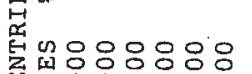

年

जिंminmm

品

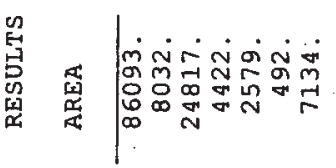

苟

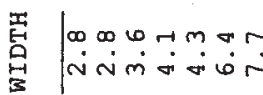

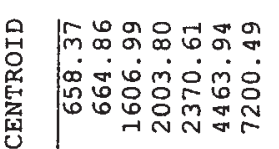

界Nกmmnm.

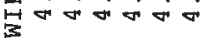

至这

昏 $\infty \infty \infty \infty \infty \infty$

๔ NNNO

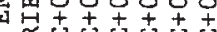

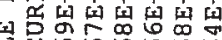

藏ror-ool

幽监

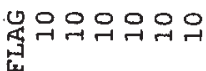

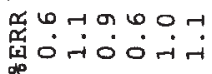

곰옹ㅇㅇㅇㅇㅇ

南南南南

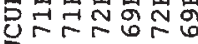

मंभiनitir

mencrisen

द्वरNiviñ

空昂

섭ㅇㅇㅇㅇㅇㅇㅇㅇㅇㅇㅇ

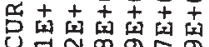

合设

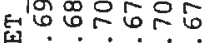

त्रतन

द्व

00000

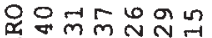

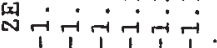

$00 \infty \infty \infty \infty$

g

㟧

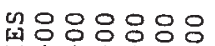

4

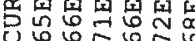

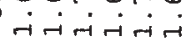

mencomor

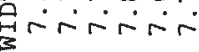

造?

$0 \infty \infty \pi \infty \infty$

Ix

我舟

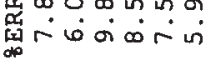

y용ㅇㅇㅇㅇㅇ

声南直血南

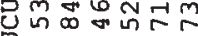

-iन्तiri

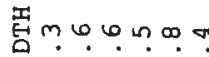

药

궁.

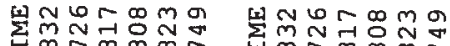

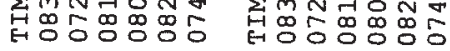

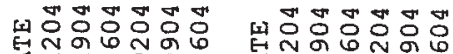

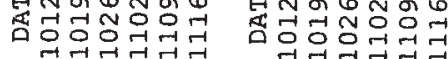




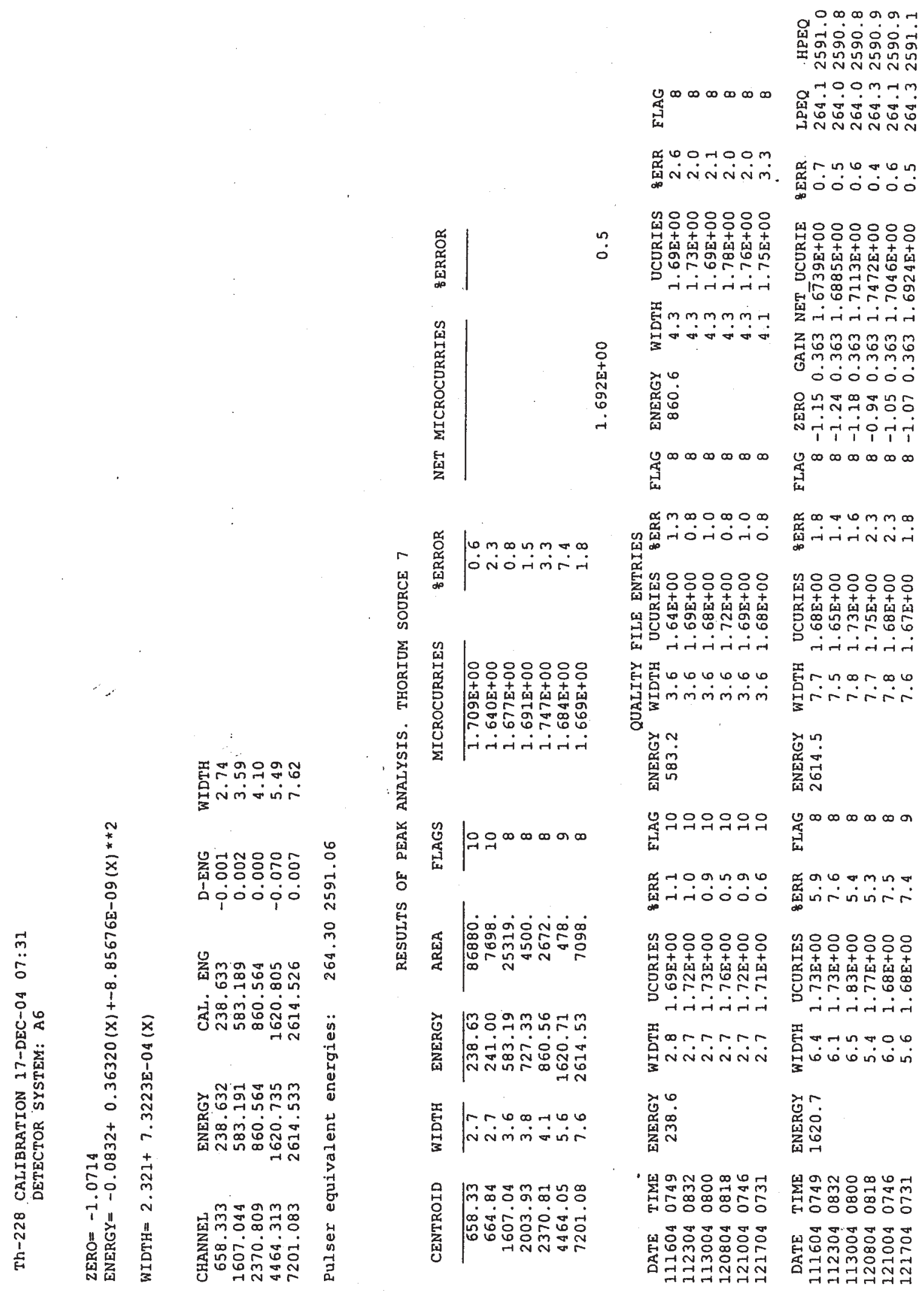




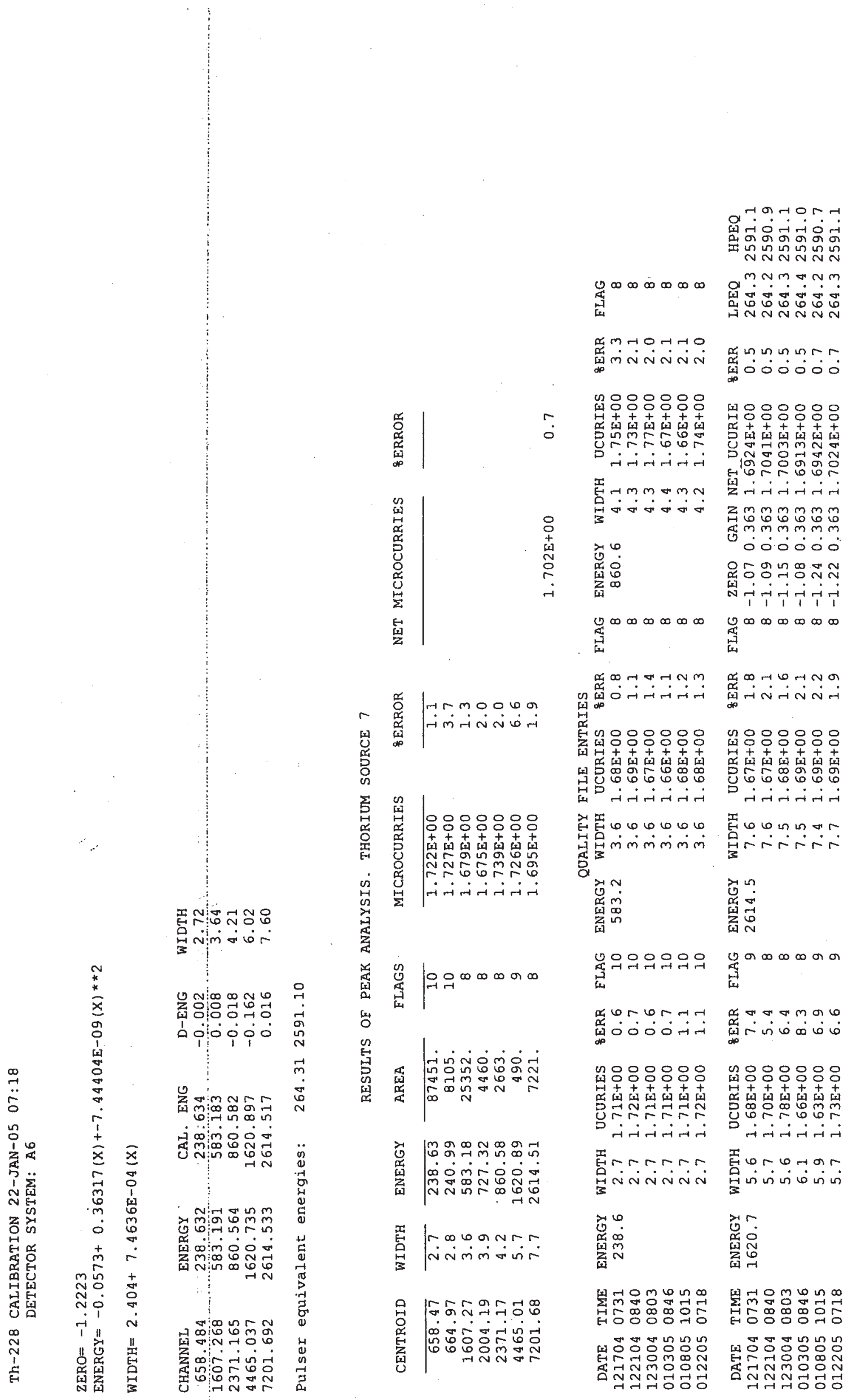




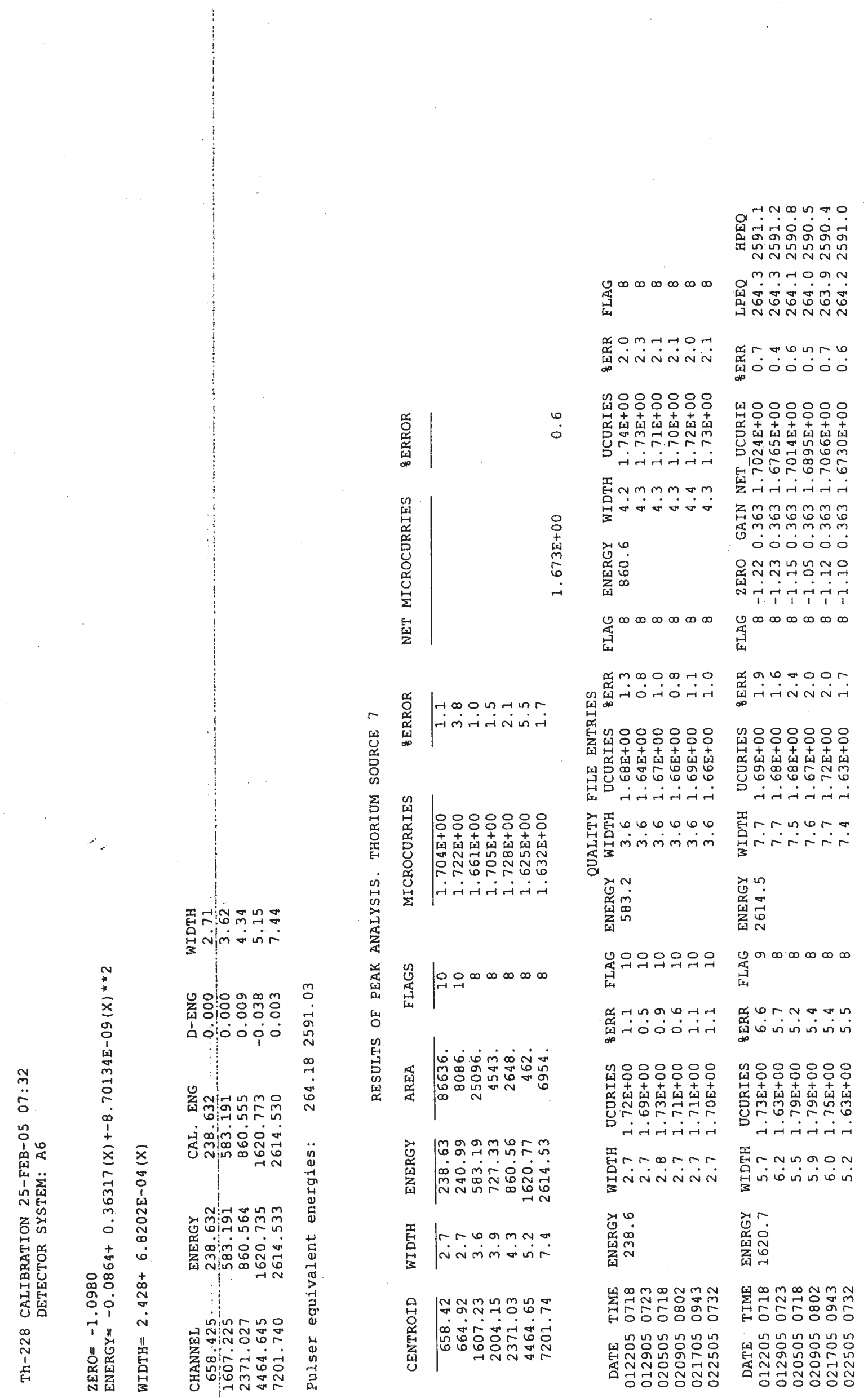


वृनmनतन

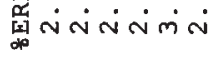

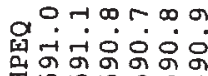

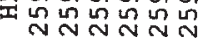

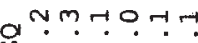

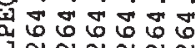

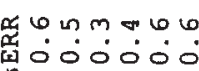

농ㅇㅇㅇㅇㅇㅇㅇㅇ (

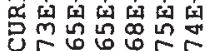
iririri

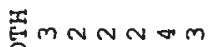

놉ㅇㅇㅇㅇㅇㅇㅇㅇㅇ

血南南南+

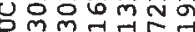

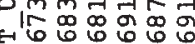

奥

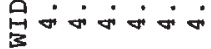

z

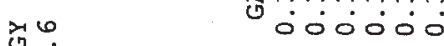

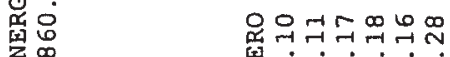

NF઼્1

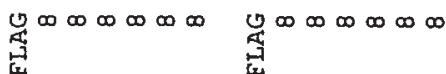

동

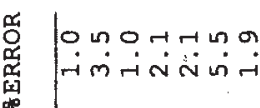

gorrao

की

xrriogor

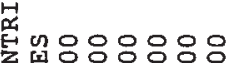

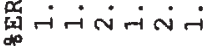

농ㅇㅇㅇㅇㅇ응

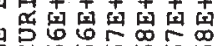

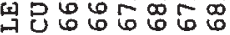

농ㅇㅇㅇㅇㅇㅇ

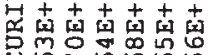

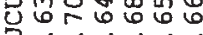

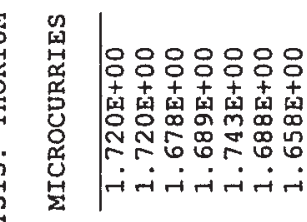

H

तं

窟 060606

目的的的

。

겅

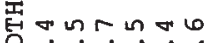

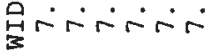

넝

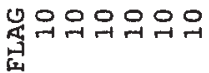

प्र $\infty \infty \infty \infty \infty$

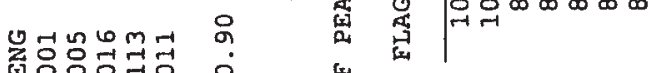

व

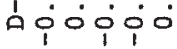

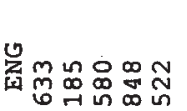

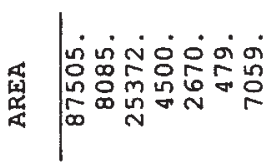

舀

य ज a

됭ㅇㅇㅇㅇㅇㅇㅇㅇ

w

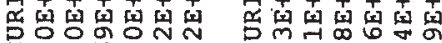

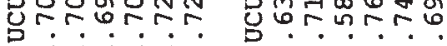

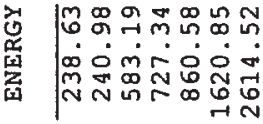

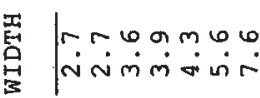

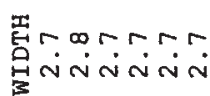
तi - i

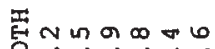

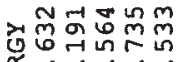

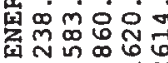

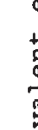

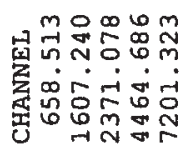

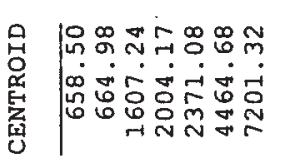

贷品

究贺

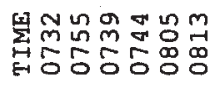

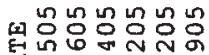

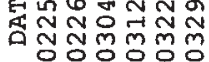

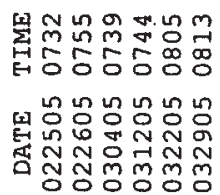




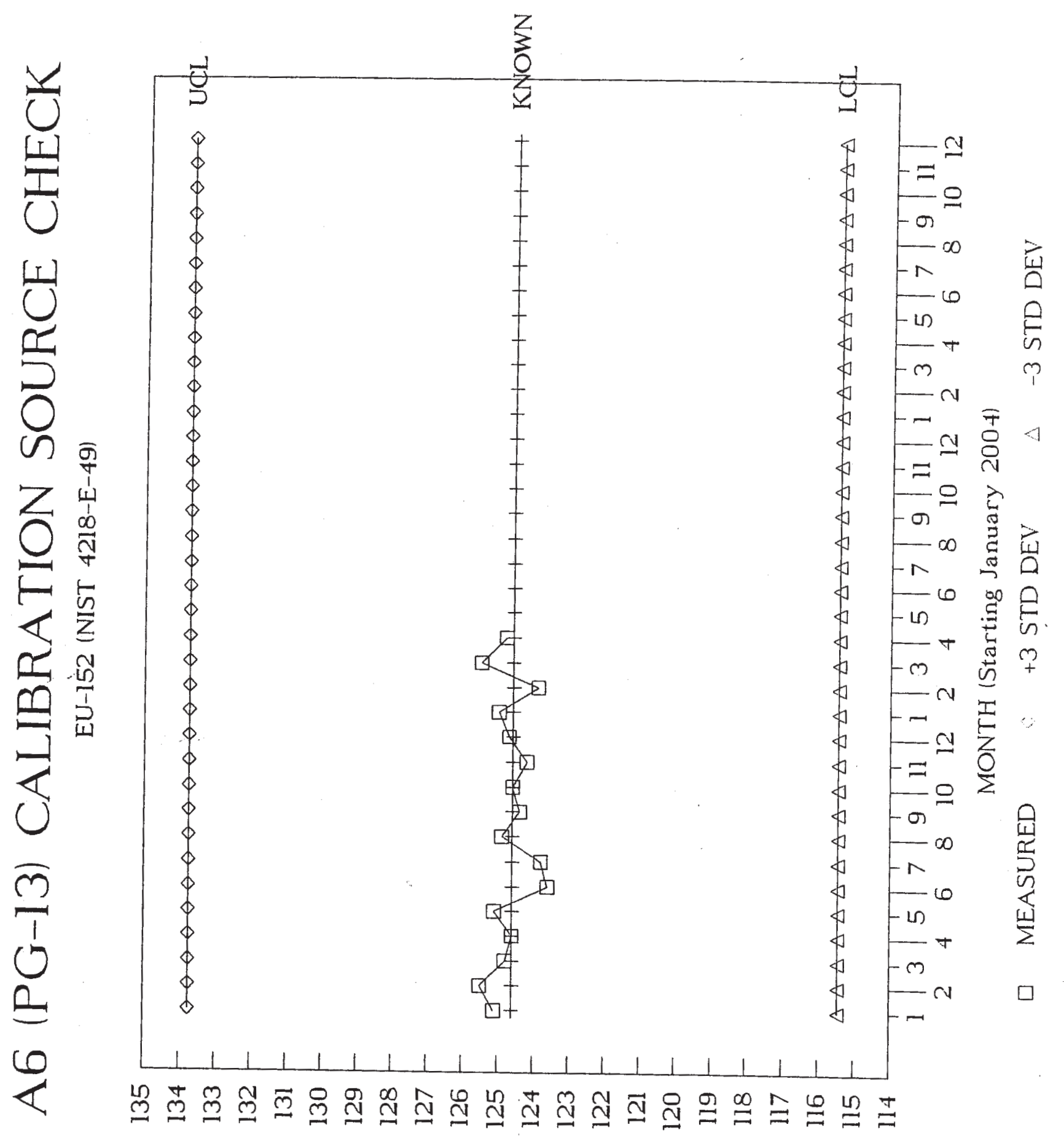




\section{RADIATION MEASUREMENTS LABORATORY}

\section{Supporting QA/QC Date}

\section{AMCHITKA ISLAND ENVIRONMENTAL ANALYSIS}

For: RML Ge Detector System: D4, Model No. PG-30

\section{INTERNAL QC CHECKS:}

Gamma-ray energy calibration (Daily or prior to detector use, pulsar equivalents are determined weekly for pulsar based detector systems).

Monthly instrument background check.

Monthly Eu-152 calibration source check.

EXTERNAL QC CHECKS (when applicable):

Mixed Analyte Performance Evaluation Program (MAPEP)

SEE RESULTS FOR RML EXTERNAL QC CHECKS IN APPENDIX D

Analyzed by: Date:

Approved by: Date:

\section{COMMENTS:}

All applicable QA/QC checks demonstrate the RML was "in control" during the time frame the samples were counted and analyzed. 
RADIATION MEASUREMENTS LABORATORY D4 (PG-30) CO-60 AMBIENT BACKGROUND
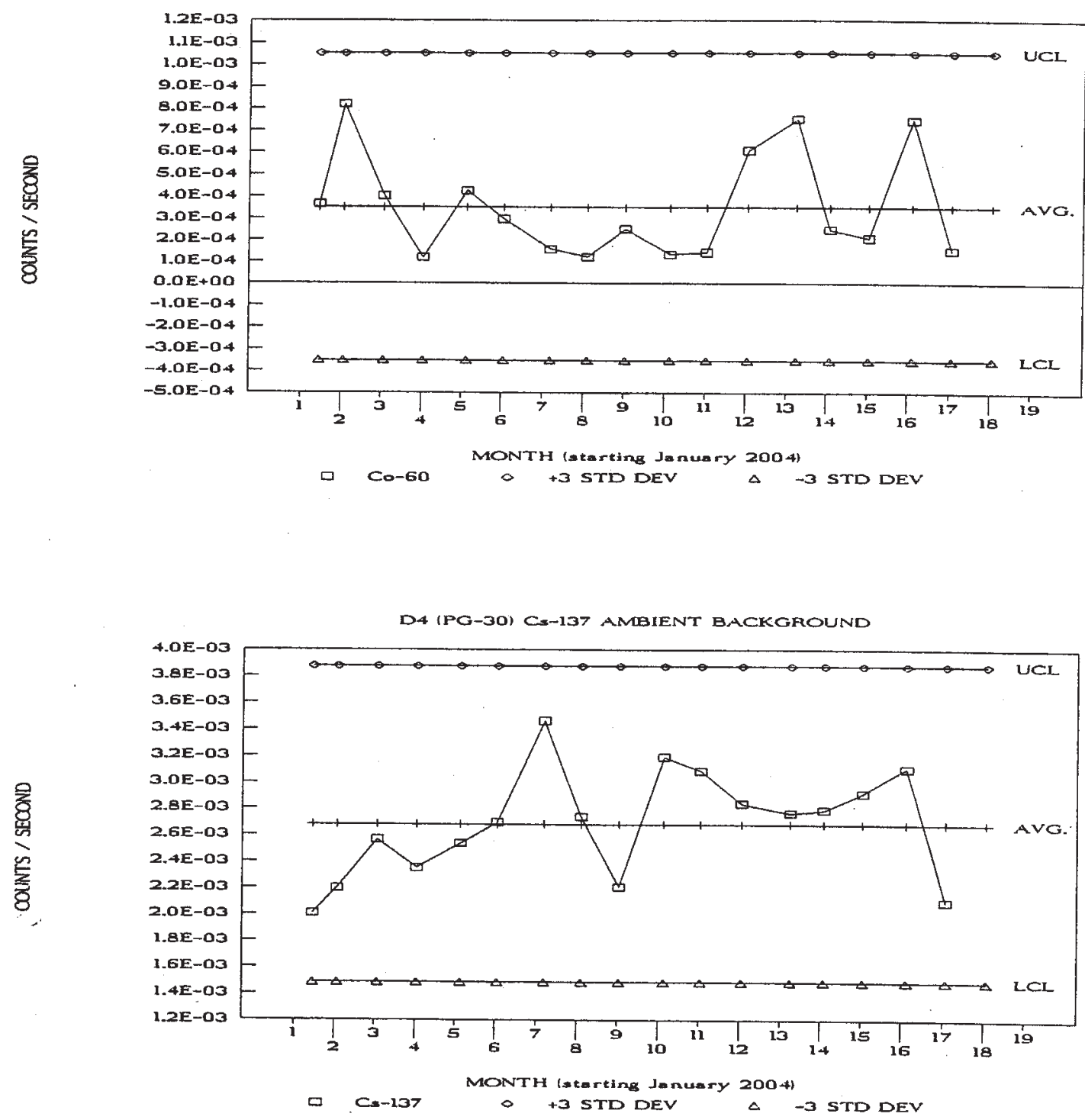

D4 (PG-30) NATURAL ACTIVITY BACKGROUND

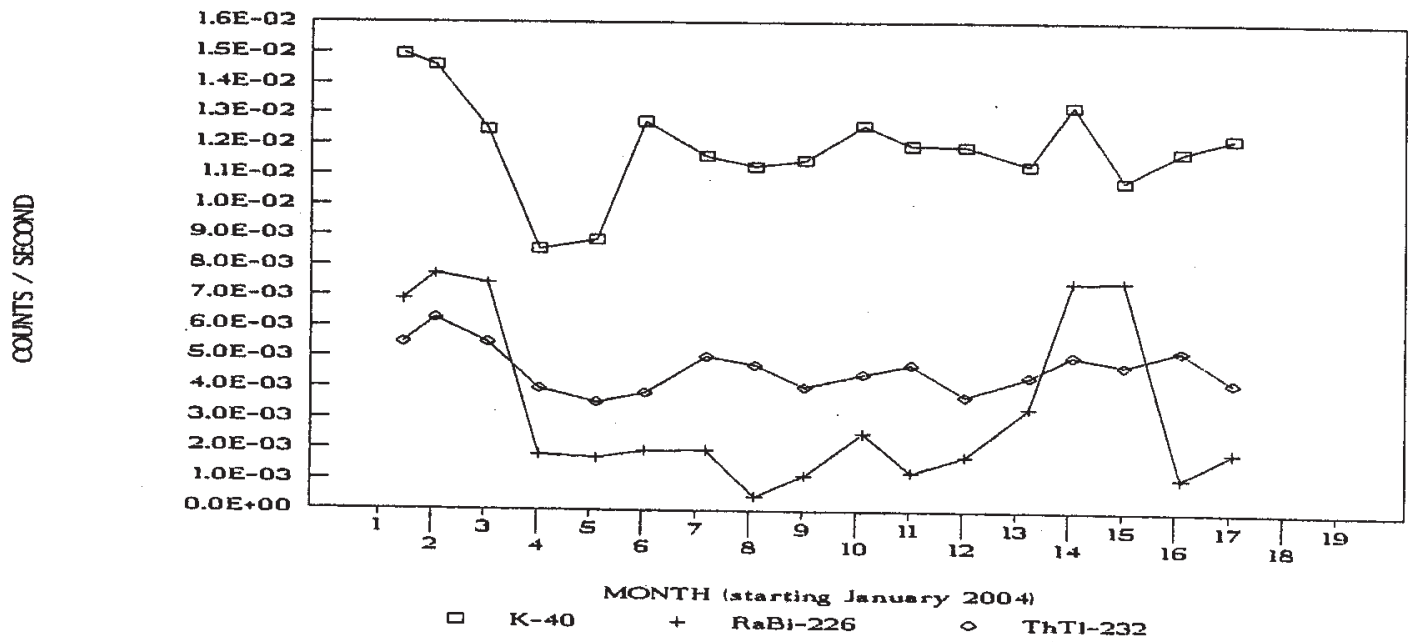



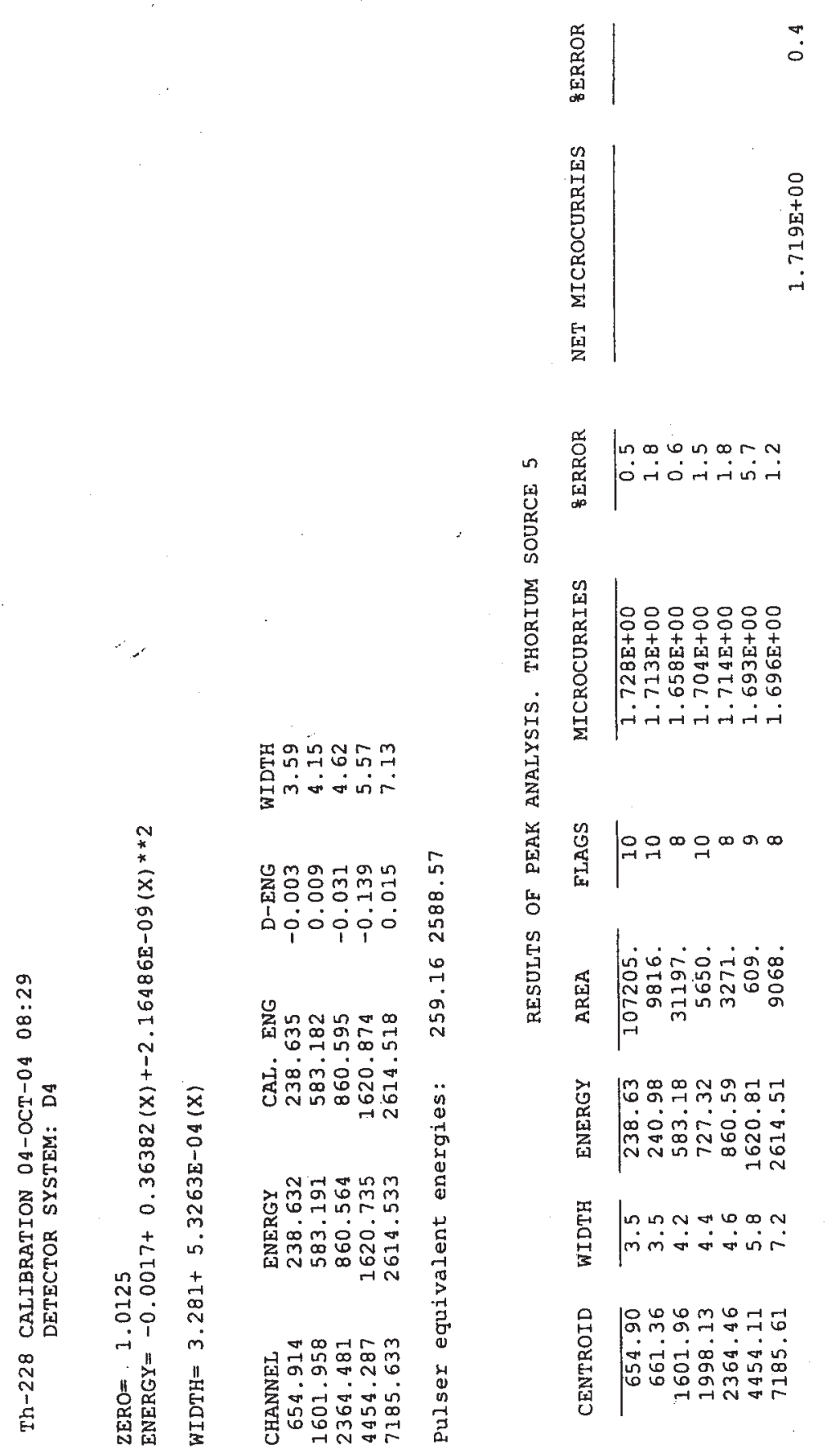

量

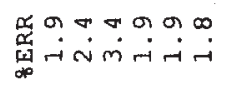

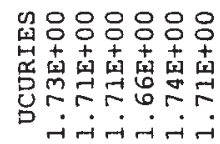

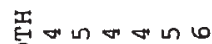

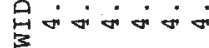

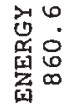

近 $\infty \infty \infty \infty \infty$

4006ro

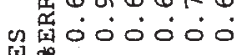

国 d

究

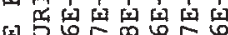

1 066666

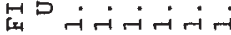

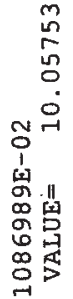

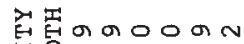

它的宁的 号

an

佫品

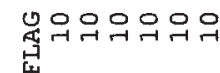

密

乐密点

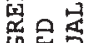

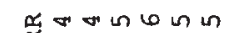

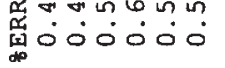

뇡ㅇㅇㅇㅇㅇㅇㅇㅇ

国国的国国利

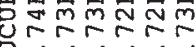
So मrifitifiti

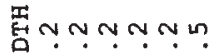
㝵的的的的

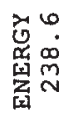

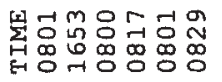

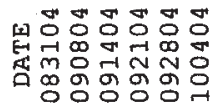

d.․․․․

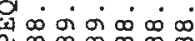

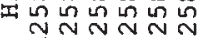

TNNNNN

घंकानंकां

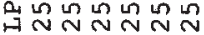

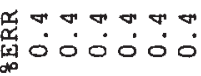

뵙ㅇㅇㅇ응응응

南南南南+

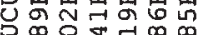

IN品

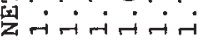

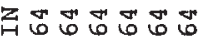

m mm mm

\%

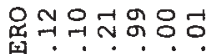

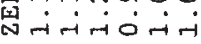

近 $\infty \infty \infty \infty \infty$

xh

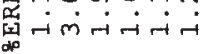

광ㅇㅇㅇㅇㅇㅇㅇㅇㅇ

南声血南声

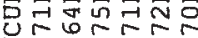

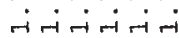

泉mommm

若ririris

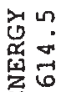

范

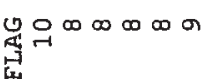

x m $r$ un or

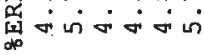

읭ㅇㅇㅇㅇㅇㅇㅇㅇㅇㅇ

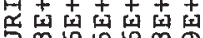

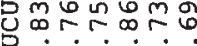

-iनi-i

蛋NNROD

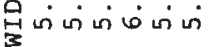

倇:

迳管

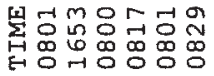

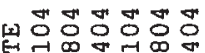

通前 


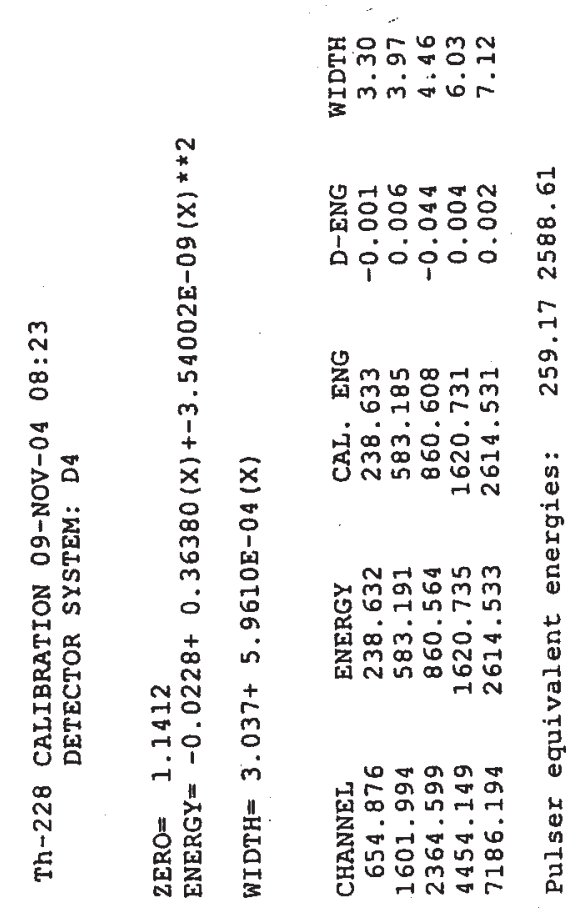

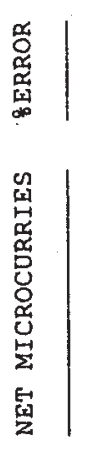

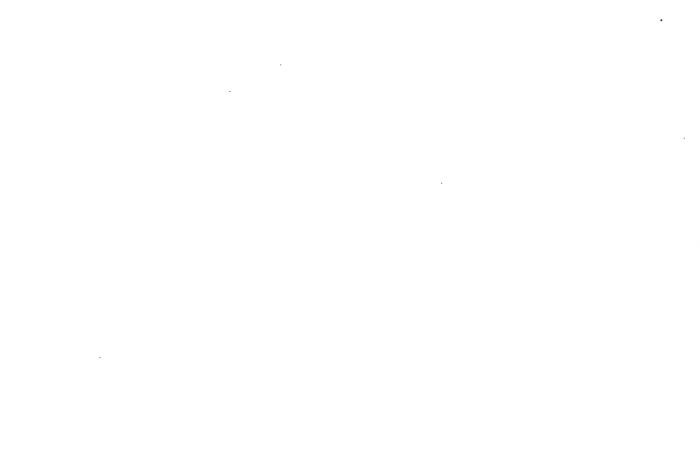

\section{동ㅇㅇㅇㅇㅇㅇㅇㅇㅇ}

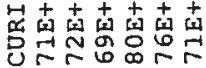

त1000 006

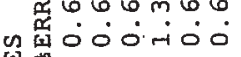
幽 茲舟응ㅇㅇㅇㅇㅇㅇ 等 골

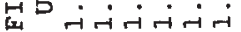

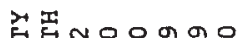

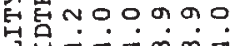
客 话N

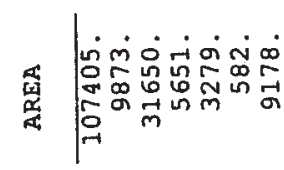

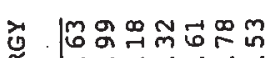
商

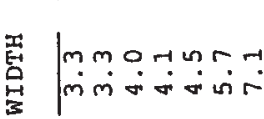

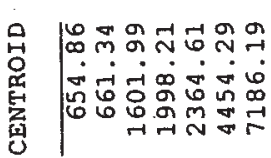

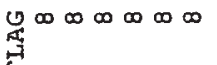

य $\infty$ \%

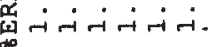
वंत्मतनंत्र

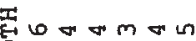

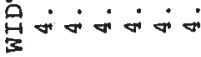

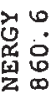

Uु $\infty \infty \infty \infty \infty \infty$ 䍃

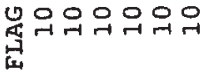
땐ำ 舟00000

6

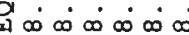

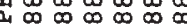
ง MNNWHN aviorio

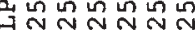

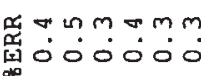

넝ㅇㅇㅇㅇㅇㅇㅇㅇ 每南南直十 Un 舟 舆唡证。 乙 정ำmmm 000000

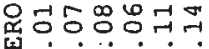

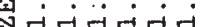
पू山 $\infty \infty \infty \infty \infty \infty$ xNAHAT

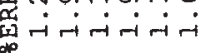
닝ㅇㅇㅇㅇㅇㅇㅇㅇㅇ 4

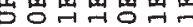
पु다달

犚nmmata 否riritio

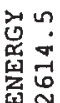

ט 舀

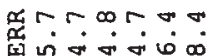

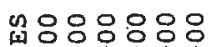

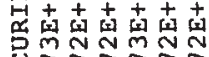

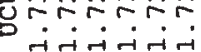

葛几nNmmm

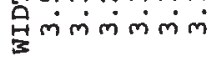

똥ㅇㅇㅇㅇㅇㅇㅛ

南南南南南

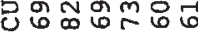

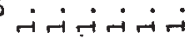

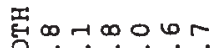

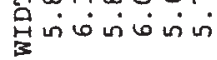
党:

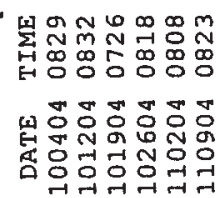

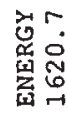

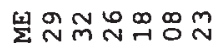

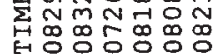

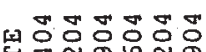

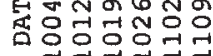




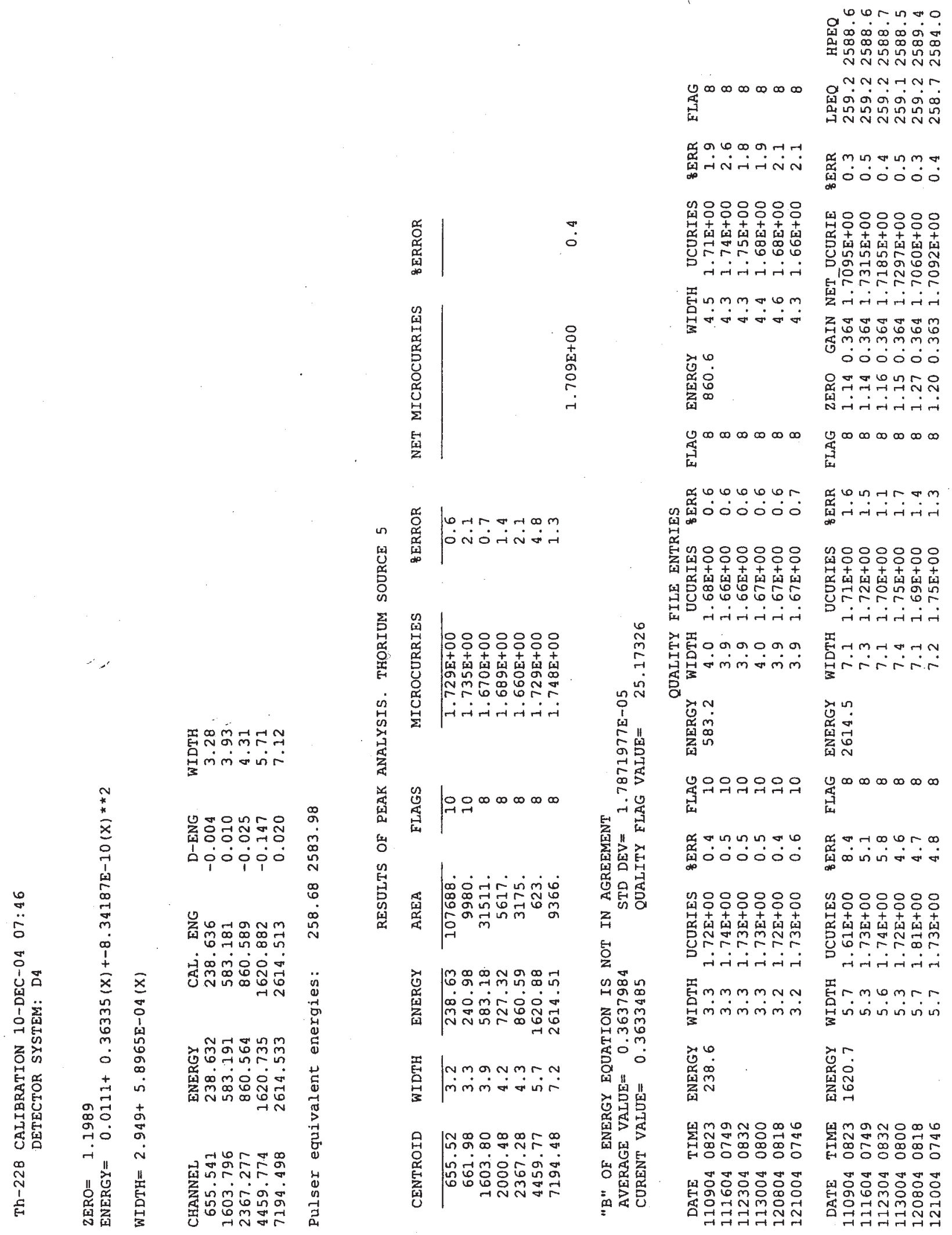




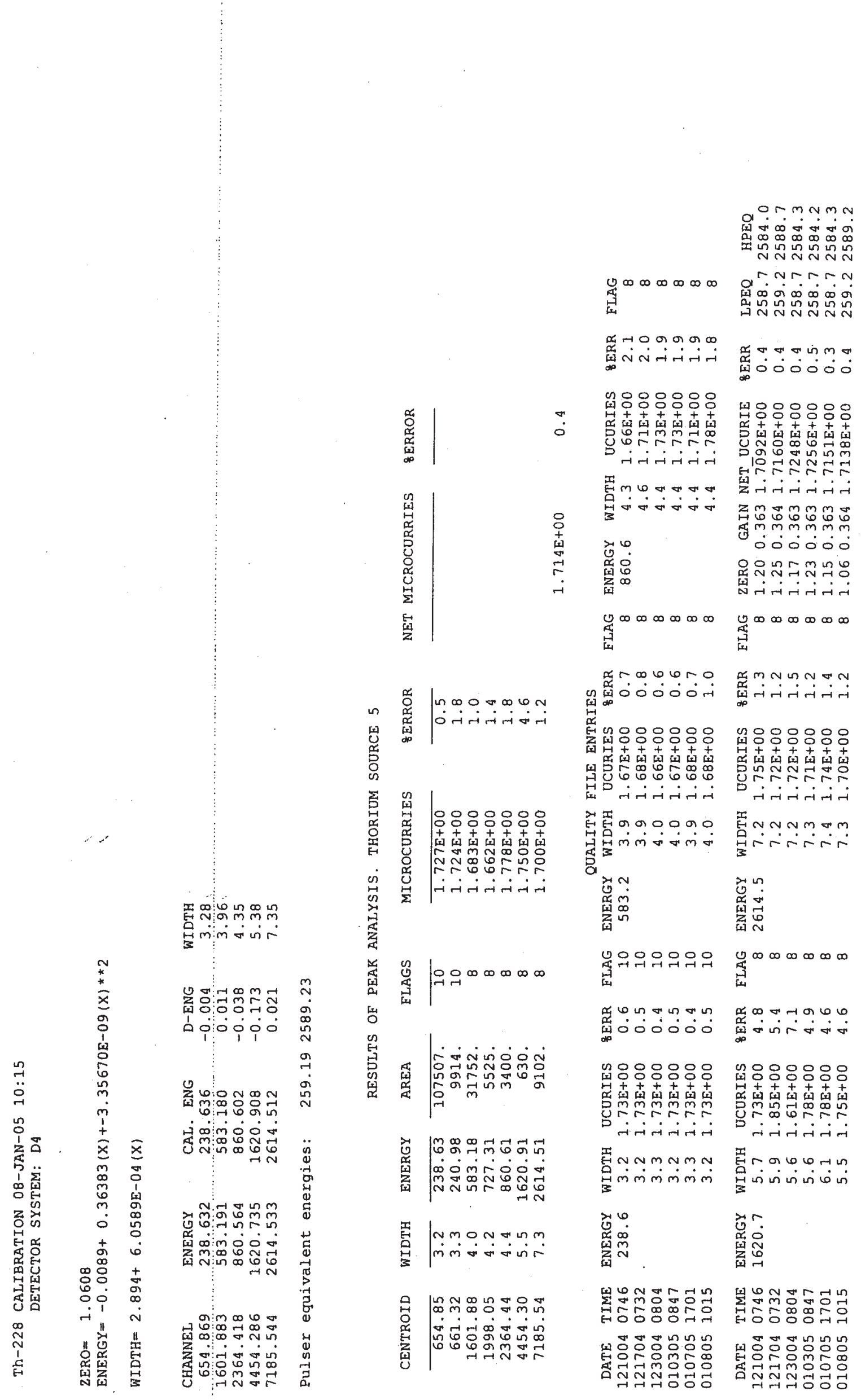




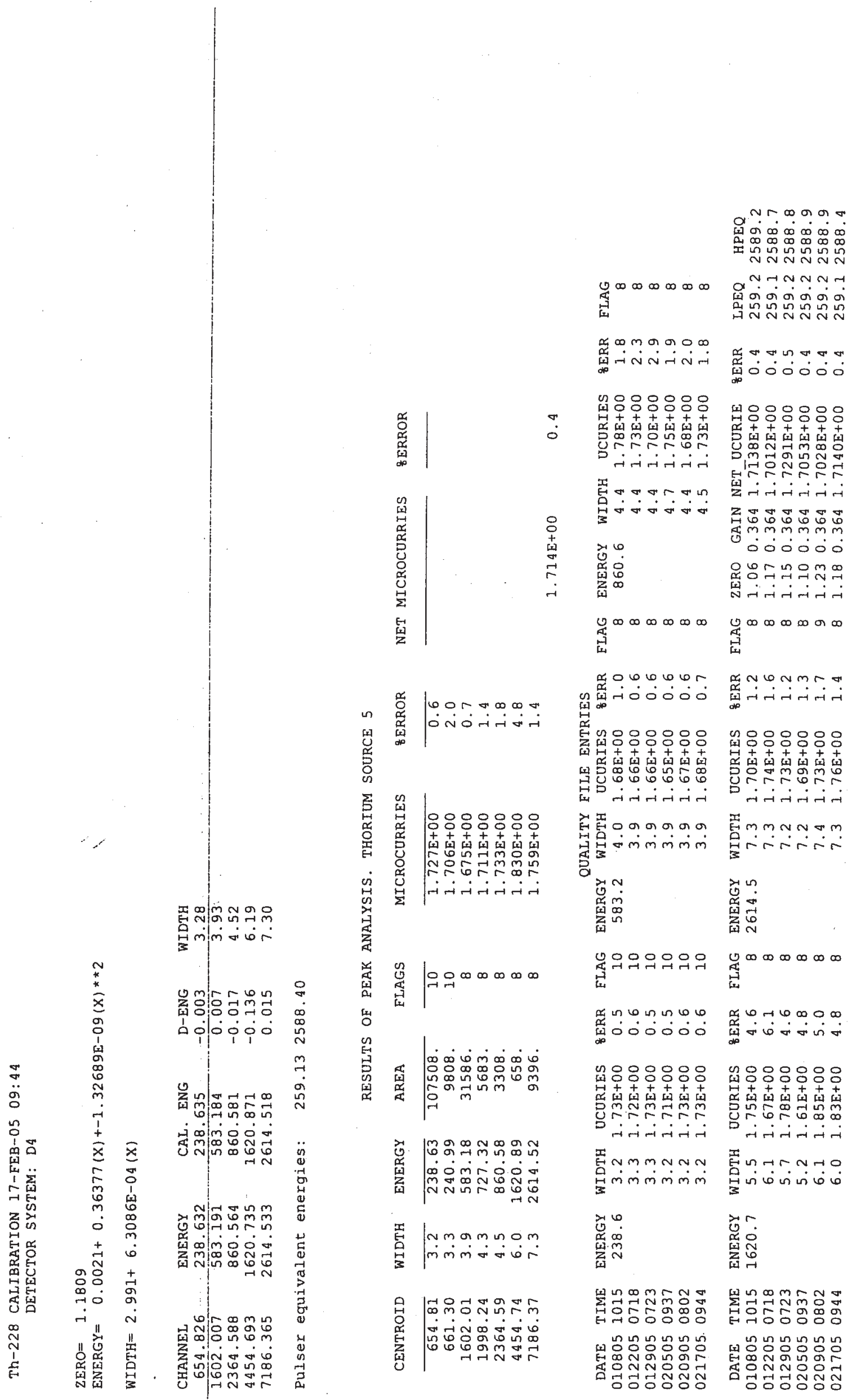




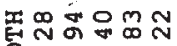
药的政

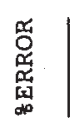

品

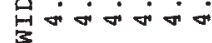

208888

ㅂ.

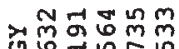

畄舟宓家:

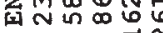

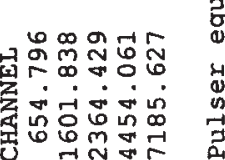

峉

$\infty \sim \infty N \sim \pi$ 覆的

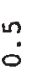

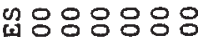

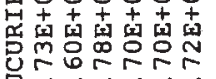
नंतनंत्रत

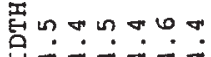

过是

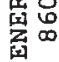

$0 \infty \infty \infty \infty$ 空

4

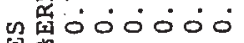

空

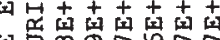

닐

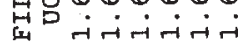

त्रिभा 000000

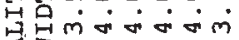

5

걸.

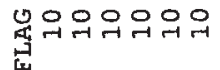

Tonnons

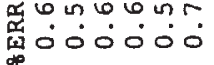

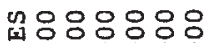

南南南南

己ृำ?

तंसंत्नंत्र

留

trr $\infty \pi \infty$

$\infty \infty \infty \infty \infty$

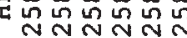

HNNNNN

बंनं कं नं

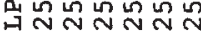

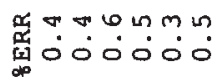

떱용ㅇㅇㅇㅇㅇㅇㅇ

tr

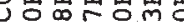

本워숭

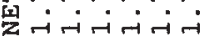

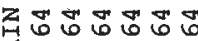

बुलmm m

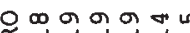

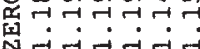

u $\infty \infty \infty \infty \infty$ on

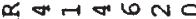

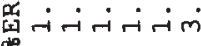

ํㅗㅇㅇㅇㅇㅇㅡ

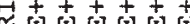

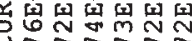

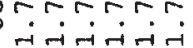

疍mNmmmm

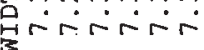

군?

$0 \infty \infty \infty \infty \infty$

㩊

का का

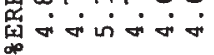

뙨ㅇㅇㅇㅇㅇㅇㅇㅛ

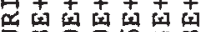

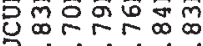
तनातन

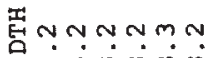

葛。oomra

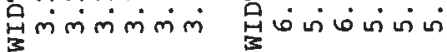

倌

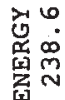

过

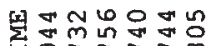
봉동ㄷㅇㅇㅇㅇ

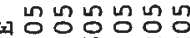

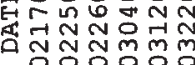

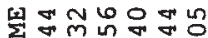
붕ㅇㅇㅇㄷㅇㅇㅇㅇ

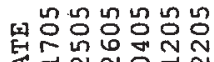
政 


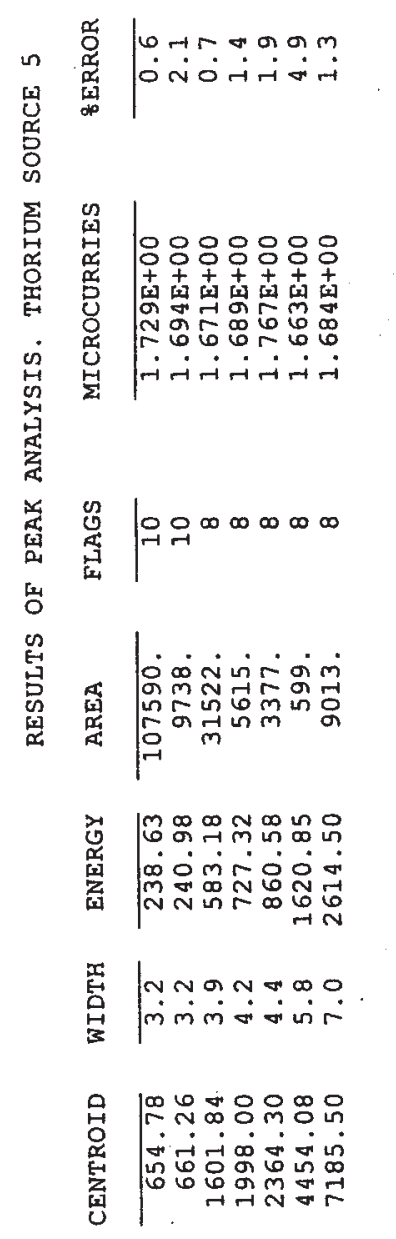

คrค

a $\infty \infty \infty \infty$

곤 $\stackrel{n}{\sim} \stackrel{n}{N} \stackrel{n}{N}$

प్ఝ

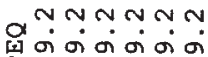

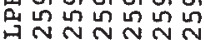

ג?ำ?

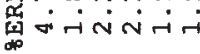

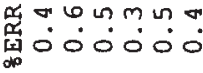
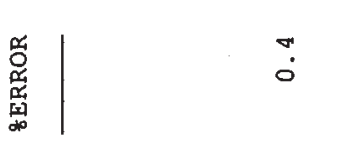

웡ㅇㅇㅇㅇㅇㅇㅇㅇ

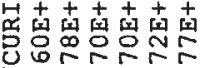
-iनiriri

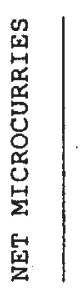

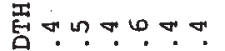

8
8
+
1
8
8
8
-

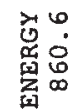

辰我 $\infty \infty \infty \infty$

봅용ㅇㅇㅇㅇㅇㅇㅇ

南南南南南

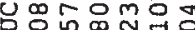

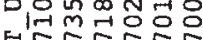

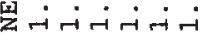

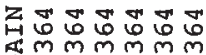

वं0ं0ं

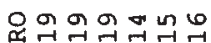

界

Uू $\infty \infty \infty \infty \infty$

प $06060 \mathrm{r}$

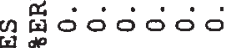

要

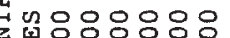

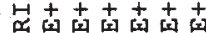

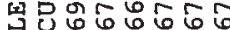

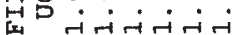

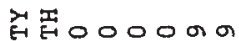

岁是的宁的

वे

怨

罢品

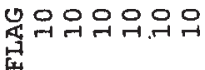

दू

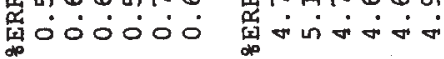

뇡ㅇㅇㅇㅇㅇㅇㅇㅇ음

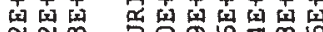

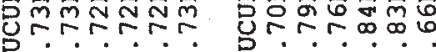

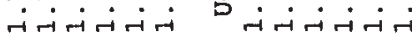

INNNMNN

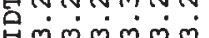

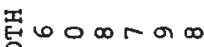
是

空星

苍员

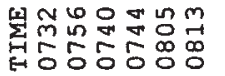

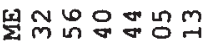

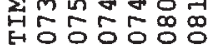

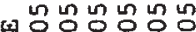

뙤융융융ㄴㅇㅇ융

TNN 


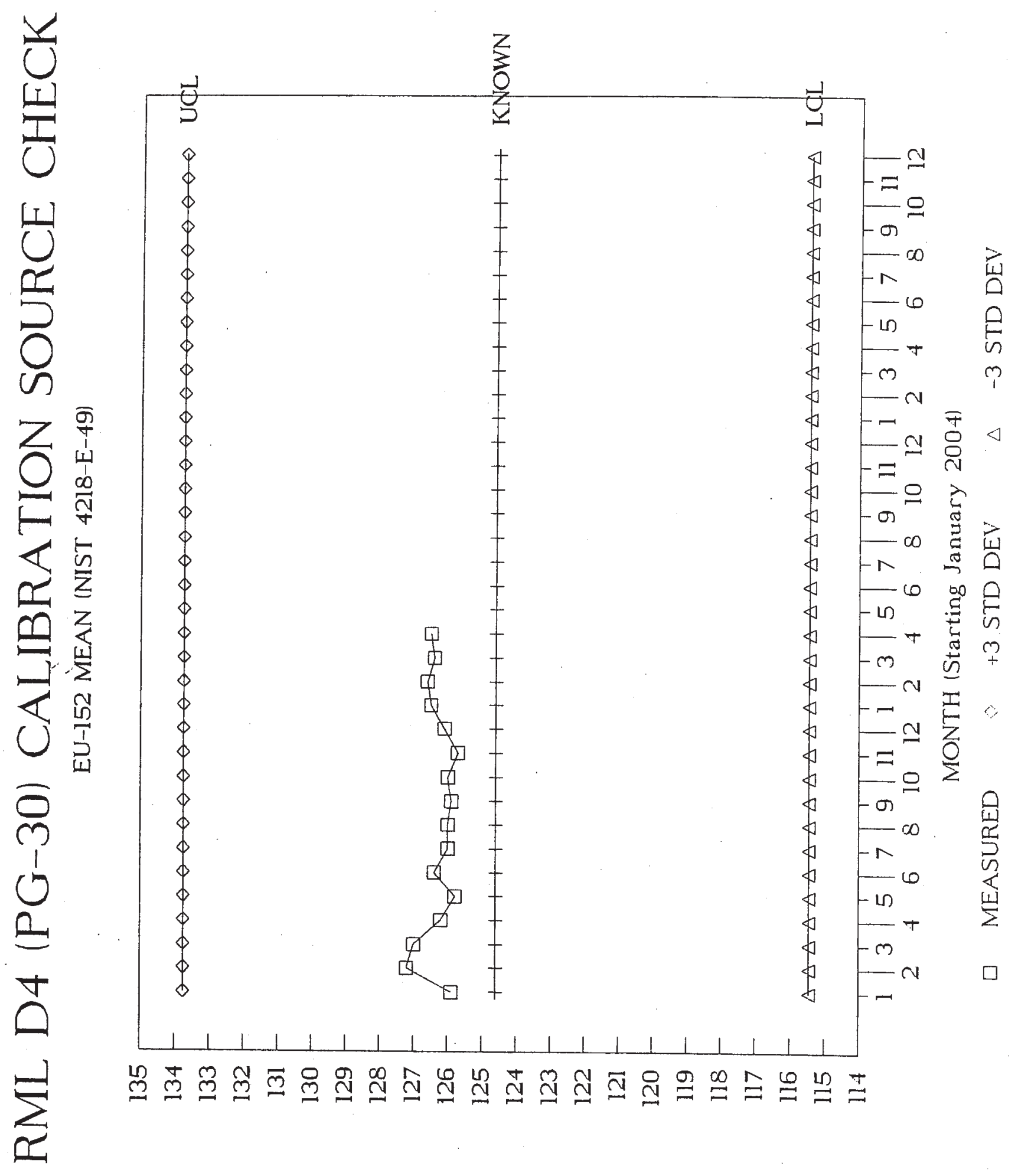

(spuesnoyL)

รางบษกอวヨ日 


\section{RADIATION MEASUREMENTS LABORATORY}

\section{Supporting QA/QC Date}

\section{AMCHITKA ISLAND ENVIRONMENTAL ANALYSIS}

For: RML LEGe (leps) Detector System: B4, Model No. CAN-41

\section{INTERNAL QC CHECKS:}

Gamma-ray energy calibration (Daily or prior to detector use, pulsar equivalents are determined weekly for pulsar based detector systems).

Monthly instrument background check.

Monthly I-129 calibration source check.

EXTERNAL QC CHECKS (when applicable):

Mixed Analyte Performance Evaluation Program (MAPEP)

\section{SEE RESULTS FOR RML EXTERNAL QC CHECKS IN APPENDIX D}

Analyzed by: Date:

Approved by: Date:

\section{COMMENT'S:}

All applicable QA/QC checks demonstrate the RML was "in control" during the time frame the samples were counted and analyzed. 


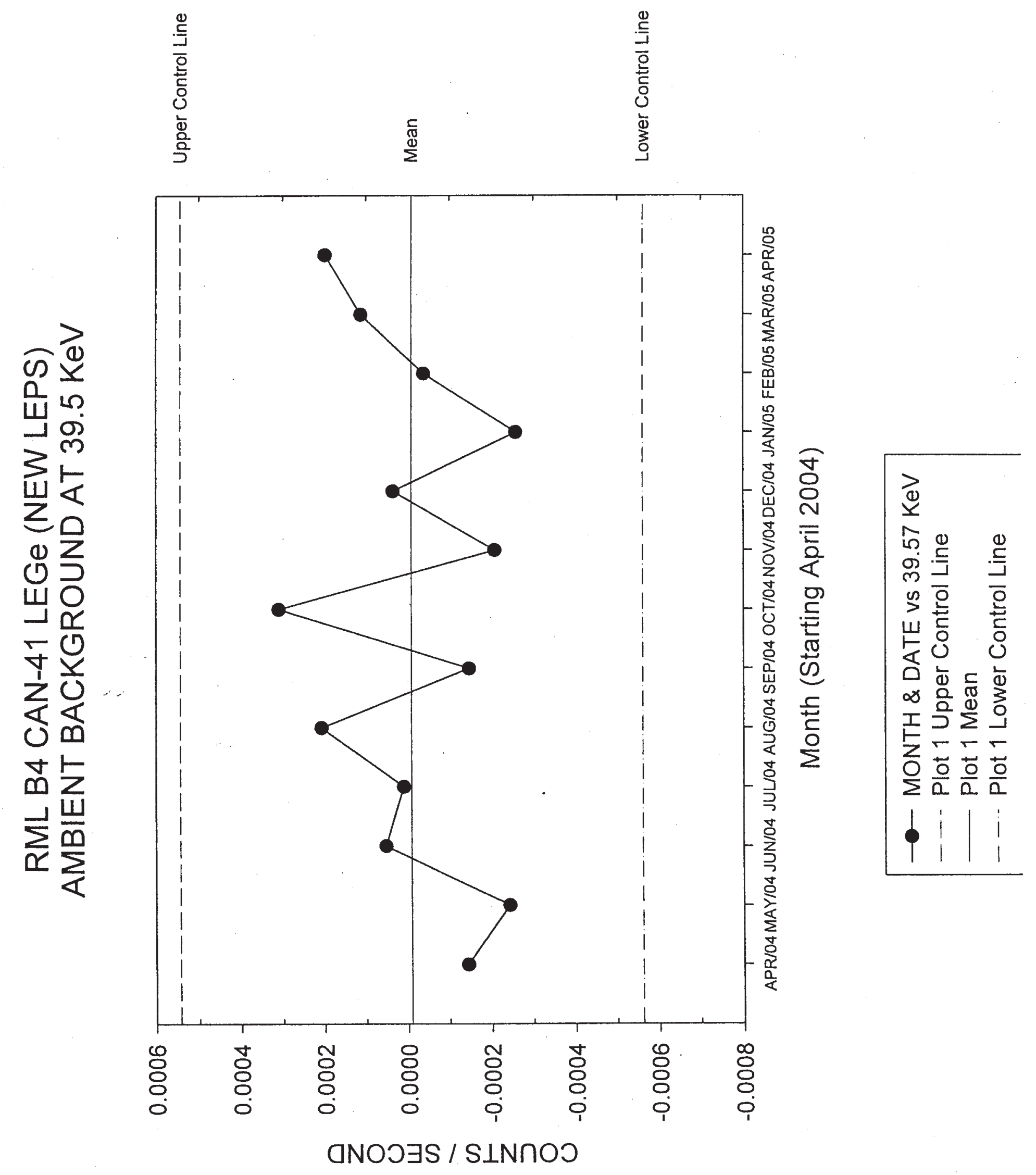



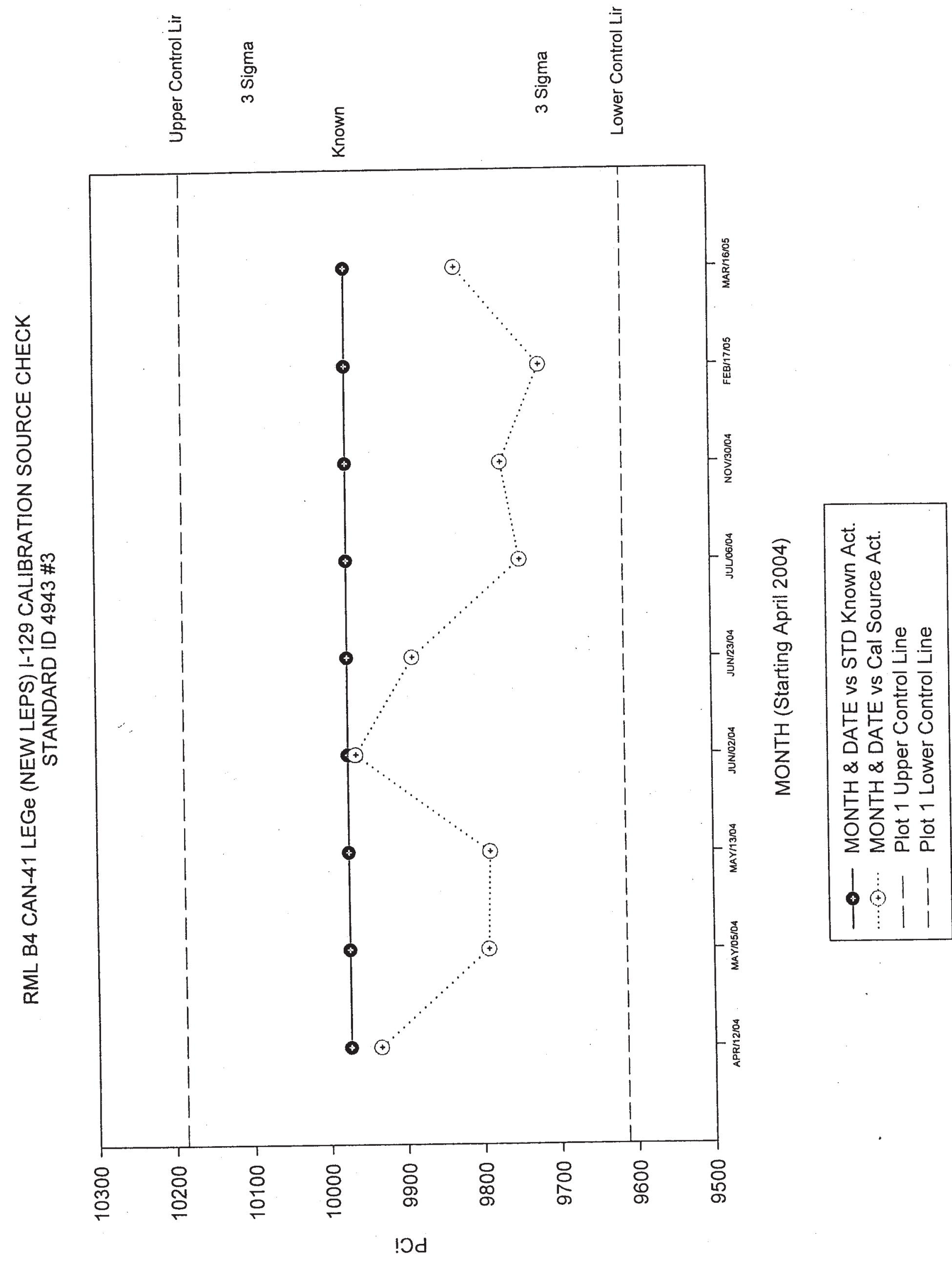


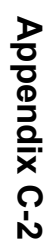


Appendix C-2

Alpha Spectroscopy and Sr-90 Internal QA/QC Program Results 


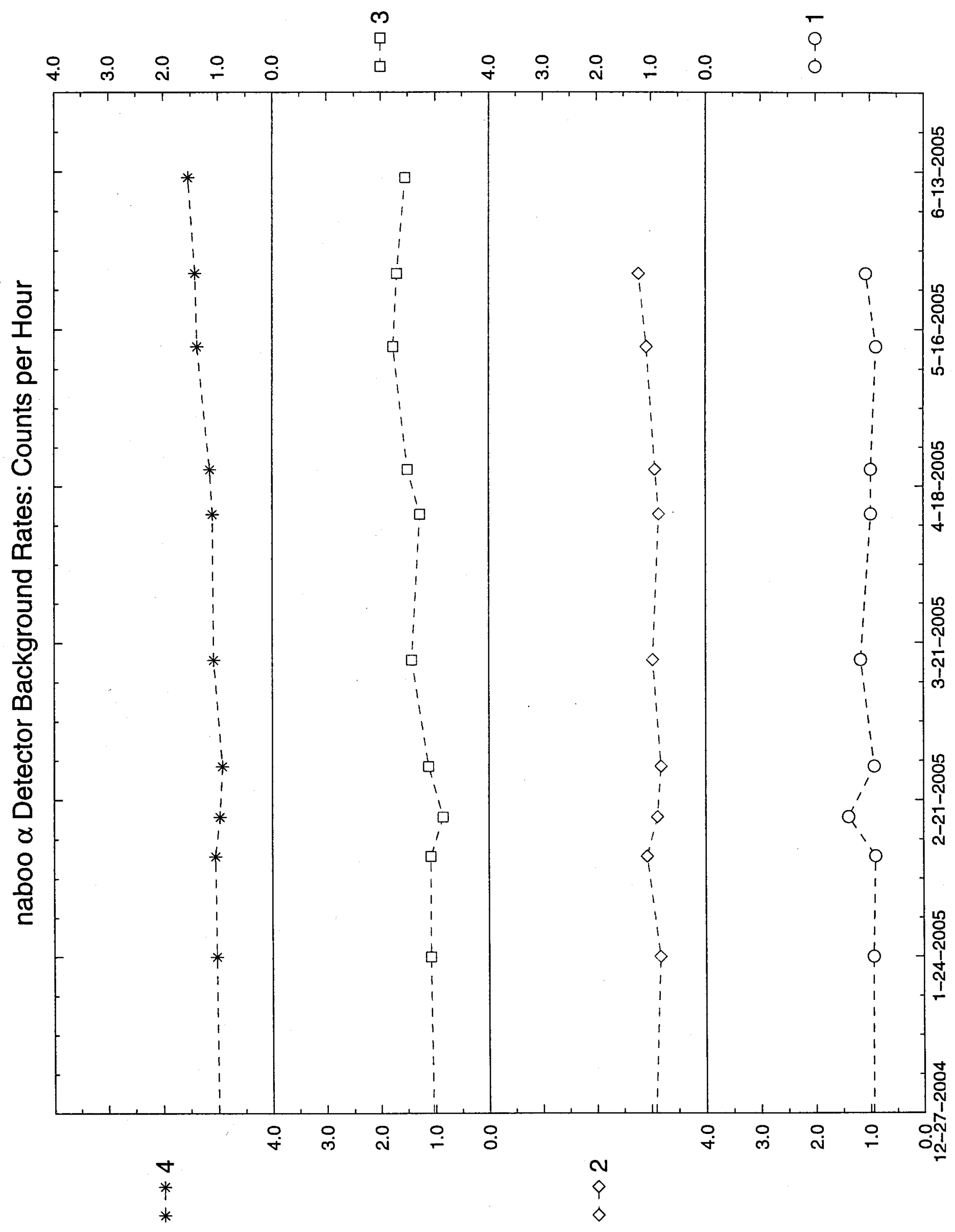




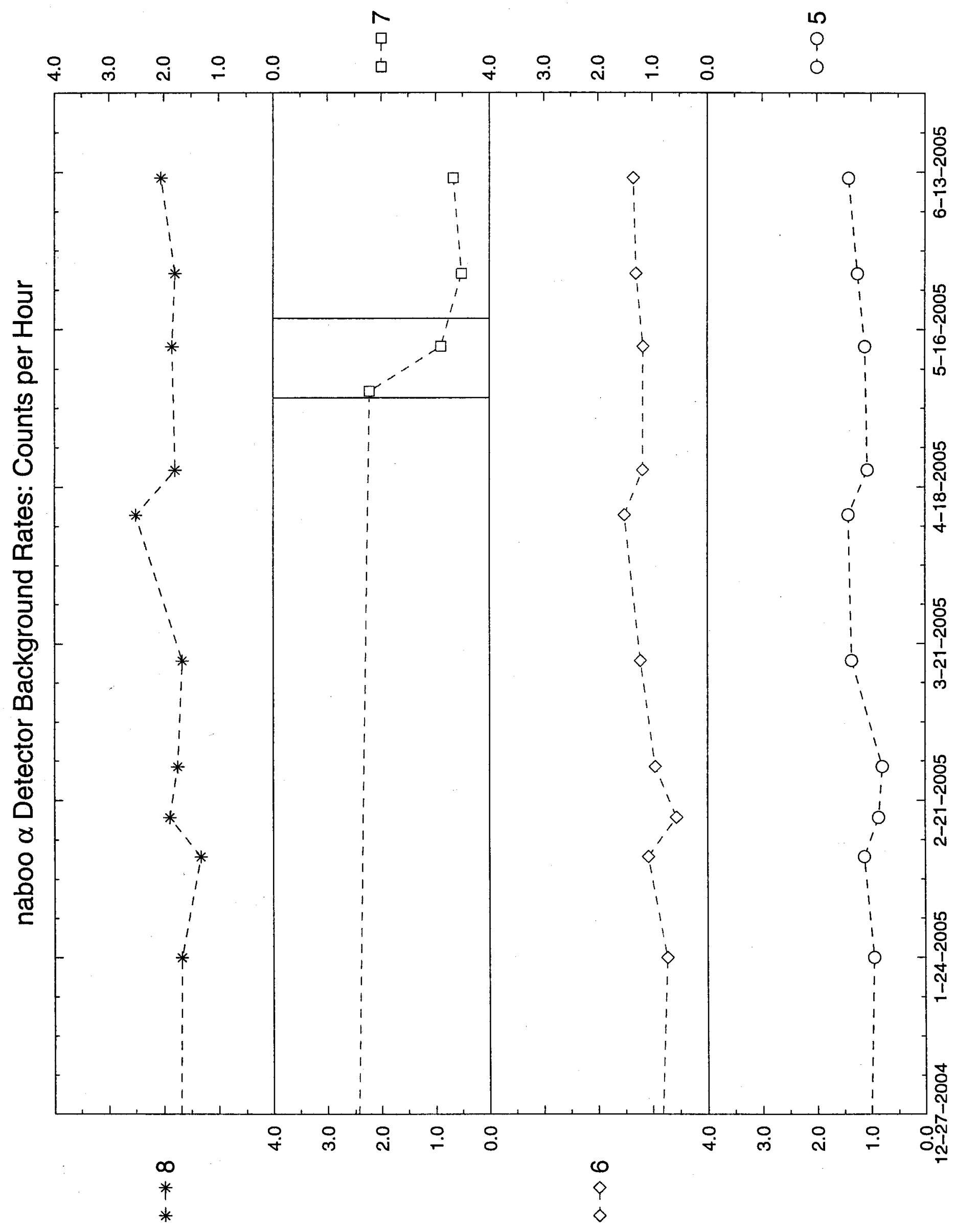




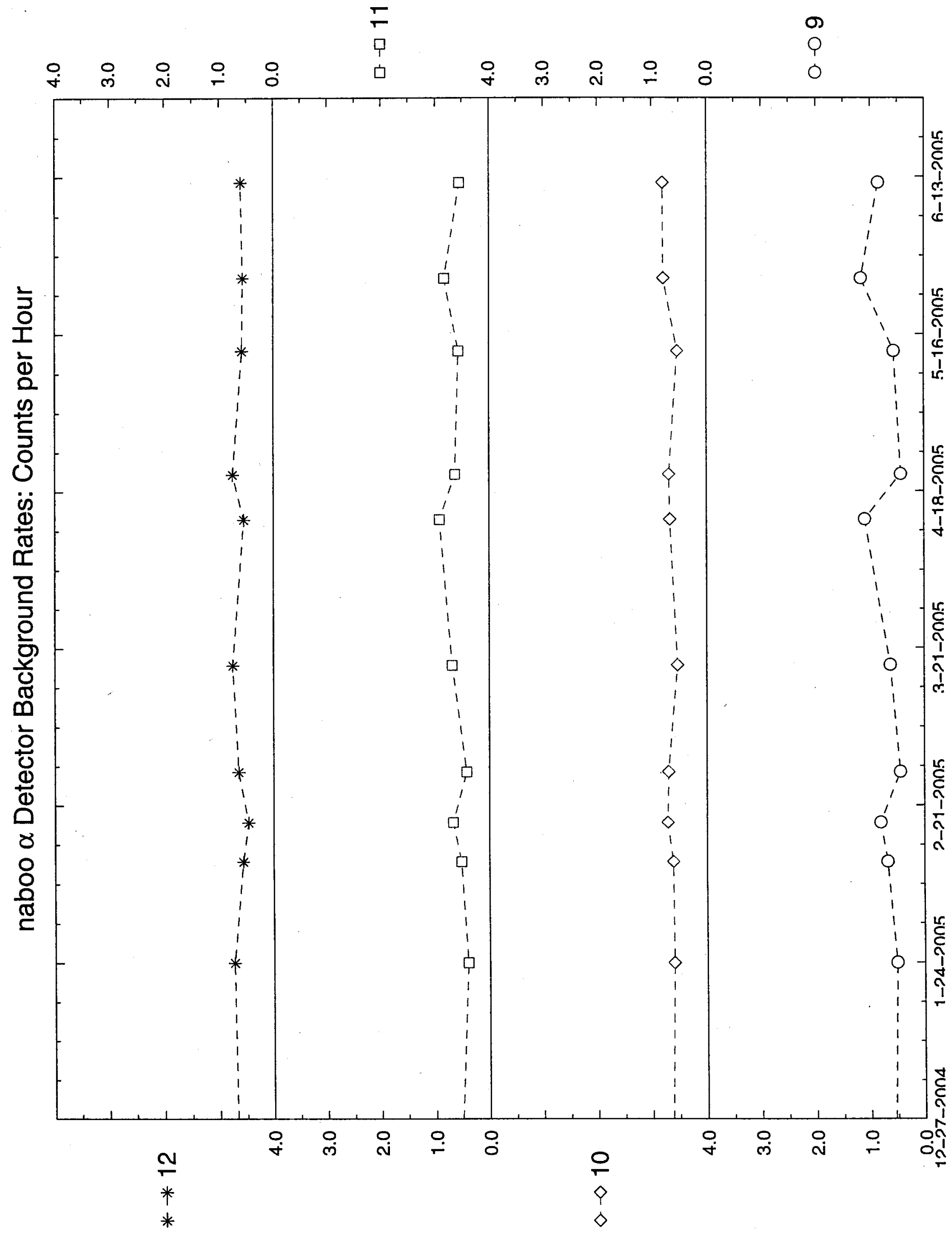




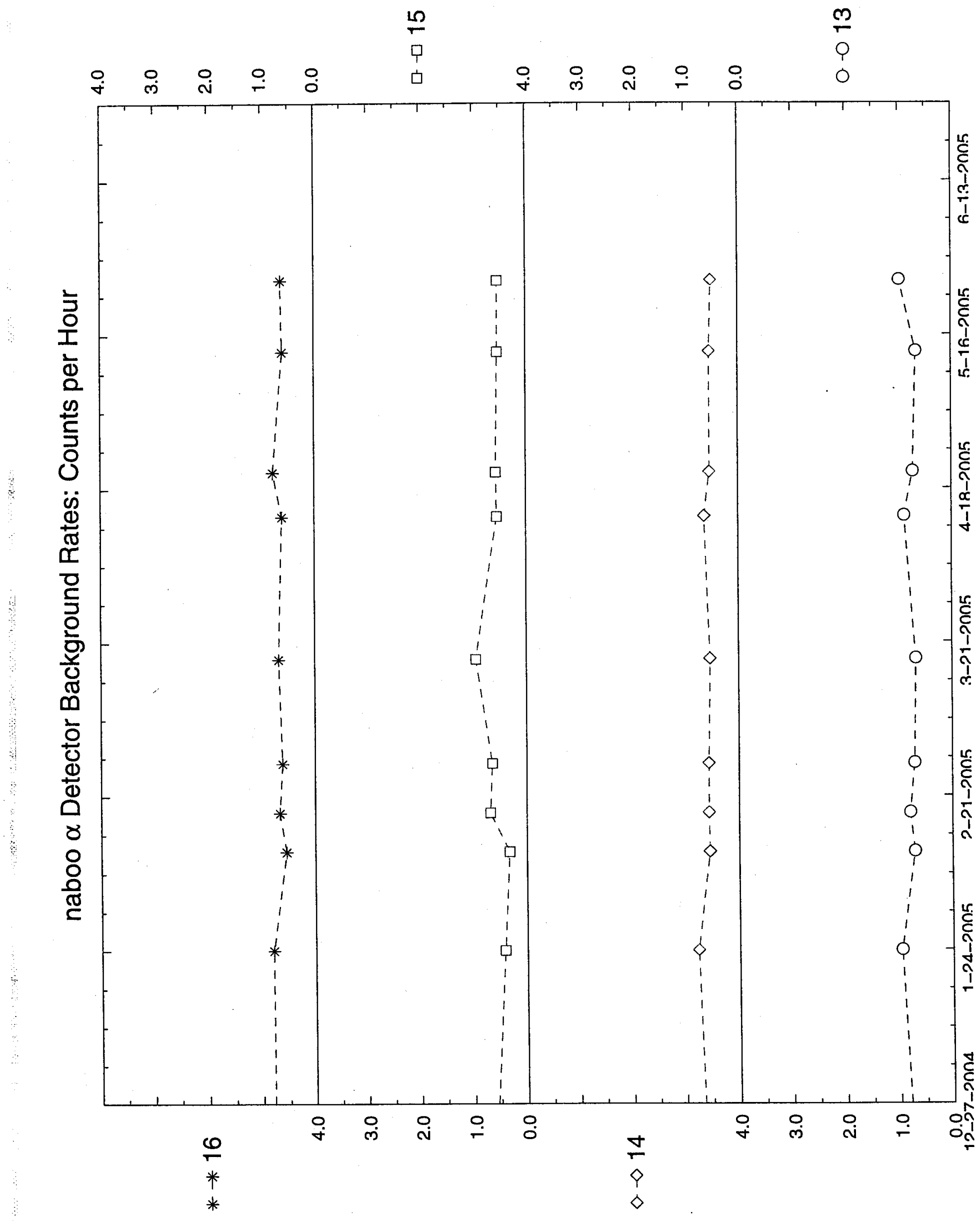


Det 04

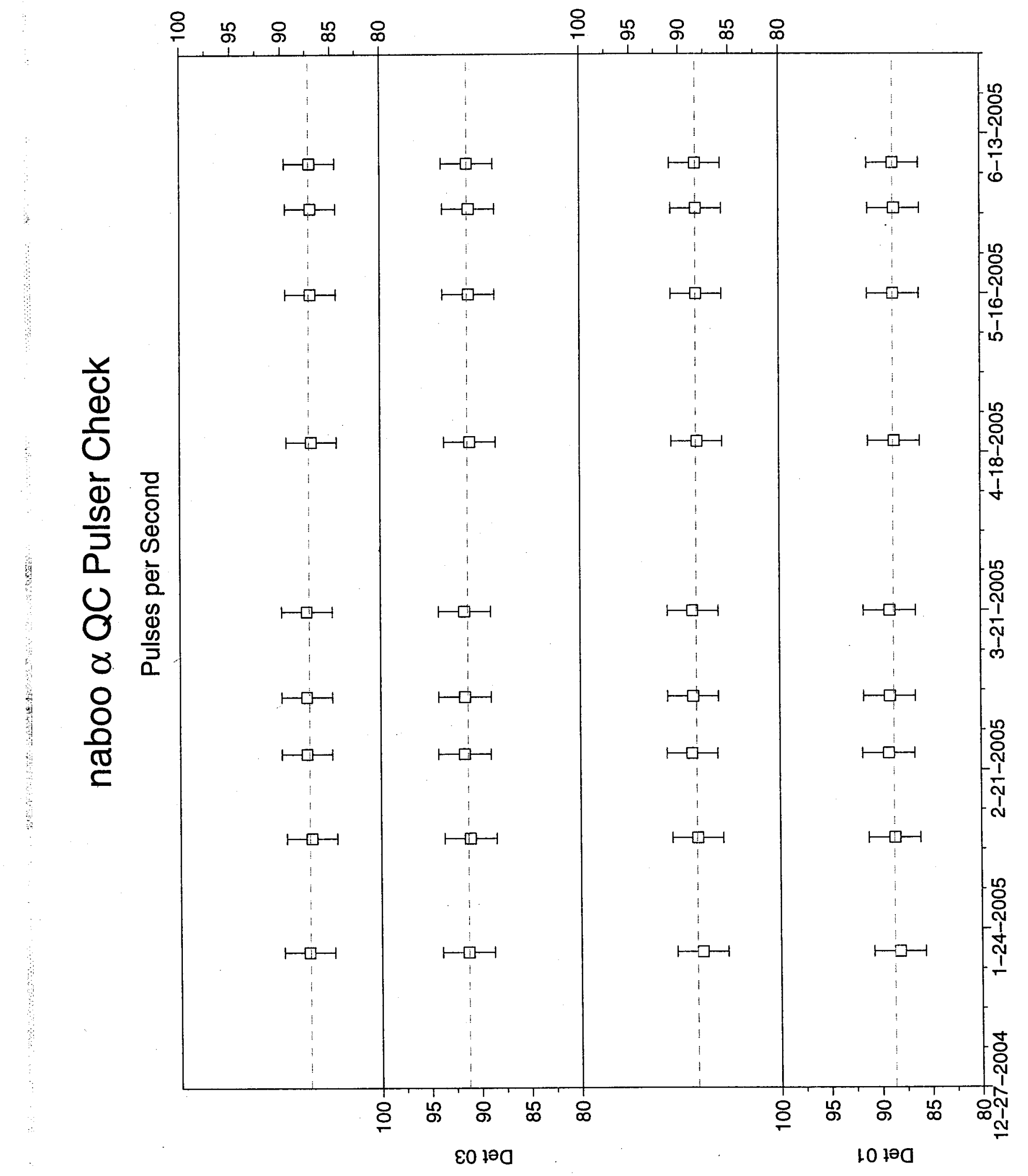

Det 02 
Det 08

Det 06

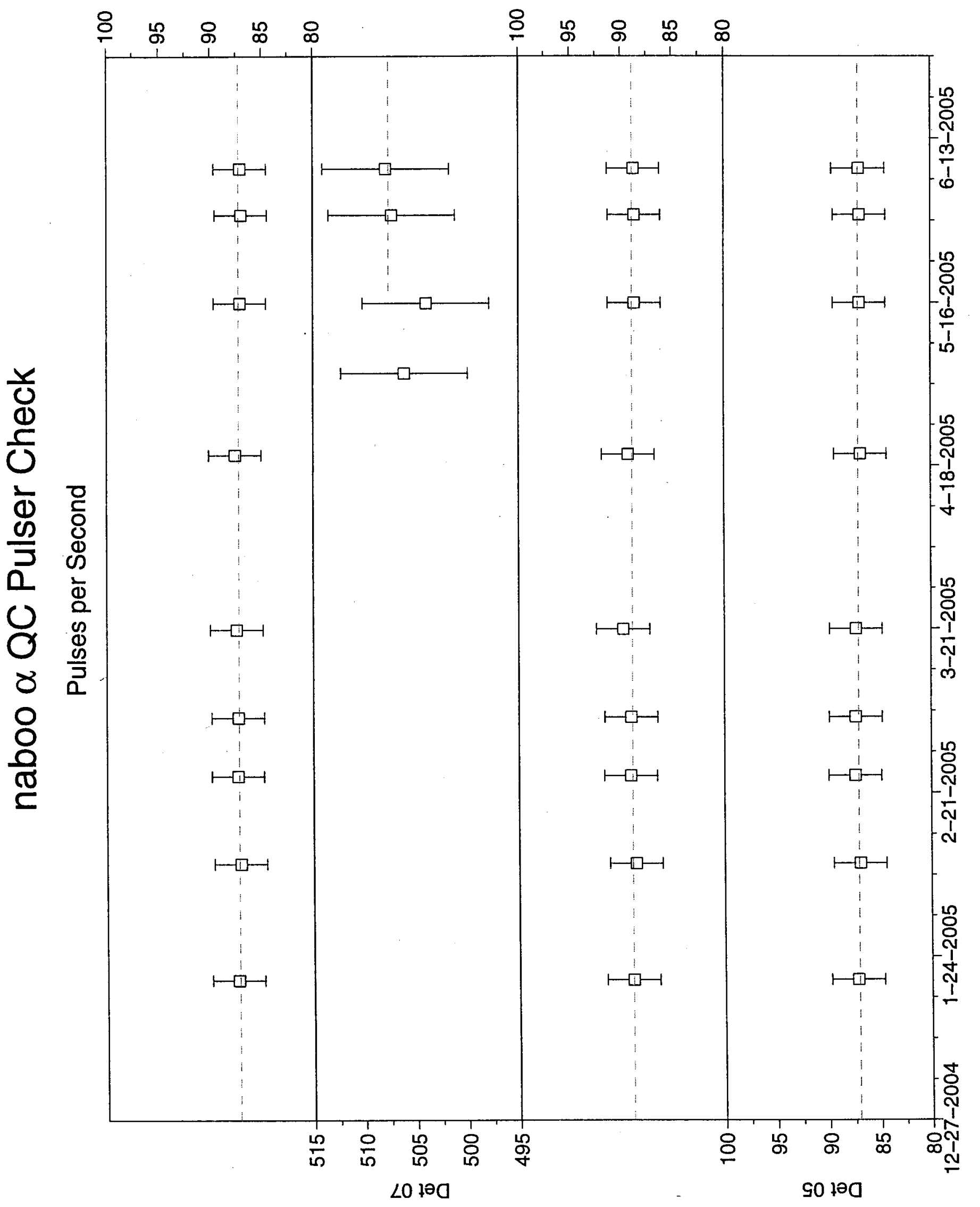




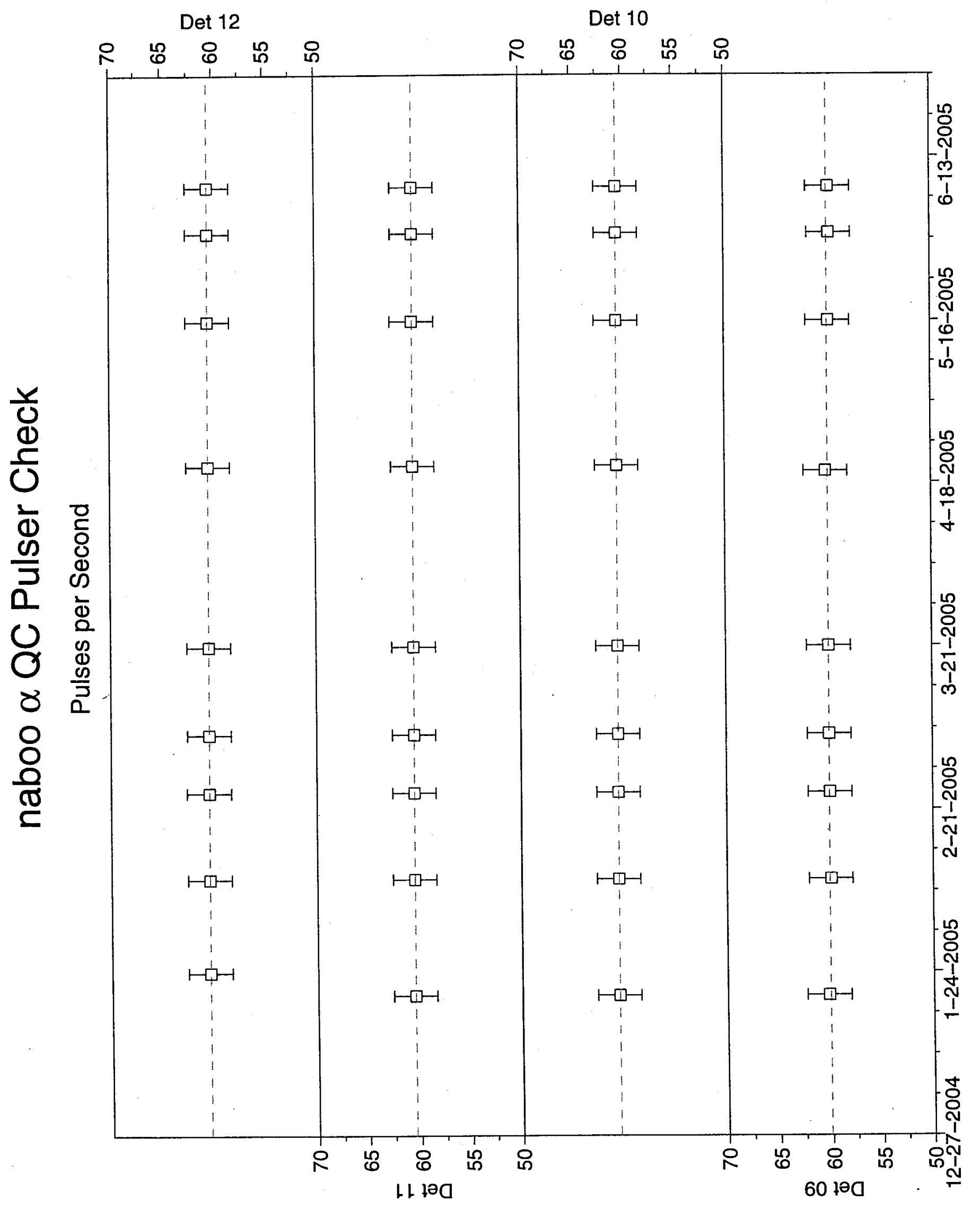




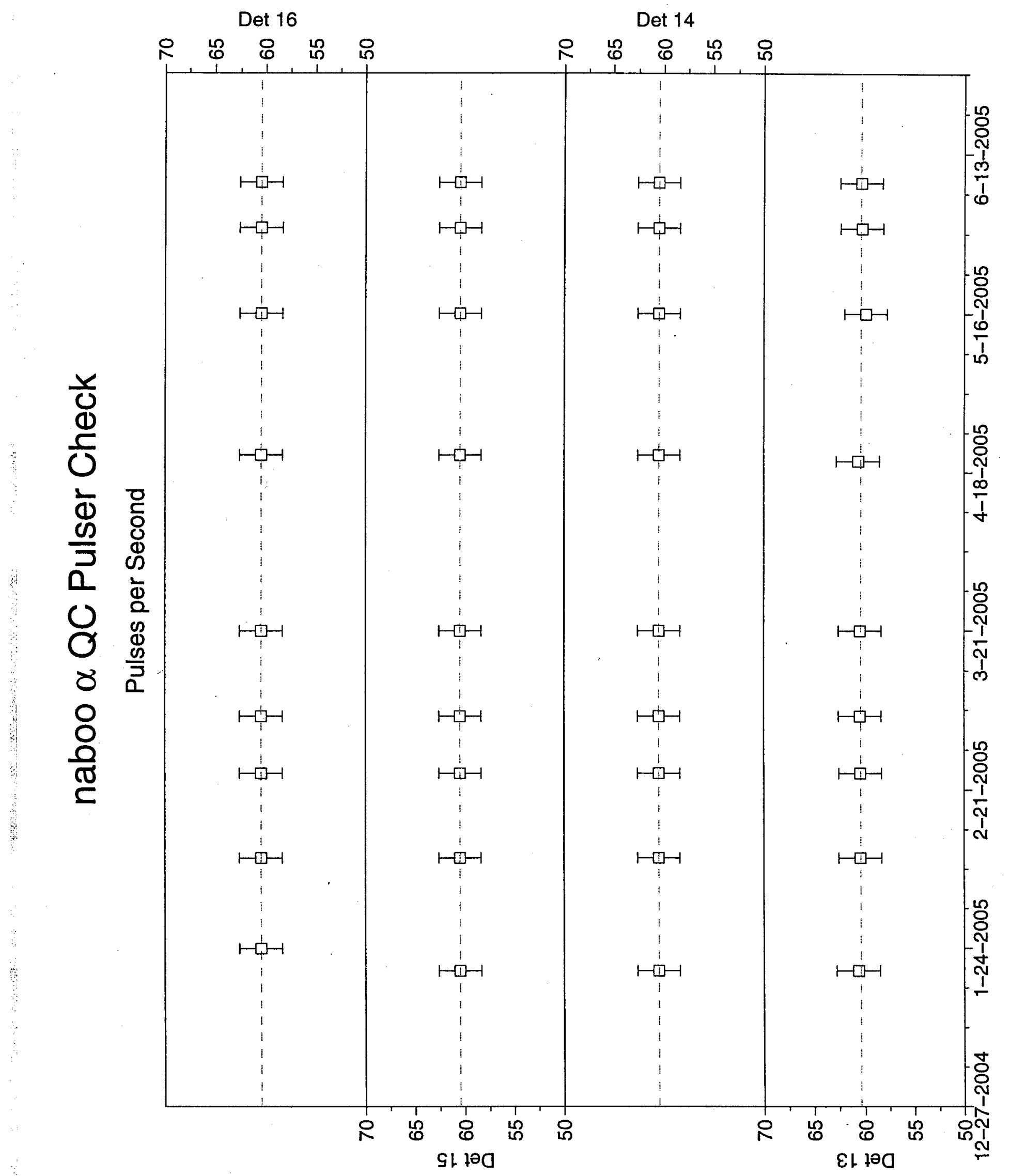




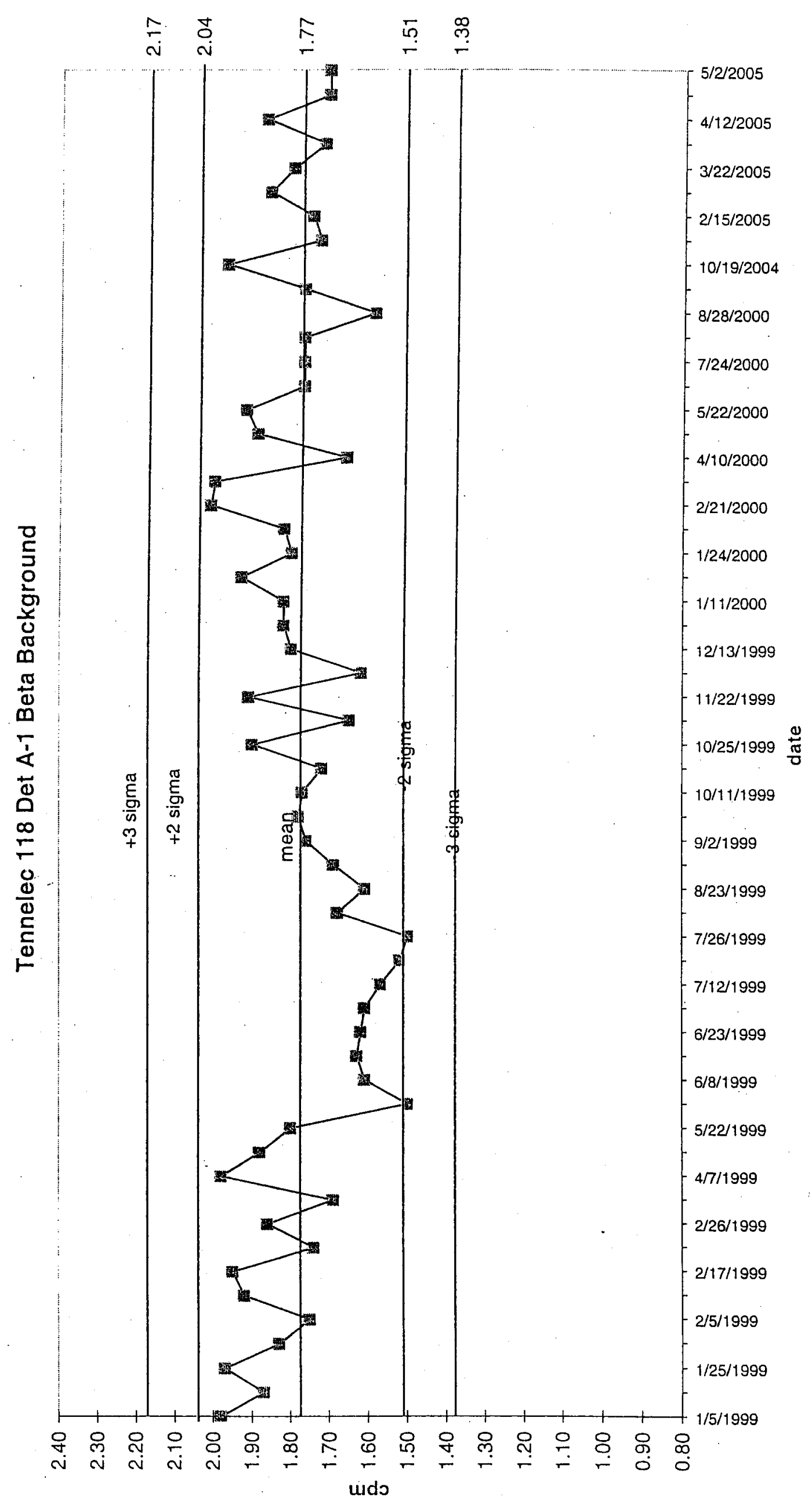




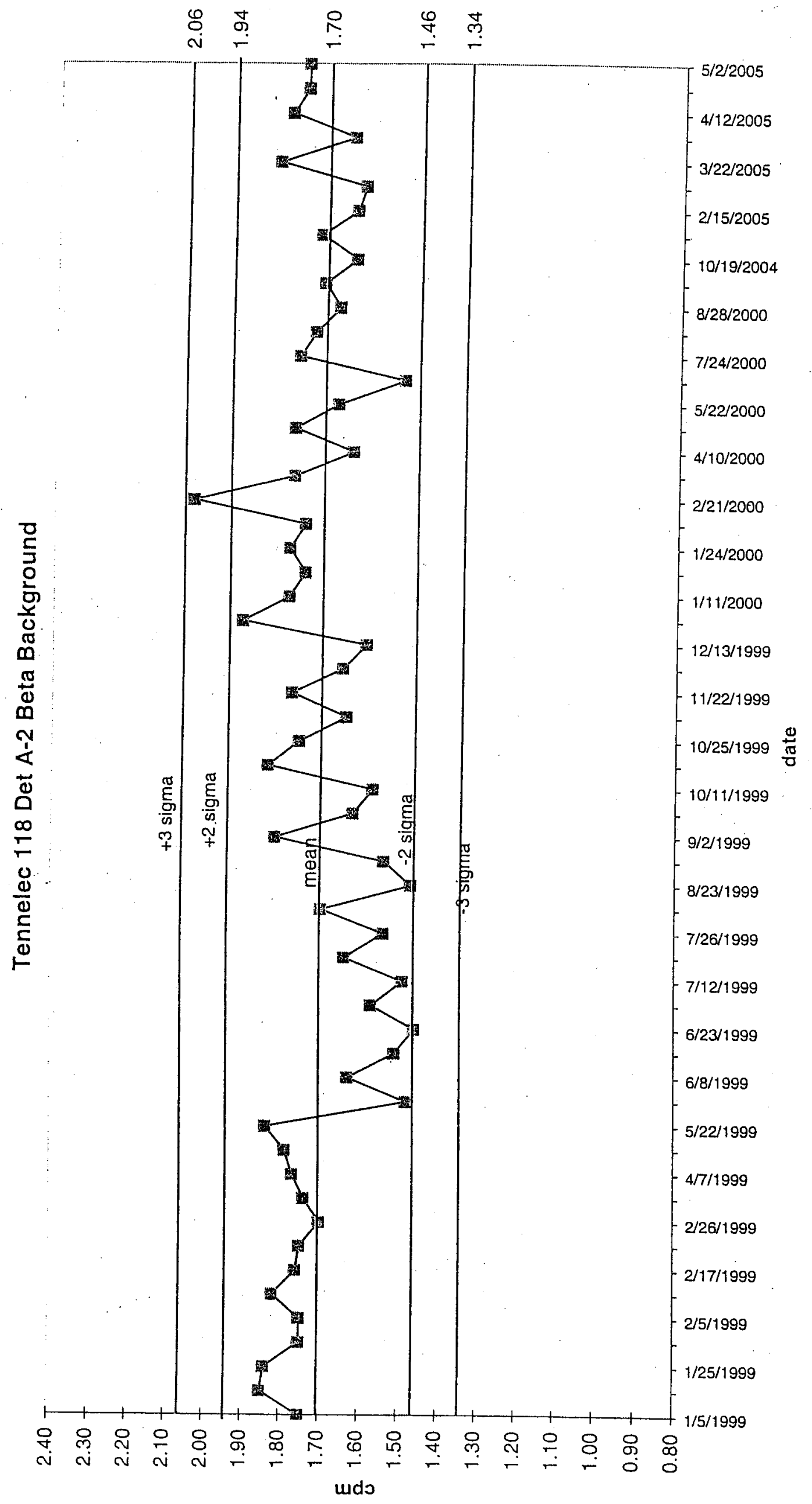




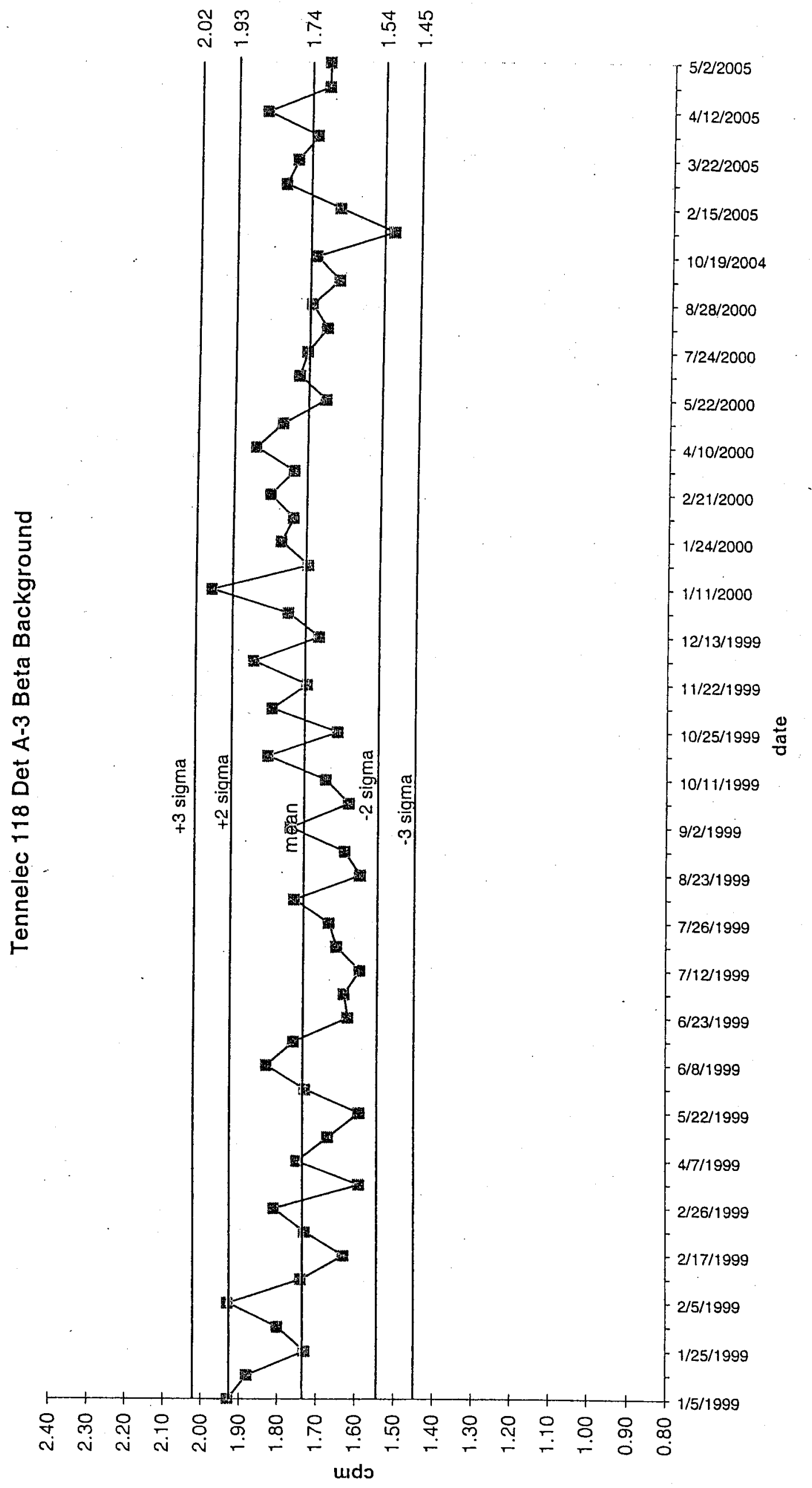




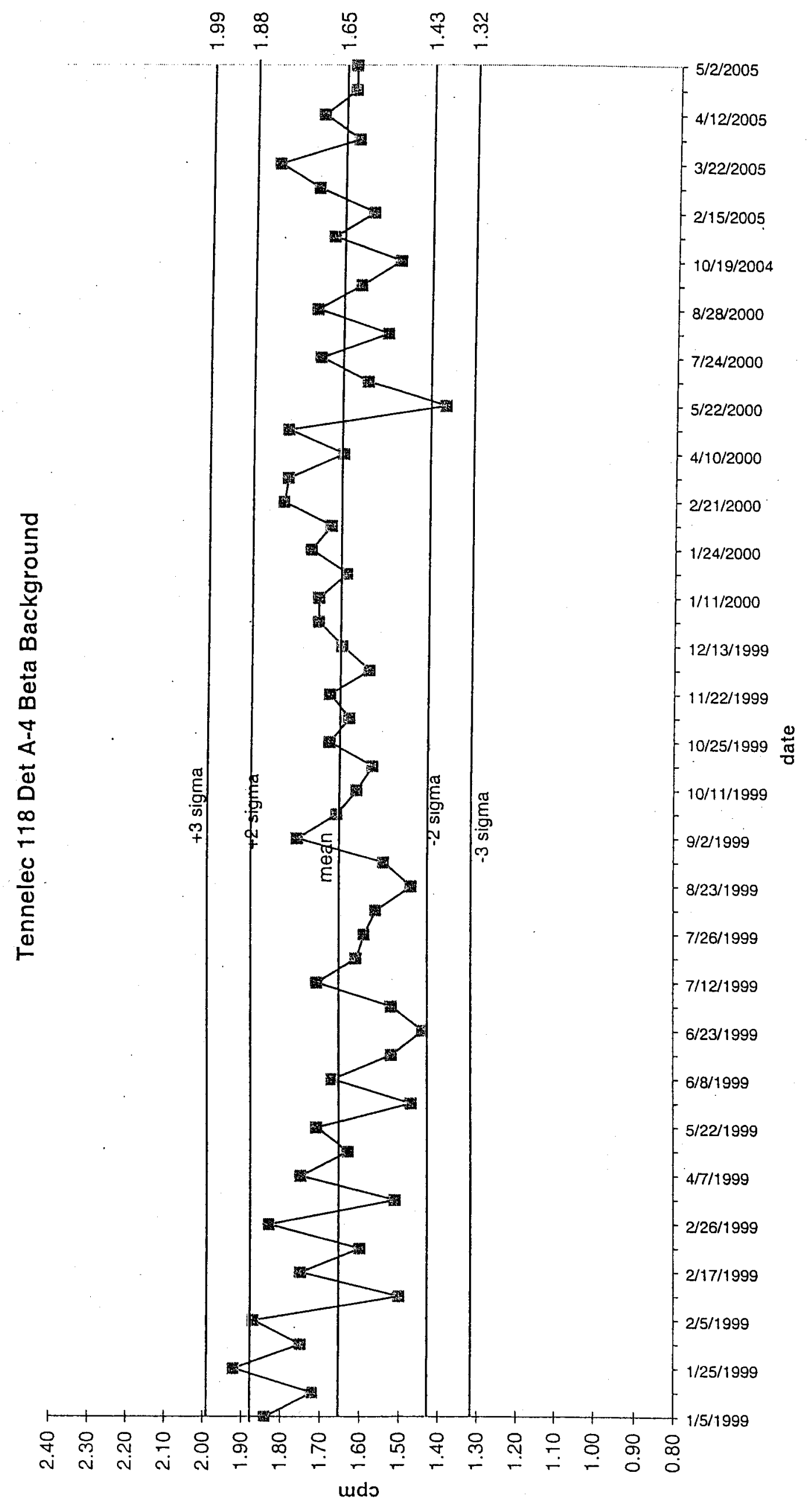




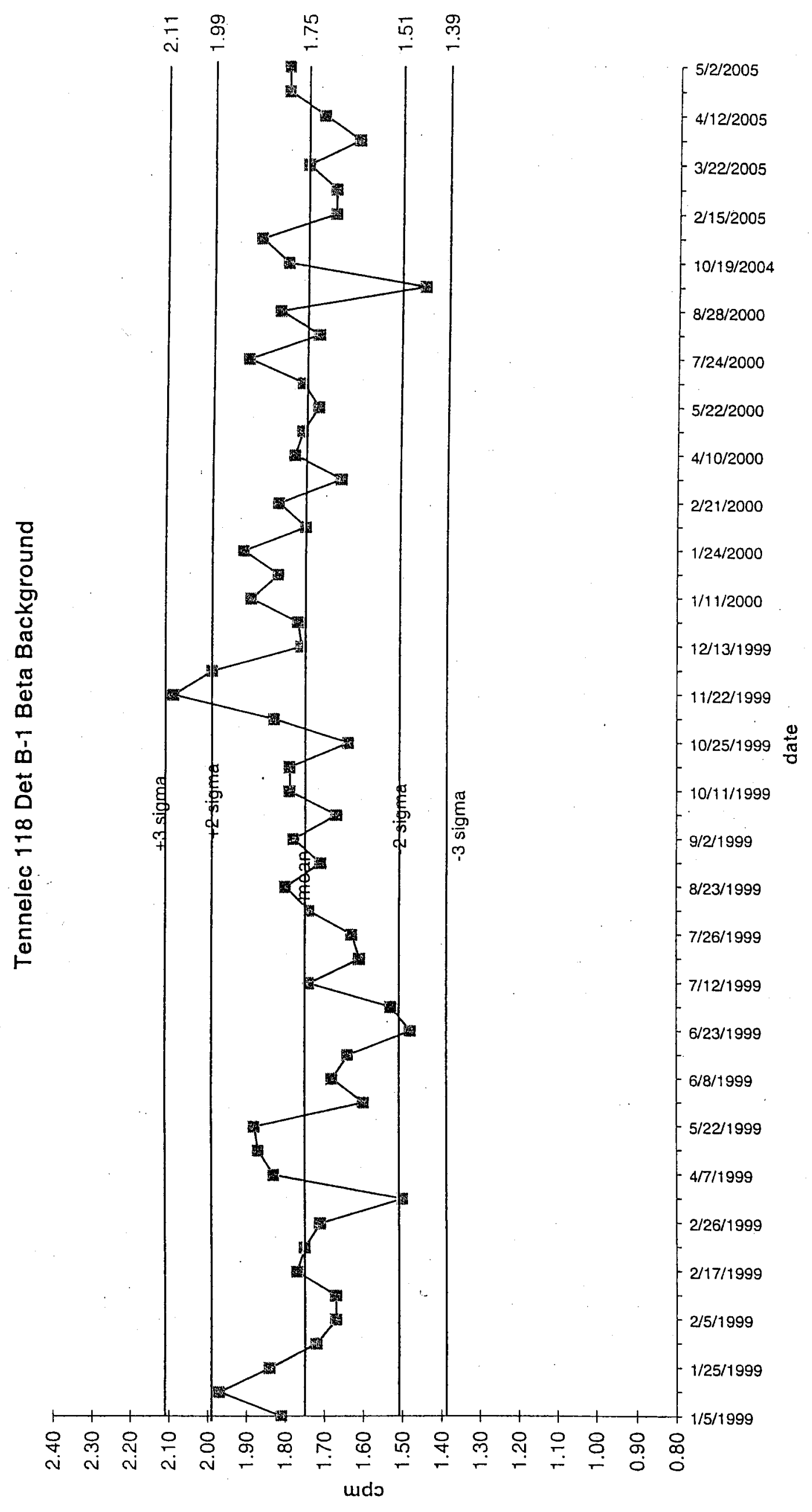




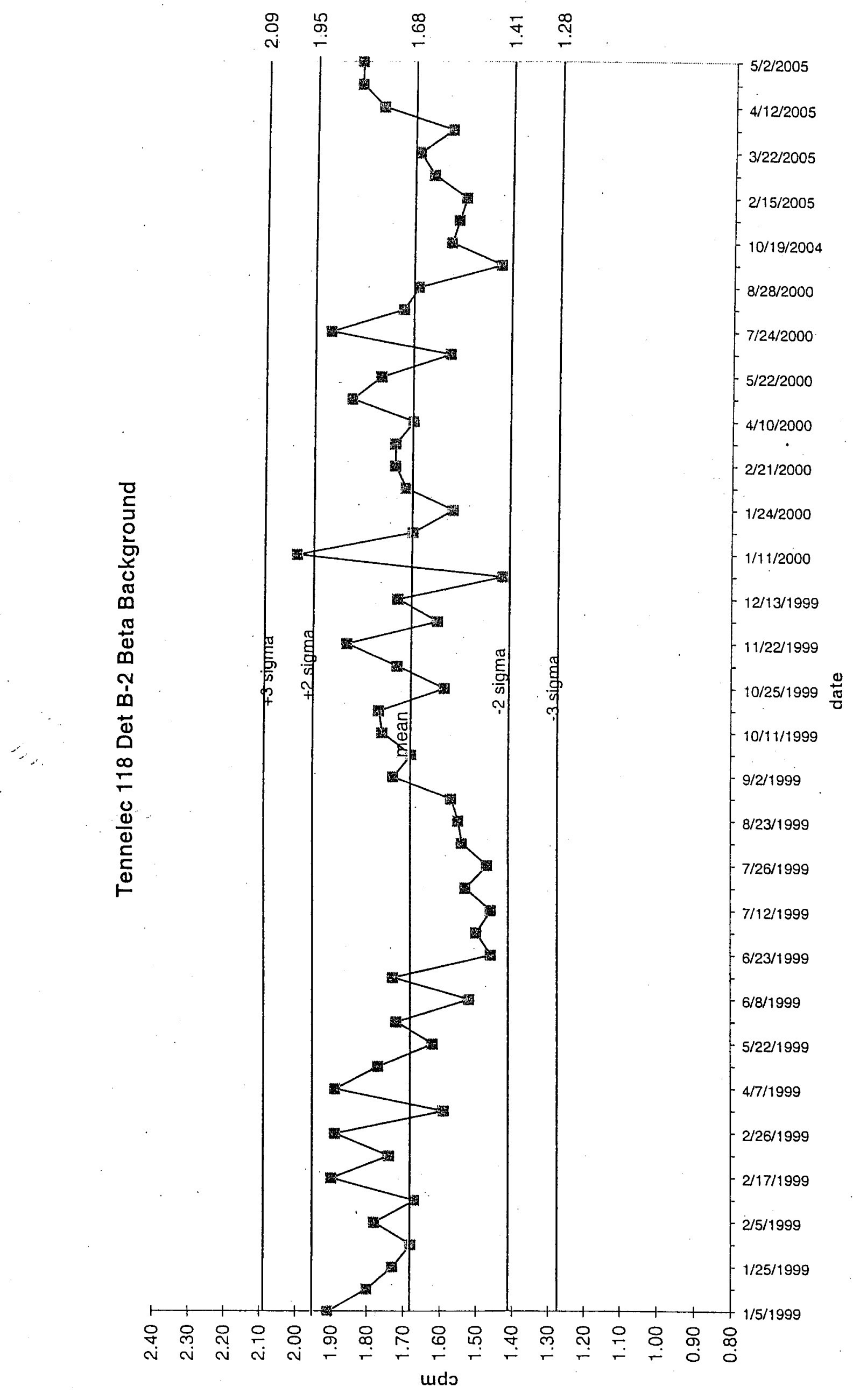




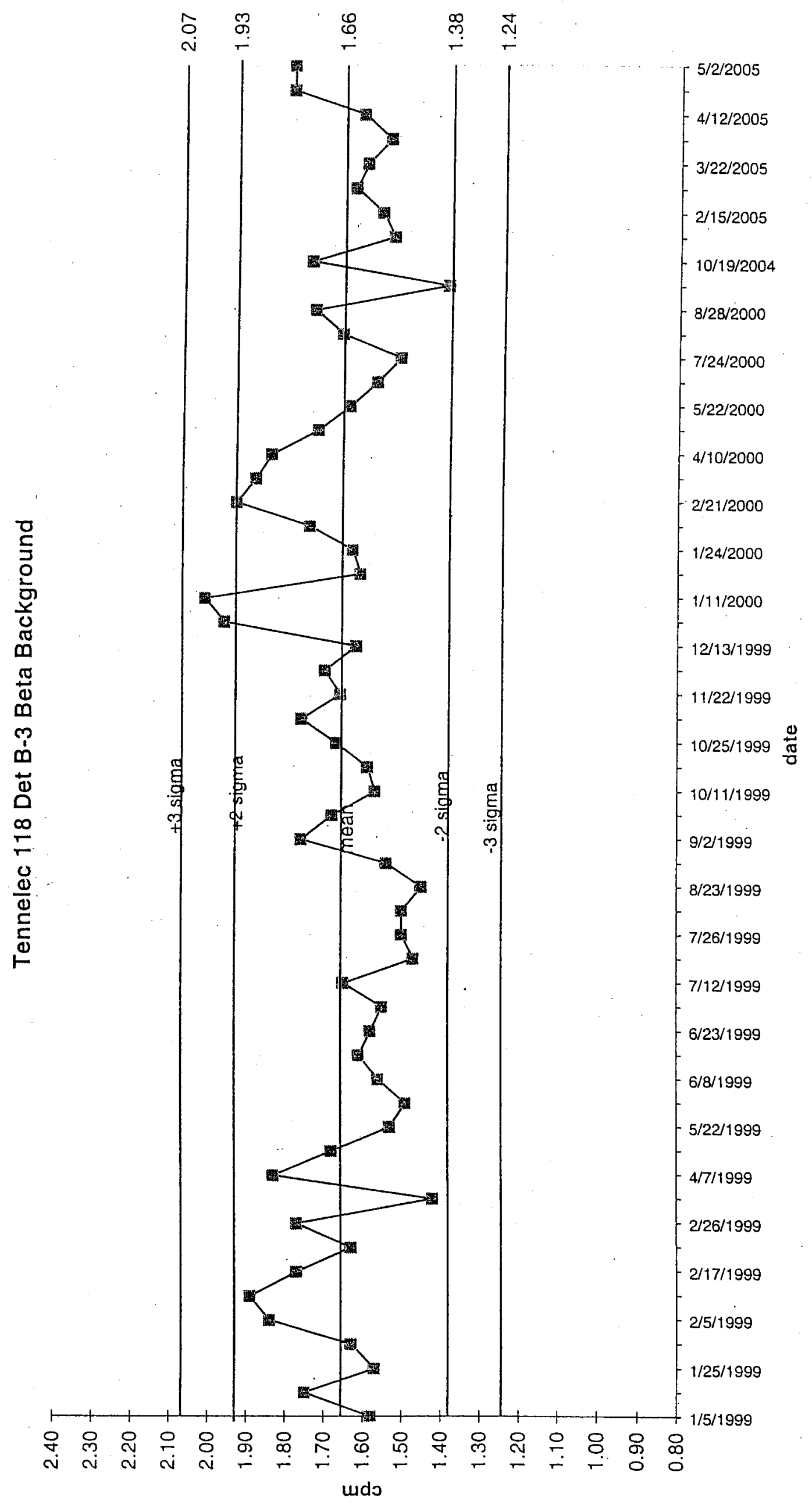




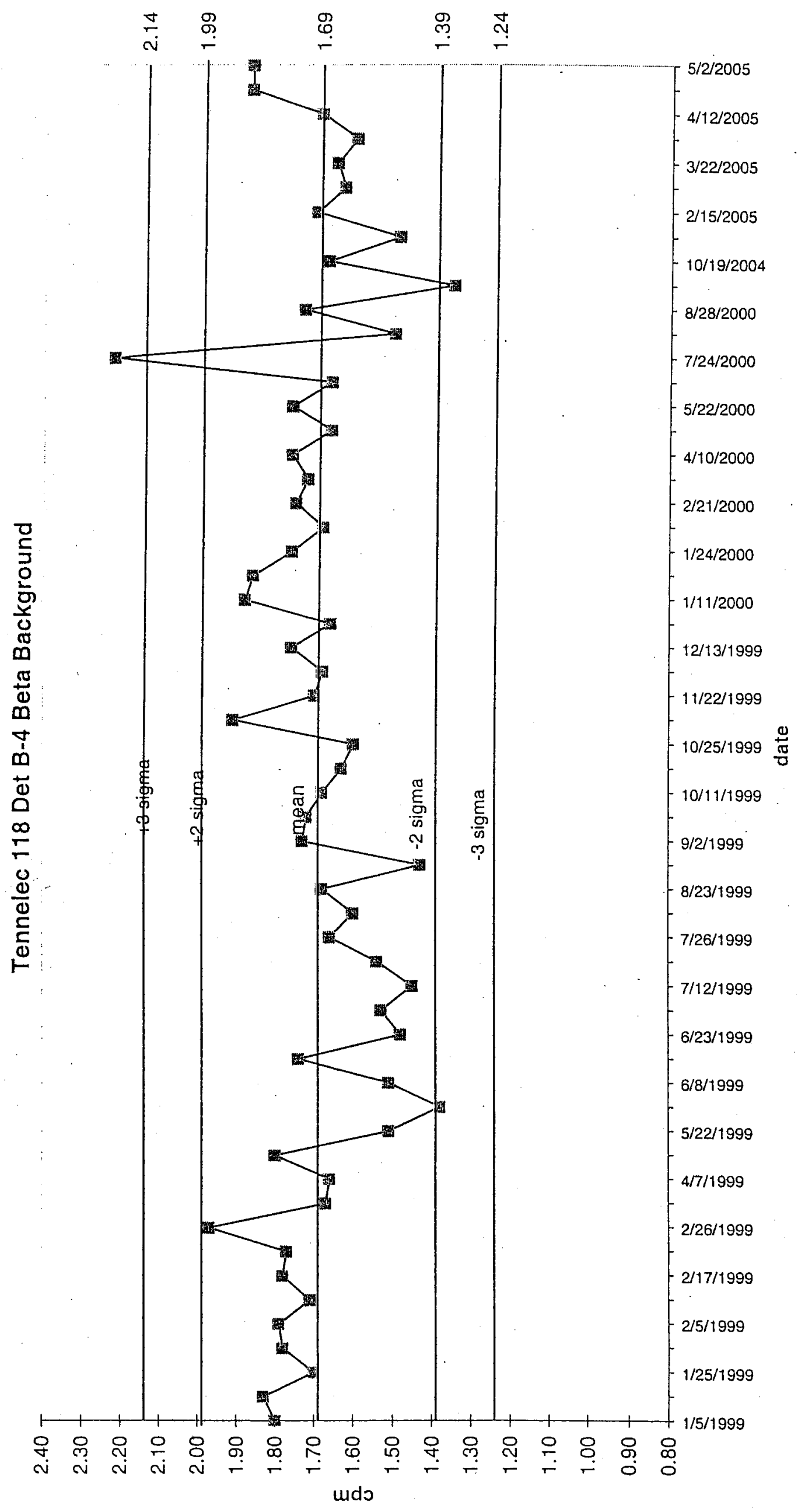




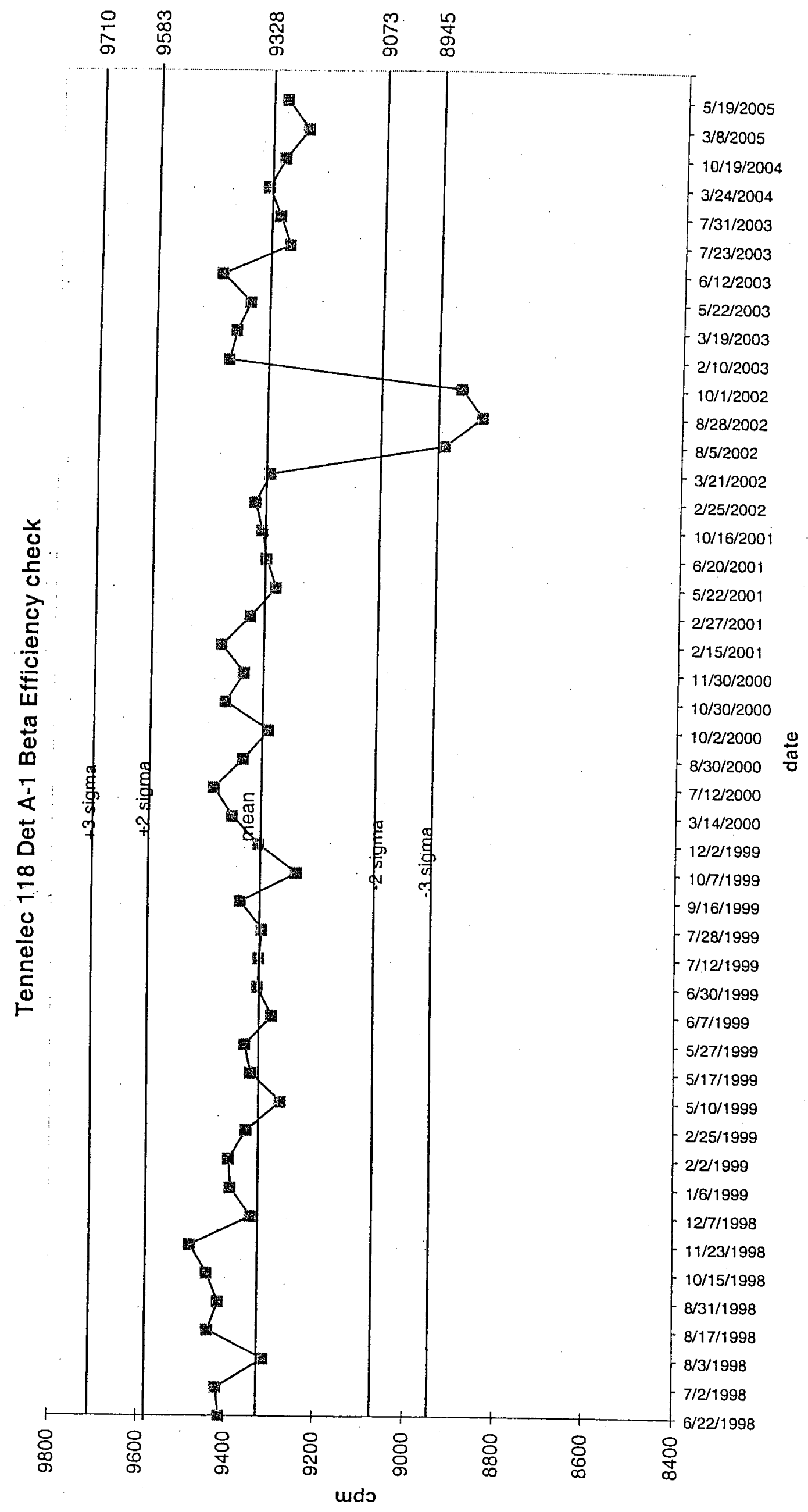




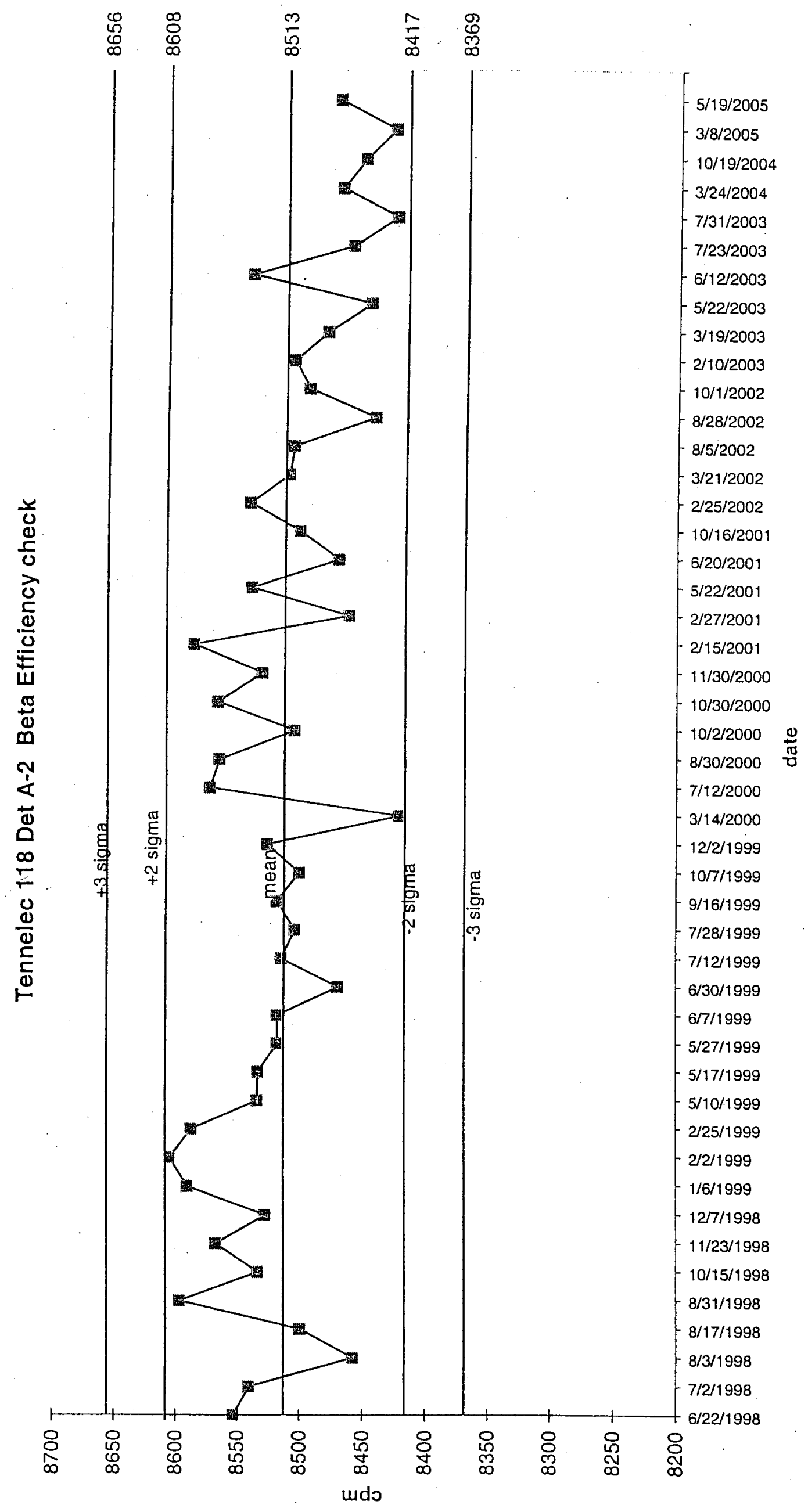




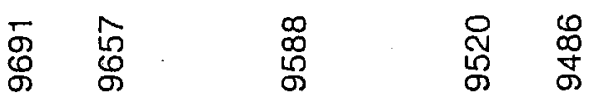

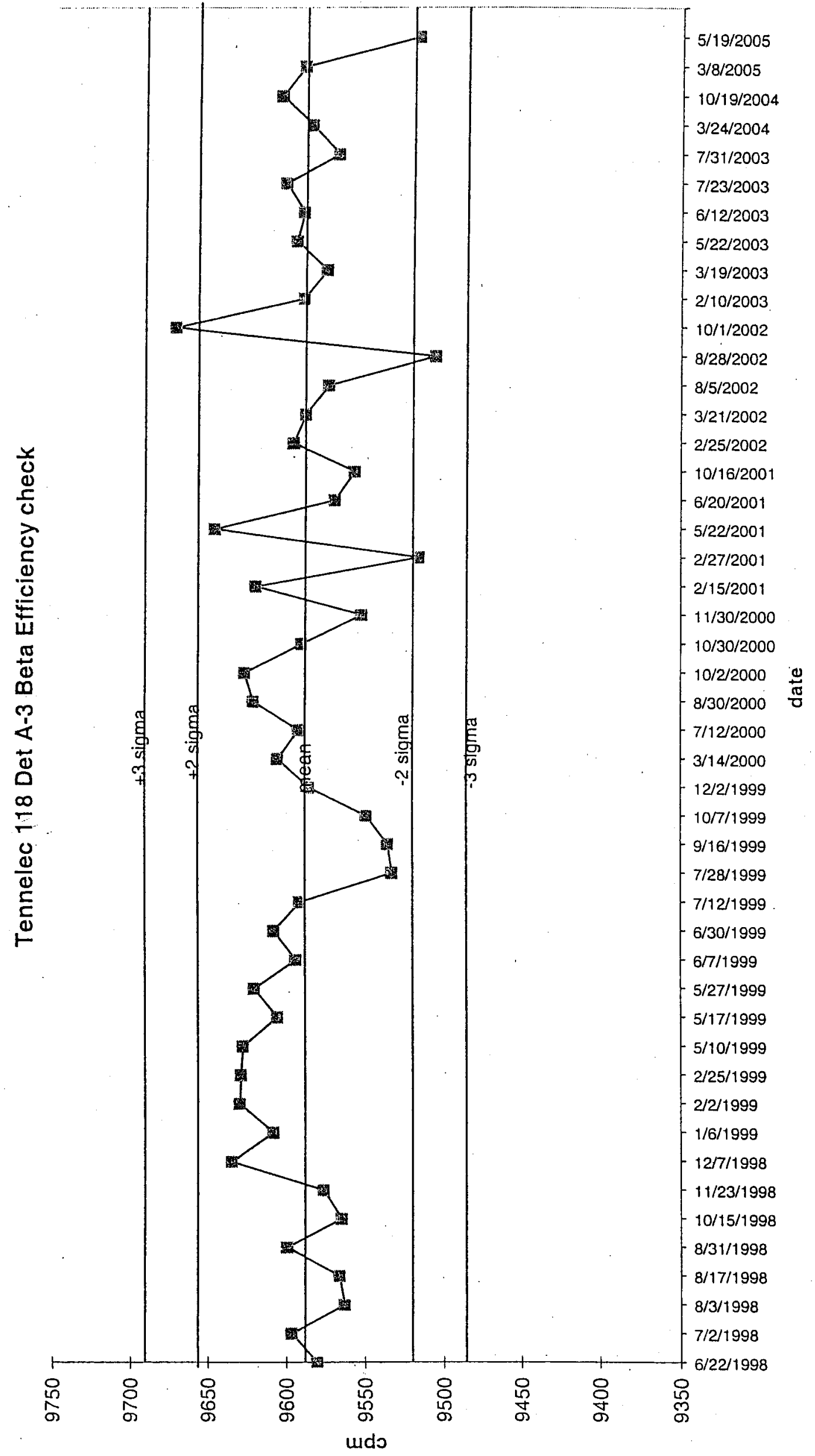




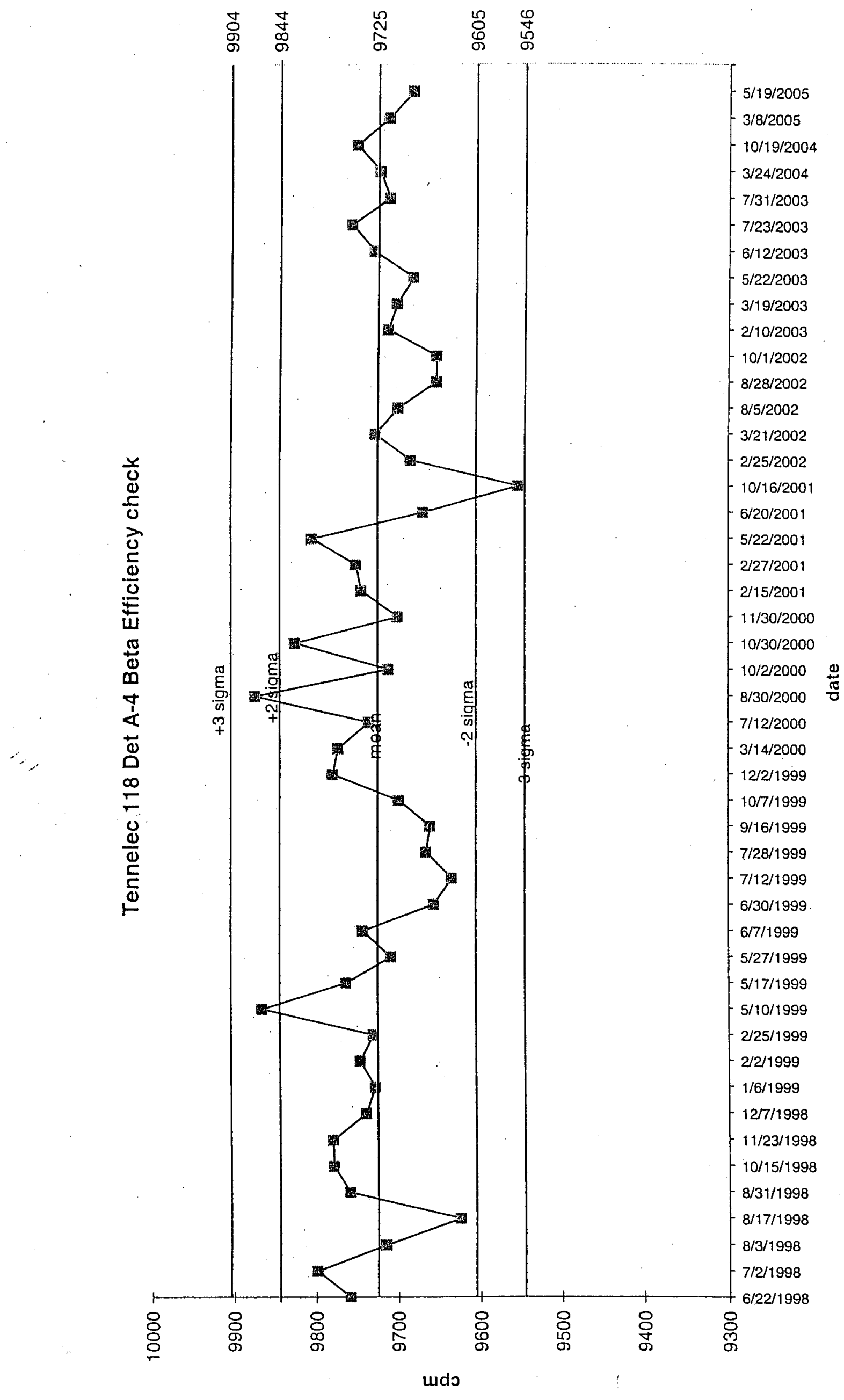




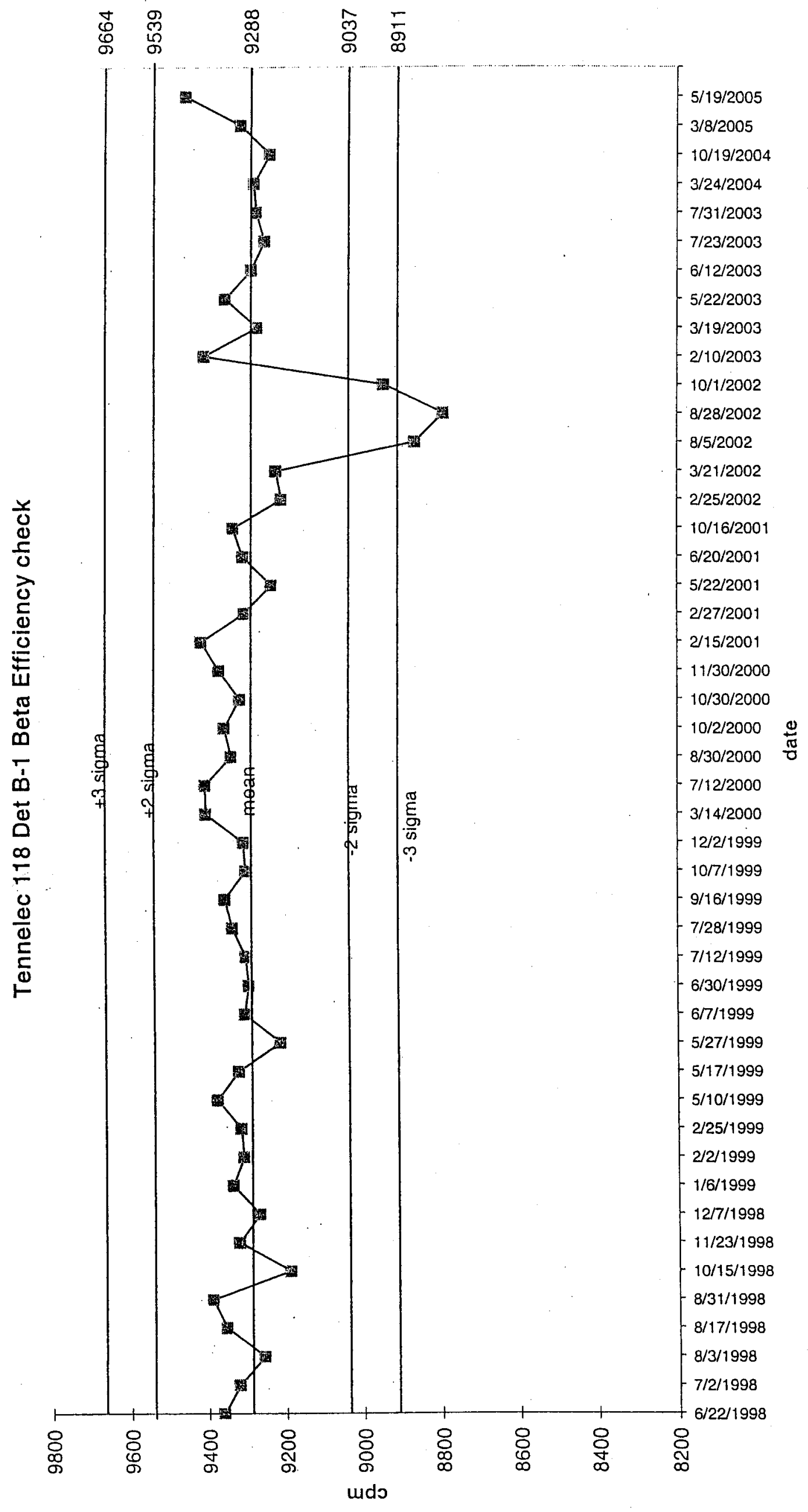




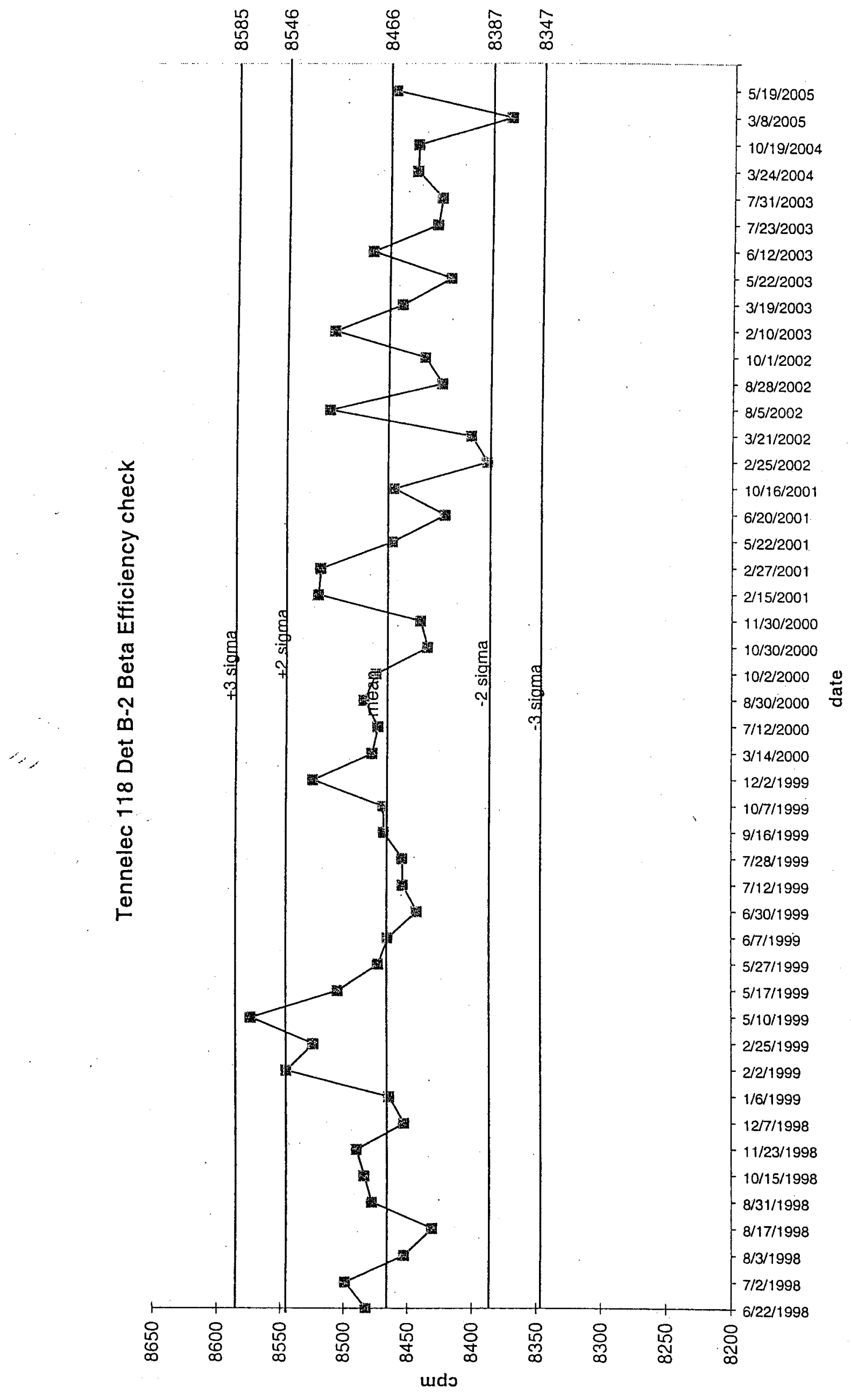




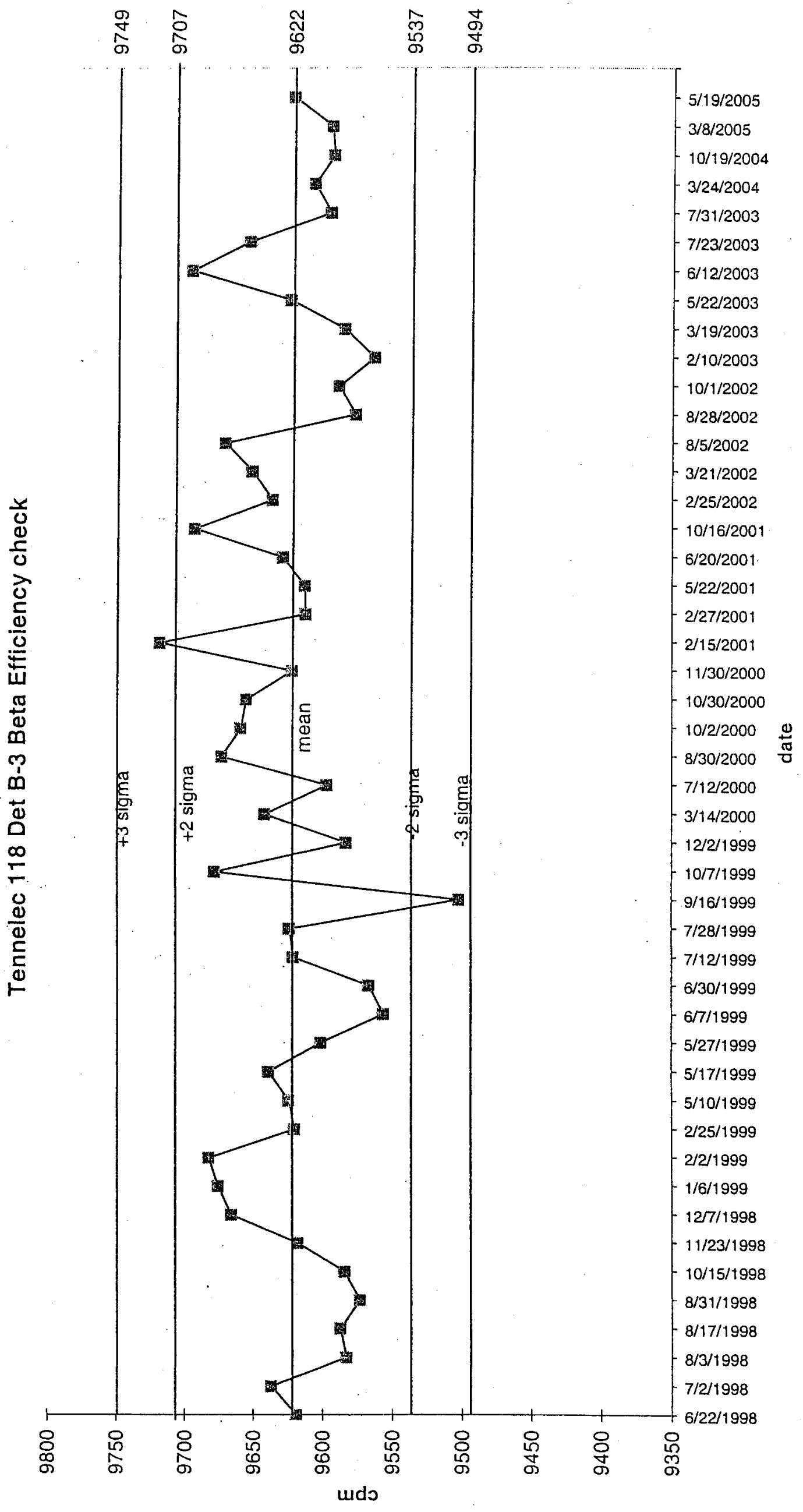




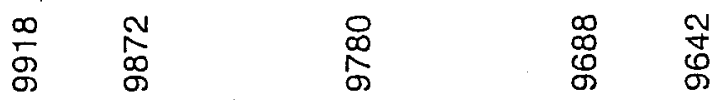

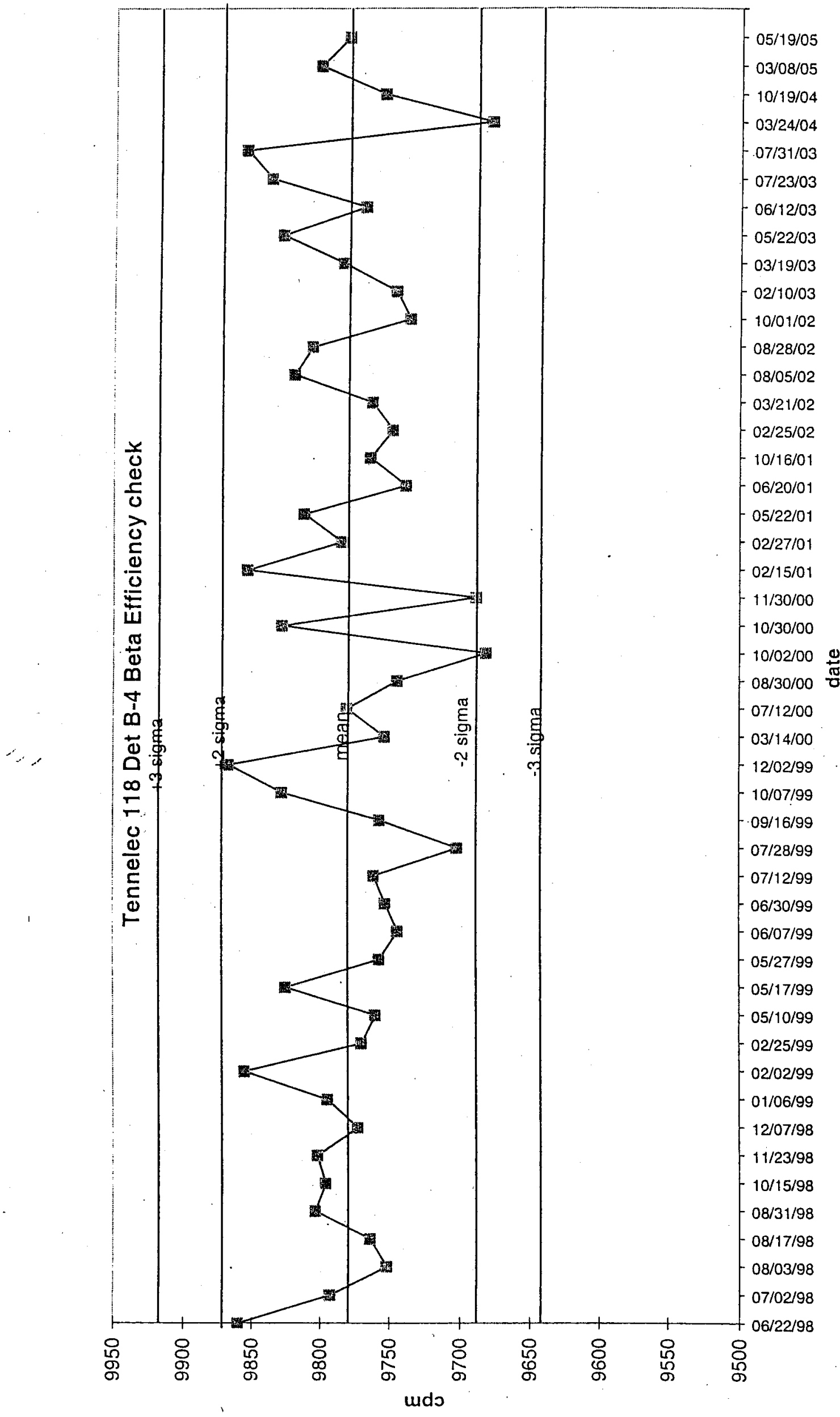


7
0
0
0
0
0
0
0
0
1 
Appendix D-1

Gamma Spectroscopy External QA/QC Program Results 


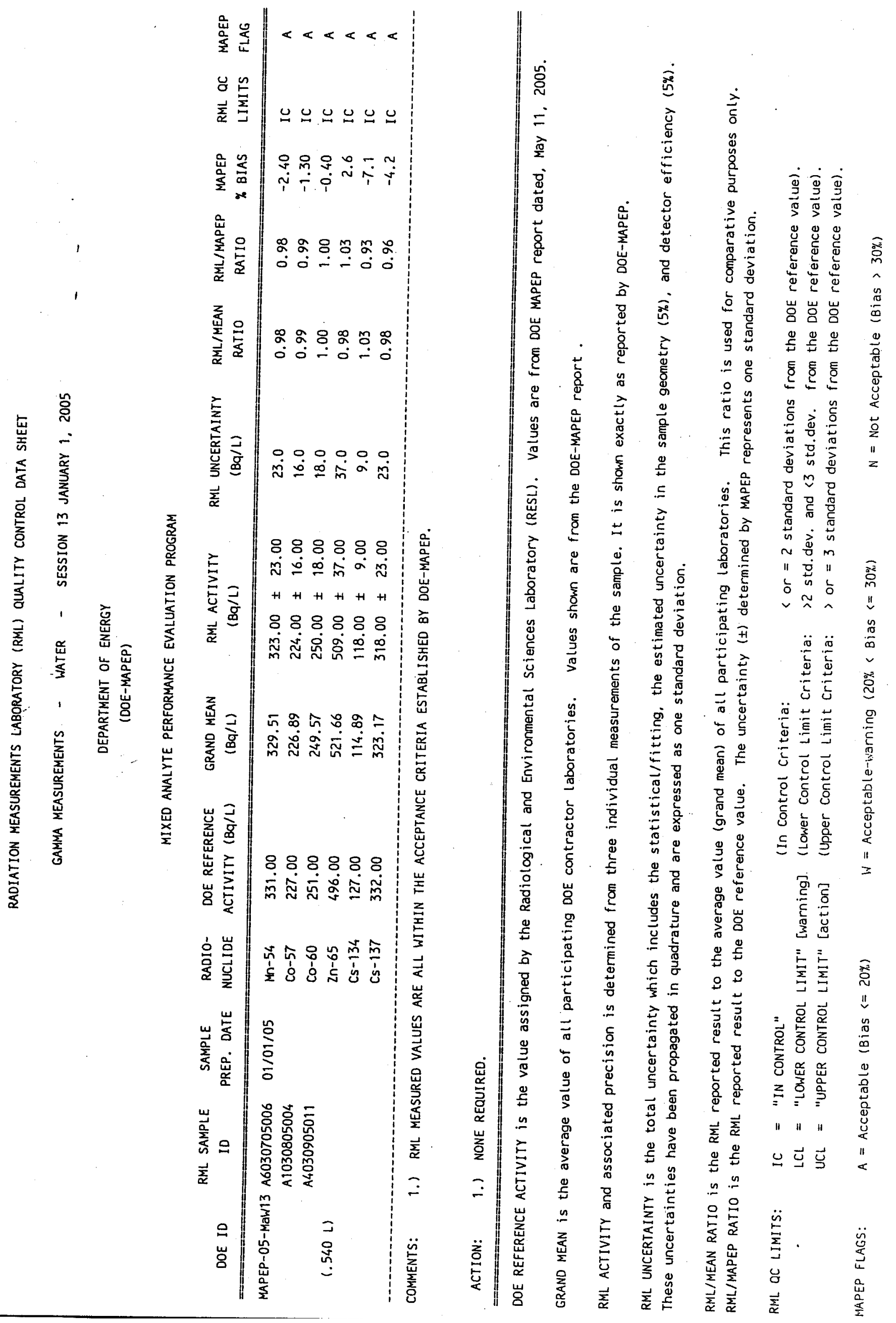




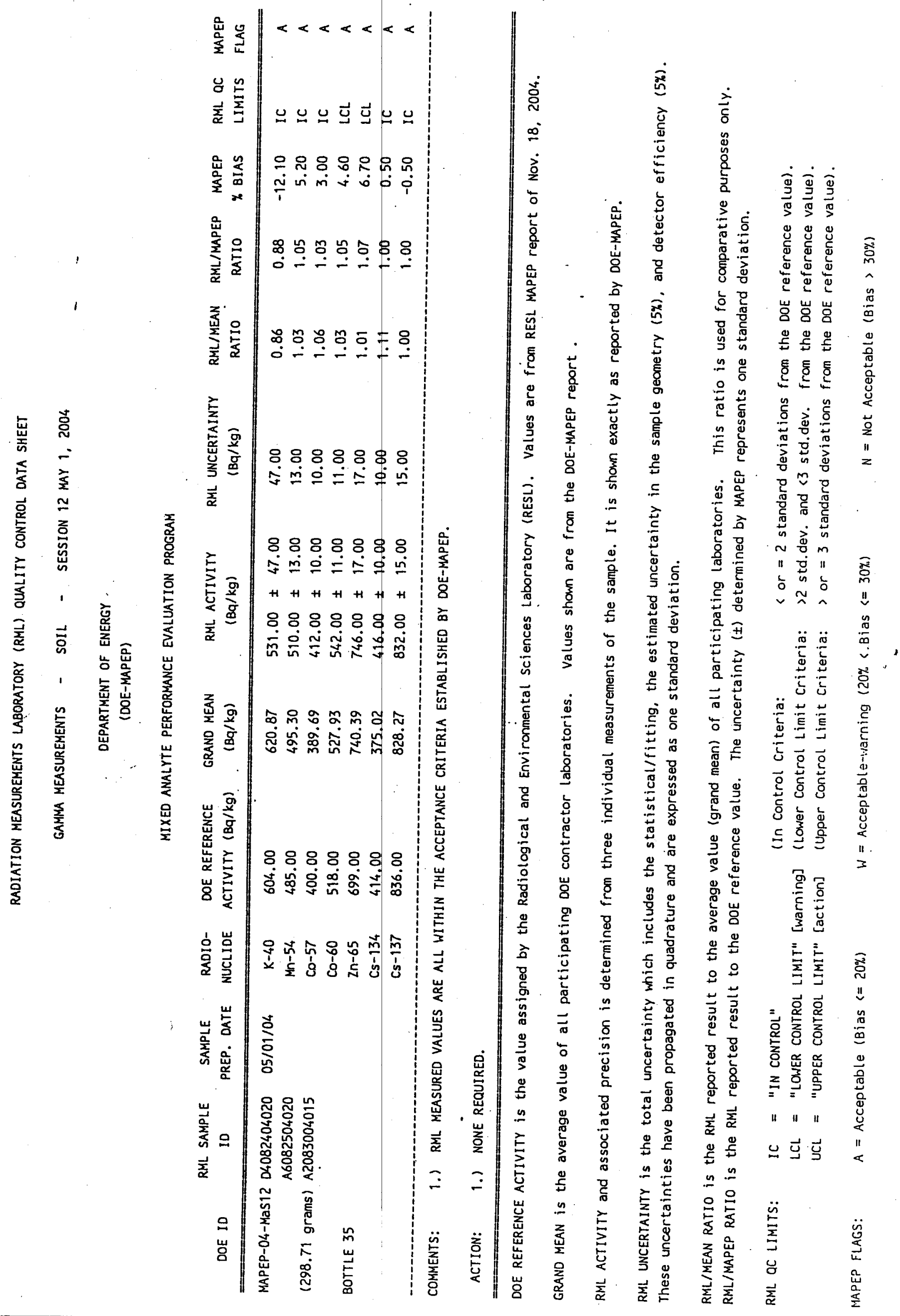




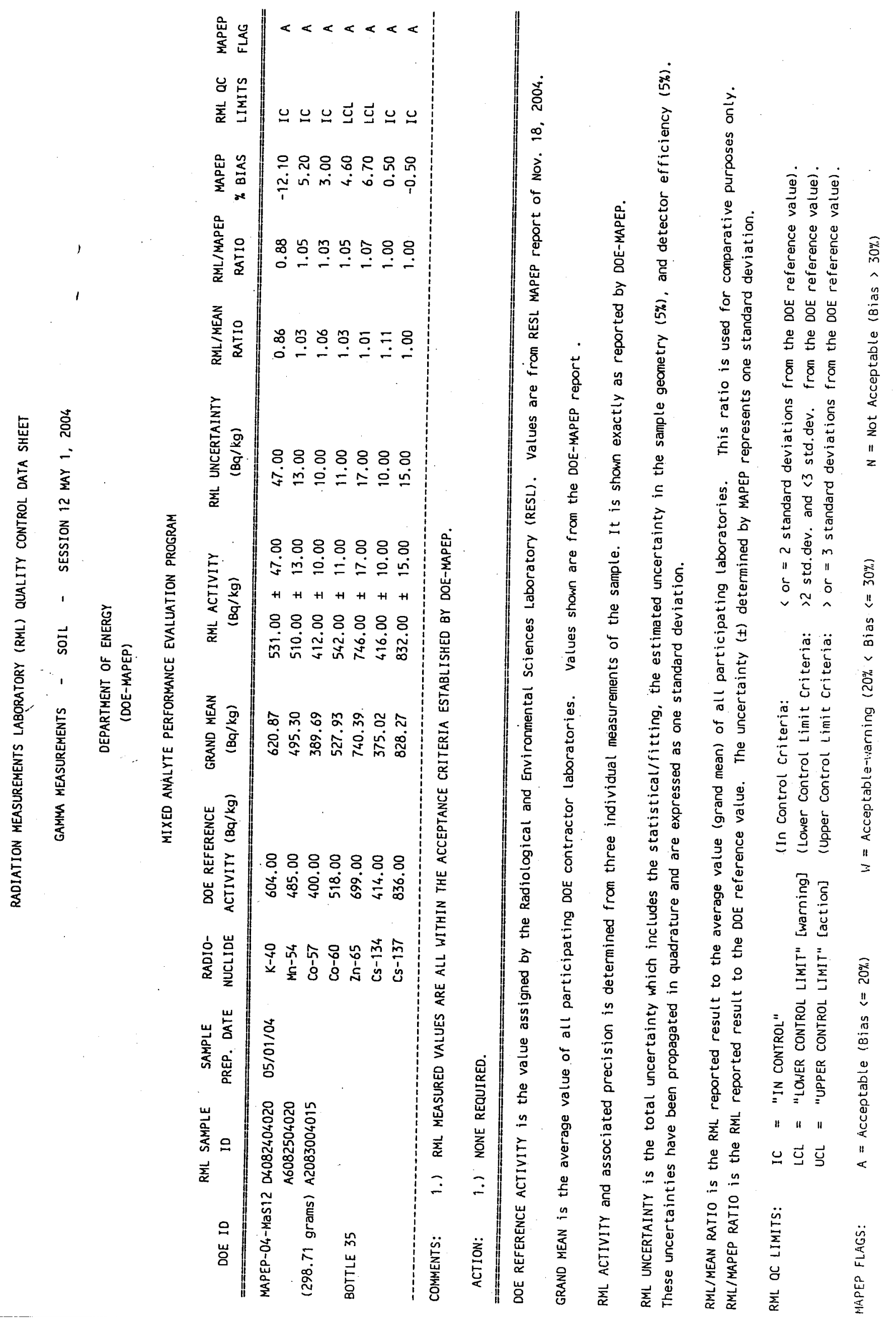


Appendix D-2

Alpha Spectroscopy and Sr-90 External QA/QC Program Results 


\section{Mixed Analyte Performance Evaluation Program}

Laboratory Results

LOCKO3 RADIATION MEASUREMENTS LABORATORYIAEDL INEEL

Sample ID: $\quad$ MAPEP-05-MaW13

Idaho Falls

83415-7111

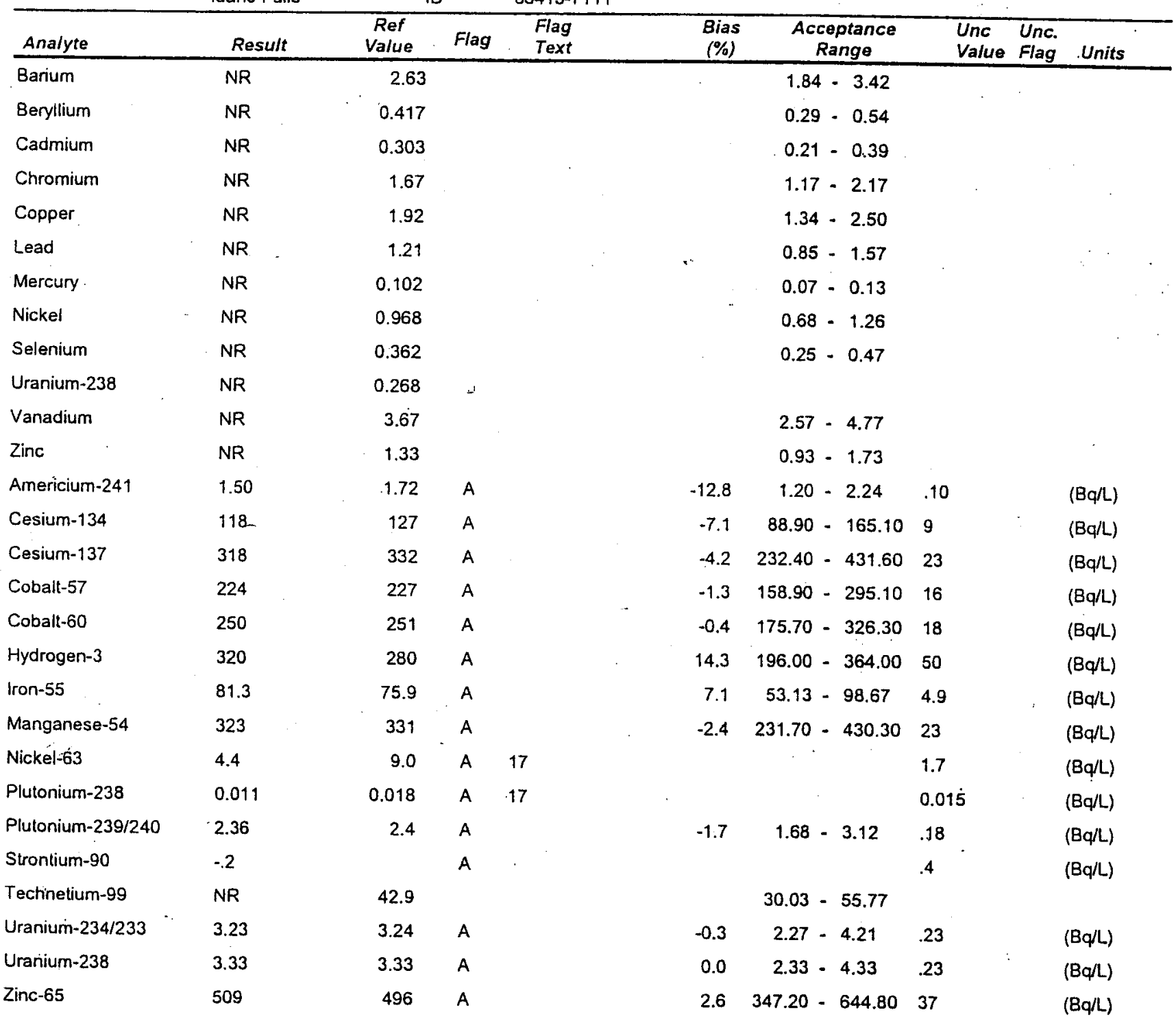




\section{Mixed Analyte Performance Evaluation Program \\ Laboratory Results}

\section{Sample ID: MAPEP-05-GrW13}

LOCKO3 RADIATION MEASUREMENTS LABORATORYIAEDL

\section{INEEL}

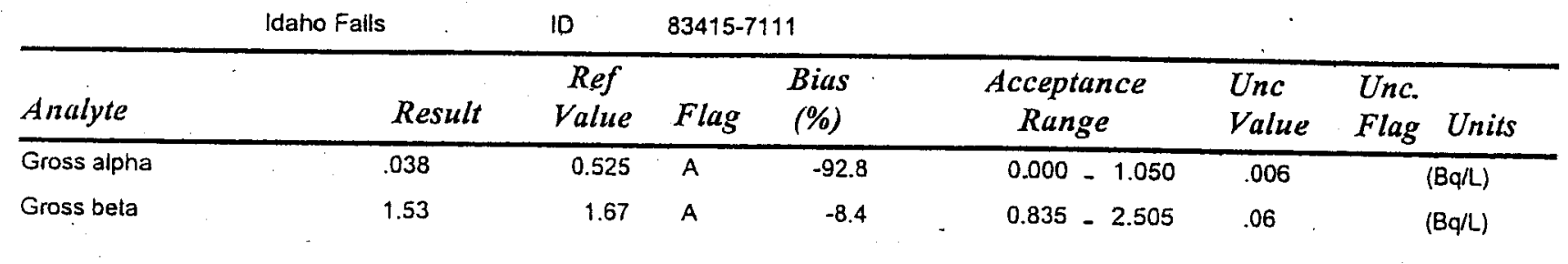

\footnotetext{
Gross Alpha Flags:

$A=$ Result acceptable, Bias $<=+/-100 \%$ with a statistically positive result at two standard deviations
}

(ResultUncertainty $>2$, i.e., the range encompassing the result, plus or minus the total uncertainty at two standard deviations, does not include zero).

$N=$ Result not acceptable. Bias $>+/-100 \%$ or the reported result is not statistically positive at two standard deviations (Result/Uncertainty $<=2$, i.e., the range encompassing the result, plus or minus the total uncertainty at two standard deviations, includes zero).

Gross Beta Flags:

$A=$ Result acceptable, Bias $<=+1-50 \%$ with a statistically positive result at two standard deviations (Result/Uncertainty $>2$, l.e., the range encompassing the result, plus or minus the total uncertainty at two standard deviations, does not include zero).

$N=$ Result not acceptable, Bias $>+1-50 \%$ or the reported result is not statistically positive at two standard deviations (ResultU Uncertainty $<=2$, i.e., the range encompassing the result, plus or minus the total uncertainty at two standard deviations, includes zero).

Flags: $\quad L=$ Uncertainty potentially too low (for infomation purposes only)

$\mathrm{H}=$ Uncertainty potentially too high (for information purposes only)

FP $=$ False Positive

$F N=$ False Negative

NR $=$ Not Reported 


\section{Mixed Analyte Performance Evaluation Program}

Laboratory Results

LOCK03 RADIATION MEASUREMENTS LABORATORYIAEDL INEEL

Sample ID: MAPEP-05-MaS13

Idaho Falls

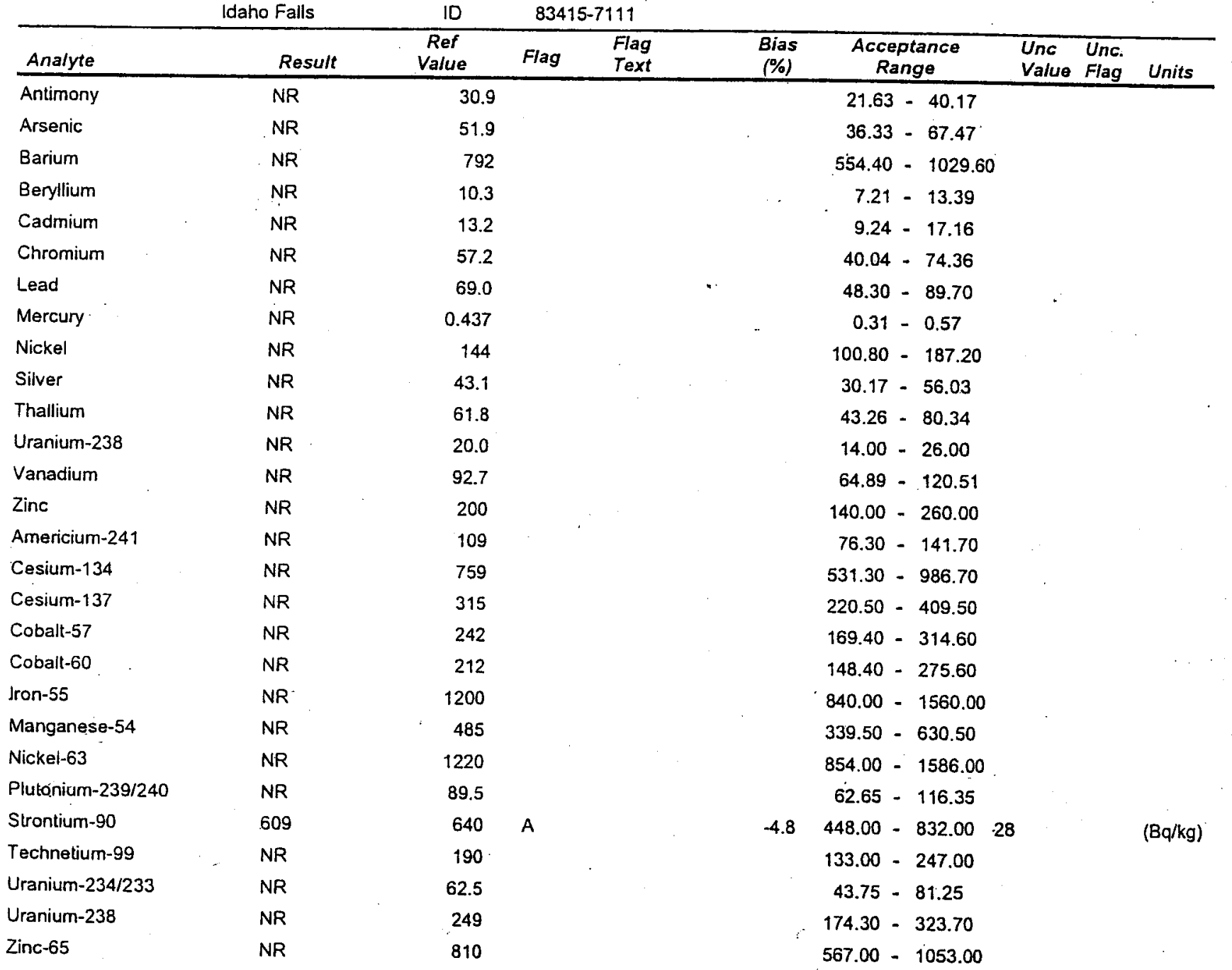

Flags: $\quad A=$ Result acceptable $\quad$ Bias $<=20 \%$

$W=$ Result acceptable with warning $20 \%<$ Bias $<=30 \%$

Flag Text 1 - False Positive

2 - False Negative

$\begin{aligned} N= & \text { Result not acceplable } \\ & L=\text { Uncertainty polentially too low }\end{aligned}$

Bias $>30 \%$

4 - Sensitivity Evaluation

5 - Total Metal

$H=$ Uncertainty potentially too high

6 - Not Evaluated

$Q=$ Particapant should evaluate reported value

$\mathrm{QL}=$ Quantitation Limit

$\mathrm{RW}=$ Report Warning

7. DL > CLP Limit

9 - Check QL

10 - Check Isomer

NiR = Not Reported

11 - False Positive Test, Value Not Reported

14 - Solubility Issue

15 - Refractory

16 - Reported zero uncertainty

17 - NOT DETECTED, reported a statistical zerc result

18 - Sensitivity evaluation, value not reported 


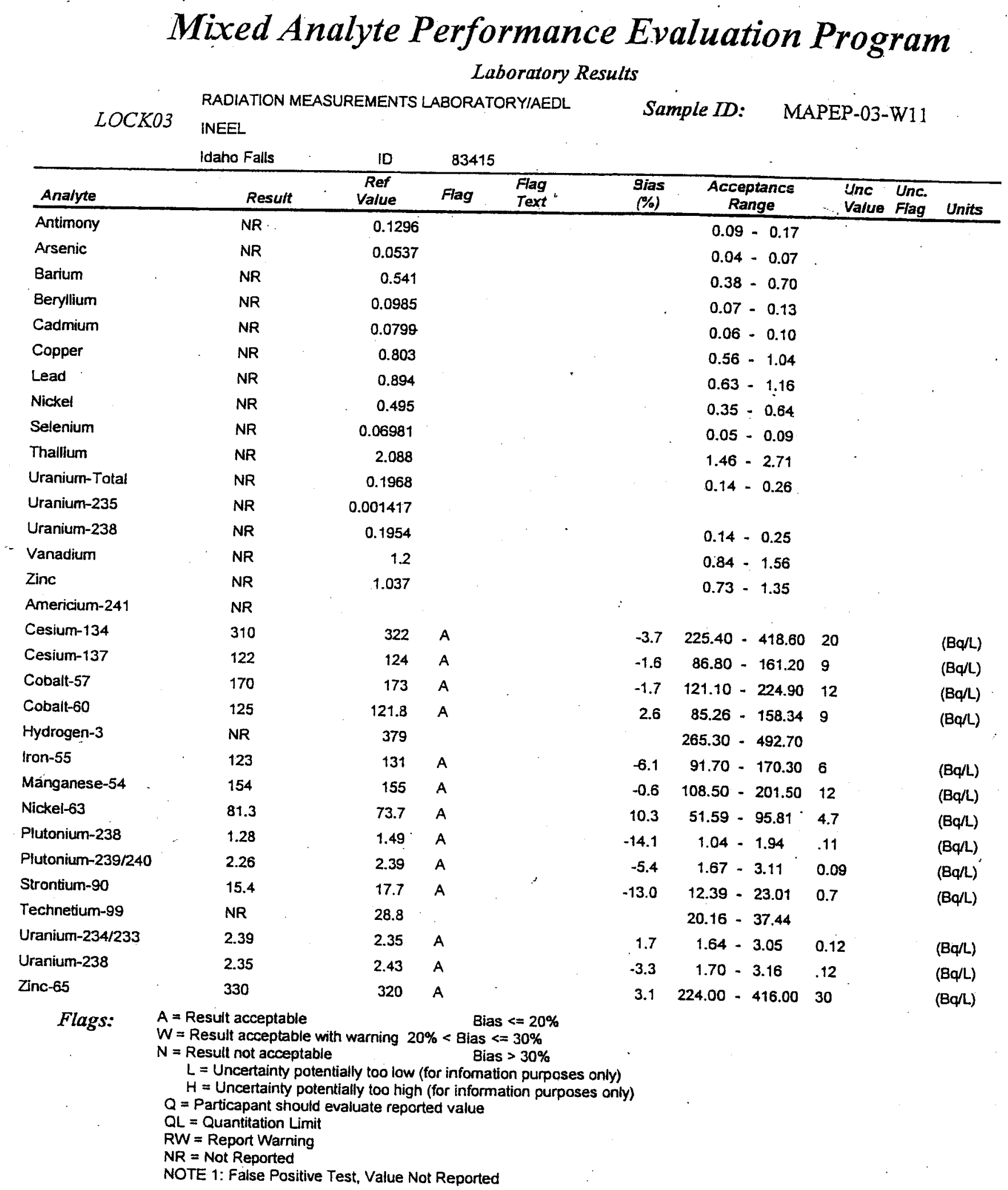




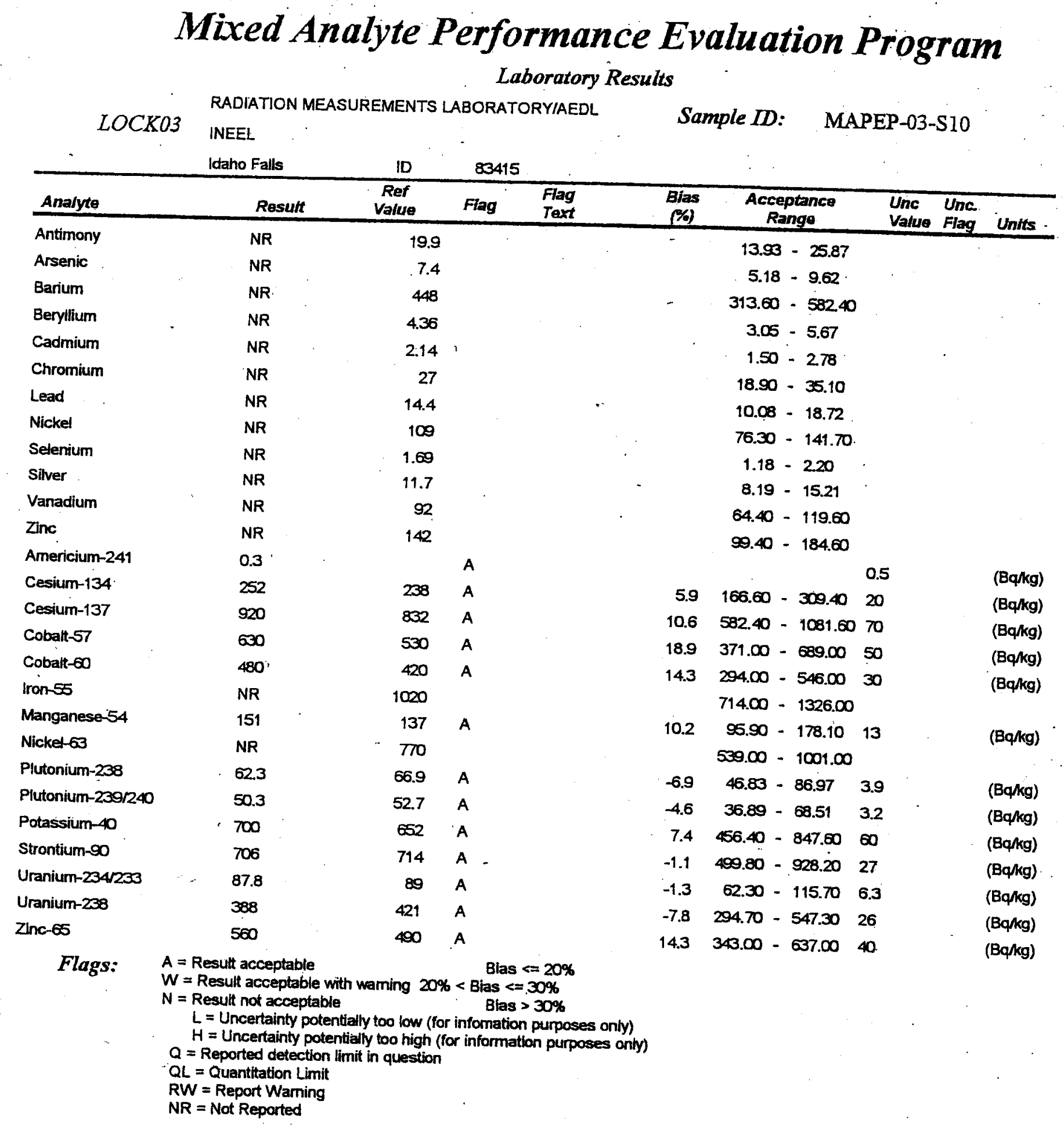




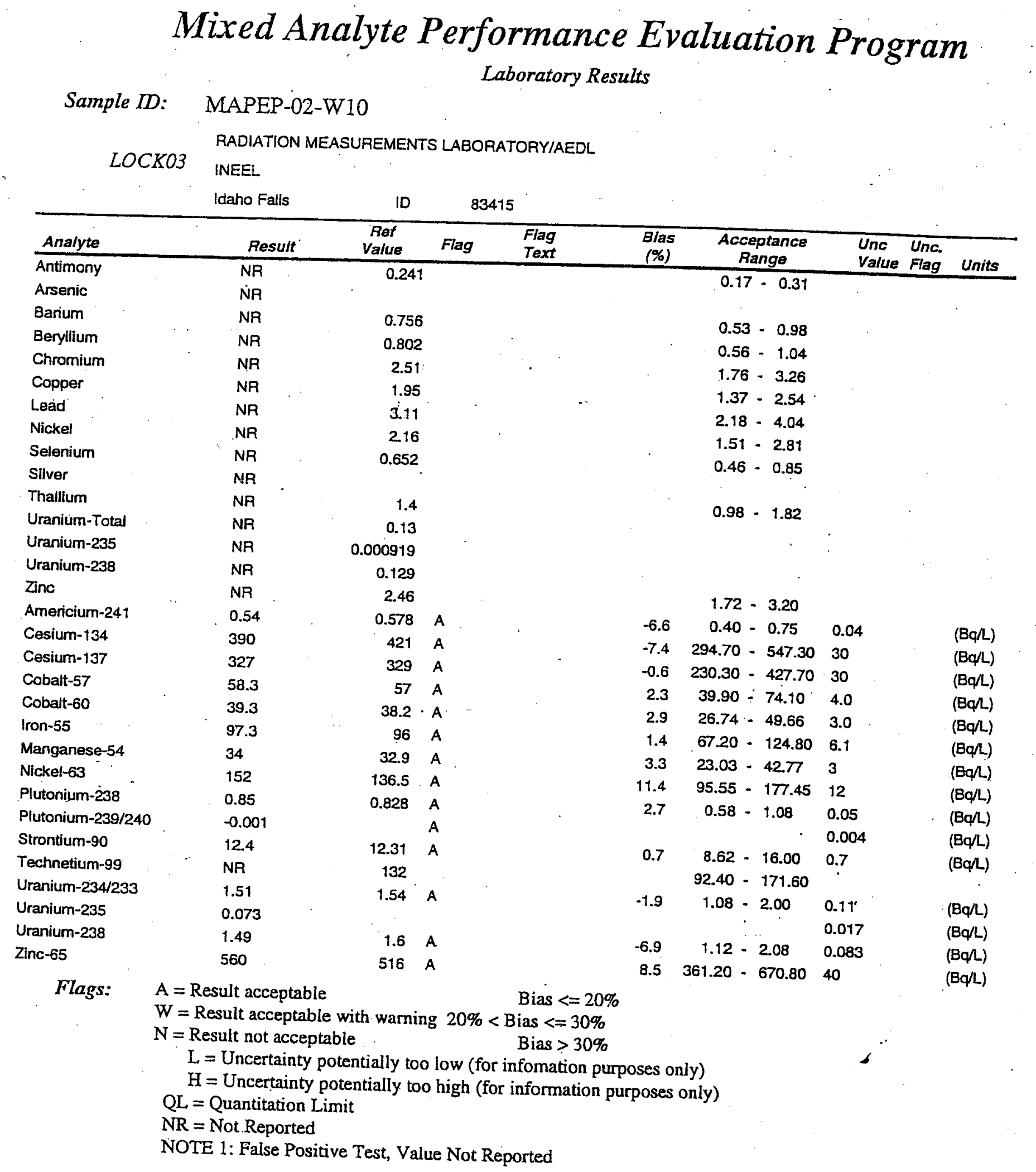




\section{Mixed Analyte Performance Evaluation Program}

Laboratory Results

Sainple ID: MAPEP-02-S9

LOCKO3 INEEL

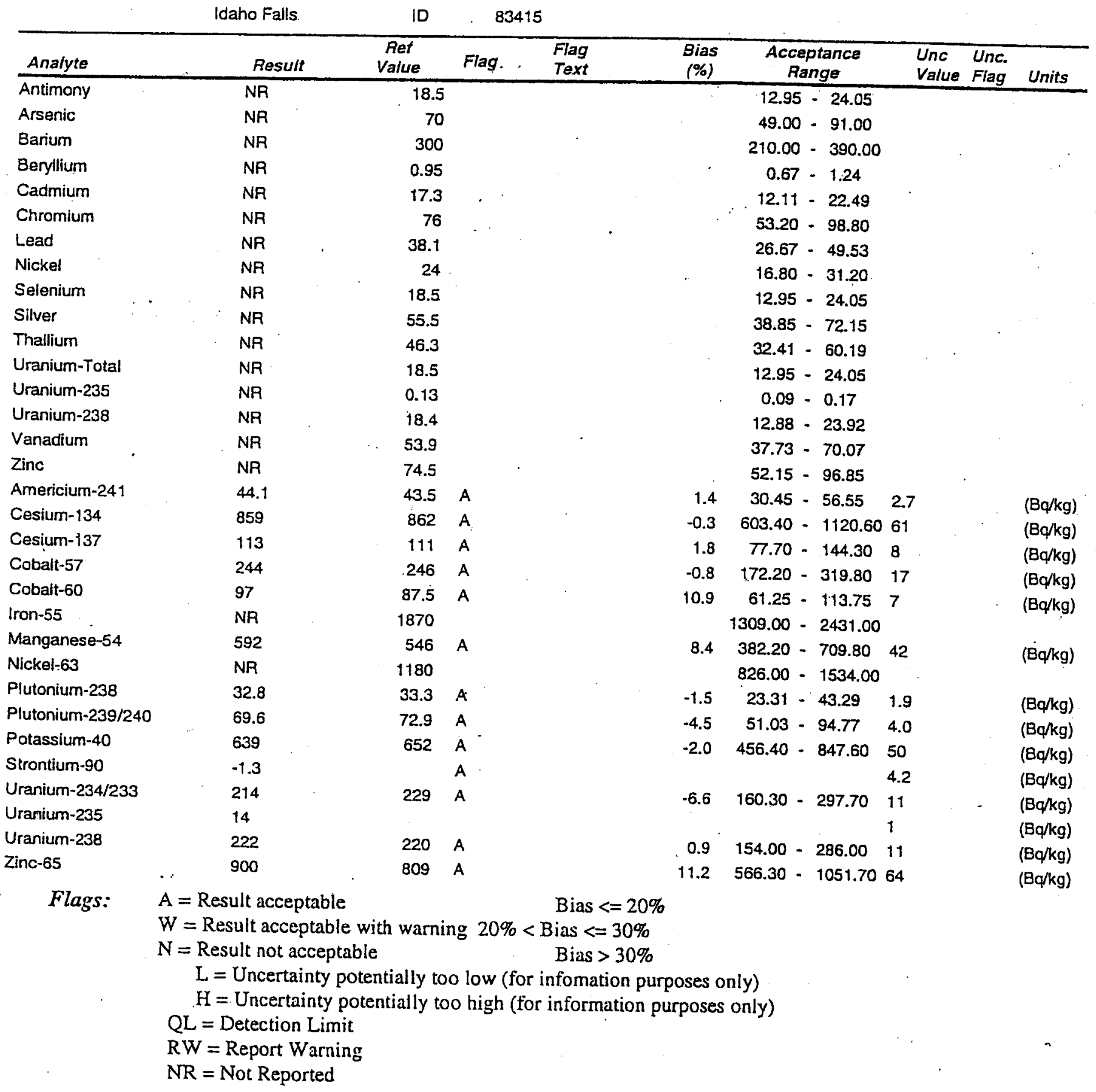


QAP 0303

EML-621

June 2003

\section{QAP 58 Results by Laboratory}

Lab: EG INEEL TRA Radioanalytical Laboratory, Scoville

\begin{tabular}{lcccccc} 
No. & $\begin{array}{c}\text { Reported } \\
\text { Test }\end{array}$ Radionuclide & $\begin{array}{c}\text { Reported } \\
\text { Value }\end{array}$ & EML & Value & EML & Reported \\
\hline
\end{tabular}

Matrix: $\mathrm{AI}$ Air Filter $\mathrm{Bq} /$ filter

\begin{tabular}{|c|c|c|c|c|c|c|}
\hline $\mathrm{AM} 241$ & 0.282 & 0.017 & 0.340 & 0.040 & 0.829 & W \\
\hline $\mathrm{CO} 60$ & 38.000 & 1.000 & 33.500 & 0.870 & 1.134 & W \\
\hline CSI37 & 118.000 & 7.000 & 99.700 & 2.300 & 1.184 & W \\
\hline MN54 & 53.000 & 1.000 & 43.800 & 1.130 & 1.210 & W \\
\hline PU238 & 0.536 & 0.027 & 0.520 & 0.010 & 1.031 & A \\
\hline PU239 & 0.345 & 0.018 & 0.330 & 0.010 & 1.045 & A \\
\hline U234 & 0.221 & 0.014 & 0.240 & 0.003 & 0.921 & $A$ \\
\hline U238 & 0.224 & 0.014 & 0.240 & 0.010 & 0.933 & A \\
\hline
\end{tabular}

Matrix: SO Soil $\mathrm{Bq} / \mathrm{kg}$

$\begin{array}{ll}1 & \mathrm{AC} 228 \\ 1 & \mathrm{~B} 1212 \\ 1 & \mathrm{~B} 1214 \\ 1 & \mathrm{CS} 137 \\ 1 & \mathrm{~K} 40 \\ 1 & \mathrm{~PB} 212 \\ 1 & \mathrm{~PB} 214 \\ 1 & \mathrm{TH} 234\end{array}$

$\begin{array}{rrrr}68.000 & 7.000 & 57.600 & 2.500 \\ 99.000 & 9.000 & 60.600 & 4.000 \\ 77.000 & 5.000 & 67.000 & 2.300 \\ 1672.000 & 11.000 & 1450.000 & 73.000 \\ 688.000 & 48.000 & 636.000 & 33.000 \\ 81.000 & 5.000 & 57.900 & 2.900 \\ 83.000 & 5.000 & 71.100 & 2.300 \\ 224.000 & 21.000 & 127.000 & 7.100\end{array}$

1.181

1.634

1.149

1.153

L.082

1.399

1.167

1.764

$\begin{array}{ll}\text { A } & \text { W } \\ \text { N } & \text { A } \\ \text { A } & \text { A } \\ \text { A } & \text { A } \\ \text { A } & \text { A } \\ \text { N } & \text { A } \\ \text { A } & \text { A } \\ \text { W } & \text { A }\end{array}$

Matrix: VE Vegetation $\mathrm{Bq} / \mathrm{kg}$

$$
\begin{array}{ll}
1 & \mathrm{CO60} \\
1 & \mathrm{CS} 137 \\
1 & \mathrm{~K} 40
\end{array}
$$

10.500
435.000
1160.000

$\begin{array}{rr}3.000 & 12.100 \\ 8.000 & 444.000 \\ 80.000 & 1120.000\end{array}$

0.700
22.000
60.000

0.868
0.980
1.036

$\begin{array}{ll}\text { W } & \text { W } \\ \text { A } & \text { A } \\ \text { A } & \text { A }\end{array}$

Matrix: WA Water $\mathrm{Bq} / \mathrm{L}$

$\begin{array}{ll}1 & \text { AM24l } \\ 1 & \text { CO60 } \\ 1 & \text { CS134 } \\ 1 & \text { CS137 } \\ 1 & \text { Gross Alpha } \\ 1 & \text { Gross Beta } \\ 1 & \text { PU238 } \\ 1 & \text { PU239 } \\ 1 & \text { U234 } \\ 1 & \text { U238 }\end{array}$

$$
\begin{array}{r}
2.260 \\
240.000 \\
30.000 \\
64.000 \\
247.000 \\
784.000 \\
3.870 \\
4.570 \\
2.050 \\
2.040
\end{array}
$$

0.120
30.000
1.000
2.000
20.000
55.000
0.200
0.230
0.110
0.150

2.130
234.000
30.500
63.800
377.500
627.500
3.330
3.920
2.050
2.160

0.150
8.400
1.090
3.400
10.000
10.000
0.300
0.300
0.190
0.210

1.061
1.026
0.984
1.003
0.654
1.249
1.162
1.166
1.000
0.944

$\begin{array}{ll}\text { A } & \text { A } \\ \text { A } & \text { A } \\ \text { A } & \text { A } \\ \text { A } & \text { A } \\ \text { W } & \text { A } \\ \text { A } & \text { A } \\ \text { W } & \text { W } \\ \text { W } & \text { W } \\ \text { A } & \text { A } \\ \text { A } & \text { A }\end{array}$

Values for elemental uranium are reported in $\mu \mathrm{g} /$ filter, $g$, or $\mathrm{mL} . \quad \mathrm{pCi} / \mathrm{g}$ or $\mathrm{mL}=\mathrm{Bq} \times 0.027$
Evatuation: $A=$ Acceptable, $W=A c c e p t a b l e$ with $W$ arning, $N=$ Not Acceptable
if the evaluation system is not appropriate for the types of analyses performed in your lab. apply a site specific evaluation. 
QAP 0209

EML-618

December 2002

\section{QAP 57 Results by Laboratory}

Lab: EG INEEL TRA Radioanalytical Laboratory, Scoville

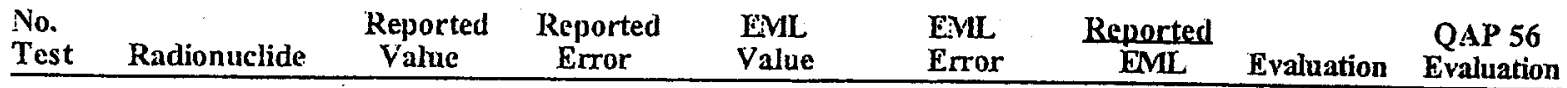

Matrix: AI Air Filter $\mathrm{Bq} /$ filter

$\begin{array}{llrlrrrrrr}1 & \text { AM241 } & 0.195 & 0.014 & 0.191 & 0.004 & 1.023 & \text { A } & \text { A } \\ 1 & \text { CO60 } & 25.000 & 2.000 & 23.000 & 0.059 & 1.087 & \text { A } & \text { A } \\ 1 & \text { CS137 } & 36.000 & 3.000 & 32.500 & 0.777 & 1.108 & \text { A } & \text { A } \\ 1 & \text { MN54 } & 60.000 & 5.000 & 52.200 & 1.170 & 1.149 & \text { A } & \text { A } \\ 1 & \text { PU238 } & 0.097 & 0.005 & 0.119 & 0.003 & 0.817 & \text { W } & \text { A } \\ 1 & \text { PU239 } & 0.183 & 0.009 & 0.206 & 0.002 & 0.889 & \text { A } & \text { A } \\ 1 & \text { SR90 } & 5.740 & 0.290 & 5.561 & 0.119 & 1.032 & \text { A } & \text { A } \\ 1 & \text { U234 } & 0.218 & 0.009 & 0.228 & 0.006 & 0.958 & \text { A } & \text { A } \\ 1 & \text { U238 } & 0.215 & 0.009 & 0.230 & 0.006 & 0.935 & \text { A } & \text { A }\end{array}$

Matrix: SO Soil Bq $/ \mathrm{kg}$

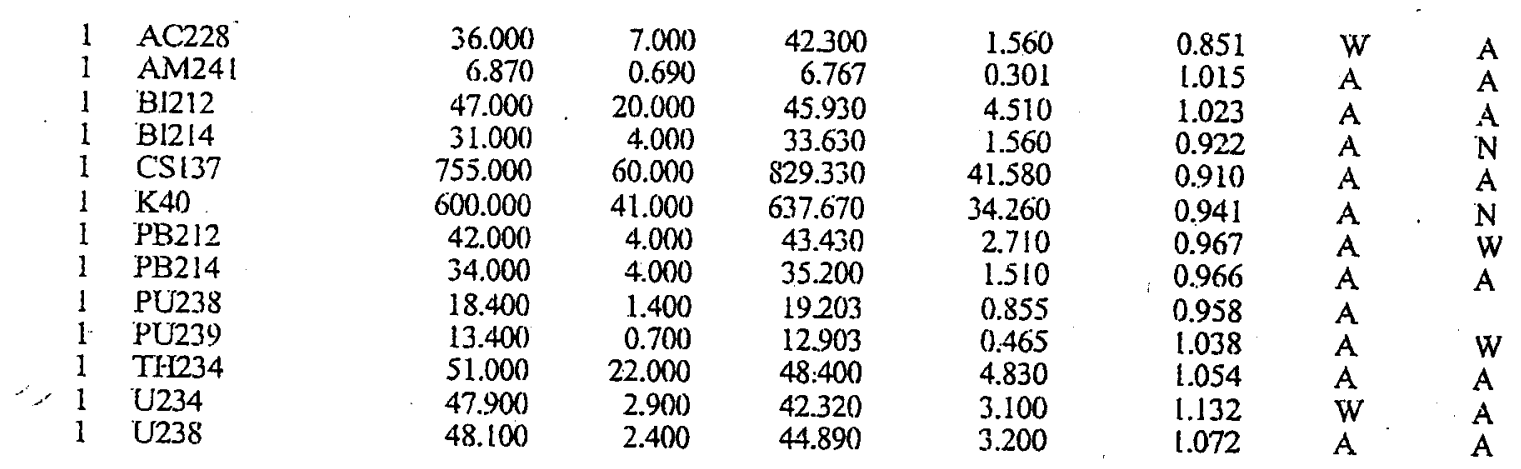

Matrix: VE Vegetation $\mathrm{Bq} / \mathrm{kg}$

$\begin{array}{ll}1 & \mathrm{CO} 60 \\ 1 & \mathrm{CS} 137 \\ 1 & \mathrm{~K} 40\end{array}$

12.000
295.000
1346.000

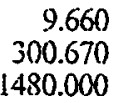

140.000
0.630
15.250

77.800
1.242
0.981
0.909

$\begin{array}{ll}\text { W } & \text { W } \\ \text { A } & \text { A } \\ \text { A } & \text { W }\end{array}$

Matrix: WA Water $\mathrm{Bq} / \mathrm{L}$

$\begin{array}{ll}1 & \text { AM241 } \\ 1 & \text { CO60 } \\ 1 & \text { CS134 } \\ 1 & \text { CS137 } \\ 1 & \text { Gross Alpha } \\ 1 & \text { Gross Beta } \\ 1 & \text { PU238 } \\ 1 & \text { PU239 } \\ 1 & \text { SR90 } \\ 1 & \text { U234 } \\ 1 & \text { U238 }\end{array}$

$\begin{array}{rrr}3.090 & 0.220 & 3.043 \\ 263.000 & 20.000 & 268.670 \\ 57.000 & 4.000 & 60.200 \\ 77.000 & 6.000 & 81.430 \\ 221.000 & 28.000 & 210.000 \\ 909.000 & 91.000 & 900.000 \\ 3.710 & 0.170 & 4.331 \\ 1.840 & 0.080 & 2.070 \\ 7.900 & 0.600 & 8.690 \\ 3.050 & 0.150 & 3.323 \\ 3.120 & 0.140 & 3.370\end{array}$

0.082
9.710
1.860
4.280
21.000
90.000
0.117
0.074
0.420
0.114
0.140

1.015
0.979
0.947
0.946
1.052
1.010
0.857
0.889
0.909
0.918
0.926

$\begin{array}{cc}\text { A } & \text { A } \\ \text { A } & \text { A } \\ \text { A } & \text { W } \\ \text { A } & \text { A } \\ \text { A } & \text { A } \\ \text { A } & \text { A } \\ \text { W } & \text { A } \\ \text { W } & \text { A } \\ \text { A } & \text { A } \\ \text { A } & \text { A } \\ \text { A } & \text { A }\end{array}$

\section{Values for elemental uranium are reported in $\mu \mathrm{g} /$ filter, $\mathrm{g}$, or $\mathrm{mL}$.}

$\mathrm{pCi} / \mathrm{g}$ or $\mathrm{mL}=\mathrm{Bq} \times 0.027$

Evaluation: $A=$ Acceptable, $W=$ Acceptable with Warning, $N=$ Not Acceptable

If the evaluation system is not appropriate for the types of analyses performed in your lab, apply a site specific evaluation. 
QAP 0203

EML-617

June 2002

\section{QAP 56 Results by Laboratory}

Lab: EG INEEL TRA Radioanalytical Laboratory, Scoville

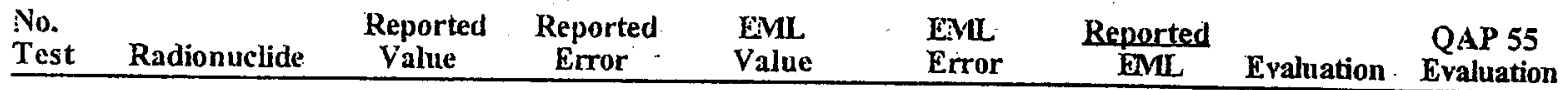

Matrix: AI Air Filter Bq/filter

\begin{tabular}{|c|c|c|c|c|c|c|}
\hline $\mathrm{AM} 241$ & 0.092 & 0.007 & 0.088 & 0.005 & 1.042 & A \\
\hline $\mathrm{CO} 060$ & 33.300 & 2.000 & 30.520 & 0.652 & 1.091 & A \\
\hline $\mathrm{CS} 137$ & 31.000 & 2.000 & 28.230 & 0.701 & 1.098 & A \\
\hline MN54 & 43.700 & 3.000 & 38.530 & 0.867 & 1.134 & A \\
\hline PU238 & 0.063 & 0.005 & 0.057 & 0.001 & 1.097 & A \\
\hline PU239 & 0.208 & 0.013 & 0.187 & 0.003 & 1.110 & A \\
\hline SR90 & 4.080 & 0.130 & 4.832 & 0.184 & 0.844 & A \\
\hline U234 & 0.289 & 0.020 & 0.297 & 0.004 & 0.972 & A \\
\hline U238 & 0.294 & 0.020 & 0.298 & 0.004 & 0.986 & A \\
\hline
\end{tabular}

Matrix: $\mathrm{SO}$ Soil $\mathrm{Bq} / \mathrm{kg}$

$\begin{array}{ll}1 & \text { AC228 } \\ 1 & \text { AM241 } \\ 1 & B 1212 \\ 1 & B 1214 \\ 1 & C S 137 \\ 1 & \text { K410 } \\ 1 & \text { PB212 } \\ 1 & \text { PB214 } \\ 1 & \text { PU239 } \\ 1 & \text { SR90 } \\ 1 & \text { TH234 } \\ 1 & \text { U234 } \\ 1 & \text { U238 }\end{array}$

$\begin{array}{rr}58.000 & 10.000 \\ 11.400 & 0.900 \\ 61.000 & 24.000 \\ 39.000 & 7.000 \\ 1370.000 & 100.000 \\ 490.000 & 80.000 \\ 62.000 & 6.000 \\ 65.000 & 8.000 \\ 22.400 & 1.900 \\ 51.800 & 2.800 \\ 124.000 & 26.000 \\ 84.200 & 5.300 \\ 86.900 & 5.600\end{array}$

$\begin{array}{rr}51.167 & 1.941 \\ 10.927 & 0.373 \\ 53.430 & 5.215 \\ 53.933 & 2.249 \\ 1326.670 & 66.510 \\ 621.670 & 33.860 \\ 51.100 & 2.753 \\ 54.367 & 2.249 \\ 19.098 & 0.706 \\ 53.756 & 1.446 \\ 89.300 & 6.837 \\ 93.885 & 7.767 \\ 96.778 & 8.410\end{array}$

$\begin{array}{ll}1.134 & A \\ 1.043 & A \\ 1.142 & A \\ 0.723 & N \\ 1.033 & A \\ 0.788 & N \\ 1.213 & \text { W } \\ 1.196 & A \\ 1.173 & \text { D } \\ 0.964 & A \\ 1.389 & A \\ 0.897 & A \\ 0.898 & A\end{array}$

$\begin{array}{ll}A & \text { W } \\ \text { A } & \\ \text { A } & A \\ N & A \\ A & A \\ N & A \\ \text { W } & A \\ A & \text { W } \\ \text { W } & \\ \text { A } & \\ \text { A } & \text { A } \\ \text { A } & \\ \text { A } & \end{array}$

Matuix: VE Vegetation $\mathrm{Bq} / \mathrm{kg}$

$\begin{array}{ll}1 & \text { AM241 } \\ 1 & C M 244 \\ 1 & C O 60 \\ 1 & C S 137 \\ 1 & \text { K40 } \\ 1 & \text { PU238 } \\ 1 & \text { PU239 } \\ 1 & \text { SR90 }\end{array}$

2.400
1.300
9.100
317.000
740.000
0.250
3.540
520.000

0.180
0.110
1.900
25.000
100.000
0.040
0.250
17.000

2.228
1.320
11.230
313.667
864.330
0.257
3.543
586.280

0.216
0.164
0.677
15.910
47.220
0.046
0.377
11.140

1.077
0.985
0.810
1.011
0.856
0.974
0.999
0.887

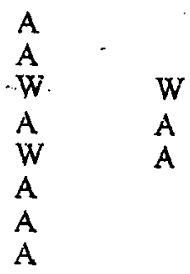

Matrix: WA Water $\mathrm{Bq} / \mathrm{L}$

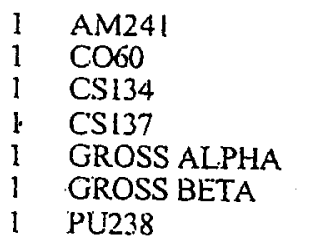

$$
\begin{array}{r}
1.570 \\
360.000 \\
3.000 \\
57.000 \\
360.000 \\
1080.000 \\
0.480
\end{array}
$$

$$
\begin{array}{r}
1.474 \\
347.330 \\
3.357 \\
56.067 \\
375.000 \\
1030.000 \\
0.490
\end{array}
$$

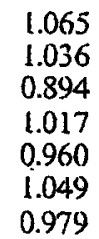

$\begin{array}{ll}\text { A } & \text { A } \\ \text { A } & \text { A } \\ \text { W } & \\ \text { A } & \text { A } \\ \text { A } & \text { A } \\ \text { A } & \text { A } \\ \text { A } & \text { A }\end{array}$

\footnotetext{
Values for elemental uranium are reported in $\mu \mathrm{g} /$ filter, $g$, or $\mathrm{mL}$. 
QAP 0203

EML-617.

June 2002

\section{QAP 56 Results by Laboratory}

Lab: EG INEEL TRA Radioanalytical Laboratory, Scoville

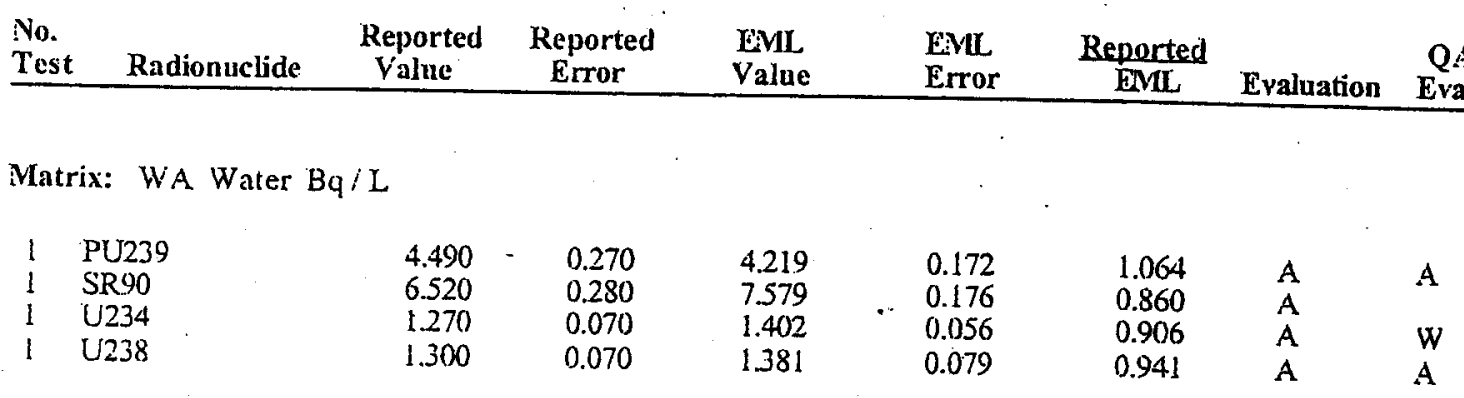

\footnotetext{
Values for elemental uranium are reported in $\mu \mathrm{g} /$ filter, $g$, or $\mathrm{mL}$.

Evaluation: $A=A c c e p t a b l e, W=A c c e p t a b l e$ with $W$ arning, $N=$ Not Acceptable

$\mathrm{pCi} / \mathrm{g}$ or $\mathrm{mL}=\mathrm{Bq} \times 0.027$

If the evaluation system is not appropriate for the types of analyses perfor
} 


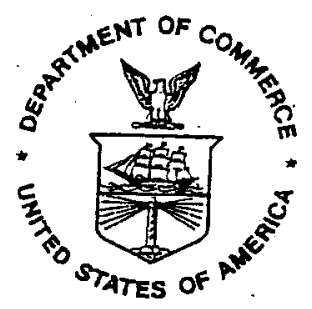

U.S. DEPARTMENT OF COMMERCE

National Institute of Standards and Technology

Gaithersburg, MD

\title{
REPORT OF TRACEABILITY
}

\author{
Bechtel BWXT Idaho, LLC \\ Idaho Falls, Idaho
}

Test Identification

NRIP02-SS

Matrix Description

Test Activity Range

Reference Time

${ }^{241} \mathrm{Am},{ }^{238} \mathrm{Pu},{ }^{230} \mathrm{Th},{ }^{90} \mathrm{Sr},{ }^{238} \mathrm{U}$ in Soil ${ }^{1}$

$30 \mathrm{mBq} \cdot$ sample $\mathrm{e}^{-1}$ to $300 \mathrm{mBq} \cdot \mathrm{sample}^{-1}$

12:00 EST, April 1; 2002

Measurement Results

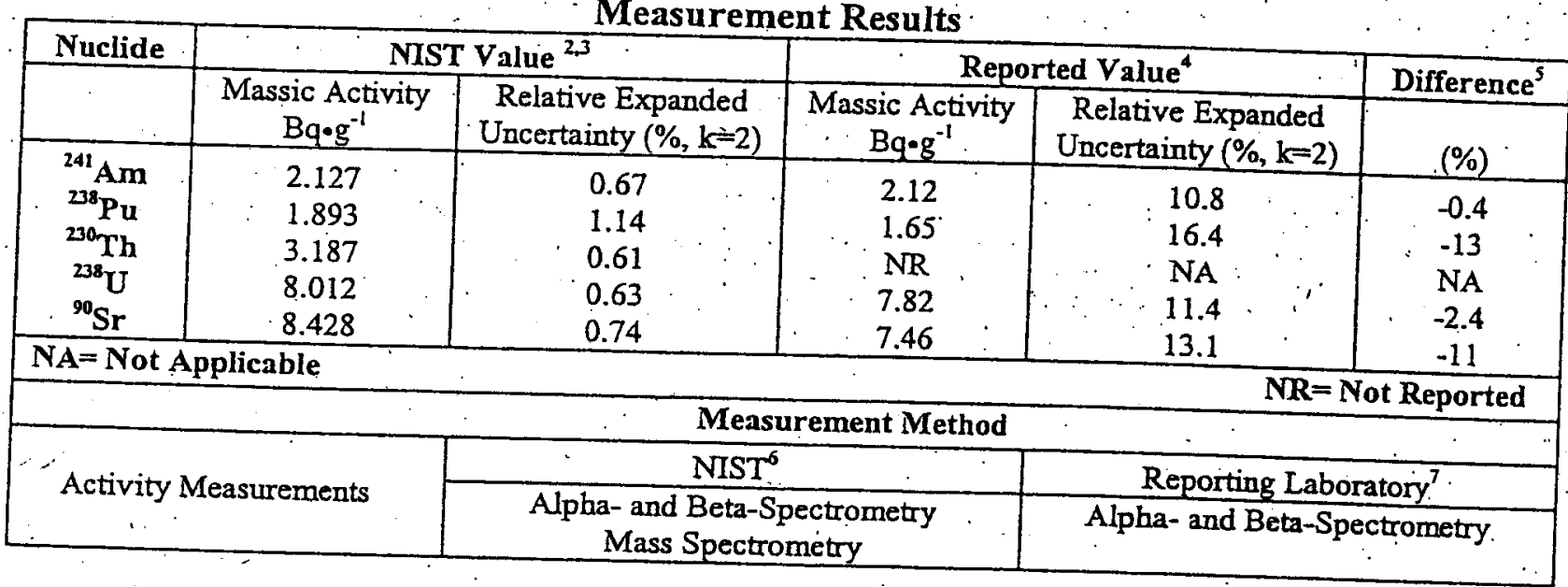

Evaluation (per ANSI N42.22)

\begin{tabular}{|c|c|c|}
\hline Nuclide & \multicolumn{2}{|c|}{ N42.22 } \\
\hline & $\begin{array}{c}\text { ANSI N42.22 } \\
\text { Traceable }\end{array}$ & $\begin{array}{c}\text { Traceability } \\
\text { Limit } \\
\text { ( } \pm \text { Percent) }\end{array}$ \\
\hline${ }^{241} \mathrm{Am}$ & Yes & 16 \\
${ }^{238} \mathrm{Pu}$ & Yes & 22 \\
${ }^{230} \mathrm{Th}$ & NA & NA \\
${ }^{238} \mathrm{U}$ & Yes & 17 \\
${ }^{90} \mathrm{Sr}$ & Yes & 17 \\
\hline
\end{tabular}

Samples Distributed

Reporting Data Received
May 17,2002

August 19, 2002
For the Director

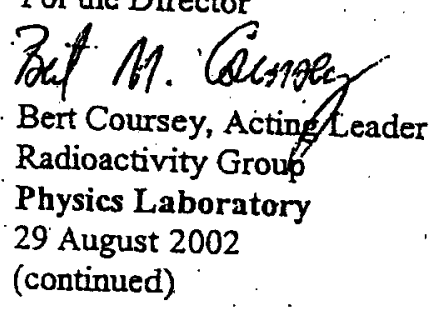




\section{U.S. DEPARTMENT OF COMMERCE \\ National Institute of Standards and Technology \\ Gaithersburg, MD}

\section{REPORT OF TRACEABILITY}

\section{Lockheed Martin Idaho Technologies Company Idaho Falls, Idaho}

Test Identification

Matrix Description

Test Activity Range

Reference Time
NRIP02-GF

${ }^{241} \mathrm{Am},{ }^{238} \mathrm{Pu},{ }^{90} \mathrm{Sr},{ }^{238} \mathrm{U},{ }^{230} \mathrm{Th}$ on Glass-Fiber Filters ${ }^{1}$

$30 \mathrm{mBq}-$ sample $^{-1}$ to $300 \mathrm{mBq} \cdot$ sample $^{-1}$

12:00 EST, April 1, 2002

\section{Measurement Results}

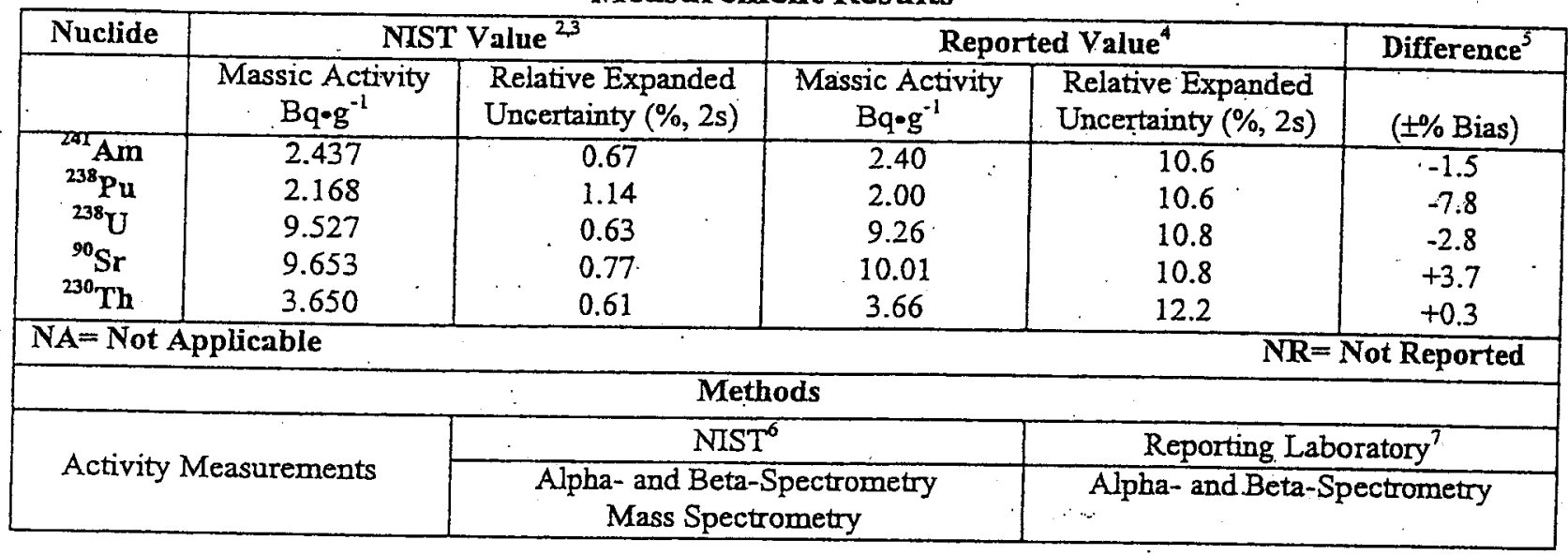

Evaluation (per ANSI N42.22)

\begin{tabular}{|c|c|c|}
\hline Nuclide & \multicolumn{2}{|c|}{ N42.22 $^{8}$} \\
\hline & $\begin{array}{c}\text { ANSI N42.22 } \\
\text { Traceable }\end{array}$ & $\begin{array}{c}\text { Traceability } \\
\text { Limit } \\
\text { (†Percent) }\end{array}$ \\
\hline${ }^{241} \mathrm{Am}$ & Yes & 16 \\
${ }^{238} \mathrm{Pu}$ & Yes & 15 \\
${ }^{238} \mathrm{U}$ & Yes & 16 \\
${ }^{90} \mathrm{Sr}$ & Yes & 17 \\
${ }^{230} \mathrm{Th}$ & Yes & 18 \\
\hline
\end{tabular}

Samples Distributed 8 March 2002

Reporting Data Received 16 May 2002
For the Director

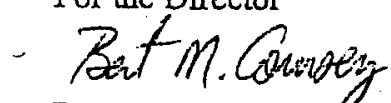

Bert M.Coursey, Ageng Leader

Radioactivity Group

Physics Laboratory

19 June 2002

(continued) 